\title{
RADIATIVE TRANSFER IN STELLAR ATMOSPHERES
}

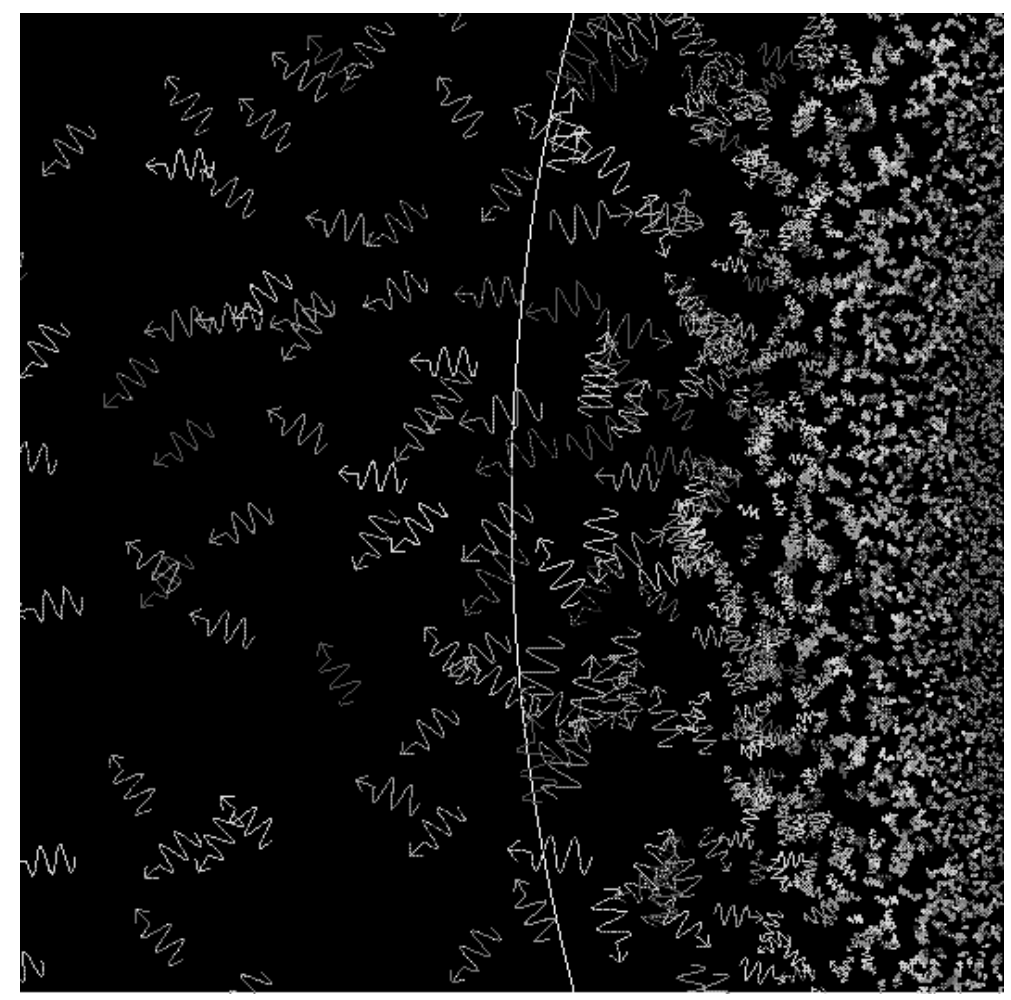

R.J. Rutten

Sterrekundig Instituut Utrecht Institute of Theoretical Astrophysics Oslo May 8, 2003

$$
\text { WWHE }
$$


Copyright (c) 1995 Robert J. Rutten, Sterrekundig Instuut Utrecht, The Netherlands.

Copying permitted for non-commercial educational purposes only. R.J. Rutten asserts the moral right to be identified as the author of these notes. In no way can he be held responsible for any liability with respect to these notes.

First Utrecht edition: March 7, 1995. Based on "Stellar Atmospheres" lecture notes by C. Zwaan. First WWW edition: June 1, 1995 for the 1995 Oslo Summer School. Figures scanned by Sake Hogeveen. Corrections from Louis Strous, Bart-Jan van Tent, Guus Oonincx, Dan Kiselman, Mats Carlsson.

Second WWW edition: June 22, 1995. Corrections from Carine Briand, Kees Dullemond, Martijn Smit.

Third edition: March 11, 1996. Corrections from Ferdi Hulleman. Section "Exercises" started.

Fourth $W W W$ edition: October 1, 1997. Corrections from Hans Akkerman, Thijs Krijger, Nils Ryde, Bob Stein.

Fourth SIU edition: January 6, 1998. Corrections from Oliver Ryan; new figures from Thijs Krijger.

Fifth SIU/WWW edition: January 4, 1999. Corrections from Mark Gieles, Jorrit Wiersma, Marc van der Sluys, Niels Zagers.

Sixth edition: May 20, 1999, for the ESMN Summer School at Oslo. Corrections from Wouter Bergmann Tiest.

Seventh edition: December 1, 2000. Corrections from Karin Jonsell, Torgny Karlsson, Hans van Rijn, Louis Strous.

Eighth edition: May 8, 2003. Corrections from Else van den Besselaar, Jacqueline Mout, Jelle de Plaa, Remco Scheepmaker.

These lecture notes are freely available as a service of the European Solar Magnetism Network (http://esmn. astro.uu.nl). There are also corresponding equation viewgraphs for classroom display.

Details on how to get printable files are given at http://www.astro.uu.nl/ rutten.

These lecture notes still evolve; the WWW information contains an update on their status. Major renewals are announced to those who request to be put on the notification email list. Corrections and additions are very welcome; please send them to R. J.Rutten @ astro.uu.nl.

You are also most welcome to cite these lecture notes. Please do so as: Rutten, R.J., 2003, Radiative Transfer in Stellar Atmospheres, Utrecht University lecture notes, 8th edition.

Cover: a stellar atmosphere is where photons leave the star, a dramatic transition from warm dense comfort in near-thermal enclosure to bare isolation in the cold emptiness of space - sufficiently traumatic to make stellar atmospheres highly interesting to astrophysicists. On average, photons get scarcer, longer, and more outwards directed further out in the atmosphere until they escape. Copied from Mats Carlsson's poster for the 1995 Oslo "Intensive Summer School on Radiative Transfer and Radiation Hydrodynamics". 


\section{Contents}

Preface

Bibliographȳy, $\quad$ xvii

1. Brief History of Stellar Spectrometry

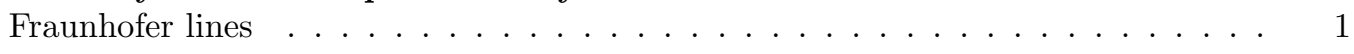

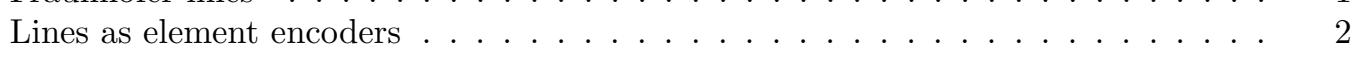

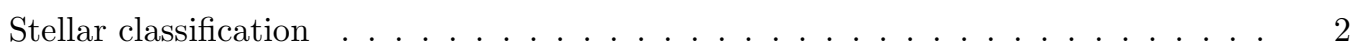

Abundance determination $\ldots \ldots \ldots \ldots \ldots \ldots$. . . . . . . . . . . . . . . . 4

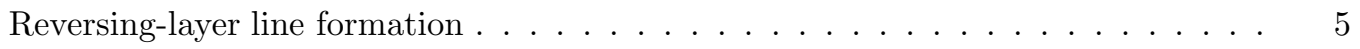

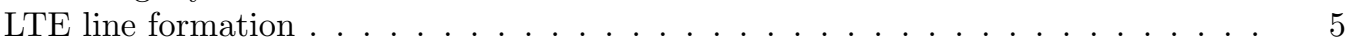

NLTE line formation . . . . . . . . . . . . . . . . . . . . 5

Numerical line formation $\ldots \ldots \ldots \ldots \ldots \ldots \ldots$

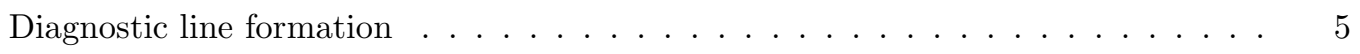

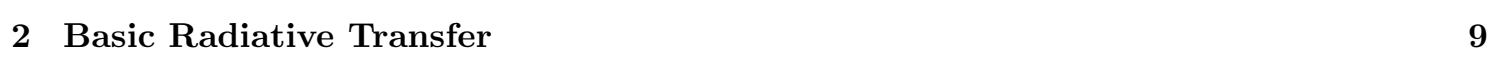

Radiation

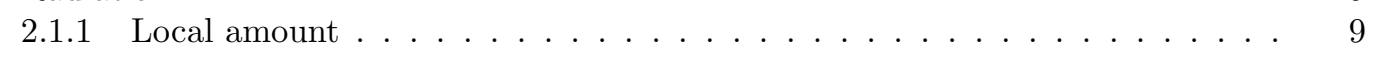

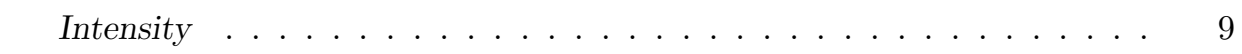

Mean intensityi . . . . . . . . . . . . . . . . . . 10

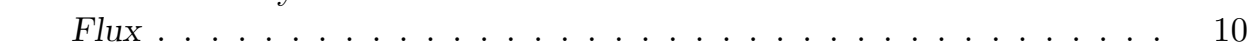

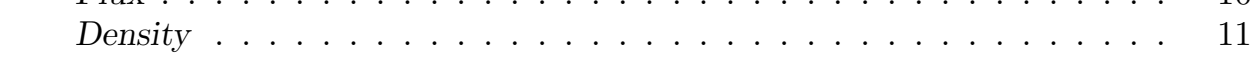

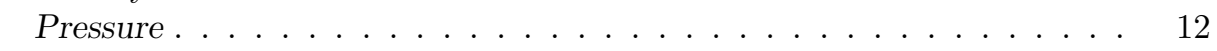

Moments of the intensity, . . . . . . . . . . . . . . . . . . . . . . 12

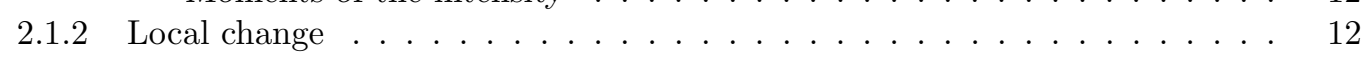

Emission . . . . . . . . . . . . . . . . . . 12

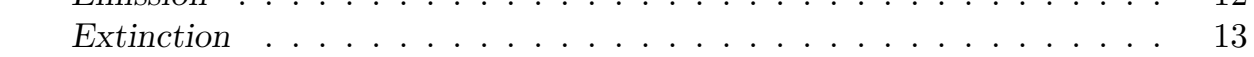

Source function . . . . . . . . . . . . . . . . . . . . 13

2.2 Transportequation

2.2 .1 Transport along a ray $\ldots \ldots \ldots \ldots$. . . . . . . . . . . . . . . . 14

Discussion

Optical length and thickness . . . . . . . . . . . . . . . . . 14

Homogeneous medium

2.2 .2 Transport through an atmosphere $\ldots \ldots \ldots \ldots \ldots . \ldots \ldots$

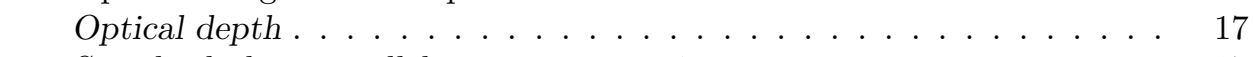

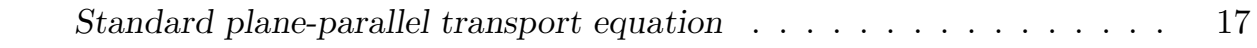

Formal solution

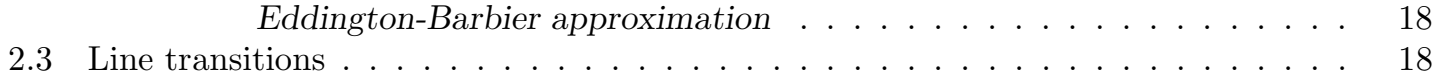

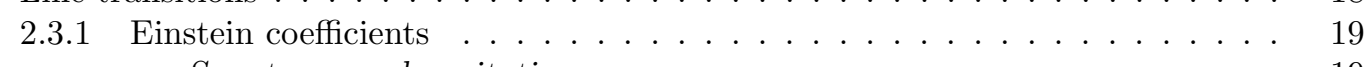

Spontaneous deexcitation! . . . . . . . . . . . . . . . . 19

Radiative excitation . . . . . . . . . . . . . . . . . . 21

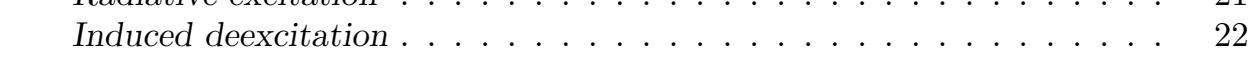




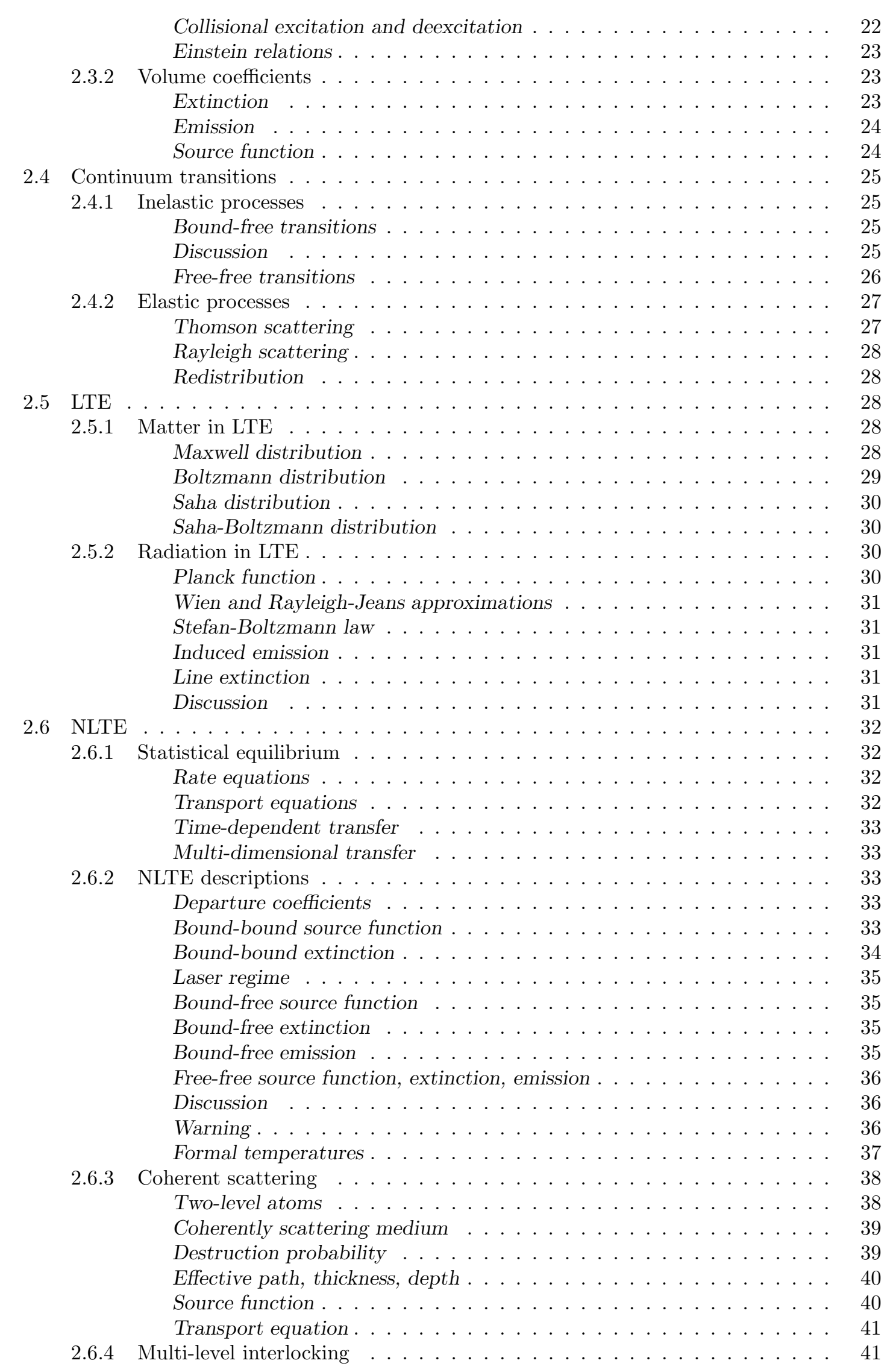




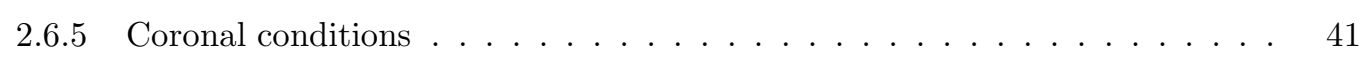

3 Bound-Bound and Bound-Free Transitions: 43

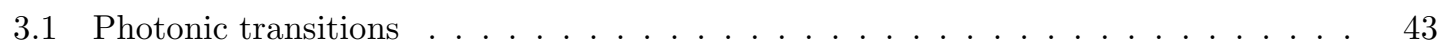

3.1 Atomic transitionsis . . . . . . . . . . . . . . . . . 43

3.1.2 Molecular transitions $\ldots \ldots \ldots \ldots \ldots \ldots \ldots$

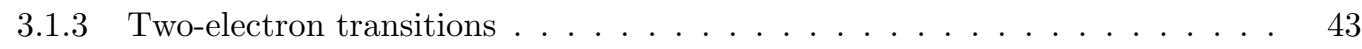

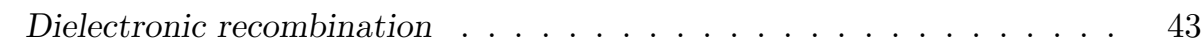

Autoionization $\ldots \ldots \ldots \ldots \ldots$. . . . . . . . . . 44

1 Bound-free resonances . . . . . . . . . . . . . . . . . . . 44

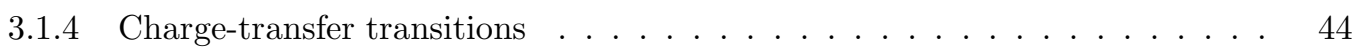

Transition rates $2 \ldots \ldots \ldots \ldots \ldots \ldots \ldots$

3.2 .1 Bound-bound radiative rates $\ldots \ldots \ldots \ldots \ldots \ldots 4 \ldots \ldots \ldots$

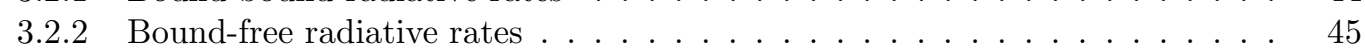

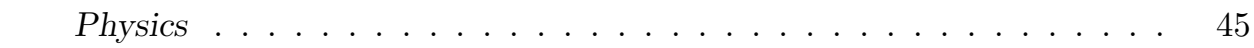

Einstein-Milne equations . . . . . . . . . . . . . . . . . . . 46

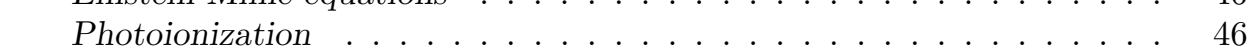

Spontaneous recombinationt . . . . . . . . . . . . . . . . . 46

Discussion . . . . . . . . . . . . . 47

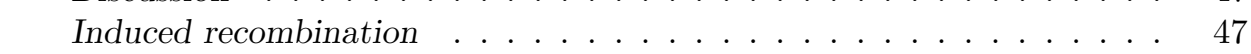

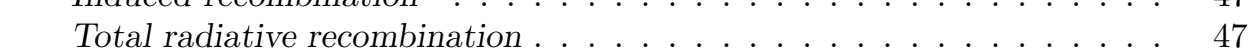

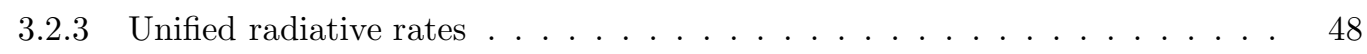

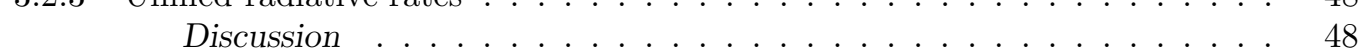

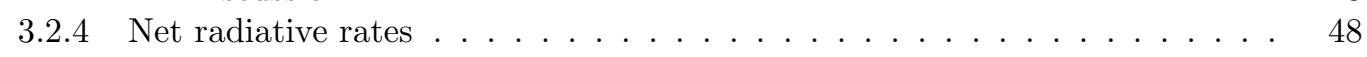

Net radiative recombination. . . . . . . . . . . . . . . . . . . . . 48

Net radiative deexcitation. . . . . . . . . . . . . . . . . . . 49

Discussion . . . . . . . . . . . . . . . . . . . 49

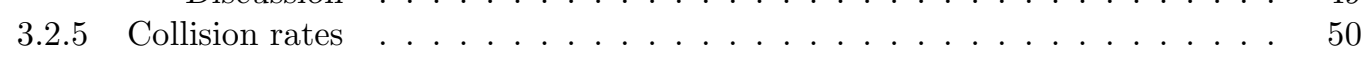

Net collision rates . . . . . . . . . . . . . . . . . . 51

Collisional coupling $\ldots \ldots \ldots \ldots$. . . . . . . . . . . . . . . . 52

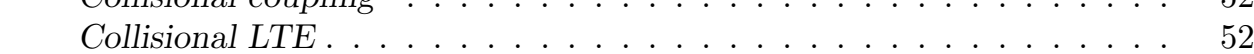

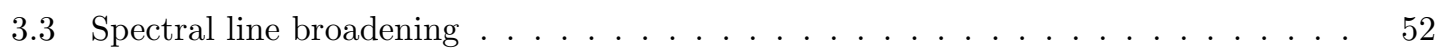

3.3.1 Radiation broadening $\ldots \ldots \ldots \ldots \ldots \ldots \ldots$

Damping profile .......................... 53

Extinction profile. . . . . . . . . . . . . . . . . . . . 53

Derivation $a_{1}$

Multiple levels and transitions $\ldots \ldots \ldots \ldots \ldots \ldots \ldots$

3.3 .2 Collision broadening $\ldots \ldots \ldots \ldots \ldots \ldots \ldots$

Elastic collisions $\ldots \ldots \ldots \ldots \ldots$. . . . . . . . . . . . . . . 54

Impact approximation $\ldots \ldots \ldots \ldots \ldots \ldots \ldots$

Quasi-static approximation . . . . . . . . . . . . . . . . . 55

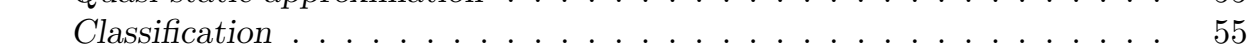

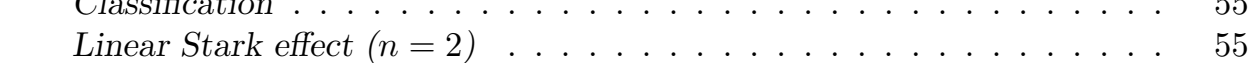

Resonance broadening $(n=3) \ldots \ldots \ldots \ldots \ldots$

Quadratic $\bar{S}$ tark effect $(n=4)$

Van der Waals broadening $(n=6) \ldots \ldots \ldots \ldots \ldots$

Van der Waals enhancement factor . . . . . . . . . . . . . 57

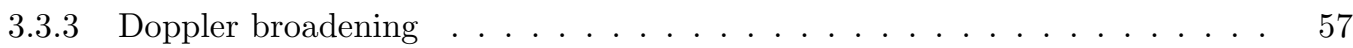

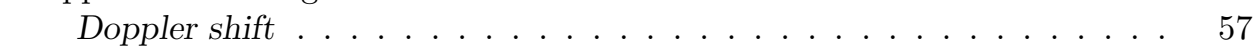

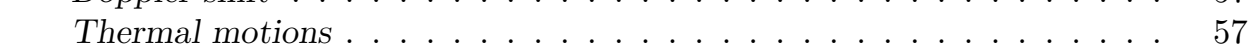

Thermal broadening . . . . . . . . . . . . . . . . . . 58

Voigtprofile

Rotational broadening . . . . . . . . . . . . . . . . . . . . 60

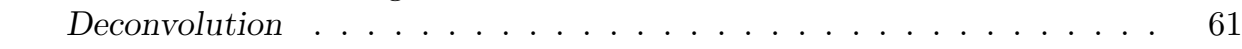


'Turbulent broadening . . . . . . . . . . . . . . . . . . . . . . . . . . . . . .

Warning . . . . . . . . . . . . . . . . 63

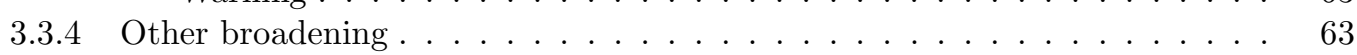

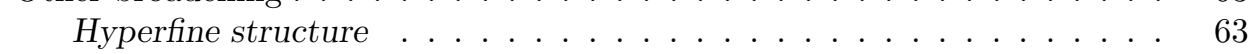

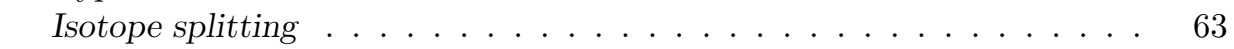

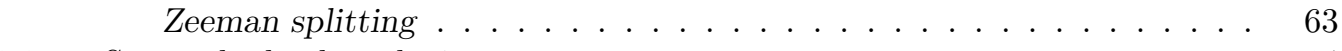

$3.3 .5-\frac{\text { Spectral edge broadening }}{1}$. . . . . . . . . . . . . . . . . . 64

\begin{tabular}{l}
3.4 \\
\hline
\end{tabular}

3.4.1 Monochromatic redistribution" . . . . . . . . . . . . . . . . . . . . . . . 64

Sharp-line atoms . . . . . . . . . . . . . . . . . . . . . . . . . . . . . . .

-

Transport equation $\ldots \ldots \ldots \ldots \ldots$

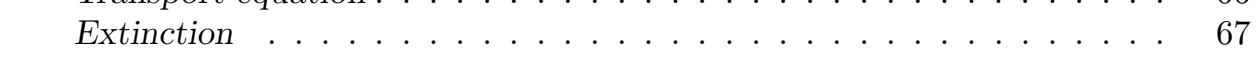

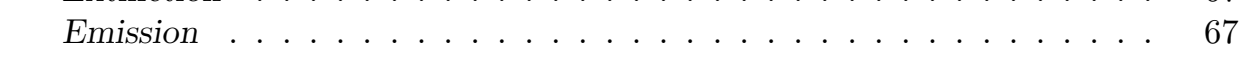

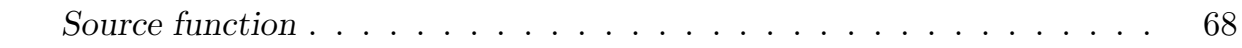

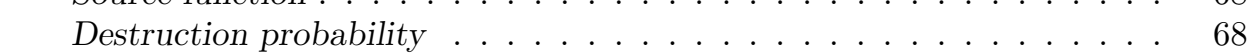

Discussion . . . . . . . . . . . . . . . . . . . . . . . . . . 69

Thomson and

3.4 .2 Completeredistribution

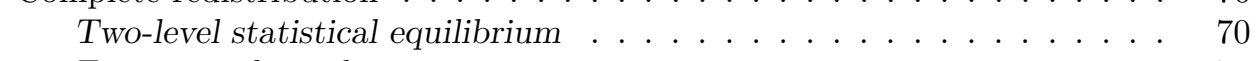

Frequency dependence . . . . . . . . . . . . . . . . . . . . . . . 71

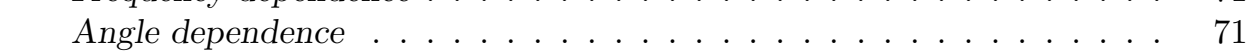

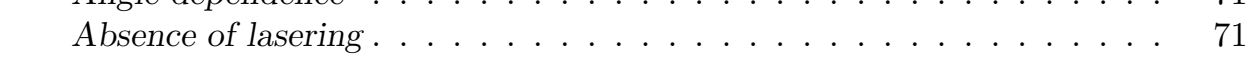

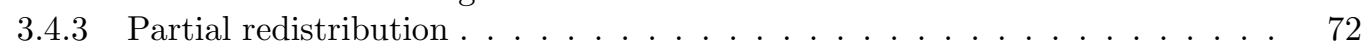

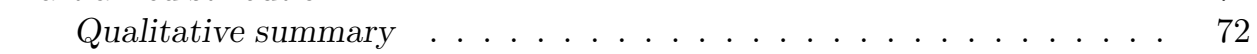

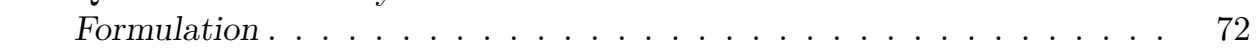

's.4.4 Angle redistribution . . . . . . . . . . . . . . . . . . . . . . . . 72

3.4.5 Spectral edge redistribution . . . . . . . . . . . . . . . . . . . . . . . 72

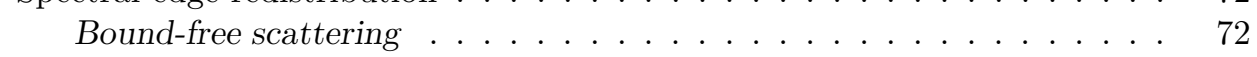

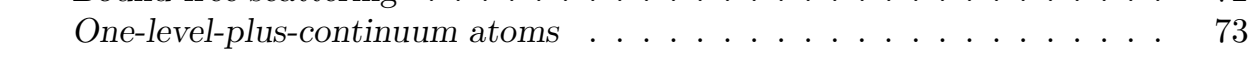

Discussion . . . . . . . . . . . . . . . . . . . 74

44 Analytical Radiative Transfer: $\quad 75$

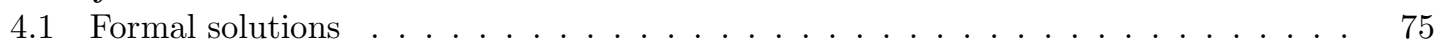

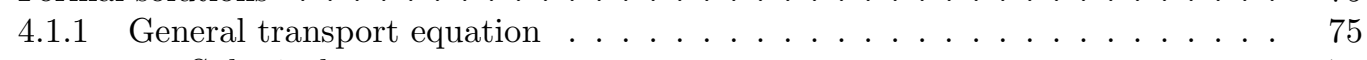

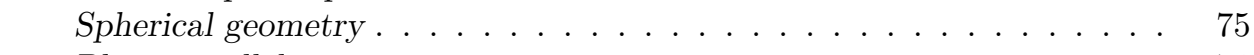

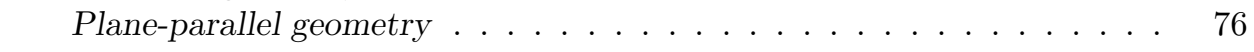

More transport equations . . . . . . . . . . . . . . . . . . . . . . . . . . . . . . . . .

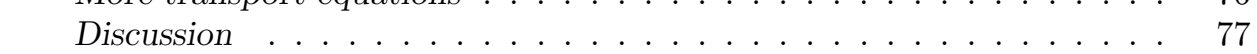

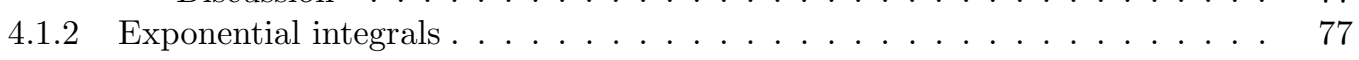

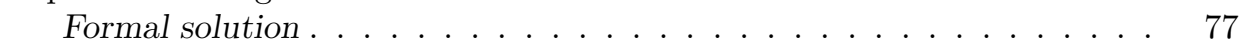

Exponential integrals . . . . . . . . . . . . . . . . . . . . . . . . . . . . . . . . .

Schwarzshild-Milne equations . . . . . . . . . . . . . . . . . . . . . 78

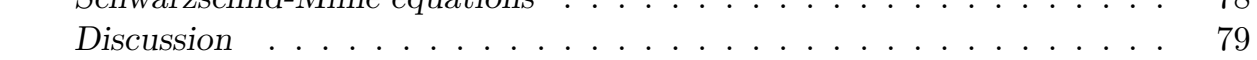

Surface values . . . . . . . . . . . . . . . . . . . . . . . . . . . . . . 81

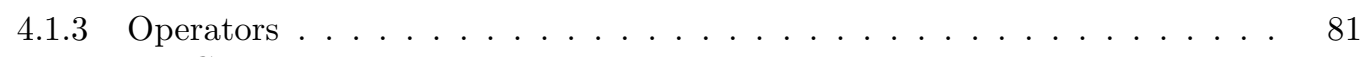

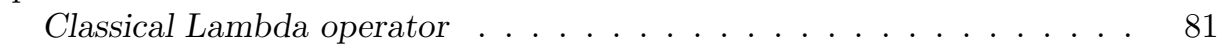

Phi and Chi operators . . . . . . . . . . . . . . . . . . . . . . . . . . 82

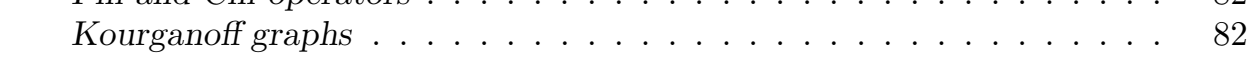

ᄂeneralized Lambda operators . . . . . . . . . . . . . . . . . . . . . . . . . . 82

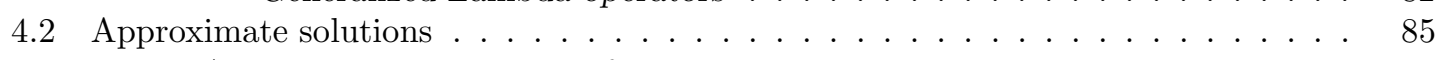

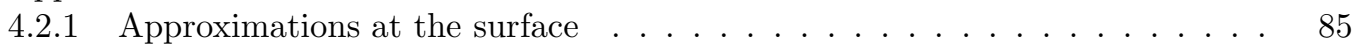

Eddington-Barbier approximations . . . . . . . . . . . . . . . . . 85 
Second Eddington approximation . . . . . . . . . . . . . . . . 86

4.2 .2 Approximations at large depth $\ldots \ldots \ldots \ldots$. . . . . . . . . . 87

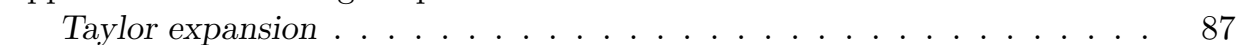

Large depth

L = Diffusion approximation . . . . . . . . . . . . . . . . . . . . . 89

$1=$ Rosseland mean extinction $\ldots \ldots \ldots$. . . . . . . . . . 90

Total radiative energy diffusion . . . . . . . . . . . . . . . . . . . . . . 90

1.2.3 The Eddington approximation . . . . . . . . . . . . . . . . . 9 91

Validity $\ldots \ldots \ldots \ldots \ldots$

i Second-order transport equation . . . . . . . . . . . . . . . . . . . . 92

4.3 Illustrative solutions

4.3 .1 Coherent scattering in the Eddington approximation . . . . . . . . . . . . 92

Transport equation

Boundary conditions . . . . . . . . . . . . . . . . . . 93

Solutionsi . . . . . . . . . . . . . . . . . 93

14.3.

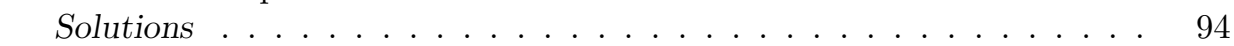

Without scattering. . . . . . . . . . . . . . . . . . . 94

With scattering . . . . . . . . . . . . . . . . . . 95

Surface values . . . . . . . . . . . . . . . . . . . . . 95

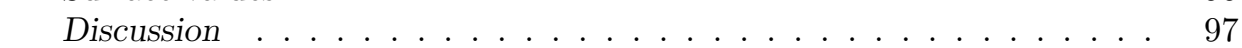

4.3 .3 Thermalization depthi . . . . . . . . . . . . . . . . . . . . . 99

Effectively thick regime . . . . . . . . . . . . . . . . . . . . 99

Optically thick regime $\ldots \ldots \ldots$. . . . . . . . . . . . . . . 100

- Optically thin regime . . . . . . . . . . . . . . . . . . . . . . . . . . 100

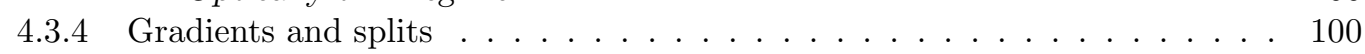

Continuum splits . . . . . . . . . . . . . . . . . . . . . 102

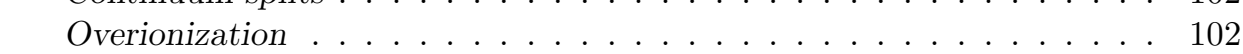

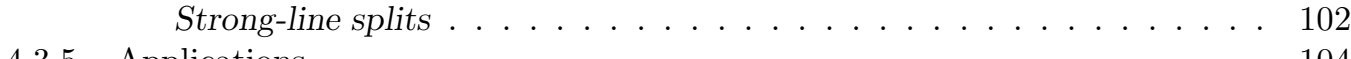

4.3 .5 Applicationg

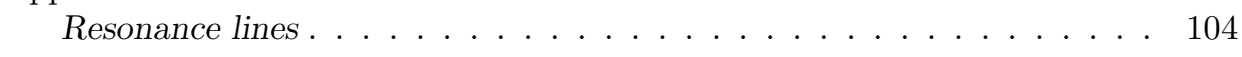

Lines with thermal background continuum . . . . . . . . . . . . . . 104

Thermalization depth . . . . . . . . . . . 106

Continuum scattering $\ldots \ldots \ldots \ldots \ldots$. . . . . . . . . . 106

Bound-bound redistribution! . . . . . . . . . . . . . . . . . . . . 107

Bound-free redistribution . . . . . . . . . . . . . . . . . . . . 110

. Discussion . . . . . . . . . . . . . . . . . 111

15 Numerical Radiative Transfer 113

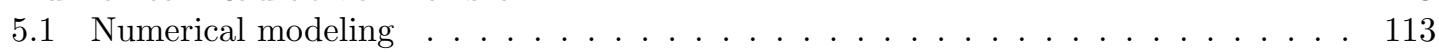

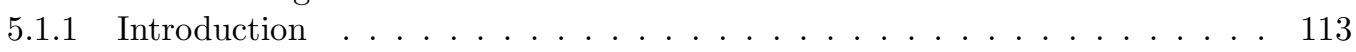

Aims . . . . . . . . . . . . . . . . . . . . 113

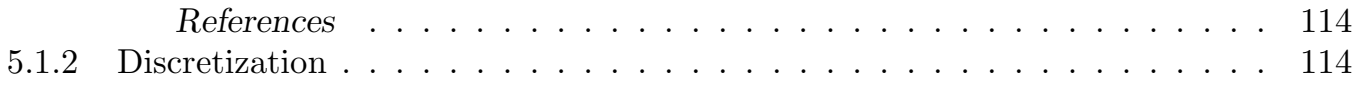

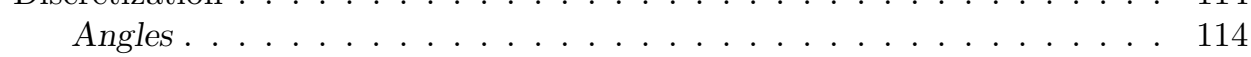

Angle quadraturè . . . . . . . . . . . . . . . . . . . 115

Frequencies . . . . . . . . . . . . . . . . . 116

Depths . . . . . . . . . . . . . . . . 116

Non-plane-parallel grids . . . . . . . . . . . . . . . . . . . . . 116

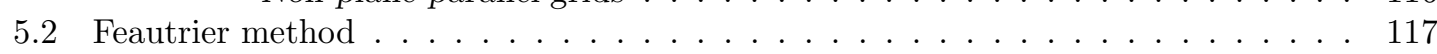

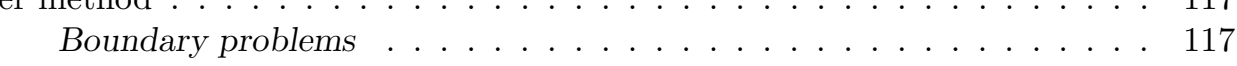

Antisymmetric averages . . . . . . . . . . . . . . . . . . . 118

Transport equation $\ldots \ldots \ldots \ldots \ldots \ldots \ldots$

Boundary conditions . . . . . . . . . . . . . . . . . . . 119 
Difference equationsi . . . . . . . . . . . . . . . . . . . . . . . 119

Forward-backward solution . . . . . . . . . . . . . . . . . . 121

Two-level solutiont . . . . . . . . . . . . . . . . . . . . 121

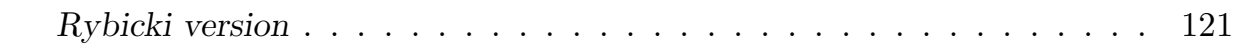

Feautrier solver as Lambda operaton . . . . . . . . . . . . . . . . . . 121

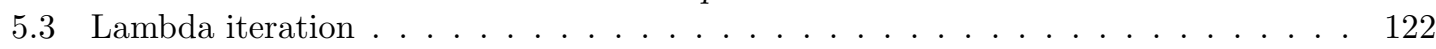

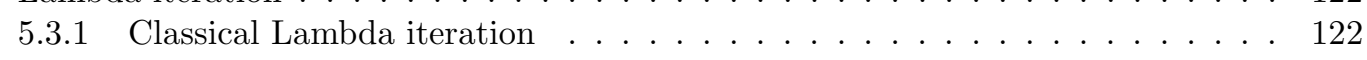

Formal solution . . . . . . . . . . . . . . . . . . . . . . . . 122

Discretization. . . . . . . . . . . . . . . . . 123

Lambda iterationi. . . . . . . . . . . . . . . . . . . . . . 123

. Multi-level iteration . . . . . . . . . . . . . . . . . . . . . . . . . . 123

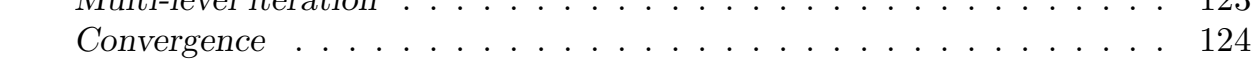

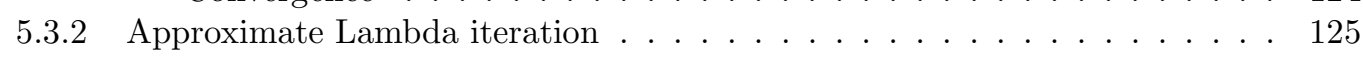

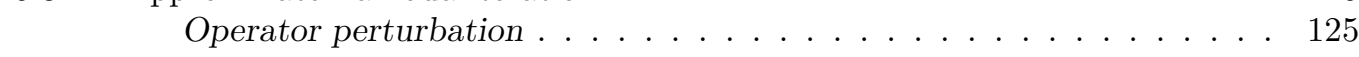

5.3 .3 Approximate Lambda operators' . . . . . . . . . . . . . . . . . . 126

Core saturation operatori $\ldots \ldots \ldots \ldots$

Scharmer operaton . . . . . . . . . . . . . . . . . 128

Partial redistribution $\ldots \ldots \ldots \ldots \ldots$

Local operator . . . . . . . . . . . . . . . . . . . . . . . . . . . . . . . . 130

5.4 Multi-level iteration

Equivalent two-level atom method . . . . . . . . . . . . . . . . 131

Complete linearization . . . . . . . . . . . . . . . . . 131

Newton-Raphson iteration. . . . . . . . . . . . . . . . . . . . . . . 131

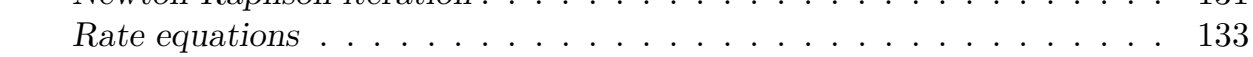

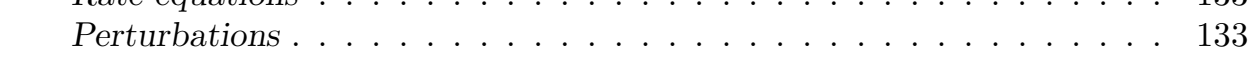

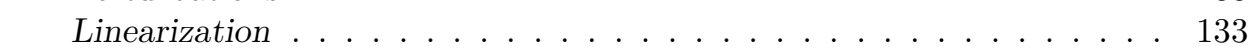

Auer-Mihalas second-order solution . . . . . . . . . . . . . . . . . . . 134

Scharmer-carlsson first-order solution, . . . . . . . . . . . . . . . . . 134

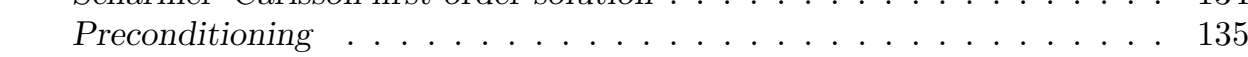

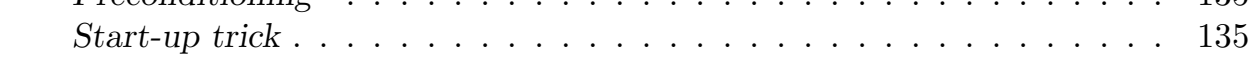

'6- Polarised_Radiative Transferi 137

6.1 Stokes parameters'

Stokes parameters for a single wavé . . . . . . . . . . . . . . . 137

Stokes parameters for actual radiation . . . . . . . . . . . . . . . . 138

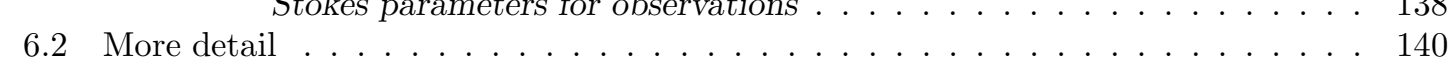

17. Atmospheres of Plane-Parallè Stars : 141

Classical modeling

Assumptions $\ldots \ldots \ldots \ldots \ldots \ldots \ldots$

. Model parameters . . . . . . . . . . . . . . . . . . . . . . . 142

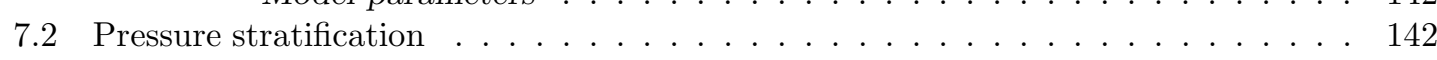

7.2 .1 Gas law . . . . . . . . . . . . . . . . . . 142

7.2. Particle densities

Chemical composition . . . . . . . . . . . . . . . . . . . . . . . . . . 143

Electron donors . . . . . . . . . . . . . . . . . . . 143

Electron and gas pressure . . . . . . . . . . . . . . . . 145

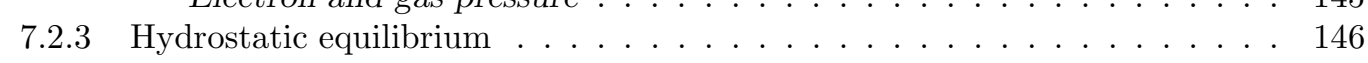

Model completion

1.. plane-parallel layers . . . . . . . . . . . . . . . . . . . . . . . . . 147

Solar $\operatorname{limb} \ldots \ldots \ldots \ldots$

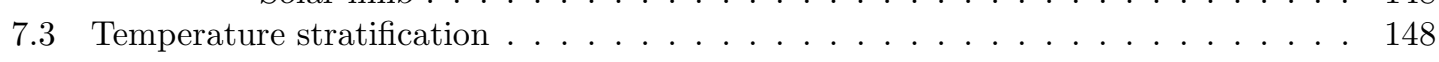

17.1 Empirical models . . . . . . . . . . . . . . . . . . . . . . . . . 148 
Center-limb variation . . . . . . . . . . . . . . . . . 150

Line intensities . . . . . . . . . . . . . . . . . . . . . . . . 150

Continuum intensities . . . . . . . . . . . . . . . . . . . 153

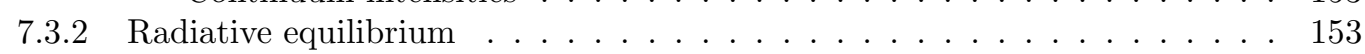

Flux constancy'

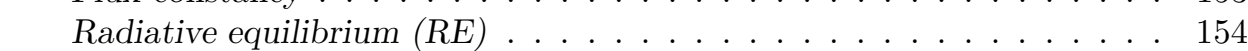

Discussion . . . . . . . . . . . . . . . . . . 155

Line cooling

Continuum cooling . . . . . . . . . . . . . . . . . 156

17.3. The grey approximation $\ldots \ldots \ldots \ldots \ldots$

Grey $R E$ source function $\ldots \ldots \ldots \ldots \ldots$

Grey RE temperature stratification . . . . . . . . . . . . . . . . . 157

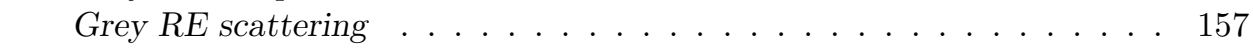

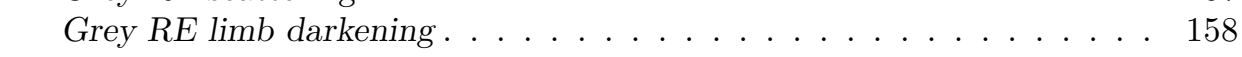

Grey extinction and mean extinction . . . . . . . . . . . . . . 158

Flux-weighted mean and Rosseland meand . . . . . . . . . . . . . . . . 159

77.4 Line blanketing $\ldots \ldots \ldots$

Backwarming . . . . . . . . . . . . . . . . . . 160

Surface effects . . . . . . . . . . . . . . . . . . . . . . 161

Strong $\bar{L} \bar{T} \overline{\mathrm{l}}$ lines $\ldots \ldots \ldots$. . . . . . . . . . . . . . . . . . . 162

Strong scattering lines . . . . . . . . . . . . . . . . . . 162

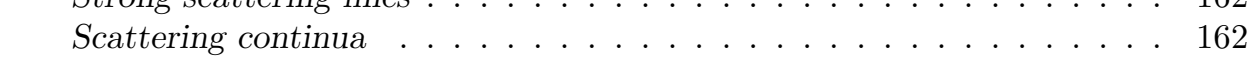

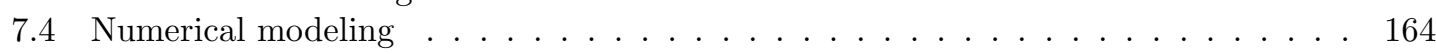

7.4.1 LTE RE modeling of cool starg . . . . . . . . . . . . . . . . . . . . . 164

Sample models . . . . . . . . . . . . . . . . . 164

Line hazét . . . . . . . . . . . . . . . . . . . . . . 164

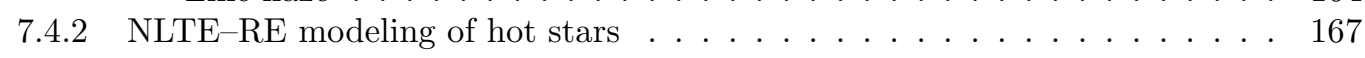

Two-level atom with Lyman alphä . . . . . . . . . . . . . . . . . . 168

Three-level atom with Balmer alphai . . . . . . . . . . . . . . 168

i8 Continua from Plane-Parallei Stars: 171

Solar continuau . $_{1}$

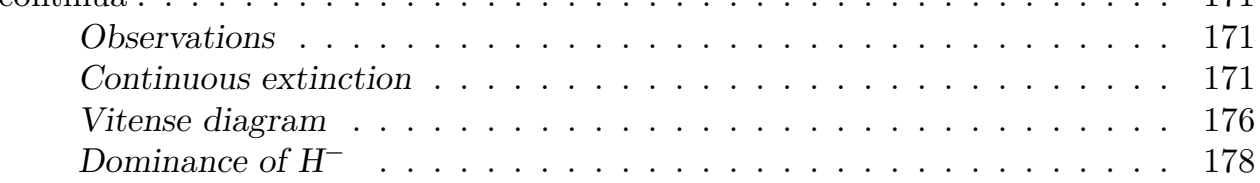

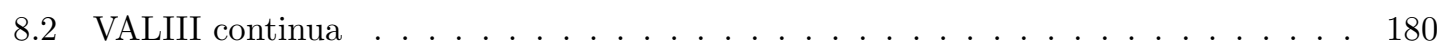

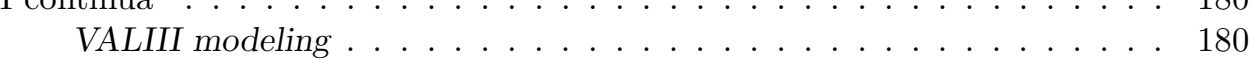

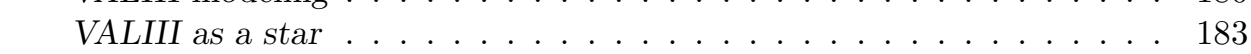

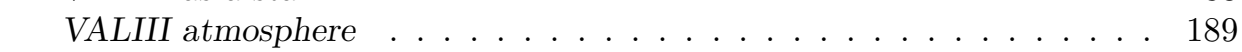

VALIII radiative transfet . . . . . . . . . . . . . . . . . . . . . . . . 189

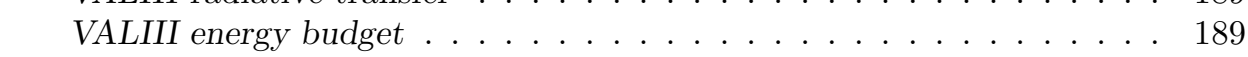

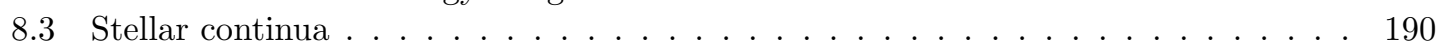

Stellar classification . . . . . . . . . . . . . . . . . . . 190

continuous extinction . . . . . . . . . . . . . . . . . . . . . . . . . 190

Vitense diagrams

Hydrogen and helium edges . . . . . . . . . . . . . . . . . . . . . . . 201

Balmer jumpi . . . . . . . . . . . . . . . . . . . 201

Thomson scattering . . . . . . . . . . . . . . . . . . . . . 202

Kurucz flux spectra $\ldots \ldots \ldots$. . . . . . . . . . . . . . . . . 202

'

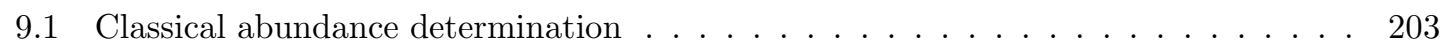

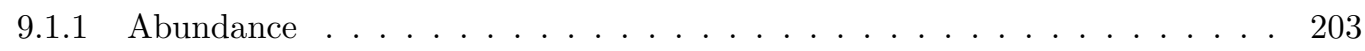




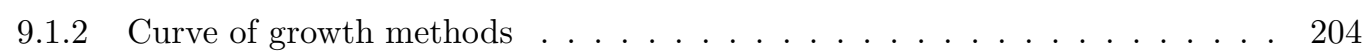

Equivalent width . . . . . . . . . . . . . . . . . . . 204

Schuster-schwarzschild atmosphere. . . . . . . . . . . . . . . . . 204

Weak lines . . . . . . . . . . . . . 205

Saturated lines . . . . . . . . . . . . . . . . . . . 206

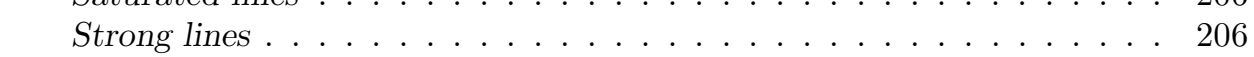

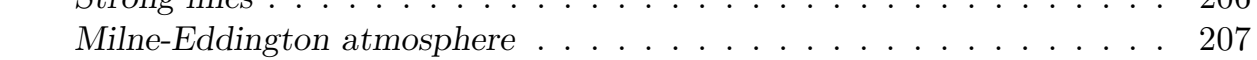

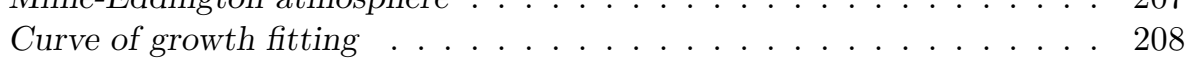

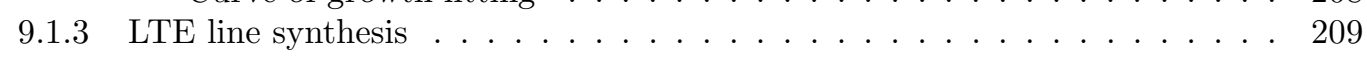

Parameters $\ldots \ldots \ldots \ldots \ldots \ldots \ldots$

HOLMUL photosphere . . . . . . . . . . . . . . . . . 210

Validity . . . . . . . . . . . . . . . . . . 210

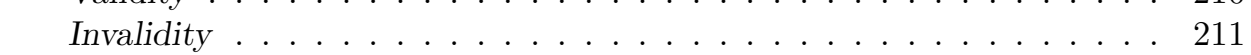

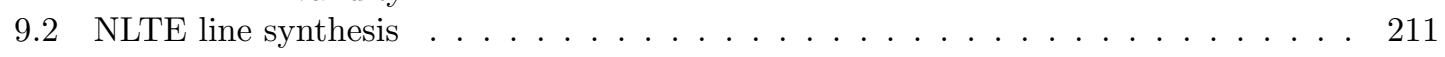

9.2 .1 Pictorial guide to solar NTLE mechanisms . . . . . . . . . . . . . 211

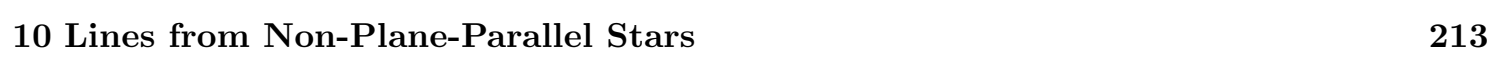

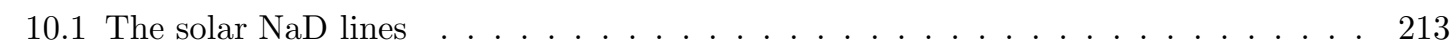

Atomic structure . . . . . . . . . . . . . . . . . . . . . . . . . 213

VALIII formation . . . . . . . . . . . . . . . . . . . . . . . . 214

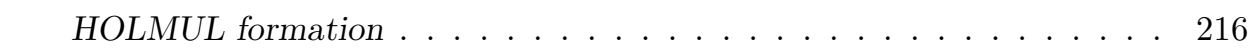

Atom-size experiments . . . . . . . . . . . . . . . . . . 216

Solar granulation . . . . . . . . . . . . . . . . . . . 218

Quasi-plane-parallel formation . . . . . . . . . . . . . . . . . . 218

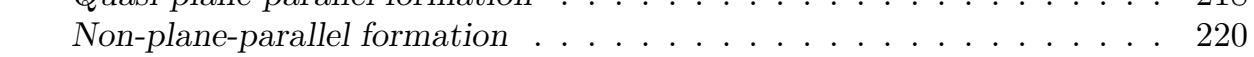

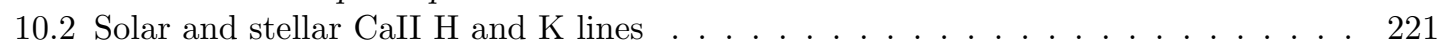

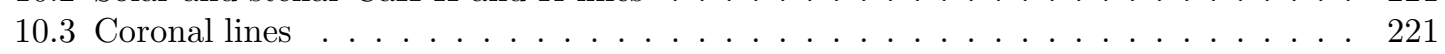

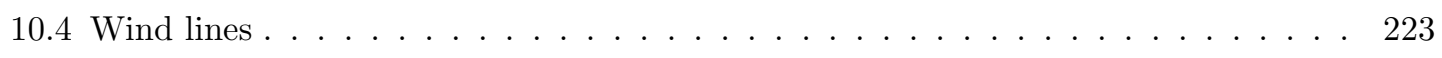

Exercises 224

Léperences 241

Index

Radiative Transfer Rapi $\quad 255$ 


\section{List of Figures}

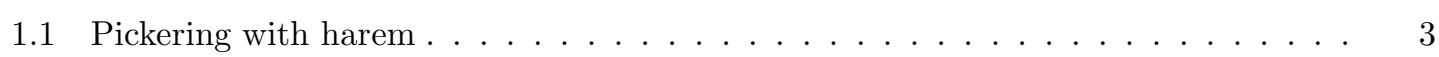

1.2 Definition of equivalent width $\ldots \ldots \ldots \ldots \ldots \ldots \ldots \ldots \ldots \ldots \ldots \ldots \ldots \ldots$

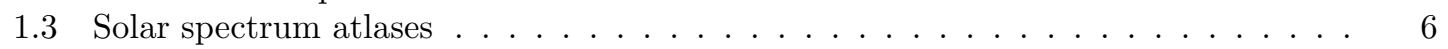

M.

1.5 Secchi and Harvard classificationg . . . . . . . . . . . . . . . . 8

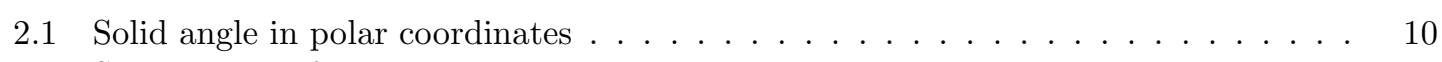

2.2 Spectral lines from a homogeneous medium . . . . . . . . . . . . . . . 16

2.3 Eddington-Barbier approximation $\ldots \ldots \ldots \ldots \ldots \ldots \ldots$

2.4 Solar limb darkeningt $\ldots \ldots \ldots$

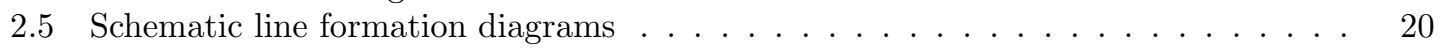

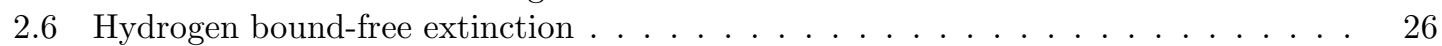

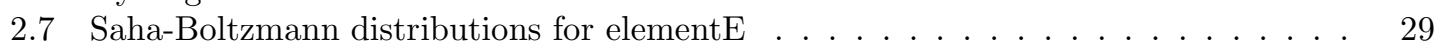

2.8 NLTE source functions.$\ldots \ldots \ldots \ldots \ldots \ldots \ldots$

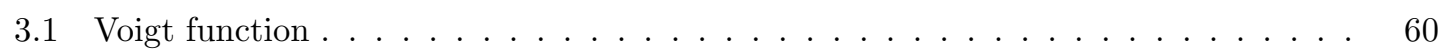

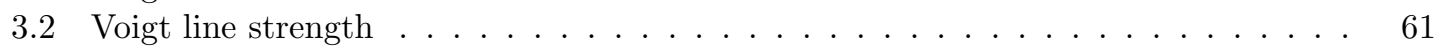

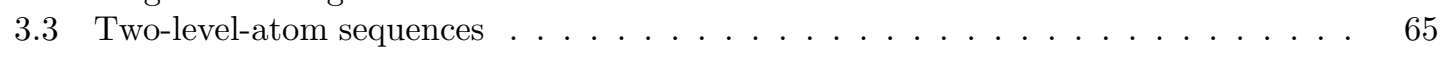

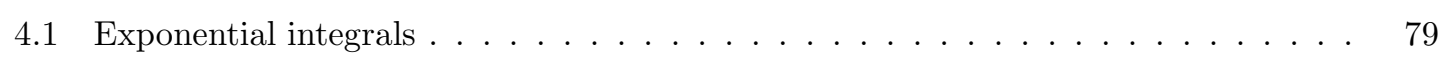

4.2 Schwarzschild equation . . . . . . . . . . . . . . . . . . . . 80

4.3 Milne equation $\ldots \ldots \ldots \ldots \ldots$. . . . . . . 81

4.4 Kourganoff plots for the Lambda operatori . . . . . . . . . . . . . . . . . . . . 83

4.5 Kourganoff plots for the phi operatorr $\ldots \ldots \ldots \ldots$. . . . . . . . . . . 84

446 Radiation field at large depthi ${ }_{4}$

4.7 Eddington approximation for an isothermal LTE atmoshere $\ldots \ldots \ldots \ldots \ldots$

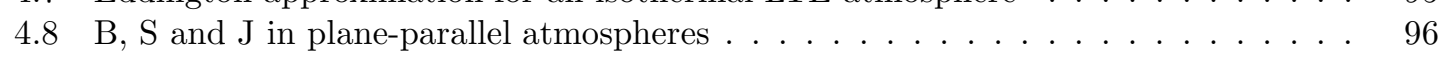

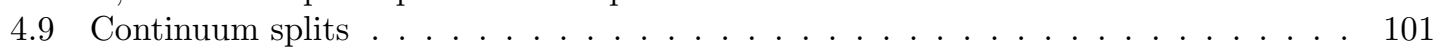

4.10 Source function gradient for strong lines . . . . . . . . . . . . . . . . . . . . . 103

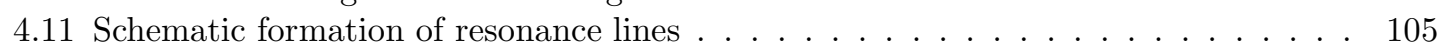

4.12 Avrett results for redistributed two-level lines $\ldots \ldots \ldots$. 108

4 A 13 -

55.1 Structure of Feautrier matrixi . . . . . . . . . . . . . . . . . . . . 122

15.2 Convergence for different ÁLI methods" . . . . . . . . . . . . . . . . . . . . . 127

5.3 Newton-Raphson iteration! $\ldots \ldots \ldots$

6.1 Elliptical polarization $\ldots \ldots \ldots$

6.2 Zeeman triplet $\ldots \ldots \ldots \ldots \ldots$

17. Ionization edges . . . . . . . . . . . . . . . . . . . . . . . . . 144

7.2 Solar flash spectrum $\ldots \ldots \ldots \ldots \ldots$. . . . . . . . . . . . . . . 148

7. 3 . Solar model atmospheres $\ldots$ 
77.4 Determination of the variation of the continum extinction with wavelength . . . . 151

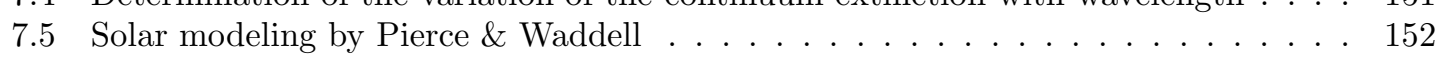

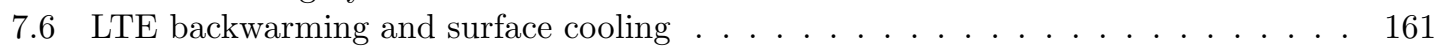

7.7 NLTE backwarming and surface cooling $\ldots \ldots$. . . . . . . . . . . . . 163

$\overline{7} 8$ Photospheric models for cool stars . . . . . . . . . . . . . . . . . 165

7.9 Solar near-ultraviolet line haze

7.10 NLTE-RE modeling of a hot start . . . . . . . . . . . . . . . . . 167

18.1 Solar irradiance spectrum

8.2 Solar continua in the far ultraviolet

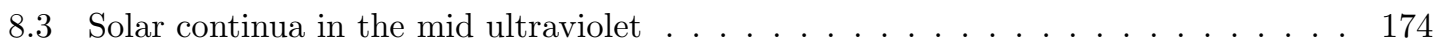

8.4 Solar continua in the near ultraviolet, visible and near infrared . . . . . . . . . . 175

8.5 Solar continua in the infrared $\ldots . .2 .177$

8.6 Vitense diagram of the continuous extinction in the solar atmosphere . . . . . . . . 179

,

8.8 VALIII electron densities and electron donors $\ldots \ldots \ldots$. . . . . . . . . . . . . 183

8.9 VÁLIII continua, radio to infrared

8 VALII continua, near infrared to mid ultraviolet . . . . . . . . . . . . . . . . 185

8.11 VALIII continua, mid ultraviolet to far ultraviolet $\ldots \ldots \ldots$. . . . . . . . . . . 186

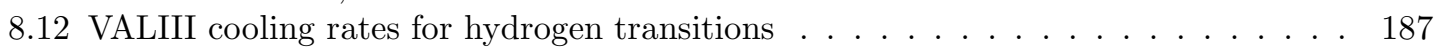

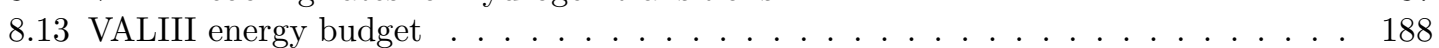

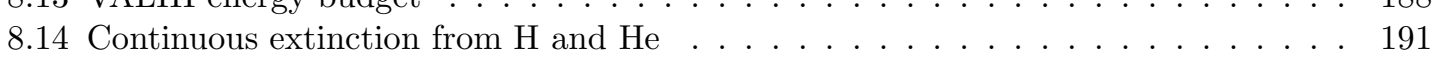

18.15 Vitense diagram of the continuous extinction in 01 stars . . . . . . . . . . . . . . . 192

18.16 Vitense diagram of the continuous extinction in 05 stars . . . . . . . . . . . . . 193

8.17 Vitense diagram of the continuous extinction in B9.5 stars . . . . . . . . . . . . . . 194

18.18 Vitense diagram of the continuous extinction in $\bar{K} 8$ stars . . . . . . . . . . . . . . . 195

18.19 Kurucz flux spectra for hot stars . . . . . . . . . . . . . . . . . 196

18.20 Kurucz flux spectra on linear scales . . . . . . . . . . . . . . . . . 197

8.21 Kurucz flux spectra redward of the Lyman limit . . . . . . . . . . . . . . . . . . . 198

8.22 Kurucz flux spectra for the optical $\ldots \ldots \ldots$

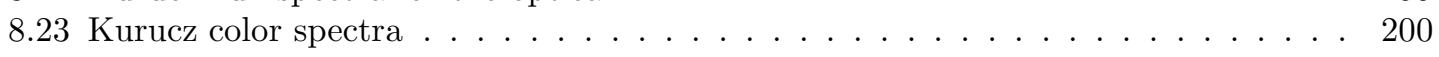

19.1 Schuster-Schwarzschild line profiles and curve of growthi $\ldots$. . . . . . . . . . . . 205

9.2 Line-to-continuum extinction ratio for solar iron lines $\ldots \ldots$. . . . . . . . 207

9.5 Sensitivities of the curve of growth . . . . . . . . . . . . . . . . . . 209

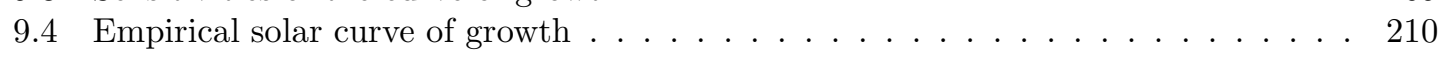

110.1 Grotrian diagram for ${ }_{0} I_{1}^{\prime}$

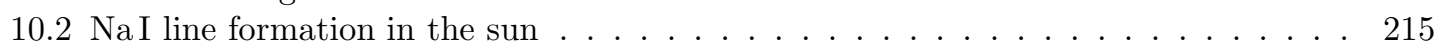

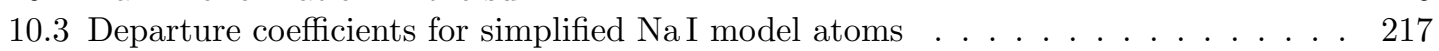

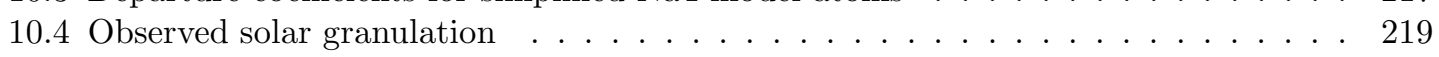

10.5 Computed solar granulation . . . . . . . . . . . . . . . . . . . . . . 220

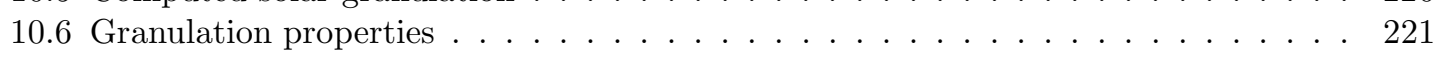

10.7 Granulation models and NaI line formation . . . . . . . . . . . . . . . . . . 222

10.8 Departure coefficient ratio for $\mathrm{NaID}_{1}$

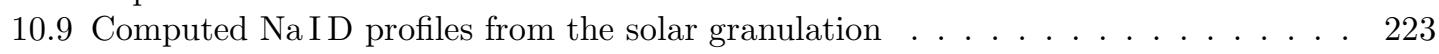

10.10Redman's eclipse spectrum $\ldots \ldots \ldots \ldots \ldots \ldots$

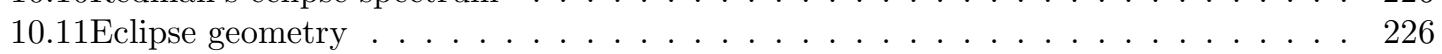

10.12Solar temperature distribution $\ldots \ldots \ldots \ldots$. . . . . . . . . . . . . . . 227

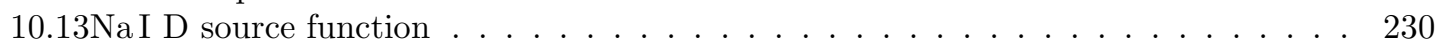

10.14Auer-Mihalas results' $\ldots \ldots \ldots \ldots$. . . . . . . . . . . . . . . . 235

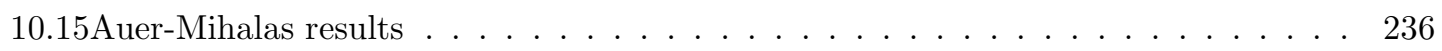




\section{List of Tables}

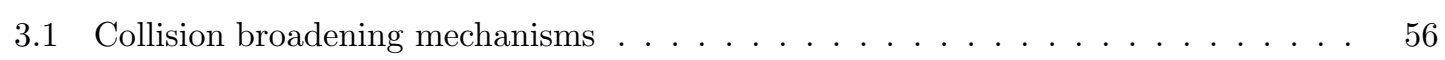

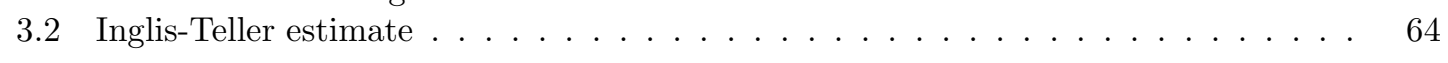

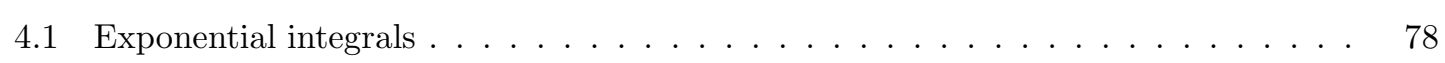

4.2 Eddington approximation for an isothermal scattering atmosphere $\ldots \ldots$. . . . . 97

$7 \overline{7} .1$ Ábundances and ionization energies of major elements . . . . . . . . . . . . . . 144

12 Solar limb darkening and energy transporti

8 spectral features . . . . . . . . . . . . . . . . . . . . . . 176

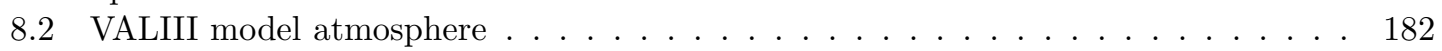

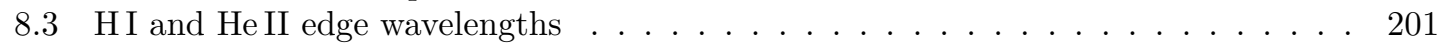




\section{Preface}

$\mathrm{T}$ his is the current version of lecture notes for the Utrecht third-year astronomy course on stellar atmospheres. The main topic treated in this 30-hour course is the classical theory of radiative transfer for explaining stellar spectra. The reason to emphasize this topic over the many newer subjects of astrophysical interest offered by stellar atmospheres is that it needs relatively much attention to be mastered. Radiative transfer in gaseous media that are neither optically thin nor completely opaque is a key part of astrophysics, but it is not a transparent subject.

This course requires familiarity with the basic quantities and processes of radiative transfer. At Utrecht I treat these in a more basic course that follows the first chapter of

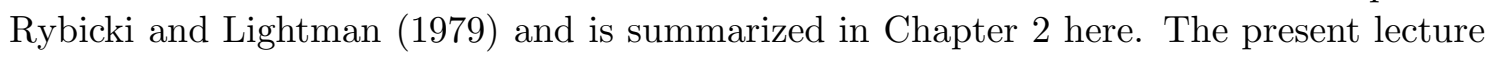
notes are roughly a middle road between

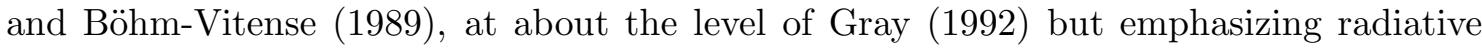
transfer rather than observational techniques and data interpretation.

In 1995 these lecture notes replaced former Dutch-language ones that were written by C. Zwaan over the many years in which he developed the Utrecht course. The approach follows Zwaan's example, much explanation was taken from him, and many equations were copied from his LaTeX files. In his course, Zwaan also paid attention to aspects of cool-star magnetism that are not treated here. They are described in Solar and Stellar Magnetic Activity by SSchrijuer and Zwaan $(2000)_{1}^{\prime}$, a book that was completed just before Zwaan's untimely death in 1999. He was my lifelong teacher and friend; these lecture notes bear his stamp.

In addition, various treatments come from other sources, especially from the books mentioned above and from unpublished Harvard course notes by E.H. Avrett. I gratefully acknowledge these debts.

These lecture notes still evolve (at present, many sections are yet empty). I welcome suggestions for improvement. 


\section{Bibliography}

$\mathrm{T}$ here is a long list of books treating topics discussed here that are worth to have, to study, or to have a look at. I frequently refer to the first four of these books in the text; in various places, the treatment follows the cited book in detail.

The first group consists of the books quoted most:

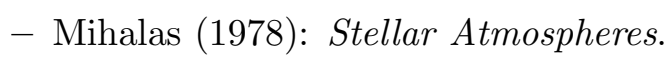

The bible on NLTE spectral line formation in stellar atmospheres. Comprehensive, highly authorative, well written. The first edition ( formal and sometimes clearer on classical topics than the 1978 edition; the latter has been expanded with the theory of expanding atmospheres, in particular the Sobolev approximation. The level is for US graduate students and researchers, higher than this course. Various sections of these lecture notes follow Mihalas closely, but are in principle self-contained. You should study this book if you start graduate research into stellar atmospheres. Out of print, but a new version is being prepared by Mihalas and Hubený.

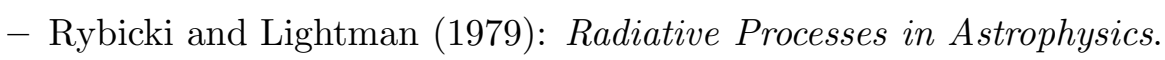

Excellent general introduction to radiative processes, in particular high-energy ones. Chapter $\overline{2}$ below gives an equation summary of the first chapter, with the same notation. In the context of the other material treated here, Chapters 9 (Atomic Structure), 10 (Radiative Transitions) and 11 (Molecular Structure) are of interest. Worth having.

- iGray (1992): Observation and Analysis of Stellar Photospheres.

Worth buying and using as complement to these lecture notes. It does not cover NLTE radiative transfer, but it adds much observational flavor which falls outside the scope of this course. It is clearly written, contains many easy-to-use formulae and recipes, and has good references to the literature.

- Novotny $(\overline{1} \overline{9} \overline{7} 3)^{\prime}:$ Introduction to Stellar Atmospheres and Interiors.

A bit oldfashioned, but still of interest for its clear and extensive low-level explanations of atomic structure, atom-photon interactions, extinction coefficients and classical atmosphere modeling. Out of print.

- Böh-1-Vitense (1989): Introduction to stellar astrophysics II. Stellar Atmospheres.

An easy to read textbook on a lower level than this course. Good on Saha-Boltzmann statistics, extinction processes, curve of growth diagnostics and observational contexts. It also discusses coronae and winds.

Then some general textbooks that also include radiative transfer: 
- Shiu (1991): The Physics of Astrophysics. I. Radiation.

Excellent general textbook on astrophysical processes, including radiative ones and atomic and molecular quantum theory. Emphasizes the underlying physics. Comparable to Rybicki \& Lightman but putting more emphasis on low-energy rather than high-energy processes. Worth buying in general but not specifically needed for this course.

- 'Ōsterbrock

About nebulae rather than about stellar atmospheres, but containing an excellent description of the physics of non-equilibrium radiative transfer.

- 'Āller $(\overline{1} 9 \overline{5} 2)$ :: The Atmospheres of the Sun and Stars.

Very readable textbook on the physics of stellar atmospheres and stellar spectra, with a good introduction to the physics of atomic and molecular spectral line formation.

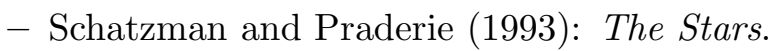

Excellent general introduction to stellar astrophysics including solar and stellar activity.

- ínarwit $(1 \overline{9} \overline{8} \overline{8}):$ Astrophysical Concepts.

A good basic astrophysics source in general, not specifically for stellar atmospheres.

- Bowers and Deeming $(1 \overline{1} \bar{g} \overline{8} \overline{4}):$ : Astrophysics I.

The two books of Bowers and Deeming are useful in that they cover a wide range of subjects in substantial detail. However, I don't like their sections on radiative transfer, nor their tendency to present results rather than explain principles. They are worth buying if you choose to possess just two books on astronomy.

The next three are compendia rather than textbooks:

- 'Ā

An authorative collection of numbers, units, data and formulae. A book to have and carry with you when you are a practising astrophysicist. The emphasis is on astronomy and astronomical spectrometry. It is getting out of date in places, especially in its references; nevertheless, it remains the book to look up the Planck constant, the distance to the nearest star, the size of Jupiter and of the Galaxy, the definition of oscillator strength, the abundance of iron, the refraction of the atmosphere, and lots more.

- íng (1974): Astrophysical formulae.

Intended as the equation counterpart to the previous book, specifying all formulae that an astrophysicist might need. Useful, but usually you want to know more about the equation you are using. Good references to the original literature.

- Baschek and Scholz (19̄9)

Part of the Landolt-Börnstein reference series giving concise but authorative summaries of whole areas of physics. This 60-page chapter represents a gründliches equation summary of the classical theory of stellar atmospheres including radiative transfer. It is particularly good in specifying equation validity limits. It also contains useful tables of various quantities.

The following group concerns books detailing radiative transfer:

- Menzel $(\overline{1} \overline{1} \overline{6} \overline{6})$ Selected Papers on the Transfer of Radiation.

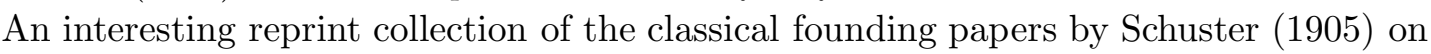


scattering, Schwarzschild (1906, 1914; both translated from German) on the equilibrium of and the radiation in the solar atmosphere, "Eddington (1916), on radiative equilibrium, Rosseland $(\overline{1} \overline{9} \overline{2} \overline{4})$ : on the Rosseland-average of stellar extinction, and the review by 'Mīinè (1930) in which he formalized the use of exponential integrals in radiative transfer theory. Recommended for more than just the historical context. Often, the original papers that laid the groundwork of a field contain well-formulated insights that become too condensed in subsequent textbooks or in very dense lecture notes such as these.

- Chandrasekhar (1950): Radiative Transfer.

Outdated and hard to read, but nevertheless still valuable as the elegant mathematical foundation of analytical radiative transfer, with precise, well-formulated definitions.

- iC̄handrasekhar (1939): Stellar Structure.

Idem. Mostly a treatment of stellar interiors, but Chapter 5 (Radiation and Equilibrium) is a beautiful and concise formulation of radiative transfer and the thermodynamics of LTE.

- 'Ǩurganoff $(19 \overline{9} 5)$ : Basic methods in transfer problems.

A readable, still worthwhile text on the mathematics of classical analytical radiative transfer, with many approximations and examples.

- 'Jefferies $(196 \overline{6})$ : Spectral Line Formation.

A carefully written account of line formation theory at the time when numerical solutions began changing the field. It was superseded by Mihalas' book and is now partially outdated. Its clear formulation of NLTE basics remains valuable, though.

- 'Āthay (1972): Radiation Transport in Spectral Lines.

The application-oriented counterpart to Jefferies' book during the 1970's. Athay, Thomas and Jefferies constituted the Boulder school of NLTE line formation, using solar lines to define NLTE physics. Athay's book has many model computations for different types of lines. It is not a good text for students, but its many numerical examples remain instructive for researchers in the field.

- 'Cannon (1985): The transfer of spectral line radiation.

A specialist book on the foundations of radiative transfer, recommended to researchers rather than to students. The first chapter is an excellent basic introduction, employing two-level atoms for insight into the physics of scattering.

- Pomraning $(\overline{1} \overline{9} \overline{7} 3):$ The Equations of Radiation Hydrodynamics.

Basics of radiation hydrodynamics. More advanced than this course.

- Mihalas and Mihălas (1984): Foundations of Radiation Hydrodynamics.

A new bible for researchers at the frontier of time-dependent radiation hydrodynamics.

Much more advanced than this course.

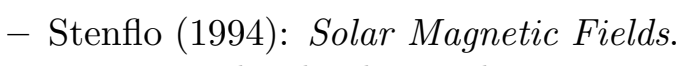

Not so much a book on solar magnetic fields as a book on polarized radiative transfer, the first authorative textbook on this intricate subject. It gives both classical and quantummechanical derivations. Solar magnetic fields set the context and the examples.

- Ũnsöld $(\overline{1} \overline{9} \overline{5} \overline{5}):$ Physik der Sternatmosphären.

This was the European stellar spectroscopy bible until the 1960's. now mostly of historical interest. Take a look at it to get a flavor of German-style stellar astrophysics 
in the first half of the 20th century. It was never officially translated into English and therefore lost its value rather rapidly. The Landölt-Bornstein chapter of Baschek and Scholz (listed above) represents a concise summary.

For numerical solution methods see:

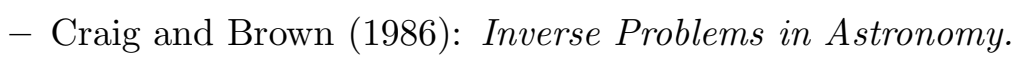

An excellent mathematical "guide to inversion strategies for remotely sensed data", needed to minimize the noise amplification inherent in astronomy's intrinsic undersampling.

- ikäkofen (1984): Methods in Radiative Transfer. KKalkofen (1987): Numerical Radiative transfer.

Two collections of review papers about the tricks of numerical radiative transfer.

The history of astronomical spectroscopy is described in detail by:

- "Hearnshaw $(\overline{1} \overline{9} \overline{8} \overline{6}):$ : The analysis of starlight.

Very readable and highly recommended. It covers all of stellar spectrometry and the people that developed it, from Newton to Mihalas, up to 1965.

Some books on solar physics, which as "the mother of astrophysics" provided the context in which most theory treated here was formulated, and from which I draw most of my examples:

- 'Foukal (1990): Solar Astrophysics.

Excellent overview of modern solar physics, well-balanced and authorative. This is the book to buy if you want one book on solar physics.

- Sitix $(1989 \overline{9})$ : The Sun: An Introduction.

Good general textbook at the undergraduate level.

- 'Ziriñ $\overline{1} \overline{1} \overline{8} 8 \overline{8}):$ : Astrophysics of the Sun.

Excellent in places, but misleading in other places; the reader has to be able to discriminate between insight and conjecture. Its strengths are its unique displays of data and lively discussions of controversial research topics.

Finally, the book that wraps up much of Kees Zwaan's work and insights on cool-star activity. It represents a natural companion to these Zwaan-inspired lecture notes:

- 'S̄chrijuer and Z̄waan $(20 \overline{0} \overline{0})$ :' Solar and Stellar Magnetic Activity.

Complete review of the field, with very strong solar-stellar links. 


\section{Chapter 1}

\section{Brief History of Stellar Spectrometry}

S tellar spectra provide our principal means to quantify stellar constitution and stellar Physics, truly "the astronomer's treasure chest" (Pannekoek). The history of astronomical spectroscopy is fairly brief, covering less than two centuries, but it is very rich. It is summarized in this introductory chapter, largely following the excellent book by Hearn'shaw $(\overline{1} \overline{9} 8 \overline{6})$ '. For the history of astronomy in wider context see Pannekoek's $(1951,1961)$ and Dijksterhuis' (1950, 1969) books (each in Dutch and English, respectively). The first is a detailed but very readable factual history of astronomy. The second is a review of the corresponding changes in philosophy. Both are highly recommended.

Fraunhofer lines. William Wollaston was to the first to observe spectral lines, in 1802. He noticed dark gaps in a solar spectrum seen through a prism fed from a narrow slit in the window shade and thought that these marked the gaps between the different colors. They included the NaI D lines and CaII H \& K. These letters do not come from him but from Joseph Fraunhofer, a glass maker who used the solar spectrum to test the quality and achromaticity of his optical products. He rediscovered the dark lines in 1814:

In a shuttered room I allowed sunlight to pass through a narrow opening in the shutters.

[...] I wanted to find out whether in the colour-image of sunlight, a similar bright stripe was to be seen, as in the colour image of lamplight. But instead of this I found with the telescope almost countless strong and weak vertical lines, which however are darker than the remaining part of the colour-image; some seem to be nearly completely black.

and labeled the darkest ones alphabetically. We still call spectral lines in stellar spectra "Fraunhofer lines", use D for NaI D, H \& K for Ca II H \& K $\lambda=430.5 \mathrm{~nm}$ and $\mathrm{b}$ for the $\mathrm{Mg} \mathrm{I} \mathrm{b}$ triplet in the green. Figure $1.3 \mathrm{isplays}$ Fraunhofer's engraving (top). The other lines present in that segment (from D to F) illustrate that he noted hundreds of fainter lines as well. He measured wavelengths for many, using an objective diffraction grating made of parallel thin wires. He also achieved spectrometry of Venus, Sirius and other stars with an objective prism, and noted in 1823 that:

The spectrum of Betelgeuse ( $\alpha$ Orionis) contains countless fixed lines which, with a good atmosphere, are sharply defined; and although at first sight it seems to have no resemblance

\footnotetext{
${ }^{1}$ Fraunhofer called them $\mathrm{H}$ together, the CaII K line was split off and called K by Henry Draper. Fraunhofer's A and B were for a telluric absorption bands starting at $\lambda=759 \mathrm{~nm}$ and $\lambda=687 \mathrm{~nm}$, C for $\mathrm{H} \alpha$ at $\lambda=656.3 \mathrm{~nm}, \mathrm{E}$ for a cluster of metal lines near $\lambda=527 \mathrm{~nm}, \mathrm{~F}$ for $\mathrm{H} \beta$ at $\lambda=486.1 \mathrm{~nm}$.
} 
to the spectrum of Venus, yet similar lines are found in the spectrum of this fixed star in exactly the places where the sunlight $\mathrm{D}$ and $\mathrm{b}$ come.

Lines as element encoders. William Herschel realized that spectra contain quantitative information on the source contents and tried to establish how and what from flame spectroscopy. A quote, also from 1823:

The colours thus communicated by the different bases to flame afford, in many cases, a ready and neat way of detecting extremely minute quantities of them.

However, the flames always contained sodium impurities and so produced the yellowish Na I D lines; for decades, these bright lines kept spectroscopists from recognizing other fainter lines as uniquely determined by other elements. In addition, Brewster and others thought that the colors of sunlight were due to interference, locally, out of the three basic colors red, yellow and blue, leaving no clear solar reason for the lines.

Becquerel succeeded in photographing the solar spectrum in 1842, recording many lines in the ultraviolet that can't be seen. Stokes and others followed his example. Quantitative solar spectroscopy came of age with Kirchhoff. He noted first that bright flame emission lines are seen as dark lines against a bright continuum background and then, with Bunsen, that wavelength coincidence between bright flame lines and dark solar lines implies that flame and sun share the same line-causing substance, whether emitting or absorbing. Kirchhoff and Bunsen recorded flame and spark spectra for many elements. The story goes that they also determined the amount of sodium in flames produced by a Mannheim fire, observed from their Heidelberg laboratory window, and that they, while discussing that measurement-at-a-distance during a stroll the evening after, realized that they had so demonstrated that spectral-line encoding is independent of distance and thus permits quantitative analysis of sources far more distant than Mannheim. Kirchhoff then ascertained that iron, calcium, magnesium, sodium nickel and chromium are certainly present in the sun, and cobalt, barium, copper and zinc probably.

Stellar classification. Stellar spectroscopy continued after Fraunhofer at no great pace until Father Secchi started a Jesuit observatory in Rome. He wrote 700 papers and two books (The Sun and The Stars), all within three decades, and started spectral classification (Figure 1.5. In the hands of Huggins (UK), Henry Draper (USA) and especially Annie Cannon (USA) stellar classification became mature. It centered at Harvard where the physicist Edward Pickering had become observatory director and was open to directing new quests on tremendous scales. He started the Harvard plate collection and was selected by the widow of Henry Draper, the first to photograph a stellar spectrum from his private observatory on the Hudson river, to embark on an ambitious spectroscopy program as a memorial to her husband. She donated large sums of money to this end. Pickering equipped a sequence of telescopes with low-dispersion objective prisms, obtaining spectra of all bright stars in the field simultaneously on one plate (at five-minute exposure for up to sixth magnitude stars per ten-degree square field with the eight-inch Bache telescope).

A sequence of women, most of them hired as "computers", developed the classification scheme (Figure 1. Memorial of 10351 stars. She classified them in a scheme assigning different letters to different types, elaborating on Secchi's original four-class division. She also noted the strength of $\mathrm{Ca}$ II $\mathrm{K}$ and $\mathrm{H} \beta$ for each spectrogram. She later revised the scheme when the element helium and its lines were identified. 


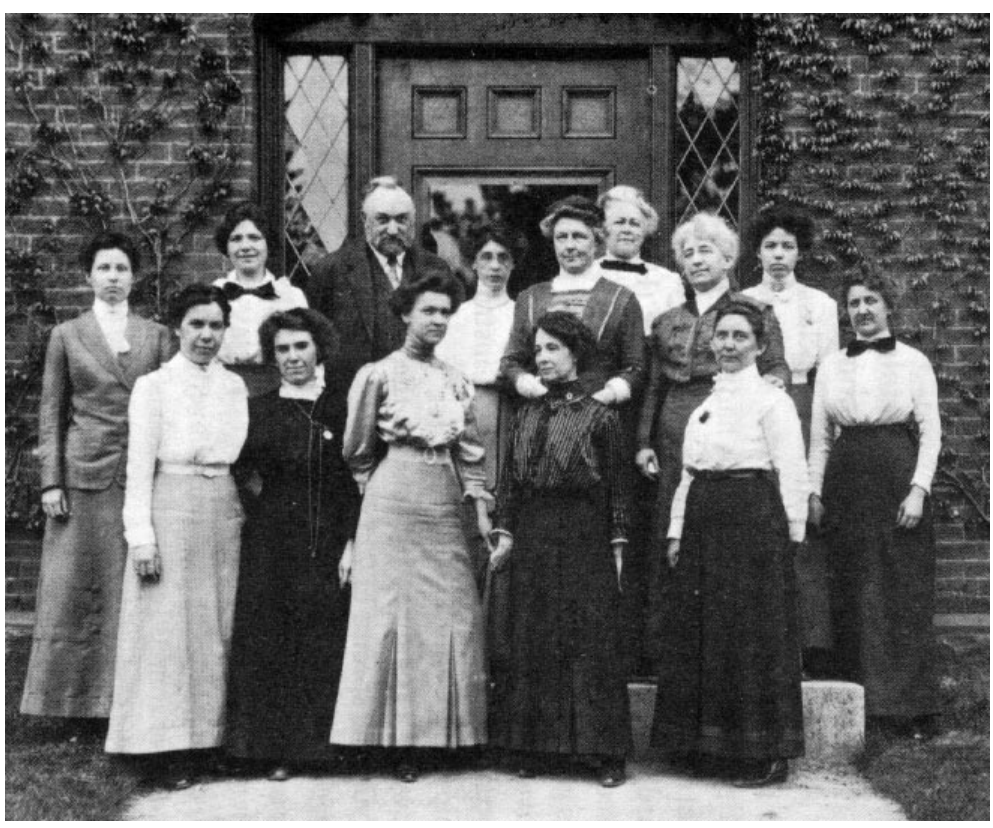

Figure 1.1: Pickering and his "harem". With his assistants, Pickering undertook spectral classification of stars on an enormous scale well before the nature of the classification was understood. Taken at Harvard in 1913. Annie Cannon is in the middle row, second to the right of Pickering. From Hearnshaw $(1986)$

Antonia Maury, a niece of Henry Draper, was the next. Pickering gave her the task to examine 5000 plates of bright stars with much higher dispersion. She came up with a new classification scheme, in twenty-two classes plus five orthogonal divisions with the line sharpness as criterion. Doppler broadening had been suggested as a mechanism but was not yet generally accepted. The large number of classes was severely criticized, especially from Potsdam. Nevertheless, some of her subsets make sense in hindsight, describing highluminosity giants and supergiants that are sharp-lined due to small collisional broadening. This distinction was not taken over by Annie Cannon (1863-1941) who

must rank among the most dedicated of astronomers of all time and certainly as one of the most illustrious from the female ranks

('i-

and updated Fleming's original classification scheme by accounting for ionized helium lines as observed from $\zeta$ Puppis. Pickering had discovered these and found that they obeyed Balmer's equation for the HI series when including half-integer values, just as for the

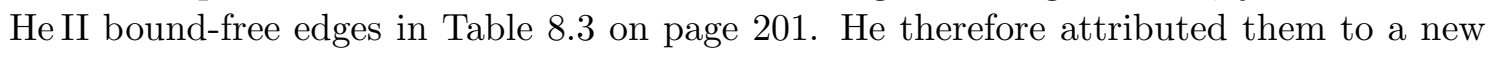
hydrogen state; only later were they identified as due to HeII by Bohr. Miss Cannon constructed the $\mathrm{O}-\mathrm{B}-\mathrm{A}-\mathrm{F}-\mathrm{G}-\mathrm{K}-\mathrm{M}$ sequence with decimal subdivisions that is still in use. After taking part in classifying some 5000 bright stars, she started on the Henry Draper Catalogue, the successor to the Henry Draper Memorial, in 1911 and completed the classification of 225300 stars within four years, at an average of 30 per working hour. She had assistants but must indeed have worked diligently. Her lifetime total amounts to 395000 classifications.

It is interesting to note that this enormous industry was strictly morphological. The classification was thought to be evolutionary, hence the terms early- and late-type stars 
that we still use. Even after Hertzsprung $q_{-1}^{z_{1}}$ and Russell plotted their diagram $m_{-1}^{\mathbf{b}_{1}}$ the nature of the spectral classicifation was unclear. That puzzle was solved after the influence of pressure had been recognized by Pannekoek, Saha had produced his equation for ionization equilibria, and Fowler and Milne had connected stellar colors with ionization differences. The crown came with the 1925 thesis of Cecilia Payne, the first woman to obtain an astronomy $\mathrm{PhD}$ at Harvard, which was later called "undoubtedly the most brilliant $\mathrm{PhD}$ thesis ever written in astronomy" by Struve. She showed that all stars more or less share the same composition, but display different line strengths from Saha-Boltzmann sensitivities to temperature and density. Stellar spectroscopy had matured from morphology to astrophysics $s_{-1}^{\pi_{1}}$

Abundance determination. Finally, quantitative spectrometry arose from work by Russell, Adams, Charlotte Moore, Unsöld, Minnaert, Pannekoek, Struve, Menzel, Allen and others. They took up the pioneering efforts in understanding stellar line formation by Schuster, Schwarzschild and Milne and turned spectral lines into a tool for stellar abundance determination.

This industry started in the first half of the 20th century; the concepts of the "equivalent width" of a spectral line and the "curve of growth" to measure its dependence on the amount of extinction were introduced by Minnaert and coworkers at Utrecht.

Measuring equivalent widths of spectral lines was an industry by its own. For the Sun, landmark Utrecht publications were the Utrecht Atlas of the solar spectrum

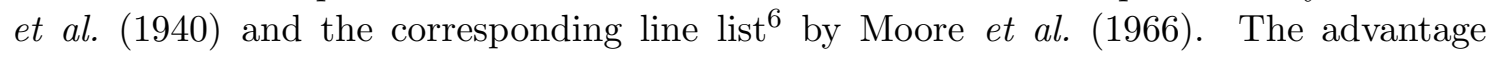

\footnotetext{
${ }^{2}$ Hertzsprung was a Danish amateur astronomer who noted that the intrinsic luminosity of the sharpline stars in Maury's classification must be large since they tend, as a group, to have much smaller proper motions than other stars of comparable apparent magnitude. He wrote in 1905 that the sharp-line stars and the "hot Orion-type" stars "shine the brightest, and among the remaining stars not the red but the yellow ones are the faintest", and two years later continued that "the bright red stars ( $\alpha$ Bootis, $\alpha$ Tauri, $\alpha$ Orionis etc.) are rare per unit volume of space, and those which belong to the normal solar series form by far the greatest number. The bright red stage is therefore quickly traversed.". He published these pioneering articles in an obscure journal on photography, but in 1908 visited Karl Schwarzschild who nearly instantaneously made him professor at Göttingen and took him along to Potsdam. After Schwarzschild's early death (from WW I military service), Hertzsprung completed his career at Leiden.

${ }^{3}$ Hertzsprung and Russell plotted their diagrams independently, Hertzsprung showing an early one to Schwarzschild already in 1908 and Russell displaying one in London in 1913, both with absolute magnitude plotted horizontally. Later in 1913 Russell showed one with absolute magnitude downwards along the yaxis, as we plot the HRD now. It was called the Russell diagram until Bengt Strömgren, two decades later, renamed it the Hertzsprung-Russell diagram.

${ }^{4}$ There is an obvious parallel with large-scale surveys of galaxies. The most ambitious one at present is the Sloan Digital Sky Survey which aims to measure redshifts for one million galaxies and quasars with multi-fiber spectrometers on a special-purpose telescope at Apache Point in New Mexico. Sofar, fewer galaxy spectra have been obtained over the years than stellar spectra inspected by Miss Cannon. Multi-fiber spectrometry now increases the efficiency of deep spectrometry to that of objective-prism spectrometry. These extragalactic efforts are yet rather morphological in nature.

${ }^{5}$ With a preface by Minnaert in Esperanto. Together with his pupils Houtgast and Mulders, Minnaert produced the Utrecht Atlas from photographic spectra taken at Mt. Wilson. Houtgast invented an ingenious microdensitometer that converted the blackness of the photographic plates into solar intensity tracings using cutout cardboard calibration curves that were scanned by galvanometer beams. The technique is described in the Atlas preface - also in English.

${ }^{6}$ An equally impressive piece of work. Lots of persons designated "computers" in the Acknowledgements measured the equivalent widths of the 24000 spectral lines in the Utrecht Atlas by counting the square millimeters of the atlas grid covered by each line. I have the original Atlas copy of Hubenet (a personal computer, as was De Jager) in my office. You can nearly smell the sweat! Each line was also meticulously identified, by checking laboratory wavelengths and multiplet membership, the multiplet measurements
} 


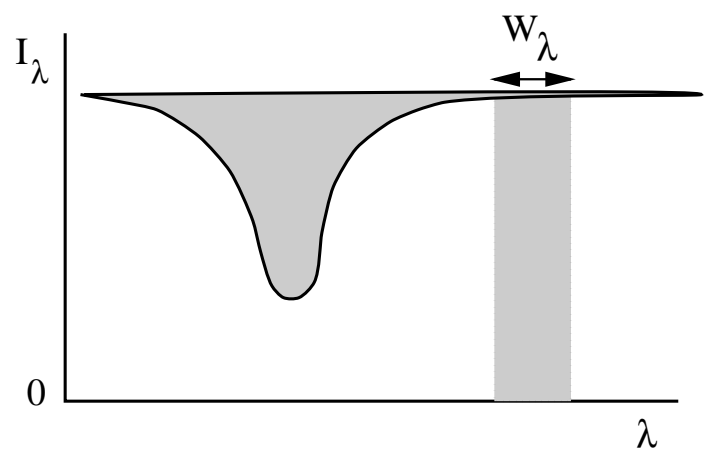

Figure 1.2: The equivalent width of a spectral line is the width of a rectangular piece of fully blocked spectrum with the same spectral area as the integrated line depression.

of using equivalent widths rather than detailed line profiles is that a bad spectrograph deforms a spectral line profile but does not (to first order) affect its area: $a_{-r}^{\pi_{1}}$

Reversing-layer line formation. The reversing layer was first proposed in the celebrated paper by Kirchhooff and Bunsen $(1860)$ :

In a memoir published by one of us [irchhoff 1859 '] it was proved from theoretical considerations that the spectrum of an incandescent gas becomes reversed (that is, the bright lines become changed into dark ones) when a source of light of sufficient intensity, giving a continuous spectrum, is placed behind the luminous gas. From this we may conclude that the solar spectrum, with its dark lines, is nothing else than the reverse of the spectrum which the sun's atmosphere alone would produce.

It was criticized by Forbes who had not found center-limb variations in solar line strengths

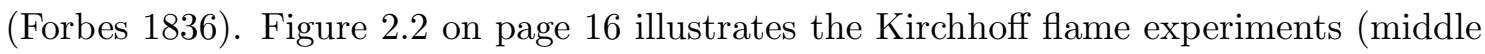
row). A slanted line of sight should indeed cause stronger lines if the Fraunhofer lines came from a thin irradiated layer.

LTE line formation. Not yet...

NLTE line formation. Not yet...

Numerical line formation. Not yet...

Diagnostic line formation. Not yet...

coming from co-author Mrs. Charlotte Moore-Sitterley at the US National Bureau of Standards.

${ }^{7}$ However, scattered light within the spectrograph affects the measured zero level and therefore also $W_{\lambda}$. In traditional grating spectrometers, irregularities in the ruling of the grating (from the ruling engine and from the gradual deterioration of the diamond cutting the grooves) caused ghosts and much stray light. Solar spectrometers were therefore made double pass later, with an intermediate slit between two grating passes to cut out the stray light. Modern gratings are made holographically from laser interference patterns that are registered in photoresist and then etched. They produce much cleaner spectra. Another solar physics trick in the use of gratings is to use échelles that are not cross-dispersed but project all the orders on top of each other. Slits in a predisperser spectrum or narrow-band filters then select the spectral lines of interest in different orders. In this way one may measure just the lines one wants, positioned side-by-side on the detector although they are far apart in the spectrum. See 'Gray (1992)! for more details on stellar spectrographs and gratings. 


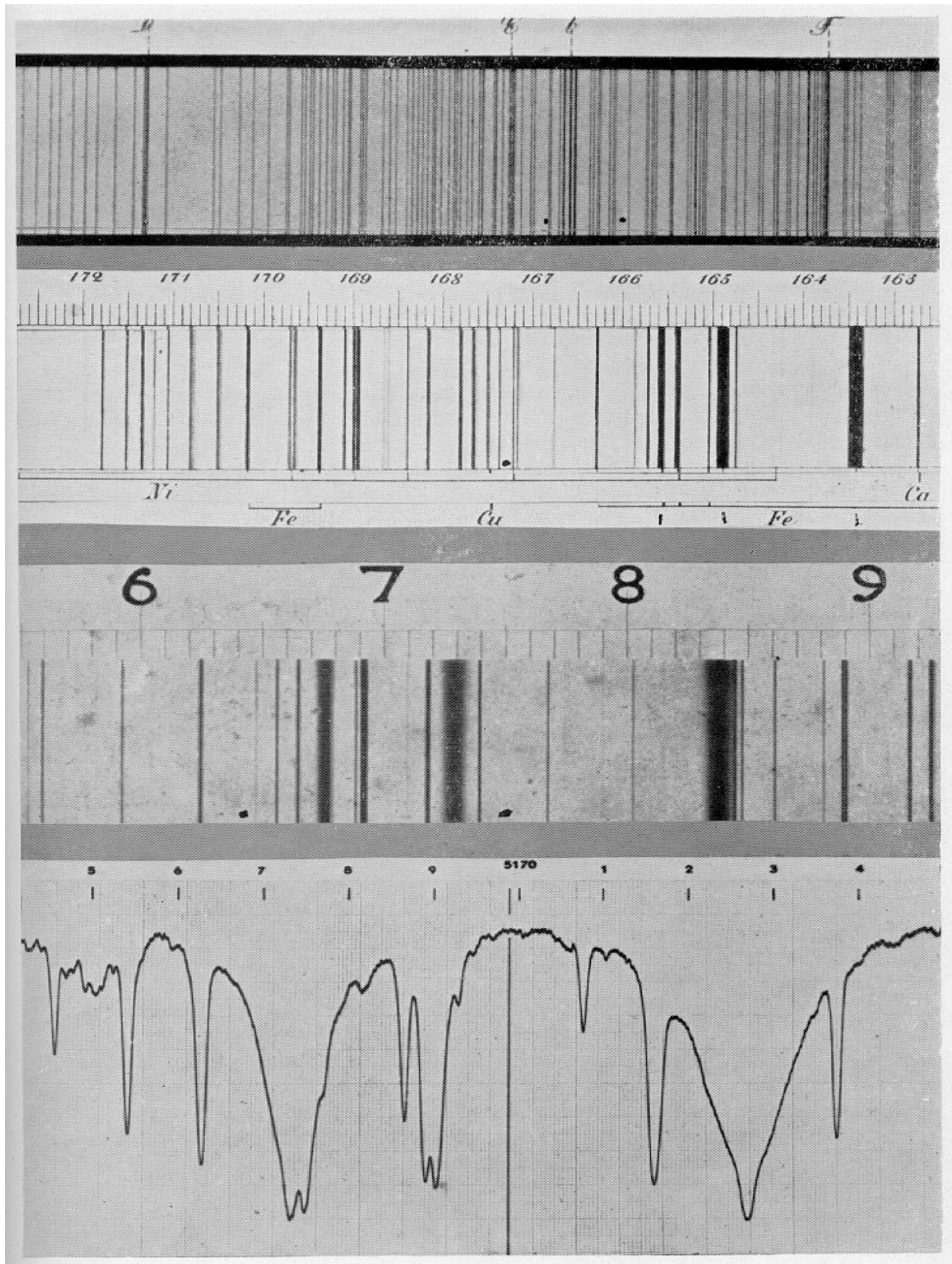

Figure 1.3: Segments of four solar spectrum atlases, respectively the engravings by Fraunhofer (top, 1815) and Kirchhoff (1861), the photographic Rowland atlas (1897) and the Utrecht intensity atlas (1940). The top segment has wavelength increasing to the left. The black dots in the first three segments mark the extent of the next segment. The three strongest lines in the second and third segments constitute the $\mathrm{Mg}$ I b triplet in the green part of the spectrum; Fraunhofer marked them $\mathrm{b}$ at he top of the figure. The $\mathrm{Na} I \mathrm{D}$ lines are marked by a beautifully written $\mathcal{D}$ at left, here cut off_by_Pannekoek__s_bounding box but present with Fraunhofer's solar energy distribution in Figure 2.4 of 'Hearnshaw (1986)! In the bottom segment, the lefthand $\mathrm{MgIb}$ line is blended with an overlapping Fe I line. - The righthand one displays a distinct transition between Doppler core and damping wings. From ㅁannekoek (1961)!' 


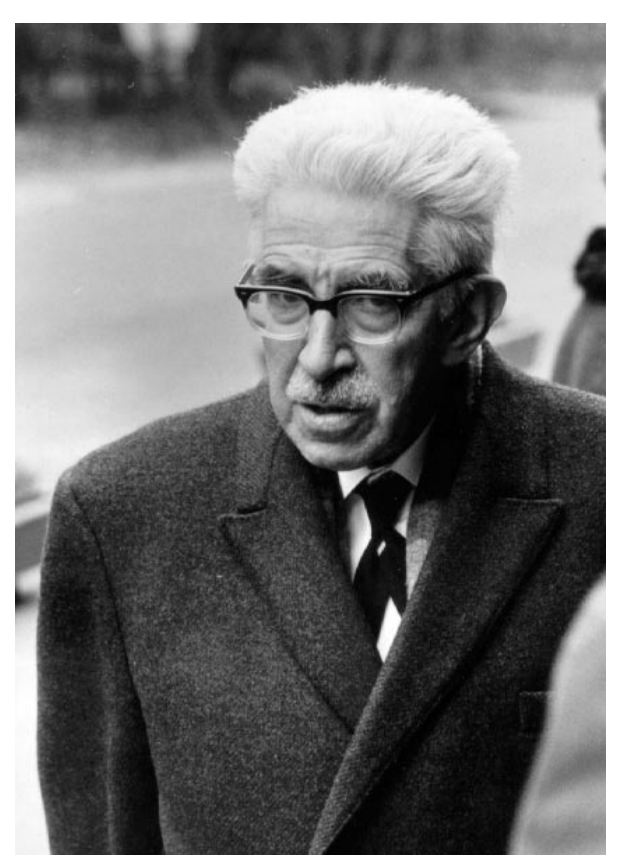

Figure 1.4: Marcel G.J. Minnaert (Brugge 1893 - Utrecht 1970). Minnaert was an idealist who had to flee Belgium after he had participated in a movement during the First World War to get Flanders independent from the Walloons. He was a biologist who wrote a thesis (Gent, 1914) about the influence of light on plant growth. At Utrecht he became a physicist, picking up W.H. Julius' interest in solar spectroscopy. He wrote another thesis (1925) on irregular diffraction, countering Julius' mistaken belief that Fraunhofer lines are due to anomalous refraction effects, and took over the solar physics department after Julius' death in the same year. In 1937 Minnaert succeeded A.A. Nijland (primarily a variable-star observer) as director of Sterrewacht Sonnenborgh and revived it into a spectroscopy-oriented astrophysical institute. In addition, he was a well-known physics pedagogue. The best-known of his books is "The nature of light and colour in the open air" (Minnaert 1954,'), a delightful, highly recommended guide to outdoors physics phenomena. I took this photograph in the Ârnhem Open Air Museum during the "Bilderberg" meeting in 1967. More portraits taken at that meeting are found at http://www.astro.uu.nl/ rutten. 



Figure 1.5: Spectral classifications. Top: Secchi's (1864) four-category scheme. The upper spectrum illustrates type 2 yellow solar-like spectra with many fine lines (Capella, Procyon, Arcturus, Aldebaran). The second spectrum (Secchi type 1) represents white or bluish-white Sirius-like stars with four strong hydrogen lines. The third (type 3 ) is Betelgeuse-like, with wide bands. The fourth (type 4) was a rare class of faint dark-red stars with fuzzy bands that Secchi correctly identified as having to do with carbon. Bottom: Harvard classification. At the bottom, $\zeta$ Puppis displays the Pickering series (at least on Pannekoek's nonfringed print). From 'Pannekoek (1961)i: 


\section{Chapter 2}

\section{Basic Radiative Transfer}

$\mathrm{T}$ his chapter presents the basic quantities and equations of radiative transfer. It is mainly a summary of Chapter 1 of Rybicki and Lightman $(19 \overline{9} \overline{9})]_{4}$ with the same notation:

- extinction is written in terms of the coefficient $\alpha_{\nu}$ per cm rather than the coefficient $\kappa_{\nu}$ per gram that is more commonly employed in books and papers on stellar atmospheres (e.g., Gray 19921$)$ and is also used here in later chapters;

- flux is written as $\mathcal{F}_{\nu}$ rather than $\pi F_{\nu}$ (the same as 'Ǵray $19 \overline{9} \overline{2} \overline{2}$; note that 'R̄ Lightman $19 \overline{9} \overline{9}_{1}^{1}$ write $F_{\nu}$ for $\mathcal{F}_{\nu}$ );

- the Planck function $B_{\nu}$ is defined in intensity units, per steradian, not as flux or energy density;

- the Einstein $A$ and $B$ transition probabilities are defined for radiation into or out of the full $4 \pi$ ster sphere (same as Rybicki and Lightman 1979 intensity (e.g., Chandrasekhar 1939, Gray 1992). The latter values are smaller by a factor of $4 \pi$.

- the photon destruction probability $\varepsilon$ is defined per extinction $\left(\varepsilon=\alpha^{\mathrm{a}} /\left(\alpha^{\mathrm{a}}+\alpha^{\mathrm{s}}\right) \approx\right.$ $\left.C_{u l} /\left(A_{u l}+C_{u l}\right)\right)$, rather than per radiative deexcitation $\left(\varepsilon^{\prime} \approx C_{u l} / A_{u l}\right)$.

\section{$2.1 \quad$ Radiation}

\subsubsection{Local amount}

Intensity. The specific intensity (or surface brightness) $I_{\nu}$ is the proportionality coefficient in:

$$
\begin{aligned}
\mathrm{d} E_{\nu} & \equiv I_{\nu}(\vec{r}, \vec{l}, t)(\vec{l} \cdot \vec{n}) \mathrm{d} A \mathrm{~d} t \mathrm{~d} \nu \mathrm{d} \Omega \\
& =I_{\nu}(x, y, z, \theta, \varphi, t) \cos \theta \mathrm{d} A \mathrm{~d} t \mathrm{~d} \nu \mathrm{d} \Omega
\end{aligned}
$$

with $\mathrm{d} E_{\nu}$ the amount of energy transported through the area $\mathrm{d} A$, at the location $\vec{r}$, with $\vec{n}$ the normal to $\mathrm{d} A$, between times $t$ and $t+\mathrm{d} t$, in the frequency band between $\nu$ and $\nu+\mathrm{d} \nu$, over the solid angle $\mathrm{d} \Omega$ around the direction $\vec{l}$ with polar coordinates $\theta$ and $\varphi$. Units: erg s${ }^{-1} \mathrm{~cm}^{-2} \mathrm{~Hz}^{-1}$ ster $^{-1}$ or $\mathrm{W} \mathrm{m}^{-2} \mathrm{~Hz}^{-1}$ ster $^{-1}$. Frequency to wavelength conversion: $I_{\lambda}=I_{\nu} c / \lambda^{2}$, with $\mathrm{d} \lambda$ and $\mathrm{d} \nu$ both positively increasing. This is the monochromatic intensity; the total intensity is $I \equiv \int_{0}^{\infty} I_{\nu} \mathrm{d} \nu$. 
By defining $I_{\nu}$ per infinitesimally small time interval, area, band width and solid angle, $I_{\nu}$ represents the macroscopic counterpart to specifying the energy carried by a bunch of identical photons along a single "ray". Since photons are the basic carrier of electromagnetic interactions, intensity is the basic macroscopic quantity to use in formulating radiative transfer $\mathbf{I L}_{\mathbf{L}}^{\mathrm{I}_{\mathbf{I}}}$ In particular, the definition per steradian ensures that the intensity along a ray in vacuum does not diminish with travel distance - photons do not decay spontaneously.

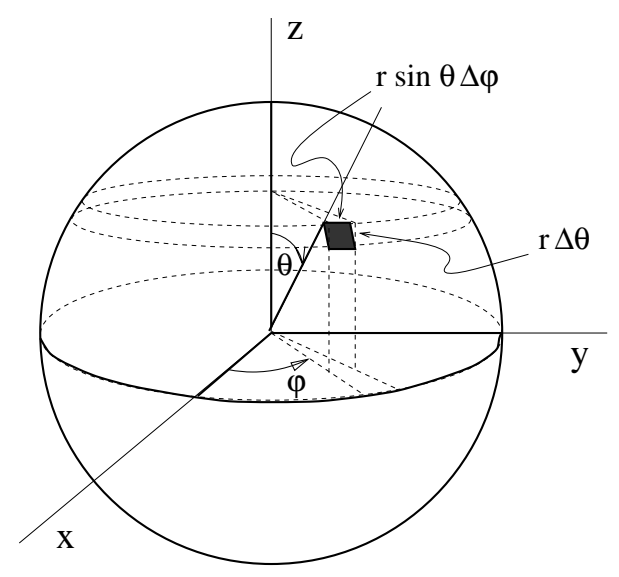

Figure 2.1: Solid angle in polar coordinates. The area of the sphere with radius $r$ limited by $(\theta, \theta+\Delta \theta)$ and $(\varphi, \varphi+\Delta \varphi)$ is $r^{2} \sin \theta \Delta \theta \Delta \phi$ so that $\Delta \Omega=\sin \theta \Delta \theta \Delta \varphi$.

Mean intensity. The mean intensity $J_{\nu}$ averaged over all directions is:

$$
J_{\nu}(\vec{r}, t) \equiv \frac{1}{4 \pi} \int I_{\nu} \mathrm{d} \Omega=\frac{1}{4 \pi} \int_{0}^{2 \pi} \int_{0}^{\pi} I_{\nu} \sin \theta \mathrm{d} \theta \mathrm{d} \varphi .
$$

Units: erg $\mathrm{cm}^{-2} \mathrm{~s}^{-1} \mathrm{~Hz}^{-1} \mathrm{ster}^{-1}$, just as for $I_{\nu}$. In axial symmetry with the $z$-axis $(\theta \equiv 0)$ along the axis of symmetry (vertical stratification only, "plane parallel layers") $J_{\nu}$ simplifies to, using $\mathrm{d} \Omega=2 \pi \sin \theta \mathrm{d} \theta=-2 \pi \mathrm{d} \mu$ with $\mu \equiv \cos \theta$ :

$$
J_{\nu}(z)=\frac{1}{4 \pi} \int_{0}^{\pi} I_{\nu}(z, \theta) 2 \pi \sin \theta \mathrm{d} \theta=\frac{1}{2} \int_{-1}^{+1} I_{\nu}(z, \mu) \mathrm{d} \mu .
$$

This quantity is the one to use when only the availability of photons is of interest, irrespective of the photon origin, for example when evaluating the amount of radiative excitation and ionization.

Flux. The monochromatic flux $\mathcal{F}_{\nu}$ is:

$$
\mathcal{F}_{\nu}(\vec{r}, \vec{n}, t) \equiv \int I_{\nu} \cos \theta \mathrm{d} \Omega=\int_{0}^{2 \pi} \int_{0}^{\pi} I_{\nu} \cos \theta \sin \theta \mathrm{d} \theta \mathrm{d} \varphi
$$

Units: erg $\mathrm{s}^{-1} \mathrm{~cm}^{-2} \mathrm{~Hz}^{-1}$ or $\mathrm{W} \mathrm{m}{ }^{-2} \mathrm{~Hz}^{-1}$. This is the net flow of energy per second through an area placed at location $\vec{r}$ perpendicular to $\vec{n}$. It is the quantity to use for specifying the energetics of radiation transfer, through stellar interiors, stellar atmospheres,

\footnotetext{
${ }^{1}$ Except for polarimetry, which needs three more Stokes parameters discussed in Section ${ }^{1} 6.1-1$.
} 
planetary atmospheres or space. In principle, flux is a vector. In stellar-atmosphere practice, the radial direction is always implied, outward positive, so that

$$
\begin{aligned}
\mathcal{F}_{\nu}(z) & =\int_{0}^{2 \pi} \int_{0}^{\pi / 2} I_{\nu} \cos \theta \sin \theta \mathrm{d} \theta \mathrm{d} \varphi+\int_{0}^{2 \pi} \int_{\pi / 2}^{\pi} I_{\nu} \cos \theta \sin \theta \mathrm{d} \theta \mathrm{d} \varphi \\
& =\int_{0}^{2 \pi} \int_{0}^{\pi / 2} I_{\nu} \cos \theta \sin \theta \mathrm{d} \theta \mathrm{d} \varphi-\int_{0}^{2 \pi} \int_{0}^{\pi / 2} I_{\nu}(\pi-\theta) \cos \theta \sin \theta \mathrm{d} \theta \mathrm{d} \varphi \\
& \equiv \mathcal{F}_{\nu}^{+}(z)-\mathcal{F}_{\nu}^{-}(z),
\end{aligned}
$$

with both the outward flux $\mathcal{F}_{\nu}^{+}$and the inward flux $\mathcal{F}_{\nu}^{-}$positive. Isotropic radiation has $\mathcal{F}_{\nu}^{+}=\mathcal{F}_{\nu}^{-}=\pi I_{\nu}$ and $\mathcal{F}_{\nu}=0$. For axial symmetry:

$$
\begin{aligned}
\mathcal{F}_{\nu}(z) & =2 \pi \int_{0}^{\pi} I_{\nu} \cos \theta \sin \theta \mathrm{d} \theta \\
& =2 \pi \int_{0}^{1} \mu I_{\nu} \mathrm{d} \mu-2 \pi \int_{0}^{-1} \mu I_{\nu} \mathrm{d} \mu \\
& =\mathcal{F}_{\nu}^{+}(z)-\mathcal{F}_{\nu}^{-}(z) .
\end{aligned}
$$

The flux emitted by a non-irradiated spherical star per $\mathrm{cm}^{2}$ of its surface at radius $r=R$ is

$$
\mathcal{F}_{\nu}^{\text {surface }} \equiv \mathcal{F}_{\nu}^{+}(r=R)=\pi \overline{I_{\nu}^{+}}
$$

with $\overline{I_{\nu}^{+}}$the intensity averaged over the apparent stellar disk that is received by a distant observer, or the intensity emitted by a Lambert-like star with isotropic $I_{\nu}^{+}$for $\mu>0$. This equality is the reason that flux is often written as $\pi F \equiv \mathcal{F}$ so that $F=\bar{I}$, with $F$ called the "astrophysical flux". The flux received at Earth ("irradiance") from a star with radius $R$ at distance $D$ is:

$$
\mathcal{R}_{\nu}=\frac{4 \pi R^{2}}{4 \pi D^{2}} \mathcal{F}_{\nu}^{\text {surface }}=\frac{\pi R^{2}}{D^{2}} \bar{I}_{\nu}
$$

Density. The radiation energy density $u_{\nu}$ is:

$$
u_{\nu}=\frac{1}{c} \int I_{\nu} \mathrm{d} \Omega
$$

with units erg $\mathrm{cm}^{-3} \mathrm{~Hz}^{-1}$ or $\mathrm{J} \mathrm{m}^{-3} \mathrm{~Hz}^{-1}$. integration over $\Delta V$ and over all beam directions gives the radiative energy $E_{\nu} \mathrm{d} \nu$ contained within $\Delta V$ across the bandwidth $\mathrm{d} \nu$ as $E_{\nu} \mathrm{d} \nu=$

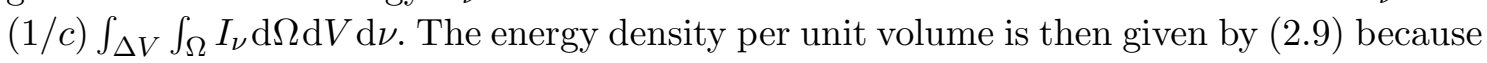
for sufficiently small volume $\Delta V$, the intensity $I_{\nu}$ is homogeneous within $\Delta V$ so that the two integrations are independent.

Isotropic radiation has $u_{\nu}=(4 \pi / c) J_{\nu}$ with $I_{\nu}=J_{\nu}$ in all directions, filling a unit sphere in $1 / c$ seconds. The monochromatic energy density has $u_{\nu}=(4 \pi / c) J_{\nu}$ and the total energy density has $u=(4 \pi / c) \int_{0}^{\infty} J_{\nu} \mathrm{d} \nu=(4 \pi / c) J$. When LTE and linear anisotropy are good approximations (as in stellar interiors) $J_{\nu} \approx B_{\nu}$ so that the total energy density is, with $(2 . \overline{9} 5)$ on page $\underline{3} \overline{1} \overline{1}:$ :

$$
u=\int u_{\nu} \mathrm{d} \nu=\frac{1}{c} \iint B_{\nu} \mathrm{d} \Omega \mathrm{d} \nu=\frac{4 \sigma}{c} T^{4}
$$

and the total photon density is, with $T$ in $\mathrm{K}$ (Bowers and Deeming $1 \overline{9} \overline{8} \overline{4}$ p. 22 ):

$$
N_{\text {photon }}=\int_{0}^{\infty} \frac{u_{\nu}}{h \nu} \mathrm{d} \nu \approx 20 T^{3} \mathrm{~cm}^{-3}
$$




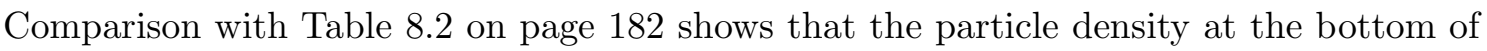
the VALIII photosphere (where $J_{\nu} \approx \bar{B}_{\nu}$ for all $\nu$ ) is much higher.

Pressure. The radiation pressure $p_{\nu}$ is (Gray p. 95; Rybicki \& Lightman p. 6):

$$
p_{\nu}=\frac{1}{c} \int I_{\nu} \cos ^{2} \theta \mathrm{d} \Omega
$$

with units dyne $\mathrm{cm}^{-2} \mathrm{~Hz}^{-1}$ or $\mathrm{N} \mathrm{m}^{-2} \mathrm{~Hz}^{-1}$. Isotropic radiation has $p_{\nu}=u_{\nu} / 3$ and $p=u / 3$. Radiation pressure is analogous to gas pressure, being the pressure of the photon gas. It is a scalar for isotropic radiation fields; a force is exerted only along a photon pressure gradient

Moments of the intensity. For axial symmetry (plane-parallel layers) the first three moments of the intensity with respect to $\mu$ are:

$$
\begin{aligned}
J_{\nu}(z) & \equiv \frac{1}{2} \int_{-1}^{+1} I_{\nu} \mathrm{d} \mu \\
H_{\nu}(z) & \equiv \frac{1}{2} \int_{-1}^{+1} \mu I_{\nu} \mathrm{d} \mu \\
K_{\nu}(z) & \equiv \frac{1}{2} \int_{-1}^{+1} \mu^{2} I_{\nu} \mathrm{d} \mu
\end{aligned}
$$

Each has the dimension of intensity and each is already familiar. The mean intensity $J_{\nu}$ was defined in (2.2). $H_{\nu}$ is called the Eddington flux and has $H_{\nu}=\mathcal{F}_{\nu} / 4 \pi=F_{\nu} / 4$, with $\mathcal{F}_{\nu}$ the real flux and $F_{\nu}=\mathcal{F}_{\nu} / \pi$ the astrophysical flux. $K_{\nu}$ is called the $K$ integral and is related to radiation pressure by $p_{\nu}=(4 \pi / c) K_{\nu} . J_{\nu}$ and $K_{\nu}$ are always positive; $H_{\nu}$ may be negative. The same definitions produce the spectrum-integrated total $J, H$ and $K$ from $I$ because the integrations over $\nu$ and $\mu$ are independent and therefore interchangeable.

\subsubsection{Local change}

Emission. The monochromatic emissivity $j_{\nu}$ per $\mathrm{cm}^{3}$ is defined by:

$$
\mathrm{d} E_{\nu} \equiv j_{\nu} \mathrm{d} V \mathrm{~d} t \mathrm{~d} \nu \mathrm{d} \Omega
$$

with $\mathrm{d} E_{\nu}$ the energy locally added to the radiation in volume $\mathrm{d} V$ per frequency bandwidth $\mathrm{d} \nu$ during time interval $\mathrm{d} t$ in directions $\mathrm{d} \Omega$. Units of $j_{\nu}$ : erg $\mathrm{cm}^{-3} \mathrm{~s}^{-1} \mathrm{~Hz}^{-1} \mathrm{ster}^{-1}$. The intensity contribution from local emission to a beam is

$$
\mathrm{d} I_{\nu}(s)=j_{\nu}(s) \mathrm{d} s
$$

where $s$ measures geometrical path length along the beam in $\mathrm{cm}$.

\footnotetext{
${ }^{2}$ The term radiation pressure is often used for the mechanical vector force on an object when it absorbs photons from a single direction. The scalar expression $\left(\mathbf{2}_{2} . \mathbf{1}_{1}^{\prime}\right)$ is only valid when the radiation behaves like a gas of particles that locally move at random. The complete definition of radiation pressure is given in Eq. (16) on page 94 of 'Baschek and Scholz (1982)! It reduces to (2.12') only if the source function is isotropic (no dependence on $\bar{\mu}$ ) and if the intensity is not too anisotropic. More precisely, the Eddington approximation (4.54, ) on page $91_{1}^{\prime}$ should hold. The latter is exact when $I_{\nu}$ obeys linear anisotropy in $\mu$.
} 
Extinction. The monochromatic extinction coefficient specifies the energy fraction taken from a beam in terms of a geometrical cross-section in $\mathrm{cm}^{2}$. It may, just as the emissivity, be defined per particle, per gram, or per $\mathrm{cm}^{3}$. Per particle:

$$
\mathrm{d} I_{\nu} \equiv-\sigma_{\nu} n I_{\nu} \mathrm{d} s
$$

with $\sigma_{\nu}$ the monochromatic extinction coefficient or cross-section per particle measured in $\mathrm{cm}^{2}$ and $n$ the absorber density (particles $\mathrm{cm}^{-3}$ ). The definition per $\mathrm{cm}$ path length is:

$$
\mathrm{d} I_{\nu} \equiv-\alpha_{\nu} I_{\nu} \mathrm{d} s
$$

with $\alpha_{\nu}=\sigma_{\nu} n$ the monochromatic linear extinction coefficient (units $\mathrm{cm}^{-1}$ ), or the monochromatic volume extinction coefficient when interpreted as cross-section per unit volume $\left(\mathrm{cm}^{2} \mathrm{~cm}^{-3}=\mathrm{cm}^{-1}\right)$. The definition per gram is:

$$
\mathrm{d} I_{\nu} \equiv-\kappa_{\nu} \rho I_{\nu} \mathrm{d} s
$$

with $\kappa_{\nu}$ the monochromatic mass extinction coefficient or the cross-section per unit mass $\left(\mathrm{cm}^{2} \mathrm{~g}^{-1}\right)$ and $\rho$ the density $\left(\mathrm{g} \mathrm{cm}^{-3}\right)$. This definition is the one usually employed in analyses of stellar atmospheres, with $\kappa_{\nu}$ usually called opacity and often absorption coefficient $t_{L \mathbf{r}}^{B_{1}}$ Usually, this coefficient includes a negative correction for the presence of induced ("stimulated") emission. In these lecture notes, $\alpha_{\nu}$ and $\kappa_{\nu}$ always include such correction while $\sigma_{\nu}$ does not.

Source function. The source function is:

$$
S_{\nu} \equiv j_{\nu} / \alpha_{\nu}
$$

Units: erg $\mathrm{cm}^{-2} \mathrm{~s}^{-1} \mathrm{~Hz}^{-1} \mathrm{ster}^{-1}$, the same as intensity. When multiple processes contribute to local emission and extinction the total source function is

$$
S_{\nu}^{\text {tot }}=\frac{\sum j_{\nu}}{\sum \alpha_{\nu}}
$$

where each pair of $j_{\nu}$ and $\alpha_{\nu}$ describes a different process. For example, the source function at a frequency $\nu$ within a spectral line is

$$
S_{\nu}^{\mathrm{tot}}=\frac{j_{\nu}^{c}+j_{\nu}^{l}}{\alpha_{\nu}^{c}+\alpha_{\nu}^{l}}=\frac{S_{\nu}^{c}+\eta_{\nu} S_{\nu}^{l}}{1+\eta_{\nu}}
$$

with $\eta_{\nu} \equiv \alpha_{\nu}^{l} / \alpha_{\nu}^{c}$ the line-to-continuum extinction ratio, $S_{\nu}^{c}$ the continuum source function and $S_{\nu}^{l}$ the line source function. Each may again be made up by different processes, $S_{\nu}^{c}$ by multiple continuum ones and $S_{\nu}^{l}$ by overlapping spectral lines. Note that the subscript $\nu$ in $S_{\nu}$ implies measurement per bandwidth interval, just as for $I_{\nu}, J_{\nu}, \mathcal{F}_{\nu}$ and $j_{\nu}$, whereas the subscript $\nu$ in $\sigma_{\nu}, \alpha_{\nu}$ and $\kappa_{\nu}$ simply expresses wavelength dependence.

\footnotetext{
${ }^{3}$ Not a good name when $\kappa_{\nu}$ also includes extinction from scattering interactions in which photons are not destroyed but only re-directed. In that case, many authors use "true absorption" for the part describing photon destruction. I prefer to follow Zwaan in using "extinction" for the total coefficient. (But I have switched from "emission coefficient" to "emissivity" in the sixth edition of these notes.)
} 


\subsection{Transport equation}

\subsubsection{Transport along a ray}

The radiation transport equation is:

$$
\mathrm{d} I_{\nu}(s)=I_{\nu}(s+\mathrm{d} s)-I_{\nu}(s)=j_{\nu}(s) \mathrm{d} s-\alpha_{\nu}(s) I_{\nu}(s) \mathrm{d} s
$$

or

or

$$
\frac{\mathrm{d} I_{\nu}}{\mathrm{d} s}=j_{\nu}-\alpha_{\nu} I_{\nu}
$$

$$
\frac{\mathrm{d} I_{\nu}}{\alpha_{\nu} \mathrm{d} s}=S_{\nu}-I_{\nu}
$$

with $s$ measured along the beam in the propagation direction.

Discussion. This basic equation expresses that photons do not decay spontaneously so that the intensity along a ray does not change unless photons are added to the beam or taken from $i_{1-1}^{\pi_{1}}$, without such processes, intensity is invariant along rays.

The versions (2.25i) and $(2.26 i)$ differ trivially in notation but drastically in their domain of application. In stellar photospheres one often meets LTE (Local Thermodynamic Equilibrium, see Section $2 . \overline{5}$ on page ${ }_{2}^{\overline{2}} \overline{8}_{-1}^{\prime} f$ ) or near-LTE conditions having $S_{\nu}=B_{\nu}(T)$ or $S_{\nu} \approx B_{\nu}(T)$ with $B_{\nu}$ the Planck function (2.92). The combination $\left(\alpha_{\nu}, S_{\nu}\right)$ then presents a much more "orthogonal" parameter space to describe radiative transfer than the combination $\left(\alpha_{\nu}, j_{\nu}\right)$. The latter two may each vary orders of magnitude over the narrow extent of a spectral line whereas their variations cancel completely or closely in the ratio $S_{\nu}=j_{\nu} / \alpha_{\nu}$. Photospheric line formation is therefore described in terms of the parameter $\alpha_{\nu}$ which details atomic particle properties (such as the gas composition, the degree of ionization and excitation, the probability of spectral line transitions at some frequency, the nature and amount of line broadening) and which sets the transparency of the medium (in particular the depth above which the gas is sufficiently transparent that photons may escape towards our telescope), and the parameter $S_{\nu}$ which describes the thermodynamic state of the medium as an ensemble of particles and photons. The two parameters may depend on each other in highly complex fashion when LTE does not hold, but they are less closely related than $\alpha_{\nu}$ and $j_{\nu}$ even then.

In contrast, one doesn't use source functions to describe radiation in or from tenuous outer atmospheres such as the solar corona. Coronal extinction is often negligible for the X-ray photons emitted there, so that the description is simply in terms of emissivities. The transport than simplifies to photon loss through escape (which constitutes the energy drain that limits the coronal temperature).

Optical length and thickness. The monochromatic optical path length $\mathrm{d} \tau_{\nu}$ measured along the beam across a layer of geometrical thickness $\mathrm{d} s$ is:

$$
\mathrm{d} \tau_{\nu}(s) \equiv \alpha_{\nu}(s) \mathrm{d} s ;
$$

\footnotetext{
${ }^{4}$ It also expresses that photons are bosons which simply add up without pushing one another aside; they actually like to join together in the same quantum state (stimulated emission). In contrast, Archimedes and the water in his bathtub were made of mutually-exclusive fermions. Neutrinos are much like photons, but they are fermions rather than bosons; they suffer inhibited emission (induced extinction) rather than induced emission because excited states cannot emit a fermion where there is one already present (Rybicki and Lightman 1979 p. 316, She 19.'p. 8).
} 
the monochromatic optical thickness ${ }_{L_{1}}^{\bar{I}_{1}}$ of a medium with total thickness $D$ is

$$
\tau_{\nu}(D)=\int_{0}^{D} \alpha_{\nu}(s) \mathrm{d} s
$$

again measured along the beam. For extinction only (no emission, $j_{\nu}=0$ ):

$$
I_{\nu}(D)=I_{\nu}(0) \mathrm{e}^{-\tau_{\nu}(D)}
$$

The transition between small and large extinction lies at the $1 /$ e value, i.e., at optical thickness $\tau_{\nu}=1$. A layer is optically thick for $\tau_{\nu}(D)>1$ and optically thin for $\tau_{\nu}(D)<1$. The optical photon mean free path $\left\langle\tau_{\nu}(s)>\right.$ is:

$$
<\tau_{\nu}(s)>\equiv \frac{\int_{0}^{\infty} \tau_{\nu}(s) \mathrm{e}^{-\tau_{\nu}(s)} \mathrm{d} \tau_{\nu}(s)}{\int_{0}^{\infty} \mathrm{e}^{-\tau_{\nu}(s)} \mathrm{d} \tau_{\nu}(s)}=1
$$

and the geometrical photon mean free path in a homogeneous medium is:

$$
l_{\nu}=\frac{<\tau_{\nu}(s)>}{\alpha_{\nu}}=\frac{1}{\alpha_{\nu}}=\frac{1}{\kappa_{\nu} \rho} .
$$

In an inhomogeneous medium this estimate represents the local free path. With $\tau_{\nu}$ and $S_{\nu}(2.26 i)$ becomes

$$
\frac{\mathrm{d} I_{\nu}}{\mathrm{d} \tau_{\nu}}=S_{\nu}-I_{\nu}
$$

from which the integral form of the transport equation follows formally:

$$
I_{\nu}\left(\tau_{\nu}\right)=I_{\nu}(0) \mathrm{e}^{-\tau_{\nu}}+\int_{0}^{\tau_{\nu}} S_{\nu}\left(t_{\nu}\right) \mathrm{e}^{-\left(\tau_{\nu}-t_{\nu}\right)} \mathrm{d} t_{\nu}
$$

Homogeneous medium. For a medium in which $S_{\nu}$ does not vary with location simplifies to:

$$
I_{\nu}(D)=I_{\nu}(0) \mathrm{e}^{-\tau_{\nu}(D)}+S_{\nu}\left(1-\mathrm{e}^{-\tau_{\nu}(D)}\right) .
$$

Thus, when the object is optically thick

$$
I_{\nu}(D) \approx S_{\nu}
$$

and when it is optically thin

$$
I_{\nu}(D) \approx I_{\nu}(0)+\left[S_{\nu}-I_{\nu}(0)\right] \tau_{\nu}(D) .
$$

These basic solutions are illustrated in Figure $2 \overline{2} .2$ and Exercise

\footnotetext{
${ }^{5}$ The term "optical" is used in this context for the whole electromagnetic spectrum, from gamma rays to radio waves, and even for neutrinos.

${ }^{6}$ If scattering or stimulated emission is important, $S_{\nu}$ depends on the local angle-averaged intensity $J_{\nu}$ and may vary with location even if the matter component of the medium is homogeneous.
} 

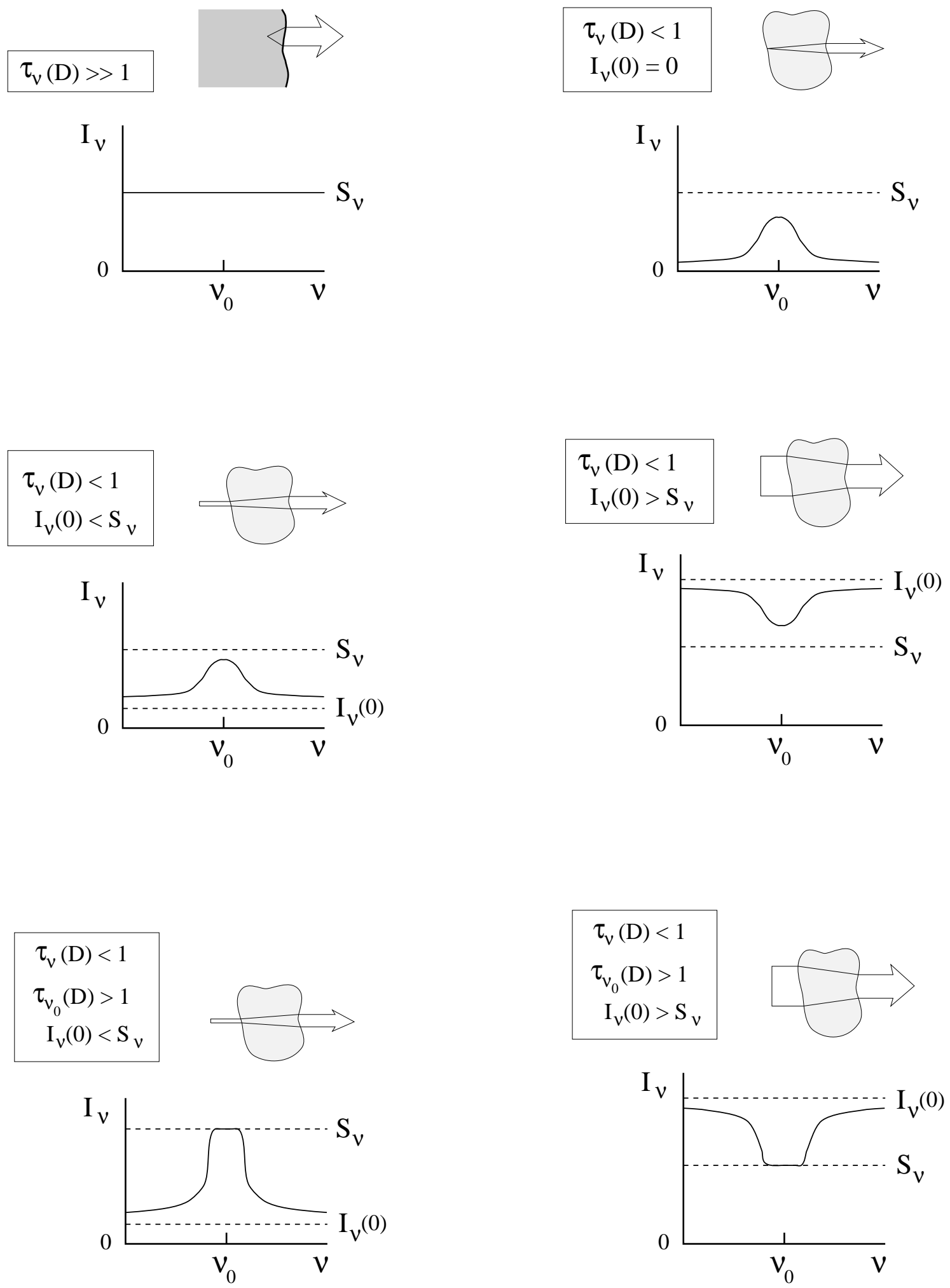

Figure 2.2: $\quad$ Spectral lines from a homogeneous object with $S_{\nu}^{l}=S_{\nu}^{c}=S_{\nu}$ everywhere, according to $\left(2.35_{1}\right)-\left(2.36_{1}^{1}\right)$. No lines emerge when the object is optically thick (top left). When it is optically thin, emission lines emerge when the object is not back-lit $\left(I_{\nu}(0)=0\right.$, top right), or when it is illuminated with $I_{\nu}(0)<S_{\nu}$. Absorption lines emerge only when the object is optically thin and $I_{\nu}(0)>S_{\nu}$. The emergent lines saturate to $I_{\nu} \approx S_{\nu}$ when the object is optically thick at line center. 


\subsubsection{Transport through an atmosphere}

Optical depth. Sofar, $\tau_{\nu}$ has denoted optical thickness, measured along the beam in the photon propagation direction. Since this course is mostly concerned with objects of which the total optical thickness along the line of sight is far too large to be of any interest, I now switch notation and use $\tau_{\nu}$ from here on for radial optical depth, as most authors do. In the context of stellar atmospheres, one often adopts axial symmetry with the $z$-axis radially outward along the axis of symmetry (perpendicular to the surface of a spherical star consisting of horizontally homogeneous shells). The viewing angle $\mu$ is then defined by $\mu \equiv \cos \theta$ where $\theta$ specifies the angle between the line of sight and the $z$-axis. In addition, plane-parallel stratification is usually assumed so that the angle $\mu$ does not vary along the line of sight as is the case for curved layers. I do the same throughout this course. In some cases, I will use the angle-dependent optical depth $\tau_{\nu \mu}$

$$
\mathrm{d} \tau_{\nu \mu} \equiv-\alpha_{\nu} \frac{\mathrm{d} z}{|\mu|}
$$

which is measured along the viewing direction, with $\mu>0$ outwards for outgoing photons and $\mu<0$ inwards for incoming photons. In most cases, however, I will use the radial optical depth $\tau_{\nu}$ which for a geometrical location with $z=z_{0}$ is given by

$$
\tau_{\nu}\left(z_{0}\right)=\int_{\infty}^{z_{0}}-\alpha_{\nu} \mathrm{d} z=\int_{z_{0}}^{\infty} \alpha_{\nu} \mathrm{d} z
$$

and which measures the optical depth along the radial line of sight with $\mu=1$, from $\tau_{\nu}=0$ at the observer's eye located at $z=\infty$. For a frequency within a spectral line the total optical depth is given by

$$
\mathrm{d} \tau_{\nu}^{\text {total }}=-\left(\alpha_{\nu}^{c}+\alpha_{\nu}^{l}\right) \mathrm{d} z=\left(1+\eta_{\nu}\right) \mathrm{d} \tau_{\nu}^{c}
$$

with $\eta_{\nu} \equiv \alpha_{\nu}^{l} / \alpha_{\nu}^{c}$ and $\tau_{\nu}^{c}$ the continuum optical depth.

Standard plane-parallel transport equation. The use of radial optical depth delivers the standard form of the radiation transport equation in plane-parallel geometry:

$$
\mu \frac{\mathrm{d} I_{\nu}}{\mathrm{d} \tau_{\nu}}=I_{\nu}-S_{\nu}
$$

Formal solution. For axial symmetry the inward directed intensity $(\mu<0)$ is, using $t_{\nu} \equiv \int_{\infty}^{z}-\alpha_{\nu}(z) \mathrm{d} z$ as $\tau_{\nu}$-like integration variable (e.g., Gray $1992 \mathrm{p}$. 114):

$$
I_{\nu}^{-}\left(\tau_{\nu}, \mu\right)=-\int_{0}^{\tau_{\nu}} S_{\nu}\left(t_{\nu}\right) \mathrm{e}^{-\left(t_{\nu}-\tau_{\nu}\right) / \mu} \mathrm{d} t_{\nu} / \mu
$$

and the outward directed intensity $(\mu>0)$ is:

$$
I_{\nu}^{+}\left(\tau_{\nu}, \mu\right)=+\int_{\tau_{\nu}}^{\infty} S_{\nu}\left(t_{\nu}\right) \mathrm{e}^{-\left(t_{\nu}-\tau_{\nu}\right) / \mu} \mathrm{d} t_{\nu} / \mu .
$$


Eddington-Barbier approximation. The emergent intensity at the stellar surface $\left(\tau_{\nu}=0, \mu>0\right)$ is given by:

$$
I_{\nu}^{+}\left(\tau_{\nu}=0, \mu\right)=\int_{0}^{\infty} S_{\nu}\left(t_{\nu}\right) \mathrm{e}^{-t_{\nu} / \mu} \mathrm{d} t_{\nu} / \mu .
$$

Substitution of

$$
S_{\nu}\left(\tau_{\nu}\right)=\sum_{n=0}^{\infty} a_{n} \tau_{\nu}^{n}=a_{0}+a_{1} \tau_{\nu}+a_{2} \tau_{\nu}^{2}+\ldots+a_{n} \tau_{\nu}^{n}
$$

and use of $\int_{0}^{\infty} x^{n} \exp (-x) \mathrm{d} x=n$ ! gives

$$
I_{\nu}^{+}\left(\tau_{\nu}=0, \mu\right)=a_{o}+a_{1} \mu+2 a_{2} \mu^{2}+\ldots+n ! a_{n} \mu^{n} .
$$

Truncation of both expansions after the first two terms produces the important EddingtonBarbier approximation

$$
I_{\nu}^{+}\left(\tau_{\nu}=0, \mu\right) \approx S_{\nu}\left(\tau_{\nu}=\mu\right)
$$

which is exact when $S_{\nu}$ varies linearly with $\tau_{\nu}$. Likewise for the emergent flux:

$$
\mathcal{F}_{\nu}^{+}(0) \approx \pi S_{\nu}\left(\tau_{\nu}=2 / 3\right) .
$$

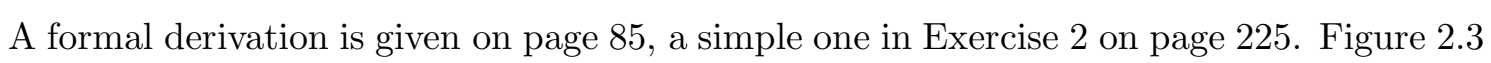
illustrates the Eddington-Barbier approximation simplistically, Figure 2.41 its application to solar limb darkening, Figure 2.5 its application to line formation at increasing sophistication.
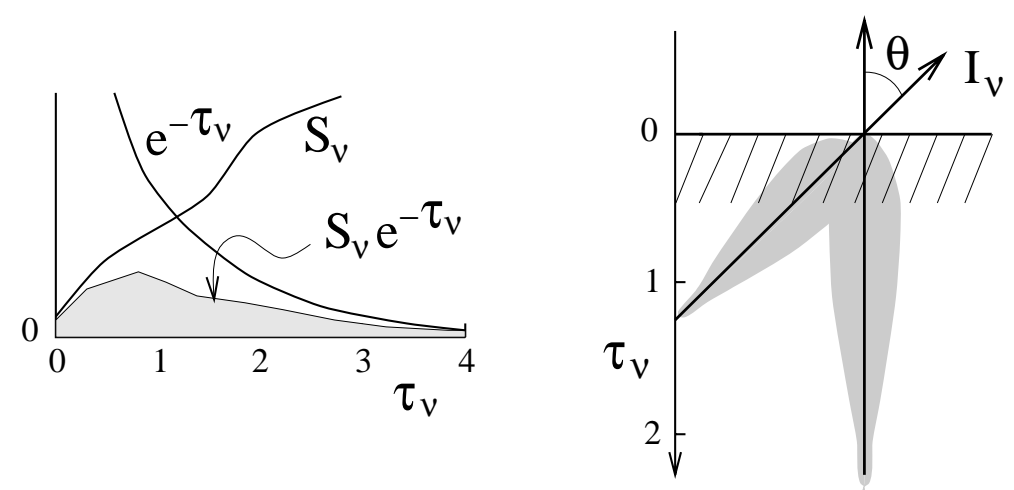

Figure 2.3: The Eddington-Barbier approximation. Left: the integrand $S_{\nu} \exp \left(-\tau_{\nu}\right)$ measures the contribution to the radially emergent intensity $I_{\nu}\left(\tau_{\nu}=0, \mu=1\right)$ from layers with different optical depth $\tau_{\nu}$. The value of $S_{\nu}$ at $\tau_{\nu}=1$ is a good estimator of the area under the integrand curve, i.e., the total contribution. Right: for a slanted beam the characteristic Eddington-Barbier depth is shallower than for a radial beam; it lies at $\tau_{\nu}=\mu$.

\subsection{Line transitions}

Bound-bound transitions between the lower $l$ and upper $u$ energy levels of a discrete electromagnetic energy-storing system such as an atom, ion or molecule may occur as:

- radiative excitation; 

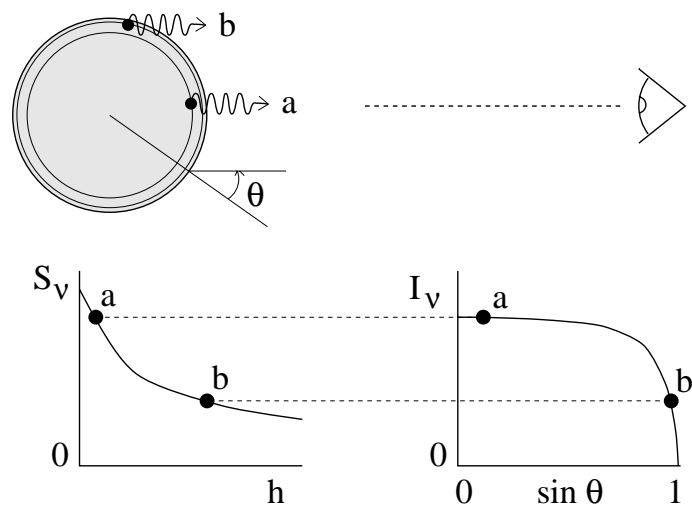

Figure 2.4: Solar limb darkening. The viewing angle $\theta$ increases with the fractional radius $r / R_{\odot}=\sin \theta$ of the apparent solar disk. The emergent intensity samples shallower layers towards the limb, with smaller source function. The final drop at $r / R_{\odot}=1$ marks the viewing angle at which the sun becomes optically thin. Note that substantial decrease of $\mu=\cos \theta$ is reached only close to the limb, for $r / R_{\odot}=\sin \theta_{-}=$ $\left(1-\mu^{2}\right)^{1 / 2}$ close to unity (Table 17.2 in on page 159 ). The off-limb extension to this sketch is given in Figure

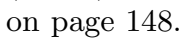

- spontaneous radiative deexcitation;

- induced radiative deexcitation;

- collisional excitation;

- collisional deexcitation.

\subsubsection{Einstein coefficients}

Spontaneous deexcitation. The Einstein coefficient for spontaneous deexcitation is:

$$
\begin{aligned}
A_{u l} \equiv & \text { transition probability for spontaneous deexcitation from } \\
& \text { state } u \text { to state } l \text { per sec per particle in state } u \text {. }
\end{aligned}
$$

In the absence of collisions and of any other transitions than the $u$ one, the mean lifetime of particles in state $u$ is $\Delta t=1 / A_{u l}$ s. The corresponding spread in energy is (Heisenberg): $\Delta E=h /(2 \pi \Delta t)$ or $\Delta \nu=\gamma^{\mathrm{rad}} /(2 \pi)$ with $\gamma^{\mathrm{rad}} \equiv 1 / \Delta t$ the radiative damping constant. This "natural" broadening process defines an emission probability distribution $\psi\left(\nu-\nu_{0}\right)$ around the line center at $\nu=\nu_{0}$ that is given by the area-normalized Lorentz profile:

$$
\psi\left(\nu-\nu_{0}\right)=\frac{\gamma^{\mathrm{rad}} / 4 \pi^{2}}{\left(\nu-\nu_{0}\right)^{2}+\left(\gamma^{\mathrm{rad}} / 4 \pi\right)^{2}} .
$$

The $A_{u l}$ coefficient is a summation over the profile, describing the transition probability for the whole line; the probability per unit of bandwidth is given by $A_{u l} \psi\left(\nu-\nu_{0}\right)$ since $\psi\left(\nu-\nu_{0}\right)$ is measured per Hertz. The spontaneous deexcitation rate per $\mathrm{cm}^{3}$ is given by the product $n_{u} A_{u l}$.

The emission-profile shape function is discussed in more detail in Section 3.31 on page ${ }_{5} 52_{1}^{1}$ ff together with other line broadening processes. The latter are usually much more important than radiative damping. For a static atmosphere and assuming that each 

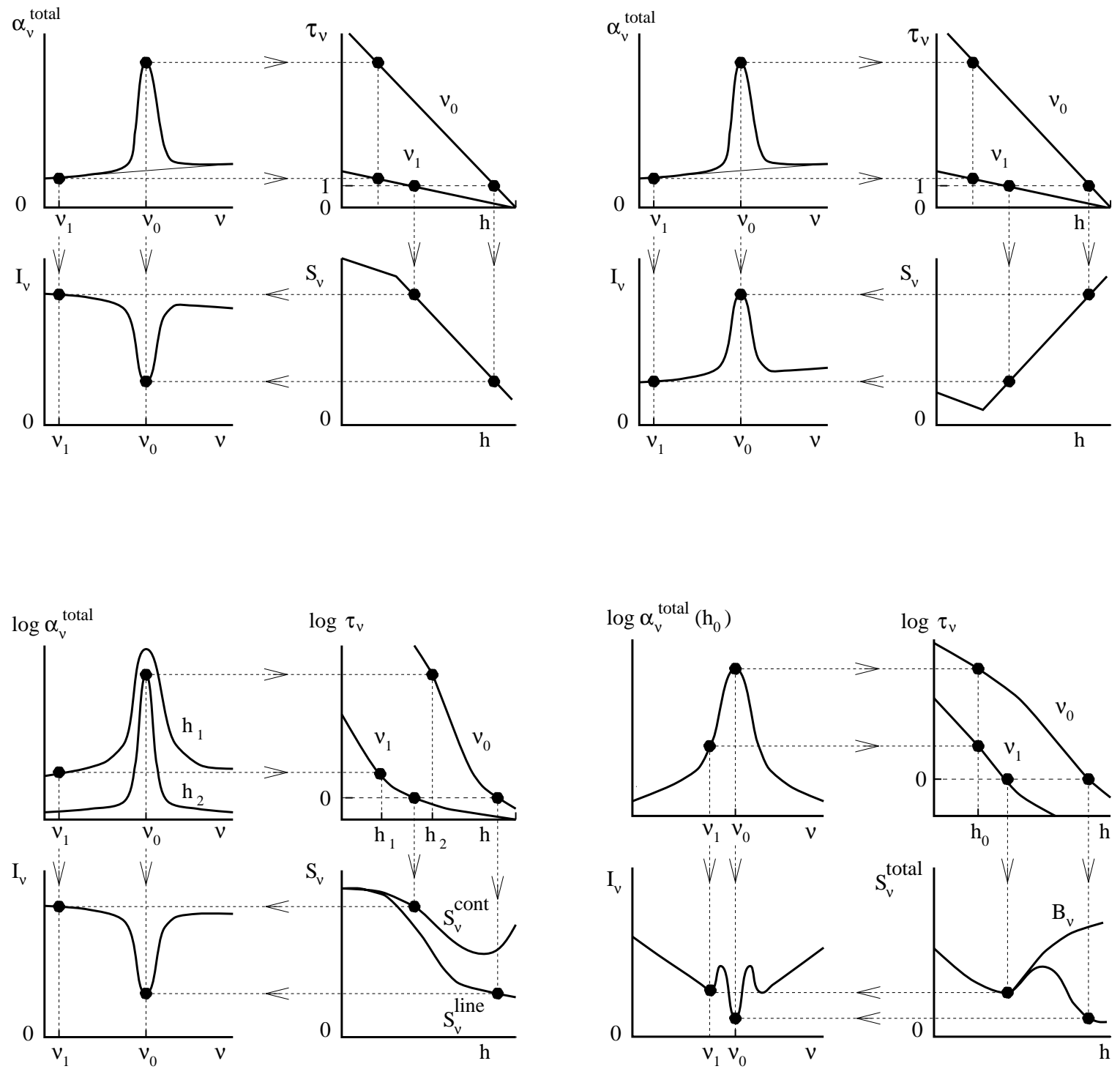

Figure 2.5: Four four-panel schematic Eddington-Barbier line formation diagrams. Top left: absorption line from a thick homogeneous medium in which the line and continuum extinction do not vary with height $h$. The extinction profile in the upper left panel sets the $\tau_{\nu}(h)$ scaling at right. In this case the scaling is linear for each frequency, with a steeper slope for larger extinction. The Eddington-Barbier $h\left(\tau_{\nu}=1\right)$ heights define the representative source function values (lower right) to which the emergent intensities correspond (lower left). The correspondence is exact where $S_{\nu}$ varies linearly with $\tau_{\nu}$ (also with $h$ in this simplified case). Top right: emission line from a similar medium. The only change is the reverse in $S_{\nu}(h)$ slope. Bottom left: formation of a strong scattering line in a more realistic atmosphere with roughly exponential density stratification and height-dependent line broadening. This case resembles the formation of the solar Na I D lines. Their line source function doesn't "feel" the chromospheric temperature rise present in the continuum source function $S_{\nu}^{c} \approx B_{\nu}$. It drops below the Planck function due to resonance scattering $\left(\sqrt{\varepsilon}\right.$ law in $\left(4.81_{1}^{\prime}\right)$ on page $197_{1}^{\prime}$ and Section $10.1_{1}^{\prime}$ on page 213$)$. At line center the total line source function is dominated by the line source function. Bottom right: formation of double emission features in the core of a very strong line with complete redistribution, in an atmosphere with a chromospheric temperature rise. This case resembles the classical explanation for the reversals in the spatially-averaged cores of the solar CaII H\&K lines. In this scheme, the intensity dip at $\nu=\nu_{1}$ maps the temperature minimum between photosphere and chromosphere. The actual formation of the reversals is much more complicated (Section 10.21 on page 221 ). 
deexcitation is independent of the preceding process(es) that put the atom in state $u$ ("complete redistribution"), the probability distribution is

$$
\psi\left(\nu-\nu_{0}\right)=\frac{H(a, v)}{\sqrt{\pi} \Delta \nu_{\mathrm{D}}}
$$

with the Doppler width $\Delta \nu_{\mathrm{D}}$ defined as

$$
\Delta \nu_{\mathrm{D}} \equiv \frac{\nu_{0}}{c} \sqrt{\frac{2 k T}{m}}
$$

where $m$ is the particle mass, and with the Voigt function $H(a, v)$ given by $(3 . \overline{6} \overline{8})$ on page $\overline{5} \bar{g} \overline{9}$ and shown in Figure shifts from Maxwellian motions ("Doppler core") and it has extended Lorentzian wings caused by collisional perturbations ("damping wings").

The emission profile is more complex when the frequency redistribution over the line profile is incomplete ("partial redistribution"), which is the case if the photon that is emitted per deexcitation has some correlation with the photon that previously excited the atom in a scattering up-down sequence. Coherent scattering, without frequency change, is the other extreme. I mostly use the two extremes of fully coherent and fully incoherent scattering in this course; partial redistribution is discussed in Section $3 . \overline{4} . \overline{3}_{1}^{1}$ on page $\overline{2}_{1}^{1}$;f.

Radiative excitation. The Einstein coefficient for radiative excitation $B_{l u}$ is defined by:

$$
\begin{aligned}
B_{l u} \bar{J}_{\nu_{0}}^{\varphi} \equiv & \text { number of radiative excitations from state } l \text { to state } u \text { per } \\
& \text { sec per particle in state } l \text {, }
\end{aligned}
$$

with the index $\nu_{0}$ defining a specific spectral line of which the extinction profile $\varphi\left(\nu-\nu_{0}\right)$ is used in the weighting of the angle-averaged exciting radiation field over the spectral extent of the lineri!

$$
\bar{J}_{\nu_{0}}^{\varphi} \equiv \int_{0}^{\infty} J_{\nu} \varphi\left(\nu-\nu_{0}\right) \mathrm{d} \nu
$$

where $\int \varphi\left(\nu-\nu_{0}\right) \mathrm{d} \nu=1$. A more general expression for this summation is

$$
\bar{J}_{\nu_{0}}^{\varphi} \equiv \frac{1}{2} \int_{0}^{\infty} \int_{-1}^{+1} I_{\nu} \varphi\left(\nu-\nu_{0}\right) \mathrm{d} \mu \mathrm{d} \nu
$$

which also holds when $\varphi\left(\nu-\nu_{0}\right)$ is anisotropic due to systematic Doppler shifts $\sum_{-1}^{\delta_{1}}$ (see page $\left.\bar{i}_{-} \overline{1}_{i}^{\prime}\right)$. In the absence of the latter (static atmosphere), the profile function $\varphi\left(\bar{\nu}-\nu_{0}\right)$

\footnotetext{
${ }^{7}$ Because $\varphi\left(\nu-\nu_{0}\right)$ is area-normalized, $\bar{J}_{\nu_{0}}^{\varphi}$ represents both the profile-weighted summation and the profile-weighted average of the radiation field over the line width. The latter is formally defined by $\bar{J}_{\nu_{0}}^{\varphi} \equiv \int J_{\nu} \varphi\left(\nu-\nu_{0}\right) \mathrm{d} \nu / \int \varphi\left(\nu-\nu_{0}\right) \mathrm{d} \nu$.

${ }^{8} \mathrm{~A}$ yet more general expression is to integrate also over the azimuthal angle $\varphi$ as in $(i 2 . \overline{2})$ on page $10_{1}^{1}$ This must be done when axial asymmetry is no longer valid (horizontal inhomogeneity). A better option is to define the Einstein coefficients in terms of the intensity. The coefficients $A$ and $B$ in $\left(2.46^{\prime}\right)$, $\left(2.50_{1}^{\prime}\right)$ and $(2.55)$ are based on emission into and extinction_out of all directions, as done by Rybicki and Lightmand '(1979),' 'Mihalas (1978)', 'Shu (1991)' and Böhm-Vitense (1989)! 'Gray (1992)! follows 'Chandrasekhar (1939), and bases the Einstein coefficients on intensity, so that they are defined per steradian and are $4 \pi$ smaller. In our case, they are divided by $4 \pi$ in (2.62) and (2.69) to produce intensity extinction and emissivities. Jefferies (1968), has the most elegant notation. He defines $A_{u l}$ for radiation into all directions, as fits a "transition probability" for deexcitations that won't care in which direction they emit photons, but defines $B_{u l}$ and $B_{l u}$ as negative and positive extinction of the intensity in a beam, per steradian. His downward radiative rate per $\mathrm{cm}^{3}$ is $n_{u} R_{u l}=n_{u} A_{u l}+4 \pi n_{u} B_{u l} \int_{0}^{\infty} J_{\nu} \chi\left(\nu-\nu_{0}\right) \mathrm{d} \nu$.
} 
is again set by random Doppler shifts and radiative plus collisional damping and given by the area-normalized Voigt function as in $(2 . \overline{2}-1)$ :

$$
\varphi\left(\nu-\nu_{0}\right)=\frac{H(a, v)}{\sqrt{\pi} \Delta \nu_{\mathrm{D}}}
$$

For small damping (Voigt parameter $a<1$ ) the line-center amplitude is

$$
\varphi\left(\nu=\nu_{0}\right)=\frac{1-a}{\sqrt{\pi} \Delta \nu_{\mathrm{D}}},
$$

where $a=0$ for a purely Gaussian line shape (pure Doppler broadening).

Induced deexcitation. The Einstein coefficient for induced deexcitation $B_{u l}$ is similarly defined by:

$$
B_{u l} \bar{J}_{\nu_{0}}^{\chi} \equiv \begin{aligned}
& \text { number of induced radiative deexcitations from state } u \text { to } \\
& \text { state } l \text { per sec per particle in state } u,
\end{aligned}
$$

similarly to $B_{l u}$, with frequency averaging

$$
\bar{J}_{\nu_{0}}^{\chi} \equiv \frac{1}{2} \int_{0}^{\infty} \int_{-1}^{+1} I_{\nu} \chi\left(\nu-\nu_{0}\right) \mathrm{d} \mu \mathrm{d} \nu=\int_{0}^{\infty} J_{\nu} \chi\left(\nu-\nu_{0}\right) \mathrm{d} \nu
$$

in which $\chi\left(\nu-\nu_{0}\right)$ is the area-normalized profile shape for induced emission. The first version is the more general one.

Collisional excitation and deexcitation. The Einstein coefficients for collisional excitation and deexcitation are:

$$
\begin{aligned}
& C_{l u} \equiv \begin{array}{l}
\text { number of collisional excitations from state } l \text { to state } u \text { per } \\
\text { sec per particle in state } l \text {. }
\end{array} \\
& C_{u l} \equiv \begin{array}{l}
\text { number of collisional deexcitations from state } u \text { to state } l \\
\text { per sec per particle in state } u
\end{array}
\end{aligned}
$$

Electron collisions (usually the most important ones) causing transitions from state $i$ to state $j$ have transition rates

$$
n_{i} C_{i j}=n_{i} N_{\mathrm{e}} \int_{v_{0}}^{\infty} \sigma_{i j}(v) v f(v) \mathrm{d} v
$$

with $N_{\mathrm{e}}$ the electron density, $\sigma_{i j}(v)$ the electron collision cross-section, $f(v)$ the areanormalized velocity distribution (usually Maxwellian) with mean value $\int v f(v) \mathrm{d} v$, and $v_{0}$ the threshold velocity with $(1 / 2) m v_{0}^{2}=h \nu_{0}$. The collision cross-section $\sigma_{i j}$ is, similarly to the radiative bb cross-section $\sigma_{\nu}^{l}$ and the corresponding Einstein coefficients $A_{u l}, B_{l u}$ and $B_{u l}$, a material property of each transition that is independent of external state parameters except the velocity (difference) $v$. 
Einstein relations. The Einstein coefficients are coupled by the Einstein relations:

$$
\frac{B_{l u}}{B_{u l}}=\frac{g_{u}}{g_{l}} \quad \frac{A_{u l}}{B_{u l}}=\frac{2 h \nu^{3}}{c^{2}}
$$

and

$$
\frac{C_{u l}}{C_{l u}}=\frac{g_{l}}{g_{u}} \mathrm{e}^{E_{u l} / k T},
$$

where $E_{u l}$ is the transition energy. The ratios (2.60) are derived for TE by equating the upward and downward radiative rates requiring detailed balance per frequency with $\varphi=\psi=\chi$ and equating the resulting expression for $J_{\nu}$ to $B_{\nu}$ at arbitrary temperature. They then hold universally since they do not depend on any medium property. The ratio $\left(\overline{2} .61_{i}\right)$ follows similarly from equating the upward and downward collisional rates in TE. It holds also outside TE if the Maxwell distribution holds.

\subsubsection{Volume coefficients}

Extinction. The monochromatic line extinction coefficient per $\mathrm{cm}$ path length expressed in Einstein coefficients is:

$$
\begin{aligned}
\alpha_{\nu}^{l} & =\frac{h \nu}{4 \pi}\left[n_{l} B_{l u} \varphi\left(\nu-\nu_{0}\right)-n_{u} B_{u l} \chi\left(\nu-\nu_{0}\right)\right] \\
& =\frac{h \nu}{4 \pi} n_{l} B_{l u} \varphi\left(\nu-\nu_{0}\right)\left[1-\frac{n_{u} g_{l} \chi\left(\nu-\nu_{0}\right)}{n_{l} g_{u} \varphi\left(\nu-\nu_{0}\right)}\right]
\end{aligned}
$$

where the term between square brackets corrects for induced emission, taken into account as negative extinction. In these lecture notes, volume coefficients $\alpha$ always contain such correction. The total line extinction coefficient is

$$
\alpha_{\nu_{0}}^{l} \equiv \int_{0}^{\infty} \alpha_{\nu}^{l} \mathrm{~d} \nu=\frac{h \nu_{0}}{4 \pi}\left(n_{l} B_{l u}-n_{u} B_{u l}\right)
$$

using $\int h \nu \varphi\left(\nu-\nu_{0}\right) \mathrm{d} \nu=h \nu_{0}$ and $\int h \nu \chi\left(\nu-\nu_{0}\right) \mathrm{d} \nu=h \nu_{0}$ assuming the profile to be symmetric or sufficiently narrow $w_{L_{1}}^{9}$ Throughout these lecture notes, the subscript $\nu_{0}$ denotes summation over the line profile ${ }_{-}^{17 !}$ and identifies the particular bound-bound transition. The coefficients $\alpha_{\nu}^{l}$ and $\alpha_{\nu_{0}}^{l}$ are rewritten with population departure coefficients in

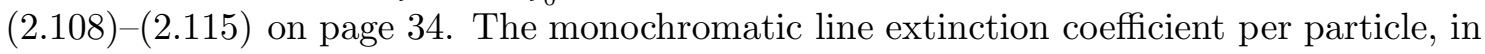
these lecture notes always without correction for induced emission, is:

$$
\sigma_{\nu}^{l}=\frac{h \nu}{4 \pi} B_{l u} \varphi\left(\nu-\nu_{0}\right)
$$

The total line extinction coefficient per particle is

$$
\sigma_{\nu_{0}}^{l} \equiv \int_{0}^{\infty} \sigma_{\nu}^{l} \mathrm{~d} \nu=\frac{h \nu_{0}}{4 \pi} B_{l u}=\frac{\pi e^{2}}{m_{\mathrm{e}} c} f_{l u}=0.02654 f_{l u} \quad \mathrm{~cm}^{2} \mathrm{~Hz}
$$

\footnotetext{
${ }^{9}$ Motion along the line of sight implies a shift of $\nu_{0}$ and local anisotropy of the monochromatic extinction coefficient. Differential motions along the line of sight imply varying shift and anisotropy. Asymmetry may be caused by hyperfine and isotope structure (page ' $633_{1}^{\prime}$ ).

${ }^{10} \mathrm{Or}$ it denotes averaging over the profile, in the case of profile-weighted summation as in (2.51) Note that (2.64) does not represent averaging of $\alpha_{\nu}^{l}$ over the line profile. The dimension of $\alpha_{\nu_{0}}^{l}$ is $\mathrm{cm}^{-1} \mathrm{~Hz}$.
} 
These coefficients are ensemble quantities, given per individual particle but sampling, respectively summed over, the ensemble distribution specified by $\varphi\left(\nu-\nu_{0}\right)$. The parameter $f_{l u}$ is the classical dimensionless oscillator strength, a quantity that was historically introduced to correct harmonic-oscillator line strength predictions for unknown quantummechanical effects. Resonance lines such as H I Ly $\alpha$ have $f_{l u} \approx 1$. The $\nu$-dependences in $\left(\overline{2} . \overline{6} 0_{1}^{*}\right)$ and $(2 . \overline{6} \cdot \overline{6})$ produce

$$
A_{u l} \sim \frac{g_{l}}{g_{u}} f_{l u}\left(\Delta E_{u l}\right)^{2}
$$

with $\Delta E_{u l}=h \nu_{0}$ the transition energy. Numerically:

$$
A_{u l}=6.67 \times 10^{13} \frac{g_{l}}{g_{u}} \frac{f_{l u}}{\lambda^{2}} \mathrm{~s}^{-1}
$$

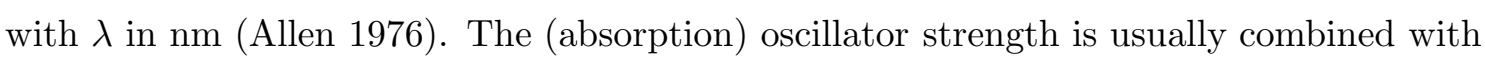
the lower-level statistical weight into the "gf-value" $g_{l} f_{l u}$ because this product defines the effective transition probability that one must knowi!l to evaluate $\alpha_{\nu}^{l}$ or $\kappa_{\nu}^{l}$. The $g_{l}$ then comes in when evaluating the lower-level population $n_{l}$, for example through its LTE Boltzmann-Saha estimate $n_{l}^{\mathrm{LTE}}$ in $(\overline{9} \cdot \overline{6})$ on page $\overline{2} \overline{0} \overline{4}$.

Emission. The monochromatic line emissivity expressed in Einstein coefficients is, without induced emission,

$$
j_{\nu}^{l}=\frac{h \nu}{4 \pi} n_{u} A_{u l} \psi\left(\nu-\nu_{0}\right)
$$

The total line emissivity is

$$
j_{\nu_{0}}^{l}=\int_{0}^{\infty} j_{\nu}^{l} \mathrm{~d} \nu=\frac{h \nu_{0}}{4 \pi} n_{u} A_{u l}
$$

using $\int h \nu \psi\left(\nu-\nu_{0}\right) \mathrm{d} \nu=h \nu_{0}$ because $\psi\left(\nu-\nu_{0}\right)$ is symmetric around $\nu=\nu_{0}$ in the absence of systematic Doppler shifts.

Source function. The monochromatic line source function expressed in Einstein coefficients is

$$
S_{\nu}^{l} \equiv j_{\nu}^{l} / \alpha_{\nu}^{l}=\frac{n_{u} A_{u l} \psi\left(\nu-\nu_{0}\right)}{n_{l} B_{l u} \varphi\left(\nu-\nu_{0}\right)-n_{u} B_{u l} \chi\left(\nu-\nu_{0}\right)}
$$

or, using the Einstein relations (2.60)

$$
S_{\nu}^{l}=\frac{\frac{A_{u l}}{B_{u l}} \frac{\psi}{\varphi}}{\frac{n_{l}}{n_{u}} \frac{B_{l u}}{B_{u l}}-\frac{\chi}{\varphi}}=\frac{2 h \nu^{3}}{c^{2}} \frac{\psi / \varphi}{\frac{g_{u} n_{l}}{g_{l} n_{u}}-\frac{\chi}{\varphi}} .
$$

The line source function may vary strongly with frequency across the line when the profile shapes are not equal due to coherent scattering or partial frequency redistribution (Section $3 . \overline{4} .3$ on page $7 \overline{2}$ ). They become equal when complete redistribution holds in which

\footnotetext{
${ }^{11}$ Direct computation of gf-values is fairly straightforward for hydrogen and hydrogen-like ions but less so for more complex atomic, ionic or molecular configurations. Chapter 10 of 'Rybicki and Lightmanı (1979)! presents the hydrogen computation and contains a table of H I gf-values on page 281 . Extensive tabulations of experimentally measured transition probabilities used to come in thick volumes produced by the US National Bureau of Standards (e.g., 'Corliss and Bozman 1962) but better values now result from large-scale computations and become available on the web, e.. $\bar{g}$., http://vizier.u-strasbg.fr/OP.html (Opacity Project), http://wwwsolar.nrl.navy.mil/chianti.html (CHIANTI database)
} 
every process takes a fresh sample of the probability distribution, without "memory" for any preceding process, so that $\varphi\left(\nu-\nu_{0}\right)=\psi\left(\nu-\nu_{0}\right)=\chi\left(\nu-\nu_{0}\right)$. The line source function then simplifies to

$$
S_{\nu_{0}}^{l}=\frac{n_{u} A_{u l}}{n_{l} B_{l u}-n_{u} B_{u l}}=\frac{2 h \nu_{0}^{3}}{c^{2}} \frac{1}{\frac{g_{u} n_{l}}{g_{l} n_{u}}-1} .
$$

The index 0 to $S_{\nu_{0}}^{l}$ signifies that the complete-redistribution version of the line source function is frequency-independent ${ }_{-1}^{172}$. The line source function simplifies yet further to to $S_{\nu_{0}}^{l}=B_{\nu_{0}}$ when the population ratio $n_{l} / n_{u}$ in (2.73i) obeys the Boltzmann distribution $(2.86 i)$ on page $2 \overline{2} \overline{9}$, as it does in LTE.

\subsection{Continuum transitions}

\subsubsection{Inelastic processes}

Bound-free transitions. For bound-free transitions of hydrogen and hydrogen-like ions the extinction cross-section in $\mathrm{cm}^{2}$ per particle is given by Kramers' formula:

$$
\sigma_{\nu}^{\mathrm{bf}}=2.815 \times 10^{29} \frac{Z^{4}}{n^{5} \nu^{3}} g_{\mathrm{bf}} \quad \text { for } \nu \geq \nu_{0},
$$

with $n$ the principal quantum number of the level $i$ from which the atom or ion is ionized, $Z$ the ion charge, $\nu$ in $\mathrm{Hz}$ and $g_{\mathrm{bf}}$ the dimensionless Gaunt factor, a quantummechanical correction factor of order unity. The Kramers cross-section decays $\sim \nu^{-3}$ above the threshold ("edge") frequency $\nu_{0}$, being zero below it because the threshold energy is the required minimum. For more complex atoms and ions than hydrogen-like ones, the bound-free cross-sections do not have such simple $\nu^{-3}$ dependence but possess peaks at "resonances" caused by other electrons in the same shell (Section $\overline{3} \cdot \overline{1} \cdot \overline{3}_{1}^{\prime}$ on page $\overline{4} \overline{3} \overline{3}$ ).

For LTE conditions the corresponding volume extinction coefficient is

$$
\alpha_{\nu}^{\mathrm{bf}}=\sigma_{\nu}^{\mathrm{bf}} n_{i}\left(1-\mathrm{e}^{-h \nu / k T}\right)
$$

with $n_{i}$ the density of particles in the ionizing level. The negative term corrects for induced

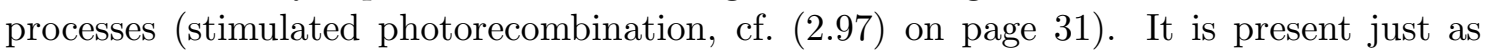
for bound-bound processes because ionization is also either radiative or collisional while recombination may also be achieved spontaneously (ion-electron collision), inducedly (ionelectron plus photon collision), or collisionally without the production of a photon (ionelectron-plus-third-particle collision). Outside LTE the more general expression (2.119i)

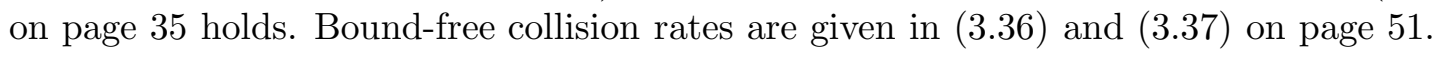

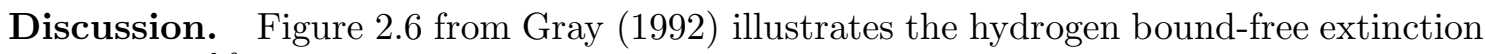
coefficient $\sigma_{\nu}^{\text {bf }}$ per particle and per bound-free feature. The peak amplitudes are of the order of $10^{-17} \mathrm{~cm}^{2}$ per particle. They increase with $n$, although Kramers' law suggests

\footnotetext{
${ }^{12}$ At least for sufficient narrow lines over which the frequency variation due to the $\nu^{3}$ scaling factor and the $n_{l} / n_{u}$ ratio can be neglected. For LTE these combine in Planckian frequency dependence. Note that $S_{\nu_{0}}^{l}=j_{\nu_{0}}^{l} / \alpha_{\nu_{0}}^{l}$ does not represent a total but the average $\int S_{\nu}^{l} \varphi\left(\nu-\nu_{0}\right) \mathrm{d} \nu / \int \varphi\left(\nu-\nu_{0}\right) \mathrm{d} \nu$ over the line. The "total"- source function is the weighted combination of line and continuous source functions as in (2.23) on page 13 . That combination varies with frequency across the line when $S^{l} \neq S^{c}$ even when $S^{l}$ and $S^{c}$ are each frequency-independent.
} 


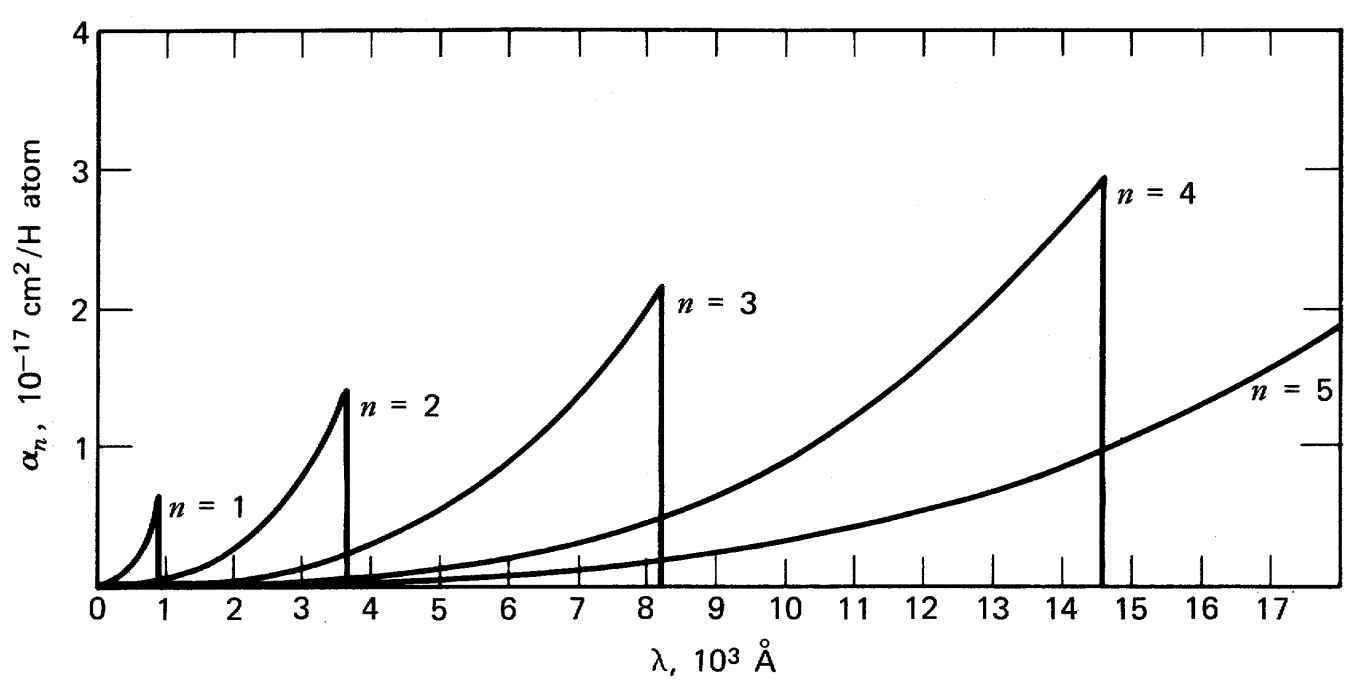

Figure 2.6: H I bound-free extinction coefficient $\sigma_{\nu}^{\text {bf }}$ per hydrogen atom in level $n$ (here written as $\alpha_{n}$ ) against wavelength. The Lyman, Balmer, Paschen, Brackett and Pfund edges are marked by the quantum number $n$ of the ionizing level. Their amplitudes increase with $n$ and have not been added up in this

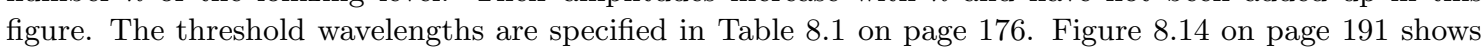
the hydrogen and helium bound-free and free-free extinction for the actual mix of particles in three stellar atmospheres. The total extinction from all continuous processes is shown for a grid of stellar atmospheres in the Vitense diagrams on page $1 \overline{1} \overline{9}$ and page 192 iff. From 'Gray $(\overline{1} \overline{9} \overline{2})$ !'

$\sigma_{\nu}^{\text {bf }} \sim 1 / n^{5}$, because the Rydberg sequence for the hydrogen ionization thresholds has $h \nu_{n}=\chi_{c n}=E_{\infty}-E_{n}=13.6 / n^{2} \mathrm{eV}$ so that the factor $\nu^{-3}$ converts into a factor $n^{6}$.

The bound-free extinction peaks are much lower than the bound-bound resonance-line peaks. For example, the Ly $\alpha$ line at $\lambda=121.5 \mathrm{~nm}$ or $\nu=2.47 \times 10^{15} \mathrm{~Hz}$ has oscillator

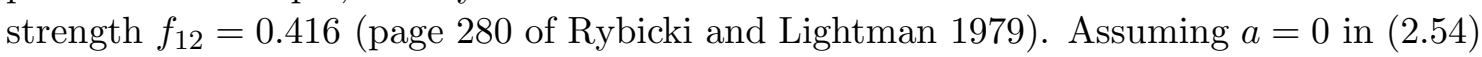

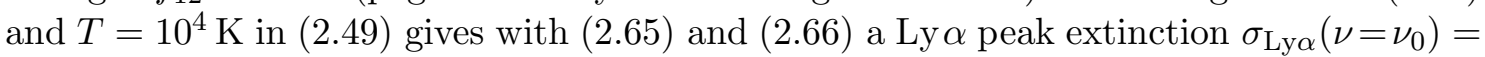
$4.0 \times 10^{-14} \mathrm{~cm}^{2}$, three orders of magnitude larger than the peaks in Figure $\overline{2} .6$. . However, the edges are much wider. The edge-integrated bound-free extinction is $\nu_{0} / 2$ times larger than (2.74), so that the full Lyman edge with threshold frequency $\nu_{0}=3.3 \times 10^{15} \mathrm{~Hz}$ has integrated cross-section $\sigma_{\text {Ly edge }}=0.01 \mathrm{~cm}^{2} \mathrm{~Hz}$, about the same as the integrated Ly $\alpha$ cross-section $\sigma_{\mathrm{Ly} \alpha}=0.011 \mathrm{~cm}^{2} \mathrm{~Hz}$ given by $(\overline{2} \cdot \overline{6})$. Note that the actual integrated radiative transiton rates in the two features depend on the radiation field, as specified by

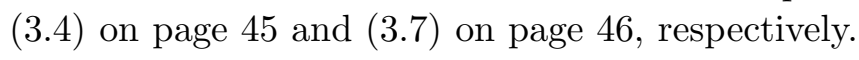

Free-free transitions. Free-free transitions ${ }_{-1}^{\prime 2}$ have $S_{\nu}=B_{\nu}$ when the Maxwell velocity distribution holds ("thermal Bremsstrahlung"). A formula for the corresponding extinction coefficient per particle is (Rybicki and Lightman 1979i p. 162):

$$
\sigma_{\nu}^{\mathrm{ff}}=3.7 \times 10^{8} N_{\mathrm{e}} \frac{Z^{2}}{T^{1 / 2} \nu^{3}} g_{\mathrm{ff}},
$$

with $Z$ the ion charge, $N_{\mathrm{e}}$ and $N_{\text {ion }}$ the electron and ion densities, and $g_{\mathrm{ff}}$ a Gaunt factor of order unity. There is no threshold frequency. This expression is derived classically;

\footnotetext{
${ }^{13}$ Note the astronomical convention: H I free-free extinction describes photon-absorbing encounters between protons and free electrons with $Z=1$ and $N_{\text {ion }}=N_{\mathrm{p}} ; \mathrm{HII}$ free-free encounters do not exist; $\mathrm{H}^{-}$ free-free encounters are between neutral hydrogen atoms and free electrons.
} 
the Gaunt factor corrects for simple errors relative to the appropriate quantummechanical result. It does not hold for more complex systems such as $\mathrm{H}^{-}$ions (which indeed would have $Z=0$ in $(2.76))$.

The corresponding volume extinction coefficient is:

$$
\alpha_{\nu}^{\mathrm{ff}}=\sigma_{\nu}^{\mathrm{ff}} N_{\mathrm{ion}}\left(1-\mathrm{e}^{-h \nu / k T}\right)
$$

which holds also out of LTE conditions (as long as the velocities are Maxwellian) because the free-free processes are always fully collisional. The negative term again corrects for induced processes. In the Wien limit defined by $\left(\overline{2} . \overline{9} \overline{3}_{1}\right)$ on page $\overline{3} \overline{1} \overline{1}$, the free-free extinction coefficient simplifies to:

$$
\alpha_{\nu}^{\mathrm{ff}} \approx 3.7 \times 10^{8} N_{\mathrm{e}} N_{\mathrm{ion}} \frac{Z^{2}}{T^{1 / 2} \nu^{3}} g_{\mathrm{ff}}
$$

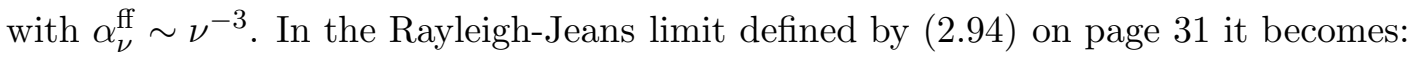

$$
\alpha_{\nu}^{\mathrm{ff}} \approx 0.018 N_{\mathrm{e}} N_{\mathrm{ion}} \frac{Z^{2}}{T^{3 / 2} \nu^{2}} g_{\mathrm{ff}}
$$

with $\alpha_{\nu}^{\mathrm{ff}} \sim \nu^{-2}$.

\subsubsection{Elastic processes}

Thomson scattering. Thomson scattering of photons by free electrons has a frequencyindependent extinction cross-section given per electron by:

$$
\sigma_{\nu}^{\mathrm{T}} \equiv \sigma^{\mathrm{T}}=\frac{8 \pi}{3} r_{\mathrm{e}}^{2}=6.65 \times 10^{-25} \mathrm{~cm}^{2} .
$$

The corresponding volume extinction coefficient is given by

$$
\alpha_{\nu}^{\mathrm{T}}=\sigma^{\mathrm{T}} N_{\mathrm{e}}
$$

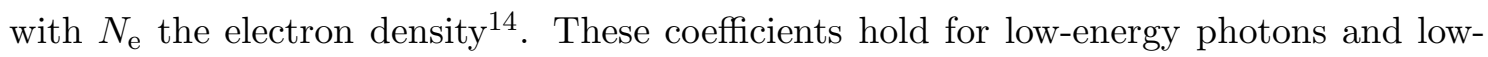
energy electrons. For high-energy photons, Thomson scattering is replaced by Compton scattering; for high-energy electrons, by inverse Compton scattering (e.g., Rybicki andi Lightman $19 \overline{7} \bar{g})$. Thomson scattering is the major source of continuous extinction in the atmospheres of hot stars where hydrogen is ionized (see discussion on page $\overline{2} \overline{0} \overline{2} \overline{2}$, of the continuous opacity diagram in Figure 8.15 on page i19.7). The much larger values of the H I bound-free peaks in (2.74) and Figure 2.6 , of order $10^{-17} \mathrm{~cm}^{2}$ per particle, win from electron scattering when hydrogen is not fully ionized (high pressure curves in Figure $8.15 i$

\footnotetext{
${ }^{14}$ There is no $(1-$ something) correction comparable to the $[1-\exp (-h \nu / k T)]$ factor used for LTE extinction processes as in $\left(2.7 \hat{T}_{1}\right)$. Induced emission processes are generally not included in elastic electron scattering (e.g., Mihalas 1978 p. 107), corresponding with the Bohr picture in which the free electron has no internal excitation energy to be released upon outside triggering. Actually, electron scattering does suffer from stimulated enhancement because photons are bosons. However, the enhancement cancels between source and sink terms when the scattering is coherent. Precisely as many photons are then stimulated to scatter monochromatically into any beam as out of it (Shu 1991 p. 71). This cancelation is discussed in more detail on page $\underline{6}$ i'.
} 
Rayleigh scattering. The extinction cross-section for Rayleigh scattering of photons with $\nu \ll \nu_{0}$ by bound electrons with characteristic bounding energy $h \nu_{0}$ is:

$$
\sigma_{\nu}^{\mathrm{R}} \approx f_{l u} \sigma^{\mathrm{T}}\left(\frac{\nu}{\nu_{0}}\right)^{4}
$$

where the oscillator strength $f_{l u}$ and the frequency $\nu_{0}$ characterize the major bound-bound "resonance transition" of the bound electron, for example the Ly $\alpha$ transition in neutral hydrogen or a weighted sum over all Lyman lines. The $\nu^{4}$ dependence makes our sky blue and sunsets red. The volume extinction coefficient for Rayleigh scattering by hydrogen atoms with density $N_{\mathrm{H}}$ is given by

$$
\alpha_{\nu}^{\mathrm{R}}=\sigma_{\nu}^{\mathrm{R}} N_{\mathrm{H}}
$$

Rayleigh scattering is included in Vitense's stellar-atmosphere opacity diagrams on page ${ }_{1}^{1} \overline{7} \overline{9} \overline{1}_{1}^{\prime}$ and page $1 \overline{1} \overline{2} \overline{2}_{1}^{\prime}$ ffr. Its contribution is usually negligible.

Redistribution. Thomson and Rayleigh scattering are coherent, meaning elastic or monochromatic; the photon gets redirected but it keeps its frequency. The redirection has phase function $\sim 1+\cos ^{2} \theta$, with sufficiently small departure from isotropy that isotropy is generally assumed. At high temperature, Thomson scattering is not truly coherent due to the Doppler shifts imposed by the electrons. They move faster by a factor 43 than

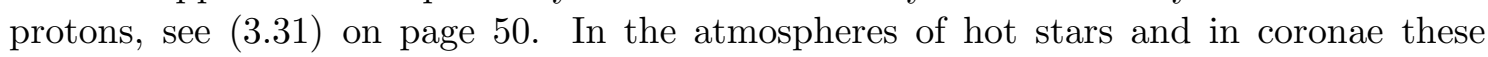
Doppler shifts are appreciable. They obliterate the Fraunhofer lines in the spectrum of the solar K corona. For hot stars, Rybicki and Hummer $(1 \overline{9} \overline{9} \overline{4})$ have formulated a radiative transfer method which includes the frequency spreading due to the appreciable thermal Doppler shifts.

\subsection{LTE}

In local thermodynamic equilibrium (LTE) all material energy partitioning, i.e., all atomic, ionic and molecular level populations, is given by Saha-Boltzmann statistics defined by the local temperature, just as if that location sits within a TE (thermodynamic equilibrium) enclosure as seen by the matter component of the ensemble (but not altogether by the photons). The definition of LTE is to assume the validity of all TE material distribution laws at the local temperature. The equality $S_{\nu_{0}}^{l}=B_{\nu_{0}}$ then follows by entering the Boltzmann distribution into (2.73i) on page 25

\subsubsection{Matter in LTE}

Maxwell distribution. Per species of particles with mass $m$ the Maxwell distribution for the velocity components in the $x$ direction is:

$$
\left[\frac{n\left(v_{x}\right)}{N} \mathrm{~d} v_{x}\right]_{\text {LTE }}=\left(\frac{m}{2 \pi k T}\right)^{1 / 2} \mathrm{e}^{-(1 / 2) m v_{x}^{2} / k T} \mathrm{~d} v_{x},
$$

\footnotetext{
${ }^{15}$ Often, the LTE equality $S_{\nu}=B_{\nu}$ is taken to be the definition of LTE. It is not; the concept requires strict coupling of the matter component to the local temperature ('Ivanov 1973). This can only be the case if the radiation is not too far off, or unimportant. Otherwise, photon processes will make the level populations depart from Saha-Boltzmann statistics. In that case $S_{\nu}^{l} \neq B_{\nu}$ as evident from (2.73). Thus, $S_{\nu}=B_{\nu}$ corresponds to requiring Boltzmann-Saha-Maxwell statistics for the material (fermion) distributions while permitting the photon (boson) distributions to depart slightly from the local TE values.
} 
with $N$ the total number of particles with mass $m$ per $\mathrm{cm}^{3}$. The subscript $[\ldots]_{\text {LTE }}$ implies evaluation of this TE distribution law at the local value of the kinetic electron temperature $T_{\mathrm{e}}$. In LTE the latter is equal to all other material temperatures (kinetic ion temperatures, excitation temperature, ionization temperature) so that we may set $T \equiv T_{\mathrm{e}}$. For the size of the particle speeds, ignoring direction, the Maxwell distribution is:

$$
\left[\frac{n(v)}{N} \mathrm{~d} v\right]_{\text {LTE }}=\left(\frac{m}{2 \pi k T}\right)^{3 / 2} 4 \pi v^{2} \mathrm{e}^{-(1 / 2) m v^{2} / k T} \mathrm{~d} v .
$$

The component distribution (2. $2 . \overline{4})$ is a Gaussian, whereas the speed distribution $(2 . \overline{8} 5)$ has a high-velocity tail due to the factor $v^{2}$. The peak location defines the most probable speed $v_{p}=\sqrt{2 k T / m}$; the average speed is $\langle v\rangle=\sqrt{3 k T / m}$.

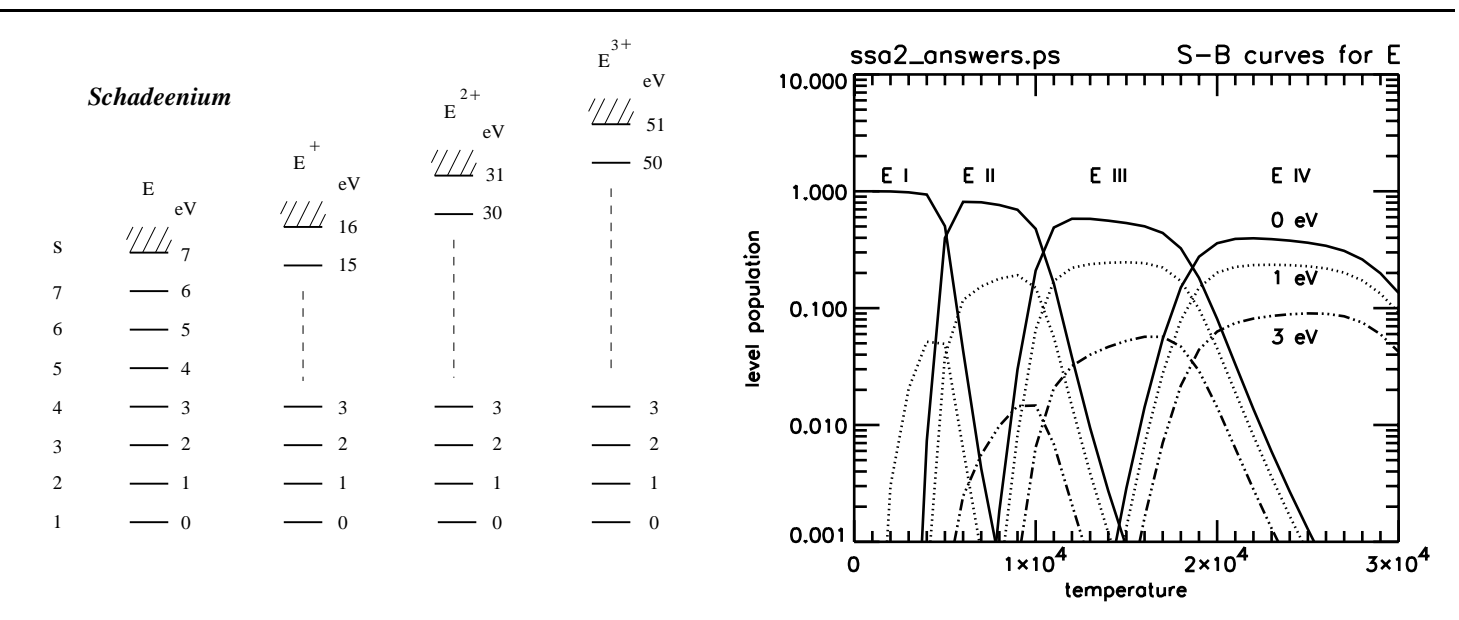

Figure 2.7: Saha-Boltzmann distributions for element E. Left: Energy level diagram for a fictitious element $\mathrm{E}$ (for Easy), showing the neutral stage (lefthand column, $r=1$ ) and the first three ionization stages $(r=2-4)$. The level energies increase in $1 \mathrm{eV}$ steps. All statistical weights $g_{r, s}$ are unity. The columns may be thought stacked on top of each other since each ion requires the previous stage to be ionized. The level counter $s$ starts at 1 within each stage. In astronomical convention the spectra of neutral schadeenium E, ionized schadeenium $\mathrm{E}^{+}$and doubly ionized schadeenium $\mathrm{E}^{2+}$ are called EI, EII, and E III, respectively. Right: Saha-Boltzmann population densities for levels 1, 2 and 4 of stages EI - EIV as function of temperature. The population of an excited level increases with temperature until its stage ionizes. Only two stages exist effectively at any temperature. Copied from my second "Stellar Spectra A" exercise available at http://www.astro.uu.nl/ rutten. Aert Schadee (1936 - 1999), who invented this didactically correct element, was an astrophysicist at Utrecht.

Boltzmann distribution. The Boltzmann excitation distribution is:

$$
\left[\frac{n_{r, s}}{n_{r, t}}\right]_{\mathrm{LTE}}=\frac{g_{r, s}}{g_{r, t}} \mathrm{e}^{-\left(\chi_{r, s}-\chi_{r, t}\right) / k T},
$$

with $n_{r, s}$ the number of atoms per $\mathrm{cm}^{3}$ in level $s$ of ionization stage $r, g_{r, s}$ the statistical weight of level $s$ in stage $r, \chi_{r, s}$ the excitation energy $y_{-}^{1} t_{1}^{\prime}$ of level $s$ in stage $r$, measured from the ground level $(r, 1)$ of stage $r$, and $\chi_{r, s}-\chi_{r, t}=h \nu$ for a radiative transition between levels $(r, s)$ and $(r, t)$, with level $s$ "higher" (more internal energy) than level $t$.

\footnotetext{
${ }^{16} \chi$ is usually not specified in ergs (as $h \nu$ usually is) but in $\mathrm{eV}$; one then uses a corresponding value for the Boltzmann constant $k$. Thus: $\exp (-\chi / k T)$ with $k=8.617 \times 10^{-5} \mathrm{eV} \mathrm{deg}^{-1}, \exp (-h \nu / k T)$ with $k=1.380 \times 10^{-16} \mathrm{erg} \mathrm{deg}^{-1}$. The physics convention is to use wavenumbers $\left(\mathrm{cm}^{-1}\right)$ and to measure these from the continuum down instead of from the ground state up.
} 
Saha distribution. The Saha ionization distribution for the population ratio between the ground levels of successive ionization stages is:

$$
\left[\frac{n_{r+1,1}}{n_{r, 1}}\right]_{\mathrm{LTE}}=\frac{1}{N_{\mathrm{e}}} \frac{2 g_{r+1,1}}{g_{r, 1}}\left(\frac{2 \pi m_{\mathrm{e}} k T}{h^{2}}\right)^{3 / 2} \mathrm{e}^{-\chi_{r} / k T},
$$

with $N_{\mathrm{e}}$ the electron density, $m_{\mathrm{e}}$ the electron mass, $n_{r+1,1}$ and $n_{r, 1}$ the population densities of the two ground states of the successive ionization stages $r$ and $r+1, \chi_{r}$ the ionization energy of stage $r$ (the minimum energy needed to free an electron from the ground state of stage $r$, with $\left.\chi_{r}=h \nu_{\text {threshold }}\right)$ and $g_{r+1,1}$ and $g_{r, 1}$ the statistical weights of the two ground levels. The freed electron has statistical weight 2 due to its choice of spin orientation. For the total population of two successive ionization stages the Saha distribution is:

$$
\left[\frac{N_{r+1}}{N_{r}}\right]_{\mathrm{LTE}}=\frac{1}{N_{\mathrm{e}}} \frac{2 U_{r+1}}{U_{r}}\left(\frac{2 \pi m_{\mathrm{e}} k T}{h^{2}}\right)^{3 / 2} \mathrm{e}^{-\chi_{r} / k T},
$$

with $N_{r+1}$ and $N_{r}$ the total population densities of the two successive ionization stages $r$ and $r+1, \chi_{r}$ the ionization energy of stage $r$ and the partition function $U_{r}$ of stage $r$ given by

$$
U_{r} \equiv \sum_{s} g_{r, s} \mathrm{e}^{-\chi_{r, s} / k T}
$$

Appendix D of 'Gray $(19 \overline{9} 2)$, contains polynomial approximations of $U_{r}$ for many atoms and singly-ionized ions. Other tables are given by Halenka and Grabowski $(1984)$.

Saha-Boltzmann distribution. Combination of the two distributions gives the LTE population ratio between a particular level $i$ and the ion state $c$ to which it ionizes as:

$$
\left[\frac{n_{c}}{n_{i}}\right]_{\mathrm{LTE}}=\frac{1}{N_{\mathrm{e}}} \frac{2 g_{c}}{g_{i}}\left(\frac{2 \pi m_{\mathrm{e}} k T}{h^{2}}\right)^{3 / 2} \mathrm{e}^{-\chi_{c i} / k T}
$$

with $n_{i}$ the total population density of level $i, n_{c}$ the number of ions in ionization level $c$ (usually the ground state of the ion term system but sometimes an excited level with excitation energy $\left.\chi_{r+1, c}\right)$ and $\chi_{c i}=\chi_{r}-\chi_{r, i}+\chi_{r+1, c}=h \nu_{\text {threshold }}$ the ionization energy from level $i$ to state $c$.

\subsubsection{Radiation in LTE}

Planck function. In LTE the Boltzmann distribution holds so that the line source function simplifies from (2.27) on page $2 \overline{2} \overline{4}$, to the Planck function:

$$
\begin{aligned}
{\left[S_{\nu}^{l}\right]_{\mathrm{LTE}} } & =\frac{2 h \nu^{3}}{c^{2}} \frac{1}{\left[\frac{g_{u} n_{l}}{g_{l} n_{u}}\right]_{\mathrm{LTE}}-1} \\
& =\frac{2 h \nu^{3}}{c^{2}} \frac{1}{\mathrm{e}^{h \nu / k T}-1} \equiv B_{\nu}(T) .
\end{aligned}
$$

This equality $S_{\nu}=B_{\nu}$ is formally derived here through the Einstein coefficients for boundbound processes, thus only for the line source function, but it holds for all LTE ("thermal") processes in which matter creates and destructs photons. 
Wien and Rayleigh-Jeans approximations. For large $h \nu / k T$ the numerator has $\exp (h \nu / k T) \gg 1$ yielding the Wien approximation:

$$
B_{\nu}(T) \approx \frac{2 h \nu^{3}}{c^{2}} \mathrm{e}^{-h \nu / k T},
$$

expressing the particle-like behavior of photons at high energy by being similar to the Boltzmann distribution. For small $h \nu / k T$ the approximation $\exp (h \nu / k T)-1 \approx h \nu / k T$ gives the Rayleigh-Jeans approximation:

$$
B_{\nu}(T) \approx \frac{2 \nu^{2} k T}{c^{2}}
$$

which is wave-like in character (Shu $\overline{1} \overline{9} \overline{1} \overline{1}, \mathrm{p} .7)$.

Stefan-Boltzmann law. Spectral integration produces the Stefan-Boltzmann law:

$$
B(T)=\int_{0}^{\infty} B_{\nu} \mathrm{d} \nu=\frac{\sigma}{\pi} T^{4},
$$

with

$$
\sigma=\frac{2 \pi^{5} k^{4}}{15 h^{3} c^{2}}=5.67 \times 10^{-5} \quad \mathrm{erg} \mathrm{cm}^{-2} \mathrm{~K}^{-4} \mathrm{~s}^{-1} .
$$

Induced emission. The LTE correction factor for bound-bound induced emission in (2.63i) on page $203 \overline{1}, 1$ is:

$$
\left[1-\frac{n_{u} B_{u l} \chi\left(\nu-\nu_{0}\right)}{n_{l} B_{l u} \varphi\left(\nu-\nu_{0}\right)}\right]_{\mathrm{LTE}}=1-\mathrm{e}^{-h \nu_{0} / k T} .
$$

The profile functions $\varphi$ and $\chi$ are equal in LTE because otherwise detailed balancing per wavelength would not be feasible in TE.

Line extinction. The LTE line extinction coefficient is:

$$
\left[\alpha_{\nu}^{l}\right]_{\mathrm{LTE}}=\frac{\pi e^{2}}{m_{\mathrm{e}} c} n_{l}^{\mathrm{LTE}} f_{l u} \varphi\left(\nu-\nu_{0}\right)\left[1-\mathrm{e}^{-h \nu_{0} / k T}\right]
$$

with $n_{l}^{\mathrm{LTE}} \equiv\left[n_{l}\right]_{\text {LTE }}$ given by the Saha-Boltzmann distributions for the local kinetic temperature $T_{\mathrm{e}}$. The classical oscillator strength $f_{l u}$ is defined by $\left(\overline{2} \cdot \overline{2} \overline{6}_{i}\right)$ on page $2 \overline{2} \overline{3}_{-1}$. It is often combined with the lower-level statistical weight $g_{l}$ (which sits in $n_{l}^{\mathrm{LTE}}$ through $\left(2.8 \overline{6} \bar{\sigma}_{1}\right)$ ) into the so-called $g f$-value measuring transition probability.

Discussion. The essential premise of LTE is that collisions control the energy partitioning of the matter in the medium more strictly than that they control the energy partitioning of the radiation. All material energy distributions (velocity, ionization, excitation, dissociation) are then fixed by the local kinetic temperature (Maxwell, Saha, Boltzmann), while the radiative energy distributions may depart slightly from the local TE values:

$$
S_{\nu}^{l}(\vec{r})=B_{\nu}[T(\vec{r})] \quad I_{\nu}(\vec{r}, \vec{l}) \neq B_{\nu}[T(\vec{r})] \quad J_{\nu}(\vec{r}) \neq B_{\nu}[T(\vec{r})] \quad \mathcal{F}_{\nu}(\vec{r}) \neq 0 .
$$


The LTE equality $S_{\nu}=B_{\nu}$ holds when the source function is dominated by collisions and/or when the frequency- and angle-averaged radiation field is Planckian, as shown for two-level atoms by $(2 . \overline{1} \overline{4} \overline{4})$ and $(2.1 \overline{4} \overline{1})$ on page $\overline{4} \overline{1}$. Deep within stars both conditions are fulfilled. There, the photons are "honorary particles" (Castor) that fully participate in the thermodynamics of the gas. Their mean free paths are much smaller than the scales over which state parameters vary appreciably. However, even there $I_{\nu}$ is not exactly isotropic (as required for $I_{\nu}=B_{\nu}$ ); therefore, (or rather, because) the net flux has $\mathcal{F}_{\nu}(\vec{r}) \neq 0$ and transports energy outward, a leak that inhibits strict TE even for the very close confinement within stars - strict TE doesn't exist in nature.

\subsection{NLTE}

Non-local thermodynamical equilibrium (NLTE or non-LTE) is a loose term which implies that the assumption of LTE fails. Often one then assumes statistical equilibrium implicitly, usually with the Maxwell distribution and complete redistribution in frequency and angle. However, the populations are now permitted to differ from the local Saha-Boltzmann equilibrium values.

\subsubsection{Statistical equilibrium}

Rate equations. Statistical equilibrium (SE) implies that the radiation fields (in all directions and on all frequencies) and level populations do not vary with time, as expressed in the statistical equilibrium equations (population equations, rate equations):

$$
\frac{\mathrm{d} n_{i}(\vec{r})}{\mathrm{d} t}=\sum_{j \neq i}^{N} n_{j}(\vec{r}) P_{j i}(\vec{r})-n_{i}(\vec{r}) \sum_{j \neq i}^{N} P_{i j}(\vec{r})=0,
$$

with $n_{i}$ the population of a particular level, $N$ the total number of levels that are important for the population of level $n_{i}$ one way or another, and $j$ stepping over all those levels. The transition rates $P_{i j}$ for radiative and collisinal processes, respectively, are given per particle in state $i$ or $j$ by:

$$
P_{i j}=R_{i j}+C_{i j}
$$

For a bound-bound transition the radiative rate per particle is:

$$
R_{i j}=A_{i j}+B_{i j} \bar{J}_{\nu_{0}}
$$

A similar expression holds for radiative bound-free rates but with $\bar{J}_{\nu_{0}}$ averaged over the ionization edge. General expressions for radiative rates $R_{i j}$ in bound-bound and bound-

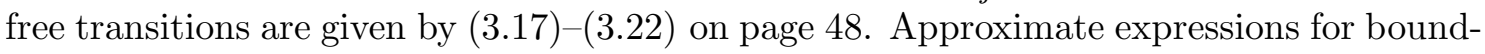

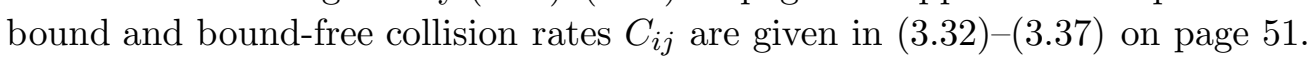

Transport equations. The population equations (2.100i) contain the mean intensities at all relevant frequencies via $\bar{J}_{\nu_{0}}$ terms as in $(2.102 i)$. The intensities are given by the radiation transport equations

$$
\mu \frac{\mathrm{d} I_{\nu}(\vec{r}, \mu)}{\mathrm{d} \tau_{\nu}(\vec{r})}=-S_{\nu}(\vec{r})+I_{\nu}(\vec{r}, \mu)
$$

at all frequencies $\nu$, directions $\mu$ and locations $\vec{r}$ that are important in $(2 \underline{2} . \overline{1} 0 \overline{0})$ one way or another. Thus, the rates $P_{i j}$ in $(2 . \overline{1} 0 \overline{0})$ depend on $\bar{J}_{\nu}$ and therefore on $I_{\nu}$ in other 
directions, whereas the optical depths $\tau_{\nu}$ and the source functions $S_{\nu}$ in $(2 . \overline{2} \overline{1} \overline{3})$ depend on the populations $n_{l}$ and $n_{u}$ of the lower and upper levels involved in transitions at the frequency $\nu$. These populations may depend on other transitions and therefore on other populations again, each dependent on radiation fields at other frequencies. Thus, a given transition of interest may be influenced by many other transitions in the same particle species, or by transitions in other atoms and molecules if these possess transitions at overlapping frequencies. The latter include all interactions that cause the continuum background at a frequency of interest. This intricate coupling between populations and radiation is non-linear and non-local. It can be very complex, except when the tremendous simplification of LTE may be assumed.

Time-dependent transfer. When SE does not hold the population equations must satisfy overall particle conservation (continuity) rather than population conservation. These equations and the transport equations then become time dependent. Systematic flows make the source function anisotropic (page $\left.\underline{1}_{\underline{1}}^{\overline{1}} \overline{1}_{1}^{1}\right)$.

Multi-dimensional transfer. Lateral inhomogeneity is likely to come with time dependence so that the geometry of stellar-atmosphere radiative transfer becomes two- or three-dimensional, instead of the one-dimensional plane-parallel simplification assumed throughout these lecture notes. Such complexity requires the full sophistication of elaborate radiative hydrodynamics ('Mīinalas and

\subsubsection{NLTE descriptions}

Departure coefficients. NLTE population departure coefficients $b_{i}$ are defined as:

$$
b_{l}=n_{l} / n_{l}^{\mathrm{LTE}} \quad b_{u}=n_{u} / n_{u}^{\mathrm{LTE}}
$$

with $n$ the actual population and $n^{\mathrm{LTE}}$ the Saha-Boltzmann values for the lower and upper

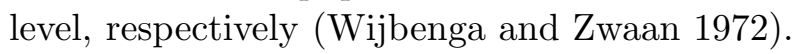

Bound-bound source function. Expressed in departure coefficients the general line source function $(2.721)$ becomes

$$
S_{\nu}^{l}=\frac{2 h \nu^{3}}{c^{2}} \frac{\psi / \varphi}{\frac{b_{l}}{b_{u}} \mathrm{e}^{h \nu / k T}-\frac{\chi}{\varphi}}
$$

and for complete redistribution with $\chi_{\nu}=\psi_{\nu}=\varphi_{\nu}$

$$
S_{\nu_{0}}^{l}=\frac{2 h \nu_{0}^{3}}{c^{2}} \frac{1}{\frac{b_{l}}{b_{u}} \mathrm{e}^{h \nu_{0} / k T}-1},
$$

where $S_{\nu_{0}}^{l}$ does not depend on frequency over the extent of a narrow line. In the Wien regime with $\left(b_{l} / b_{u}\right) \exp (h \nu / k T) \gg 1$ the fractional departure of the source function from the Planck function is given by the inverse fugacity ratio $b_{u} / b_{l}$ :

$$
S_{\nu_{0}}^{l} \approx \frac{b_{u}}{b_{l}} B_{\nu_{0}}
$$


Bound-bound extinction. The monochromatic line extinction coefficient (2.63i) on page $\overline{2} \overline{3}$ becomes:

$$
\begin{aligned}
\alpha_{\nu}^{l} & =\frac{h \nu}{4 \pi} b_{l} n_{l}^{\mathrm{LTE}} B_{l u} \varphi\left(\nu-\nu_{0}\right)\left[1-\frac{b_{u} n_{u}^{\mathrm{LTE}} B_{u l} \chi}{b_{l} n_{l}^{\mathrm{LTE}} B_{l u} \varphi}\right] \\
& =\frac{h \nu}{4 \pi} b_{l} n_{l}^{\mathrm{LTE}} B_{l u} \varphi\left(\nu-\nu_{0}\right)\left[1-\frac{b_{u}}{b_{l}} \frac{\chi}{\varphi} \mathrm{e}^{-h \nu / k T}\right] \\
& =b_{l} n_{l}^{\mathrm{LTE}} \sigma_{\nu}^{l}\left[1-\frac{b_{u}}{b_{l}} \frac{\chi}{\varphi} \mathrm{e}^{-h \nu / k T}\right] \\
& =\frac{\pi e^{2}}{m_{\mathrm{e}} c} b_{l} n_{l}^{\mathrm{LTE}} f_{l u} \varphi\left(\nu-\nu_{0}\right)\left[1-\frac{b_{u}}{b_{l}} \frac{\chi}{\varphi} \mathrm{e}^{-h \nu / k T}\right]
\end{aligned}
$$

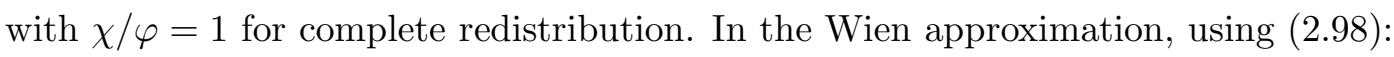

$$
\alpha_{\nu}^{l} \approx b_{l}\left[\alpha_{\nu}^{l}\right]_{\mathrm{LTE}} .
$$

Similarly, the total line extinction coefficient $\left(\overline{2} 2 . \overline{6} 4_{i}\right)$ on page $2 \overline{2} \overline{3} \overline{3}$ becomes

$$
\begin{aligned}
\alpha_{\nu_{0}}^{l} & =\frac{h \nu_{0}}{4 \pi} b_{l} n_{l}^{\mathrm{LTE}} B_{l u}\left[1-\frac{b_{u}}{b_{l}} \mathrm{e}^{-h \nu_{0} / k T}\right] \\
& =\frac{\pi e^{2}}{m_{\mathrm{e}} c} b_{l} n_{l}^{\mathrm{LTE}} f_{l u}\left[1-\frac{b_{u}}{b_{l}} \mathrm{e}^{-h \nu_{0} / k T}\right] \\
& \approx b_{l}\left[\alpha_{\nu_{0}}^{l}\right]_{\mathrm{LTE}} .
\end{aligned}
$$

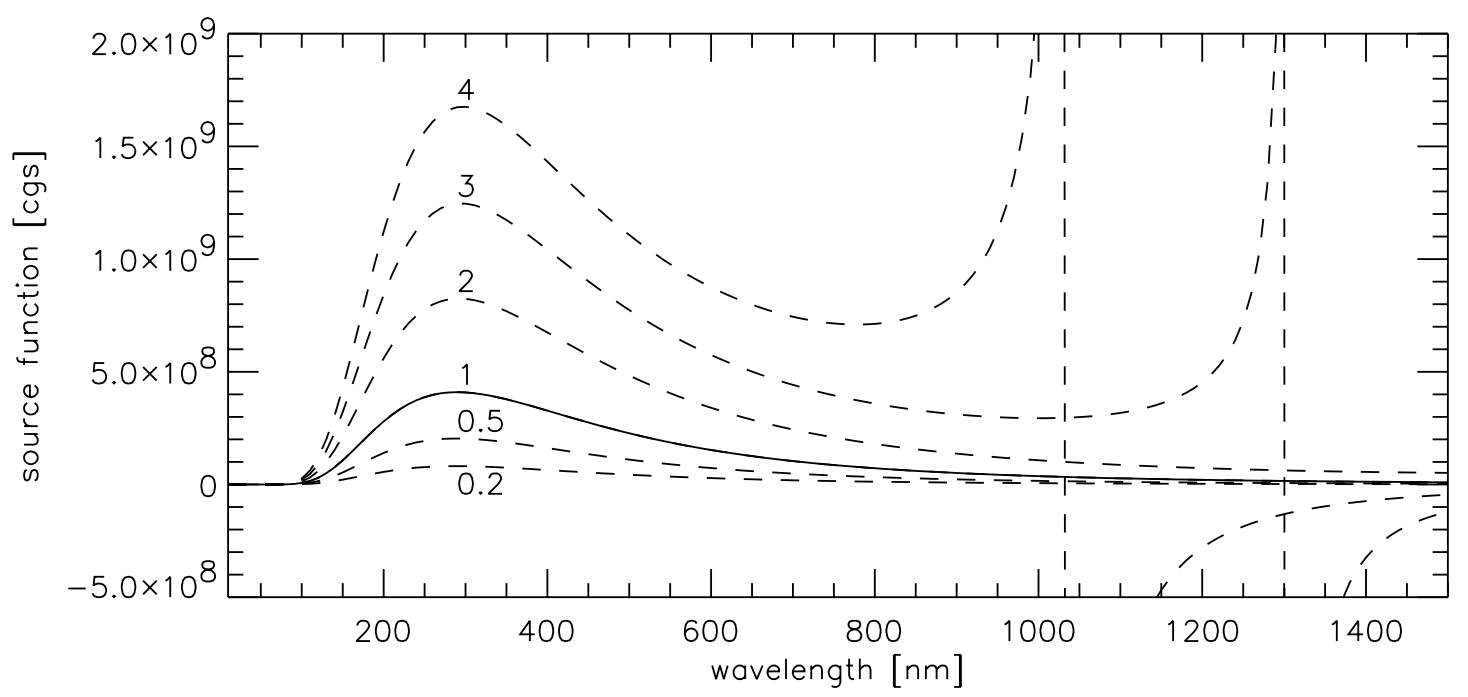

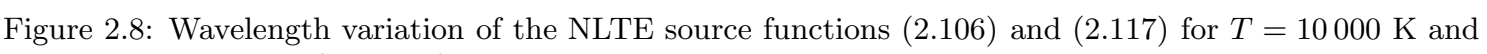
the specified ratios $b_{u} / b_{l}$ or $b_{c} / b_{i}$, respectively, in cgs units with $\bar{\Delta} \bar{\lambda}=1 \mathrm{~nm}$. The NLTE source function scales with the Planck function (solid curve) in the Wien part at left, but reaches the laser regime for large $b_{u} / b_{l}$ in the Rayleigh-Jeans part at right. 
Laser regime. In the Rayleigh-Jeans part $(h \nu \ll k T)$ the volume extinction coefficient $\alpha_{\nu_{0}}^{l}$ becomes negative for sufficient excess $b_{u}>b_{l}$ due to the correction for stimulated emission. Light amplification rather than extinction then occurs along the beam (lasering). The line source function $S_{\nu_{0}}^{l}$ also goes negative when $1-\left(b_{u} / b_{l}\right) \exp \left(-h \nu_{0} / k T\right)<0$ while the line emissivity $j_{\nu_{0}}^{l}=\alpha_{\nu_{0}}^{l} S_{\nu_{0}}^{l}$ remains positive. The NLTE source function correction factor is about the reverse of the NLTE line extinction correction factor:

$$
\frac{S_{\nu_{0}}^{l}}{B_{\nu_{0}}}=\frac{1-\mathrm{e}^{-h \nu_{0} / k T}}{\left(b_{l} / b_{u}\right)\left[1-\left(b_{u} / b_{l}\right) \mathrm{e}^{\left.-h \nu_{0} / k T\right]}\right.}=b_{u} \frac{\left[\alpha_{\nu_{0}}^{l}\right]_{\mathrm{LTE}}}{\alpha_{\nu_{0}}^{l}},
$$

so that the source function blows up to large values before it becomes negatives. illustrated in Figure 2.8 .

Bound-free source function. The general monochromatic bound-free source function is given by

$$
S_{\nu}^{\mathrm{bf}}=\frac{2 h \nu^{3}}{c^{2}} \frac{1}{\frac{b_{i}}{b_{c}} \mathrm{e}^{h \nu / k T}-1}
$$

where the index $i$ denotes the ionizing level and the index $c$ the level of the next stage of ionization into which it ionizes (the "parent", usually the ion ground state, as in (2.90)

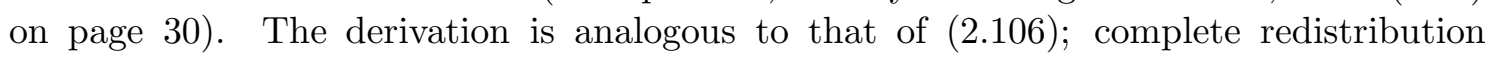
holds because the collisional capture of a free electron represents a fresh sampling without memory for the kinetic energy bestowed in preceding ionization. In this case the spectral feature may be quite wide. In the Wien regime (negligible stimulated recombination) the monochromatic source function has Planckian frequency dependence over the feature width:

$$
S_{\nu}^{\mathrm{bf}} \approx \frac{b_{c}}{b_{i}} B_{\nu}
$$

The edge-averaged bound-free source function is given by $\left(3 . \overline{1} 08_{i}\right)$ on page ${ }_{1}^{1} \overline{3}$.

Bound-free extinction. The monochromatic bound-free extinction coefficient per $\mathrm{cm}$ including correction for induced emission is similarly given by:

$$
\alpha_{\nu}^{\mathrm{bf}}=b_{i} n_{i}^{\mathrm{LTE}} \sigma_{i c}(\nu)\left(1-\frac{b_{c}}{b_{i}} \mathrm{e}^{-h \nu / k T}\right) .
$$

Bound-free emission. The monochromatic bound-free emissivity can be written with $(2.116 i)$ as

$$
j_{\nu}^{\mathrm{bf}}=\alpha_{\nu}^{\mathrm{bf}} S_{\nu}^{\mathrm{bf}}=b_{c}\left[\alpha_{\nu}^{\mathrm{bf}}\right]_{\mathrm{LTE}} B_{\nu}
$$

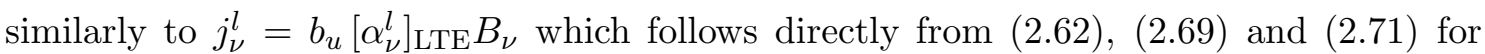
complete redistribution. In the Wien part $j_{\nu}^{\text {bf }}$ scales $\sim \sigma_{\nu}^{\text {bf }} \nu^{3} \exp (-h \nu / k T)$, for hydrogen or hydrogenic ions $\sim \exp (-h \nu / k T)$, so that the emission edge is much sharper than the extinction edge. In the Rayleigh-Jeans part the emissivity is not affected by lasering because the stimulated emission went to $\alpha_{\nu}^{\text {bf }}$ in (2.119i). Note that $j_{\nu}^{\text {bf }} \sim n_{c} N_{\mathrm{e}}$ as expected

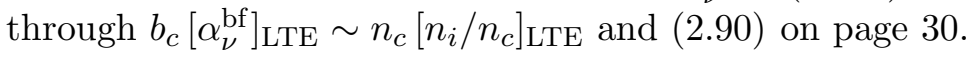

\footnotetext{
${ }^{17} \mathrm{Such}$ increase explains the strong solar emission lines of $\mathrm{Mg} \mathrm{I}$ near $\lambda=12 \mu \mathrm{m}$ ('Carlsson et al. $1992 \mathrm{i}$ ). They would laser for $b_{u} / b_{l}>1.27$ at $T=5000 \mathrm{~K}$ but do not reach such large overexcitation. Lasering goes with population inversion since $\left(b_{u} / b_{l}\right) \exp \left(-h \nu_{0} / k T\right)>1$ makes $n_{u} / n_{l}=\left(b_{u} / b_{l}\right)\left(g_{u} / g_{l}\right) \exp \left(-h \nu_{0} / k T\right)>$ $g_{u} / g_{l}$ and usually $g_{u}>g_{l}$, for example $g=2 n^{2}$ hydrogenically.
} 
Free-free source function, extinction, emission. For completeness the corresponding free-free expressions:

$$
\begin{aligned}
S_{\nu}^{\mathrm{ff}} & =B_{\nu} \\
\alpha_{\nu}^{\mathrm{ff}} & =b_{c} n_{c}^{\mathrm{LTE}} \sigma_{\nu}^{\mathrm{ff}}\left(1-\mathrm{e}^{-h \nu / k T}\right) \\
j_{\nu}^{\mathrm{ff}} & =b_{c}\left[\alpha_{\nu}^{\mathrm{ff}}\right]_{\mathrm{LTE}} B_{\nu}
\end{aligned}
$$

where $\sigma_{\nu}^{\mathrm{ff}}$ is given by (2.7\%) on page $\overline{2} \overline{6}_{\mathrm{r}}^{\mathrm{r}} \quad$ The free-free source function and stimulated emission correction are thermal. Formally, this follows by setting $b_{i}=b_{c}$ in $(2.1 \overline{1} \bar{i})$ and (2.119i). Physically, it follows from the assumption of the Maxwell distribution for the kinetic energy partitioning, sampled afresh by each new bremsstrahlung photon. Departures from LTE in the ion population that are caused by other transitions affect the free-free extinction but not the amount of emission per extinction $=$ source function.

Discussion. These formal expressions do not specify what part of the source function is controlled by $B_{\nu}$ or by $J_{\nu}$ (at this frequency or at other frequencies); they only express the overall result in term of population departures relative to the LTE equilibrium values. The actual values of $n_{l}$ and $n_{u}$ may depend on $B_{\nu}$ and $J_{\nu}$ at widely different frequencies through other transitions that may feed excess population into these levels or deplete them excessively. Bound-free transitions contain at least partial thermalization, since part of the photon energy produces kinetic energy (ionization) or uses kinetic energy (recombination), but they may also depend on $J_{\nu}$ in the ionization edge or at other frequencies. This partial thermalization and sensitivity to radiation is also hidden in the resulting population departures.

Only free-free photon emission and extinction are strictly kinetic, respectively creating and destroying photons in every interaction, with $S_{\nu}^{\mathrm{ff}}=B_{\nu}$ and $\alpha_{\nu}^{\mathrm{ff}}=b_{c}\left[\alpha_{\nu}^{\mathrm{ff}}\right]$ LTE wherever the Maxwell distribution holds.

Lasering occurs at sufficient bound-bound overexcitation or bound-free overionization, but only when other transitions provide a mechanism to overpopulate the higher level (see

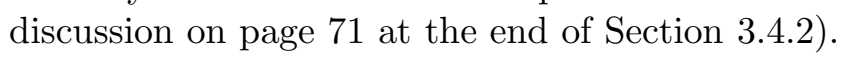

Warning. I should add a warning here that the departure coefficients $b_{i}$ are often de-

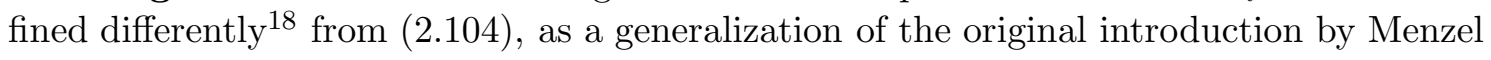
iand Cillié (1937) for H I in which the departure of the neutral hydrogen population was normalized by the HII density (free protons). In the Menzel convention, the $b_{i}$ coefficients are normalized to the LTE population of the next ionization stage by a partial Saha-Boltzmann evaluation, whereas in the preferable Zwaan convention of 'W' 'Zwaan (1972)!' used here the $b_{i}$ are normalized by the total particle density (abundance) of the element through the complete Saha-Boltzmann relations. The two conventions are:

$$
\begin{gathered}
b_{i}^{\text {Zwaan }} \equiv n_{i} / n_{i}^{\mathrm{LTE}} \\
b_{i}^{\mathrm{Menzel}} \equiv \frac{n_{i} / n_{i}^{\mathrm{LTE}}}{n_{C} / n_{C}^{\mathrm{LTE}}}
\end{gathered}
$$

where $n_{C}$ is the total population of the next ion. Sometimes its ground state is used instead

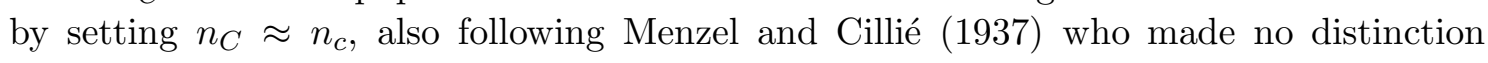

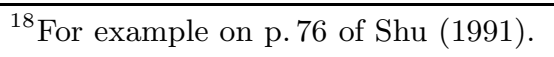


because free protons do not possess excited statesit. When the atom (or ion) containing level $i$ is predominantly ionized so that $n_{C} \approx n_{C}^{\mathrm{LTE}}{ }^{-}$because most particles of the species sit in that stage, then

$$
b_{i}^{\mathrm{Menzel}} \approx n_{i} / n_{i}^{\mathrm{LTE}} \approx b_{i}^{\mathrm{Zwaan}},
$$

but when most of the element sits in level $i$ itself

$$
b_{i}^{\mathrm{Menzel}} \approx n_{C}^{\mathrm{LTE}} / n_{C} \approx 1 / b_{C}^{\text {Zwaan }} .
$$

The continuum has $b_{C}^{\text {Menzel }} \equiv 1$ and the ratio $b_{i} / b_{C} \approx b_{i} / b_{c}$ is the same in both definitions.

The warning is thus that one sometimes has to reinterpret plots of $b_{i}$ coefficients in publications to obtain the actual departures from Saha-Boltzmann populations. Sometimes the authors themselves aren't aware that they actually plot $1 / b_{C}$ instead of $b_{i}$. The reader then has to reverse their interpretations and perhaps their conclusions $s_{-}^{\prime 2}$

Formal temperatures. Another way to formalize the deviation of the source function from the Planck function is to introduce formal NLTE temperatures $T_{x}$ with $T_{x}=T_{\mathrm{e}}$ in LTE and $T_{x} \neq T_{\mathrm{e}}$ outside LTE. They are useful when comparing radiation or source function behavior at different wavelengths by cancelling the Planck function variation with

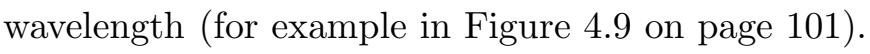

The excitation temperature $T_{\mathrm{exc}}$ is defined by

$$
\frac{n_{u}}{n_{l}} \equiv \frac{g_{u}}{g_{l}} \mathrm{e}^{-h \nu / k T_{\text {exc }}}
$$

as the temperature to be entered into the Boltzmann distribution to obtain the actual population ratios between levels within the same stage of ionization. The general line source function (2.73i) for complete redistribution then becomes

$$
S_{\nu_{0}}^{l}=\frac{2 h \nu_{0}^{3}}{c^{2}} \frac{1}{\frac{g_{u} n_{l}}{g_{l} n_{u}}-1}=\frac{2 h \nu_{0}^{3}}{c^{2}} \frac{1}{\mathrm{e}^{h \nu_{0} / k T_{\mathrm{exc}}}-1}=B_{\nu_{0}}\left(T_{\mathrm{exc}}\right) .
$$

The ionization temperature $T_{\text {ion }}$ may similarly be defined as the temperature that must be formally entered instead of $T_{\mathrm{e}}$ in the Saha distribution (2.88) to obtain the actual ionization balance between two successive stages of ionization. Note that this $T_{\text {ion }}$ does not necessarily specify the bound-free source function $S_{\nu}^{\text {bf }}$ of $(2.117 i)$ when entered in the

\footnotetext{
${ }^{19}$ Nor do protons possess a yet higher ionization stage. The higher ionization stages are formally also included in the Zwaan definition but usually devoid of population for any transition of interest and therefore neglected in the generalized Menzel definition. This is permitted because NLTE departure coefficients are used in situations where departures from LTE are not so excessive that Saha-Boltzmann partitioning is not a reasonable first approximation. In that case there are only two adjacent ionization stages with significant population. There is not much use for NLTE departure coefficients in describing, for example, coronal conditions in which multiple ionization stages co-exist, all very far out of LTE. For these, ratioing to non-existing far-off LTE populations does not make sense.

${ }^{20}$ For example, various authors have taken the VALIII $b_{1}$ plot for solar H I on p. 663 of 'Vernazza et al.' (1981)! as evidence that the hydrogen ground state is underpopulated by a factor three in the solar temperature minimum region. That would be strange because all hydrogen sits in that ground state at such low temperature. The reason is simply that Nernazza et al. (1981), use the Menzel definition (as they in fact state carefully, specifying (12.125i)-(2.127i) on the same page). Their $b_{1}$ curve plots the Zwaan departure coefficient for the free proton population on an inverted scale. The proton density is larger than the Saha-Boltzmann value in the VALIII temperature minimum region due to overionization by the Balmer continuum which has $J_{\nu}>B_{\nu}$.
} 
Planck function because (2.117i) describes bound-free transitions between a specific level and a specific ion state, not the summed populations of two whole stages as in (2.88). The level-to-continuum combined Saha-Boltzmann equation (2.90i) must be used to define a $T_{\text {ion }}$ per bound-free transition that has

$$
S_{\nu}^{\mathrm{bf}} \equiv \frac{2 h \nu^{3}}{c^{2}} \frac{1}{\mathrm{e}^{h \nu / k T_{\text {ion }}}-1}=B_{\nu}\left(T_{\mathrm{ion}}\right) .
$$

The radiation temperature $T_{\text {rad }}$ expresses the mean intensity into the Planck function by setting

$$
B_{\nu}\left(T_{\text {rad }}\right) \equiv J_{\nu}
$$

The brightness temperature $T_{\mathrm{b}}$ expresses the observed intensity into the Planck function through

$$
B_{\nu}\left(T_{\mathrm{b}}\right) \equiv I_{\nu}
$$

with $T_{\mathrm{b}}=T_{\mathrm{e}}\left(\tau_{\nu}=\mu\right)$ for the observed intensity that emerges from an optically thick plane-parallel LTE medium when the Eddington-Barbier approximation holds. Finally, the effective temperature $T_{\text {eff }}$ of a star is defined by

$$
\pi B\left(T_{\text {eff }}\right)=\sigma T_{\text {eff }}^{4} \equiv \mathcal{F}_{\text {surface }}
$$

and expresses the spectrum-integrated flux $\mathcal{F}^{+}$leaving the star per $\mathrm{cm}^{2}$ of its surface as a

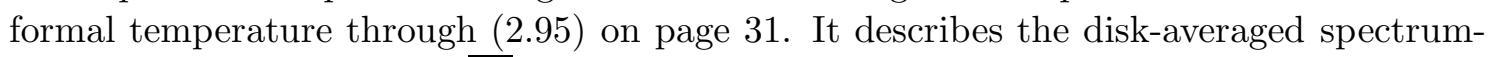

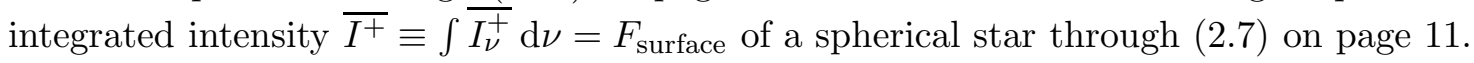

\subsubsection{Coherent scattering}

A principal NLTE situation in stellar atmosphere occurs at locations and wavelengths where scattering is important ${ }_{-}^{27 !}$ The easiest case of photon scattering is when it is isotropic and monochromatic, where the latter term means that there is no frequency shift between the incoming and the outgoing photon. Such monofrequent scattering is usually called "coherent". Examples are Thomson scattering off free electrons (neglecting Dopplershifts) and resonance scattering in atomic bound-bound transitions. Neither process is strictly

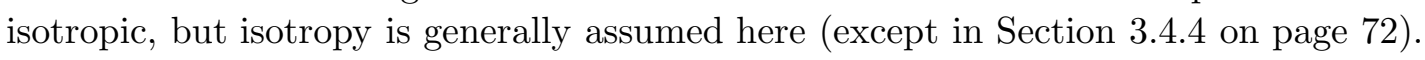

Two-level atoms. Two-level atoms are a useful idealization that permits detailed discussion of spatial non-locality due to photon scattering processes without having to bother with spectral non-locality due to photon conversion processesi- ${ }^{22 !}$. For two-level atoms the five bound-bound processes may be combined in different pairs:

\footnotetext{
${ }^{21}$ In planetary atmospheres NLTE scattering is the rule. "Most of the light we see reaches our eyes in an indirect way. Looking at a tree, or a house, we see diffusely reflected sunlight. Looking at_a cloud, or at the sky, we see scattered sunlight." says the introduction to the standard book by van de Hulst (1957)! A very NLTE situation: the light is last scattered nearby but yet possesses non-local solar color temperature and even the solar line spectrum (a spectrum of my nose showing the solar Ca II H\& K lines). When droplets and dust particles are involved, scattering requires more complex mathematical formulation than the (nearly) isotropical scattering in stellar atmospheres. Van de_Hulst's book treats single scattering; he has added detailed recipes for multiple scattering in van de Hulst (1980). Scattering is also becoming important in illumination simulations, for example in computer visualization of human skin which gets its pink tint from subsurface scattering on blood vessels.

${ }^{22}$ The classical example of spectral non-locality (non-monochromaticity) due to photon conversion is the Zanstra mechanism. It makes cold planetary nebulae appear bright in H I Balmer- $\alpha$ in the red part of the spectrum thanks to ultraviolet H I Lyman continuum irradiation followed by radiative recombination and radiative cascade through the $\mathrm{H}$ I energy levels. The mechanism is also spatially non-local since the Lyman photons come from the hot star at the center of the nebula, often not even seen in the visible.
} 
- photon scattering: radiative excitation followed by spontaneous or induced radiative deexcitation. The new photon is often described as still being the old one, but it has been re-directed and has possibly been slightly shifted (redistributed) in frequency;

- photon creation: collisional excitation followed by spontaneous or induced radiative deexcitation. This pair makes a new photon out of kinetic energy;

- photon destruction: radiative excitation followed by collisional deexcitation. This pair thermalizes a photon into kinetic energy.

Similar pairs hold for bf, $\mathrm{ff}$ and other processes. The split is treated in more detail in

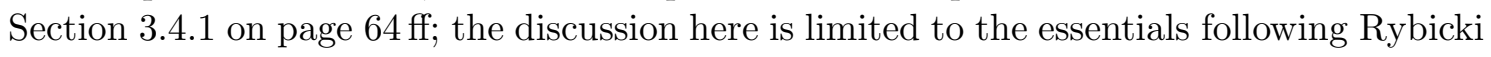
i. and Lightman $(1 \overline{9} \overline{7} \overline{9})$ i

Coherently scattering medium. A useful simplification of resonance scattering is to assume that the medium consists purely of two-level atoms and that the scattering is coherent. Each radiative excitation is then necessarily followed by deexcitation in the same transition, either a radiative one at exactly the same frequency (spontaneous or induced; photon scattering) or a collisional one (photon destruction). In repeated scattering, photons step in random walk through the medium without coupling their energy to the local conditions. On the other hand, the photon creation and photon destruction pairs couple the radiation energy to the local kinetic energy; these pairs constitute "thermal" processes $_{1}^{233}$.'

The strength of the coupling depends on the relative frequency of the thermal and scattering sequences. The monochromatic bound-bound extinction coefficient $\alpha_{\nu}^{l}$ may in this case be written as the sum of partial extinction coefficients $\alpha_{\nu}^{\mathrm{a}}$ for photon destruction (absorption, often called "true absorption" when "absorption" is used for extinction) and $\alpha_{\nu}^{\mathrm{s}}$ for photon scattering:

$$
\alpha_{\nu}^{l}=\alpha_{\nu}^{\mathrm{a}}+\alpha_{\nu}^{\mathrm{s}} .
$$

The continuous extinction coefficient may similarly be split into a coherent scattering part due to Thomson or Rayleigh scattering and a thermal destruction part due to free-frea . $_{\text {I }}$ processes.

Destruction probability. The photon destruction probability per extinction for coherently scattering two-level atoms is given by

$$
\varepsilon_{\nu} \equiv \frac{\alpha_{\nu}^{\mathrm{a}}}{\alpha_{\nu}^{\mathrm{a}}+\alpha_{\nu}^{\mathrm{s}}}
$$

Its complement is the scattering probability per extinction:

$$
1-\varepsilon_{\nu}=\frac{\alpha_{\nu}^{\mathrm{s}}}{\alpha_{\nu}^{\mathrm{a}}+\alpha_{\nu}^{\mathrm{s}}}
$$

\footnotetext{
${ }^{23}$ When the Maxwell distribution holds so that the local kinetic energy of all material particle species (fermions) is parametrized by the local electron temperature. This is generally the case in stellar atmospheres and is assumed tacitly throughout these lecture notes - together with the assumption that all fermions occur exclusively as a gas of free atoms and/or ions plus electrons and/or molecules, without aggregation into fluid or solid states or boson condensates. This quite reasonable assumption makes radiative transfer in stellar atmospheres again simpler than radiative transfer in planetary atmospheres.

${ }^{24}$ Or bound-free processes, but these may mix thermal and scattering behavior since the part of the energy jump above the ionization limit is converted into kinetic energy while the remainder represents internal atomic energy rather like a bound-bound transition. More on this in Section 3.2 .2 ' on page ' 15 ' and Section 3.4 .5 on page 172
} 
Effective path, thickness, depth. The effective path length which a photon has come away from its origin after $N$ randomly directed scattering steps through a coherently scattering, homogeneous medium is:

$$
l_{\nu}^{*} \approx \sqrt{N} l_{\nu}
$$

with free path per step (Eq.

$$
l_{\nu}=\frac{\left\langle\tau_{\nu}\right\rangle}{\alpha_{\nu}}=\frac{1}{\alpha_{\nu}^{\mathrm{a}}+\alpha_{\nu}^{\mathrm{s}}} .
$$

Since the destruction probability is $\varepsilon_{\nu}$, a photon travels on average $N=1 / \varepsilon_{\nu}$ scattering steps between its creation and its destruction. The characteristic diffusion length or thermalization length or effective free path $l_{\nu}^{*}$ of scattered photons is therefore

$$
l_{\nu}^{*} \approx l_{\nu} / \sqrt{\varepsilon_{\nu}}
$$

and the effective optical thickness $\tau_{\nu}^{*}$ of a homogeneous layer is

$$
\tau_{\nu}^{*}=\sqrt{\varepsilon_{\nu}} \tau_{\nu},
$$

where $\tau_{\nu}$ is the optical thickness of the layer. In an optically thick object the effective radial optical depth $\tau_{\nu}^{*}$ is again defined the other way by

$$
\mathrm{d} \tau_{\nu}^{*}=\sqrt{\varepsilon_{\nu}} \mathrm{d} \tau_{\nu}
$$

where $\tau_{\nu}$ measures radial optical depth. In a homogeneous medium (constant $\varepsilon_{\nu}$ ), the value $\tau_{\nu}^{*} \approx 1$ marks the characteristic depth where newly created photons may embark on scattering sequences that eventually bring them to the surface and out, whereas the value $\tau_{\nu \mu} \approx 1$ marks the characteristic depth where they have their last scattering interaction and then escape in direction $\mu$. For $\varepsilon_{\nu} \ll 1$ the $\tau_{\mu \nu} \approx 1$ characteristic escape depth is much shallower than the $\tau_{\nu}^{*} \approx 1$ characteristic creation depth which lies at $\tau_{\nu} \approx 1 / \sqrt{\varepsilon_{\nu}}$. In that case, the escaping photons do not portray the conditions at the location from where they are observed. Reversely, the effect of photon escape at the object's surface on the source function and on the energy balance is "transported" by such scattering to deep layers,

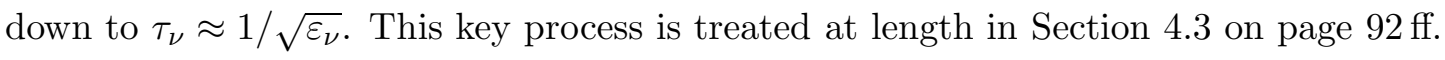

Source function. When the velocity distribution is Maxwellian, the monochromatic two-level line source function for collisional processes equals the Planck function so that

$$
j_{\nu}^{\mathrm{a}}=\alpha_{\nu}^{\mathrm{a}} B_{\nu}
$$

For pure coherent scattering, each photon that is redirected into the beam represents a photon taken out of a beam with arbitrary direction ${ }_{-}^{\prime 25}$. The process source function then equals the photon supply per steradian as specified by the mean intensity $J_{\nu}$ :

$$
j_{\nu}^{\mathrm{s}}=\alpha_{\nu}^{\mathrm{s}} J_{\nu} .
$$

\footnotetext{
${ }^{25}$ Stimulated scattering (radiative excitation followed by induced deexcitation) into the beam requires an additional triggering photon in the beam direction so that its probability scales with the beam intensity. However, this contribution cancels against its reverse, loss of photons out of the beam due to stimulated scattering (Figure 13.3' on page ' 65 '). Only the spontaneous re-emission part counts, so that resonance scattering is an isotropic producer of "new" photons even where the radiation field is highly anisotropic - as in the outer atmosphere of a star.
} 
The combined two-level-atom line source function for a mixture of thermal absorption and coherent scattering is with $(2.22 i),(2.134)$ and $(2.135 i)$ :

$$
S_{\nu}^{l}=\frac{j_{\nu}^{\mathrm{a}}+j_{\nu}^{\mathrm{s}}}{\alpha_{\nu}^{\mathrm{a}}+\alpha_{\nu}^{\mathrm{s}}}=\left(1-\varepsilon_{\nu}\right) J_{\nu}+\varepsilon_{\nu} B_{\nu}
$$

An elaborate derivation in terms of Einstein coefficients is given in Section $3 . \overline{4} .11$ The expression holds also for monochromatic (elastic, coherent) Thomson and Rayleigh scattering in the presence of thermal bound-free and free-free continuum processes that cause photon destruction at the frequency $\nu$.

A similar expression holds for the case of complete redistribution:

$$
S_{\nu_{0}}^{l}=\left(1-\varepsilon_{\nu_{0}}\right) \bar{J}_{\nu_{0}}^{\varphi}+\varepsilon_{\nu_{0}} B_{\nu_{0}}
$$

where $\varepsilon_{\nu_{0}}$ is the profile-summed photon destruction probability defined by

$$
\varepsilon_{\nu_{0}} \equiv \frac{\alpha_{\nu_{0}}^{\mathrm{a}}}{\alpha_{\nu_{0}}^{\mathrm{a}}+\alpha_{\nu_{0}}^{\mathrm{s}}}
$$

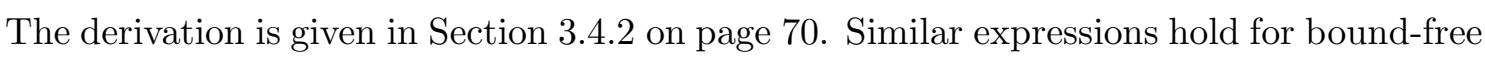
scattering (Section $3 . \overline{4} \cdot \overline{I_{1}^{\prime}}$ ) on page

Transport equation. For a medium made up of two-level atoms, the transport equation along the propagation direction

$$
\mathrm{d} I_{\nu}=-\alpha_{\nu}^{\mathrm{a}} I_{\nu} \mathrm{d} s-\alpha_{\nu}^{\mathrm{s}} I_{\nu} \mathrm{d} s+\alpha_{\nu}^{\mathrm{a}} B_{\nu} \mathrm{d} s+\alpha_{\nu}^{\mathrm{s}} J_{\nu} \mathrm{d} s
$$

is, with optical thickness $\mathrm{d} \tau_{\nu} \equiv \alpha_{\nu}^{l} \mathrm{~d} s=\left(\alpha_{\nu}^{\mathrm{a}}+\alpha_{\nu}^{\mathrm{s}}\right) \mathrm{d} s$, once more given by

$$
\frac{\mathrm{d} I_{\nu}}{\mathrm{d} \tau_{\nu}}=\frac{\mathrm{d} I_{\nu}}{\left(\alpha_{\nu}^{\mathrm{a}}+\alpha_{\nu}^{\mathrm{s}}\right) \mathrm{d} s}=S_{\nu}^{l}-I_{\nu}
$$

and when using $\tau_{\nu}$ for radial optical depth in axial symmetry by

$$
\mu \frac{\mathrm{d} I_{\nu}}{\mathrm{d} \tau_{\nu}}=I_{\nu}-S_{\nu}^{l}
$$

In the case of complete redistribution the monofrequent line source function has $S_{\nu}^{l}=S_{\nu_{0}}^{l}$ and does not vary over the line profilie $\mathrm{g}_{-}^{\prime \prime}$

\subsubsection{Multi-level interlocking}

Not yet...

\subsubsection{Coronal conditions}

Not yet...

\footnotetext{
${ }^{26}$ In the more realistic case of a medium containing not only two-level atoms but also other particles which contribute continuous extinction at the line wavelength, the total source function replaces $S_{\nu}^{l}$ in the transport equation. It varies over the line profile, even if $S^{l}$ does not in the complete-redistribution case, due to the frequency-dependent weighting of any difference between $S^{l}$ and $S^{c}$ in $\left(2{ }^{-} \cdot 23_{1}^{\prime}\right)$ on page ${ }_{-1}^{-1} 3_{-}^{\prime}$.
} 


\section{Chapter 3}

\section{Bound-Bound and Bound-Free Transitions}

\footnotetext{
$\mathrm{T}$
} his chapter discusses bound-bound and bound-free transitions in more detail. It starts with summarizing pertinent physics, continues with formal expressions for radiative and collisional transition rates, and ends with spectral line broadening and redistribution.

\subsection{Photonic transitions}

Not yet...

\subsubsection{Atomic transitions}

Not yet...

\subsubsection{Molecular transitions}

Not yet...

\subsubsection{Two-electron transitions}

So far we have discussed transitions involving a single valence electron only. Two-electron processes are sometimes important, namely for recombination when the particles have much higher energy than the radiation fields, or for ionization in the reverse situation.

Dielectronic recombination. Two-electron recombination is important in hot stellar coronae because the free electrons there move so fast. The $C_{c i} \sim 1 / T$ dependence in $(\overline{3} .37 i)$ below diminishes the collisional recombination rate for a given transition $c i$. The reason is that the peak of the Maxwell distribution moves away from $v_{p}=0$ as $v_{p}=$ $\sqrt{2 k T / m} \sim \sqrt{T}$ whereas slow electrons are captured more easily. The same holds for radiative recombination. The bound-free extinction cross-section diminishes rapidly above the threshold frequency $\nu_{0}$, hydrogenically $\sim\left(\nu-\nu_{0}\right)^{-3}$ according to Kramers' law (2.74)

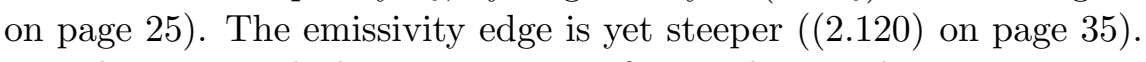

Thus, coronal electrons are too fast to be caught. For atoms and ions with other electrons in their outer shell dielectronic recombination uses a large fraction of the too-large kinetic energy of the incoming electron for bound-bound excitation of another electron in 
the outer shell, cutting down the leftover amount above threshold. This process dominates the recombination rates in the solar corona (iMihalas 1978. 134).

The low coronal density permits the excited second electron to deexcite spontaneously. The product is at least two photons, one from the bound-free radiative recombination and one from the second electron, usually a resonance-line photon. The bound-free recombination favors the highest levels. Due to the low density and the absence of radiation in optically-thin coronal conditions, a many-photon cascade then follows. All outward-bound photons escape and so provide the line-rich coronal X-ray spectrum.

Autoionization. The reverse two-electron process, dielectronic ionization, employs overly energetic photons rather than electrons. It is not important in coronal conditions where the mean intensity is very low, but may be so when the ambient photons are much hotter than the thermal particle speeds. Photon extinction may then increase the ion fraction by using part of the photon energy for bound-bound excitation of a second electron, so cutting the photon energy down to a value closer to the threshold ionization energy of the first electron. This process can be important when cool matter is embedded in hot radiation. It is sometimes called autoionization but strictly speaking that is the transition from doubly-excited state to ionized singly-excited state.

Bound-free resonances. Both processes are taken into account as resonances in bound-free radiative cross-sections, taking the form of humps in the cross-section frequency dependence that lie well above the cutoff frequency. The cross-sections of bound-free transitions in complex spectra such as Fe I and Fe II that result from outer shells with many electrons and many holes contain many such resonances. Some elements (Al I for example) have large resonances close to their ground-state ionization limit.

\subsubsection{Charge-transfer transitions}

Not yet...

\subsection{Transition rates}

Evaluation of the monochromatic extinction coefficients $\alpha_{\nu}$ for each bound-bound and continuum process requires knowledge of the particle densities. In the case of LTE, these follow from the chemical composition and the use of the Saha-Boltzmann distributions $\left(\overline{2}-\overline{8} \overline{6}_{i}\right)-\left(\overline{2}-\overline{2}^{\prime}\right)$ for the local temperature $T=T_{\mathrm{e}}$ and electron pressure $P_{e}$ as discussed in Section 17.2 .2 on page $1143 \overline{3}$ ff. When departures from LTE are important, the statistical equilibrium rate equations (2.100i) must be solved for the pertinent particles and wavelengths along with the radiative transfer equations (2.103i). This section discusses such NLTE rates.

\subsubsection{Bound-bound radiative rates}

We start by reviewing the bound-bound case. The statistical equilibrium equations were written in (2.100i) on page $32 \overline{2}$ as

$$
\frac{\mathrm{d} n_{i}}{\mathrm{~d} t}=\sum_{j \neq i}^{N} n_{j} P_{j i}-n_{i} \sum_{j \neq i}^{N} P_{i j}=0
$$


with $N$ the total number of pertinent levels, including continua. For bound-bound transitions, the rates $P_{i j}$ per particle in state $i$ or $j$ are given by (2.102i) on page 32

$$
P_{i j}=A_{i j}+B_{i j} \bar{J}_{\nu_{0}}+C_{i j}
$$

For a spectral line the radiative excitation rate per $\mathrm{cm}^{3}$ may with $(2.6 \overline{2}-6)$ on page $2 \overline{2} \overline{3}$, be written as:

$$
\begin{aligned}
n_{l} R_{l u} & =n_{l} B_{l u} \bar{J}_{\nu_{0}} \\
& =n_{l} \int_{0}^{\infty} B_{l u} J_{\nu} \varphi\left(\nu-\nu_{0}\right) \mathrm{d} \nu \\
& =4 \pi n_{l} \int_{0}^{\infty} \frac{\sigma_{\nu}^{l}}{h \nu} J_{\nu} \mathrm{d} \nu
\end{aligned}
$$

which holds also for very wide lines. The radiative deexcitation rate may similarly be rewritten, using the Einstein relations $(2.600)-(2.61)$, the line extinction coefficient (2.65i) and assuming complete redistribution with $\chi=\psi=\varphi$ :

$$
\begin{aligned}
n_{u} R_{u l} & =n_{u} A_{u l}+n_{u} B_{u l} \bar{J}_{\nu_{0}} \\
& =n_{u} \int_{0}^{\infty} A_{u l} \varphi\left(\nu-\nu_{0}\right) \mathrm{d} \nu+n_{u} \int_{0}^{\infty} B_{u l} J_{\nu} \varphi\left(\nu-\nu_{0}\right) \mathrm{d} \nu \\
& =n_{u} \frac{g_{l}}{g_{u}} \int_{0}^{\infty} B_{l u} \varphi\left(\nu-\nu_{0}\right)\left(\frac{2 h \nu^{3}}{c^{2}}+J_{\nu}\right) \mathrm{d} \nu \\
& =4 \pi n_{u} \frac{g_{l}}{g_{u}} \int_{0}^{\infty} \frac{\sigma_{\nu}^{l}}{h \nu}\left(\frac{2 h \nu^{3}}{c^{2}}+J_{\nu}\right) \mathrm{d} \nu .
\end{aligned}
$$

Induced emission is here counted as positive contribution to the downward rate, rather then entered as negative upward rate as is done in $\alpha_{\nu}^{l}$. The notation convention is that the rates $R_{l u}$ and $R_{u l}$ measure the radiative part of $P_{i j}$ as the number of radiative transitions per second per particle rather than per $\mathrm{cm}^{3}$. Both notations are used in the literature; this one follows Mihalas (1970, 1978).

\subsubsection{Bound-free radiative rates}

Physics. Bound-free processes always involve a second particle (the free electron) with partial exchange between kinetic and radiative energy (the part of $h \nu$ representing the kinetic energy above the ionization limit) even in radiative transitions, in addition to a possibly non-thermalizing part given by the threshold energy. The latter represents a discrete memory that may be transported non-locally in scattering sequences similarly to the bound-bound excitation energy of a resonance transition ${ }_{-1}^{\mathrm{I}_{1}}$. The part above the edge has no memory, sampling the Maxwell distribution anew at each electron capture. This

\footnotetext{
${ }^{1}$ Note that the total source function is usually a mixture of $J_{\nu}$ and $B_{\nu}$ terms anyhow. The two-level case has $S_{\nu}=\left(1-\varepsilon_{\nu}\right) J+\varepsilon_{\nu} B_{\nu}$ with $J=J_{\nu}$ for coherent scattering, $J=\bar{J}_{\nu_{0}}$ for complete redistribution, and $J$ a more complicated frequency-weighted function of $J_{\nu}$ for partial redistribution, but for actual lines there is an extra term from the continuum background at the line frequency, at least with $S_{\nu}^{c}=B_{\nu}$ as in $\left(4.94_{1}^{\prime}\right)$ on page 106 and possibly including $S_{\nu}^{c}=J_{\nu}$ for electron scattering as in (4.100). Additional $J_{\nu}, \bar{J}_{\nu_{0}}$ and $B_{\nu}$ terms with other frequencies come from multi-level processes. For example, if photoexcitation in a resonance line is followed by radiative deexcitation in a subordinate line to an intermediate level and the atom deexcites collisionally from that level back to the original one, part of the incoming photon has been thermalized and part has been emitted at a longer wavelength (photon conversion).
} 
local sampling is analogous to the collisional broadening of bound-bound transitions. Both are described by redistribution over the extinction profile.

In the limit of negligible Maxwell contribution (a sharp extinction edge resembling a spectral line in the spectrum) bound-free scattering behaves just as bound-bound scatter-

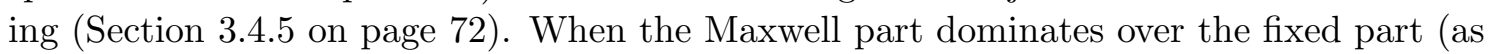
is the case for $\mathrm{H}^{-}$ionization), recombination tends toward the thermal nature of free-free bremsstrahlung?

Einstein-Milne equations. Relations similar to the Einstein relations (흔. exist to relate the upward and downward bound-free transition probabilities; these are the

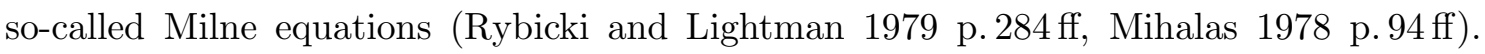
Formulation of the latter in terms of Einstein-like coefficients for bound-free transitions is evaded here by application of Einstein's trick to obtain general relations from their TE limits to the bound-free rates themselves. It is used below to obtain a bound-free analogon to $\left(\overline{3}^{-} . \overline{6}\right)$ without defining bound-free equivalents to $A_{u l}$ and $B_{u l}$ in $\left(\overline{3} \cdot \overline{3} \cdot \bar{l}^{\prime}\right)$.

Photoionization. I follow Mihalas' treatment (1970 p. 141, 1978 p. $130 \mathrm{ff}$ ) except that I keep writing $\sigma_{i c}$ for the monochromatic bound-free extinction coefficient per particle, rather than $\alpha_{i c}$ which keeps its meaning here of extinction per cm path length (or volume coefficient with cross-section $\mathrm{cm}^{2}$ per $\mathrm{cm}^{3}$ ). It is comparable to the bound-bound extinction coefficient $\sigma_{\nu}^{l}$ in $(2.6 \overline{2})$ on page $2 \overline{2} \overline{3}$, and does not include correction for induced emission. It enters the bound-free rate similarly to $\sigma_{\nu}^{l}$ in $(\overline{3}-\overline{1})$, under the integral since photoionization edges are relatively wide. The photoionization rate per $\mathrm{cm}^{3}$ from a bound level $i$ to the continuum $c$ is therefore:

$$
n_{i} R_{i c}=4 \pi n_{i} \int_{\nu_{0}}^{\infty} \frac{\sigma_{i c}(\nu)}{h \nu} J_{\nu} \mathrm{d} \nu
$$

with $\nu_{0}$ the threshold frequency (series limit). For hydrogenic transitions $\sigma_{i c}(\nu)$ is given by Kramers' formula (2.74) on page $2 \overline{2} \overline{5}_{-r}^{\prime}$ Other transitions may have very complicated $\sigma_{i c}(\nu)$ frequency dependence, with humps from resonances caused by other electrons (holes) in the outer shell (Section $3 . \overline{1} . \overline{3}$, on page $\left.\overline{4}_{1} \overline{3}\right)$.

Spontaneous recombination. An Einsteinian detailed balancing argument delivers the photorecombination rate $R_{c i}$. In TE the radiative ionizations at any frequency are balanced exactly by the radiative recombinations. Since $J_{\nu}=B_{\nu}$ in TE, the total radiative recombination rate in TE per $\mathrm{cm}^{3}$ is therefore, with $n_{c}$ the ion density:

$$
\left[n_{c} R_{c i}\right]_{\mathrm{TE}}=\left[n_{i} R_{i c}\right]_{\mathrm{TE}}=4 \pi n_{i}^{\mathrm{TE}} \int_{\nu_{0}}^{\infty} \frac{\sigma_{i c}(\nu)}{h \nu} B_{\nu} \mathrm{d} \nu
$$

The total TE radiative recombination rate is made up by spontaneous and induced recombinations and may be split into these components with the TE correction for induced

\footnotetext{
${ }^{2}$ Even free-free processes may transport non-thermal energy in scattering sequences. Imagine an intense beam of high-energy photons hitting a cooler ionised hydrogen gas. Free-free absorptions then impart large kinetic energy to electrons that may lose that energy again through free-free emission in subsequent encounters. Their excess kinetic energy would then act as memory; the source function for such sequences would follow $\overline{J_{\nu}}$ rather than $B_{\nu}(T)$. However, such scattering is excluded by postulating the velocity distribution to be Maxwellian. That assumption implies that the kinetic memory of overly intense radiation is washed out in collisional encounters before re-emission occurs. Each new photon then samples the local kinetic equilibrium temperature, so that free-free processes have $S_{\nu}=B_{\nu}$.
} 
recombination (2.97i) on page $3 \overline{3} 1$ :

$$
\begin{aligned}
{\left[_{c} R_{c i}\right]_{\mathrm{TE}} } & =4 \pi n_{i}^{\mathrm{TE}} \int_{\nu_{0}}^{\infty} \frac{\sigma_{i c}(\nu)}{h \nu} B_{\nu}\left[\left(1-\mathrm{e}^{-h \nu / k T}\right)+\mathrm{e}^{-h \nu / k T}\right] \mathrm{d} \nu \\
& =\left[n_{c} R_{c i}^{\mathrm{spon}}\right]_{\mathrm{TE}}+\left[n_{c} R_{c i}^{\mathrm{ind}}\right]_{\mathrm{TE}}
\end{aligned}
$$

with the TE spontaneous recombination rate given by

$$
\left[n_{c} R_{c i}^{\mathrm{spon}}\right]_{\mathrm{TE}}=4 \pi n_{i}^{\mathrm{TE}} \int_{\nu_{0}}^{\infty} \frac{\sigma_{i c}(\nu)}{h \nu} B_{\nu}\left(1-\mathrm{e}^{-h \nu / k T}\right) \mathrm{d} \nu .
$$

However, spontaneous recombination requires a free electron but no knowledge about possible departures from TE in the local radiation field. Also when TE (or LTE) is not valid the TE spontaneous recombination rate holds per ion, as long as the velocity distribution is Maxwellian. We therefore have only to rescale (3.11 ) from the LTE ion density $n_{c}^{\mathrm{LTE}}$ to the actual ion density $n_{c}$ to obtain the general rate:

$$
\begin{aligned}
n_{c} R_{c i}^{\mathrm{spon}} & =4 \pi \frac{n_{c}}{n_{c}^{\mathrm{LTE}}} n_{i}^{\mathrm{LTE}} \int_{\nu_{0}}^{\infty} \frac{\sigma_{i c}(\nu)}{h \nu} B_{\nu}\left(1-\mathrm{e}^{-h \nu / k T}\right) \mathrm{d} \nu \\
& =4 \pi n_{c}\left[\frac{n_{i}}{n_{c}}\right]_{\mathrm{LTE}} \int_{\nu_{0}}^{\infty} \frac{\sigma_{i c}(\nu)}{h \nu} \frac{2 h \nu^{3}}{c^{2}} \mathrm{e}^{-h \nu / k T} \mathrm{~d} \nu .
\end{aligned}
$$

Discussion. Although the spontaneous recombination rate per ion does not sense radiation, the latter may have been instrumental in producing the ions. Such non-local sensitivity sits in $n_{c}$. The process is less "spontaneous" than spontaneous bound-bound deexcitation because the ion must wait for a free electron to come by. That electron sits hidden in the LTE ratio $\left[n_{i} / n_{c}\right]_{\text {LTE }}$ given by the Saha-Boltzmann distribution (2.90i) on page $\overline{3} \overline{3}_{0}$, which also contains the temperature dependence due to the Maxwell part above the threshold energy. Note the equality of $(3.12 i)$ and $(2.120 i)$ on page $3 \overline{5}, 1$ apart from the rate/intensity conversion (factor $4 \pi / h \nu$ ), the summing over frequency, and the contribution by stimulated emission.

Expression (3.13i) describes the total recombination rate in coronal equilibrium, where the three-body collisional and stimulated-emission contributions may both be neglected.

Induced recombination. A similar argument serves for the stimulated recombination. For TE it is given by the second term in (19.3.

$$
\left[n_{c} R_{c i}^{\mathrm{ind}}\right]_{\mathrm{TE}}=4 \pi n_{i}^{\mathrm{TE}} \int_{\nu_{0}}^{\infty} \frac{\sigma_{i c}(\nu)}{h \nu} B_{\nu} \mathrm{e}^{-h \nu / k T} \mathrm{~d} \nu,
$$

and it is generalized to the actual rate per $\mathrm{cm}^{3}$ by rescaling it from the LTE ion density $n_{c}^{\mathrm{LTE}}$ to the actual ion density $n_{c}$ and replacing the TE radiation field $B_{\nu}$ by the actual radiation field $J_{\nu}$ :

$$
n_{c} R_{c i}^{\text {ind }}=4 \pi n_{c}\left[\frac{n_{i}}{n_{c}}\right]_{\text {LTE }} \int_{\nu_{0}}^{\infty} \frac{\sigma_{i c}(\nu)}{h \nu} J_{\nu} \mathrm{e}^{-h \nu / k T} \mathrm{~d} \nu
$$

Total radiative recombination. The end result for the total recombination rate is:

$$
n_{c} R_{c i}=4 \pi n_{c}\left[\frac{n_{i}}{n_{c}}\right]_{\mathrm{LTE}} \int_{\nu_{0}}^{\infty} \frac{\sigma_{i c}(\nu)}{h \nu}\left(\frac{2 h \nu^{3}}{c^{2}}+J_{\nu}\right) \mathrm{e}^{-h \nu / k T} \mathrm{~d} \nu,
$$

corresponding to $\left(\overline{3}_{3}^{-}, \overline{6}_{i}\right)$ for the bound-bound case with $g_{l} / g_{u}=\left[n_{l} / n_{u}\right]_{\mathrm{LTE}} \exp \left(-h \nu_{0} / k T\right)$. 


\subsubsection{Unified radiative rates}

The bound-bound and bound-free upward and downward radiative rates per particle between a level $i$ and a higher-energy level $j$ may now be written in a compact unified notation (Mihalas 1978 pag. 131; 'Ćarlsson $19 \overline{9} \overline{8} \overline{6}$ pag. 3):

$$
\begin{aligned}
\text { upward } i \rightarrow j: \quad R_{i j} & =\frac{1}{2} \int_{-1}^{1} \int_{0}^{\infty} \frac{4 \pi}{h \nu} \sigma_{i j} I_{\nu \mu} \mathrm{d} \nu \mathrm{d} \mu \\
& =\int_{0}^{\infty} \frac{4 \pi}{h \nu} \sigma_{i j} J_{\nu} \mathrm{d} \nu \\
\text { downward } j \rightarrow i: \quad R_{j i} & =\frac{1}{2} \int_{-1}^{1} \int_{0}^{\infty} \frac{4 \pi}{h \nu} \sigma_{i j} G_{i j}\left(\frac{2 h \nu^{3}}{c^{2}}+I_{\nu \mu}\right) \mathrm{d} \nu \mathrm{d} \mu \\
& =\int_{0}^{\infty} \frac{4 \pi}{h \nu} \sigma_{i j} G_{i j}\left(\frac{2 h \nu^{3}}{c^{2}}+J_{\nu}\right) \mathrm{d} \nu
\end{aligned}
$$

with for bound-bound transitions

$$
\sigma_{i j}=\sigma_{\nu}^{l}=\frac{h \nu_{i j}}{4 \pi} B_{i j} \varphi_{\nu \mu} \quad G_{i j}=\frac{g_{i}}{g_{j}}=\left[\frac{n_{i}}{n_{j}}\right]_{\mathrm{LTE}} \mathrm{e}^{-h \nu / k T}
$$

and for bound-free transitions

$$
\sigma_{i j}=\sigma_{i c}(\nu) \quad G_{i j}=\left[\frac{n_{i}}{n_{c}}\right]_{\mathrm{LTE}} \underset{\mathrm{e}^{-h \nu / k T}}{ }
$$

Discussion. The unified notation illustrates that bound-free transitions behave as bound-bound transitions, with the recombination dependence on the electron density and velocity distribution accounted for through the Saha ratio $\left[n_{i} / n_{c}\right]$ LTE. Reversely, the integrals that are needed for wide bound-free edges also account for slow frequency variations

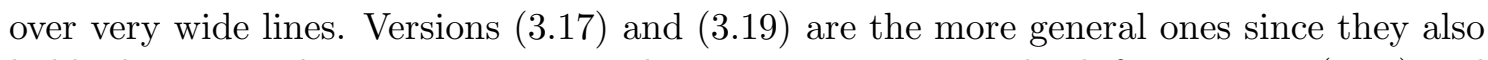
hold when $\sigma_{i j}$ and $\sigma_{i c}$ are anisotropic due to systematic Dopplershifts; versions (3.18) and $\left(\overline{3}_{2} \cdot 0_{i}^{\prime}\right)$ assume isotropic extinction. The radiative rates per $\mathrm{cm}^{3}$ are obtained by multiplication with the particle densities $\left(n_{i}\right.$ for $i \rightarrow j, n_{j}$ for $j \rightarrow i$ ), which are affected by departures from LTE and quantify the latter. Complete redistribution holds over the bound-free edges and is assumed for the lines.

\subsubsection{Net radiative rates}

Net radiative recombination. The net radiative recombination per $\mathrm{cm}^{3}$ is given by the difference between total radiative recombination and photoionization. Using the NLTE

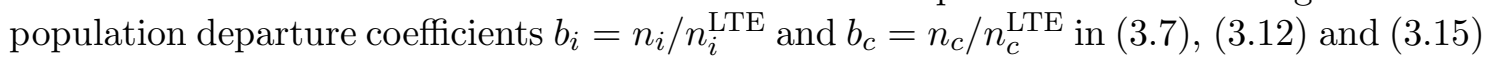
it becomes:

$$
\begin{aligned}
& n_{c} R_{c i}-n_{i} R_{i c} \\
& \quad=4 \pi \int_{\nu_{0}}^{\infty} \frac{\sigma_{i c}(\nu)}{h \nu}\left[n_{i}^{\mathrm{LTE}} b_{c} B_{\nu}\left(1-\mathrm{e}^{-h \nu / k T}\right)+n_{i}^{\mathrm{LTE}} b_{c} J_{\nu} \mathrm{e}^{-h \nu / k T}-n_{i} J_{\nu}\right] \mathrm{d} \nu \\
& =4 \pi n_{i}^{\mathrm{LTE}} b_{c} \int_{\nu_{0}}^{\infty} \frac{\sigma_{i c}(\nu)}{h \nu}\left[B_{\nu}\left(1-\mathrm{e}^{-h \nu / k T}\right)-\frac{b_{i}}{b_{c}} J_{\nu}\left(1-\frac{b_{c}}{b_{i}} \mathrm{e}^{-h \nu / k T}\right)\right] \mathrm{d} \nu .
\end{aligned}
$$

In the Wien limit:

$$
n_{c} R_{c i}-n_{i} R_{i c}=4 \pi n_{i}^{\mathrm{LTE}} b_{c} \int_{\nu_{0}}^{\infty} \frac{\sigma_{i c}(\nu)}{h \nu}\left(B_{\nu}-\frac{b_{i}}{b_{c}} J_{\nu}\right) \mathrm{d} \nu .
$$


Net radiative deexcitation. For comparison I derive the corresponding expressions for the net radiative rate in bound-bound transitions using ( $(3)$ and assuming complete redistribution so that $(2.71)$ reduces to $(2,2.73)$ :

$$
\begin{aligned}
n_{u} & R_{u l}-n_{l} R_{l u} \\
& =n_{u} A_{u l}+n_{u} B_{u l} \bar{J}_{\nu_{0}}-n_{l} B_{l u} \bar{J}_{\nu_{0}} \\
& =S_{\nu_{0}}^{l}\left(n_{l} B_{l u}-n_{u} B_{u l}\right)-\bar{J}_{\nu_{0}}\left(n_{l} B_{l u}-n_{u} B_{u l}\right) \\
& =n_{l} B_{l u}\left[S_{\nu_{0}}^{l}\left(1-\frac{n_{u} B_{u l}}{n_{l} B_{l u}}\right)-\bar{J}_{\nu_{0}}\left(1-\frac{n_{u} B_{u l}}{n_{l} B_{l u}}\right)\right] \\
& =\frac{4 \pi}{h \nu_{0}} n_{l} \sigma_{\nu_{0}}^{l}\left[S_{\nu_{0}}^{l}\left(1-\frac{b_{u}}{b_{l}} \mathrm{e}^{-h \nu_{0} / k T}\right)-\bar{J}_{\nu_{0}}\left(1-\frac{b_{u}}{b_{l}} \mathrm{e}^{-h \nu_{0} / k T}\right)\right] \\
& =\frac{4 \pi}{h \nu_{0}} n_{l}^{\mathrm{LTE}} b_{u} \sigma_{\nu_{0}}^{l}\left[B_{\nu_{0}}\left(1-\mathrm{e}^{-h \nu_{0} / k T}\right)-\frac{b_{l}}{b_{u}} \bar{J}_{\nu_{0}}\left(1-\frac{b_{u}}{b_{l}} \mathrm{e}^{-h \nu_{0} / k T}\right)\right]
\end{aligned}
$$

where the last step uses the equality

$$
\begin{aligned}
& S_{\nu_{0}}^{l}\left(1-\frac{b_{u}}{b_{l}} \mathrm{e}^{-h \nu_{0} / k T}\right)=\frac{2 h \nu_{0}^{3} / c^{2}}{\left(b_{l} / b_{u}\right) \mathrm{e}^{h \nu_{0} / k T}-1} \frac{b_{u}}{b_{l}}\left(\frac{b_{l}}{b_{u}}-\mathrm{e}^{-h \nu_{0} / k T}\right) \\
& \quad=\frac{b_{u}}{b_{l}} \frac{2 h \nu_{0}^{3} / c^{2}}{\left(b_{l} / b_{u}\right) \mathrm{e}^{h \nu_{0} / k T}-1} \frac{\left(b_{l} / b_{u}\right) \mathrm{e}^{h \nu_{0} / k T}-1}{\mathrm{e}^{h \nu_{0} / k T}}=\frac{b_{u}}{b_{l}} \frac{2 h \nu_{0}^{3} / c^{2}}{\mathrm{e}^{h \nu_{0} / k T}}=\frac{b_{u}}{b_{l}} B_{\nu_{0}}\left(1-\mathrm{e}^{-h \nu_{0} / k T}\right) .
\end{aligned}
$$

The Wien simplification of $(\overline{3} . \overline{2}-\overline{1})$ is:

$$
n_{u} R_{u l}-n_{l} R_{l u} \approx \frac{4 \pi}{h \nu_{0}} n_{l}^{\mathrm{LTE}} b_{u} \sigma_{\nu_{0}}^{l}\left(B_{\nu_{0}}-\frac{b_{l}}{b_{u}} \bar{J}_{\nu_{0}}\right) .
$$

These expressions recover the form of $(3.23)$ and $(3.24)$. A faster derivation of $(3.2 \overline{1})$ is:

$$
\begin{aligned}
n_{u} R_{u l}-n_{l} R_{l u} & =n_{u} A_{u l}+n_{u} B_{u l} \bar{J}_{\nu_{0}}-n_{l} B_{l u} \bar{J}_{\nu_{0}} \\
& =\frac{4 \pi}{h \nu_{0}}\left(j_{\nu_{0}}^{l}-\alpha_{\nu_{0}}^{l} \bar{J}_{\nu_{0}}\right) \\
& =\frac{4 \pi}{h \nu_{0}} \alpha_{\nu_{0}}^{l}\left(S_{\nu_{0}}^{l}-\bar{J}_{\nu_{0}}\right) \\
& \approx \frac{4 \pi}{h \nu_{0}} b_{u}\left[\alpha_{\nu_{0}}^{l}\right]_{\mathrm{LTE}}\left(B_{\nu_{0}}-\frac{b_{l}}{b_{u}} \bar{J}_{\nu_{0}}\right)
\end{aligned}
$$

where the last step uses the Wien approximation to set $\alpha_{\nu_{0}}^{l} \approx b_{l}\left[\alpha_{\nu_{0}}^{l}\right]_{\text {LTE }}$ and $S_{\nu_{0}}^{l} \approx$ $\left(b_{u} / b_{l}\right) B_{\nu_{0}}$. This result equals ( $\left(\overline{3} \cdot 2 \overline{2}_{i}\right)$ since $\alpha_{\nu_{0}}^{l} \approx n_{l} \sigma_{\nu_{0}}^{l}$ in the Wien approximation.

Discussion. The net rate vanishes in $(\overline{3} .25 i)$ and $(\overline{3} .29 i)$ for pure resonance scattering with $S_{\nu_{0}}^{l}=\bar{J}_{\nu_{0}}$ because in that case all new photons in the line are old line photons and precisely as many electrons go up as go down (monochromatic radiative equilibrium). When $b_{l}=b_{u}$ and therefore $S_{\nu_{0}}^{l}=B_{\nu_{0}}$ a net radiative rate exists for $\bar{J}_{\nu_{0}} \neq B_{\nu_{0}}$ because the LTE equality $S_{\nu_{0}}^{l}=B_{\nu_{0}}$ describes overall balancing in the transition but not detailed balancing as takes place in $\mathrm{TE}_{1-\mathrm{r}}^{3 \mathrm{~B}}$ The same holds for the bound-free rates given by $(\overline{3} . \overline{2} \overline{4})$. Even when LTE holds with $b_{i}=b_{c}=1$ net radiative recombination occurs at locations

\footnotetext{
${ }^{3}$ Deep within a stellar atmosphere (in terms of the optical depth within the line) detailed balancing does occur so that the net radiative rate is zero. This has often been used in older NLTE line synthesis codes, defining a Net Radiative Bracket $Z_{u l}$ by $n_{u} R_{u l}-n_{l} R_{l u}=n_{u} A_{u l} Z_{u l}$ so that $Z_{u l}=1-\bar{J}_{\nu_{0}} / S_{\nu_{0}}^{l}$ for
} 
with $J_{\nu_{0}}<B_{\nu_{0}}$ and net radiative ionization occurs for $J_{\nu_{0}}>B_{\nu_{0}}$. Detailed balancing (TE) requires that radiative up equals radiative down monochromatically, thus $J_{\nu}=B_{\nu}$ at every $\nu$ in addition to $b_{i}=b_{c}$ or $b_{l}=b_{u}$. LTE permits radiative imbalances on the condition that these do not spoil the equilibrium Saha-Boltzmann population ratios, i.e., as long as collisional transitions dominate the total rates sufficiently that the resulting populations don't care about photons.

The final result (3.27) for the bound-bound net rate in the Wien approximation is basically transparent - we might have written it down without derivation. The net rate vanishes in TE and therefore departs from zero when $\bar{J}_{\nu_{0}}$ departs from $B_{\nu}$, a basic non-TE phenomenon requiring a directional gradient (anisotropy) in $I_{\nu}$. It departs even more when the population ratio between the lower and upper levels lies above the LTE value. In that case, there are extra atoms available to get excited by photons present in a $\bar{J}_{\nu_{0}}$ overdose, or to not get excited due to a lack of photons in $\bar{J}_{\nu_{0}}$, adding to the number $n_{l}^{\mathrm{LTE}}$ that the Saha-Boltzmann equipartition laws prescribe. Thus, a $b_{l} / b_{u}$ population excess enhances a $B_{\nu}-\bar{J}_{\nu_{0}}$ inequality. The role of the reversed departure ratio in the correction for induced emission to the $\bar{J}_{\nu_{0}}$ term in $(\overline{3} . \overline{2} \overline{6})$ is obvious too. So is the similarity between $\left(\overline{3} . \overline{2} \overline{6}_{i}\right)$ and the bound-free net rate $(3.23 i)$. In both, the $J_{\nu}$ contribution to the net downward rate turns positive for $b_{u} / b_{l}>\exp (+h \nu / k T)$ (lasering).

\subsubsection{Collision rates}

For completeness I add standard formulae here without derivation for the electron collision rates per particle per second, both for the bound-bound and the bound-free cases. Free electrons generally dominate in causing collisional transitions due to their large collision frequency with

$$
\frac{\text { electron collision frequency }}{\text { ion collision frequency }} \sim \frac{N_{\mathrm{e}}<v_{\mathrm{e}}>}{N_{\text {ion }}<v_{\text {ion }}>} \sim \frac{N_{\mathrm{e}}}{N_{\text {ion }}}\left(\frac{m_{\mathrm{H}} A}{m_{\mathrm{e}}}\right)^{1 / 2}
$$

with $A$ the atomic weight of the ion. The frequency ratio is already 43 for an ionized hydrogen gas and increases further for ions that are heavier than protons, or when second or higher ionization gives $N_{\mathrm{e}}>N_{\text {ion }}$. In cool photospheres the density of neutral hydrogen atoms is much larger than the electron density, but the cross-sections for H I collisions are smaller (though badly known); they are usually neglected. According to a dipoleapproximation derivation by 'Van Regemorter $(1962)$, the electron collision rates per $\mathrm{cm}^{3}$ for bound-bound collisional transitions in atoms may be estimated by $(\S 6.2 .1-\S 6.2 .2$ of Jefferies $1 \overline{9} \overline{\bar{c}} \overline{\mathrm{B}})$ :

$$
\begin{aligned}
& n_{l} C_{l u} \approx 2.16\left(\frac{E_{0}}{k T}\right)^{-1.68} T^{-3 / 2} \mathrm{e}^{-E_{0} / k T} n_{l} N_{\mathrm{e}} f \\
& n_{u} C_{u l} \approx 2.16\left(\frac{E_{0}}{k T}\right)^{-1.68} T^{-3 / 2} \frac{g_{l}}{g_{u}} n_{u} N_{\mathrm{e}} f
\end{aligned}
$$

and for ions by

$$
n_{l} C_{l u} \approx 3.9\left(\frac{E_{0}}{k T}\right)^{-1} T^{-3 / 2} \mathrm{e}^{-E_{0} / k T} n_{l} N_{\mathrm{e}} f
$$

complete redistribution. The ratio of $\bar{J}_{\nu_{0}}$ to $S_{\nu_{0}}^{l}$ is often known with more precision within the computer than each quantity itself, so that it pays to code the iteration in this parameter. In addition, exceptionally strong lines such as Ly $\alpha$ are often "put in detailed balance" a priori for large optical depths (in the line, but already high in the atmosphere) by setting $Z_{u l}=0$, to keep the computer from evaluating large numbers that cancel in the end. 


$$
n_{u} C_{u l} \approx 3.9\left(\frac{E_{0}}{k T}\right)^{-1} T^{-3 / 2} \frac{g_{l}}{g_{u}} n_{u} N_{\mathrm{e}} f
$$

where $f$ is the oscillator strength. The bound-free rates are:

$$
\begin{aligned}
& n_{i} C_{i c} \approx 2.7 \zeta\left(\frac{E_{0}}{k T}\right)^{-2} T^{-3 / 2} \mathrm{e}^{-E_{0} / k T} n_{i} N_{\mathrm{e}} \\
& n_{c} C_{c i} \approx 5.6 \times 10^{-16} \zeta\left(\frac{E_{0}}{k T}\right)^{-2} T^{-3} \frac{g_{i}}{g_{c}} n_{c} N_{\mathrm{e}}^{2}
\end{aligned}
$$

with $\zeta$ the number of electrons in the outer shell. The frequency $\nu_{0}$ with $E_{0}=h \nu_{0}$ represents the bound-bound frequency or the bound-free threshold frequency of the transition, respectively. The colliding electrons need to possess, or they gain, at least this energy, with $E_{0}=(1 / 2) m_{\mathrm{e}} v_{0}^{2}$ and $v_{0}$ the corresponding threshold velocity of the electron if we regard the ion or atom as stationary. The term $T^{-3 / 2} \exp \left(-E_{0} / k T\right)$ comes from the Maxwell distribution. The bound-bound up and down pairs are related through the LTE Boltzmann ratio $(2 . \overline{6} \overline{1})$. The bound-free pairs $(3 . \overline{3} \overline{6})$ and $(3 . \overline{3} \bar{i})$ are similarly related by the LTE ratio $\left(\overline{2} \overline{2} \overline{9} 0_{i}^{i}\right)$ on page $\overline{3} \overline{0} \bar{i}$

$$
\frac{C_{c i}}{C_{i c}}=\left[\frac{n_{i}}{n_{c}}\right]_{\mathrm{LTE}}=2.06 \times 10^{-16}\left(g_{i} / g_{c}\right) \mathrm{e}^{+E_{0} / k T} T^{-3 / 2} N_{\mathrm{e}}
$$

which holds equally well outside LTE (as long as the Maxwell distribution is valid).

Note that the downward probabilities per particle $C_{u l}$ and $C_{c i}$ are less sensitive to temperature than the upward probabilities $C_{l u}$ and $C_{i c}$. Roughly speaking, any encounter with a free electron will do for speeding it up by transferring excitation or ionization energy to it. The upward probabilities scale roughly as $\exp \left(-E_{0} / k T\right)$ and increase rapidly with increasing temperature. Up and down rate balancing as in TE then follows from the Boltzmann-Saha factor, with the same temperature sensitivity. The downward bound-free rate $n_{c} C_{c i}$ describes a three-body encounter and therefore scales with $N_{\mathrm{e}}^{2}$. It is larger for recombination to higher-lying levels (smaller $E_{0}$ and larger $g_{i}$ ).

Note that the bound-bound rates scale with the oscillator strength $f$ and hold only for permitted transitions. The collision rates in forbidden transitions (very small $f$ ) are generally badly known, but not as small as these expressions would predict.

Net collision rates. With the departure coefficients $b_{i}$ in the Zwaan definition (2.124) the ratios of the collision rates become simply:

$$
\begin{aligned}
& \frac{n_{u} C_{u l}}{n_{l} C_{l u}}=\frac{b_{u}}{b_{l}} \\
& \frac{n_{c} C_{c i}}{n_{i} C_{i c}}=\frac{b_{c}}{b_{i}} .
\end{aligned}
$$

The net downward collision rates are:

$$
\begin{gathered}
n_{u} C_{u l}-n_{l} C_{l u}=n_{l} C_{l u}\left(\frac{b_{u}}{b_{l}}-1\right)=b_{u} n_{l}^{\mathrm{LTE}} C_{l u}\left(1-\frac{b_{l}}{b_{u}}\right) \\
n_{c} C_{c i}-n_{i} C_{i c}=n_{i} C_{i c}\left(\frac{b_{c}}{b_{i}}-1\right)=b_{c} n_{i}^{\mathrm{LTE}} C_{i c}\left(1-\frac{b_{i}}{b_{c}}\right),
\end{gathered}
$$

where the righthand versions have the same form as $(3.27 i)$ on page $\underline{4} \overline{9} \cdot 1$. Collisional balancing occurs for $b_{u}=b_{l}$ and $b_{c}=b_{i}$, respectively. 
Collisional coupling. At a given temperature all rates increase for decreasing $E_{0}=$ $(1 / 2) m_{\mathrm{e}} v_{0}^{2}$ because at lower cutoff energy $E_{0}$ more of the Maxwell tail is not cut off and contributes. The bound-bound rate increase is steeper for atoms than for ions. High levels (high in the term diagram, close to the continuum) have small level-to-level $E_{l u}$ separations. They are therefore strongly coupled by collisions.

The coupling is yet stronger for the bound-free collision rates with small $E_{i c}$ since these increase even more rapidly with decreasing $E_{0}$. In addition, these transitions concern high-lying levels of which the large statistical weight ratio $g_{i} / g_{c} \sim n^{2}$ enhances the ionization rate even more. Therefore, the highest levels in an atom are not only coupled strongly together by collisions, but they are even more strongly coupled by collisions to the population of the ion ground state $n_{c}$.

Collisional LTE. The bound-bound estimates (3.32)-(3.35) are only valid for permitted radiative dipole transitions with $f \simeq 10^{-3}-1$. For forbidden lines with smaller $f$ the collision rates do not drop much below the values typical of permitted lines, and therefore far outweigh the small radiative rates. The resulting collisional dominance implies that forbidden lines have LTE population ratios between their upper and lower levels.

This holds in particular for the fine-structure levels within a term (a group of levels with the same orbital angular momentum $L$ and spin angular momentum $S$ ). They are close together in energy and therefore have large collision rates between them. The radiative rates are negligible because radiative transitions between them are strictly forbidden. Thus, the levels within a term nearly always share their populations in Boltzmann equilibrium. The collective term population may be out of LTE, though.

A multiplet consists of permitted (or reasonably permitted) transitions between various combinations of upper levels in one term and lower levels in another term. The different lines in a multiplet therefore tend to share their upper level population departures and their lower-level population departures, and therefore have the same ratio $b_{u} / b_{l}$ and (if they are not too far apart in wavelength) also the same line source function $S_{\nu_{0}}^{l}$.

There are also forbidden transitions at larger energy separation, mostly equal-parity intersystem lines from the ground state in complex spectra, especially Fe I and Fe II.

\subsection{Spectral line broadening}

Spectral lines map the line extinction profile through the source function, both heightdependent, into the emergent spectrum. We now specify the shape of the line extinction coefficient. There is a large literature on stellar line broadening. The basic aspects are:

- natural broadening or "radiation damping", due to the limited lifetimes of excited states;

- collisional broadening, due to collisions with or perturbations by other particles;

- Doppler broadening by thermal motions;

- Doppler broadening by non-thermal motions; often split between microturbulence and macroturbulence for scales that are small or large compared to photon mean free paths;

- rotational Doppler broadening, of lines in the flux spectrum of a non-resolved star;

- partial frequency redistribution, a situation between the limits of coherent scattering and complete redistribution. 


\subsubsection{Radiation broadening}

Spontaneous deexcitation limits the lifetime of excited states with a statistical distribution function and therefore causes uncertainty in the energy of any transitions out of them according to the uncertainty relation. This line broadening is called natural broadening or radiative damping; "natural" means that a line is broadened even in the absence of other particles and "damping" comes from the classical description of a spectral line as a damped driven harmonic oscillator ('Gray 1992' p. $203 \mathrm{ff}$ ). In a two-level atom the single transition has

$$
\gamma^{\mathrm{rad}}=\gamma_{u}^{\mathrm{rad}}=A_{u l}
$$

because the lower level of a two-level atom has infinite lifetime and does not contribute existential uncertainty.

Damping profile. The shape of the corresponding broadening function is given by the Lorentz damping profile

$$
\psi\left(\nu-\nu_{0}\right)=\frac{\gamma^{\mathrm{rad}} / 4 \pi^{2}}{\left(\nu-\nu_{0}\right)^{2}+\left(\gamma^{\mathrm{rad}} / 4 \pi\right)^{2}}
$$

which has $\int_{0}^{\infty} \psi\left(\nu-\nu_{0}\right) \mathrm{d} \nu=1$, full width at half maximum $\operatorname{FWHM}(\nu)=\gamma^{\mathrm{rad}} / 2 \pi$ in frequency units, and $\operatorname{FWHM}(\lambda)=\lambda^{2} \gamma^{\mathrm{rad}} / 2 \pi c$ in wavelength units.

In the absence of other broadening agents this is the emission profile function. An estimate of its width: the CaII K line near $\lambda \approx 400 \mathrm{~nm}$ has frequency $\nu=c / \lambda \approx 10^{15} \mathrm{~Hz}$, transition probability $A \approx 10^{8} \mathrm{~s}^{-1}$ and therefore a natural width of order $\Delta \lambda=0.008 \mathrm{pm}$. The classical value for the damped harmonic oscillator is:

$$
\gamma^{\mathrm{rad}}=\frac{8 \pi e^{2}}{3 m_{\mathrm{e}} c \lambda_{0}^{2}}
$$

with FWHM $=0.012 \mathrm{pm}$ independent of wavelength. These values are very small. The other broadening agents are usually more effective. However, radiation damping does not depend on location and therefore exceeds collisional damping in the low-density outer layers of stellar atmospheres.

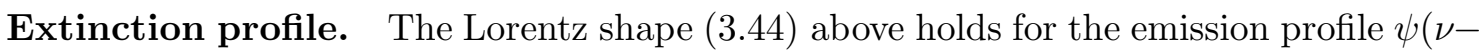
$\nu_{0}$ ) since this is the shape function defined for spontaneous bound-bound transitions. However, atoms deexcite spontaneously the same way whether they are inside or outside a TE enclosure. We may therefore simply take the TE equality $\psi\left(\nu-\nu_{0}\right)=\varphi\left(\nu-\nu_{0}\right)$ that is required to have TE detailed balancing at every individual frequency and generalize it to non-TE conditions. Thus, (3.4) describes also the radiative-damping constituent of the extinction profile $\varphi\left(\nu-\nu_{0}\right)$.

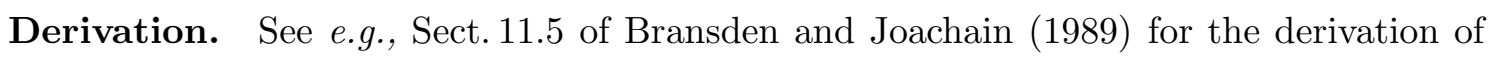
$(3.44)$. Basically, the profile describes the convolution of two Fourier transforms. The first is the delta function $\delta\left(\nu-\nu_{0}\right)$ that represents the Fourier transform of an infinitely long, monofrequent wave $\exp \left(-i 2 \pi \nu_{0} t\right)$ with frequency $\nu_{0}$. The second is the Fourier transform of the $\exp \left(-A_{u l} t\right)=\exp \left(-\gamma^{\mathrm{rad}} t\right)$ decay distribution. The actual wave function consists of the infinite wave multiplied by the decay distribution. The energy amplitude distribution 
is given by the Fourier transform of the perturbed wave function, or the convolution the two separate component transforms. The latter convolution recovers the transform of the decay distribution centered at $\nu=\nu_{0}$.

Multiple levels and transitions. In real atoms and ions the lower level of a given line may also have finite lifetime (when it isn't the ground state) and there may also be multiple downward transitions from each level. Multiple transition probabilities add up as

$$
\gamma_{u}^{\mathrm{rad}}=\sum_{l<u} A_{u l}
$$

because they measure deexcitations per second, an additive quantity. In other words, the corresponding decay functions multiply as $\exp \left(-A_{u 1} t\right) \exp \left(-A_{u 2} t\right)=\exp \left[-\left(\gamma_{1}^{\mathrm{rad}}+\gamma_{2}^{\mathrm{rad}}\right) t\right]$ so that the $\gamma^{\text {rad }}$ broadening parameters add linearly. Or in yet other words, the convolution of two Lorentz profiles with halfwidth parameters $\gamma_{1}$ and $\gamma_{2}$ delivers a new Lorentz profile with halfwidth parameter $\gamma=\gamma_{1}+\gamma_{2}$. The total natural damping width is therefore given by:

$$
\gamma^{\mathrm{rad}}=\gamma_{l}^{\mathrm{rad}}+\gamma_{u}^{\mathrm{rad}}=\sum_{i<l} A_{l i}+\sum_{i<u} A_{u i},
$$

or better yet, by the sum of the actual total radiative transition rates per particle out of the lower and upper level as determined in the computer. In that case extinction and stimulated emission out of each level are automatically taken into account ('Gray 1992 p. 209).

\subsubsection{Collision broadening}

Collisional broadening tends to present large problems in spectral line modeling and large complexity in formulating appropriate formalisms to treat it properly. Such formalisms exist but often only in rough form. A physicist may easily spend a whole career on collisional line broadening. The standard texts are the book on electron collisions by

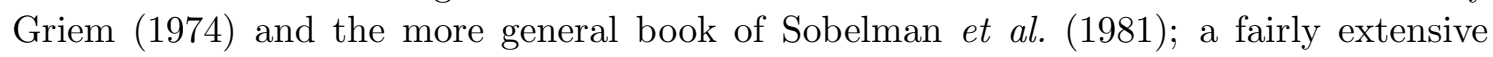
summary is found in 'Mihalas $(19 \overline{7} \overline{8})$. Gray (1992) gives a much simpler summary and specifies useful approximations for applications.

Elastic collisions. Collisional broadening or pressure broadening results from other particles in the neighborhood. They may be electrons, ions, atoms or molecules. Their charge affects the radiating or extincting atom or ion of interest through the Coulomb interaction and therefore affects the frequency of a bound-bound transition between perturbed levels. These collisional encounters are often termed elastic although the energies are slightly changed momentarily; the term inelastic is reserved for collisions involving bound-bound transitions between different energy levels.

Neutral atoms take part to some extent as perturber because they are polarizable, having a net electric field at close quarters. Of these, neutral H I atoms have the largest polarizability due to the bad shielding of the proton by the single electron. They are therefore important spectral line broadeners in cool atmospheres in which hydrogen is not ionized and which contain few free electrons.

\footnotetext{
${ }^{4}$ The convolution of two functions $f(x)$ and $g(x)$ is defined as $h(a) \equiv f * g(a) \equiv \int f(x) g(a-x) \mathrm{d} x$. Convolution is equivalent to multiplication in the Fourier domain: $H=F \cdot G$ with $H, F$ and $G$ the Fourier transforms of $h, f$ and $g$.
} 
There are two extreme formalisms to describe the changes in the term structure, the impact approximation and the quasi-static approximation.

Impact approximation. The perturber comes by at large speed and causes a momentary disruption of the wave train emitted by a deexciting atom. Such disruptions are described as phase jumps in the wave train (Lindholm theory) and cause broadening with a Lorentz profile just as the natural-broadening decay functions do; the resulting broadening parameters $\gamma^{\mathrm{col}}$ are then simply added to $\gamma^{\mathrm{rad}}$. See Fig. 11.4 of 'Gray $(1 \overline{1} 9 \underline{9} 2)^{\prime}$ and his

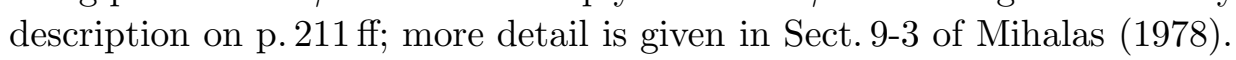

Such fast disruptions are primarily caused by free electrons due to their large collision frequency and high thermal speed. However, perturbations by neutral hydrogen atoms are also well described by the impact approximation. The duration of their encounters is brief although they are slower because the spatial extent of this interaction is very short (see below).

Quasi-static approximation. The other extreme is to have the surrounding particles at rest, which finds its application in describing the broadening by the slow-moving ions (protons when hydrogen is partially ionized). The ion density then defines an electric field that resets the internal term structure of the radiating (extinguishing) atom. The profile shape then depends on the frequency with which different surrounding fields are realized; a statistical description is needed to describe them. In the nearest-neighbor approximation one accounts only for the binary encounters and neglects the more distant perturbers.

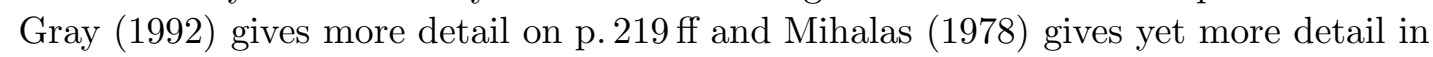
Sect. 9.4. A profile function results which is not Lorentzian but has the so-called Holtsmark shape. It has a dip at line center and decays in the far wings $\sim \Delta \nu^{-5 / 2}$, whereas the Lorentz profile in (3.44) decays as $\Delta \nu^{-2}$. See Fig. 11.9 of 'Gray $(19 \overline{9} 92)$, Fig. 9-3 of 'Mihalas and Fig. 9-1 of 'Mihalas $19 \overline{1} \overline{7})$, for examples.

Classification. The classical classification is to split the various interactions by their schematic dependence on the separation $r$ between the absorbing (photon-extinguishing) atom or ion and the perturber in the form

$$
\Delta \nu=\frac{\Delta E}{h} \equiv \frac{C_{n}}{r^{n}}
$$

with $C_{n}$ the interaction constant and $r$ the distance at the moment of closest encounter (the impact parameter) for the impact approximation and the distance to the next ion in the quasi-static nearest-neighbor approximation. This scheme assumes that the interaction potentials may to first order be described as power laws in $r$, an approximation which holds to some extent but not perfectly. The power index $n$ defines the name and type of the interaction specified in Table 3.1.

Linear Stark effect $(n=2)$. The lowest-order broadening is called the linear Stark effect. It is important for H I lines and explains their very large width in spectra from hot stars. It is also important for hydrogenic lines such as He II lines and Rydberg lines (lines with high principal quantum number $n$ obeying the Rydberg formula for hydrogen levels) of other elements. These interactions are with protons and electrons as perturbers. Their spatial extent $\sim r^{-2}$ is so large because hydrogen has a permanent dipole moment. The 


\begin{tabular}{llllll}
\hline$n$ & name & profile & atom, ion & perturbers & spectral type \\
\hline 2 & linear Stark & Holtsmark & hydrogen, hydrogenic & ions & hot \\
2 & linear Stark & Lorentz? & hydrogen, hydrogenic & electrons & hot \\
3 & resonance & Lorentz & hydrogen & hydrogen & solar \\
4 & quadratic Stark & Lorentz & non-hydrogenic & electrons, ions & hot \\
6 & van der Waals & Lorentz & non-hydrogenic & hydrogen & cool \\
\hline
\end{tabular}

Table 3.1: Collision broadening mechanisms.

decay is the same as for the Coulomb field of a point source so that the energy change is proportional to the surrounding field strength,

The linear Stark effect from proton collisions is best described by quasi-static theory and the Holtsmark profile shape. The surrounding electric field splits the normally degenerate $2 n^{2}$ sublevels of each H I level into pairs of separate Stark components, each shifted from line center over a distance that is proportional to the field strength in a symmetrical pattern. Statistically averaging over field realizations produces Holtsmark broadening for each component; all these profiles must be added together to derive the total broadening.

The electron broadening component of the linear Stark effect is usually described with the impact approximation, although its validity is questionable. "Unified" quantumtheoretical approaches exist also; Mihalas gives a summary. The resulting Lorentz profiles must be convolved with the summed Holtsmark shapes from the ions.

Resonance broadening $(n=3)$. The $n=3$ decay describes the interaction scale for collisions between neutral hydrogen atoms themselves. In order to see Balmer lines (from the $n=2$ level, where $n$ is the principal quantum number, another $n$ ) the atmosphere should be not too cool. It shouldn't be too hot or neutral hydrogen is not the major perturber. It seems to be important for the solar $\mathrm{H} \alpha$ line.

Quadratic Stark effect $(n=4)$. Most lines other than H I lines are broadened by electron and ion impacts with spatial extent $\sim r^{-4}$ because they arise from systems without dipole moment. The short interaction length makes the impact approximation applicable. Therefore the broadening function is a Lorentzian and the corresponding $\gamma_{4}$ adds to $\gamma^{\mathrm{rad}}$. Electron broadening dominates for non-hydrogenic atoms and ions in the atmospheres of hot stars where the electron density is high and the neutral hydrogen density small. It is described extensively by Griem (1974)!

Van der Waals broadening $(n=6)$. The overwhelming numbers of neutral hydrogen atoms makes them the dominant broadener of spectral lines from cool-star atmospheres. The short scale length of the interaction makes the encounter brief and the impact approximation valid, so that the profile shape is again Lorentzian and the corresponding line

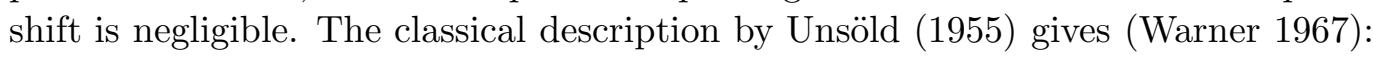

$$
\log \gamma_{6} \approx 6.33+0.4 \log \left(\overline{r_{u}^{2}}-\overline{r_{l}^{2}}\right)+\log P_{g}-0.7 \log T
$$


where the difference in atomic mean square radii $\overline{r^{2}}$ between the upper and the lower level is often estimated from the Bates-Damgaard hydrogenic approximation

$$
\overline{r^{2}}=\frac{n^{* 2}}{2 Z^{2}}\left(5 n^{* 2}+1-3 l(l+1)\right)
$$

The mean square radius is measured in atomic units, $l$ is the angular quantum number of the level and $n^{*}$ is its effective (hydrogen-like) principal quantum number of the level given by

$$
n^{* 2}=R \frac{Z^{2}}{E_{\infty}-E_{n}}
$$

in which the Rydberg constant $R=13.6 \mathrm{eV}=2.18 \times 10^{-11} \mathrm{erg}, Z$ is the ionization stage ( $Z=1$ for Fe I, $Z=2$ for Fe II, etc) and $E_{\infty}-E_{n}$ is the ionization energy from the level.

Van der Waals enhancement factor. For other lines than H I and hydrogenic lines (HeII, Rydberg lines) the major contribution is from van der Waals broadening with $n=6$. It typically produces $\gamma_{6} \approx 10^{9} \mathrm{~s}^{-1}$ near $\tau^{\text {cont }}=1$ in the visible, exceeding $\gamma_{\text {rad }}$ even for resonance lines (see Fig. 11.6 of 'Gray 1992 for $\mathrm{NaI} \mathrm{D}_{1}$ ). But it is usually not large enough, in the sense that observed line profiles from cool stars seem to suffer more damping than $(\overline{3} \cdot \overline{4})$ indicates. Most spectroscopists therefore apply a fudge factor called damping enhancement factor that increases the computed Van der Waals damping by a factor of $2-5$. There have been efforts to describe this interaction with more complicated potentials than simple $r^{-6}$ dependence (Lennard-Jones, Smirnov-Roueff potentials). The best current recipe is available at http://www.astro.uu.se/ barklem/ and implements the

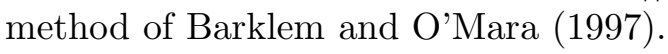

\subsubsection{Doppler broadening}

Doppler shift. The motion of a radiating particle along the line of sight produces a Doppler shift given by (for $\xi \ll c$ ):

$$
\frac{\Delta \nu}{\nu}=-\frac{\Delta \lambda}{\lambda}=\frac{\xi}{c}
$$

with $\xi$ the velocity component along the line of sight. Its sign is usually taken positive towards the observer, so that positive Doppler shift means blueshift and upward motion in the stellar atmosphere. A photon that is emitted at frequency $\nu^{\prime}$ in the frame of the emitting atom is detected by the observer as blueshifted to the frequency $\nu$ with

$$
\nu=\nu^{\prime}(1+\xi / c) \approx \nu^{\prime}+\nu_{0} \xi / c
$$

where $\nu^{\prime}$ is replaced by the line-center frequency $\nu_{0}$ in the second term since $\xi / c \ll 1$. The same result is obtained for an absorbing atom that moves with velocity $\xi$ towards the observer and extincts radiation that it sees redshifted in its own frame to

$$
\nu^{\prime}=\nu(1-\xi / c) \approx \nu-\nu_{0} \xi / c .
$$

Thermal motions. For purely thermal motions the distribution of velocities in the line of sight is given by the component form of the Maxwell distribution:

$$
\frac{n(\xi)}{N} \mathrm{~d} \xi=\frac{1}{\xi_{0} \sqrt{\pi}} \mathrm{e}^{-\xi^{2} / \xi_{0}^{2}} \mathrm{~d} \xi
$$


which is an area-normalized Gaussian distribution with variance

$$
\xi_{0}=\sqrt{\frac{2 k T}{m}}=12.85 \sqrt{\frac{T}{10^{4} A}}
$$

with $k$ the Boltzmann constant, $m$ the mass of the atom, $A$ the atomic weight and $\xi_{0}$ in $\mathrm{km} \mathrm{s}^{-1}$. The root-mean-square velocity component in the line of sight is:

$$
<\xi^{2}>^{1 / 2}=\sqrt{\frac{k T}{m}}=\frac{\xi_{0}}{\sqrt{2}} .
$$

Thermal broadening. What is the line extinction profile when thermal motions are taken into account? Remember that the monochromatic extinction coefficient per particle $\sigma_{\nu}^{l}$ in $\left(\overline{2} \cdot \overline{6} \overline{6}_{1}\right)$ on page ${ }_{2}^{2} \overline{2} \overline{3}$ is an ensemble probability, representing the average value for all particles that may cause extinction through that bound-bound transition at that wavelength. Write it as $\sigma_{\nu}^{l} \equiv \sigma^{l}\left(\nu-\nu_{0}\right)$. The total over the line is given by

$$
\sigma_{\nu_{0}}^{l} \equiv \int_{0}^{\infty} \sigma^{l}\left(\nu-\nu_{0}\right) \mathrm{d} \nu=\int_{0}^{\infty} \frac{h \nu}{4 \pi} B_{l u} \varphi\left(\nu-\nu_{0}\right) \mathrm{d} \nu=\frac{\pi e^{2}}{m_{\mathrm{e}} c} f \int_{0}^{\infty} \varphi\left(\nu-\nu_{0}\right) \mathrm{d} \nu=\frac{\pi e^{2}}{m_{\mathrm{e}} c} f
$$

with $f \equiv f_{l u}$ the classical oscillator strength in $\left(2.66^{i}\right)$. The ensemble-averaged extinction coefficient per particle in the particle frame is per line-of-sight velocity $\xi$ using $(3.54)$ :

$$
\sigma_{\nu^{\prime}}^{l}=\sigma^{l}\left(\nu^{\prime}-\nu_{0}\right)=\sigma^{l}\left(\nu-\xi \nu_{0} / c-\nu_{0}\right) .
$$

This must be averaged over the velocity distribution $n(\xi)$ to obtain the ensemble-averaged extinction coefficient in the frame of the observer:

$$
\sigma^{l}\left(\nu-\nu_{0}\right)=\frac{\int \sigma^{l}\left(\nu-\xi \nu_{0} / c-\nu_{0}\right) n(\xi) \mathrm{d} \xi}{\int n(\xi) \mathrm{d} \xi}=\int_{-\infty}^{+\infty} \sigma^{l}\left(\nu-\xi \nu_{0} / c-\nu_{0}\right) \frac{n(\xi)}{N} \mathrm{~d} \xi
$$

which represents a convolution (see footnote on page $\left.\overline{5} \overline{5} \overline{4}_{-1}^{\prime}\right)$. In the case that thermal Doppler shifts constitute the only broadening $\delta_{-1}^{51}$ the local extinction profile shape per particle $\varphi\left(\nu^{\prime}-\right.$ $\left.\nu_{0}\right)$ is replaced by a delta function $\delta\left(\bar{\nu}^{\prime}-\nu_{0}\right)$ because in that case both levels are sharp and each extinction concerns a single (shifted) frequency only. The convolution is then, using

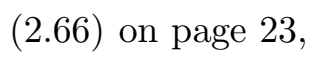

$$
\sigma^{l}\left(\nu-\nu_{0}\right)=\frac{\pi e^{2}}{m_{\mathrm{e}} c} f \int_{-\infty}^{+\infty} \delta\left(\nu-\xi \nu_{0} / c-\nu_{0}\right) \frac{n(\xi)}{N} \mathrm{~d} \xi
$$

and produces the monochromatic extinction coefficient in the frame of the observer, measured per particle but averaged over all line-of-sight particle velocities, by setting $\xi=\left(\nu-\nu_{0}\right) c / \nu_{0}$ in the Maxwell distribution $n(\xi) / N$ :

$$
\sigma_{\nu}^{l}=\frac{\pi e^{2}}{m_{\mathrm{e}} c} f \frac{n\left[\left(\nu-\nu_{0}\right) c / \nu_{0}\right]}{N}=\frac{\sqrt{\pi} e^{2}}{m_{\mathrm{e}} c} \frac{f}{\Delta \nu_{\mathrm{D}}} \mathrm{e}^{-\left(\Delta \nu / \Delta \nu_{\mathrm{D}}\right)^{2}}
$$

with the Doppler width $\Delta \nu_{\mathrm{D}}$ defined by

$$
\Delta \nu_{\mathrm{D}} \equiv \frac{\xi_{0}}{c} \nu_{0}=\frac{\nu_{0}}{c} \sqrt{\frac{2 k T}{m}}
$$

\footnotetext{
${ }^{5}$ Pure Doppler broadening is valid at high temperature and low density.
} 
and the Gaussian extinction profile given by

$$
\varphi\left(\nu-\nu_{0}\right)=\frac{1}{\sqrt{\pi} \Delta \nu_{\mathrm{D}}} \mathrm{e}^{-\left(\Delta \nu / \Delta \nu_{\mathrm{D}}\right)^{2}}
$$

In wavelength units the monochromatic extinction coefficient is:

$$
\sigma_{\lambda}^{l}=\frac{\sqrt{\pi} e^{2}}{m_{\mathrm{e}} c} \frac{\lambda^{2}}{c} \frac{f}{\Delta \lambda_{\mathrm{D}}} \mathrm{e}^{-\left(\Delta \lambda / \Delta \lambda_{\mathrm{D}}\right)^{2}}
$$

with

$$
\Delta \lambda_{\mathrm{D}} \equiv \frac{\xi_{0}}{c} \lambda_{0}=\frac{\lambda_{0}}{c} \sqrt{\frac{2 k T}{m}}
$$

and $\Delta \lambda / \Delta \lambda_{\mathrm{D}}=\Delta \nu / \Delta \nu_{\mathrm{D}}$. The full width at half maximum of the profile is FWHM $=$ $2 \ln (2) \Delta \lambda_{\mathrm{D}}=1.38 \Delta \lambda_{\mathrm{D}}$.

Voigt profile. When the collisional damping has the Lorentz profile shape (as in the impact approximation), the total damping profile is the convolution of the constituents and is given by a Lorentz profile with $\gamma=\gamma^{\mathrm{rad}}+\gamma^{\mathrm{col}}$. Assuming that collisional broadening and thermal Doppler shifting are independent processes, this shape function must be convolved with (3.62) for thermal broadening to obtain the total extinction coefficient. The damping profile $(3 . \overline{4})$ is area-normalized and therefore takes the place of the $\delta$ function in $(3.61)$, again with the frame-of-the-atom frequency shift $\nu^{\prime}-\nu_{0}=\nu-\xi \nu_{0} / c-\nu_{0}$ as argument. Thus:

$$
\begin{aligned}
\sigma_{\nu}^{l} & =\left[\frac{\sqrt{\pi} e^{2}}{m_{\mathrm{e}} c} \frac{f}{\Delta \nu_{\mathrm{D}}} \mathrm{e}^{-\left(\Delta \nu / \Delta \nu_{\mathrm{D}}\right)^{2}}\right] *\left[\frac{\gamma / 4 \pi^{2}}{\left(\nu^{\prime}-\nu_{0}\right)^{2}+(\gamma / 4 \pi)^{2}}\right] \\
& =\frac{\sqrt{\pi} e^{2}}{m_{\mathrm{e}} c} \frac{f}{\Delta \nu_{\mathrm{D}}} \int_{-\infty}^{+\infty} \frac{\left(\gamma / 4 \pi^{2}\right) \mathrm{e}^{-\left(\Delta \nu / \Delta \nu_{\mathrm{D}}\right)^{2}}}{\left(\nu^{\prime}-\nu_{0}\right)^{2}+(\gamma / 4 \pi)^{2}} \mathrm{~d} \nu \\
& =\frac{\sqrt{\pi} e^{2}}{m_{\mathrm{e}} c} \frac{f}{\Delta \nu_{\mathrm{D}}} H(a, v)
\end{aligned}
$$

with

$$
\begin{aligned}
H(a, v) & \equiv \frac{a}{\pi} \int_{-\infty}^{+\infty} \frac{\mathrm{e}^{-y^{2}}}{(v-y)^{2}+a^{2}} \mathrm{~d} y \\
y & \equiv \frac{\xi}{\xi_{0}}=\frac{\xi}{c} \frac{\nu_{0}}{\Delta \nu_{\mathrm{D}}}=\frac{\xi}{c} \frac{\lambda_{0}}{\Delta \lambda_{\mathrm{D}}} \\
v & \equiv \frac{\nu-\nu_{0}}{\Delta \nu_{\mathrm{D}}}=\frac{\lambda-\lambda_{0}}{\Delta \lambda_{\mathrm{D}}} \\
a & \equiv \frac{\gamma}{4 \pi \Delta \nu_{\mathrm{D}}}=\frac{\lambda^{2}}{4 \pi c} \frac{\gamma}{\Delta \lambda_{\mathrm{D}}} .
\end{aligned}
$$

The function $H(a, v)$ is called the Voigt function. Examples are shown in Fig. 1 i; ; a simple numerical approximation and references to more elaborate routines are given on page 107 of Baschek and Scholz $(1982)_{1}^{1}$. The Voigt function is not normalized but has area $\sqrt{\pi}$ in $v$ units; the maximum value in the center has $H(a, v=0) \approx 1-a$ for $a<1$. The corresponding area-normalized extinction profile is:

$$
\varphi\left(\nu-\nu_{0}\right)=\frac{H(a, v)}{\sqrt{\pi} \Delta \nu_{\mathrm{D}}} .
$$


The rough approximation for $a \ll 1$

$$
H(a, v) \approx \mathrm{e}^{-v^{2}}+\frac{a}{\sqrt{\pi} v^{2}}
$$

shows that the shape of the Voigt function approximates a Gaussian near line center but possesses $\Delta \nu^{-2}$ damping decay in the far wings. The cross-over between the two can be graphically read off as the intersections for various $a$ with the $a=0$ parabola in Fig. Usually, $a$ is rather small, less than $a=0.1$. For $a \approx 0.01$ the change between Gaussian and Lorentzian character lies near $v=2.7$ and has $H(a, v) \approx 10^{-3}$. Thus, the extent of the Doppler core of the extinction profile is quite wide. Only very strong lines have sufficient extinction far enough from line center to possess damping wings $S_{1-1}^{6 !}$ without sensitivity to the Doppler width:

$$
\varphi\left(\nu-\nu_{0}\right) \approx \frac{1}{\sqrt{\pi} \Delta \nu_{\mathrm{D}}} \frac{a}{\sqrt{\pi} v^{2}}=\frac{1}{\sqrt{\pi} \Delta \nu_{\mathrm{D}}} \frac{\gamma}{4 \pi \Delta \nu_{\mathrm{D}}} \frac{\Delta \nu_{\mathrm{D}}^{2}}{\sqrt{\pi} \Delta \nu^{2}}=\frac{\gamma}{4 \pi^{2} \Delta \nu^{2}} .
$$

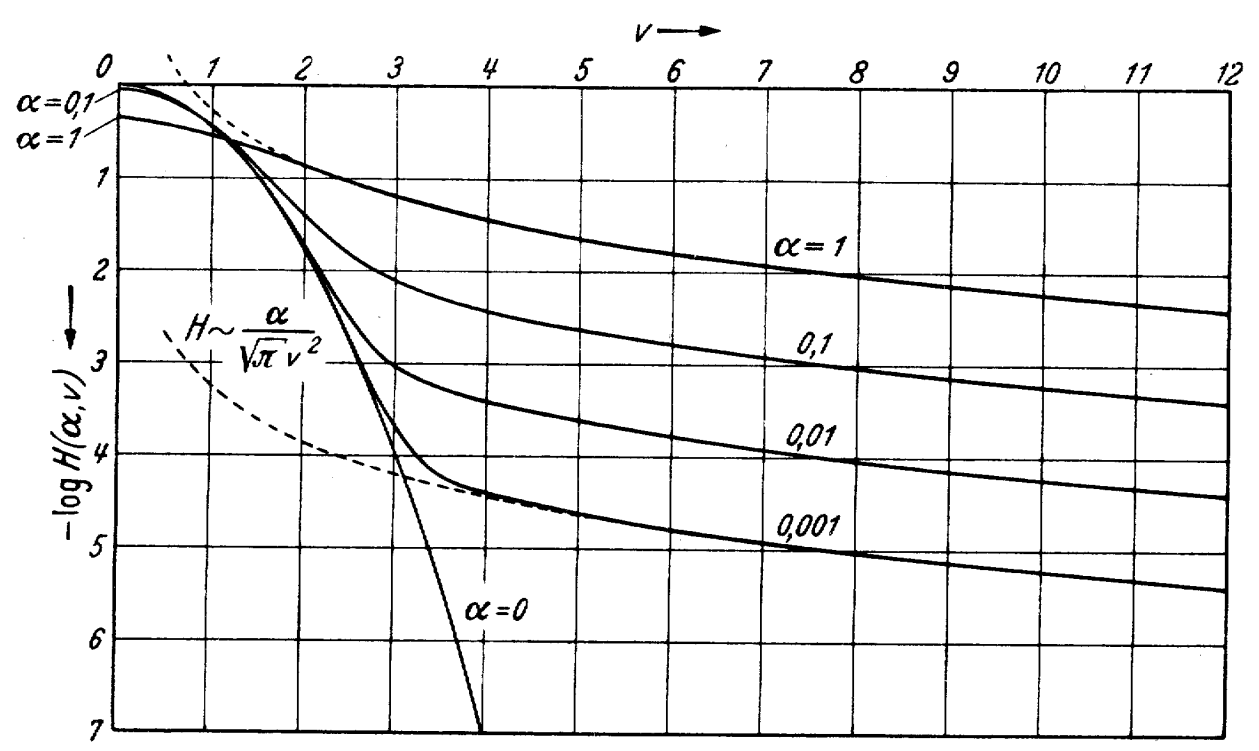

Figure 3.1: The Voigt function $H(a, v)$ for different values of the damping parameter $a$ (written as $\alpha$ ). From 'Unsöld (1955)!

Rotational broadening. Thermal broadening describes the microscopic motion of individual particles in the stellar atmosphere. The other scale extreme is the macroscopic broadening of the lines in a stellar flux spectrum caused by the rotation of the whole star. See Gray (1992)! p. $370 \mathrm{ff}$. Assume that the star is spherical and rotates rigidly. Choose the coordinate system such that the $z$-axis points towards the observer (as always in this

\footnotetext{
${ }^{6}$ Of course, any bound-bound extinction profile has damping wings far from line center. What counts is whether the line extinction there is still competitive with the local continuum extinction. Spectral lines only show damping wings in the emergent spectrum when their line extinction exceeds the continuum extinction appreciably at $\Delta \lambda \gg 3 \Delta \lambda_{\mathrm{D}}$.
} 

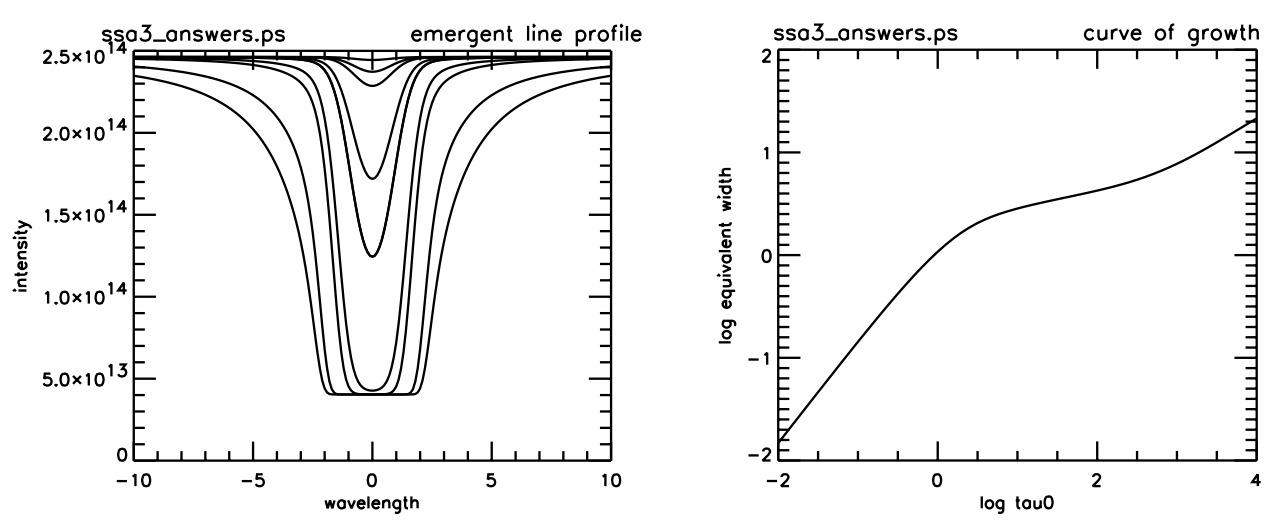

Figure 3.2: Left: absorption profiles from a Schuster-Schwarzschild layer with Voigt-shaped extincton, computed from (2.34) on page 15 using $S_{\lambda}^{l}=S_{\lambda}^{c}=B_{\lambda}(T)$ with $T=5700 \mathrm{~K}$ for the continuous irradiation from behind and $\bar{T}=4200 \overline{\mathrm{K}}$ for the line-extincting layer. The Voigt profile has $a=0.1$. The $x$ axis is in dimensionless units $\Delta \lambda / \Delta \lambda_{\mathrm{D}}$. The wavelength is $\lambda=500 \mathrm{~nm}$. The different profiles are for increasing line-center optical thickness of the Schuster-Schwarzschild layer. Right: corresponding "curve of growth" of the equivalent width (area of the absorption dip normalized by the continuum intensity) with the layer opacity. In the initial "Doppler part" the area of the absorption line increases linearly with the layer opacity. Saturation is reached when the layer becomes optically thick at line center. For yet larger layer opacity, the "damping branch" shows renewed area growth thanks to the layer's optical thinness in the extended wings. An observational curve of growth is shown in Figure $9 . \overline{4}$ on page $210 \mathrm{~s}$ analytical analysis is given in Section 9.1 .2 Copied from my third "Stellar Spectra A" exercise available at http://www.astro.uu.nl/ rutten.

course) and that it contains the rotation axis in the $(y, z)$ plane. The apparent disk of the star then consists of $y$-strips per $x$ value that have the same projected rotation velocity

$$
v_{z}(x)=x \Omega \sin i,
$$

with $i$ the axis inclination and $\Omega$ the angular rotation velocity. The center strip with $x=0$ has no component in the line of sight because all its elements move perpendicular to the line of sight. The largest Doppler shift occurs for $x=R$ at the limb:

$$
\Delta \lambda_{R}=(\lambda / c) R \Omega \sin i=(\lambda / c) v \sin i
$$

with $v$ the equatorial velocity. The flux spectrum that passes through the stellar surface is given by

$$
\mathcal{F}_{\nu}=\int I_{\nu} \cos \theta \mathrm{d} \Omega=\iint \frac{I_{\nu}(x, y)}{R^{2}} \mathrm{~d} x \mathrm{~d} y
$$

where the first integral is over all outward directions and the second over the apparent stellar disk in the $(x, y)$ plane. The irradiance spectrum observed at Earth is $\mathcal{R}_{\nu}=$ $\mathcal{F}_{\nu} R^{2} / D^{2}$ with $D$ the distance to the star. The problem arises that $I_{\nu}$ differs at different locations $(x, y)$ due to limb darkening, non-rigid rotation and other symmetry breaks. In that case $I_{\nu}$ must be evaluated at many $(x, y)$ locations to integrate $(\overline{3} \overline{3} \overline{7} \overline{7} \bar{i})$ numerically.

Deconvolution. A shortcut is feasible when the relative line profile in the emergent intensity

$$
H(\nu) \equiv I(\nu) / I_{\nu}^{\mathrm{cont}}
$$


does not vary across the stellar disk. The relative flux profile may then be written as a convolution (Gray 1992 p. 373)

$$
\begin{aligned}
\frac{\mathcal{F}_{\nu}}{\mathcal{F}_{\nu}^{\text {cont }}} & =\frac{\int H(\nu-\Delta \nu) I_{\nu}^{\text {cont }} \cos \theta \mathrm{d} \Omega}{\int I_{\nu}^{\text {cont }} \cos \theta \mathrm{d} \Omega} \\
& =\frac{\iint\left(H(\nu-\Delta \nu) I_{\nu}^{\text {cont }} / R^{2}\right) \mathrm{d} x \mathrm{~d} y}{\iint\left(I_{\nu}^{\text {cont }} / R^{2}\right) \mathrm{d} x \mathrm{~d} y} \\
& =\int_{-\infty}^{+\infty} H(\nu-\Delta \nu) G(\Delta \nu) \mathrm{d} \nu \\
& =H(\nu) * G(\nu)
\end{aligned}
$$

with $\Delta \nu$ at each location $(x, y)$ the Doppler shift that corresponds to $(\overline{3}-\overline{3} . \overline{5} ;)$ and the function $G(\Delta \nu)$ describing the rotation profile. It has the shape of a half ellipse. Equation 17.12 of 'Ḡ'Gray $(1 \overline{1} 9 \overline{9} 2)$ ' gives a similar expression for such direct convolution with a function $G(\Delta \nu)$ for a fairly general limb darkening law on the assumption that it does not differ between the continuum and the line.

Since convolution implies multiplication of Fourier transforms, the splitting of observed flux profiles between the rotation profile and the intrinsic intensity profile is best done in the Fourier domain, see Gray $(1992)$, p. $378 \mathrm{ff}$. The elliptical shape of $G(\Delta \nu)$ causes a distinct side-lobe $\sin x / x$ pattern in the transform. The locations of the corresponding dips between the humps are not very sensitive to noise and furnish a good diagnostici!r

Turbulent broadening. There are other motions in stellar atmospheres than just the microscopic thermal particle motions and the macroscopic rotation of the whole star. Examples are waves of various sorts and turbulent convection. They are neglected when stars are assumed to possess time-independent plane-parallel geometry. In that case one also neglects stationary inhomogeneities and magnetic structuring. In order to make up for this glaring deficiency in their modeling, stellar spectroscopists follow Struve's trick of introducing two additional fudge parameters called microturbulence and macroturbulence. As the names indicate, they supposedly have random nature and supposedly operate on microscopic scales and macroscopic scales, respectively. They are usually defined as convolutions with Gaussian velocity distributions, respectively of the local line extinction coefficient (microturbulence) and of the emergent intensity or flux profile (macroturbulence).

The microturbulence is simply entered by redefining the Doppler width (3.63i) on page $\overline{5} \bar{\alpha}$ as

$$
\Delta \nu_{\mathrm{D}} \equiv \frac{\nu_{0}}{c} \sqrt{\frac{2 k T}{m}+\xi_{\text {micro }}^{2}}
$$

\footnotetext{
${ }^{7}$ Such zeroes in a Fourier transform are useful to fit a known transform shape with zeroes in the presence of other signals without transform zeroes. Examples of the latter are white noise, noise with a Gaussian frequency distribution, or in this case the Voigt-like intrinsic shape of the non-rotationally broadened spectral lines. See 'Gray (1992)! for examples. However, Fourier transform zeroes are a nuisance for restoring data by deconvolution using the convolution theorem (convolution in the measurement domain transforms into multiplication in the Fourier domain, and vice versa). If restoration is done by dividing the transform $S(f)$ of the signal $s(x)$ by the transform $A(f)$ of the smearing function $A(x)$ (for example the point spread function of a telescope or the instrumental profile of a spectrograph), zeroes in $A(t)$ make the corrected transform $S(t) / A(t)$ infinity. Much more sophisticated methods are generally needed to restore images, spectra, time sequences etc. for instrumental effects.
} 
to describe the convolution of the two Gaussians convolving the computed emergent intensity profile numerically with a Gaussian velocity distribution:

$$
\frac{I_{\mathrm{c}}-I_{\lambda}}{I_{\mathrm{c}}}=\left[\frac{I_{\mathrm{c}}-I_{\lambda}}{I_{\mathrm{c}}}\right]_{\text {comp }} * \frac{1}{\xi_{\text {macro } \sqrt{\pi}}} \mathrm{e}^{-\xi^{2} / \xi_{\text {macro }}^{2}}
$$

The value of these parameters is derived by requiring that the computed line profiles fit the observed ones. Since a single choice will usually not work for more than one line, they are often assumed to vary with height and/or with viewing angle, effectively enlarging the free parameter space.

Warning. Although the names imply random motions, the turbulence parameters are really ad-hoc fitting parameters that serve to correct for any deficiencies in plane-parallel modeling, including not or badly modeled departures from LTE and wrong collision broadening in addition to dynamical effects and inhomogeneities and whatever else is wrong in the modeling. There have been efforts to produce a more physical description from real theories of real stochastic turbulence $e_{-\mathbf{I}}$. I don't think these descriptions make much more sense than the classical definitions do; all use of these parameters remains rather suspect since they mostly do not describe actual turbulence. The problem is that the fitted turbulence needs to be fairly large to explain observed line profiles, typically larger than the thermal broadening and even supersonic in hot stars. See Fig. 18.9 of 'Gray (19.92)', for an overview.

\subsubsection{Other broadening}

Hyperfine structure. The angular momentum of the nucleus in atoms and ions couples with the angular momentum of the electron cloud and so causes a slight splitting of energy levels into hypermultiplets. It is usually negligible, with the component separations well below the thermal Dopplerwidth, except in some optical spectra (Mn I, V I, CoI, CuI). The patterns are found in physics handbooks.

Isotope splitting. Different isotopes have different nuclear mass and therefore slightly different term energies. The effect is most outspoken for the light elements (H I, Li I) but also evident in BaII.

Zeeman splitting. Magnetic fields split magnetically sensitive lines in patterns of polarized components of which the Zeeman triplet is the simplest case. At optical wavelengths the splitting is seen as line broadening. Towards the infrared the Zeeman splitting becomes more noticeable because it increases as $\Delta \lambda_{\mathrm{Z}} \sim \lambda^{2}$ whereas the thermal line width increases as $\Delta \lambda_{\mathrm{D}} \sim \lambda$. In the solar spectrum, the famous $\mathrm{Mg}$ I emission lines near $12 \mu \mathrm{m}$ are fully split for fields as weak as 300 Gauss (Rutten and Carlsson 1994i). The book by Ŝtenflo (1994), treats polarized radiative transfer and solar magnetic field diagnostics extensively.

\footnotetext{
${ }^{8}$ If there really are Gaussian-distributed turbulent motions in the stellar atmosphere that broaden the local line extinction coefficient, the value $\xi_{\text {micro }}$ would be larger than the rms velocities_ $\leq v_{ \pm t u r b}^{2}>^{1 / 2}$ by $\sqrt{2}$.

${ }^{9}$ Also, De Jager has introduced mesoturbulence for intermediate scales and 'Gray (1992)! uses a radialtangential model assuming that the actual motions in the atmosphere are either radial or horizontal but not slanted.
} 


\subsubsection{Spectral edge broadening}

Doppler shifts and collisional perturbations affect any radiating particle, not only for bound-bound transitions but also for bound-free and free-free transitions. The corresponding extinction coefficients are similarly smeared by the broadening distribution. In particular, the sharp extinction edge at the ionization threshold frequency gets just as blurred as a bound-bound extinction peak.

Near-edge line blending. Due to their Stark broadening the hydrogen lines in a series overlap close to the series limit and with the broadened limit itself, so that they and the edge merge into a quasi-continuum at frequencies well below the nominal threshold.

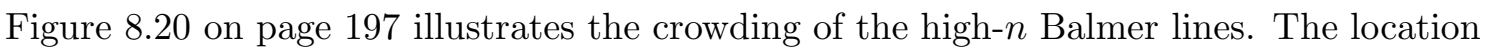
of the Balmer threshold is well to the right of the peak marking the continuum opacity windows between lower- $n$ Balmer lines. The classical Inglis-Teller estimate for the number of the highest-frequency Balmer line that is still separately discernible has (page 82 of

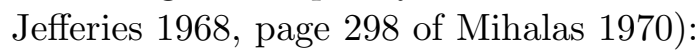

$$
\log N_{\mathrm{e}}=23.2-7.5 \log n_{\max }^{\text {Balmer }}
$$

assuming that the line width depends only on the electron density through Stark broadening. It has often been used as a rough first estimate to find $N_{\mathrm{e}}$. Table the large range in Balmer line visibility and corresponding $N_{\mathrm{e}}$ between different spectral types.

\begin{tabular}{llrc}
\hline star & spectral type & $n_{\max }^{\text {Balmer }}$ & $\log N_{\mathrm{e}}$ \\
\hline$\alpha$ Cyg & A2 I & 29 & 12.2 \\
Sirius & A2 V & 18 & 13.8 \\
$\tau$ Sco & B0 V & 14 & 14.6 \\
white dwarf & DA & 8 & 16.4 \\
\hline
\end{tabular}

Table 3.2: Inglis-Teller estimates for various stars. From Mihalas $(1970) !$

\subsection{Spectral line redistribution}

\subsubsection{Monochromatic redistribution}

Sharp-line atoms. We now return to coherent scattering by two-level atoms in order

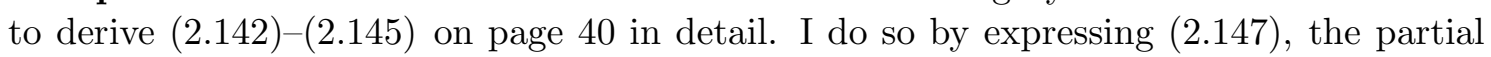
extinction coefficients $\alpha_{\nu}^{\mathrm{a}}$ and $\alpha_{\nu}^{\mathrm{s}}$ and the destruction probability $\varepsilon_{\nu}$ for two-level atoms into the Einstein coefficients. Their definitions $(2.46)-(2.58)$ specify transition probabilities for the whole line, but for radiative transport we are interested in what happens monochromatically at each frequency within the line. We already avoid cross-talk between different transitions by discussing the idealized case of a two-level-atom medium; I will now also avoid spectral cross-talk within the line, between frequencies across the line width, by further simplification to two-level atoms with infinitely sharp upper and lower levels, having no line broadening whatsoever. Such sharp-line atoms supposedly have $\varphi\left(\nu-\nu_{0}\right)=\psi\left(\nu-\nu_{0}\right)=\chi\left(\nu-\nu_{0}\right)=\delta\left(\nu-\nu_{0}\right)$, where $\delta$ is the delta function with the required area normalization $\int \delta\left(\nu-\nu_{0}\right) \mathrm{d} \nu=1$. The total radiation field in the line is then 
given by $J_{\nu_{0}} \equiv \int J_{\nu} \delta\left(\nu-\nu_{0}\right) \mathrm{d} \nu=J\left(\nu=\nu_{0}\right)$, and the Einstein coefficients specify transition probabilities at the single frequency $\nu=\nu_{0}$. This idealized case resembles coherent scattering in the sense that the transport equation concerns only a single frequency, without cross-talk to other frequencies, and it also resembles complete redistribution because all transitions are spread over the full "profile", each process taking a fresh sample of the same probability distribution!

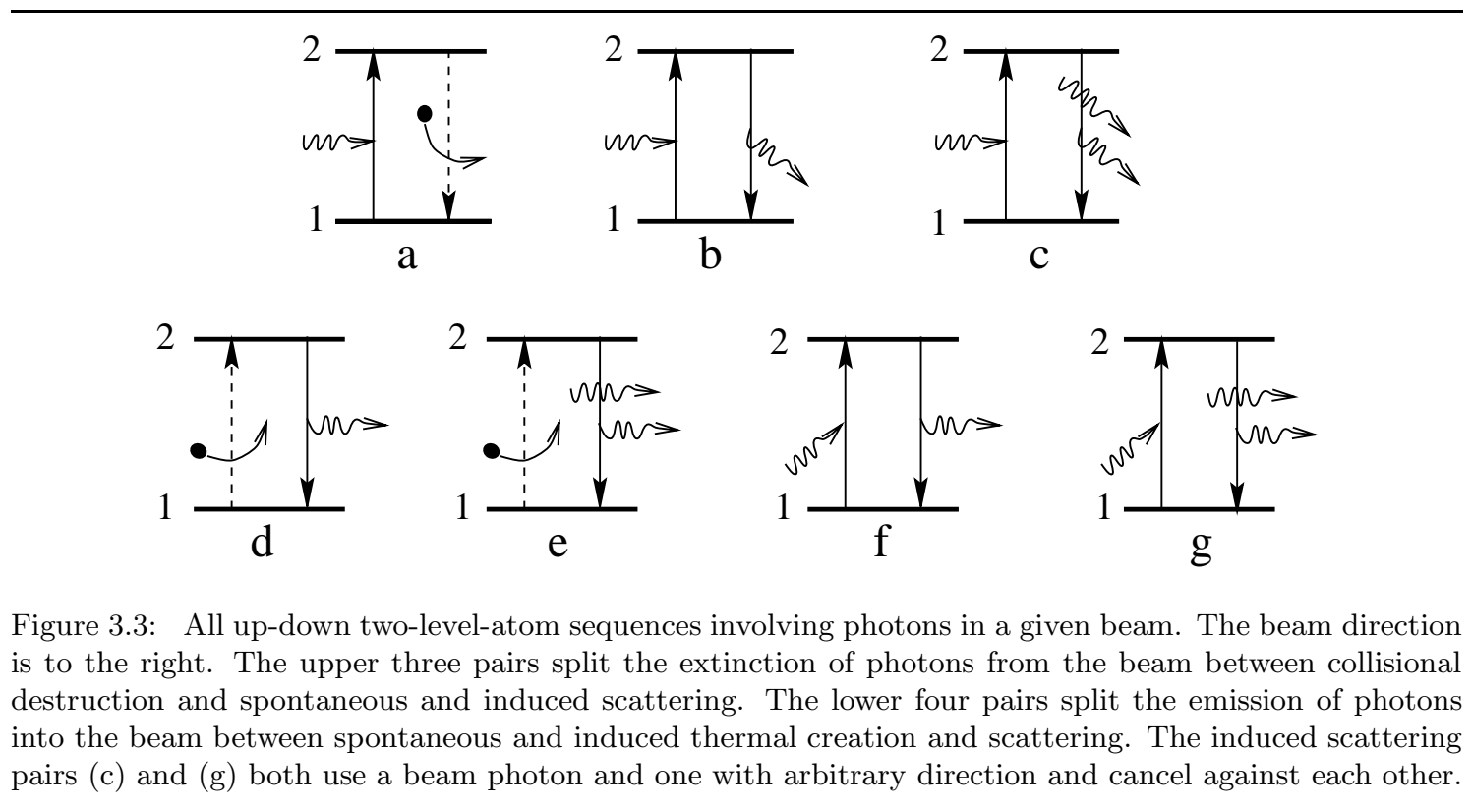

Up-down sequences. The line extinction was split in (2.1. $\overline{1} \overline{3} \overline{4})$ in thermal and scattering components depending on the nature of the two-level up-down sequences. For two level atoms, each extinction (radatiative excitation) is followed either by collisional deexcitation (photon destruction) or radiative deexcitation (photon scattering). This split depends on what happens after the initial extinction. I now detail such splitting not only for the extinction of photons from the beam but also for the emission of photons into the beam. For sharp-line two-level atoms, the full set of up and down process combinations involving photons $s_{-}^{117}$ in the beam direction consists of the following seven sequences, schematically shown in Figure 3.3

(a) thermal extinction $=$ radiative excitation by a beam photon followed by collisional deexcitation,

(b) spontaneous scattering extinction = radiative excitation by a beam photon followed by spontaneous deexcitation,

(c) induced scattering extinction $=$ radiative excitation by a beam photon followed by induced deexcitation,

(d) spontaneous thermal emission $=$ collisional excitation followed by spontaneous emission of a photon into the beam,

\footnotetext{
${ }^{10}$ References: 'Milne (1928)', Milne (1930)', also reprinted in 'Menzel (1966)', Thomas $(1957)$;

${ }^{11}$ Collisional excitation followed by collisional dexcitation does not involve photons, but couples the level populations to the kinetic energy partitioning (Maxwell distribution).
} 
(e) induced thermal emission $=$ collisional excitation followed by induced emission of a photon into the beam,

(f) spontaneous scattering emission $=$ radiative excitation followed by spontaneous emission of a photon into the beam,

(g) induced scattering emission $=$ radiative excitation followed by induced emission of a photon into the beam.

Same-direction scattering sequences (radiative excitation by a photon from the beam followed by radiative deexcitation producing a photon also in the beam direction) contribute both extinction through (b) and (c) and emission through (f) and (g). They replace old by new photons and are therefore counted in each, even though they do not change the beam intensity.

Transport equation. These seven sequences combine in the profile-integrated transport equation for radiation with frequency $\nu=\nu_{0}$ along a beam through a medium of sharp-line two-level atoms as:

$$
\begin{aligned}
\frac{\mathrm{d} I_{\nu_{0}}=-}{\mathrm{d} s}= & -\underbrace{n_{1} \sigma_{\nu_{0}}^{l} I_{\nu_{0}} \frac{C_{21}}{P_{21}}}_{(\mathrm{a})}-\underbrace{n_{1} \sigma_{\nu_{0}}^{l} I_{\nu_{0}} \frac{A_{21}}{P_{21}}}_{(\mathrm{b})}-\underbrace{n_{1} \sigma_{\nu_{0}}^{l} I_{\nu_{0}} \frac{B_{21} J_{\nu_{0}}}{P_{21}}}_{(\mathrm{c})} \\
& +\underbrace{n_{1} C_{12} \frac{\left(h \nu_{0} / 4 \pi\right) A_{21}}{P_{21}}}_{(\mathrm{d})}+\underbrace{n_{1} C_{12} \frac{\left(h \nu_{0} / 4 \pi\right) B_{21} I_{\nu_{0}}}{P_{21}}}_{(\mathrm{e})} \\
& +\underbrace{n_{1} B_{12} J_{\nu_{0}} \frac{\left(h \nu_{0} / 4 \pi\right) A_{21}}{P_{21}}}_{(\mathrm{g})}+\underbrace{n_{1} B_{12} J_{\nu_{0}} \frac{\left(h \nu_{0} / 4 \pi\right) B_{21} I_{\nu_{0}}}{P_{21}}}_{P_{21}}
\end{aligned}
$$

with $P_{21}$ the total deexcitation probability per particle in level 2 given by

$$
P_{21} \equiv A_{21}+B_{21} J_{\nu_{0}}+C_{21} .
$$

Each term in (3. the product of the excitation probability per $\mathrm{cm}^{3}$ for the upward process and the fractional deexcitation probability of the downward process. The three negative contributions (a) (c) make up the total extinction of photons from the beam per centimeter path length. The four positive contributions (d) - (g) make up the total emission of new photon energy into the beam. The factors $h \nu_{0} / 4 \pi$ convert isotropic emission probability into energy emitted per steradian. The specific intensity $I_{\nu_{0}}$ enters in (a) - (c) as the quantity to be extincted, but also in the fractions in (e) and (g) because the stimulating photon has to be in the beam under consideration to induce photon emission along it.

Equation (3.83i) is monochromatic but describes total profile-integrated extinction and emission with $\int \varphi\left(\nu-\nu_{0}\right) \mathrm{d} \nu=\int \delta\left(\nu-\nu_{0}\right) \mathrm{d} \nu=1$, so that the extinction coefficient per particle in level 1 is given by $(2.66 i)$ on page $2 \overline{2} \overline{3}_{1}^{\prime}$ as $\sigma_{\nu_{0}}^{l}=\left(h \nu_{0} / 4 \pi\right) B_{12}$ and (3.83i) may also be written as:

$$
\frac{\mathrm{d} I_{\nu_{0}}}{\mathrm{~d} s}=\frac{h \nu_{0}}{4 \pi} n_{1}[-\underbrace{B_{12} I_{\nu_{0}} \frac{C_{21}}{P_{21}}}_{\text {(a) }}-\underbrace{B_{12} I_{\nu_{0}} \frac{A_{21}}{P_{21}}}_{\text {(b) }}-\underbrace{B_{12} I_{\nu_{0}} \frac{B_{21} J_{\nu_{0}}}{P_{21}}}_{\text {(c) }}
$$




$$
+\underbrace{C_{12} \frac{A_{21}}{P_{21}}}_{\text {(d) }}+\underbrace{C_{12} \frac{B_{21} I_{\nu_{0}}}{P_{21}}}_{(\mathrm{e})}+\underbrace{B_{12} J_{\nu_{0}} \frac{A_{21}}{P_{21}}}_{\text {(f) }}+\underbrace{B_{12} J_{\nu_{0}} \frac{B_{21} I_{\nu_{0}}}{P_{21}}}_{\text {(g) }}]
$$

Extinction. Conventionally, the volume extinction coefficient $\alpha_{\nu_{0}}^{l}$ includes correction for induced emission. The total sharp-line extinction coefficient $\alpha_{\nu_{0}}^{l}\left(\right.$ dimension $\mathrm{cm}^{-1} \mathrm{~Hz}$ ) is therefore obtained by subtracting contributions (e) and (g) from the summed extinction terms (a), (b) and (c):

$$
\begin{aligned}
\alpha_{\nu_{0}}^{l}= & \frac{(\mathrm{a})-(\mathrm{e})+(\mathrm{b})+(\mathrm{c})-(\mathrm{g})}{I_{\nu_{0}}} \\
= & \frac{h \nu_{0}}{4 \pi} n_{1}\left[B_{12} \frac{C_{21}}{P_{21}}-C_{12} \frac{B_{21}}{P_{21}}\right. \\
& \left.\quad+B_{12} \frac{A_{21}}{P_{21}}+B_{12} \frac{B_{21} J_{\nu_{0}}}{P_{21}}-B_{12} J_{\nu_{0}} \frac{B_{21}}{P_{21}}\right] \\
\equiv & \alpha_{\nu_{0}}^{\mathrm{a}}+\alpha_{\nu_{0}}^{\mathrm{s}} .
\end{aligned}
$$

The thermal part $\alpha_{\nu_{0}}^{\mathrm{a}}$ consists of the difference (a) $-(\mathrm{e})$ :

$$
\begin{aligned}
\alpha_{\nu_{0}}^{\mathrm{a}} & =\frac{h \nu_{0}}{4 \pi} n_{1} B_{12} \frac{C_{21}}{P_{21}}\left[1-\frac{C_{12} B_{21}}{C_{21} B_{12}}\right] \\
& =\frac{h \nu_{0}}{4 \pi} n_{1} B_{12} \frac{C_{21}}{P_{21}}\left[1-\mathrm{e}^{-h \nu_{0} / k T}\right],
\end{aligned}
$$

where the reformulation is obtained from the Einstein relations $(2.600)-(2.61)$. The resulting TE correction factor $\left[1-\exp \left(-h \nu_{0} / k T\right)\right]$ befits the thermal origin of the induced photons. In the absence of any scattering, so that $\alpha_{\nu_{0}}^{\mathrm{s}}=0$ and $C_{21} / P_{21}=1$, this expression recovers the LTE version with $b_{l}=b_{u}=1$ of the general line extinction coefficient (2.113i).

The scattering part $\alpha_{\nu_{0}}^{\mathrm{s}}$ of $\alpha_{\nu_{0}}^{l}$ consists of (b) $+(\mathrm{c})-(\mathrm{g})$ and has

$$
\begin{aligned}
\alpha_{\nu_{0}}^{s} & =\frac{h \nu_{0}}{4 \pi} n_{1} B_{12}\left[\frac{A_{21}}{P_{21}}+\frac{B_{21} J_{\nu_{0}}}{P_{21}}-J_{\nu_{0}} \frac{B_{21}}{P_{21}}\right] \\
& =\frac{h \nu_{0}}{4 \pi} n_{1} B_{12} \frac{A_{21}}{P_{21}}
\end{aligned}
$$

where contributions (c) and (g) cancel because both sequences require a photon with arbitrary direction $\left(J_{\nu_{0}}\right)$ in addition to the photon from the beam $\left(I_{\nu_{0}}\right)$. Their probabilities are equal; just as many photons are induced to scatter into the beam as out of the beam. Only the spontaneous part of the scattering remains.

Emission. The total volume emissivity $j_{\nu_{0}}^{l}$ is made up by the left-over terms (d) and (f), respectively describing the thermal and scattering parts of the spontaneous emission:

$$
\begin{aligned}
j_{\nu_{0}}^{l} & =\frac{h \nu_{0}}{4 \pi} n_{1} C_{12} \frac{A_{21}}{P_{21}}+\frac{h \nu_{0}}{4 \pi} n_{1} B_{12} J_{\nu_{0}} \frac{A_{21}}{P_{21}} \\
& \equiv j_{\nu_{0}}^{\mathrm{a}}+j_{\nu_{0}}^{\mathrm{s}} .
\end{aligned}
$$


Source function. The source function $S_{\nu_{0}}^{\mathrm{a}}$ for the thermal sequences becomes, using all

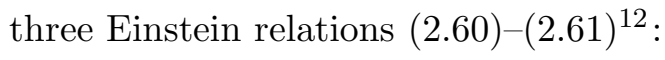

$$
\begin{aligned}
S_{\nu_{0}}^{\mathrm{a}} & \equiv j_{\nu_{0}}^{\mathrm{a}} / \alpha_{\nu_{0}}^{\mathrm{a}} \\
& =\frac{\left(h \nu_{0} / 4 \pi\right) n_{1} C_{12} A_{21} / P_{21}}{\left(h \nu_{0} / 4 \pi\right) n_{1} B_{12} C_{21}\left[1-\mathrm{e}^{-h \nu_{0} / k T}\right] / P_{21}} \\
& =\frac{A_{21}}{B_{12}} \frac{C_{12}}{C_{21}} \frac{\mathrm{e}^{h \nu_{0} / k T}}{\mathrm{e}^{h \nu_{0} / k T}-1} \\
& =\frac{2 h \nu_{0}^{3}}{c^{2}} \frac{1}{\mathrm{e}^{h \nu_{0} / k T}-1} \\
& =B_{\nu_{0}} .
\end{aligned}
$$

The source function $S_{\nu_{0}}^{\mathrm{s}}$ for the remaining scattering sequences is:

$$
\begin{aligned}
S_{\nu_{0}}^{\mathrm{s}} & \equiv j_{\nu_{0}}^{\mathrm{s}} / \alpha_{\nu_{0}}^{\mathrm{s}} \\
& =\frac{\left(h \nu_{0} / 4 \pi\right) n_{1} B_{12} J_{\nu_{0}} A_{21} / P_{21}}{\left(h \nu_{0} / 4 \pi\right) n_{1} B_{12} A_{21} / P_{21}} \\
& =J_{\nu_{0}} .
\end{aligned}
$$

The combined sharp-line source function is again given by (2.144i), using $j_{\nu_{0}}^{l}=\alpha_{\nu_{0}}^{\mathrm{a}} B_{\nu_{0}}+$ $\alpha_{\nu_{0}}^{s} J_{\nu_{0}}$ and $\varepsilon_{\nu_{0}} \equiv \alpha_{\nu_{0}}^{\mathrm{a}} /\left(\alpha_{\nu_{0}}^{\mathrm{a}}+\alpha_{\nu_{0}}^{\mathrm{s}}\right)$ :

$$
S_{\nu_{0}}^{l} \equiv \frac{j_{\nu_{0}}^{l}}{\alpha_{\nu_{0}}^{l}}=\left(1-\varepsilon_{\nu_{0}}\right) J_{\nu_{0}}+\varepsilon_{\nu_{0}} B_{\nu_{0}}
$$

Destruction probability. The profile-summed photon destruction probability for these sharp-line two-level atoms follows from $(3.87)-(3.89)$ :

$$
\begin{aligned}
\varepsilon_{\nu_{0}} & \equiv \frac{\alpha_{\nu_{0}}^{\mathrm{a}}}{\alpha_{\nu_{0}}^{\mathrm{a}}+\alpha_{\nu_{0}}^{\mathrm{s}}} \\
& =\frac{C_{21}\left[1-\exp \left(-h \nu_{0} / k T\right)\right]}{C_{21}\left[1-\exp \left(-h \nu_{0} / k T\right)\right]+A_{21}} \\
& =\frac{C_{21}}{C_{21}+A_{21} /\left[1-\exp \left(-h \nu_{0} / k T\right)\right]} \\
& =\frac{C_{21}}{C_{21}+A_{21}+B_{21} B_{\nu_{0}}} .
\end{aligned}
$$

The equality $A_{21} /\left[1-\exp \left(-h \nu_{0} / k T\right)\right]=A_{21}+B_{21} B_{\nu_{0}}$ used in the last step rewrites the increase of the spontaneous emission by the TE correction factor as thermal contribution by induced emission.

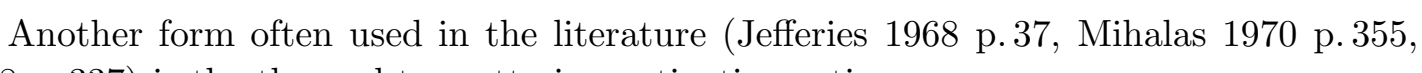
1978 p. 337) is the thermal-to-scattering extinction ratio

$$
\varepsilon_{\nu_{0}}^{\prime} \equiv \alpha_{\nu_{0}}^{\mathrm{a}} / \alpha_{\nu_{0}}^{\mathrm{s}}
$$

\footnotetext{
${ }^{12}$ This derivation produces the Wien approximation rather than $B_{\nu_{0}}$ if the stimulated emission process (e) is not included, illustrating that Einstein needed the latter to obtain the -1 in the denominator of $B_{\nu_{0}}$.
} 


$$
\begin{aligned}
& =\frac{\varepsilon_{\nu_{0}}}{1-\varepsilon_{\nu_{0}}} \\
& =\frac{C_{21}}{A_{21}}\left[1-\mathrm{e}^{-h \nu_{0} / k T}\right],
\end{aligned}
$$

with which the sharp-line source function becomes

$$
S_{\nu_{0}}^{l}=\frac{J_{\nu_{0}}+\varepsilon_{\nu_{0}}^{\prime} B_{\nu_{0}}}{1+\varepsilon_{\nu_{0}}^{\prime}}
$$

Discussion. The $(\mathrm{c})-(\mathrm{g})$ cancelation of $(\overline{3} . \overline{8} \overline{9})$ is implicitly achieved by including the stimulated emission as negative correction to the line extinction. Contribution (c) counts no longer in the volume extinction coefficient, nor does (g) count in the volume emissivity. This cancelation is desirable because, if one would instead add (c) to the extinction and (e) to the emission, the non-corrected extinction coefficient would overestimate the actual attenuation of the beam across the volume so that the optical depth scaling would not measure the actual opacity of the medium $\mathrm{m}_{1-}^{\mathrm{I}{ }_{1}{ }_{1}}$. The $(\mathrm{c})-(\mathrm{g})$ cancelation also removes the stimulated-emission sensitivity to $I_{\nu_{0}}$, leaving the source function for the remaining spontaneous scattering $S_{\nu_{0}}^{\mathrm{s}}=J_{\nu_{0}}$ isotropic.

Expression (3.94) for $S_{\nu_{0}}^{l}$ splits the production of new photons per extinction between the scattering and thermal contributions. The terms $J_{\nu_{0}}$ and $B_{\nu_{0}}$ represent the radiation and thermal reservoirs from which new photons may be obtained. Their contributions are weighted by the relative probabilities of scattering $\left(1-\varepsilon_{\nu_{0}}\right)$ and thermal interaction $\left(\varepsilon_{\nu_{0}}\right)$. The weighting factors and the thermal reservoir are locally controlled quantities, but the local photon reservoir $J_{\nu_{0}}$ has non-local sensitivity to what happens elsewhere.

Version $(\overline{3} . \overline{9})$ for the destruction probability is the standard definition seen in the literature, often in the approximation $\varepsilon_{\nu_{0}} \approx C_{21} /\left(C_{21}+A_{21}\right)$ neglecting stimulated emission (or as $\varepsilon_{\nu_{0}}^{\prime} \approx C_{21} / A_{21}$ ). The Planck function appears in the $B_{21} B_{\nu_{0}}$ term in the numerator of (13.98. scatterings in $\alpha_{\nu_{0}}^{l}$. In version (3.96i) the thermal remainder of the induced deexcitations shows up as a correction for induced photon creation applied to the photon destruction contribution $C_{21}$.

Thomson and Rayleigh scattering. Coherent Thomson and Rayleigh scattering may be seen as special sharp-line scattering, without contributions (a), (d) or (e). The classical Thomson cross-section, as derived from the harmonic oscillator, corresponds to the spontaneous scattering sequence in contribution (b). This is the right measure for the volume extinction since addition of contribution (c) would again add unwanted extinction due to the exact cancelation by contribution (g). Thus, $\alpha^{\mathrm{T}}=\sigma^{\mathrm{T}} N_{\mathrm{e}}$ and $j_{\nu}^{\mathrm{T}}=\alpha^{\mathrm{T}} J_{\nu}$, ignoring the stimulated scatterings as is indeed done in $(\overline{2} . \overline{8} \overline{1})$ and $(2 . \overline{8} \overline{3})$.

In hot-star atmospheres, where thermal Dopplershifts are substantial, the corresponding $J_{\nu}$ differences are yet small so that induced scattering may still be neglected as is always done (e.g., Rybicki and Hummer $\left.\overline{1} \overline{9} \overline{9} \overline{2}_{1}^{\prime}\right)$. Only when the photons lose their identity through large frequency shifts must the induced continuum scattering processes be included, as in the Kompaneets version of the Fokker-Planck approximation (Sect.7.6 of Rybicki and Lightman 1979

\footnotetext{
${ }^{13}$ Same-direction scattering sequences also add extinction without reducing the intensity, but their contribution vanishes in the limit $\Delta \Omega \rightarrow 0$ implied by $\mathrm{d} \Omega$ in definition ( $(2 . \overline{2} \cdot \overline{1})$.
} 


\subsubsection{Complete redistribution}

We now treat the case in which the single transition of the two-level atom is broadened, but with the simplification that the scattering obeys complete frequency redistribution over the line profile. In this case the three profile functions are still equal, having $\psi\left(\nu-\nu_{0}\right)=$ $\chi\left(\nu-\nu_{0}\right)=\varphi\left(\nu-\nu_{0}\right)=H(a, v) / \sqrt{\pi} \Delta \nu_{\mathrm{D}}$.

Two-level statistical equilibrium. The corresponding line source function $S_{\nu_{0}}^{l}$ may now be derived directly, for the transition as a whole, from the condition of statistical equilibrium for the two-level atoms:

$$
\frac{\mathrm{d} n_{2}}{\mathrm{~d} t}=n_{1} P_{12}-n_{2} P_{21}=0 .
$$

Reorder into an expression for $n_{1} / n_{2}$, substitute it into (2.73) and multiply with $g_{1} / g_{2}$ :

$$
\begin{aligned}
S_{\nu_{0}}^{l} & =\frac{2 h \nu_{0}^{3}}{c^{2}} \frac{1}{\frac{g_{2} n_{1}}{g_{1} n_{2}}-1} \\
& =\frac{\left(2 h \nu_{0}^{3} / c^{2}\right)\left[\left(g_{1} / g_{2}\right) B_{12} \bar{J}_{\nu_{0}}^{\varphi}+\left(g_{1} / g_{2}\right) C_{12}\right]}{A_{21}+B_{21} \bar{J}_{\nu_{0}}^{\varphi}+C_{21}-\left(g_{1} / g_{2}\right) B_{12} \bar{J}_{\nu_{0}}^{\varphi}-\left(g_{1} / g_{2}\right) C_{12}} .
\end{aligned}
$$

The denominator sums the extinction, again splitting it between the spontaneous, scattering and thermal sequels. The negative terms come from the -1 and represent the correction for stimulated emission, split into scattering and thermal parts. Thus, the two terms containing $\bar{J}_{\nu_{0}}^{\varphi}$ represent the stimulated-scattering parts of the extinction and of the emission, respectively. Conversion of $B_{12}$ into $B_{21}$ with (2.60) shows again that they cancel:

$$
\begin{aligned}
S_{\nu_{0}}^{l} & =\frac{\left(2 h \nu_{0}^{3} / c^{2}\right)\left[B_{21} \bar{J}_{\nu_{0}}^{\varphi}+\left(g_{1} / g_{2}\right) C_{12}\right]}{A_{21}+B_{21} \bar{J}_{\nu_{0}}^{\varphi}+C_{21}-B_{21} \bar{J}_{\nu_{0}}^{\varphi}-\left(g_{1} / g_{2}\right) C_{12}} \\
& =\frac{\left(2 h \nu_{0}^{3} / c^{2}\right)\left[B_{21} \bar{J}_{\nu_{0}}^{\varphi}+\left(g_{1} / g_{2}\right) C_{12}\right]}{A_{21}+C_{21}-\left(g_{1} / g_{2}\right) C_{12}} .
\end{aligned}
$$

Now transform $C_{12}$ into $C_{21}$, divide by $A_{21}$ and use (2.60i) for $B_{21} / A_{21}$ :

$$
\begin{aligned}
S_{\nu_{0}}^{l} & =\frac{\left(2 h \nu_{0}^{3} / c^{2}\right)\left[\left(B_{21} / A_{21}\right) \bar{J}_{\nu_{0}}^{\varphi}+\left(C_{21} / A_{21}\right) \exp \left(-h \nu_{0} / k T\right)\right]}{1+C_{21} / A_{21}-\left(C_{21} / A_{21}\right) \exp \left(-h \nu_{0} / k T\right)} \\
& =\frac{\bar{J}_{\nu_{0}}^{\varphi}+\left(C_{21} / A_{21}\right)\left(2 h \nu_{0}^{3} / c^{2}\right) \exp \left(-h \nu_{0} / k T\right)}{1+C_{21} / A_{21}-\left(C_{21} / A_{21}\right) \exp \left(-h \nu_{0} / k T\right)} .
\end{aligned}
$$

The standard expressions are now regained by defining the total destruction probabilities

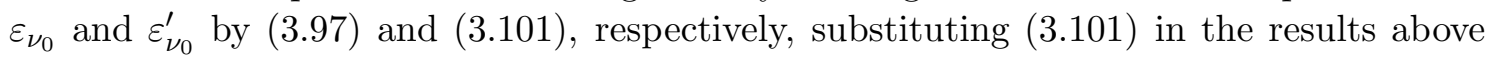
and using $\left(3.1000^{\prime}\right)$ :

$$
\begin{aligned}
S_{\nu_{0}}^{l} & =\frac{\bar{J}_{\nu_{0}}^{\varphi}+\varepsilon_{\nu_{0}}^{\prime} B_{\nu_{0}}}{1+\varepsilon_{\nu_{0}}^{\prime}} \\
& =\left(1-\varepsilon_{\nu_{0}}\right) \bar{J}_{\nu_{0}}^{\varphi}+\varepsilon_{\nu_{0}} B_{\nu_{0}} .
\end{aligned}
$$


Frequency dependence. The coherent-scattering line source function $(3.94)=(2.144)$ varies with frequency across the line, but the complete-redistribution version (3.105il) does not $_{-}^{174}$.- In the more realistic case where additional background extinction is supplied by other particles than these two-level atoms, the total source function given by

$$
S_{\nu}^{\mathrm{tot}}=\frac{\alpha_{\nu}^{l} S_{\nu_{0}}^{l}+\alpha_{\nu}^{c} S_{\nu}^{c}}{\alpha_{\nu}^{l}+\alpha_{\nu}^{c}}
$$

varies over the line profile even if $S_{\nu_{0}}^{l}, S_{\nu}^{c}$ and $\alpha_{\nu}^{c}$ do not, due to the large variation of $\alpha_{\nu}^{l}$ over the profile ${ }_{-.}^{115 !}$

Angle dependence. The resonance-scattering source functions $S_{\nu}^{l}$ and $S_{\nu_{0}}^{l}$ are isotropic since $B_{\nu}, \varepsilon_{\nu}, \varepsilon_{\nu_{0}}, J_{\nu}$ and $\bar{J}_{\nu_{0}}^{\varphi}$ are all isotropic. Even at small optical depth, where the radiation field $I_{\nu}(\mu)$ is highly anisotropic on its way out, the line source function does not vary with direction thanks to the cancelation of the stimulated scattering contributions in $\left(\overline{3} .88^{i}\right)$ and the absence of such contribution in (3.90). However, the total source function $S_{\nu}^{\text {tot }}$ is anisotropic when systematic motions cause different Doppler shifts of $\alpha^{l}$ in different directions. This may even be the case in LTE with $S_{\nu_{0}}^{l}=S_{\nu_{0}}^{c}=B_{\nu_{0}}$.

Absence of lasering. The cancelation of the stimulated scattering sequences in (3.8.8i) and in the derivation of ( $3 . \overline{1} \overline{0} \bar{i})$ also inhibits lasering. The line source functions $(\overline{3} \overline{9} \overline{4})$ and $(3.105)$ are always positive, and so are the extinction coefficients $\alpha_{\nu_{0}}^{\mathrm{a}}$ and $\alpha_{\nu_{0}}^{\mathrm{s}}$ in $(3.87 \mathrm{i})$ and $(3.89)$. The remaining stimulated emission is exclusively of thermal origin (pair (e) in Figure $3 . \overline{3}$ ), without possibility of amplification. Thus, the poor two-level atoms can't laser by themselves. That takes richer atomic structure, providing pathways without the exact up-down cancelation that results from the linkage in (3.83) between $2 \rightarrow 1$ downward processes and preceding $1 \rightarrow 2$ upward excitations of the same atom. Adding other levels (or pumping transitions of other particles) provides roundabout ways to feed electrons into the upper state that upset this one-to-one linkage, and may provide a feeding mechanism! that results in the $b_{2}>b_{1}$ overexcitation required by $\left(2 . \overline{1} \overline{1} \overline{6} \bar{\sigma}_{1}\right)$ on page $3 \overline{3} \bar{s}_{-}$to produce $S_{\nu_{0}}^{l}<\overline{0}$. Atoms with only two levels cannot build up such large overexcitation at any value of $I_{\nu_{0}}$ or $J_{\nu_{0}}$. On the contrary, the $\sqrt{\varepsilon}$ law discussed at length in Section $\overline{4} .31$ on page $\overline{9} \overline{2}$ iff says that two-level atoms tend towards $b_{2} / b_{1}=\sqrt{\varepsilon_{\nu_{0}}}$, severe underexcitation when scattering dominates, unless the Planck function increases steeply with optical depth. It is even worse for optically thin objects which have $b_{2} / b_{1} \approx \varepsilon_{\nu_{0}}$ (Wien limit) unless they are irradiated $d_{-}^{17}$.

\footnotetext{
${ }^{14}$ Unless the line is very wide; $S_{\nu_{0}}^{l}$ then varies with $\nu_{0}=\nu$ as in $\left(\overline{2} . \overline{10} 6_{i}\right)$ and Figure $\overline{2} . \overline{8}$.

${ }^{15}$ Unless $S_{\nu_{0}}^{l}=S_{\nu}^{c}$ but this is unlikely for strong lines at the height where the line-center radiation escapes.

${ }^{16}$ Solid-state lasers such as the one in your CD player overpopulate the upper level of the lasering transition through semi-conductor pumping. There isn't a wall socket in the two-level-atom gas to provide surplus electrons from power lines. Optical pumping uses an overdose of radiation in a higher-energy transition to feed the upper level. The solar Mg I $12 \mu \mathrm{m}$ lines analysed by "Carlsson et al. (1992)!' have $b_{u}>b_{l}$ due to a collision-dominated population diffusion flow from the ion state with $\bar{b}_{c} \approx \overline{1}$ down to lower levels with $b_{i}<1$. The latter underpopulations are caused by photon losses in near-infrared lines (optical suction) and by radiative overionization in ultraviolet edges (optical pumping).

${ }^{17}$ For example the solar corona. The off-limb corona that is visually observed during a total eclipse has two components: the outer $\mathrm{F}$ corona consisting of photospheric light that is scattered by micron-size interplanetary dust particles, and the inner $\mathrm{K}$ corona which is sunlight that is Thomson-scattered by coronal electrons. Equation (3.94, ) holds for both types of scattering with $S_{\nu} \approx J_{\nu}$ apart from Dopplershifts (through which the fast electrons wash out the Fraunhofer lines, as first diagnosed by Grotrian - but he
} 


\subsubsection{Partial redistribution}

So far, we have split the issue of frequency redistribution in elastic scattering into its two extremes, adopting either purely coherent scattering or complete redistribution over the line profile. The topic of frequency and angle redistribution in spectral line formation is an old and complicated one ${ }_{-}^{\prime 18 !}$ requiring much mathematics that is treated extensively by

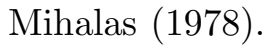

Qualitative summary. Thermal motions cause frequency redistribution over the Doppler core of the extinction profile. The scattering is coherent in the frame of the atom, but the frequencies are redistributed over the Doppler core of about $3 \Delta \nu_{\mathrm{D}}$ in the frame of the observer. It is usually assumed that the microturbulence acts the same, in keeping with its name, but this is a dangerous assumption to which the results are quite sensitive.

In the wings of the line, coherent scattering occurs when radiative damping exceeds collisional damping whereas line-broadening collisions cause frequency reshuffling. In the outer layers $\gamma^{\text {rad }}$ is set by the Einstein $A$ coefficients and can be large compared to the collisional damping which decays outward with the perturber density. In deep layers the collisional damping is usually far larger than the radiative damping. The outer line wings are formed deep, due to their small line extinction.

Thus, generally we may expect Doppler redistribution over the cores of strong resonance lines, coherent scattering in the inner wings, and collisional redistribution over the more deeply formed outer wings. In the far wings, line photons are also lost in continuum extinctions that redistribute them across the spectrum. Complete redistribution is generally a good assumption for weaker lines in stellar spectra.

Formulation. When the scattering is neither coherent nor completely redistributed ("partial redistribution"), the probability of scattering photons from one frequency to another must be accounted for. The monochromatic $S_{\nu}^{l}$ may then sensitively depend on the monochromatic radiation field $J_{\nu}$ at other frequencies in the line. Across strong spectral lines $J_{\nu}$ can vary a lot, so that cross-talk between different parts of the line profile can be very important.

Not yet...

\subsubsection{Angle redistribution}

Not yet...

\subsubsection{Spectral edge redistribution}

Bound-free scattering. Bound-free edges may suffer from scattering just like resonance lines. The analogous process combination consists of photoionization followed by radiative recombination back to the original level of the ion, producing a photon in the same spectral

didn't dare to propose the required million-degree coronal temperature then but waited until his identification of the coronal lines from Edlén's term diagrams for highly ionized iron made the high temperature inevitable). The EUV emission lines from coronal loops have $S_{\nu}^{l} \approx \varepsilon_{\nu} B_{\nu}$ because the photospheric irradiation $\left(J_{\nu} \approx B_{\nu}\left[T_{\text {eff }}\right] / 3\right)$ is negligible at EUV wavelengths while the loops are optically thin in these lines and cannot hold the thermally created photons.

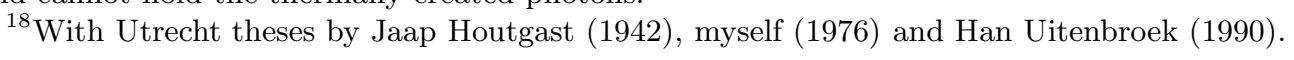


feature from which one was taken. As mentioned earlier, complete redistribution over the edge profile holds since the free electron that is caught for recombination doesn't know with what kinetic energy the previously liberated electron took off. Bound-free scattering is therefore in principle the same as redistributed bound-bound scattering 19!", sensing only $\bar{J}_{\nu_{0}}$. The differences are qualitative: the bound-free edges tend to be wider in the spectrum, they tend to have much smaller peak extinction, and recombination tends to favor pathways down through the energy level structure that differ from the way up taken by the preceding ionization.

One-level-plus-continuum atoms. I describe such bound-free scattering sequences through analogy with the two-level atom description of Section $\overline{3} . \overline{4} . \overline{2}$ by assuming a medium of one-level-plus-continuum atoms. The bound-free counterpart to (3.98), describing bound-free photon destruction per bound-free extinction by subsequent three-body recombination, is then obtained by writing $\varepsilon_{\nu_{0}}^{\mathrm{bf}}$ as the collisional fraction of all recombination rates back to the single level:

$$
\varepsilon_{\nu_{0}}^{\mathrm{bf}}=\frac{C_{c i}}{C_{c i}+R_{c i}^{\mathrm{spon}}+\left[R_{c i}^{\mathrm{ind}}\right]_{\mathrm{LTE}}}
$$

where the collisional recombination rate per ion $C_{c i}$ is given by $(\overline{3} \cdot \overline{3} \bar{i})$ on page $\overline{5} \overline{1}$, , the spontaneous rate $R_{c i}^{\text {spon }}$ by (3.13) on page $\overline{4} \overline{7}_{v}^{\prime}$ and the thermally induced rate $\left[R_{c i}^{\text {ind }}\right]_{\text {LTE }}$ by (1).15i) but with $B_{\nu}$ instead of $J_{\nu}$ under the integral. Defining the edge-averaged quantities:

$$
\bar{S}_{\nu_{0}}^{\sigma} \equiv \frac{\int_{\nu_{0}}^{\infty} S_{\nu} \sigma_{i c}(\nu) \mathrm{d} \nu}{\int_{\nu_{0}}^{\infty} \sigma_{i c}(\nu) \mathrm{d} \nu} \quad \bar{J}_{\nu_{0}}^{\sigma} \equiv \frac{\int_{\nu_{0}}^{\infty} J_{\nu} \sigma_{i c}(\nu) \mathrm{d} \nu}{\int_{\nu_{0}}^{\infty} \sigma_{i c}(\nu) \mathrm{d} \nu} \quad \bar{B}_{\nu_{0}}^{\sigma} \equiv \frac{\int_{\nu_{0}}^{\infty} B_{\nu} \sigma_{i c}(\nu) \mathrm{d} \nu}{\int_{\nu_{0}}^{\infty} \sigma_{i c}(\nu) \mathrm{d} \nu}
$$

with $\nu_{0}$ the bound-free threshold frequency then yields as bound-free analogon to (3.105i) for scattering in a gas of one-level-plus-continuum atoms:

$$
\bar{S}_{\nu_{0}}^{\sigma}=\left(1-\varepsilon_{\nu_{0}}^{\mathrm{bf}}\right) \bar{J}_{\nu_{0}}^{\sigma}+\varepsilon_{\nu_{0}}^{\mathrm{bf}} \bar{B}_{\nu_{0}}^{\sigma} .
$$

The frequency variation of the monochromatic source function $S_{\nu}^{\text {bf }}$ is given by (2.117i) on page $3 \overline{5}_{1}^{1}$ and is Planckian in the Wien regime ${ }_{-}^{27 !}$

\footnotetext{
${ }^{19}$ In bound-bound scattering the excited atom must deexcite radiatively before a deexciting collision thermalizes its memory of its prior radiative excitation. Resonance lines with large $A_{u l}$ have brief decay times and are therefore prone to scattering. In radiative recombination the ion has to wait for a passing electron that it can capture, but collisional recombination requires a three-body collision which may take much longer yet.

${ }^{20}$ The quantities $S_{\nu_{0}}$ and $B_{\nu_{0}}$ in $\left({ }_{3} .105_{1}\right)$ are similarly averaged over $\varphi\left(\nu-\nu_{0}\right)$, but for symmetric and narrow $\varphi\left(\nu-\nu_{0}\right)$ profiles they equal the line-center values $S\left(\nu=\nu_{0}\right)$ and $B\left(\nu=\nu_{0}\right)$. The bound-free averages, however, do not equal the values at the threshold frequency.

${ }^{21}$ But in the Rayleigh-Jeans regime the one-level-plus-continuum gas cannot laser, just as a two-level gas_cannot laser. The buildup of $b_{c} / b_{i}>1$ overionization that would produce bound-free lasering in $\left(2.117_{1}\right)$ on page 35 requires additional pathways through the term structure or through other particles to upset the cancelation of stimulated scattering extinction and emission inherent in (3.109.1). In essence, this is the same result as derived for synchrotron emission and absorption by Rybicki and Lightmant '(1979)' in their Section 6.9 "The impossibility of a synchrotron maser in vacuum", where "vacuum" stands for gases with refractive index unity as the ones discussed here. Synchrotron continua are ensembles of discrete transitions that represent higher harmonics of the cyclotron frequency and overlap in energy. Photon creation and destruction occur by exchange of kinetic Larmor energy rather than kinetic thermal energy, but the interactions are again spontaneous, induced, and collisional, and they resemble bound-free
} 
Discussion. The destruction probability increases with the electron density both for two-level atoms and for one-level-plus-continuum atoms. Scattering is therefore most important for lines and edges with large opacity so that the representative $\tau=1$ escape depth is high up in the atmosphere, at low density. Recombination edges have opacities that are comparable to resonance lines when integrated over the feature (see the evaluation for Ly $\alpha$ and the Lyman continuum in Section $2 . \overline{4} . \overline{1}_{1}^{\prime}$ on page $\left.2 \overline{2} \overline{5}_{1}^{\prime}\right)$, but the lines reach much higher peak opacity.

For actual multi-level atoms the collisional recombination probability $C_{c i}$ is larger for

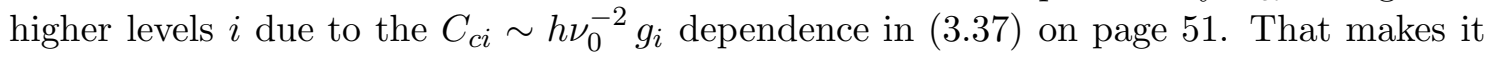
likelier that photionizations from a low level are followed by three-body recombination into a high level and subsequent cascade (collisional or radiative) through the term diagram than that photoionizations are followed by direct three-body recombination back to the low level. Radiative recombination into higher levels plus subsequent cascade (as in the Zanstra mechanism for planetary nebulae) also represents a photon loss term that is not included in the simple one-level-plus-continuum description. Nevertheless, the $S_{\nu} \approx J_{\nu}$ behavior of the major ultraviolet and EUV edges portrayed in Figures 8.10 and 8.11 on pages $1 \overline{1} \overline{1}-1 \overline{1} \overline{6} \overline{6}_{1}^{1}$ demonstrates that resonance scattering affects the bound-free continua of the VÁLIII atmosphere much as described by $(3.109)$ with small $\varepsilon_{\nu_{0}}^{\mathrm{bf}}$.

emission and extinction with $\nu_{0}=e|\vec{B}| / 2 \pi m_{\mathrm{e}}$ the cyclotron frequency. The synchrotron source function may be expressed in an equation similar to (3.109i), with the Planck function replaced by the boson equivalent of the non-Maxwellian kinetic energy distribution (proportional to $\nu^{2}\langle E\rangle \sim \nu^{-5 / 2}$ for a power law distribution $N(E) \mathrm{d} E \sim E^{-p} \mathrm{~d} E$ ). Synchrotron photon destruction occurs when an electron carrying Larmor-energy memory of a photonic interaction collides with other particles and so returns to the kinetic energy distribution before releasing that memory as another photon. Synchrotron radiation from optically thick media may therefore be affected by scattering, while lasering is again inhibited by the absence of external states (or wall sockets) that might provide uppel-level population to feed noncanceling stimulated scattering. By the way, the same holds for nonthermal free-free scattering (footnote on page ' ${ }^{4} 6_{i}$ ). When the Maxwell distribution holds, which is asumed throughout these lecture notes, $\varepsilon_{\nu}^{\mathrm{ff}} B_{\nu}=\bar{B}_{\nu}$ since every new free-free photon samples the thermal distribution, but if nonthermal energetic radiation would take a non-negligible role in the kinetic energy partitioning and impart a kinetic memory to photon-absorbing electrons, the free-free source function may also be written as (i.109i) with another boson energy distribution than the Planck function and with $\varepsilon_{\nu}$ measuring the relative occurrence of non-radiative collisional interactions. 


\section{Chapter 4}

\section{Analytical Radiative Transfer}

I $\mathrm{n}$ this chapter the formalisms of radiative transfer are expanded for the special case of optically thick objects with axial symmetry, i.e., for "plane-parallel" stellar interiors and stellar photospheres. There is a large literature on this subject, often of mathematical nature, which concentrated on analytical approximations during the first half of the twentieth century. This chapter represents a very condensed summary.

\subsection{Formal solutions}

We limit the discussion of the transport equation and its formal solutions here to the case of plane-parallel geometry with an isotropic source function. The restriction to static planeparallel geometry is an idealization to which actual stellar atmospheres do not adhere?! However, although the principles of stellar radiative transfer are easiest formulated for plane-parallel static conditions, they hold more generally and are readily applicable in much wider context.

\subsubsection{General transport equation}

The general transport equation along a ray is (iMihalas $19 \overline{9} \overline{8}_{1}^{\prime}$ p. 30;

$$
\frac{\mathrm{d} I_{\nu}}{\mathrm{d} s}=\frac{\partial I_{\nu}}{\partial t} \frac{\mathrm{d} t}{\mathrm{~d} s}+\frac{\partial I_{\nu}}{\partial s}=\frac{1}{c} \frac{\partial I_{\nu}}{\partial t}+\frac{\partial I_{\nu}}{\partial s}=j_{\nu}-\alpha_{\nu} I_{\nu}
$$

with $s$ the geometrical path along a ray and

$$
\frac{\partial I_{\nu}}{\partial s}=\frac{\partial I_{\nu}}{\partial x} \frac{\mathrm{d} x}{\mathrm{~d} s}+\frac{\partial I_{\nu}}{\partial y} \frac{\mathrm{d} y}{\mathrm{~d} s}+\frac{\partial I_{\nu}}{\partial z} \frac{\mathrm{d} z}{\mathrm{~d} s} .
$$

Spherical geometry. Assuming time independence (steady state) so that $\partial I_{\nu} / \partial t=0$, adopting polar coordinates with $\mathrm{d} r=\cos \theta \mathrm{d} s$ and $r \mathrm{~d} \theta=-\sin \theta \mathrm{d} s$ and taking a spherical star without azimuthal $(\varphi)$ intensity variations so that $\partial I_{\nu} / \partial \varphi=0$, the transport equation becomes (Mīihāas 1978' p. 33; Gray p. 113):

$$
\frac{\partial I_{\nu}}{\partial s}=\cos \theta \frac{\partial I_{\nu}}{\partial r}-\frac{\sin \theta}{r} \frac{\partial I_{\nu}}{\partial \theta}=\mu \frac{\partial I_{\nu}}{\partial r}+\frac{1-\mu^{2}}{r} \frac{\partial I_{\nu}}{\partial \mu}=j_{\nu}-\alpha_{\nu} I_{\nu}
$$

\footnotetext{
${ }^{1}$ Rather, they abound in fine structure to an astonishing degree. The sudden transition from near-TE confinement in the stellar interior to open-ended interstellar emptiness represents a pathological chasm in order and entropy to which stars adapt with sufficient complexity to keep you and me busy for years to come.
} 
and with $S_{\nu} \equiv j_{\nu} / \alpha_{\nu}$

$$
\frac{\mu}{\kappa_{\nu} \rho} \frac{\partial I_{\nu}}{\partial r}+\frac{1-\mu^{2}}{\kappa_{\nu} \rho r} \frac{\partial I_{\nu}}{\partial \mu}=S_{\nu}-I_{\nu}
$$

This version must be used for stars with extended atmospheres.

Plane-parallel geometry. Most stellar photospheres are sufficiently thin compared with the stellar radius (see $(\overline{7} . \overline{2} \overline{3})$ on page $\left.1 \overline{4} \overline{4} \overline{7}_{1}\right)$ that the plane-parallel approximation $\partial \theta / \partial r \approx 0$ holds (apart from inhomogeneities). The second term of the lefthand side then vanishes, giving the standard form with $\mathrm{d} \tau_{\nu}=-\kappa_{\nu} \rho \mathrm{d} r$ measuring radial optical depth:

$$
\mu \frac{\mathrm{d} I_{\nu}}{\mathrm{d} \tau_{\nu}}=I_{\nu}-S_{\nu}
$$

More transport equations. The assumption that $S_{\nu}$ is isotropicil, delivers variations of the transport equation expressed in the moments of $I_{\nu}$ defined by $(2.13 i)-(2.15 i)$. The first one is obtained by angle averaging $\left[(1 / 4 \pi) \int \mathrm{d} \Omega=(1 / 2) \int \mathrm{d} \mu\right]$ both sides of $(1, .51)$ :

$$
\begin{aligned}
\frac{1}{2} \int_{-1}^{+1} \mu \frac{\mathrm{d} I_{\nu}}{\mathrm{d} \tau_{\nu}} \mathrm{d} \mu & =\frac{1}{2} \int_{-1}^{+1} I_{\nu} \mathrm{d} \mu-\frac{1}{2} \int_{-1}^{+1} S_{\nu} \mathrm{d} \mu \\
\frac{\mathrm{d} H_{\nu}\left(\tau_{\nu}\right)}{\mathrm{d} \tau_{\nu}} & =J_{\nu}\left(\tau_{\nu}\right)-S_{\nu}\left(\tau_{\nu}\right)
\end{aligned}
$$

or

$$
-\frac{\mathrm{d} H_{\nu}(z)}{\mathrm{d} z}=\kappa_{\nu} \rho J_{\nu}(z)-\kappa_{\nu} \rho S_{\nu}(z)
$$

which says that flux divergence equals the difference between angle-averaged emitted en-

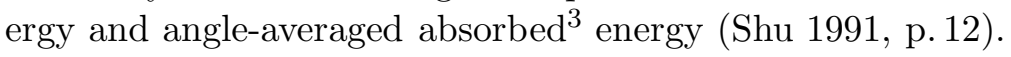

A second version follows from multiplying both sides of (14.5in by $\mu$ before the angle averaging $\left[(1 / 4 \pi) \int \mu \mathrm{d} \Omega=(1 / 2) \int \mu \mathrm{d} \mu\right]$ :

$$
\begin{aligned}
\frac{1}{2} \int_{-1}^{+1} \mu^{2} \frac{\mathrm{d} I_{\nu}}{\mathrm{d} \tau_{\nu}} \mathrm{d} \mu & =\frac{1}{2} \int_{-1}^{+1} \mu I_{\nu} \mathrm{d} \mu-\frac{1}{2} \int_{-1}^{+1} \mu S_{\nu} \mathrm{d} \mu \\
\frac{\mathrm{d} K_{\nu}\left(\tau_{\nu}\right)}{\mathrm{d} \tau_{\nu}} & =H_{\nu}\left(\tau_{\nu}\right)
\end{aligned}
$$

since $\int_{-1}^{+1} \mu S_{\nu} \mathrm{d} \mu=0$ for isotropic $S_{\nu}$. Further left and right differentiation gives, with (4)

$$
\frac{\mathrm{d}^{2} K_{\nu}\left(\tau_{\nu}\right)}{\mathrm{d} \tau_{\nu}^{2}}=J_{\nu}\left(\tau_{\nu}\right)-S_{\nu}\left(\tau_{\nu}\right)
$$

The $K$ integral represents photon pressure in which a gradient produces a photon flux; the second derivative measures the flux divergence.

\footnotetext{
${ }^{2}$ Isotropy of $S_{\nu}$ does not require that $I_{\nu}$ is isotropic, see page ${ }^{\prime} \overline{7} \bar{l}_{1}^{\prime}$.

3 "Absorbed" is wrong terminology; $\kappa_{\nu}$ describes extinction, whēther due to scattering, photon conversion, or "true" absorption. I should use "extinguished" rather than "absorbed". I use "extincted" as if it were an existing verb. Perhaps it will exist if you use it too.
} 
Discussion. Rewriting the transport equations into these higher-moment versions does not imply that the basic problem $\left(I_{\nu}\right.$ is needed to obtain $J_{\nu} ; J_{\nu}$ is needed to obtain $I_{\nu}$ unless LTE holds) goes away. Shu (1991), compares these higher-moment transport equations to Fourier decomposition in which only the infinite series contains all the information present in the original function. Thus, a general angular expansion of the transport equation should have an infinite number of terms in $\mu$ and also in the azimuthal angle $\varphi$, i.e., expansion in spherical harmonics $Y_{l m}(\theta, \varphi)$. The lower moments of $I_{\nu}$ in (2.13 2.15) are so often used in the literature because they function as the first few Fourier coefficients in frequency analysis, containing basic properties while ignoring the finer-structure details given by higher terms (high-frequency "noise").

The basic simplification assumed throughout these lecture notes (except the final chapter) is that of static plane-parallel geometry. The static part does away with time dependence and guarantees source function isotropy (page $\left.\underline{n}_{1}^{1} \underline{1}_{1}^{\prime}\right)$. The plane-parallel part does away with spherical geometry and with lateral inhomogeneity. The latter assumption is a very basic one because it limits the non-localness of the radiative transfer problem to only one dimension: just the variation with depth. The actual source function along the line of sight is non-locally influenced by the local radiation field $d_{-1}^{t_{1}}$, sensing the different intensities in different directions that together make up the local value of $J_{\nu}$. In the one-dimensional case $I_{\nu}$ may be found by solving (4.5i) per $\mu$ direction, or the intensity evaluation may be skipped by finding $J_{\nu}$ directly from (4. ity, the intensities at any location and in any direction depend on what happens in the surrounding volume, at least over the extent of the effective mean free photon path at any relevant frequency.

\subsubsection{Exponential integrals}

Formal solution. With the boundary conditions $S_{\nu}\left(\tau_{\nu}\right) \exp \left(-\tau_{\nu}\right) \rightarrow 0$ for $\tau_{\nu} \rightarrow \infty$ (always the case, see Gray p. 114, Mihalas 1978 p. 37, Kourganoff 1952 p. 27) and $I_{\nu}^{-}(0, \mu)=0$ (no irradiation from outside) the formal solution of the transfer equation is given by (Eqs. $2 . \overline{1} \cdot \overline{2} \cdot \overline{2} ;$, Gray p. 114):

$$
\begin{aligned}
& I_{\nu}^{+}\left(\tau_{\nu}, \mu\right)=+\int_{\tau_{\nu}}^{\infty} S_{\nu}\left(t_{\nu}\right) \mathrm{e}^{-\left(t_{\nu}-\tau_{\nu}\right) / \mu} \mathrm{d} t_{\nu} / \mu \\
& I_{\nu}^{-}\left(\tau_{\nu}, \mu\right)=+\int_{0}^{\tau_{\nu}} S_{\nu}\left(t_{\nu}\right) \mathrm{e}^{-\left(t_{\nu}-\tau_{\nu}\right) / \mu} \mathrm{d} t_{\nu} /|\mu|,
\end{aligned}
$$

saying that intensity measures the source function as weighted exponentially by $\exp \left(-t_{\nu} / \mu\right)$ along the beam up to the location of interest. Note that $\mu<0$ in the second equation.

Exponential integrals. Similar expressions follow for the moments of $I_{\nu}$ by assuming $S_{\nu}$ isotropic, with some non-exam algebra. Write

$$
\begin{aligned}
& \int_{-1}^{+1} I_{\nu}\left(\tau_{\nu}, \mu\right) \mu^{n} \mathrm{~d} \mu \\
& =\int_{0}^{+1} \mu^{n} \mathrm{~d} \mu \int_{\tau_{\nu}}^{\infty} S_{\nu}\left(t_{\nu}\right) \mathrm{e}^{-\left(t_{\nu}-\tau_{\nu}\right) / \mu} \frac{\mathrm{d} t_{\nu}}{\mu}+\int_{-1}^{0} \mu^{n} \mathrm{~d} \mu \int_{0}^{\tau_{\nu}} S_{\nu}\left(t_{\nu}\right) \mathrm{e}^{-\left(\tau_{\nu}-t_{\nu}\right) /-\mu} \frac{\mathrm{d} t_{\nu}}{-\mu},
\end{aligned}
$$

\footnotetext{
${ }^{4}$ Not in the trivial LTE case, but that is not what these lecture notes are about. The non-localness includes the sensitivity of $S_{\nu}$ to $J_{\nu}$ at other frequencies except in the case of coherent scattering.
} 
substitute $1 / \mu=w$ in the first term on the righthand side, with $|\mathrm{d} \mu / \mathrm{d} w|=\mu / w, \mu^{n}=$ $1 / w^{n}, \mu \rightarrow 0$ giving $w \rightarrow \infty$ and $w=1$ for $\mu=1$. Similarly, substitute $-1 / \mu=w$ in the second term on the righthand side, with $|\mathrm{d} \mu / \mathrm{d} w|=-\mu / w, \mu^{n}=(-1)^{n} / w^{n}, w=1$ for $\mu=-1$, and $\mu=0$ giving $w \rightarrow \infty$. Exchanging the order of integration then produces:

$$
\begin{aligned}
& \int_{-1}^{+1} I_{\nu}\left(\tau_{\nu}, \mu\right) \mu^{n} \mathrm{~d} \mu \\
& =\int_{\tau_{\nu}}^{\infty} S_{\nu}\left(t_{\nu}\right) \mathrm{d} t_{\nu} \int_{\infty}^{1} \frac{\mathrm{e}^{-\left(t_{\nu}-\tau_{\nu}\right) w}}{w^{n+1}} \mathrm{~d} w+(-1)^{n} \int_{0}^{\tau_{\nu}} S_{\nu}\left(t_{\nu}\right) \mathrm{d} t_{\nu} \int_{+1}^{\infty} \frac{\mathrm{e}^{-\left(\tau_{\nu}-t_{\nu}\right) w}}{w^{n+1}} \mathrm{~d} w \\
& =\int_{\tau_{\nu}}^{\infty} S_{\nu}\left(t_{\nu}\right) E_{n+1}\left(t_{\nu}-\tau_{\nu}\right) \mathrm{d} t_{\nu}+(-1)^{n} \int_{0}^{\tau_{\nu}} S_{\nu}\left(t_{\nu}\right) E_{n+1}\left(\tau_{\nu}-t_{\nu}\right) \mathrm{d} t_{\nu},
\end{aligned}
$$

where the exponential integrals $E_{n}\left(t_{\nu}\right)$ are defined by

$$
E_{n}(x) \equiv \int_{1}^{\infty} \frac{\mathrm{e}^{-x w}}{w^{n}} \mathrm{~d} w=\int_{0}^{1} \mathrm{e}^{-x / \mu} \mu^{n-1} \frac{\mathrm{d} \mu}{\mu}
$$

with $n=1,2, \ldots$ and $x>0$. Properties of these integrals are described in Appendix 1

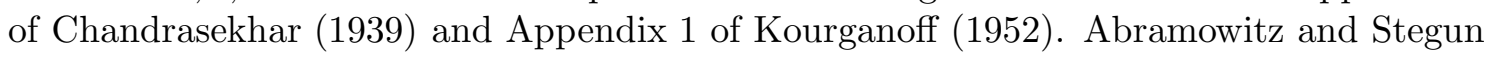
- (1964) specify useful polynomial fits for $E_{1}(x)$ from which $E_{n}(x)$ with higher $n$ can be found with a recursion formula; they are also given by Gray (p.118). A sampling is given in Table $\overline{4}$ and Figure because $\mu \leq 1$. The asymptotic value for $x \gg 1$ is

$$
E_{n}(x)=\frac{\mathrm{e}^{-x}}{x}\left[1-\frac{n}{x}+\frac{n(n+1)}{x^{2}}+\cdots\right]
$$

so that all exponential integrals obey $E_{n} \approx(1 / x) \exp (-x)$ at large $x$.

\begin{tabular}{lllllll}
\hline$x$ & $\mathrm{e}^{-x}$ & $E_{1}(x)$ & $E_{2}(x)$ & $E_{3}(x)$ & $E_{4}(x)$ & $E_{n}(x)$ \\
\hline 0 & 1 & $\infty$ & 1.0000 & 0.5000 & $1 / 3$ & $1 /(n-1)$ \\
0.01 & 0.9900 & 4.0379 & 0.9497 & 0.4903 & 0.3284 & \\
0.1 & 0.9048 & 1.8229 & 0.7225 & 0.4163 & 0.2877 & \\
0.5 & 0.6065 & 0.5598 & 0.3266 & 0.2216 & 0.1652 & \\
1 & 0.3679 & 0.2194 & 0.1485 & 0.1097 & 0.0860 & \\
3 & 0.0498 & 0.0131 & 0.0106 & 0.0089 & 0.0077 & \\
\hline
\end{tabular}

Table 4.1: Exponential_integrals. Note that $E_{n}(0)=1 /(n-1) . E_{1}(x)$ has a logarithmic singularity at $x=0$ (p. 374 of Chandrasekhar 1939').

Schwarzschild-Milne equations. The moment equations (2.13i)-(2.15i) are rewritten with exponential integrals into the Schwarzschild equation for the mean intensity

$$
\begin{aligned}
J_{\nu}\left(\tau_{\nu}\right) & \equiv \frac{1}{2} \int_{-1}^{+1} I_{\nu}\left(\tau_{\nu}, \mu\right) \mathrm{d} \mu \\
& =\frac{1}{2} \int_{\tau_{\nu}}^{\infty} S_{\nu}\left(t_{\nu}\right) E_{1}\left(t_{\nu}-\tau_{\nu}\right) \mathrm{d} t_{\nu}+\frac{1}{2} \int_{0}^{\tau_{\nu}} S_{\nu}\left(t_{\nu}\right) E_{1}\left(\tau_{\nu}-t_{\nu}\right) \mathrm{d} t_{\nu} \\
& =\frac{1}{2} \int_{0}^{\infty} S_{\nu}\left(t_{\nu}\right) E_{1}\left(\left|t_{\nu}-\tau_{\nu}\right|\right) \mathrm{d} t_{\nu}
\end{aligned}
$$




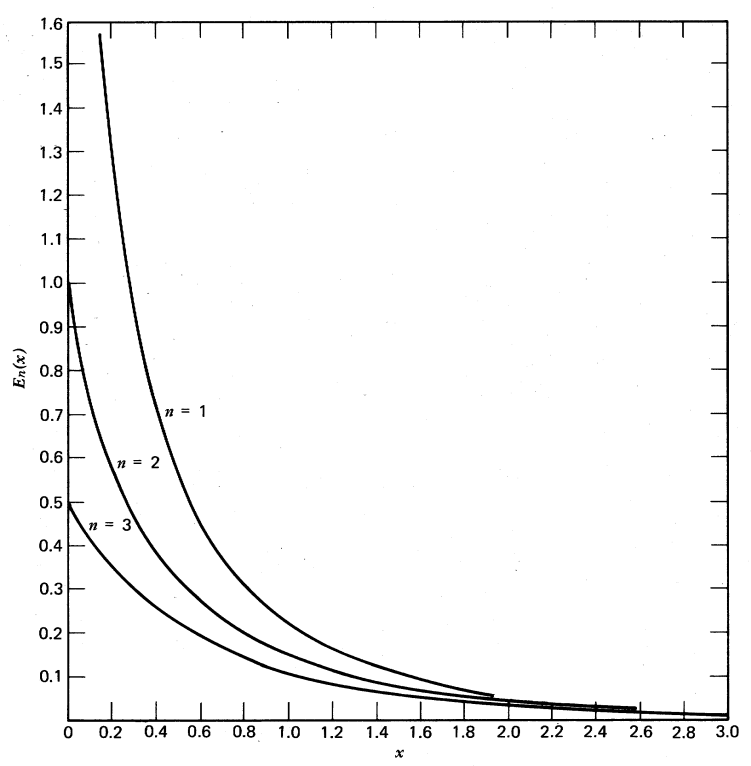

Figure 4.1: The first three exponential integrals $\underline{E}_{\underline{n}}(x) . E_{1}(x)$ has a singularity at $x=0$. For large $x$ all $E_{n}(x)$ have $E_{n}(x) \approx \exp (-x) / x$. From 'Gray $(1992) !$ '.

the Milne equation for flux

$$
\begin{aligned}
\mathcal{F}_{\nu}\left(\tau_{\nu}\right) & =\mathcal{F}_{\nu}^{+}\left(\tau_{\nu}\right)-\mathcal{F}_{\nu}^{-}\left(\tau_{\nu}\right) \\
& =2 \pi \int_{0}^{1} \mu I_{\nu}\left(\tau_{\nu}\right) \mathrm{d} \mu-2 \pi \int_{0}^{-1} \mu I_{\nu}\left(\tau_{\nu}\right) \mathrm{d} \mu \\
& =2 \pi \int_{\tau_{\nu}}^{\infty} S_{\nu}\left(t_{\nu}\right) E_{2}\left(t_{\nu}-\tau_{\nu}\right) \mathrm{d} t_{\nu}-2 \pi \int_{0}^{\tau_{\nu}} S_{\nu}\left(t_{\nu}\right) E_{2}\left(\tau_{\nu}-t_{\nu}\right) \mathrm{d} t_{\nu}
\end{aligned}
$$

and for the $K$ integral

$$
K_{\nu}\left(\tau_{\nu}\right)=\frac{1}{2} \int_{0}^{\infty} S_{\nu}\left(t_{\nu}\right) E_{3}\left(\left|t_{\nu}-\tau_{\nu}\right|\right) \mathrm{d} t_{\nu}
$$

Gray (p. 115) obtains (4.15) by writing out the transformation with $(4.12 i)$ for $\mathcal{F}_{\nu}$ in detail.

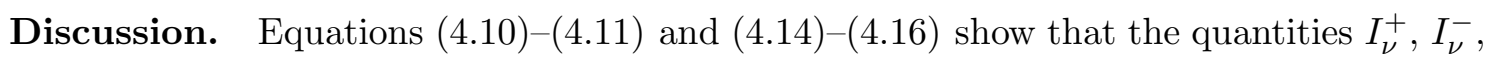
$J_{\nu}, \mathcal{F}_{\nu}^{+}, \mathcal{F}_{\nu}^{-}$and $K_{\nu}$ all represent depth-weighted samplings of the source function $S_{\nu}$, with the weighting extending over a region around (or below or above) the depth of interest $\tau_{\nu}$ and with differences in the steepness of the weighting functions. The flux $\mathcal{F}_{\nu}\left(\tau_{\nu}\right)$ measures the difference between the contributions from below and above, whereas $J_{\nu}$ and $K_{\nu}$ average them. $I_{\nu}^{+}$and $I_{\nu}^{-}$sample the contribution from below and from above separately, slanted along the line of sight.

Figure 14.21 illustrates the effect of the weighting of $S_{\nu}$ by $E_{1}\left(\left|t_{\nu}-\tau_{\nu}\right|\right)$ in (4.14.14). It makes $J_{\nu}$ depart from $S_{\nu}$ near the surface of the atmosphere by sensing both the absence of incoming photons from outside and the size of the compensating photon supply from the deeper layers. The bottom panels show that $J_{\nu}$ exceeds $S_{\nu}$ at the surface when $S_{\nu}$ increases steeply with depth but drops below $S$ for flatter gradients $\mathrm{d} B_{\nu} / \mathrm{d} \tau_{\nu}$. Figure illustrates the subtraction of the two $E_{2}\left(\tau_{\nu}-t_{\nu}\right)$ weightings in the Milne equation (4.15i 

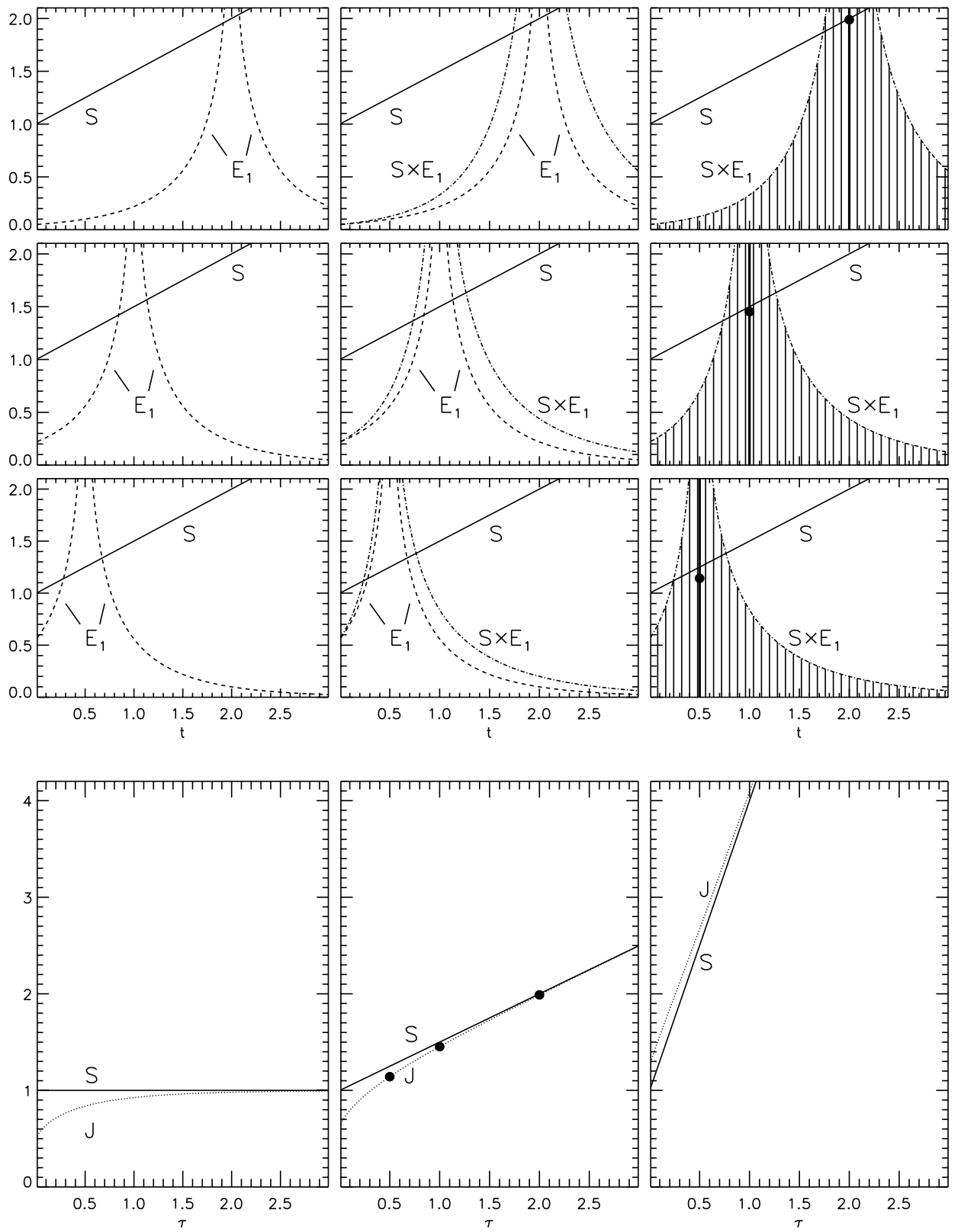

Figure 4.2: The working of the Schwarzschild equation (4.14). The upper part illustrates the non-local weighting of $S=1+0.5 t$ by the $E_{1}(|t-\tau|)$ kernel. Lefthand column: $S$ and $E_{1}(|t-\tau|)$ for $\tau=2.0$ (top), $\tau=1.0$ (middle) and $\tau=0.5$ (bottom). Middle column: corresponding integrands $S(t) \times E_{1}(|t-\tau|)$. Righthand column: The value of $J$ at $t=\tau$ (black dot) is obtained by integration (shading) over the leftand righthand integrands and taking their average (summation and division by 2). The lefthand integrand tails are cut off at the $t=0$ surface, while the righthand integrands gain larger amplitude from the inward increase of $S$ (their tails extend beyond the figure limit to $t=\infty$ ). At sufficiently large depth $\tau$ the surface cutoff does not affect the lefthand integrand so that $J(t=\tau) \approx S(t=\tau)$ for linear $S(t)$ dependence. The lower part shows the behavior of $J$ for three different source function steepnesses $\mathrm{d} S / \mathrm{d} \tau$. Lefthand panel: homogeneous plane-parallel atmosphere with depth-independent source function (Lambert radiator). The mean intensity drops to $J=0.5 S$ at the surface. Middle panel: $\mathrm{d} S / \mathrm{d} \tau=0.5$ as in the upper three rows, with the same dots. The integrand cutoff at the surface wins yet from the inward $S$ increase so that $J<S$ at the surface. Righthand panel: steeper inward increase $(\mathrm{d} S / \mathrm{d} \tau=3)$ produces $J>S$ at the surface. More examples in Figure $\overline{4} \cdot{ }_{4}^{4}$. Thijs Krijger production. 


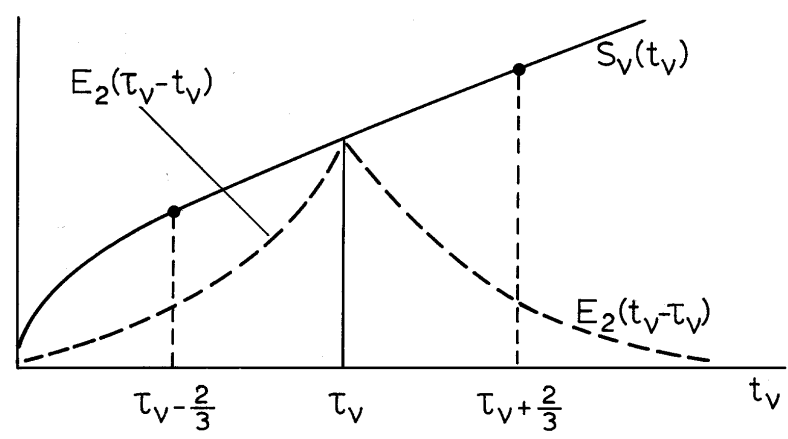

Figure 4.3: The working of the Milne equation $\left(\overline{4} . \overline{1} \overline{5}_{1}^{\prime}\right)$. The flux $\mathcal{F}_{\nu}$ represents the local difference between outward and inward source function variations, weighted by symmetrical faster-than-exponential decay functions $E_{2}$. The difference with Figure 'A.2', apart from the steeper $E_{2}$ decay, is that the lefthand integrand is now subtracted from the righthand one. At sufficiently large depth $\mathcal{F}_{\nu}$ becomes constant for linear $S_{\nu}\left(t_{\nu}\right)$ dependence. The $t_{\nu}=\tau_{\nu} \pm 2 / 3$ locations mark representative Eddington-Barbier locations at which $S_{\nu}$ characterizes the net outward and net inward flux at $t_{\nu}=\tau_{\nu}$ (Section $4.2 .1{ }^{1}$ ). Examples in Figure $4.5_{r}^{\prime}$ From Żwaan $(1993) i$

Surface values. In particular, the emergent intensity and flux at the stellar surface are:

$$
\begin{aligned}
I_{\nu}^{+}(0, \mu) & =\int_{0}^{\infty} S_{\nu}\left(\tau_{\nu}\right) \mathrm{e}^{-\tau_{\nu} / \mu} \mathrm{d} \tau_{\nu} / \mu \\
\mathcal{F}_{\nu}^{+}(0) & =2 \pi \int_{0}^{\infty} S_{\nu}\left(\tau_{\nu}\right) E_{2}\left(\tau_{\nu}\right) \mathrm{d} \tau_{\nu} .
\end{aligned}
$$

The latter result holds only when $S_{\nu}$ is isotropic; when that is not the case the solution for $I_{\nu}(0, \mu)$ must be replaced by similar equations for $I_{\nu}(0, \theta, \varphi)$ and then be integrated over the apparent stellar disk. The same must be done when $S_{\nu}\left(\tau_{\nu}\right)$ differs between different surface locations, so that one cannot simply equate the observed total flux from the star to $4 \pi R^{2} \mathcal{F}_{\nu}(0)$ to obtain the observed irradiance from $\left(\overline{2} . \overline{2}^{\prime}\right)$.

\subsubsection{Operators}

Equations (4.14)-(4.1) are of so much use that each has been converted into operator form. Equation (

$$
\mathcal{L}_{1 / \mu}\left[S_{\nu}\left(\tau_{\nu}\right)\right] \equiv \int_{0}^{\infty} S_{\nu}\left(\tau_{\nu}\right) \mathrm{e}^{-\tau_{\nu} / \mu} \mathrm{d} \tau_{\nu} / \mu=I_{\nu}^{+}(0, \mu) .
$$

Classical Lambda operator. The classical Lambda operator $\boldsymbol{\Lambda}_{\tau}$ is defined by the righthand side of the Schwarzschild equation (4.14):

$$
\boldsymbol{\Lambda}_{\tau}[f(t)] \equiv \frac{1}{2} \int_{0}^{\infty} f(t) E_{1}(|t-\tau|) \mathrm{d} t .
$$

A few properties (Chapter 2 of

$$
\begin{aligned}
& \boldsymbol{\Lambda}_{\tau}[1]=1-\frac{1}{2} E_{2}(\tau) \\
& \boldsymbol{\Lambda}_{\tau}[t]=\tau+\frac{1}{2} E_{3}(\tau)
\end{aligned}
$$




$$
\begin{aligned}
& \boldsymbol{\Lambda}_{\tau}\left[t^{2}\right]=\frac{2}{3}+\tau^{2}-E_{4}(\tau) \\
& \boldsymbol{\Lambda}_{\tau}\left[t^{p}\right]=\frac{1}{2} p !\left[\sum_{k=0}^{p} \frac{\tau^{k}}{k !} \delta_{\alpha}+(-1)^{p+1} E_{p+2}(\tau)\right]
\end{aligned}
$$

with $\delta_{\alpha}=0$ for even $\alpha \equiv p+1-k$ and $\delta_{\alpha}=2 / \alpha$ for odd $\alpha$.

With the Lambda operator the Schwarzschild equation becomes:

$$
J_{\nu}\left(\tau_{\nu}\right)=\frac{1}{2} \int_{0}^{\infty} S_{\nu}\left(t_{\nu}\right) E_{1}\left(\left|t_{\nu}-\tau_{\nu}\right|\right) \mathrm{d} t_{\nu}=\boldsymbol{\Lambda}_{\tau_{\nu}}\left[S_{\nu}\left(t_{\nu}\right)\right]
$$

It represents a double integration in angle $\left(E_{1}\right)$ and optical depth $h_{-1}^{151}$ over the source function. It delivers the mean intensity $J_{\nu}$ from a known source function $S_{\nu}$ as in Figure 4.2.

Phi and Chi operators. For completeness I add the $\boldsymbol{\Phi}$ and $\chi$ operators defined by

$$
\begin{aligned}
\mathbf{\Phi}_{\tau_{\nu}}\left[S_{\nu}\left(t_{\nu}\right)\right] & \equiv 2 \int_{\tau_{\nu}}^{\infty} S_{\nu}\left(t_{\nu}\right) E_{2}\left(t_{\nu}-\tau_{\nu}\right) \mathrm{d} t_{\nu}-2 \int_{0}^{\tau_{\nu}} S_{\nu}\left(t_{\nu}\right) E_{2}\left(\tau_{\nu}-t_{\nu}\right) \mathrm{d} t_{\nu} \\
& =F_{\nu}\left(\tau_{\nu}\right)
\end{aligned}
$$

and

$$
\begin{aligned}
\chi_{\tau_{\nu}}\left[S_{\nu}\left(t_{\nu}\right)\right] & \equiv 2 \int_{0}^{\infty} S_{\nu}\left(t_{\nu}\right) E_{3}\left(\left|t_{\nu}-\tau_{\nu}\right|\right) \mathrm{d} t_{\nu} \\
& =4 K_{\nu}\left(\tau_{\nu}\right),
\end{aligned}
$$

where astrophysical flux $F_{\nu}=\mathcal{F}_{\nu} / \pi$ is used. The factor 4 before $K$ serves to have, with ('יis'),

$$
\mathbf{\Phi}_{\tau}[f(t)]=\frac{\mathrm{d}}{\mathrm{d} \tau} \chi_{\tau}[f(t)] .
$$

Kourganoff graphs. Figure 4 shows results of the Schwarzschild equation for various source functions $S \equiv B$ with prescribed depth dependence. Figure $\overline{4} .5$ shows corresponding results of the Milne equation. These classical plots are taken from "Kourganoff (1 $19 \overline{9} 5 \overline{2})$. He assumed LTE with $S=B$, but his graphs illustrate the general behavior of $J$ and $\mathcal{F}$ that result through the $\boldsymbol{\Lambda}_{\tau}$ and $\boldsymbol{\Phi}$ transforms of the source function, respectively. The emergent intensity is given exactly by $I_{\nu}(0,1)=S_{\nu}\left(\tau_{\nu}=1\right)$ for the first four graphs with linear $S(\tau)$ dependence. It exceeds the mean intensity at the surface in each case. The top-left graph is for a Lambert radiator with $I_{\nu}(0, \mu)=S=1$ in all outward directions $\mu$, so that $\boldsymbol{\Lambda}_{\tau}[S]=J_{\nu}(0)=(1 / 2) \int_{0}^{1} S \mathrm{~d} \mu=S / 2=1 / 2$. The pulse function at bottom right illustrates that localized source function structure of small spatial extent produces washed-out radiation signature.

Generalized Lambda operators. The classical Lambda operator $\boldsymbol{\Lambda}_{\tau}$ produces $J_{\nu}$ from $S_{\nu}$ through (4.20). In a more general sense, any procedure that delivers $J_{\nu}$ from $S_{\nu}$ may be regarded as a $\boldsymbol{\Lambda}_{\tau}$ operator. A yet more general version of such an operator is one that delivers the angle-dependent specific intensity $I_{\nu}(\mu)$ from $S_{\nu}$, rather than just the angle-averaged intensity $J_{\nu}$. It has become common practice to call such operators also $\boldsymbol{\Lambda}$

\footnotetext{
${ }^{5}$ Or even a triple integration, adding a frequency integral, if one computes $\bar{J}_{\nu_{0}}$ for a spectral line in complete redistribution.
} 

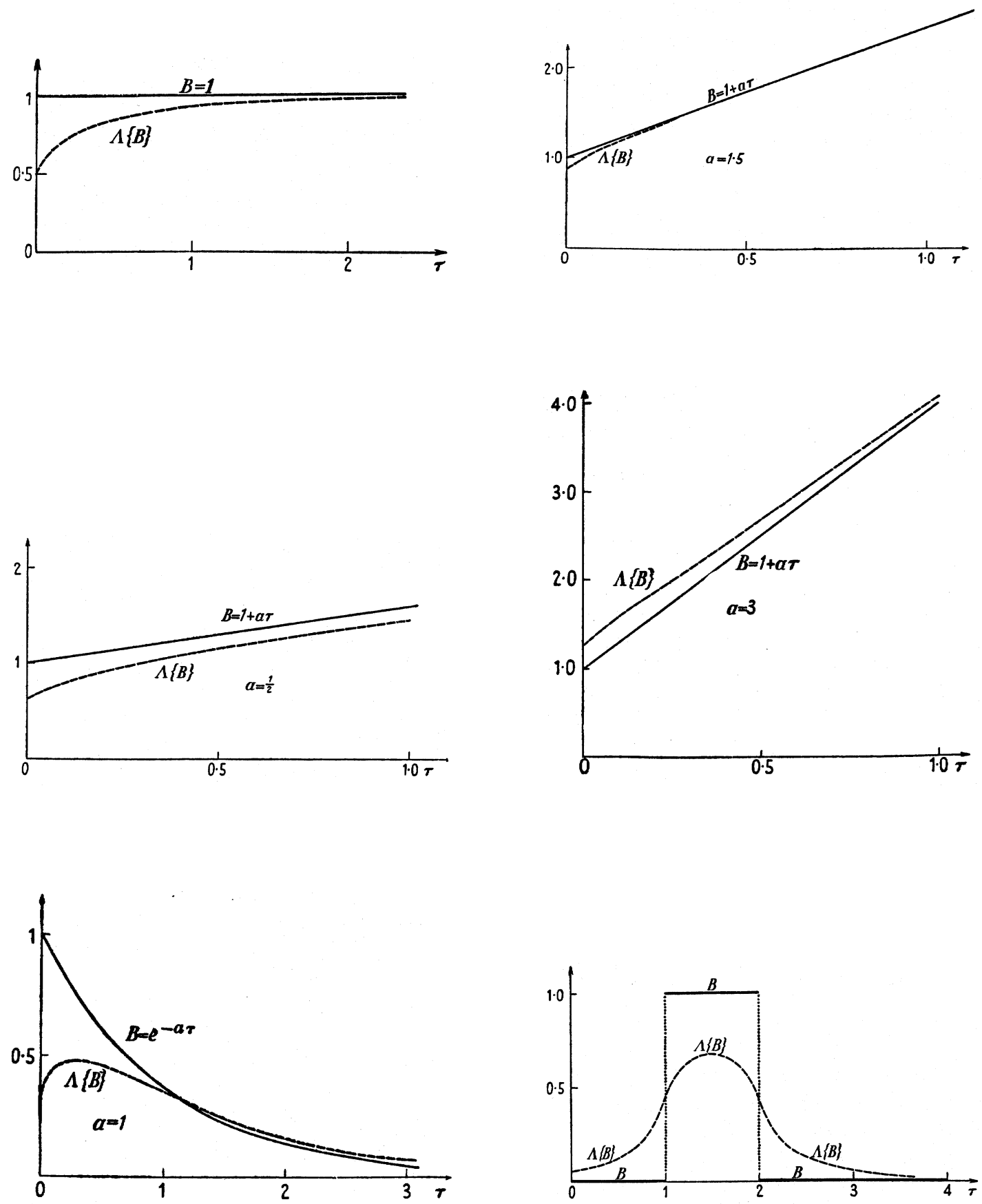

Figure 4.4: The effect of the $\boldsymbol{\Lambda}_{\tau}$ operator (Schwarzschild equation) on specified functions $B(\tau)$. Each graph plots $\boldsymbol{\Lambda}_{\tau}[B]$ against optical depth. The top-left graph is for constant source function (isothermal in the case of LTE, as Kourganoff assumed by writing $S=B$ ) as in a homogeneous medium. At the surface, $\boldsymbol{\Lambda}_{\tau}[B]=(1 / 2) B$; for $\tau \rightarrow \infty, \boldsymbol{\Lambda}_{\tau}[B] \rightarrow B$. The next three graphs are for linearly outward decaying $B=1+a \tau$ with the value of $a$ specified in each graph. $\boldsymbol{\Lambda}_{\tau}[B]$ drops below $B$ or stays above it depending on the steepness of $B$ (second row), while $\boldsymbol{\Lambda}_{\tau}[B] \approx B$ for $B=1+1.5 \tau$ (top right). The bottom graphs are for exponential inward decay (left) and for a pulse function (right). From KKourganoff (1952)! 

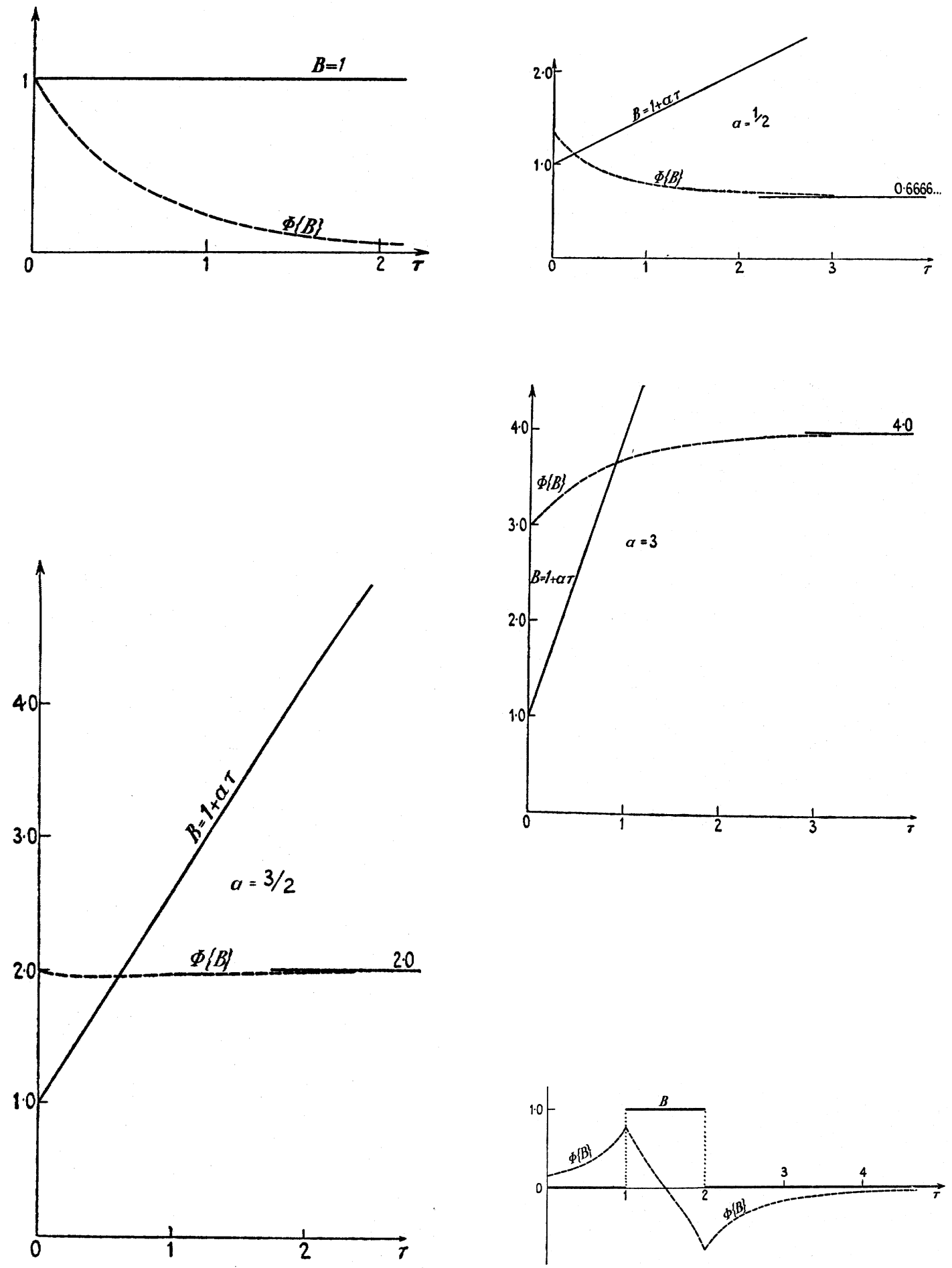

Figure 4.5: The effect of the $\boldsymbol{\Phi}$ operator ( $\left(\overline{4} .22_{1}\right)$ for the Milne equation (4.15) on specified functions $B(\tau)$ ). $\boldsymbol{\Phi}[B]$ becomes constant for $\tau \gg 1$ (zero for constant_B $B_{2}$ upper-left graph) and is nearly constant at all depths when $B=1+1.5 \tau$ (lower-left graph). From Kourganoff $(1952)$ ! 
operators, using the indices $\mu$ and $\nu$ for intensity-producing operators and dropping the index $\mu$ for $J_{\nu}$-producing operators. Thus, in the notation of "Hubeny. $\left(19922_{1}^{6}\right.$.

$$
\begin{aligned}
I_{\nu}\left(\tau_{\nu}, \mu\right) & =\boldsymbol{\Lambda}_{\mu \nu}\left[S_{\nu}\left(t_{\nu}\right)\right] \\
J_{\nu}\left(\tau_{\nu}\right) & =\boldsymbol{\Lambda}_{\nu}\left[S_{\nu}\left(t_{\nu}\right)\right],
\end{aligned}
$$

where

$$
\boldsymbol{\Lambda}_{\nu}=\frac{1}{2} \int_{-1}^{+1} \boldsymbol{\Lambda}_{\mu \nu} \mathrm{d} \mu
$$

In these definitions, $\boldsymbol{\Lambda}$ operates on the quantity within [ ], the argument $t_{\nu}$ ranges over all optical depth values that are needed in the $\boldsymbol{\Lambda}$ evaluation, and $\boldsymbol{\Lambda}$ may actually stand for a long computer program rather than an explicit mathematical operator. For example, the Feautrier method described in Section $5.2 \bar{j}$ on page $\overline{1} \mathbf{1} \overline{1} \bar{z}_{1}$ ff delivers $J_{\nu}$ from a given $S_{\nu}$ and may be seen as a $\boldsymbol{\Lambda}_{\nu}$ operator. Similarly, a $\boldsymbol{\Lambda}_{\mu \nu}$ operator may be constructed from

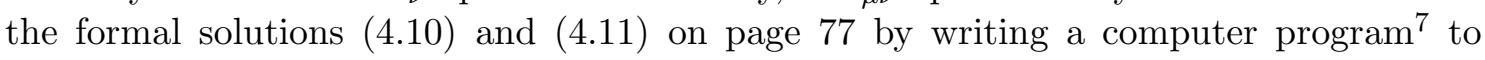
evaluate:

$$
\begin{aligned}
\Lambda_{+\mu \nu}\left[S_{\nu}\right]=I_{\nu}^{+}\left(\tau_{\nu}, \mu\right) & =\int_{\tau_{\nu}}^{\infty} S_{\nu}\left(t_{\nu}\right) \mathrm{e}^{-\left(t_{\nu}-\tau_{\nu}\right) / \mu} \mathrm{d} t_{\nu} / \mu \\
& =\mathrm{e}^{\tau_{\nu \mu}} \int_{\tau_{\nu \mu}}^{\infty} S_{\nu} \mathrm{e}^{-t_{\nu \mu}} \mathrm{d} t_{\nu \mu}
\end{aligned}
$$

and

$$
\begin{aligned}
\Lambda_{-\mu \nu}\left[S_{\nu}\right]=I_{\nu}^{-}\left(\tau_{\nu},-|\mu|\right) & =\int_{0}^{\tau_{\nu}} S_{\nu}\left(t_{\nu}\right) \mathrm{e}^{-\left(\tau_{\nu}-t_{\nu}\right) /|\mu|} \mathrm{d} t_{\nu} /|\mu| \\
& =\mathrm{e}^{-\tau_{\nu \mu}} \int_{0}^{\tau_{\nu \mu}} S_{\nu} \mathrm{e}^{t_{\nu \mu}} \mathrm{d} t_{\nu \mu},
\end{aligned}
$$

where (4.29) and (4.31) use optical depth $\tau_{\mu \nu}$ along the viewing direction $|\mu|$ rather than radial optical depth $\tau_{\nu}$.

\subsection{Approximate solutions}

Optically-thick radiative transfer has analytical solutions only at large depth, where LTE holds and the deviations from isotropy are small. In shallower layers, approximations are inevitable. The most important one is the (first) Eddington approximation; it is used extensively below. This section is largely taken from Zwaan's (1993) lecture notes.

\subsubsection{Approximations at the surface}

\footnotetext{
${ }^{6}$ Hubeny drops all arguments $\tau_{\nu}$, uses $\mu$ also as index to the intensity $I_{\nu}\left(\tau_{\nu}, \mu\right)$ by writing it as $I_{\nu \mu}$, uses $I_{-\mu \nu}$ for inward-directed beams, and drops the $\nu$ index from the line source function $S_{\nu}^{l}\left(\tau_{\nu}\right)$ by writing $S$ when complete distribution holds so that the line source function is independent of frequency over the width of a (narrow) line. He also introduces $\bar{J}_{\nu}\left(\tau_{\nu}\right)=\overline{\boldsymbol{\Lambda}}\left[S_{\nu}\left(t_{\nu}\right)\right]$ with $\overline{\boldsymbol{\Lambda}}=\int \boldsymbol{\Lambda}_{\nu} \varphi\left(\nu-\nu_{0}\right) \mathrm{d} \nu$ as frequency-

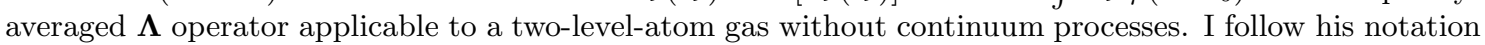
in Chapter

${ }^{7}$ Such a program won't be fast because the exponential integrals are costly to compute. Much faster methods based on "operator splitting" are discussed in Chapter 5.
} 
Eddington-Barbier approximations. The Eddington-Barbier approximation (2.44) for the emergent intensity is based on the polynomial expansion

$$
S_{\nu}\left(\tau_{\nu}\right)=\sum_{n=0}^{\infty} a_{n} \tau_{\nu}^{n}
$$

which produces, using the linearity of the operators:

$$
\begin{aligned}
I_{\nu}^{+}(0, \mu) & =\mathcal{L}_{1 / \mu}\left\{S_{\nu}\left(\tau_{\nu}\right)\right\} \\
& =\sum_{n=0}^{\infty} n ! a_{n} \mu^{n} \\
J_{\nu}\left(\tau_{\nu}\right) & =\boldsymbol{\Lambda}_{\nu}\left[S_{\nu}\right] \\
& =a_{0} \boldsymbol{\Lambda}_{\nu}[1]+a_{1} \boldsymbol{\Lambda}_{\nu}[t]+a_{2} \boldsymbol{\Lambda}_{\nu}\left[t^{2}\right]+\cdots \\
& \approx a_{0}\left[1-\frac{1}{2} E_{2}\left(\tau_{\nu}\right)\right]+a_{1}\left[\tau_{\nu}+\frac{1}{2} E_{3}\left(\tau_{\nu}\right)\right]+a_{2}\left[\frac{2}{3}+\tau_{\nu}^{2}-E_{4}\left(\tau_{\nu}\right)\right] \\
& \approx a_{0}+a_{1} \tau_{\nu}+a_{2} \tau_{\nu}^{2}+\frac{2}{3} a_{2}-\frac{a_{0}}{2} E_{2}\left(\tau_{\nu}\right)+\frac{a_{1}}{2} E_{3}\left(\tau_{\nu}\right)-a_{2} E_{4}\left(\tau_{\nu}\right) \\
F_{\nu}\left(\tau_{\nu}\right) & =\mathbf{\Phi}_{\tau_{\nu}}\left[S_{\nu}\right] \\
& =2 a_{0} E_{3}\left(\tau_{\nu}\right)+a_{1}\left[\frac{4}{3}-2 E_{4}\left(\tau_{\nu}\right)\right]+a_{2}\left[\frac{8}{3} \tau_{\nu}+4 E_{5}\left(\tau_{\nu}\right)\right]+\cdots
\end{aligned}
$$

The surface values are approximately given by:

$$
\begin{aligned}
I_{\nu}^{+}(0, \mu) & \approx a_{0}+a_{1} \mu \\
& \approx S_{\nu}\left(\tau_{\nu}=\mu\right) \\
J_{\nu}(0) & \approx a_{0}+\frac{2 a_{2}}{3}-\frac{a_{0}}{2}+\frac{a_{1}}{4}-\frac{a_{2}}{3} \\
& \approx \frac{a_{0}}{2}+\frac{a_{1}}{4}+\frac{a_{2}}{3} \\
& \approx \frac{1}{2} S_{\nu}\left(\tau_{\nu}=1 / 2\right) \\
F_{\nu}(0) & =a_{0}+\frac{2}{3} a_{1}+a_{2}+\cdots \\
& \approx S_{\nu}\left(\tau_{\nu}=\frac{2}{3}\right)
\end{aligned}
$$

which are the Eddington-Barbier approximations for the emergent intensity, mean intensity and net flux at the surface in the absence of irradiation $\left(I^{-}(0, \mu)=0\right)$. They are exact for a linear source function $S_{\nu}\left(\tau_{\nu}\right)=a_{0}+a_{1} \tau_{\nu}$ and are then easily found directly from the definitions (Exercise $\overline{2}$ on page $\left.\overline{2} \overline{2} \overline{2} \bar{y}_{i}^{\prime}\right)$. Note once more that $J_{\nu}(0)>S_{\nu}(0)$ when the source function increases steeply inwards and that $J_{\nu}(0)<S_{\nu}(0)$ for a slow inward increase of $S_{\nu}\left(\tau_{\nu}\right)$.

Second Eddington approximation. A homogeneous medium with $S_{\nu}=a_{0}$ is a Lambert radiator having $I_{\nu}^{+}(0, \mu)=S_{\nu}=a_{0}$ for all outward directions $\mu>0, J_{\nu}(0)=S_{\nu} / 2=$ $a_{0} / 2=I_{\nu}(0) / 2, F_{\nu}(0)=S_{\nu}=I_{\nu}(0)=a_{0}$ and therefore $F_{\nu}(0)=2 J_{\nu}(0)=4 H_{\nu}(0)$ or $\mathcal{F}_{\nu}(0)=2 \pi J_{\nu}(0)$. The latter relation is called the second Eddington approximation. It 
follows directly from the definition of astrophysical flux by setting

$$
\begin{aligned}
F_{\nu}(0) & \equiv 2 \int_{-1}^{+1} I_{\nu}(0, \mu) \mu \mathrm{d} \mu \\
& =2 \int_{0}^{1} I_{\nu}(0, \mu) \mu \mathrm{d} \mu \\
& \approx 2<I_{\nu}^{+}(0, \mu)>\int_{0}^{1} \mu \mathrm{d} \mu \\
& \approx 2 J_{\nu}(0)
\end{aligned}
$$

using the absence of incoming radiation in the first step and recognizing that $J_{\nu}(0)=$ $(1 / 2)<I_{\nu}^{+}(0, \mu)>$ for the same reason. As noted above, this approximation is exact for a Lambert radiator, simply expressing that $F_{\nu}(0)=F_{\nu}^{+}(0)$ represents an average only over the outward directions $\mu>0$ while $J_{\nu}(0)$ is an average over all $\mu$. In general, it is a coarse approximation. It doesn't even hold for a non-constant linear source function

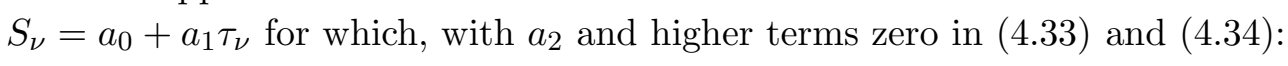

$$
\frac{F_{\nu}(0)}{2 J_{\nu}(0)}=\frac{a_{0} / 2+a_{1} / 3}{a_{0} / 2+a_{1} / 4} \neq 1 \text {. }
$$

Most stars have limb darkening at optical wavelengths, with $a_{1}$ positive. In that case, $J_{\nu}(0) \approx(1 / 2) S_{\nu}\left(\tau_{\nu}=1 / 2\right)<(1 / 2) S_{\nu}\left(\tau_{\nu}=1\right)$ and $F_{\nu}(0) / J_{\nu}(0)>2$ or $J_{\nu}(0)<2 H_{\nu}(0)$.

\subsubsection{Approximations at large depth}

Taylor expansion. At large depth $\left(\tau_{\nu} \gg 1\right)$ radiative transfer becomes simple because all scale lengths become larger than the effective photon mean free path, so that photons are locally trapped in a near-homogeneous environment even while random-walking about in scattering sequences. The radiation fields therefore approach isotropy. In addition, the density is large enough that collisional photon destruction far outweighs photon scattering. The conditions therefore approach TE, making LTE a valid assumption. Expansion of $S_{\nu}$ in a Taylor-McLaurin series gives (Mihāas 1970' p. 28, 1978 p. 50):

$$
S_{\nu}\left(\tau_{\nu}\right)=\sum_{n=0}^{\infty} \frac{\left(t_{\nu}-\tau_{\nu}\right)^{n}}{n !}\left[\frac{\mathrm{d}^{n} S_{\nu}\left(t_{\nu}\right)}{\mathrm{d} t_{\nu}^{n}}\right]_{\tau_{\nu}}
$$

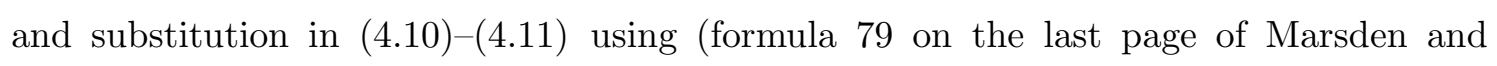
Tromba 1976i)

$$
\begin{aligned}
\int_{x_{1}}^{x_{2}} x^{n} \mathrm{e}^{a x} \mathrm{~d} x & =\left.\frac{x^{n} \mathrm{e}^{a x}}{a}\right|_{x_{1}} ^{x_{2}}-\frac{n}{a} \int_{x_{1}}^{x_{2}} x^{n-1} \mathrm{e}^{a x} \mathrm{~d} x \\
\int_{x_{1}}^{x_{2}} x^{n} \mathrm{e}^{-x} \mathrm{~d} x & \left.=-\mathrm{e}^{-x}\left[x^{n}+n x^{n-1}+n(n-1) x^{n-2}+\cdots+n !\right]\right]_{x_{1}}^{x_{2}} \\
\int_{0}^{\infty} x^{n} \mathrm{e}^{-x} \mathrm{~d} x & =n !
\end{aligned}
$$

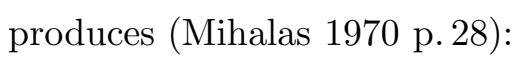

$$
I_{\nu}^{+}\left(\tau_{\nu}, \mu\right)=\sum_{n=0}^{\infty} \frac{1}{n !}\left[\frac{\mathrm{d}^{n} S_{\nu}\left(t_{\nu}\right)}{\mathrm{d} t_{\nu}^{n}}\right]_{\tau_{\nu}} \int_{\tau_{\nu}}^{\infty}\left(t_{\nu}-\tau_{\nu}\right)^{n} \mathrm{e}^{-\left(t_{\nu}-\tau_{\nu}\right) / \mu} \mathrm{d} t_{\nu} / \mu
$$




$$
\begin{aligned}
& =\sum_{n=0}^{\infty}\left[\frac{\mathrm{d}^{n} S_{\nu}\left(t_{\nu}\right)}{\mathrm{d} t_{\nu}^{n}}\right]_{\tau_{\nu}} \frac{1}{n !} \int_{0}^{\infty} x^{n} \mathrm{e}^{-x / \mu} \mathrm{d} x / \mu \\
& =\sum_{n=0}^{\infty}\left[\frac{\mathrm{d}^{n} S_{\nu}\left(t_{\nu}\right)}{\mathrm{d} t_{\nu}^{n}}\right]_{\tau_{\nu}} \frac{\mu^{n}}{n !} \int_{0}^{\infty} x^{n} \mathrm{e}^{-x} \mathrm{~d} x \\
& =\sum_{n=0}^{\infty} \mu^{n}\left[\frac{\mathrm{d}^{n} S_{\nu}\left(t_{\nu}\right)}{\mathrm{d} t_{\nu}^{n}}\right]_{\tau_{\nu}}
\end{aligned}
$$

and

$$
\begin{aligned}
I_{\nu}^{-}\left(\tau_{\nu}, \mu\right) & =\sum_{n=0}^{\infty} \frac{1}{n !}\left[\frac{\mathrm{d}^{n} S_{\nu}\left(t_{\nu}\right)}{\mathrm{d} t_{\nu}^{n}}\right]_{\tau_{\nu}}\left[-\int_{0}^{\tau_{\nu}}\left(t_{\nu}-\tau_{\nu}\right)^{n} \mathrm{e}^{-\left(t_{\nu}-\tau_{\nu}\right) / \mu} \mathrm{d} t_{\nu} / \mu\right] \\
& =\sum_{n=0}^{\infty} \frac{1}{n !}\left[\frac{\mathrm{d}^{n} S_{\nu}\left(t_{\nu}\right)}{\mathrm{d} t_{\nu}^{n}}\right]_{\tau_{\nu}}(-1)^{n}\left[+\int_{0}^{\tau_{\nu}}\left(\tau_{\nu}-t_{\nu}\right)^{n} \mathrm{e}^{-\left(\tau_{\nu}-t_{\nu}\right) /|\mu|} \mathrm{d} t_{\nu} /|\mu|\right] \\
& =\sum_{n=0}^{\infty}\left[\frac{\mathrm{d}^{n} S_{\nu}\left(t_{\nu}\right)}{\mathrm{d} t_{\nu}^{n}}\right]_{\tau_{\nu}}(-1)^{n} \frac{|\mu|^{n}}{n !}\left[\int_{0}^{\tau_{\nu} /|\mu|} x^{n} \mathrm{e}^{-x} \mathrm{~d} x\right] \\
& =\sum_{n=0}^{\infty} \mu^{n}\left[\frac{\mathrm{d}^{n} S_{\nu}\left(t_{\nu}\right)}{\mathrm{d} t_{\nu}^{n}}\right]_{\tau_{\nu}}\left[1-\frac{\mathrm{e}^{-\left(\tau_{\nu} /|\mu|\right)}}{n !}\left\{\left(\tau_{\nu} /|\mu|\right)^{n}+n\left(\tau_{\nu} /|\mu|\right)^{n-1}+\cdots+n !\right\}\right]
\end{aligned}
$$

where the term $[1-\cdots]$ in the last expression goes to unity for large $\tau_{\nu}$. Thus:

$$
I_{\nu}\left(\tau_{\nu}, \mu\right)=S_{\nu}\left(\tau_{\nu}\right)+\mu\left[\frac{\mathrm{d} S_{\nu}\left(t_{\nu}\right)}{\mathrm{d} t_{\nu}}\right]_{\tau_{\nu}}+\mu^{2}\left[\frac{\mathrm{d}^{2} S_{\nu}\left(t_{\nu}\right)}{\mathrm{d} t_{\nu}^{2}}\right]_{\tau_{\nu}}+\cdots
$$

holds in all directions $-1 \leq \mu \leq 1$ when $\tau_{\nu} \gg 1$, and holds also for $\mu>0$ at smaller depth.

Large depth. For $\tau_{\nu} \gg 1$ substitution in (2.1.13i) gives

$$
J_{\nu}\left(\tau_{\nu}\right)=\frac{1}{2} \sum_{n=0}^{\infty}\left[\frac{\mathrm{d}^{n} S_{\nu}\left(t_{\nu}\right)}{\mathrm{d} t_{\nu}^{n}}\right]_{\tau_{\nu}} \int_{-1}^{+1} \mu^{n} \mathrm{~d} \mu=\sum_{k=0}^{\infty} \frac{1}{2 k+1}\left[\frac{\mathrm{d}^{(2 k)} S_{\nu}\left(t_{\nu}\right)}{\mathrm{d} t_{\nu}^{(2 k)}}\right]_{\tau_{\nu}}
$$

and, with similar expressions for $F_{\nu}$ and $K_{\nu}$ :

$$
\begin{aligned}
J_{\nu}\left(\tau_{\nu}\right) & =S_{\nu}\left(\tau_{\nu}\right)+\frac{1}{3}\left[\frac{\mathrm{d}^{2} S_{\nu}\left(t_{\nu}\right)}{\mathrm{d} t_{\nu}^{2}}\right]_{\tau_{\nu}}+\cdots \\
F_{\nu}\left(\tau_{\nu}\right) & =\frac{4}{3}\left[\frac{\mathrm{d} S_{\nu}\left(t_{\nu}\right)}{\mathrm{d} t_{\nu}}\right]_{\tau_{\nu}}+\frac{4}{5}\left[\frac{\mathrm{d}^{3} S_{\nu}\left(t_{\nu}\right)}{\mathrm{d} t_{\nu}^{3}}\right]_{\tau_{\nu}}+\cdots \\
K_{\nu}\left(\tau_{\nu}\right) & =\frac{1}{3} S_{\nu}\left(\tau_{\nu}\right)+\frac{1}{5}\left[\frac{\mathrm{d}^{2} S_{\nu}\left(t_{\nu}\right)}{\mathrm{d} t_{\nu}^{2}}\right]_{\tau_{\nu}}+\cdots
\end{aligned}
$$

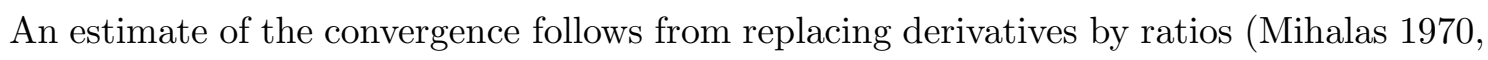
p. 29):

$$
\left|\frac{\mathrm{d}^{n} S_{\nu}}{\mathrm{d} t_{\nu}^{n}}\right| \sim \frac{S_{\nu}}{t_{\nu}^{n}}
$$

so that

$$
\frac{\left|\mathrm{d}^{n+2} S_{\nu} / \mathrm{d} t_{\nu}^{n+2}\right|}{\left|\mathrm{d}^{n} S_{\nu} / \mathrm{d} t_{\nu}^{n}\right|} \sim \frac{S_{\nu} / t_{\nu}^{n+2}}{S_{\nu} / t_{\nu}^{n}} \sim \frac{1}{t_{\nu}^{2}}
$$


which shows that all three expansions converge rapidly for $\tau_{\nu} \gg 1$. Thus, at sufficiently large depth:

$$
\begin{aligned}
I_{\nu}\left(\tau_{\nu}, \mu\right) & \approx S_{\nu}\left(\tau_{\nu}\right)+\mu\left[\frac{\mathrm{d} S_{\nu}\left(t_{\nu}\right)}{\mathrm{d} t_{\nu}}\right]_{\tau_{\nu}} \\
J_{\nu}\left(\tau_{\nu}\right) & \approx S_{\nu}\left(\tau_{\nu}\right) \\
F_{\nu}\left(\tau_{\nu}\right) & \approx \frac{4}{3}\left[\frac{\mathrm{d} S_{\nu}\left(t_{\nu}\right)}{\mathrm{d} t_{\nu}}\right]_{\tau_{\nu}} \\
K_{\nu}\left(\tau_{\nu}\right) & \approx \frac{1}{3} S_{\nu}\left(\tau_{\nu}\right) .
\end{aligned}
$$

The isotropic component of the radiation field $J_{\nu}$ is set by the value of the source function whereas the anisotropic component $F_{\nu}$ is determined by the gradient $\mathrm{d} S_{\nu} / \mathrm{d} \tau_{\nu}$, as sketched in Fig. "1. $\overline{1}^{\prime}$. No wonder, since $F_{\nu}$ measures net flux, i.e., the difference between $F_{\nu}^{+}$and $F_{\nu}^{-}$ at depth $\tau_{\nu}$. The radial outward intensity is $I_{\nu}\left(\tau_{\nu}, 1\right) \approx S_{\nu}+\mathrm{d} S_{\nu} / \mathrm{d} \tau_{\nu}$ and is slightly larger than the radial inward intensity $I_{\nu}\left(\tau_{\nu},-1\right) \approx S_{\nu}-\mathrm{d} S_{\nu} / \mathrm{d} \tau_{\nu}$. The gradient is relatively small, the flux therefore also, since the relative anisotropy decreases for increasing depth as:

$$
\frac{\mathrm{d} S_{\nu} / \mathrm{d} \tau_{\nu}}{S_{\nu}} \sim \frac{S_{\nu} / \tau_{\nu}}{S_{\nu}} \sim \frac{1}{\tau_{\nu}}
$$

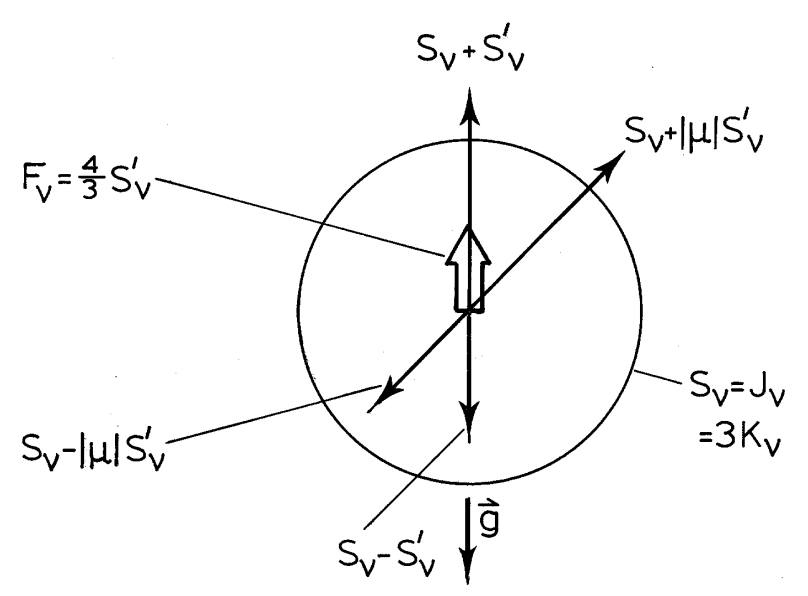

Figure 4.6: The radiation field for $\tau_{\nu} \gg 1$. Inwards is down, as denoted by the gravity vector $\vec{g}$. The gradient $S_{\nu}^{\prime}=\mathrm{d} S_{\nu} / \mathrm{d} \tau_{\nu}$ is small compared with the value of $S_{\nu}$. As a result, the intensity (thin arrows) is nearly isotropic, $J_{\nu}=S_{\nu}$, and the net flux (broad arrow) is small. From Żwaan (1993)!

Diffusion approximation. The Rosseland or diffusion approximation holds sufficiently deep inside a star where $I_{\nu}$ is nearly isotropic and where LTE holds so that $S_{\nu}=B_{\nu}$. This is generally the case at depths with $\tau_{\nu}^{*}>1$, with $\tau_{\nu}^{*}$ denoting effective radial optical depth defined by $(\overline{2} . \overline{1} \overline{4} \overline{1})$ on page $\overline{4} \overline{0}$. We then have, with $(\overline{4} . \overline{4} \overline{2})-(\overline{4} . \overline{4} \overline{4})$ :

$$
\begin{aligned}
I_{\nu}\left(\tau_{\nu}, \mu\right) & \approx B_{\nu}\left(\tau_{\nu}\right)+\mu\left[\frac{\mathrm{d} B_{\nu}\left(t_{\nu}\right)}{\mathrm{d} t_{\nu}}\right]_{\tau_{\nu}} \\
J_{\nu}(z) & \approx B_{\nu}(z) \\
\mathcal{F}_{\nu}(z) & \approx 2 \pi \int_{-1}^{+1} \mu I_{\nu} \mathrm{d} \mu \approx \frac{4 \pi}{3} \frac{\mathrm{d} B_{\nu}(z)}{\mathrm{d} \tau_{\nu}} .
\end{aligned}
$$


The monochromatic flux is now expressed in the gradient of $B_{\nu}$ in optical depth. This equation has the general form of a diffusion process, in which the transported flux of a quantity equals the product of a diffusion coefficient and a spatial gradient in that quantity.

Rosseland mean extinction. In order to recast (4.48) into the familiar expression for the total flux $\mathcal{F}$ as a function of the geometrical radial temperature gradient $\mathrm{d} T / \mathrm{d} z$ we use the Rosseland mean extinction coefficient

$$
\begin{aligned}
\frac{1}{\alpha_{\mathrm{R}}} & \equiv \frac{\int_{0}^{\infty}\left(1 / \alpha_{\nu}\right)\left(\mathrm{d} B_{\nu} / \mathrm{d} T\right) \mathrm{d} \nu}{\int_{0}^{\infty}\left(\mathrm{d} B_{\nu} / \mathrm{d} T\right) \mathrm{d} \nu} \\
\frac{1}{\kappa_{\mathrm{R}}} & \equiv \frac{\int_{0}^{\infty}\left(1 / \kappa_{\nu}\right)\left(\mathrm{d} B_{\nu} / \mathrm{d} T\right) \mathrm{d} \nu}{\int_{0}^{\infty}\left(\mathrm{d} B_{\nu} / \mathrm{d} T\right) \mathrm{d} \nu}
\end{aligned}
$$

with $\kappa_{\mathrm{R}}(z)=\alpha_{\mathrm{R}}(z) / \rho(z)$. This harmonic mean serves below to obtain (4.52i) from (4.51); it is also derived as flux-weighted mean on page $\mathbf{i} \overline{6} \overline{0}_{1}^{1}$. It averages the extinction similarly to the formula for combining parallel resistors $1 / \stackrel{R}{=}=1 / R_{1}+1 / R_{2}$, extinction representing resistance to the photon flux which favors the more transparent spectral windows. The monochromatic Planck function temperature sensitivity $\mathrm{d} B_{\nu} / \mathrm{d} T$ enters as weighting function for the same reason. It produces larger flux from a given spatial temperature gradient at frequencies where it is large.

Total radiative energy diffusion. The total energy flow is now given by:

$$
\begin{aligned}
\mathcal{F}(z) & \equiv \int_{0}^{\infty} \mathcal{F}_{\nu}(z) \mathrm{d} \nu \\
& \approx-\frac{4 \pi}{3} \int_{0}^{\infty} \frac{1}{\alpha_{\nu}} \frac{\mathrm{d} B_{\nu}}{\mathrm{d} z} \mathrm{~d} \nu \\
& \approx-\frac{4 \pi}{3} \int_{0}^{\infty} \frac{1}{\alpha_{\nu}} \frac{\mathrm{d} B_{\nu}}{\mathrm{d} T} \frac{\mathrm{d} T}{\mathrm{~d} z} \mathrm{~d} \nu \\
& \approx-\frac{16}{3} \frac{\sigma T^{3}}{\alpha_{\mathrm{R}}} \frac{\mathrm{d} T}{\mathrm{~d} z} \\
& \approx-\frac{1}{3} \frac{c}{\kappa_{\mathrm{R}} \rho} \frac{\mathrm{d} u}{\mathrm{~d} z}
\end{aligned}
$$

where the total energy density $u$ is given by $(2.10)$ on page $1 \overline{1}^{1}$ as $u=(4 \sigma / c) T^{4}$. This diffusion equation is also often called the radiation conduction equation. It says that a negative temperature gradient is required to let net radiative flux diffuse outwards through a star by thermal absorptions and re-emissions with a mean free photon path $l \equiv 1 / \rho \kappa_{\mathrm{R}}$. In the solar interior $l$ measures only a few millimeters, making the optical depth from the surface $\tau_{\nu} \approx 10^{11}$ so that $(4 . \overline{4} 2)-(4.45)$ hold with high accuracy. A quote from 'SThu' $(1999 \overline{1}):$

A "random walk" slows down the free-flight speed $c$ by a typical factor of $l / R_{\odot}$, so that the time $R_{\odot}^{2} / \mathcal{D}$ (with the radiative diffusivity $\mathcal{D}=c / 3 \rho \kappa_{\mathrm{R}}$ ) is roughly $3 R_{\odot} / l$ times longer than the free-flight time $R_{\odot} / c$ of about $2 \mathrm{~s}$. This process prevents the Sun from releasing its considerable internal reservoir of photons in one powerful blast, but instead regulates it to the stately observed luminosity of $L_{\odot}=3.86 \times 10^{33} \mathrm{erg} \mathrm{s}^{-1}$. In any case, apart from being useful for rough order-of-magnitude arguments, this accurate equation constitutes one of the fundamental equations underlying the whole theory of stellar structure and evolution.

\footnotetext{
${ }^{8}$ The original paper by "Rosseland $(1924)$ ', is reprinted in 'Menzel $(1966)$.'.
} 


\subsubsection{The Eddington approximation}

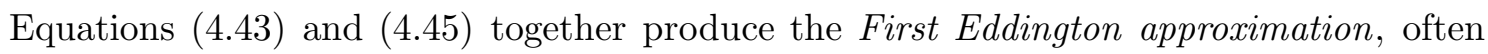
called the Eddington or the Milne-Eddington approximation:

$$
K_{\nu}\left(\tau_{\nu}\right) \approx \frac{1}{3} J_{\nu}\left(\tau_{\nu}\right)
$$

It may also be derived directly by setting

$$
\begin{aligned}
K_{\nu}\left(\tau_{\nu}\right) & \equiv \frac{1}{2} \int_{-1}^{+1} I_{\nu}\left(\tau_{\nu}, \mu\right) \mu^{2} \mathrm{~d} \mu \\
& \approx \frac{1}{2}<I_{\nu}\left(\tau_{\nu}, \mu\right)>\int_{-1}^{+1} \mu^{2} \mathrm{~d} \mu \\
& \approx \frac{1}{3} J_{\nu}\left(\tau_{\nu}\right)
\end{aligned}
$$

where $\left\langle I_{\nu}\right\rangle=J_{\nu}$ because all directions count, not only the outgoing ones as in (4.

Validity. The Eddington approximation is exact for isotropic radiation. It is also exact, at any depth $\tau_{\nu}$, when $I_{\nu}\left(\tau_{\nu}, \mu\right)$ can be expanded as $I_{\nu}\left(\tau_{\nu}, \mu\right)=\sum_{i=0}^{n} a_{i}\left(\tau_{\nu}\right) \mu^{i}$ in only odd powers of $\mu$, with all even coefficients $a_{i}=0$. This is easily derived from the definitions of $J_{\nu}$ and $K_{\nu}$ since all primitives with even powers of $\mu$ drop out of $\int_{-1}^{+1} \mathrm{~d} \mu$. It implies that the Eddington approximation may also hold for $\tau_{\nu}<1$, in contrast to the approximations in Eqs. (

In particular, the Eddington approximation is exact when $I_{\nu}$ possesses linear anisotropy in $\mu$. If

$$
I_{\nu}\left(\tau_{\nu}, \mu\right) \equiv a_{0}\left(\tau_{\nu}\right)+a_{1}\left(\tau_{\nu}\right) \mu
$$

then

$$
\begin{aligned}
J_{\nu}\left(\tau_{\nu}\right) & \equiv \frac{1}{2} \int_{-1}^{+1} I_{\nu}\left(\tau_{\nu}, \mu\right) \mathrm{d} \mu=a_{0}\left(\tau_{\nu}\right), \\
H_{\nu}\left(\tau_{\nu}\right) & \equiv \frac{1}{2} \int_{-1}^{+1} \mu I_{\nu}\left(\tau_{\nu}, \mu\right) \mathrm{d} \mu=a_{1}\left(\tau_{\nu}\right) / 3 \\
K_{\nu}\left(\tau_{\nu}\right) & \equiv \frac{1}{2} \int_{-1}^{+1} \mu^{2} I_{\nu}\left(\tau_{\nu}, \mu\right) \mathrm{d} \mu=a_{0}\left(\tau_{\nu}\right) / 3 .
\end{aligned}
$$

Note that these expressions do not hold when only $I_{\nu}^{+}=a_{0}+a_{1} \mu$ but $I_{\nu}^{-}$is not. In particular, the Eddington approximation is not exact at the surface even when $S_{\nu}$ is linear in $\tau_{\nu}$ and the Eddington-Barbier approximation $I_{\nu}^{+}(0, \mu)=a_{0}+a_{1} \mu$ is therefore exact. On the other hand, it is again exact when $I_{\nu}^{+}$and $I_{\nu}^{-}$are independently isotropic, including the Lambert case that $I_{\nu}^{+}(0)=a_{0}$ and $I_{\nu}^{-}(0)=0$ (corresponding to depth-independent $\left.S_{\nu}=a_{0}\right)$.

The difference with the diffusion approximation is that the Eddington approximation does not require LTE, only linear anisotropy (deviations in $I_{\nu}\left(\tau_{\nu}, \mu\right)$ from isotropy only to first order). It therefore tends to hold quite well in the layers between $\tau_{\nu}=1$ and $\tau_{\nu}^{*}=1$ with $\tau_{\nu}^{*}$ the effective optical depth (page $\left.\overline{4} \bar{q}_{-}\right)$. It may be useful even for $\tau_{\nu}<1$. In contrast, the diffusion approximation requires LTE and therefore holds only for $\tau_{\nu}^{*}>1$. 
Second-order transport equation. Substitution of the Eddington approximation into

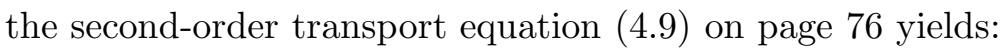

$$
\frac{1}{3} \frac{\mathrm{d}^{2} J_{\nu}\left(\tau_{\nu}\right)}{\mathrm{d} \tau_{\nu}^{2}}=J_{\nu}\left(\tau_{\nu}\right)-S_{\nu}\left(\tau_{\nu}\right)
$$

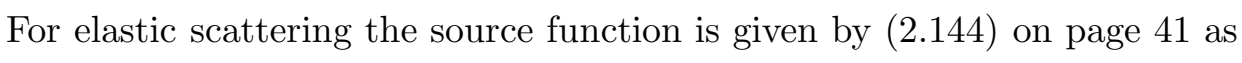

$$
S_{\nu}=\left(1-\varepsilon_{\nu}\right) J_{\nu}+\varepsilon_{\nu} B_{\nu}
$$

so that the second-order form of the transport equation becomes:

$$
\frac{1}{3} \frac{\mathrm{d}^{2} J_{\nu}\left(\tau_{\nu}\right)}{\mathrm{d} \tau_{\nu}^{2}}=\varepsilon_{\nu}\left[J_{\nu}\left(\tau_{\nu}\right)-B_{\nu}\left(\tau_{\nu}\right)\right] .
$$

From given $T(z)$ and $\varepsilon_{\nu}(z)$ and with suitable boundary conditions this equation may be solved to determine $J_{\nu}\left(\tau_{\nu}\right)$, then $S_{\nu}\left(\tau_{\nu}\right)$ and finally, with the formal integral solutions of

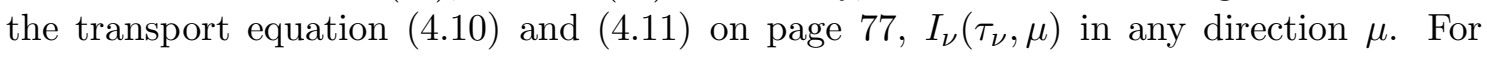
non-irradiated stellar atmospheres the boundary conditions are the absence of incoming radiation at the surface and the Rosseland approximation (4.46) at large depth.

\subsection{Illustrative solutions}

In this key section of these lecture notes I discuss analytical solutions of the transport equation (4.61) for the case of the two-level source function with elastic scattering as given by (4.60). Thus, the assumption is that photons are, per extinction, either destroyed into the thermal pool or redirected elastically, without change of frequency (and with isotropic angle redistribution). The photons are created in thermal processes as specified by the source term $\varepsilon_{\nu} B_{\nu}$ in (4.60ij). This situation may describe a gas of two-level atoms with $\varepsilon_{\nu}$ the destruction probability for line photons, or a scattering line with a thermal background continuum (page 10.019 ), or Thomson or Rayleigh continuum scattering with additional thermal bound-free or free-free processes (page $\left.10 \overline{1} \overline{6}^{4}\right)$.

\subsubsection{Coherent scattering in the Eddington approximation}

For tractability I adopt the Eddington approximation, assume the Planck function $B_{\nu}\left(\tau_{\nu}\right)$ to depend linearly on depth, and assume that the destruction probability $\varepsilon_{\nu}$ has the same value at all depths. These assumptions make the problem analytically solvable. The assumption of the Eddington approximation in (4.6. are really $\approx$ signs. The assumption of depth-independent $\varepsilon_{\nu}$ is unrealistic, since in any stellar atmosphere in hydrostatic equilibrium the density decreases roughly exponentially outwards and the collision probability therefore increases rapidly inwards. Nevertheless, the simplified case of constant $\varepsilon_{\nu}$ represents a landmark test in radiative transfer theory. It is also treated by "Rybicki and Lightman $(1979)$, in a two-beam approximation for an isothermal atmosphere. The treatment below for depth-dependent $B_{\nu}\left(\tau_{\nu}\right)$ is from Zwaan (cf. page $153 \mathrm{ff}$ of "Mihalas 1970') and is a bit more complex. Its advantage is that it permits to quantify $J_{\nu}-B_{\nu} \gtrless 0$ "splits" between $J_{\nu}$ and $B_{\nu}$ (Section 4.3. inequalities depend on the temperature gradient and are important in NLTE radiative transfer because they characterize the non-local nature of the local radiation field. 
Transport equation. By using (4.61) we adopt the (first) Eddington approximation. In addition, we assume that the Planck function varies linearly with optical depth

$$
B_{\nu}\left(\tau_{\nu}\right) \equiv B_{\nu, 0}+b_{\nu} \tau_{\nu}
$$

so that $\mathrm{d}^{2} B_{\nu} / \mathrm{d} \tau_{\nu}^{2}=0$ and $(4.61)$ may be rewritten into the simple form

$$
\frac{1}{3} \frac{\mathrm{d}^{2}}{\mathrm{~d} \tau_{\nu}^{2}}\left(J_{\nu}-B_{\nu}\right)=\varepsilon_{\nu}\left(J_{\nu}-B_{\nu}\right)
$$

Since we assume $\varepsilon_{\nu}$ depth-independent we directly obtain the general solution

$$
J_{\nu}-B_{\nu}=C_{1} \mathrm{e}^{-\sqrt{3 \varepsilon_{\nu}} \tau_{\nu}}+C_{2} \mathrm{e}^{+\sqrt{3 \varepsilon_{\nu}} \tau_{\nu}} .
$$

Boundary conditions. The integration constants $C_{1}$ and $C_{2}$ follow from the boundary conditions that $J_{\nu}=B_{\nu}$ for $\tau_{\nu} \rightarrow \infty$ in the Rosseland approximation (4.4.7. is no incident radiation at $\tau_{\nu}=0$. The first condition results in $C_{2}=0$. To get the constant $C_{1}$ we might adopt the second Eddington approximation $(4.35) J_{\nu}(0)=2 H_{\nu}(0)$, but this is exact only for an unrealistic Lambert radiator. We therefore set $J_{\nu}(0)=a_{\nu} H_{\nu}(0)$ and keep $a_{\nu}$ as a free parameter. It depends on $b_{\nu}$ since it is set by the limb darkening and is therefore formally frequency-dependent, but its value ranges only between 1 and 2 for realistic atmospheres. As we will see below, $a_{\nu}=\sqrt{3}=1.73$ is a good choice. This boundary condition produces with $\left(\begin{array}{l}4 \\ 4\end{array} .81\right)$, again assuming (4.54):

$$
\begin{aligned}
J_{\nu}(0) & =B_{\nu, 0}+C_{1} \\
& =a_{\nu} H_{\nu}(0)=a_{\nu}\left[\frac{\mathrm{d} K_{\nu}}{\mathrm{d} \tau_{\nu}}\right]_{\tau_{\nu}=0}=\left(a_{\nu} / 3\right)\left[\frac{\mathrm{d} J_{\nu}}{\mathrm{d} \tau_{\nu}}\right]_{\tau_{\nu}=0}=-\left(a_{\nu} / 3\right) \sqrt{3 \varepsilon_{\nu}} C_{1}+a_{\nu} b_{\nu} / 3 \\
C_{1} & =-\frac{B_{\nu, 0}-a_{\nu} b_{\nu} / 3}{1+\left(a_{\nu} / 3\right) \sqrt{3 \varepsilon_{\nu}}}
\end{aligned}
$$

Solutions. Substitution in (4.6. $\overline{4})$ gives, again with $H_{\nu}=\mathrm{d} K_{\nu} / \mathrm{d} \tau_{\nu} \approx(1 / 3) \mathrm{d} J_{\nu} / \mathrm{d} \tau_{\nu}$ :

$$
\begin{aligned}
J_{\nu}\left(\tau_{\nu}\right) & =B_{\nu}\left(\tau_{\nu}\right)+C_{1} \mathrm{e}^{-\sqrt{3 \varepsilon_{\nu}} \tau_{\nu}} \\
& =B_{\nu, 0}+b_{\nu} \tau_{\nu}-\frac{B_{\nu, 0}-a_{\nu} b_{\nu} / 3}{1+\left(a_{\nu} / 3\right) \sqrt{3 \varepsilon_{\nu}}} \mathrm{e}^{-\sqrt{3 \varepsilon_{\nu}} \tau_{\nu}} \\
S_{\nu}\left(\tau_{\nu}\right) & =B_{\nu}\left(\tau_{\nu}\right)+\left(1-\varepsilon_{\nu}\right) C_{1} \mathrm{e}^{-\sqrt{3 \varepsilon_{\nu}} \tau_{\nu}} \\
& =B_{\nu, 0}+b_{\nu} \tau_{\nu}-\left(1-\varepsilon_{\nu}\right) \frac{B_{\nu, 0}-a_{\nu} b_{\nu} / 3}{1+\left(a_{\nu} / 3\right) \sqrt{3 \varepsilon_{\nu}}} \mathrm{e}^{-\sqrt{3 \varepsilon_{\nu}} \tau_{\nu}} \\
H_{\nu}\left(\tau_{\nu}\right) & =b_{\nu} / 3+\sqrt{\varepsilon_{\nu}} \frac{B_{\nu, 0}-a_{\nu} b_{\nu} / 3}{\sqrt{3}+a_{\nu} \sqrt{\varepsilon_{\nu}}} \mathrm{e}^{-\sqrt{3 \varepsilon_{\nu}} \tau_{\nu}} \\
I_{\nu}^{+}(0, \mu) & =B_{\nu, 0}+b_{\nu} \mu-\frac{\left(1-\varepsilon_{\nu}\right)\left(B_{\nu, 0}-a_{\nu} b_{\nu} / 3\right)}{\left(1+\left(a_{\nu} / 3\right) \sqrt{3 \varepsilon_{\nu}}\right)\left(1+\mu \sqrt{3 \varepsilon_{\nu}}\right)}
\end{aligned}
$$

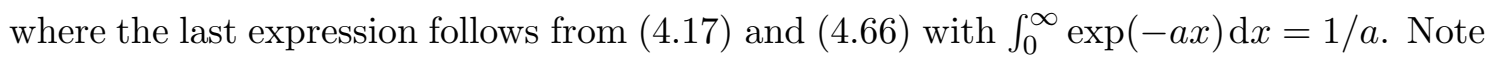
that at large depth $S_{\nu} \approx B_{\nu}$ (LTE) and that $J_{\nu} \approx B_{\nu}$ and $H_{\nu}=\mathcal{F}_{\nu} / 4 \pi=F_{\nu} / 4 \approx b_{\nu} / 3 \approx$

\footnotetext{
${ }^{9}$ Note that the source function is not assumed to vary linearly with $\tau_{\nu}$, only its thermal constituent. Thus, we are not assuming that the Eddington-Barbier relation holds exactly.
} 
$(1 / 4)(4 / 3) \mathrm{d} S_{\nu} / \mathrm{d} \tau_{\nu}=(1 / 3) \mathrm{d} S_{\nu} / \mathrm{d} \tau_{\nu}$, obeying (4.43) and (4.44) on page $\overline{8} \overline{4}$. These analytic solutions are compared with exact numerical results in Figure 4.8.

The factor $\sqrt{3}$ in the term $\exp \left(-\sqrt{3 \varepsilon_{\nu}} \tau_{\nu}\right)$ has the character of a viewing angle with $\mu=1 / \sqrt{3}$. The same solutions may also be obtained from the two-beam approximation!17! in which photons travel exclusively in the two directions $\mu= \pm 1 / \sqrt{3}$, these values being chosen to obtain the Eddington approximation when evaluating the intensity mo-

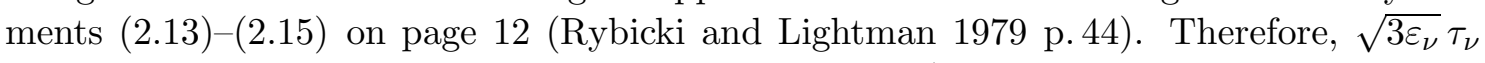
corresponds to the effective optical depth $\tau_{\mu \nu}^{*} \equiv \sqrt{\varepsilon_{\nu}} \tau_{\nu} / \mu$ as measured along the viewing direction $\mu=1 / \sqrt{3}$. See Exercise $\overline{5}$, on page $\overline{2} 2 \overline{2} \overline{2}$.

\subsubsection{Isothermal atmosphere}

Solutions. We first study the results above for the simplified case of an isothermal atmosphere by setting $b_{\nu}=0$. The solutions (4.4.65i) $\left(1 \overline{4}-\overline{6} 8_{i}\right)$ then become:

$$
\begin{aligned}
J_{\nu}\left(\tau_{\nu}\right) & =B_{\nu, 0}\left[1-\frac{1}{1+\left(a_{\nu} / 3\right) \sqrt{3 \varepsilon_{\nu}}} \mathrm{e}^{-\sqrt{3 \varepsilon_{\nu}} \tau_{\nu}}\right] \\
S_{\nu}\left(\tau_{\nu}\right) & =B_{\nu, 0}\left[1-\frac{1-\varepsilon_{\nu}}{1+\left(a_{\nu} / 3\right) \sqrt{3 \varepsilon_{\nu}}} \mathrm{e}^{-\sqrt{3 \varepsilon_{\nu}} \tau_{\nu}}\right] \\
I_{\nu}^{+}(0, \mu) & =B_{\nu, 0}\left[1-\frac{1-\varepsilon_{\nu}}{\left(1+\left(a_{\nu} / 3\right) \sqrt{3 \varepsilon_{\nu}}\right)\left(1+\mu \sqrt{3 \varepsilon_{\nu}}\right)}\right]
\end{aligned}
$$

with $J_{\nu} \leq B_{\nu}, S_{\nu} \leq B_{\nu}$ and $I_{\nu}^{+}(0, \mu) \leq B_{\nu}$ for $\varepsilon_{\nu}<1$. For $\varepsilon_{\nu}=1$ the source function is constant with $S_{\nu}=B_{\nu, 0}$, the mean intensity $J_{\nu}$ increases rapidly with the optical depth $\tau_{\nu}$ to the value $J_{\nu}\left(\tau_{\nu}\right)=B_{\nu, 0}$, and the flux $H_{\nu}$ decreases as rapidly to zero. This case is illustrated in Fig. 14 and detailed below. For small $\varepsilon_{\nu}$ the inward exponential rise of $J_{\nu}$ and decay of $H_{\nu}$ are less rapid.

Without scattering. For the special case that $\varepsilon_{\nu}=1$, implying collision-dominated LTE conditions without scattering, these solutions recover ${ }_{-1}^{n 1}$ the simple case of a homogeneous medium shown in the upper-left graphs of Figs. Setting $a_{\nu}$ to $\sqrt{3}$ then gives:

$$
\begin{aligned}
J_{\nu}\left(\tau_{\nu}\right) & =\left[1-\frac{\mathrm{e}^{-\sqrt{3} \tau_{\nu}}}{1+\left(a_{\nu} / 3\right) \sqrt{3}}\right] B_{\nu, 0}=\left[1-(1 / 2) \mathrm{e}^{-\sqrt{3} \tau_{\nu}}\right] B_{\nu, 0} \\
S_{\nu}\left(\tau_{\nu}\right) & =B_{\nu, 0} \\
H_{\nu}\left(\tau_{\nu}\right) & =\frac{\mathrm{e}^{-\sqrt{3} \tau_{\nu}}}{\sqrt{3}+a_{\nu}} B_{\nu, 0}=\frac{\mathrm{e}^{-\sqrt{3} \tau_{\nu}}}{2 \sqrt{3}} B_{\nu, 0} \\
I_{\nu}^{+}(0, \mu) & =B_{\nu, 0} \\
\mathcal{F}_{\nu}(0) & =\frac{2 \pi}{\sqrt{3}} B_{\nu, 0} .
\end{aligned}
$$

\footnotetext{
${ }^{10}$ Historically, the two-stream approximation was introduced by Schuster $(190 \overline{0})$ 'in his article "Radiation through a foggy atmosphere" (reprinted in 'Menzel 1966) for two-sided isotropy, $I^{+}$and $I^{-}$different but each $\mu$-independent. The Eddington approximation is then exact. Rybicki \&_ Light_man's two-beam_case is the simplest version of what is called the method of discrete ordinates (Mihalas 1970.' $47 \mathrm{ff}$; 'Kourganoff 1952 p. $101 \mathrm{ff}$.)

Without effects from the density stratification; that doesn't matter since we measure optical depths rather than geometrical depths.
} 


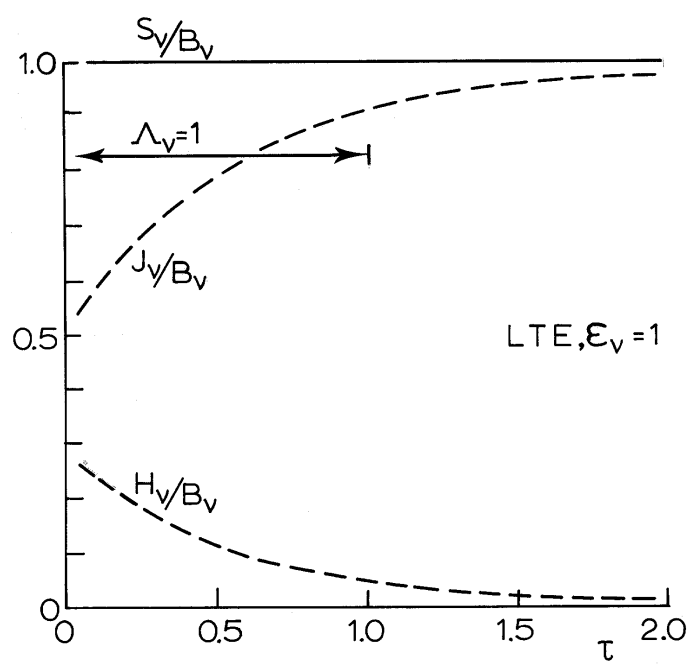

Figure 4.7: Solutions of the transport equation for an isothermal LTE atmosphere as given by $\left(\overline{4} \overline{4} . \overline{7} \overline{2}^{\prime}\right)$ and (4. $\left..6_{1}^{\prime \prime}\right)$. The two dashed curves are the same (apart from the factor 4 in $H_{\nu}=F_{\nu} / 4$ and the approximation

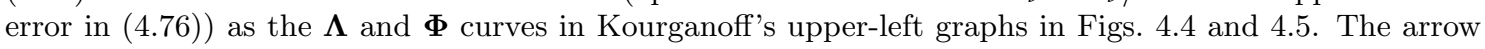

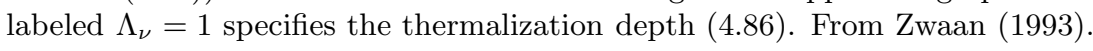

It is illustrated in Fig. $\overline{4} . \overline{7}$, the same as the constant $-B$ panels in Figs. $\overline{4} .4$ and $\overline{4} .5$. . The emergent flux is $\mathcal{F}_{\nu}(0)=(2 / \sqrt{3}) \pi B_{\nu, 0}, 15 \%$ larger than the correct value $\overrightarrow{\mathcal{F}}_{\nu}(0) \stackrel{=}{=} \pi B_{\nu, 0}$. It comes out correct when one takes $a_{\nu}=2$ as in the second Eddington approximation which is indeed exact for isotropic $I_{\nu}^{+}(0)=B_{\nu, 0}$, but than the result for $J_{\nu}(0)$ in (4.72i) becomes incorrect with $J_{\nu}(0) \neq(1 / 2) B_{\nu, 0}$. They can't be both right for one value of $a_{\nu}$ due to the initial assumption of the first Eddington approximation. The latter is also exact at the surface for two-sided isotropy $I_{\nu}^{+}(0)=B_{\nu, 0}$ and $I_{\nu}^{-}(0)=0$, but it is not exactly valid in the subsurface layers that contribute to $J_{\nu}(0)$ and $\mathcal{F}_{\nu}(0)$ through the $\boldsymbol{\Lambda}_{\nu}$ and $\boldsymbol{\Phi}$ weighting.

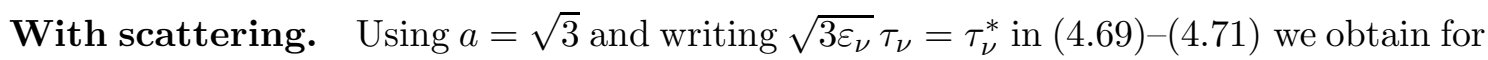
an isothermal atmosphere with elastic scattering (cf. 'R

$$
\begin{aligned}
J_{\nu}\left(\tau_{\nu}\right) & =\left[1-\frac{1}{1+\sqrt{\varepsilon_{\nu}}} \mathrm{e}^{-\tau_{\nu}^{*}}\right] B_{\nu, 0} \\
S_{\nu}\left(\tau_{\nu}\right) & =\left[1-\left(1-\sqrt{\varepsilon_{\nu}}\right) \mathrm{e}^{-\tau_{\nu}^{*}}\right] B_{\nu, 0} \\
H_{\nu}\left(\tau_{\nu}\right) & =\frac{1}{\sqrt{3}} \frac{\sqrt{\varepsilon_{\nu}}}{1+\sqrt{\varepsilon_{\nu}}} \mathrm{e}^{-\tau_{\nu}^{*}} B_{\nu, 0} .
\end{aligned}
$$

Examples of this case (isothermal atmosphere with $b_{\nu}=0$, depth-independent $\varepsilon_{\nu}, a_{\nu}=$ $\sqrt{3})$ are given in the top panels of Figure $\overline{4} . \overline{8}$ and in Table $\overline{4} .21$.

Surface values. The emergent radiation at the surface of an isothermal atmosphere has, again with $a=\sqrt{3}$ :

$$
J_{\nu}(0)=\frac{\sqrt{\varepsilon_{\nu}}}{1+\sqrt{\varepsilon_{\nu}}} B_{\nu, 0}
$$



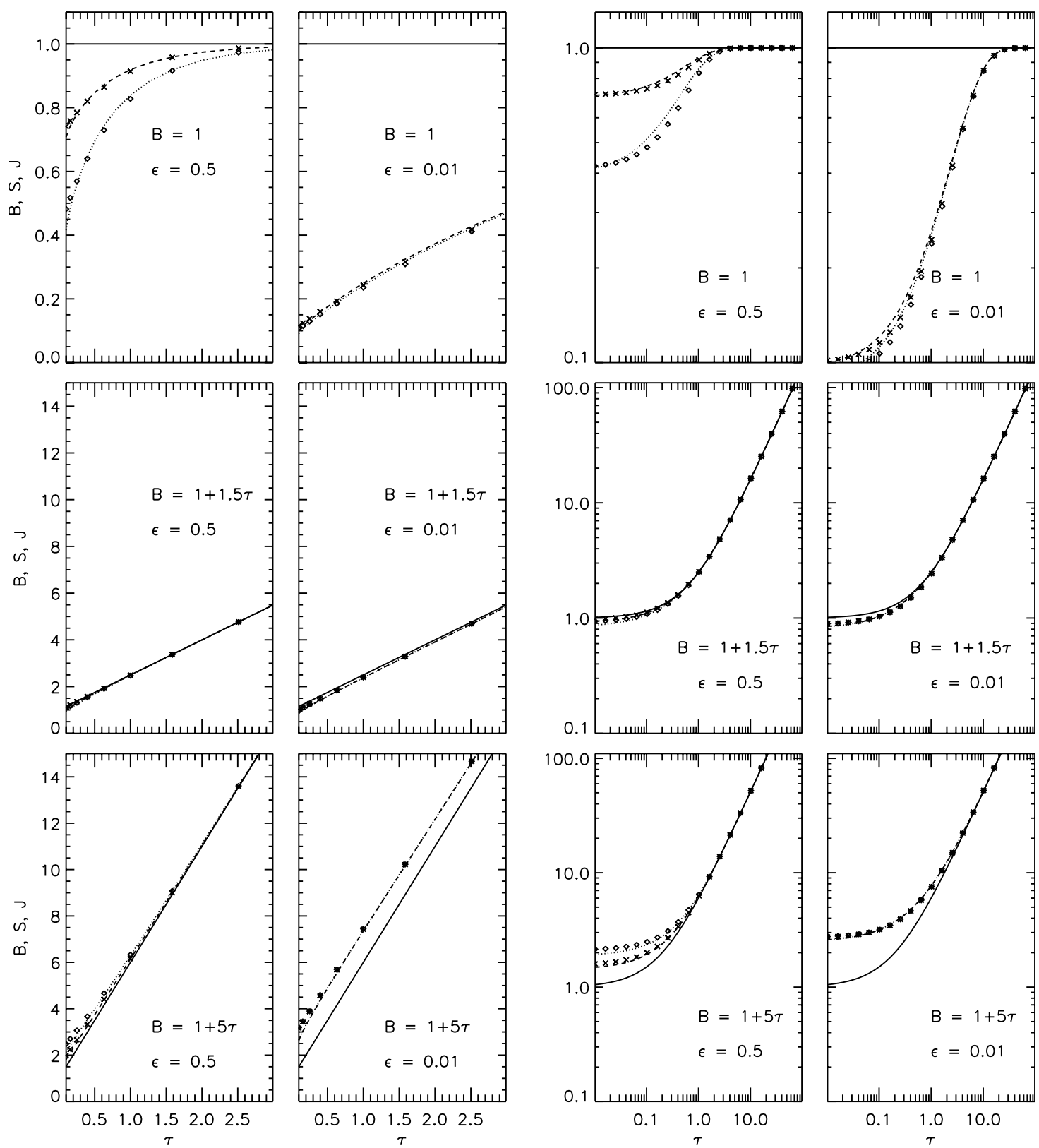

Figure 4.8: Variation of $B, S$ and $J$ with depth in a plane-parallel atmosphere for coherent scattering with depth-independent $\varepsilon$ and linearly depth-dependent $B$ as in Section ' $\overline{4} . \bar{*}$ ', for different combinations of $\varepsilon$ and $\mathrm{d} B / \mathrm{d} \tau$. The relation between $B, S$ and $J$ is given by $(\overline{4} .60$. Lefthand part: numerical results plotted on linear scales. Righthand part: the same results on logarithmic scales. Solid: Planck function B. Dashed: source function $S$. Dotted: mean intensity $J$. Top row: isothermal atmosphere producing $J<B$. Middle row: "radiative-equilibrium gradient" $\mathrm{d} B / \mathrm{d} \tau=1.5$ producing $J \approx B$ (see Section 7.3 .3 on page 156 ). Bottom row: steep inward increase of the Planck function producing $J>B$ (Section 4.3.4'). Diamonds and crosses: corresponding Eddington approximation results from (4.65)-(4.66). The approximations fit the exact solutions very well, implying that the radiation fields in these atmospheres do not get too anisotropical. The exact solutions were obtained through accelerated Lambda iteration as described in Section 5.3 .2 . The linear displays at left follow the format of Figure 14.2', on page '80' and Figure '4.4' on page 83 . The logarithmic versions at right follow the format used for similar demonstration results in Figure 4.9 on page 101 and Figures such as the VALIII ones on page 184 iff. Thijs Krijger production. 


\begin{tabular}{l|ccc|ccc|c|cc}
\hline & \multicolumn{5}{|c|}{ Radiation fields } & \multicolumn{2}{c}{ Limb darkening } \\
\hline & \multicolumn{3}{|c|}{$\varepsilon_{\nu}=1 / 100$} & \multicolumn{3}{c|}{$\varepsilon_{\nu}=9 / 16$} & \multicolumn{2}{c}{$I_{\nu}^{+}(0) / B_{\nu, 0}$} \\
\hline$\tau_{\nu}$ & $J_{\nu} / B_{\nu}$ & $S_{\nu} / B_{\nu}$ & $H_{\nu} / B_{\nu}$ & $J_{\nu} / B_{\nu}$ & $S_{\nu} / B_{\nu}$ & $H_{\nu} / B_{\nu}$ & $\mu$ & $\varepsilon_{\nu}=1 / 100$ & $\varepsilon_{\nu}=9 / 16$ \\
\hline 0 & 0.091 & 0.100 & $5.2510^{-2}$ & 0.429 & 0.750 & 0.274 & 0 & 0.100 & 0.750 \\
0.1 & 0.107 & 0.116 & $5.1610^{-2}$ & 0.498 & 0.781 & 0.217 & 0.1 & 0.115 & 0.779 \\
0.5 & 0.166 & 0.175 & $4.8110^{-2}$ & 0.702 & 0.869 & 0.129 & 0.5 & 0.172 & 0.848 \\
1 & 0.235 & 0.243 & $4.4110^{-2}$ & 0.844 & 0.932 & 0.068 & 1 & 0.233 & 0.891 \\
2 & 0.357 & 0.363 & $3.7110^{-2}$ & 0.958 & 0.981 & 0.018 & & & \\
5 & 0.618 & 0.621 & $2.2110^{-2}$ & 0.999 & 0.9996 & & & & \\
10 & 0.839 & 0.841 & $0.9310^{-2}$ & & & & & & \\
20 & 0.971 & 0.972 & $0.1610^{-2}$ & & & & & & \\
\hline
\end{tabular}

Table_4.2: $J_{\nu}, S_{\nu}$ and $H_{\nu}$ from the Eddington approximation for an isothermal atmosphere $\left(b_{\nu}=0\right)$ given

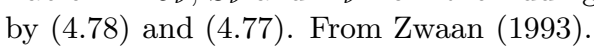

$$
\begin{aligned}
S_{\nu}(0) & =\sqrt{\varepsilon_{\nu}} B_{\nu, 0} \\
I_{\nu}^{+}(0, \mu) & =\frac{1+\mu \sqrt{3}}{1+\mu \sqrt{3 \varepsilon_{\nu}}} \sqrt{\varepsilon_{\nu}} B_{\nu, 0} \\
\mathcal{F}_{\nu}^{+}(0) & =\frac{4 \pi}{\sqrt{3}} \frac{\sqrt{\varepsilon_{\nu}}}{1+\sqrt{\varepsilon_{\nu}}} B_{\nu, 0} .
\end{aligned}
$$

Equation (4.81) is the well-known $\sqrt{\varepsilon}$ law. It actually holds exactly for an isothermal atmosphere with depth-independent $\varepsilon_{\nu}$, making $a_{\nu}=\sqrt{3}$ the right choice here. Note that $J_{\nu}(0)=S_{\nu}(0) /\left(1+\sqrt{\varepsilon_{\nu}}\right)$ varies between $J_{\nu}(0)=B_{\nu, 0} / 2$ for LTE and $J_{\nu}(0) \approx S_{\nu}(0)=$ $\sqrt{\varepsilon_{\nu}} B_{\nu, 0} \ll B_{\nu, 0}$ for small $\varepsilon_{\nu}$. For $\varepsilon_{\nu} \ll 1$ the emergent intensity and flux are correspondingly small:

$$
\begin{aligned}
I_{\nu}^{+}(0,1) & \approx(1+\sqrt{3}) \sqrt{\varepsilon_{\nu}} B_{\nu, 0}=2.7 \sqrt{\varepsilon_{\nu}} B_{\nu, 0} \ll B_{\nu, 0} \\
\mathcal{F}_{\nu}^{+}(0) & \approx \frac{4 \pi}{\sqrt{3}} \sqrt{\varepsilon_{\nu}} B_{\nu, 0}=7.3 \sqrt{\varepsilon_{\nu}} B_{\nu, 0} \ll B_{\nu, 0} .
\end{aligned}
$$

Discussion. The $\sqrt{\varepsilon}$ law $S_{\nu}(0)=\sqrt{\varepsilon_{\nu}} B_{\nu, 0}$ requires discussion. Poetically, it says that when the source of photons (the thermal furnace at large depth) is veiled by an opaque fog, translucent but not transparent, its brilliance is hidden from sight. The basic reason for this lack of emergent photons is that a photon on its way out in the direction to the observer suffers the chance of being scattered back to larger depths, where its random-walk steps are much shorter. It may well be confined there until it is eventually: $\mathbf{L}_{-}^{12}$ destroyed. Thus, photons experience more difficulty in escaping. Compensation would occur if photons would enter from outside the atmosphere and be scattered back into the direction towards the observer, but such photons are absent since $\mathcal{F}_{\nu}^{-}=0$ at the surface.

There is asymmetry between creation and destruction of photons near the surface because photons may leave the star without returning into the thermal pool. The photon

\footnotetext{
${ }^{12}$ Eventually betekent uiteindelijk, niet eventueel; alle Fransen en sommige Hollanders gebruiken dit woord verkeerd. Voor twee-niveau atomen betekent het hier: totdat het foton, met door $\varepsilon_{\nu}$ gegeven waarschijnlijkheid per extinctie, in een deëxciterende botsing het loodje legt. Voor Thomson verstrooiing betekent het hier: totdat het foton in plaats van een vrij elektron een foton-opslokkend atoom of ion ontmoet.
} 
losses to outer space represent an unbalanced "destruction" term in terms of population rates. In LTE the atmosphere doesn't care: the photon leak is simply ignored by requiring thermal equilibrium between photon emission (= creation) and photon extinction $(=$ destruction) all the way out to $\tau_{\nu}=0$. The loss of escaping photons is postulated not to affect the populations, so that the two-level atoms create and destroy photons TE-wise without being aware of the proximity of interstellar space. LTE assumes that photoexcitations do not contribute significantly to the population partitioning between upper and lower level. Equation (2.10 $\overline{1}$ ) on page $\overline{3} \overline{3}$, says that the line source function scales with the ratio of upper-level to lower-level population departure, linearly for the Wien approximation: $S^{l} \approx\left(b_{u} / b_{l}\right) B_{\nu}$. When photoexcitations are actually important, the $\sqrt{\varepsilon}$ law says that the LTE excitation estimate $b_{u} / b_{l}=1$ is much too large by ignoring photon losses.

Dropping the LTE condition means that the transport of photons is coupled selfconsistently with the "transport" of lack of atomic excitations. The scattering part of the source function senses the anisotropy of the radiation field, which increases towards the surface. Scattering also propagates the knowledge of the lack of incoming photons at the surface towards subsurface layers, resulting in $b_{u} / b_{l}<1$ below $\tau_{\nu}=1$. The upper-level population density $n_{u}$ is smaller than it would be in LTE since there is a lack of excitations: $b_{u} \equiv n_{u} / n_{u}^{\mathrm{LTE}}<1$. In a rigorous two-level situation, the lower-level population density $n_{l}$ increases by the same amount ${ }_{1}^{1{ }^{\prime \prime}}\left(\Delta n_{l}=-\Delta n_{u}\right)$ so that $b_{l} \equiv n_{l} / n_{l}^{\text {LTE }}>1$. The source function therefore has $S^{l} \approx\left(b_{u} / b_{l}\right) B_{\nu}<B_{\nu}$.

An appropriate "Gedankenexperiment" is to add a thin layer of two-level atoms with $\varepsilon_{\nu} \ll 1$ on top of the atmosphere. It creates a few additional photons and destroys a few existing ones, but its main effect is that it scatters many more. These came all from below; scattering them isotropically implies that fewer escape. Thus, the scattering impedes the photon flux; extra scattering reduces the emergent intensity. The same must hold for the next thin surface layer that was already present, according to the principle of invariance ('Chandrasekhar 19501$)$. A quantitative analysis of this experiment, producing the $\sqrt{\varepsilon}$ law

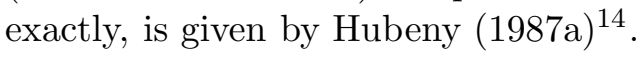

The effect of the surface may also be illustrated by considering the extreme case

\footnotetext{
${ }^{13}$ Note that usually the lower-level increase represents a smaller fraction in $b_{l}=\left(n_{l}^{\mathrm{LTE}}+\Delta n_{l}\right) / n_{l}^{\mathrm{LTE}}$ than the fractional $b_{u}$ decrease $b_{u}=\left(n_{u}^{\mathrm{LTE}}-\Delta n_{u}\right) / n_{u}^{\mathrm{LTE}}$, because lower levels have larger populations due to the Boltzmann factor $n_{l} / n_{u} \sim \exp (h \nu / k T) \gg 1$. However, this is not the case for Rydberg states high in the term diagram. These tend to have $n_{u} \approx n_{l}$. Their Boltzmann populations increase with their principal quantum number $n$ because their statistical weights go as $n^{2}$ while their energy separations are small (they lie at infrared and radio wavelengths). For Rydberg transitions a two-level atom is not the right description anyhow because such high levels are strongly coupled together and are also strongly coupled to the next ion. That coupling is collision dominated because these levels are close in energy; Rydberg transitions therefore have $\varepsilon_{\nu} \approx 1$ (see Section 3.2 .5 on page 50 ' $\mathrm{ff}$ ). Strongly scattering resonance lines (small $\varepsilon_{\nu}$ ) are found at shorter wavelengths, where collisions cannot easily bridge the large upper-lower energy jump $h \nu$. They therefore also have $n_{l} \gg n_{u}$. For two-level resonance lines the NLTE reduction in the population departure ratio therefore has $b_{u} / b_{l} \sim b_{u}$ and $S_{\nu}^{l} \approx b_{u} B_{\nu}$. Real resonance lines tend to have two-level-like outward decreasing $b_{u} / b_{l}$ (increasing drop from unity), corresponding to outward increasing split between $S_{\nu}^{l}=\left(1-\varepsilon_{\nu}\right) J_{\nu}+\varepsilon_{\nu} B_{\nu}$ and $B_{\nu}$ with $S_{\nu}^{l}<B_{\nu}$, while $b_{l}$ and $b_{u}$ each may share an additional variation with height, set by non-two-level effects such as over- or underionization. For an example see Figure 110.21 on page 215

14 "Hubený" means "thin" in Czech. When dividing astronomers between optically thin (grosso modo most radio and X-ray astronomers) and optically thick (near-UV, optical and infrared astronomers), a division which separates astronomers into two distinct classes with a deep chasm in between, Hubený is anything but thin. At Goddard he has developed a major code for stellar-atmosphere radiative transfer that includes large numbers of lines in the ultraviolet. The code is called TLUSTY, meaning "thick" in Czech.
} 
of complete photon losses from an optically thin object. Take the EUV line radiation from a coronal loop as example, schematizing the latter as a hot tenuous isothermal slab above the photosphere. Each EUV photon from the loop is created through collisional excitation followed by spontaneous deexcitation. It flies off into space or it gets absorbed by the photosphere, being lost to the loop in both cases. Since the irradiation of the loop by the much cooler photosphere is negligible at EUV wavelengths, $J_{\nu}$ remains negligible and the two-level EUV line source function is given by $S_{\nu}^{l} \approx\left(b_{u} / b_{l}\right) B_{\nu} \approx \varepsilon_{\nu} B_{\nu}$ with $b_{u} / b_{l} \approx \varepsilon_{\nu} \ll 1$. Thus, all EUV line photons are thermally created but the excitation and the source function remain very far below their LTE values. The ions cannot build up thermal population ratios because there are only collisional excitations, all of those produce photons, and all the photons escape immediately without a chance of thermal destruction. In contrast, a thick atmosphere inevitably has $J_{\nu} \approx B_{\nu}$ at depth and part of all that radiation manages to diffuse outward and escape.

The optically thin case with $S_{\nu} \approx \varepsilon_{\nu} B_{\nu}$ may be used, as in Eq. 1.103 of 'R̂. :- Lightman 1 (1979) to obtain a first-order estimate of the radiation emitted by a thick stellar atmosphere by assuming the emitted amount proportional to the depth $\tau_{\nu}=1$, taken to delineate the volume from which photons escape. First assume large collision frequency so that LTE holds with $S_{\nu}=B_{\nu}$. All photons that leave that volume are collisionally created by the atoms that emit them, just as every photon extinction represents a destruction. If $\varepsilon_{\nu}$ is then reduced from unity to a small value by having fewer collisions at constant particle density, the amount of extinction and the $\tau_{\nu}=1$ depth do not change but the photon creation, given by $\varepsilon_{\nu} B_{\nu}$, goes down by a factor $\varepsilon_{\nu}$. On the other hand, the effective photon-emitting volume is now larger because scattering photons may diffuse outward from below the $\tau_{\nu}=1$ depth and then escape as well. The emitting volume now reaches effectively to about effective optical depth $\tau^{*}=1$ and is of order $1 / \sqrt{\varepsilon_{\nu}}$ larger than the LTE emitting volume. Thus, the total photon emission is roughly diminished by $\varepsilon_{\nu} / \sqrt{\varepsilon_{\nu}}=\sqrt{\varepsilon_{\nu}}$.

\subsubsection{Thermalization depth}

Figures the outer part of an isothermal atmosphere. It reaches $B_{\nu}$ only near $\tau_{\nu}=1$ for the LTE case, and near $\tau_{\nu}=1 / \sqrt{\varepsilon_{\nu}}$ for $\varepsilon_{\nu}<1$. This behavior stems from the $\exp \left(-\sqrt{3 \varepsilon_{\nu}} \tau_{\nu}\right)$ term in (4.65), which also affects the source function when $\varepsilon_{\nu} \neq 1$ as seen in (4.66i). Physically, these patterns reflect the photon mean free path, the effective mean free path and their relative scaling by $\sqrt{\varepsilon_{\nu}}$ in $(\overline{2} . \overline{1} \overline{3} \overline{1})$ on page $\overline{4} \overline{0}$. The mean intensity drops where photons escape, the more so when that also occurs via scattering sequences. We therefore introduce the thermalization depth $\Lambda_{\nu}$

$$
\Lambda_{\nu}=1 / \sqrt{\varepsilon_{\nu}}
$$

as the location where the radiation field $J_{\nu}$ "thermalizes" $\left(J_{\nu} \approx B_{\nu}\right)$ in units of optical depth!-

Effectively thick regime. For $\tau_{\nu}>\Lambda_{\nu}$ the radiation field is thermalized. At these depths, the overlying atmosphere is not only non-transparent but also non-translucent

\footnotetext{
${ }^{15}$ This definition holds only for depth-independent $\varepsilon_{\nu}$ as is assumed in this whole section. In a real atmosphere, $\varepsilon_{\nu}$ increases roughly exponentially with geometrical depth, along with the particle density. The thermalization depth is then loosely defined as the depth at which $J_{\nu}$ reaches $B_{\nu}$ as seen from outside, or where $J_{\nu}$ starts deviating from $B_{\nu}$ as seen from inside.
} 
for random-walk scattering sequences since it is not only optically thick $\left(\tau_{\nu}>1\right)$, but effectively thick $\left(\sqrt{\varepsilon_{\nu}} \tau_{\nu}>1\right)$ as well. At large depth, photons are fully enclosed by local matter so that the photon energy partitioning is fully coupled to the local matter energy partitioning (Maxwell and Saha-Boltzmann; $S_{\nu} \approx B_{\nu}$ ). The photons are "honorary particles" of the gas, just as confined to the local conditons as the more massive particles.

Optically thick regime. For $1<\tau_{\nu}<\Lambda_{\nu}$ the overlying atmosphere is effectively thin. It is not transparent, but, as a fog, effectively translucent; photons may diffuse through it. Likewise, the information about a non-irradiated surface (anisotropy in $I_{\nu}$ ) diffuses inward. When scattering dominates the source function, the latter follows $J_{\nu}$ and expresses the lack of incoming photons as lack of excitations $\left(b_{u}<1\right)$. When collisions dominate, $\Lambda_{\nu} \approx 1$ without difference between opacity and effective opacity.

Optically thin regime. Only for $\tau_{\nu}<1$ are the overlying layers transparent. Photons may escape immediately when emitted in directions $\mu>0$ from depths $\tau_{\nu} \lesssim \mu$. When $\varepsilon_{\nu}$ is small, the Eddington-Barbier depth $\tau_{\nu}=\mu$ with $S_{\nu}\left(\tau_{\nu}=\mu\right) \approx(1+\mu \sqrt{3}) \sqrt{\varepsilon_{\nu}} B_{\nu, 0}$ characterizes their last interaction, not the location where they were created originally. The latter location lies, characteristically, at $\tau_{\nu}=\Lambda_{\nu}$.

\subsubsection{Gradients and splits}

We now return to the more general case of a non-isothermal atmosphere, with $b_{\nu} \neq 0$ in $(4.65)-(4.68)$. The concept of thermalization depth $\Lambda_{\nu}$ remains valid, with the same meaning, but we now find that the sign of the outward split between $J_{\nu}$ and $B_{\nu}$ depends on the ratio $b_{\nu} / B_{\nu, 0}$. For the LTE case in Fig. 4.4 this $J_{\nu}-B_{\nu}$ sign sensitivity is already present. Scattering enhances the magnitude of the split between $J_{\nu}$ and $B_{\nu}$. From (4.65i) the difference is given by:

$$
\frac{J_{\nu}\left(\tau_{\nu}\right)-B_{\nu}\left(\tau_{\nu}\right)}{B_{0, \nu}}=\frac{a_{\nu} b_{\nu} / 3 B_{0, \nu}-1}{1+\left(a_{\nu} / 3\right) \sqrt{3 \varepsilon_{\nu}}} \mathrm{e}^{-\sqrt{3 \varepsilon_{\nu}} \tau_{\nu}},
$$

with $J_{\nu} \approx B_{\nu}$ for $\tau_{\nu} \gg 1$ and $J_{\nu}(0)=B_{\nu, 0} / 2$ for $b_{\nu}=0, \varepsilon_{\nu}=1$ and $a_{\nu}=\sqrt{3}$. For small $\varepsilon_{\nu} \ll 1$ and again writing $\tau_{\nu}^{*}=\sqrt{3 \varepsilon_{\nu}} \tau_{\nu}$ we obtain

$$
J_{\nu}\left(\tau_{\nu}\right)-B_{\nu}\left(\tau_{\nu}\right) \approx\left[a_{\nu} b_{\nu} / 3-B_{\nu, 0}\right] \mathrm{e}^{-\tau_{\nu}^{*}}
$$

with $J_{\nu}(0) \approx 0$ for $b_{\nu}=0$. The $J_{\nu} \neq B_{\nu}$ split is zero when the Planck function has $b_{\nu} / B_{0}=3 / a_{\nu}$, that is, when $b_{\nu} / B_{0}=1.5$ for $a_{\nu}=2$ and $b_{\nu} / B_{0}=1.7$ for $a_{\nu}=\sqrt{3}$. In that case $J_{\nu}=B_{\nu}$ at all depths, for any value of $\varepsilon_{\nu}$, and $S_{\nu}=J_{\nu}=B_{\nu}$. This case is shown in the upper-right panel of Figure 4.4 . Figure approximately constant with depth. Thus, if a star wants to deliver constant flux at some frequency through its atmosphere, it has to adjust its temperature stratification to obtain $b_{\nu} / B_{\nu, 0} \approx 1.5$ at that frequency (Section 77.3 .21 on page $115 \overline{3}_{1}^{\prime}$ ).

A flatter $B_{\nu}$ gradient with $b_{\nu}<3 B_{\nu, 0} / a_{\nu}$ produces $J_{\nu}<B_{\nu}$, whereas a steeper $B_{\nu}$ gradient produces $J_{\nu}>B_{\nu}$. In the first case, the production of photons in deeper layers is not sufficient to maintain $J_{\nu} \approx B_{\nu}$ higher up; in the second case, there are more photons arriving from deeper layers than given by the local TE prediction. The size of the split depends on $\varepsilon_{\nu}$, for example varying for $b_{\nu}=0$ and $a_{\nu}=\sqrt{3}$ between $J_{\nu}(0) \approx$ $-0.5 B_{\nu}(0)+B_{\nu}(0)=0.5 B_{\nu}(0)$ for $\varepsilon_{\nu} \approx 1$ and $J_{\nu}(0) \approx-B_{\nu}(0)+B_{\nu}(0)=0$ for $\varepsilon_{\nu} \approx 0$ in $\left(\overline{4}_{1}^{4} . \overline{7}_{1}\right)$ and $(4.80)$. So does its depth scaling, since the split extends to $\tau_{\nu} \approx \Lambda_{\nu}$. 

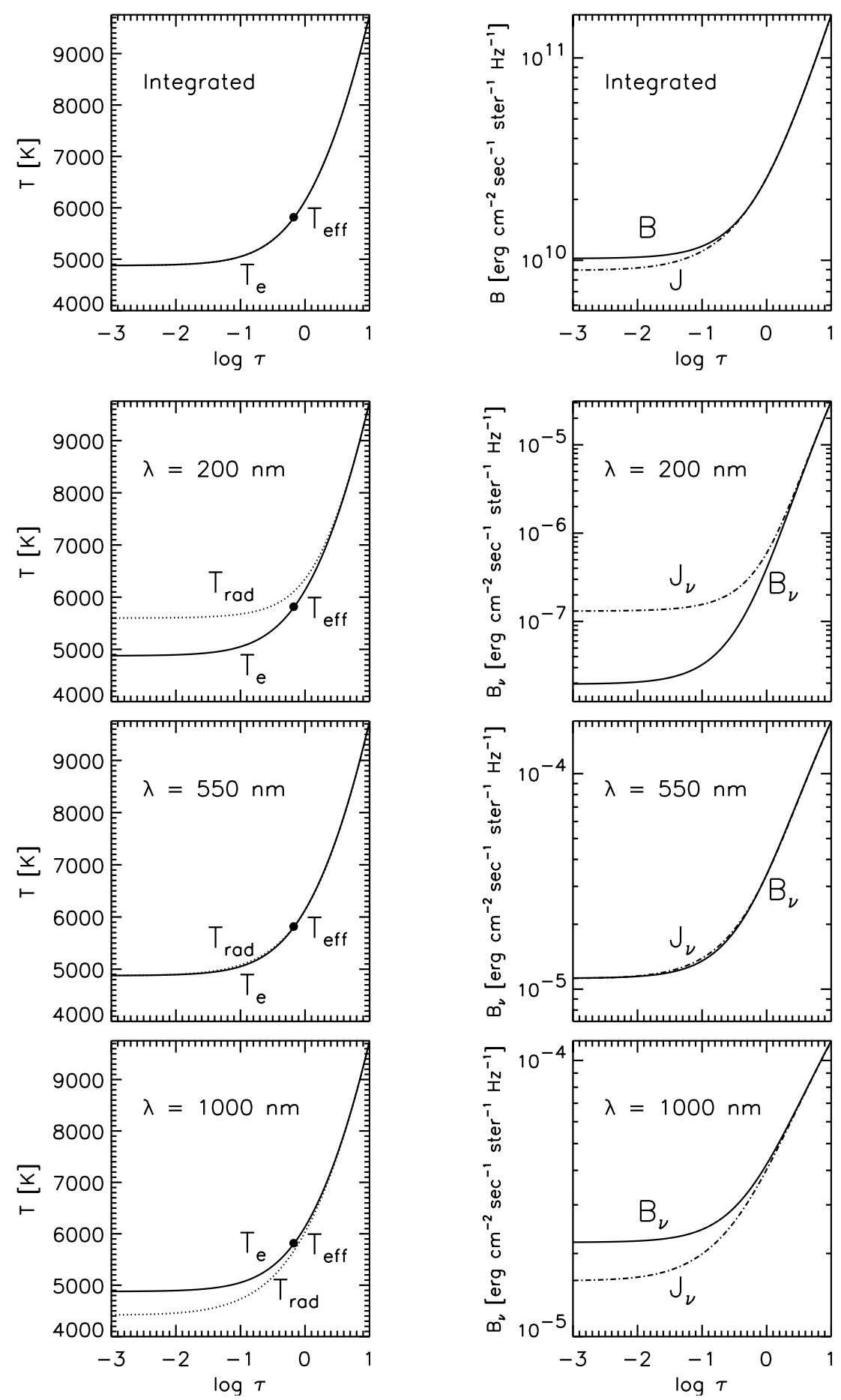

Figure 4.9: $J_{\nu}-B_{\nu}$ continuum splits for a stellar atmosphere in LTE with the solar effective temperature

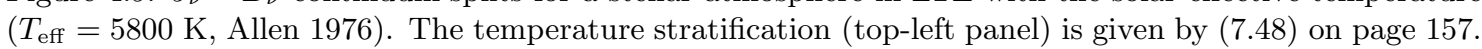
The top-right panel shows the corresponding spectrum-integrated variations of the total source function $S=B$ and the total mean intensity $J$. The atmosphere is close to radiative equilibrium $(S=J)$. The other panels show the corresponding variations of $B_{\nu}$ and $J_{\nu}$ at three wavelengths. The corresponding temperature representations in the lefthand panels enable comparison on a fixed ordinate scale. Thijs Krijger production. 
Continuum splits. Splits between $J_{\nu}$ and $B_{\nu}$ as in (4.87) occur across the spectrum even in LTE situations because the Planck function sensitivity to temperature varies with wavelength. The temperature-depth relations that are characteristic for stellar photospheres are set by radiative equilibrium in the part of the spectrum where the stars radiate the bulk of their energy away, for solar-type stars in the visible. We will discuss the physics of radiative equilibrium in more detail in Chapter $\bar{\tau}_{1}$, but the discussion above already shows that flux constancy requires $b_{\nu} / B_{\nu, 0} \approx 1.5$ for an LTE atmosphere with linear depth dependence $B_{\nu}=B_{\nu, 0}+b_{\nu} \tau_{\nu}$. If the temperature gradient is set in this manner in the visible, it produces $J_{\nu}(0) \approx B_{\nu}(0)$ there. In the infrared, however, the Planck function sensitivity to temperature $\partial B_{\nu} / \partial T$ is much weaker so that the given temperature gradient results in a much flatter Planck function gradient $\mathrm{d} B_{\nu} / \mathrm{d} \tau_{\nu}$, producing $J_{\nu}(0)<B_{\nu}(0)$. Likewise, setting the gradient to $b_{\nu} / B_{\nu, 0} \approx 1.5$ in the visible produces $J_{\nu}(0)>B_{\nu}(0)$ in the ultraviolet where $\partial B_{\nu} / \partial T$ is much larger.

Figure 4.91 illustrates such continuum splits at three different wavelengths for an LTE atmosphere which is close to radiative equilibrium. The $\partial B_{\nu} / \partial T$ sensitivity is evident from the large differences between the ordinate scales at right. It does not affect the

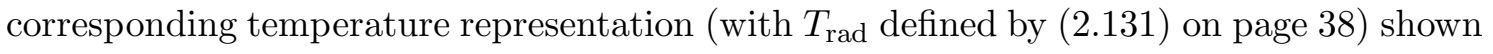
in the lefthand panels, so that these have the same ordinate scale.

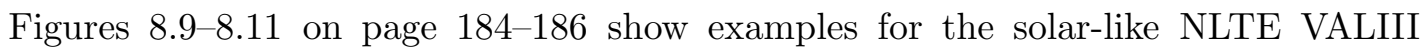
atmosphere. The upper-right panel in Figure 10.10 has $J_{\nu} \approx B_{\nu}$ in the region where the continuum at $\lambda=500 \mathrm{~nm}$ escapes, representative of the bulk of the emergent flux. The lower-right panel of Figure $\bar{s} .9^{\prime}$ shows the corresponding $J_{\nu}(0)<B_{\nu}(0)$ behavior in the infrared while the lower panels of Figure 8.10 show that $J_{\nu}(0)>B_{\nu}(0)$ in the ultraviolet. The split in the lower-right panel of Figure 8.100 exhibits departure from LTE in its deep thermalization $\left(\log \tau_{\nu}^{c}\left(\Lambda_{\nu}\right)>0\right)$.

Overionization. Often, departures from LTE line formation in stellar atmospheres have to do with $J_{\nu} \neq B_{\nu}$ splits in the ultraviolet bound-free continua of minority ionization stages. For these, such overabundance of radiation leads to overionization of the minority stage ${ }_{-}^{\prime \prime b_{1}^{\prime \prime}}$ which may affect the opacities of all lines since these scale with $b_{l}$. A classic example is the solar Fe I spectrum as computed by Athay and Lites $(1972)$ '. They found that most Fe I levels are underpopulated in the upper photosphere because the ultraviolet $J_{\nu}-B_{\nu}$ excesses in the Fe I bound-free edges produce radiative overionization. The lowerright panel of the VALIII plot Figure 8.10 on page 185i holds for such an Fe I edge, as indicated by the top part of the plot. As a result, all Fe I lines, also those in the visible and infrared, have smaller opacity than LTE would predict.

\footnotetext{
${ }^{16} \mathrm{~A} J_{\nu}>B_{\nu}$ radiation excess may also produce overionization for the majority ionization stage of an element, but in that case the line opacities will not be much affected. A change in the net rate to the next stage is not important if that whole stage is not important.
} 

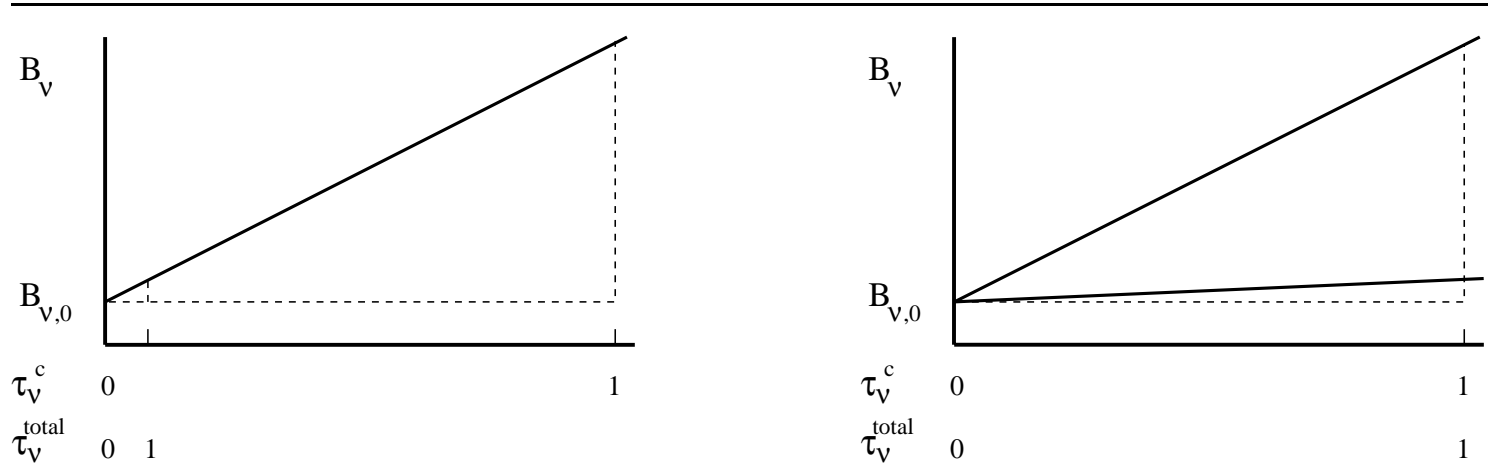

Figure 4.10: Schematic illustration of the flat Planck function gradient experienced by strong lines. The stellar atmosphere property that the total flux does not vary with height (radiative equilibrium) requires a temperature gradient $\mathrm{d} B_{\nu} / \mathrm{d} \tau_{\nu}^{c} \approx 1.5 B_{\nu, 0}$ near the frequency of maximum flux (lower-left panel of Figure $4 . \bar{y}^{\prime}$

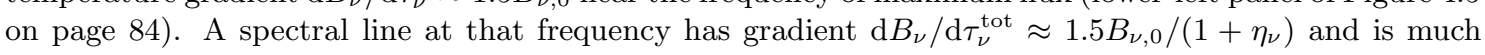
shallower for $\eta \gg 1$, as demonstrated in the righthand panel by plotting $B_{\nu}\left(\tau_{\nu}\right)$ separately for equal $\Delta \tau_{\nu}^{c}$ and $\Delta \tau_{\nu}^{\text {tot }}$ segments.

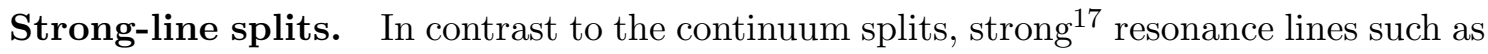
the hydrogen and helium Lyman lines in hot stars and metal resonance lines as Mg II h \& $\mathrm{k}$ in cool stars always have $J_{\nu}<B_{\nu}$ and therefore $S_{\nu}^{l}<B_{\nu}$, also at short wavelengths. The reason is that they "see" the Planck function gradient much flatter than the continuum does. Again, when the temperature-depth relation is set by radiative equilibrium, it

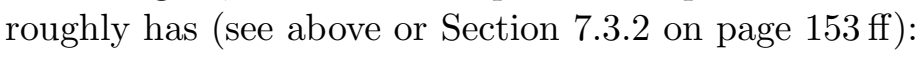

$$
B_{\nu}\left(\tau_{\nu}^{c}\right) \approx B_{\nu, 0}\left(1+1.5 \tau_{\nu}^{c}\right)
$$

in the wavelength region where the stellar flux peaks. Strong spectral lines have additional opacity $\alpha_{\nu}^{l}$ that is much larger than the continuum opacity $\alpha_{\nu}^{c}$, with $\eta_{\nu} \equiv \alpha_{\nu}^{l} / \alpha_{\nu}^{c}$ as large as $10^{6}$. At their wavelength the Planck function gradient on the total optical depth scale

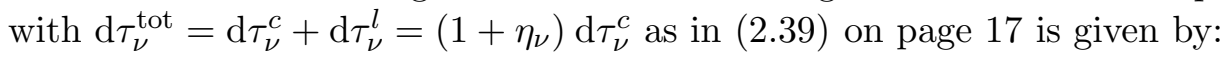

$$
B_{\nu}\left(\tau_{\nu}^{\mathrm{tot}}\right) \approx B_{\nu, 0}\left(1+\frac{1.5}{1+\eta_{\nu}} \tau_{\nu}^{\mathrm{tot}}\right) .
$$

For very strong lines with $\eta_{\nu} \gg 1$ the Planck function gets very flat the way they see it

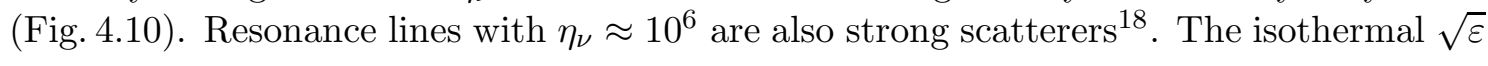

\footnotetext{
${ }^{17}$ When physicists talk about strong lines they mean large $A_{u l}$. When astronomers talk about strong lines they mean large relative observed intensity for emission lines $\left(I_{\nu_{0}} \gg I_{\nu}^{\text {cont }}\right)$ or large relative observed line depth for absorption lines $\left(I_{\nu}^{\text {cont }}-I_{\nu_{0}} \ll I_{\nu}^{\text {cont }}\right.$ ); usually, they mean large equivalent width (Figure $1.2_{1}^{\prime}$ on page continuum opacity $\alpha_{\nu}^{c}$ so that $\eta_{\nu} \equiv \alpha_{\nu}^{l} / \alpha_{\nu}^{c} \gg 1$. Large transition probability helps to make lines strong, but large line strength also requires large abundance of the element and a large population fraction in the lower level of the line. Lines may be astronomically strong without being physically strong and the other way around. The strongest lines of an element are the resonance lines out of the ground level of the dominant ionization stage. In the visible part of the solar spectrum the Ca II H \& K lines are by far the strongest ones because the dominant stages of the more abundant elements (H I, He I, C I, N I, O I, Mg II, Si II, S II L have their resonance lines in the ultraviolet. Sodium has the same solar abundance as calcium (Table 1.1, on page 144) and is also largely ionized, so that the Na ID lines are far weaker than $\mathrm{H} \& \mathrm{~K}$. Hydrogen is much more abundant and is not ionized, but the $\mathrm{H} \alpha$ line suffers from the small Boltzmann population factor of its $n=2$ lower level.

${ }^{18}$ Due to the combination of their large intrinsic transition probability (being resonance lines, they have $f_{l u} \approx 1$ ) and their large opacity (product of large $f_{l u}$, large Saha-Boltzmann population fraction and
} 
law therefore applies very well to them, so that their line-center intensity is given by (4.82i) on page $\overline{9} \overline{\overline{7}}$, far below the limiting LTE value $I_{\nu}^{+}(0, \mu) \approx B_{\nu, 0}$. Thus, strong scattering lines have very dark cores even at wavelengths for which the $B_{\nu}\left(\tau_{\nu}^{c}\right)$ gradient is steep (to the short wavelength side of the stellar flux peak). Figure 10.20 on page 212 for the solar NaI D lines.

\subsubsection{Applications}

Resonance lines. The solutions in the previous section describe monochromatic radiative transfer of photons that are created thermally and that, at the end of a scattering sequence, are either destroyed back into the thermal pool or leave the star (a highly non-local non-thermal process). The description is valid for coherently scattering lines of

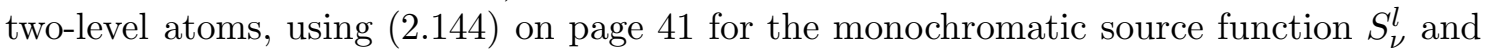
$\left(\overline{3} \cdot 9 \overline{7}_{i}\right)$ for the monochromatic destruction probability $\varepsilon_{\nu}$. It may also be seen as describing monochromatic transfer for the non-broadened two-level lines described by ( $3 . \overline{9} \overline{4})$ on page $6 \overline{8}$. This simple case holds well for actual resonance lines if they are very strong (both large transition probability and large opacity). Schematic examples are shown in Figure $\overline{4}_{1}^{-1} \overline{1}$ and discussed in Exercise $\overline{6}_{1}^{\prime}$ on page $2 \overline{2} \overline{2} \overline{9}_{r}^{\prime}$ The formation of the VALIII Na I D lines is shown in Figure 10.0

Lines with thermal background continuum. Real stars do not consist of two-level atoms alone. Photon creation and photon destruction can also be achieved by the continuum processes that occur at the wavelength of a spectral line. For example, the background continuum in the visible solar spectrum is caused mainly by $\mathrm{H}^{-}$bound-free extinction which represents an LTE process to a high degree of accuracy. A photon at the line frequency may therefore be destroyed by converting ("ionizing") a $\mathrm{H}^{-}$ion into a neutral hydrogen atom. If $\alpha_{\nu}^{c}$ is made up by such LTE processes, the monochromatic probability of such continuum destruction per extinction is given by the ratio

$$
\delta_{\nu} \equiv \frac{\alpha_{\nu}^{c}}{\alpha_{\nu}^{l}+\alpha_{\nu}^{c}}
$$

Using the line-to-continuum extinction ratio

$$
\eta_{\nu} \equiv \frac{\alpha_{\nu}^{l}}{\alpha_{\nu}^{c}}
$$

so that $\delta_{\nu}=1 /\left(1+\eta_{\nu}\right)$, the combined photon destruction probability $\lambda_{\nu}$ per extinction for coherently scattering two-level atoms in an atmosphere with LTE background continuum processes is

$$
\lambda_{\nu}=\delta_{\nu}+\left(1-\delta_{\nu}\right) \varepsilon_{\nu}=\varepsilon_{\nu}+\left(1-\varepsilon_{\nu}\right) \delta_{\nu}=\frac{\alpha_{\nu}^{c}+\varepsilon_{\nu} \alpha_{\nu}^{l}}{\alpha_{\nu}^{c}+\alpha_{\nu}^{l}}=\frac{1+\varepsilon_{\nu} \eta_{\nu}}{1+\eta_{\nu}},
$$

where the first version sums continuum destruction and collisional destruction of the remaining photons, the second vice-versa. The total monochromatic source function is given

large element abundance). The large $A_{u l}$ gives them two-level character. The large opacity causes high formation, with $\tau_{\nu}^{\text {tot }}=1$ far out in the atmosphere where collisions are scarce due to the small density so that $\varepsilon_{\nu} \approx C_{u l} /\left(A_{u l}+C_{u l}\right) \ll 1$. 

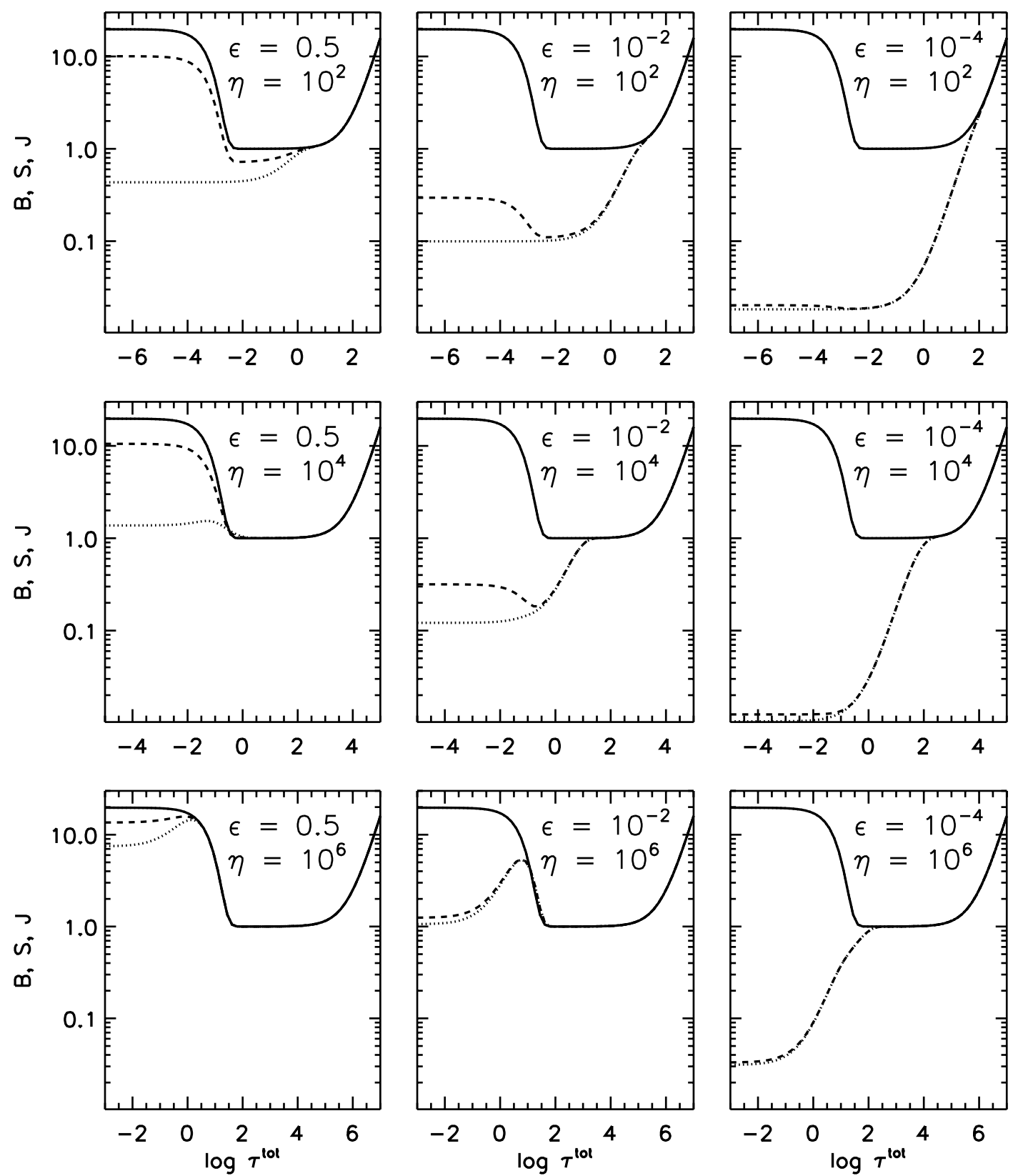

Figure 4.11: Schematic formation of resonance lines in a stellar atmosphere with a chromospheric temperature rise. The Planck function stratification is defined by $B=1+1.5 \tau^{c}+18.7 \exp \left(-14.410^{4} \tau^{c}\right)$ to have a radiative-equilibrium gradient $\mathrm{d} B / \mathrm{d} \tau^{c}=1.5$ in the layers below $\tau^{c}=10^{-4}$ and to reflect an outward temperature rise above this height, rather like the VALIII atmosphere tabulated on page 182. Each panel shows the variation of $B, S$ and $J$ for coherent scattering with depth-independent $\varepsilon$ and depth-independent line strength $\eta=\alpha^{l} / \alpha^{c}$ as specified, against total optical depth $\tau^{\text {tot }}=(1+\eta) \tau^{c}$. The x-axis scales are shifted over $\log \eta$ per row in order to keep the $B\left(\tau^{c}\right)$ stratification in place. Solid: Planck function $B$ in arbitrary units. Dashed: source function $S$. Dotted: mean intensity $J$. The relation between $B, S$ and $J$ is given by (4.60 
by:

$$
\begin{aligned}
S_{\nu}^{\text {tot }} & =\frac{S_{\nu}^{c}+\eta_{\nu} S_{\nu}^{l}}{1+\eta_{\nu}}=\frac{B_{\nu}+\eta_{\nu}\left[\left(1-\varepsilon_{\nu}\right) J_{\nu}+\varepsilon_{\nu} B_{\nu}\right]}{1+\eta_{\nu}} \\
& =\left(1-\lambda_{\nu}\right) J_{\nu}+\lambda_{\nu} B_{\nu} \\
& =\left(1-\lambda_{\nu}\right) J_{\nu}+\left(\lambda_{\nu}-\varepsilon_{\nu}\right) B_{\nu}+\varepsilon_{\nu} B_{\nu} .
\end{aligned}
$$

The last version shows that the new photons that are added to the beam per extinction are made up by scattered line photons (first term), photons fed from the continuum into the line (second term), and collisionally created photons (third term). Version (4.94) regains the form of (

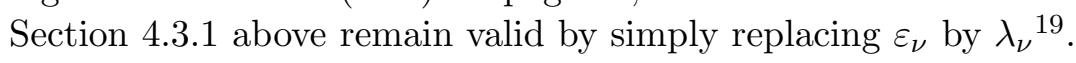

Thermalization depth. The monochromatic thermalization depth $\Lambda_{\nu}$ for a coherently scattering line with an LTE background continuum is similarly rewritten by using the combined photon destruction probability $\lambda_{\nu}$ in the place of $\varepsilon_{\nu}$ into

$$
\Lambda_{\nu}=\frac{1}{\sqrt{\lambda_{\nu}}}=\sqrt{\frac{1+\eta_{\nu}}{1+\varepsilon_{\nu} \eta_{\nu}}}
$$

with $\Lambda_{\nu} \geq 1$ and $\Lambda_{\nu} \gg 1$ for $\varepsilon_{\nu} \ll 1$ and $\eta_{\nu} \gg 1$. This depth is measured in units of the total optical depth scale $\tau_{\nu}^{\text {tot }}$ with $\mathrm{d} \tau_{\nu}^{\text {tot }}=\mathrm{d} \tau_{\nu}^{c}+\mathrm{d} \tau_{\nu}^{l}=\left(1+\eta_{\nu}\right) \mathrm{d} \tau_{\nu}^{c}$ as in (2.39i) on page $1 \overline{1} \bar{z}_{-}$. The value of the continuum optical depth $\tau_{\nu}^{c}$ that corresponds to the thermalization depth is:

$$
\tau_{\nu}^{c}\left(\Lambda_{\nu}\right)=\frac{1}{1+\eta_{\nu}} \Lambda_{\nu}=\frac{1}{\left[\left(1+\eta_{\nu}\right)\left(1+\varepsilon_{\nu} \eta_{\nu}\right)\right]^{1 / 2}}
$$

with $\tau_{\nu}^{c}\left(\Lambda_{\nu}\right) \ll 1$ for $\eta_{\nu} \gg 1$. It moves inward for smaller $\varepsilon_{\nu}$. For $\eta_{\nu} \gg 1$ the extremes are given by $\operatorname{LTE}\left(\varepsilon_{\nu} \approx 1\right)$, for which the location of the thermalization is given by

$$
\tau_{\nu}^{c}\left(\Lambda_{\nu}\right) \approx 1 / \eta_{\nu} \approx \tau_{\nu}^{c}\left[\tau_{\nu}^{\mathrm{tot}}=1\right]
$$

and by strong scattering with $\varepsilon_{\nu} \ll 1 / \eta_{\nu}$ for which

$$
\tau_{\nu}^{c}\left(\Lambda_{\nu}\right) \approx 1 / \sqrt{\eta_{\nu}} \approx \tau_{\nu}^{c}\left[\tau_{\nu}^{\mathrm{tot}}=\sqrt{\eta_{\nu}}\right]
$$

Thus, the continuous destruction processes limit the large thermalization depth that coherent scattering would otherwise put at $\tau_{\nu}=\Lambda_{\nu}=1 / \sqrt{\varepsilon_{\nu}}$. For $\eta_{\nu} \approx 1$ the thermalization depth varies between $\tau_{\nu}^{c}\left(\Lambda_{\nu}\right)=1$ and $\tau_{\nu}^{c}\left(\Lambda_{\nu}\right)=1 / 2$, depending on the amount of scattering. For $\eta_{\nu} \ll 1$ the thermalization depth is fully set by the continuum processes at $\tau_{\nu}^{c}\left(\Lambda_{\nu}\right)=1$. For coherently scattering lines the thermalization depth therefore varies across the profile between $\tau_{\nu}^{c}\left(\Lambda_{\nu}\right) \approx 1 / \sqrt{\eta_{\nu}}$ and $\tau_{\nu}^{c}\left(\Lambda_{\nu}\right) \approx 1$.

Continuum scattering. The treatment above for coherent two-level scattering with a background continuum also describes the case of elastic Thomson and/or Rayleigh scattering in continua. The creation and destruction processes are then physically different

\footnotetext{
${ }^{19}$ On the condition that $\lambda_{\nu}$ does not vary with depth, since the demonstration required that $\varepsilon_{\nu}$ is constant with depth. Either assumption is unrealistic. Solar examples of the actual depth-variation of $\eta_{\nu}$ are shown in Figure $9.2,10$ page 207
} 
from the scattering process. A free electron and a photon cannot destroy or create each other when they meet, only redistribute their combined energy and momentum between them. In the low-energy elastic limit, Thomson scattering only redirects existing photons. The same holds for Rayleigh scattering. The scattered photons are created by other interactions (bound-free or free-free) and are similarly destroyed eventually if they don't leave the star:- The Thomson and Rayleigh cross-sections are nearly isotropic (phase function of the form $\left.1+\cos ^{2} \theta\right)$; the two-level source function for coherent scattering in (2.144) on page $\overline{4}_{1}^{1} \overline{1}$, therefore also describes such elastic continuum scattering quite well. For the

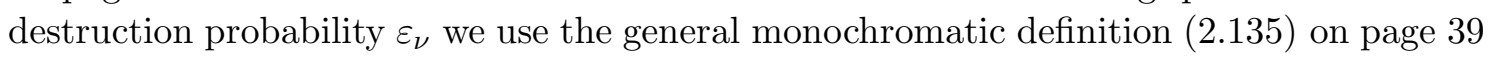
and redefine it for continuum processes as:

$$
\varepsilon_{\nu} \equiv \frac{\sum \alpha_{\nu}^{\mathrm{bf}}+\sum \alpha_{\nu}^{\mathrm{ff}}}{\alpha_{\nu}^{\mathrm{T}}+\alpha_{\nu}^{\mathrm{R}}+\sum \alpha_{\nu}^{\mathrm{bf}}+\sum \alpha_{\nu}^{\mathrm{ff}}}
$$

where the assumption is made that the bound-free processes are thermal, as is the case for $\mathrm{H}^{-}$extinction. The demonstration results of Section 4.3.1' then apply to this case as well.

Bound-bound redistribution. For the case of complete redistribution over the line profile of a scattering line with a thermal background continuum the term $J_{\nu}$ in (4.94) is replaced by $\bar{J}_{\nu_{0}}, \varepsilon_{\nu}$ in is replaced by $\varepsilon_{\nu_{0}}$, and $\delta_{\nu}(4.93)$ is replaced by its profile-averaged value

$$
\delta_{\nu_{0}} \equiv \int_{0}^{\infty} \delta_{\nu} \varphi\left(\nu-\nu_{0}\right) \mathrm{d} \nu=\int_{0}^{\infty} \frac{\varphi\left(\nu-\nu_{0}\right)}{1+\eta_{\nu_{0}} \varphi\left(\nu-\nu_{0}\right)} \mathrm{d} \nu=\frac{1}{\eta_{\nu_{0}}} \int_{0}^{\infty} \frac{\eta_{\nu_{0}} \varphi\left(\nu-\nu_{0}\right)}{1+\eta_{\nu_{0}} \varphi\left(\nu-\nu_{0}\right)} \mathrm{d} \nu,
$$

using $\delta_{\nu}=1 /\left(1+\eta_{\nu}\right)$ and defining $\eta_{\nu_{0}} \equiv \int \eta_{\nu} \mathrm{d} \nu$ with dimension $\mathrm{Hz}$, so that $\eta_{\nu}=\eta_{\nu_{0}} \varphi\left(\nu-\nu_{0}\right)$ and $\eta_{\nu_{0}}=\left(h \nu_{0} / 4 \pi\right)\left(n_{l} B_{l u}-n_{u} B_{u l}\right) / \alpha_{\nu_{0}}^{c}$ as follows by dividing left, middle and right of $\left(\overline{2} .64{ }^{4}\right)$ on page $\overline{2} \overline{3}_{1}^{\prime}$ by $\alpha_{\nu}^{c}$ and assuming the latter constant (or linearly varying) over the extent of the line. The profile-summed (or profile-averaged) total destruction probability $\lambda_{\nu_{0}}$ is then given by

$$
\lambda_{\nu_{0}} \equiv \int_{0}^{\infty} \lambda_{\nu} \varphi\left(\nu-\nu_{0}\right) \mathrm{d} \nu=\varepsilon_{\nu_{0}}+\left(1-\varepsilon_{\nu_{0}}\right) \delta_{\nu_{0}}
$$

and the total source function $S_{\nu_{0}}^{\text {tot }}$ for two-level scattering with complete redistribution on a thermal background is given by

$$
S_{\nu_{0}}^{\text {tot }}=\left(1-\lambda_{\nu_{0}}\right) \bar{J}_{\nu_{0}}+\lambda_{\nu_{0}} B_{\nu_{0}}
$$

This source function is more complex than the coherent-scattering case in (14.94i) or in Section $4 . \overline{3}$ i' because it obtains its frequency independence by depending on $\bar{J}_{\nu}$ at all

\footnotetext{
${ }^{20}$ The continuum processes are discussed in Chapter $\overline{8}$. In hot-star photospheres, the photon creation and destruction is primarily by HI bound-free transitions, for example the Paschen continuum for the visible $\lambda=365-821 \mathrm{~nm}$ wavelength region. Compared to cool stars, Thomson scattering replaces $\mathrm{H}^{-}$as major source of extinction because $\mathrm{H}^{-}$vanishes at higher $T_{\text {eff }}$ while partial hydrogen ionization frees many electrons. Rayleigh scattering by hydrogen atoms is important in the ultraviolet for cool stars; very_ cool stars also suffer from Rayleigh scattering by molecules. For Thomson scattering in hot stars, 象ybicki and Hummer (1994)! have dropped the elastic limit by formulating a radiative transfer method which includes the frequency spreading due to the substantial Doppler shifts from the thermal electron motions.
} 

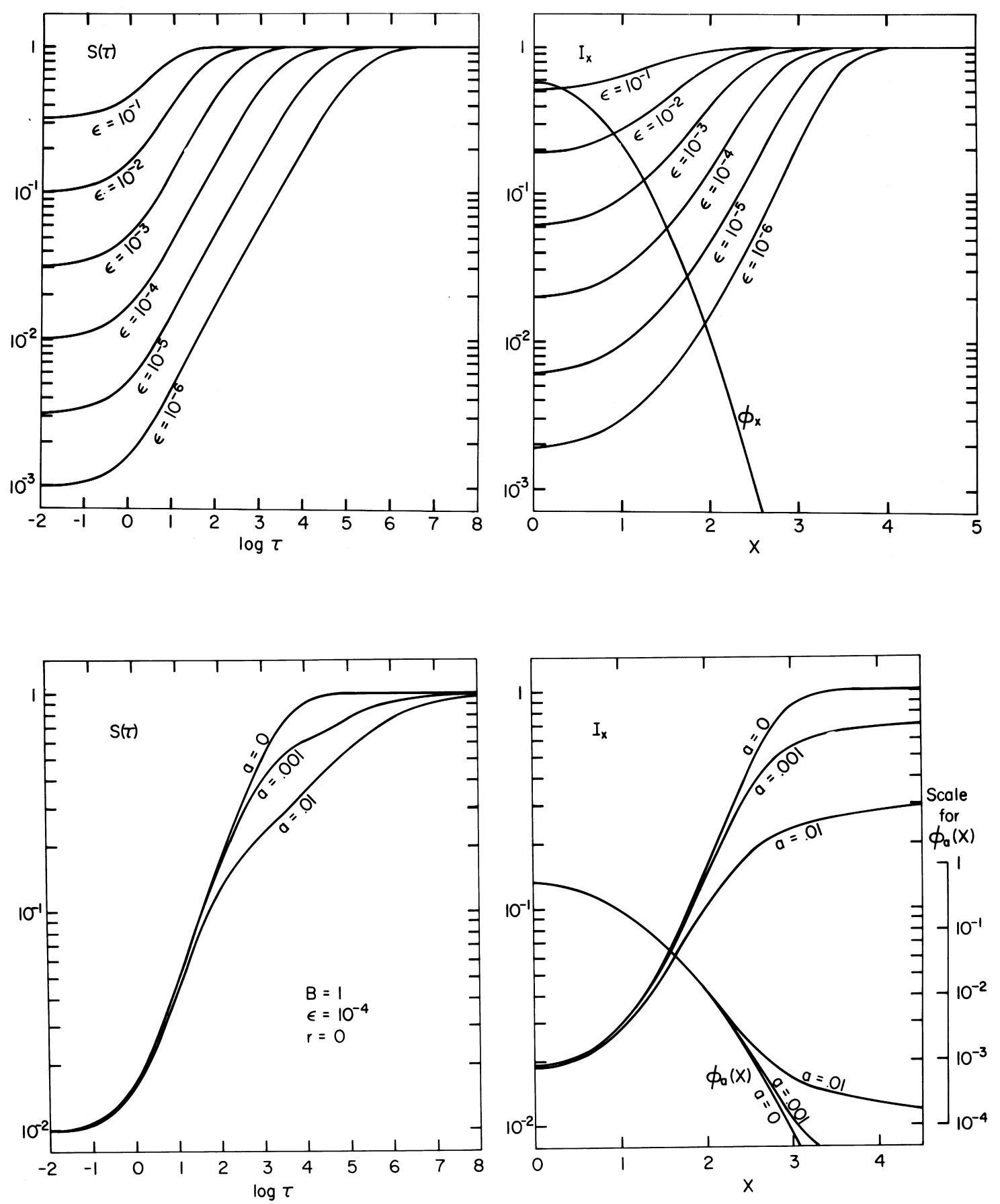

Figure 4.12: Avrett results for two-level-atom lines with complete redistribution from an isothermal atmosphere. The lefthand panels show the source function, the righthand panels corresponding emergent line profiles (only half profiles since they are symmetric) in dimensionless units $x=\left(\nu-\nu_{0}\right) / \Delta \nu_{\mathrm{D}}$ where the Dopplerwidth $\Delta \nu_{\mathrm{D}}$ is defined by $\left(\overline{2} .4 \overline{9}_{1}^{\prime}\right)$ on page $21^{-1}$. The extinction profile $\varphi(x)$ is shown as well. It is Gaussian with $\varphi=(1 / \sqrt{\pi}) \exp \left(-x^{2}\right)^{-}$when $a=\overline{0}$, and has extended wings for larger $a$ as given by $\varphi=(1 / \sqrt{\pi}) H(a, x)$ with the Voigt function $H(a, x)$ defined by $\left(\bar{B} . \overline{6}_{1}^{\prime}\right)$ on page $\bar{p}^{\prime} \bar{l}_{\mathbf{k}}^{\prime}$ The optical depth scale has $\tau=\sqrt{\pi} \tau\left(\nu=\nu_{0}\right)$. The parameter $r \equiv \alpha_{\nu}^{c} / \alpha_{\nu_{0}}^{l}$ is set to zero (no background continuum). The Planck function, the profile-summed collisional destruction probability $\varepsilon_{\nu_{0}} \equiv \epsilon$ and the damping parameter $a$ are assumed constant throughout the atmosphere. Upper panels: Gaussian line, no background extinction. The $\sqrt{\varepsilon}$ law applies. The thermalization depth has $\Lambda_{\nu_{0}} \approx 1 / \varepsilon_{\nu_{0}}$. Lower panels: $\epsilon=10^{-4}$, no background continuum, three values of $a$. For larger $a$ the extinction and emergent profiles possess extended wings and the thermalization deepens even further. From 'Avrett $(1965) !$ 

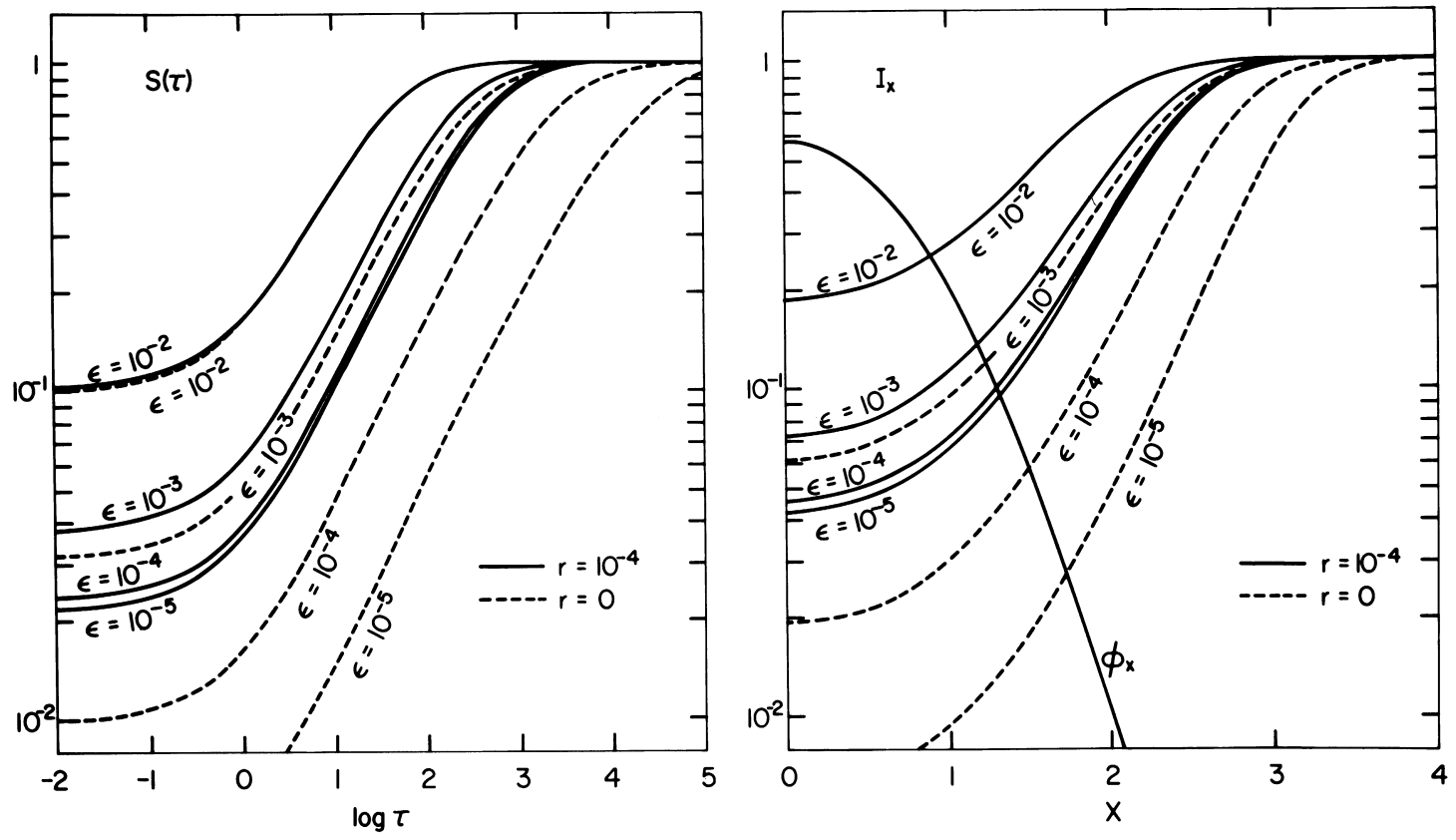

Figure 4.13: Avrett results for two-level-atom lines with complete redistribution and a background continuum. The atmosphere is isothermal. Axis labeling and parameters as for the upper panels of Figure $12^{1}$ the extinction profile $\varphi(x)$ is again Gaussian (righthand panel). Dashed curves: $r \equiv \alpha_{\nu}^{c} / \alpha_{\nu_{0}}^{l}$ set to $r=\overline{0}$, describing pure resonance scattering without background continuum. Solid curves: $r=10^{-4}$ or $\eta_{\nu_{0}}=10^{4}$, describing fairly strong lines. Lack of continuum thermalization is unimportant when $r \ll \varepsilon_{\nu_{0}}$. Lack of collisional destruction is unimportant when $\varepsilon_{\nu_{0}} \ll r$. From îtvrett $(1965)$.

frequencies within the line, averaged together into $\bar{J}_{\nu_{0}}$. The second-order Eddingtonapproximation transport equation (4.59) becomes:

$$
\frac{1}{3} \frac{\mathrm{d}^{2} J_{\nu}\left(\tau_{\nu}\right)}{\mathrm{d} \tau_{\nu}^{2}}=J_{\nu}\left(\tau_{\nu}\right)-\left(1-\lambda_{\nu_{0}}\right) \bar{J}_{\nu_{0}}-\lambda_{\nu_{0}} B_{\nu_{0}}
$$

mixing monochromatic radiation $J_{\nu}$ with profile-averaged radiation $\bar{J}_{\nu_{0}}$. The physics of the transfer therefore differs from the monochromatic demonstration above.

The line photons now make up an ensemble across the line width and collectively feel the average properties of the atmosphere - in particular the fact that they may escape from it. Photons in the line wings have less opacity to traverse towards the surface than photons in the line core do; this difference is frequency-averaged over the profile when complete redistribution over the local extinction profile occurs. A single thermalization depth holds for the whole line, of which the value depends on the shape of the extinction profile. Compared with the monochromatic behavior of a coherently scattering line with the same extinction profile, the line-center photons of a redistributed line may escape from deeper layers by exiting via the wings through the frequency reshuffling, whereas the wing photons escape less easily because they stand a large chance of being redistributed into the opaque core. Lines with extended low-extinction wings offer more escape opportunity and therefore thermalize deeper than boxy profiles without wide wings. An extreme example of a wing-less profile is given by the sharp-line two-level atoms of Section 3.4. $\overline{1}_{1}^{\prime}$ on page 64', which have complete redistribution over the monochromatic delta function. 
The monofrequent demonstration of Section $4 . \overline{3} \overline{1}_{1}^{\prime}$ holds also for these atoms, resulting in $\Lambda_{\nu_{0}}=1 / \sqrt{\lambda_{\nu_{0}}}$. More realistic profile functions have wings, thus have larger profileaveraged escape probability, thus have deeper thermalization than $\Lambda_{\nu_{0}}=1 / \sqrt{\lambda_{\nu_{0}}}$.

I skip the analytical analysis; it is given in Section 11-2 of 'Mihalas $(1 \overline{1} \overline{7} \overline{8})_{1}^{1}$ following Hummer $(1968) ! \quad$ Figure 4.12 shows numerical results that were obtained early in the development of NLTE methodology by Âvrett (1965), and Arett and Hummer (1965), for a two-level scattering line without continuous background. They show that the $\sqrt{\epsilon}$ law also holds exactly for two-level scattering with complete redistribution, with $S_{\nu_{0}}=\sqrt{\varepsilon_{\nu_{0}}} B_{\nu}$ at the top of an isothermal constant $-\varepsilon_{\nu_{0}}$ atmosphere.

Line broadening is discussed in more detail in Section 3.31 on page $\overline{2} \overline{2}_{1}^{2} \mathrm{ff}$; the two extreme profile shapes are given by the Gaussian and Lorentzian profile functions, respectively. The corresponding thermalization depths are, measured in units of profile-summed optical depth with $\mathrm{d} \tau_{\nu_{0}}^{l}=\alpha_{\nu_{0}} \mathrm{~d} z=\sqrt{\pi} \Delta \nu_{\mathrm{D}} \mathrm{d} \tau\left(\nu=\nu_{0}\right)$ (dimension $\mathrm{Hz}$ ) and repeating the sharpline case for reference (Mihalas 1978 p. 342):

$$
\begin{array}{ll}
\Lambda_{\nu_{0}}=1 / \varepsilon_{\nu_{0}}^{1 / 2} & \text { delta function } \\
\Lambda_{\nu_{0}} \approx 1 / \varepsilon_{\nu_{0}} & \text { Gauss profile } \\
\Lambda_{\nu_{0}} \approx 1 / \varepsilon_{\nu_{0}}^{2} & \text { Lorentz profile. }
\end{array}
$$

Thus, thermalization is reached at line-center optical depth $\tau^{l}\left(\nu=\nu_{0}\right) \approx \Lambda_{\nu_{0}} /\left(\sqrt{\pi} \Delta \nu_{\mathrm{D}}\right)$.

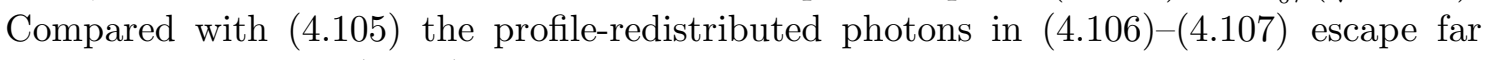
deeper, especially in (4.107i) due to the extended wings of the Lorentz profile. The upper panels of Figure 4.12 illustrate this behavior for a Gaussian line and various values of $\varepsilon_{\nu_{0}}$; the lower panels show results for $\varepsilon_{\nu_{0}}=10^{-4}$ and increasingly large damping specified by the Voigt parameter $a=\gamma /\left(4 \pi \Delta \nu_{\mathrm{D}}\right)$.

In the presence of a background continuum, the thermalization depths are given by

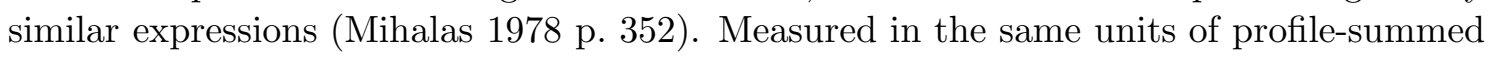
line optical depth (neglecting the continuum contribution by assuming $\eta \gg 1$ ) they are:

$$
\begin{array}{ll}
\Lambda_{\nu_{0}}=1 / \lambda_{\nu_{0}}^{1 / 2} & \text { delta function } \\
\Lambda_{\nu_{0}} \approx 1 / \lambda_{\nu_{0}} & \text { Gauss profile } \\
\Lambda_{\nu_{0}} \approx 1 / \lambda_{\nu_{0}}^{2} & \text { Lorentz profile }
\end{array}
$$

so that thermalization is reached again at line-center optical depth $\tau^{l}\left(\nu=\nu_{0}\right) \approx$ $\Lambda_{\nu_{0}} /\left(\sqrt{\pi} \Delta \nu_{\mathrm{D}}\right)$, corresponding to continuum optical depth $\tau^{c}\left(\nu=\nu_{0}\right) \approx \Lambda_{\nu_{0}} /\left(\sqrt{\pi} \Delta \nu_{\mathrm{D}} \eta(\nu=\right.$ $\left.\left.\nu_{0}\right)\right)$. These dependencies are illustrated by Figure 14.13! Lack of collisional thermalization is now bounded by continuum thermalization and vice-versa through ( $(\bar{A} . \overline{1} \overline{0} \overline{2} \overline{2})$. The largest of the parameters $\varepsilon_{\nu_{0}}$ and $\delta_{\nu_{0}}$ controls the thermalization depth.

Bound-free redistribution. The bound-free processes are not necessarily thermal, as discussed on page $\overline{4}_{4} \overline{5}_{1}$ and in terms of one-level-plus-continuum atoms in Section $3 . \overline{4} . \overline{5}$ on page $72 \mathrm{ff}$. Their description is analogous to bound-bound scattering with complete redistribution. Examples of the resonance-line-like effect of such bound-free scattering on the behavior of $J_{\nu}$ and $S_{\nu}$ are shown for the VALIII Si I, C I and He I edges in Figure 8.11 on page $1 \overline{1} 8 \overline{6}_{1}^{1}$. The VALIII H I Lyman continuum is also a scattering one $(\lambda=90.7 \mathrm{~nm}$ panel in Fig. 36 of 'Vernazza et al. $19 \overline{8} \overline{1}$ i i). 
Discussion. The extensive demonstration above assumed the Eddington approximation, coherent scattering, linear depth-dependence of the Planck function, and constant destruction probability $\varepsilon_{\nu}$. Since the density in a stellar atmosphere increases roughly exponentially with depth, the actual collision probability also increases rapidly with depth. The demonstration results $(4.651)-(4.66 i)$ and Avrett's numerical results in Figures 4.13 have $S_{\nu}$ approach $B_{\nu}$ at large depth because $J_{\nu}$ approaches $B_{\nu}$ (enforced by setting $C_{2}=0$ in $\left.(4.64)\right)$, but in real atmospheres $\varepsilon_{\nu}$ goes to unity with depth and so makes $S_{\nu}$ approach $B_{\nu}$ as well. 


\section{Chapter 5}

\section{Numerical Radiative Transfer}

$\mathrm{T}$ he analytical demonstration in Section 4.31 for constant- $\varepsilon$ scattering in the Eddington approximation comes about as far as one may fruitfully push analytical solutions. There are more refined analytical solutions in the literature, often highly intricate, but most of these date back to the pre-computer era when problem solving had to be analytical by sheer necessity. We wouldn't gain much physical insight here from treating them in detail, and turn to numerical methods.

\section{$5.1 \quad$ Numerical modeling}

\subsubsection{Introduction}

This chapter reviews numerical solution roughly in historical order while emphasizing the underlying physics. It is necessarily incomplete, very condensed, and again restricted to the classical problem of a static plane-parallel stellar atmosphere. However, this is not a severe limitation because the formalisms described here work similarly for more complex geometries and for time-dependent situations.

The discussion is also restricted to methods in which the atmospheric structure (temperature and density stratifications, plus the chemical composition) is known, requiring that the spectral lines and continua that are being computed do not affect the atmosphere. However, the methods discussed here apply also to the more complex problems in which atmospheric structure and NLTE radiative transfer are locked together. The computation of atmospheric models employs the same tricks.

The recipes discussed below are treated in some detail not only because they are an integral part of modern stellar-atmosphere modeling but also because they supply physical insights. I emphasize the earlier tricks (Rybicki's core saturation approximation on

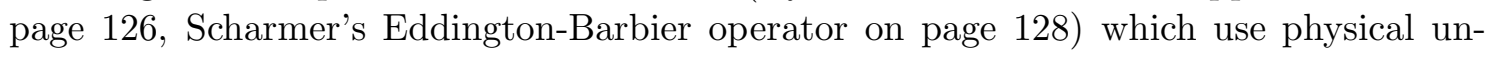
derstanding of how photons migrate through a stellar atmosphere to speed up their computational emulation. More recently, progress has become more mathematical in nature (matrix simplification, convergence acceleration).

Aims. The basic problem of radiative transfer is that evaluation of a particular $I_{\nu}\left(\tau_{\nu}, \mu\right)$ requires $S_{\nu}$, and therefore $J_{\nu}$, and therefore $I_{\nu}$ in many directions, and therefore $S_{\nu}$ at many locations, and often also at many frequencies. The non-linear coupling between the radiative transfer equations and the statistical equilibrium (or time-dependent!) rate equations and the complexity introduced by many different atomic processes ask for computer 
processing. The aim is to do that not only for the simple geometry of static plane-parallel atmospheres, but also for the real world with complex three-dimensional time-dependent hydrodynamics, atmospheric expansion, stellar winds, MHD structuring, etc. This means that the development of fast computer methods is essential.

References. The initial methods were developed in the 1960's and early 1970's and are well described by iMihalas $(19 \overline{1} 78)$.' They made stellar radiative transfer into one of the first fields of physics in which complex non-linear systems were habitually solved.

Renewed interest in basic methods came in the late 1980's when operator perturbation techniques, originally $y_{-1}^{T_{1}}$ proposed for radiative transfer problems by 'Cannon usually called ALI (for Accelerated Lambda Iteration or Approximate Lambda Iteration) came into regular use. These are not a single trick but rather a class of ideas, with the use of an approximate operator in operator splitting as common element. Their description below is not a textbook-fashion presentation of logical development along well-defined lines, but rather a collection of tricks and strategies that is partially taken from the review

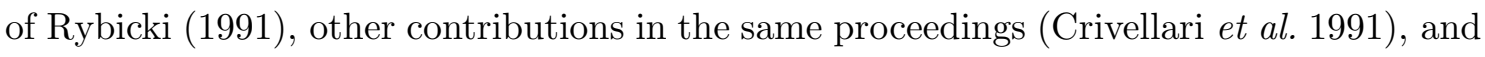
the review by Hubeny $(1992)$ !. Two review collections were published earlier by Kalkofen (1984, 1987). The more recent papers of Rybicki and Hummer (1991, 1992, 1994) also make good reading.

\subsubsection{Discretization}

Putting equations in computers requires discretization, in this case in location (only radial depth for plane-parallel atmospheres), frequency and angle.

Angles. Let there be $2 m$ angles $\mu_{j}$, with one set $j=1, \ldots, m$ covering the $\mu>0$ hemisphere and the other set $j=1, \ldots, m$ the opposite directions $-\left|\mu_{j}\right|$. The mean intensity is then numerically approximated by

$$
\begin{aligned}
J & \equiv \frac{1}{2} \int_{-1}^{+1} I \mathrm{~d} \mu \\
& =\frac{1}{2} \int_{0}^{+1} I^{+} \mathrm{d} \mu+\frac{1}{2} \int_{-1}^{0} I^{-} \mathrm{d} \mu \\
& =\frac{1}{2} \int_{0}^{+1} I^{+} \mathrm{d} \mu+\frac{1}{2} \int_{0}^{+1} I^{-} \mathrm{d}(-\mu) \\
& \approx \frac{1}{2} \sum_{j=1}^{m} a_{j} I_{j}^{+}+\frac{1}{2} \sum_{j=1}^{m} a_{j} I_{j}^{-},
\end{aligned}
$$

where the intensities are split between outgoing rays $I^{+}$and incoming rays $I^{-}$. In the remainder of this chapter we take $\mu$ positive and use $-\mu \equiv-|\mu<0|$ to denote inward directions, as in $\left(\overline{5}^{5}-1_{1}^{1}\right)$.

\footnotetext{
${ }^{1}$ Such operator splitting methods had long been known in numerical analysis outside radiative transfer. Hubeny (1992), comments that Cannon introduced these methods during the period of greatest development of Feautrier-type methods with complete linearization (by Auer, Heasley, Mihalas, Milkey, Rybicki and others) so that they were overlooked because the other radiative transfer specialists were far too busy at the time employing their nice new tool to solve, realistically for the first time, multi-level line formation problems. One wonders what tricks are being missed right now?
} 
Angle quadrature. The sums in $(\overline{5} .2 i)$ represent numerical integration, often called "quadrature". The quadrature weights $a_{j}$ depend on the choice of directions $\mu_{i}$. Let me briefly review numerical integration. An example of one-point quadrature is:

$$
\int_{a}^{b} f(x) \mathrm{d} x \approx(b-a) f(x=0.5) .
$$

The trapezium rule or Euler two-point quadrature is:

$$
\int_{a}^{b} f(x) \mathrm{d} x \approx \frac{1}{2}(b-a)[f(a)+f(b)] .
$$

The three-point Simpson rule with equidistant sampling $x_{3}-x_{2}=x_{2}-x_{1}=\Delta x$ is:

$$
\int_{x_{1}}^{x_{3}} f(x) \mathrm{d} x \approx \frac{\Delta x}{3}\left[f\left(x_{1}\right)+4 f\left(x_{2}\right)+f\left(x_{3}\right)\right],
$$

This is a closed Newton-Cotes quadrature. The next two are

$$
\int_{x_{1}}^{x_{4}} f(x) \mathrm{d} x \approx \frac{3 \Delta x}{8}\left[f\left(x_{1}\right)+3 f\left(x_{2}\right)+3 f\left(x_{3}\right)+f\left(x_{4}\right)\right]
$$

and

$$
\int_{x_{1}}^{x_{5}} f(x) \mathrm{d} x \approx \frac{2 \Delta x}{45}\left[7 f\left(x_{1}\right)+32 f\left(x_{2}\right)+12 f\left(x_{3}\right)+32 f\left(x_{4}\right)+7 f\left(x_{5}\right)\right] .
$$

Thus, the integral is approximated by $\int_{x_{1}}^{x_{n}} f(x) \mathrm{d} x \approx \sum_{1}^{n} a_{i} f\left(x_{i}\right)$. These approximations are not very precise. Much better results are obtained by choosing the sampling locations $x_{i}$ non-equidistant and clever. This is done in Gaussian quadrature, for example GaussLegendre or Gauss-Laguerre integration. Another approach is to use spline functions which are piecewise segments of polynomial (usually cubic) fits which minimize abruptness at their connections by requiring derivative continuity there. See chapter "Integration of

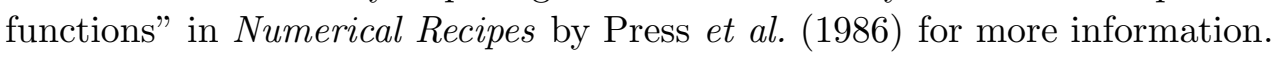

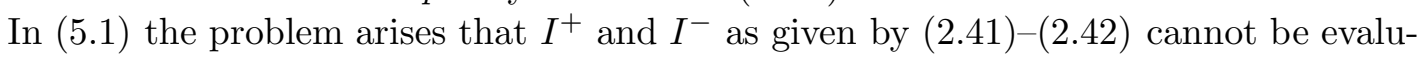
ated at $\mu=0$. The integrals in $\left(\overline{5}-\bar{l}^{\prime}\right)$ must therefore be evaluated with "open quadrature" neglecting the $\mu=0$ endpoint. There are open Newton-Cotes quadrature formulae like the expressions above, but it is again much better to use non-equidistant Gaussian quadrature. The defining formula is:

$$
\int_{-1}^{+1} f(x) \mathrm{d} x \approx \sum_{i=1}^{n} w_{i} f\left(x_{i}\right)
$$

and the required abscissa values $x_{i}$ and weights $w_{i}$ are tabulated for $n=2-10$ and even higher orders on page 916 of integration is often sufficiently accurate for angle integrations as in (1. 1.

$$
\begin{array}{rllll}
x_{i} & = & -0.7745966692, & 0.0000000000 & 0.7745966692 \\
w_{i} & = & 0.55555555555, & 0.8888888888, & 0.5555555555
\end{array}
$$

for the $[-1,+1]$ interval. Rescaling that to $[0,1]$ requires halving the weights. 
Frequencies. Most of the discussions below concern the simple case of purely coherent scattering, so that a single frequency suits to demonstrate numerical methods. However, in actual stellar atmosphere and emergent spectrum modeling one needs to specify frequency grids across all lines and continua of interest, and integrate over these to obtain profilesummed quantities. For spectral lines distinction is often made between the Gaussian Doppler core and the Lorentzian damping wings of the local extinction profile (shown on page $\left.\overline{\underline{6}}_{\underline{1}}^{\prime}\right)$, using equidistant sampling of $\Delta \nu=\nu-\nu_{0}$ in the core and logarithmically spaced sampling (equidistant in $\log \Delta \nu$ ) in the extended wings. Usually, the spacings

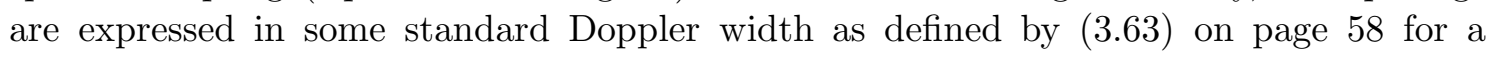
representative temperature. For symmetric lines half the profile suffices.

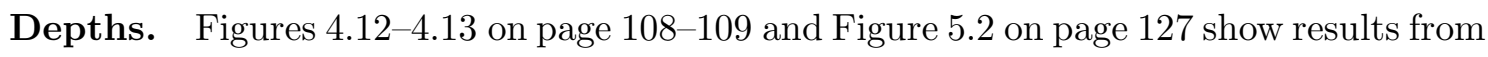

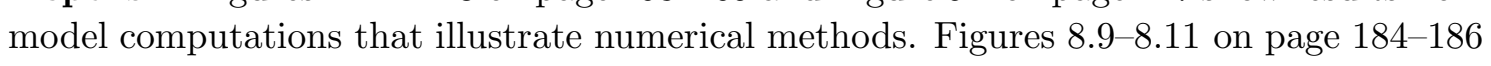
show results from detailed modeling of observed solar continua. These graphs demonstrate that when studying radiative transfer in stellar atmospheres or emergent spectra from stellar atmospheres, a wide range in optical depth is required in the computations and for displaying results. Discretization of the optical depth scale into logarithmically spaced points is therefore a good choice, say $5-10$ per decade with $\Delta_{i} \equiv \log \tau_{i+1}-\log \tau_{i}=0.2-0.1$.

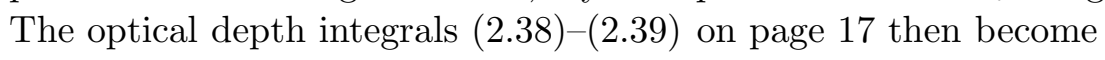

$$
\begin{aligned}
\tau_{\nu}^{c} & =\int_{z_{0}}^{\infty} \alpha_{\nu}^{c} \mathrm{~d} z=\int_{0}^{\tau_{0}} \mathrm{~d} t^{c}=\int_{-\infty}^{\log \tau_{0}} \frac{\mathrm{d} \log t^{c}}{\log e} \\
\tau_{\nu}^{\text {total }} & =\int_{z_{0}}^{\infty}\left(\alpha_{\nu}^{c}+\alpha_{\nu}^{l}\right) \mathrm{d} z=\int_{0}^{\tau_{0}}\left(1+\eta_{\nu}\right) \mathrm{d} t^{c}=\int_{-\infty}^{\log \tau_{0}}\left(1+\eta_{\nu}\right) \frac{\mathrm{d} \log t^{c}}{\log e}
\end{aligned}
$$

respectively for the continuum optical depth scale on which a grid may be defined and for a frequency within a spectral line at which the total monochromatic optical depth is integrated on the continuum grid. These integrals may be implemented as simple trapezoidal summations, adding grid points when more accuracy is required.

In plane-parallel modeling one usually uses a reference grid in continuum optical depth at $\lambda=500 \mathrm{~nm}$, or the Rosseland optical depth as defined by $(7.62)$ on page 1600 column density scale as listed in Table $\overline{8} . \overline{2}$ on page $11 \overline{2} \overline{2}$. Even if the reference grid has equidistant sampling $\Delta_{i} \equiv \Delta$, the sampling of the $\log \tau_{\nu}^{\text {total }}$ scale will not be equidistant

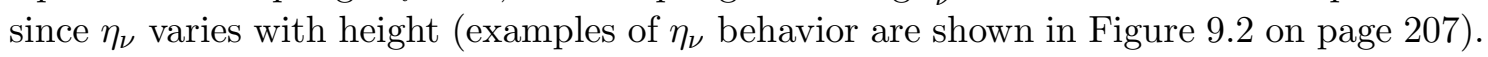
It may therefore be necessary to add grid points in order to sample fast variations that occur at specific frequencies. For example, the VALIII grid in Table 8.22 samples the range $\log \tau_{500}=[-7,-6]$ with many grid points in order to follow the steep temperature increase just above $h=2000 \mathrm{~km}$ adequately, but has only few grid points per $\log \tau_{500}$ decade in deeper layers.

Non-plane-parallel grids. In multi-dimensional and/or time-dependent modeling the spatial grid becomes a major matter of concern. For example, even in dynamical modeling with only one dimension it is worthwhile to optimize the grid distribution continuously, following features where and when they develop with just the appropriate resolution in grid points. See 'Dorfi and Drury $(1 \overline{9} \overline{8} 7)$ ', for such "adaptive mesh" techniques.

Another issue is the fundamental choice between laboratory-frame formulation and comoving-frame formulation. The latter has the advantage that the local extinction coef-

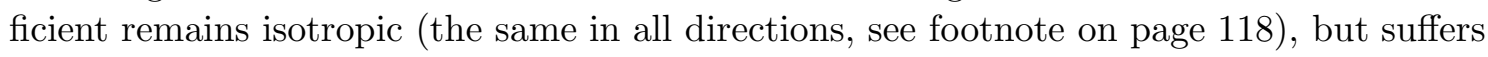


from complex coordinate transformations. See Chapter 14 of Mihalas (1978) and îinalas and Mihalas $(1984)$.

\subsection{Feautrier method}

Boundary problems. A general problem with numerical stellar-atmosphere radiative transfer has to do with the boundary conditions. Again, dropping indices $\nu, \mu$ and arguments $\tau$, the general transfer equation along any direction $\mu$ is:

$$
\mu \frac{\mathrm{d} I}{\mathrm{~d} \tau}=I-S
$$

For the illustrative monofrequent case of pure coherent scattering it becomes:

$$
\mu \frac{\mathrm{d} I}{\mathrm{~d} \tau}=I-\varepsilon B-\frac{(1-\varepsilon)}{2} \int_{-1}^{+1} I \mathrm{~d} \mu .
$$

Let's discretize into $n$ depth points $\tau_{i}$ with $i=1, \ldots, n$ and $m$ angles $\mu_{j}$ covering one hemisphere. The pure-scattering transport equation at a given depth $\tau_{i}$ in a given direction $\mu$ (one of the discrete $\mu_{j}$ 's) then becomes, with ( incoming rays:

$$
\begin{aligned}
\mu \frac{\mathrm{d} I_{\mu}^{+}}{\mathrm{d} \tau} & =I_{\mu}^{+}-\varepsilon B-\frac{(1-\varepsilon)}{2}\left[\sum_{j=1}^{m} a_{j} I_{j}^{+}+\sum_{j=1}^{m} a_{j} I_{j}^{-}\right] \\
(-\mu) \frac{\mathrm{d} I_{\mu}^{-}}{\mathrm{d} \tau} & =I_{\mu}^{-}-\varepsilon B-\frac{(1-\varepsilon)}{2}\left[\sum_{j=1}^{m} a_{j} I_{j}^{+}+\sum_{j=1}^{m} a_{j} I_{j}^{-}\right] .
\end{aligned}
$$

The boundary conditions are again that $I_{\mu}^{-}=0$ for all inward directions $-\mu_{j}$ at the outer boundary $\tau_{1}=\tau_{\min }$ (the first discrete $\tau$ value, with $\tau_{\min } \ll 1$ ) and that at the inner boundary $\tau_{n}=\tau_{\max }$ (with $\tau_{\max } \gg 1$ ) the Rosseland approximation holds with $I_{\mu}^{+}=B+\mu \mathrm{d} B / \mathrm{d} \tau$ for all outward $\mu_{j}$.

The problem is that these boundary conditions fix only half of the $I\left(\mu_{i}\right)$ values at each boundary. At the surface they deliver $I_{1}^{-}$and at depth $I_{n}^{+}$, but the summation that produces $J$ requires all $I_{\mu}^{+}$and $I_{\mu}^{-}$values per $\mu_{j}$ at every depth. One cannot simply guess the lacking values to start integrating from one end and then use the boundary condition at the other end as check to improve the guess (the shooting method, like Robin Hood and Wilhelm Tell), because first-order linear differential equations such as these possess general solutions which contain terms $\sum_{m} \exp \left(-k_{m} \tau\right)$ and $\sum_{m} \exp \left(+k_{m} \tau\right)$ where

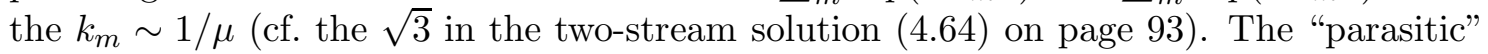
terms $\sum_{m} \exp \left(+k_{m} \tau\right)$ grow rapidly with depth, as $\exp (2 \tau) \sim 10^{\tau}$ needing at least $\tau_{\text {max }}$ significant digits. If we integrate $\left(\begin{array}{l}5 \\ -5\end{array}\right)$ from the surface inward, guessing the emerging $I_{\mu}^{+}$ values for the $J$ summation, errors in these guesses grow exponentially with $\tau$ and become infinite long before we get to the depth where the inner boundary condition might be used to check the guesses. The same problem occurs for the second-order transport equation

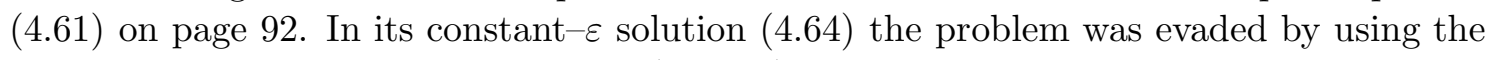
inner boundary condition analytically $\left(C_{2}=0\right)$ to set the outer one. 
Antisymmetric averages. In a method devised by Feautrier (1) $\overline{1} \overline{9} \overline{4}) \overline{4}$ the upper and lower boundary conditions are coupled together by combining the radiation fields in opposite directions per angle $\mu_{j}$ (Mīihalas 1970' p. $164 \mathrm{ff} ;$ 'Mihalas $197 \overline{8}_{1}^{\prime}$ p. $156 \mathrm{ff} ;$ Appendix A of Rybicki and Hummer 1991 ). This is done by introducing the Feautrier variables

$$
\begin{aligned}
P_{\nu}\left(\tau_{\nu}, \mu\right) & \equiv \frac{1}{2}\left[I_{\nu}\left(\tau_{\nu}, \mu\right)+I_{\nu}\left(\tau_{\nu},-\mu\right)\right]=\frac{1}{2}\left[I_{j}^{+}+I_{j}^{-}\right] \\
R_{\nu}\left(\tau_{\nu}, \mu\right) & \equiv \frac{1}{2}\left[I_{\nu}\left(\tau_{\nu}, \mu\right)-I_{\nu}\left(\tau_{\nu},-\mu\right)\right]=\frac{1}{2}\left[I_{j}^{+}-I_{j}^{-}\right],
\end{aligned}
$$

where the righthand versions are in discretized notation. The quantity $P$ is $J$-like in character by averaging the outgoing and incoming intensities along the given direction. Integrating $P$ over all outward directions delivers $J_{\nu}(\tau)$ :

$$
\begin{aligned}
J_{\nu}\left(\tau_{i}\right) & =\int_{0}^{+1} P_{\nu}\left(\tau_{i}, \mu\right) \mathrm{d} \mu \\
& \approx \sum_{j=1}^{m} a_{j} P\left(\tau_{i}, \mu_{j}\right)
\end{aligned}
$$

where (15it) is the numerical addition of known $P_{\nu}\left(\tau_{i}, \mu_{j}\right)$ over the set of $m$ positive (outward) directions $\mu_{j}$ with numerical integration weights $a_{j}$. Thus, the $m$ Feautrier parameters $P\left(\tau_{i}, \mu_{j}\right)$ are the quantities we seek in order to obtain $J_{\nu}\left(\tau_{i}\right)$. Note that the Feautrier parameter $R$ is flux-like in character.

Transport equation. We again split the general transport equation between outgoing and incoming directions (again dropping indices):

$$
\begin{aligned}
\mu \frac{\mathrm{d} I^{+}}{\mathrm{d} \tau} & =I^{+}-S^{+} \\
-\mu \frac{\mathrm{d} I^{-}}{\mathrm{d} \tau} & =I^{-}-S^{-} .
\end{aligned}
$$

Adding the two together, respectively subtracting the second from the first, together with the assumption that the source function is isotropic $\mathbf{L}_{-1}^{\mathcal{Q}_{1}}$ with $S^{+}=S^{-}$, delivers respectively:

$$
\begin{aligned}
& \mu \frac{\mathrm{d} R}{\mathrm{~d} \tau}=P-S \\
& \mu \frac{\mathrm{d} P}{\mathrm{~d} \tau}=R .
\end{aligned}
$$

\footnotetext{
${ }^{2}$ As noted on page $171_{-1}^{\prime}$ the total source function is no longer isotropic when the line extinction profile is Doppler-shifted by systematic motion at the given depth. The total source function has $S^{\text {tot }}=\left(\alpha^{l} S^{l}+\right.$ $\left.\alpha^{c} S^{c}\right) /\left(\alpha^{l}+\alpha^{c}\right)$ so that different Doppler-shifts of $\alpha^{l}$ for different directions cause anisotropy, shifting the profile one way along an outgoing direction $\mu$ and the other along the reversed ingoing direction $-\mu$. For example, for radial expansion with velocity $v$ the extinction profile shifts with $\mu$ as $\varphi\left[\Delta \nu-\mu \nu_{0}(v / c)\right]$ (page $5 \overline{7}_{1}$ ), over $2 \nu_{0}(v / c)$ between the radially outward and inward directions. In that case, the Feautrier method is not valid. However, a trick is possible (Mihalas 1978, page $451 \mathrm{ff}$ ) using the two-sided symmetry of the static extinction profile (when there are no blends or other profile asymmetries for a non-moving medium). One then sets up a frequency grid covering the full line (plus sufficient room for the shifts) and uses the frequencies $\nu_{i}>\nu_{0}$ to cover the profile in outgoing rays and the frequencies $\nu_{i}<\nu_{0}$ to cover the profile for the incoming rays. By flipping between the two sets of frequencies between the two directions, the extinction profile is effectively not shifted between the two so that isotropy holds and the Feautrier method keeps its validity. Thus, one redefines the Feautrier parameters as $P(\tau, \Delta \nu, \mu)=(1 / 2)\left[I^{+}(\tau, \Delta \nu, \mu)+\right.$ $I^{-}(\tau,-\Delta \nu,-\mu)$ and $\left.R(\tau, \Delta \nu, \mu)\right]=(1 / 2)\left[I^{+}(\tau, \Delta \nu, \mu)-I^{-}(\tau,-\Delta \nu,-\mu)\right]$. An alternative is to adopt the "comoving" frame of the fluid rather than the static "laboratory" frame. Within the comoving frame the source function is again isotropic, but the transport equation then suffers from Lorentz transformations or even non-inertial coordinate transformations.
} 
Elimination of $R$ then produces the Feautrier transport equation:

$$
\mu^{2} \frac{\mathrm{d}^{2} P(\tau, \mu)}{\mathrm{d} \tau^{2}}=P(\tau, \mu)-S(\tau)
$$

again a second-order transport equation which may be solved for the Feautrier variable $P$ when the source function $S$ and appropriate boundary conditions are known, per angle $\mu$ and depth $\tau$. The advantage over ( $\left(\overline{4} . \overline{9}_{1}^{\prime}\right)$ on page $\overline{7} \overline{6}_{1}$ and its Eddington-approximation reformulation into the closed form (4.61 inward and outward directions. Note that it is often written as

$$
\frac{\mathrm{d}^{2} P(\tau, \mu)}{\mathrm{d} \tau^{2}}=P(\tau, \mu)-S(\tau)
$$

where $\tau \equiv \tau_{\nu \mu}$ then designates the inward optical depth along a ray with direction $\mu$, instead of the radial optical depth $\tau=\tau_{\nu}$ used here.

Boundary conditions. The outer boundary condition at $\tau=\tau_{1} \ll 1$ is again that $I^{-}=I\left(\tau_{1},-\mu\right)=0$ so that $R\left(\tau_{1}, \mu\right)=P\left(\tau_{1}, \mu\right)=(1 / 2) I\left(\tau_{1}, \mu\right)$ and with (15.16):

$$
\mu\left[\frac{\mathrm{d} P(\tau, \mu)}{\mathrm{d} \tau}\right]_{\tau_{1}}=P\left(\tau_{1}, \mu\right)
$$

expressing the $P$ gradient at the boundary for every direction $\mu$ in $P\left(\tau_{1}, \mu\right)$ and using radial optical depth $\tau=\tau_{\nu}$. The inner boundary condition at $\tau=\tau_{n} \gg 1$ is again that $I^{+}=I\left(\tau_{n}, \mu\right)=B\left(\tau_{n}\right)+\mu \mathrm{d} B\left(\tau_{n}\right) / \mathrm{d} \tau$ with $J_{\nu}\left(\tau_{n}\right)=B_{\nu}\left(\tau_{n}\right)$. With (15.16i) and the definitions $\left(\overline{5}^{\prime}, 9_{1}^{\prime}\right)$ and $\left(\overline{5}^{\prime}, 10_{i}^{\prime}\right)$ the $P$ gradients at $\tau_{n}$ then are:

$$
\begin{aligned}
\mu\left[\frac{\mathrm{d} P(\tau, \mu)}{\mathrm{d} \tau}\right]_{\tau_{n}} & =R\left(\tau_{n}, \mu\right) \\
& =\frac{1}{2}\left[I\left(\tau_{n}, \mu\right)-I\left(\tau_{n},-\mu\right)\right] \\
& =I\left(\tau_{n}, \mu\right)-\frac{1}{2}\left[I\left(\tau_{n}, \mu\right)+I\left(\tau_{n},-\mu\right)\right] \\
& =I\left(\tau_{n}, \mu\right)-P\left(\tau_{n}, \mu\right) \\
& =B\left(\tau_{n}\right)+\mu\left[\frac{\mathrm{d} B}{\mathrm{~d} \tau}\right]_{\tau_{n}}-P\left(\tau_{n}, \mu\right)
\end{aligned}
$$

We now have first-order boundary conditions $s_{-1}^{3,1}$ which express the $\mathrm{d} P / \mathrm{d} \tau$ gradients in the boundary values of $P$ per $\mu$.

Difference equations. We now replace integrals by sums and derivatives by differences. The simplest scheme is to approximate the latter by using

$$
\begin{aligned}
& {[\Delta \tau]_{i+1 / 2} \approx \tau_{i+1}-\tau_{i} \equiv \Delta \tau_{i}} \\
& {[\Delta \tau]_{i-1 / 2} \approx \tau_{i}-\tau_{i-1} \equiv \Delta \tau_{i-1}}
\end{aligned}
$$

\footnotetext{
${ }^{3}$ Second-order boundary conditions are more appropriate to second-order problems. At the surface, a second-order condition follows from a Taylor expansion of $P_{1}$ with $\tau$. A more general boundary_condition also admits illumination from outside the atmosphere. At depth, the second-order term of $\left(4.38_{1}^{\prime}\right)$ may be included. See 'Áuer $(1967)$ '.
} 
for $i=2, \ldots, n-1$. The gradients are then given by

$$
\begin{aligned}
{\left[\frac{\mathrm{d} P\left(\tau, \mu_{j}\right)}{\mathrm{d} \tau}\right]_{i+1 / 2} } & \equiv \lim _{\Delta \tau \rightarrow 0} \frac{\left[\Delta P\left(\tau, \mu_{j}\right)\right]_{i+1 / 2}}{[\Delta \tau]_{i+1 / 2}} \\
& \approx \frac{P\left(\tau_{i+1}, \mu_{j}\right)-P\left(\tau_{i}, \mu_{j}\right)}{\tau_{i+1}-\tau_{i}}=\frac{P_{i+1}-P_{i}}{\Delta \tau_{i}}
\end{aligned}
$$

where evaluation midway the sampling interval helps to obtain the second derivatives needed for $(\overline{5}-17)$ at the sample locations. They are given by

$$
\begin{aligned}
{\left[\frac{\mathrm{d}^{2} P\left(\tau, \mu_{j}\right)}{\mathrm{d} \tau^{2}}\right]_{i} } & \approx \frac{\left[\Delta P\left(\tau, \mu_{j}\right) / \Delta \tau\right]_{i+1 / 2}-\left[\Delta P\left(\tau, \mu_{j}\right) / \Delta \tau\right]_{i-1 / 2}}{[\Delta \tau]_{i}} \\
& \approx \frac{\left[\Delta P\left(\tau, \mu_{j}\right) / \Delta \tau\right]_{i+1 / 2}-\left[\Delta P\left(\tau, \mu_{j}\right) / \Delta \tau\right]_{i-1 / 2}}{\frac{1}{2}\left([\Delta \tau]_{i+1 / 2}+[\Delta \tau]_{i-1 / 2}\right)} \\
& \approx \frac{2}{\Delta \tau_{i-1}+\Delta \tau_{i}}\left[\frac{P_{i+1}}{\Delta \tau_{i}}-\frac{P_{i}}{\Delta \tau_{i}}-\frac{P_{i}}{\Delta \tau_{i-1}}+\frac{P_{i-1}}{\Delta \tau_{i-1}}\right] \\
& =\frac{2 P_{i-1}}{\Delta \tau_{i-1}\left(\Delta \tau_{i-1}+\Delta \tau_{i}\right)}-\frac{2 P_{i}}{\Delta \tau_{i} \Delta \tau_{i-1}}+\frac{2 P_{i+1}}{\Delta \tau_{i}\left(\Delta \tau_{i-1}+\Delta \tau_{i}\right)}
\end{aligned}
$$

With these (

$$
\mu^{2}\left[\frac{\mathrm{d}^{2} P}{\mathrm{~d} \tau^{2}}\right]_{i}-P_{i}=A_{i} P_{i-1}-B_{i} P_{i}+C_{i} P_{i+1}=-S_{i}
$$

with

$$
\begin{aligned}
A_{i} & =\frac{2 \mu^{2}}{\Delta \tau_{i-1}\left(\Delta \tau_{i-1}+\Delta \tau_{i}\right)} \\
B_{i} & =1+\frac{2 \mu^{2}}{\Delta \tau_{i} \Delta \tau_{i-1}} \\
C_{i} & =\frac{2 \mu^{2}}{\Delta \tau_{i}\left(\Delta \tau_{i-1}+\Delta \tau_{i}\right)} .
\end{aligned}
$$

which represents second-order coupling of the locations $\tau_{i-1}, \tau_{i}$ and $\tau_{i+1}$, hence a tridiagonal equation system. It is numerically advantageous to subtract the 1 in $(5.24)$.

The source function values $S_{i}$ are assumed known for all depths $\tau_{i}$. There are $m$ sets of such linear equations, one per angle $\mu_{j}$, at each depth point $\tau_{i}$ with $i=2, \ldots, n-1$. Write the $P\left(\tau_{i}, \mu_{j}\right)$ at each $\tau_{i}$ into vectors $\mathbf{P}_{i}$ with $m$ elements each; we then have $n$ linear equations between $n$ vectors which may be written in matrix notation as

$$
\mathbf{T} \mathbf{P}=\mathbf{S}
$$

where $\mathbf{T}$ is a tridiagonal matrix with elements $A_{i, j}, B_{i, j}$ and $C_{i, j}$ according to (5.5. $\overline{3}$ )$(15.25 i)$. Thus, the $\mathbf{P}$ vectors with $2 \leq i \leq n-1$ are related by $n-2$ linear equations $(5.22 i)$ containing $\mathbf{P}_{i-1}, \mathbf{P}_{i}$, and $\mathbf{P}_{i+1}$. For $i=1,2$ there is a linear equation between $\mathbf{P}_{1}$ and $\mathbf{P}_{2}$ which is found by substituting the difference expression for the gradient into (5.18) and assuming that the gradient $\mathrm{d} P / \mathrm{d} \tau$ does not change between $i=1$ and $i=3 / 2$ (as is the case if $\tau_{1}$ is sufficiently far out, well in thin conditions). Substitution of the gradient into $\left(\overline{5} .19_{1}^{i}\right)$ similarly produces a linear equation between $\mathbf{P}_{n-1}$ and $\mathbf{P}_{n}$. 
Forward-backward solution. Numerical solution proceeds by a forward elimination backward substitution scheme. Start using the outer boundary condition by expressing $\mathbf{P}_{1}$ in $\mathbf{P}_{2}$. The result is put into the equation between $\mathbf{P}_{1}, \mathbf{P}_{2}$, and $\mathbf{P}_{3}$ to express $\mathbf{P}_{2}$ in terms of $\mathbf{P}_{3}$. Proceeding in this manner expresses each $\mathbf{P}_{i}$ in terms of $\mathbf{P}_{i+1}$. We finally reach $i=n$ where the inner boundary condition delivers $\mathbf{P}_{n}$. Back substitutions then produce $\mathbf{P}_{n} \rightarrow \mathbf{P}_{n-1} \rightarrow \mathbf{P}_{n-2} \rightarrow \ldots \rightarrow \mathbf{P}_{2} \rightarrow \mathbf{P}_{1}$, so that all $\mathbf{P}_{i}$ and with (5.12) all $J_{\nu}\left(\tau_{i}\right)$ are known.

The Feautrier solution is a good example of an implicit scheme: all variables are found together from local evaluations - no blind shooting. The forward-backward solving exploits the one-dimensional nature of the problem.

Two-level solution. For the illustration case of pure coherent scattering the secondorder Feautrier transport equation (5.17i) becomes:

$$
\begin{aligned}
\mu^{2} \frac{\mathrm{d}^{2} P(\tau, \mu)}{\mathrm{d} \tau^{2}} & =P(\tau, \mu)-\varepsilon(\tau) B(\tau)-(1-\varepsilon(\tau)) J(\tau) \\
& =P(\tau, \mu)-\varepsilon(\tau) B(\tau)-(1-\varepsilon(\tau)) \sum_{j=1}^{m} a_{j} P_{j}\left(\tau, \mu_{j}\right),
\end{aligned}
$$

where the second version again specifies the discrete quadrature needed to obtain $J$ from

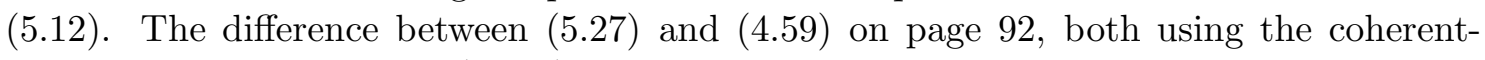
scattering source function $(2.14 \overline{4}+1)$, is that the inner and outer boundary conditions are now specified for the single quantity $P$ rather than for $I^{+}$and $I^{-}$separately. In this case, $S_{\nu}$ is linear in $P_{\nu}$ so that the transport equation may be solved directly by the scheme described above, provided one solves it along all directions $\mu$ simultaneously in order to sum the $P\left(\tau_{i}, \mu_{j}\right)$ at each $\tau_{i}$ for the $J$ evaluation. Thus, at each angle $\mu$ there are contributions from all other angles. This means that the diagonal of the $\mathbf{T}$ matrix in $(\overline{5} .26)$ consists of $m \times m$ submatrices. The structure is shown in Figure

After solution all $P_{i, j}$ are known; so are the mean intensities $J_{i}$ and the emergent intensities $I_{1, j}^{+}$(from $\left({ }_{0}^{-} .9_{1}^{*}\right)$ since $I_{1, j}^{-}=0$ ). Thus, in the case of coherent scattering the Feautrier method may serve to determine all desired quantities from known $\varepsilon(\tau)$ and $B(\tau)$ per frequency $\nu$. As shown in Section 4.3 .5 on page $10 \overline{1}$ iff, this formalism may also be used for two-level scattering lines with a thermal background and/or for the combination of thermal and scattering continuous processes.

Rybicki version. Sofar, the Feautrier method has been discussed for a single frequency. For spectral lines, a frequency grid covering each line must be used, adding another dimension to the problem. For most lines, complete frequency redistribution over the line profile is usually a better assumption than coherent scattering (Section $3 . \overline{4} . \overline{2}$ on page $\overline{1} \bar{z}_{1}^{0} \mathrm{ff}$ ). In that case, $J_{\nu}$ is replaced by $\bar{J}_{\nu}$ in the source function as in ('A. $\left.\overline{1} \overline{0} \overline{3}_{1}\right)$ on page $\overline{1} \overline{1}_{\overline{7}}$. It should no longer be necessary to achieve separate Feautrier solutions for each frequency in the line separately since the line source function feels only the redistributed total radiation across

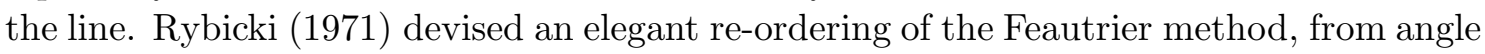
vectors per frequency to depth vectors per frequency, in which only the frequency-averaged mean intensity $\bar{J}_{\nu}$ is used within the forward elimination-back substitution scheme. It is described by Mihalas (1978) on page $158 \mathrm{ff}$. 


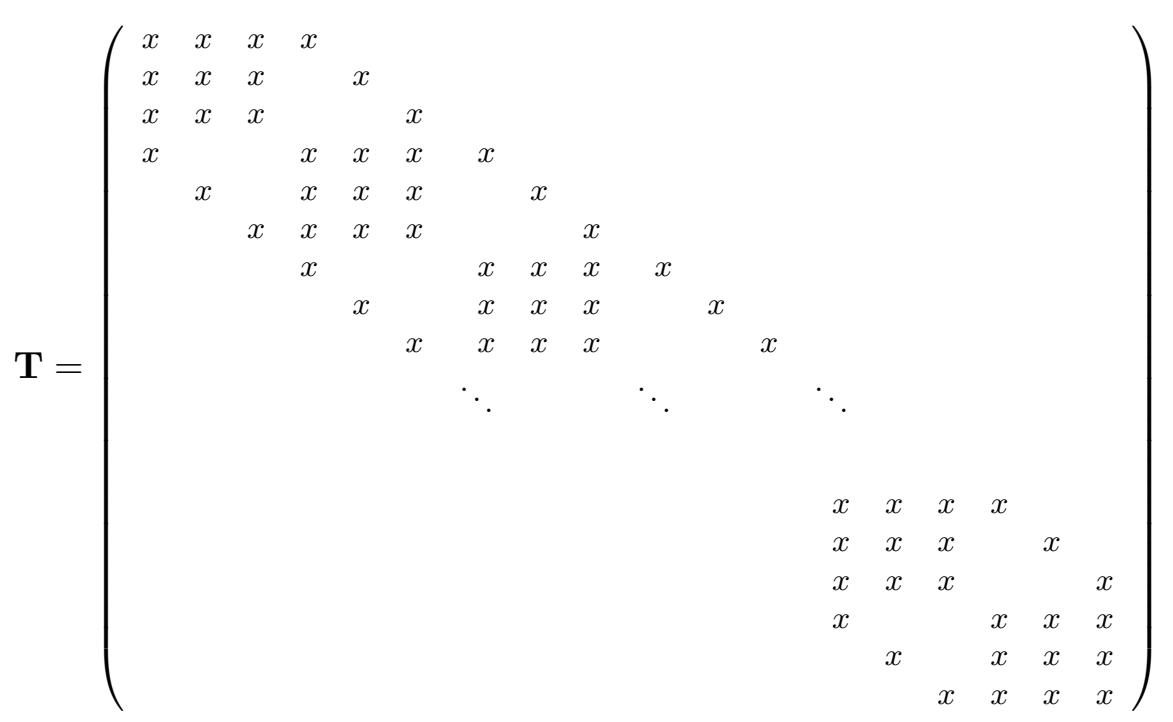

Figure 5.1: The structure of the monofrequent Feautrier matrix for coherent scattering with $m=3$. It consists of $3 \times 3$ submatrices, one per depth point. All empty elements are zero.

Feautrier solver as Lambda operator. The Feautrier method is a general and numerically robust method to compute $J_{\nu}\left(\tau_{\nu}\right)$ from a given source function $S_{\nu}\left(\tau_{\nu}\right)$. The two-level atom line source function was used in $(5,27)$ to illustrate a case where $S_{\nu}$ is not known but where (5.1.i) may be rewritten in closed form because the two-level line source function is linear in $J_{\nu}$. In general, the Feautrier method is a differential realization of the $\boldsymbol{\Lambda}_{\nu}$ operator defined by (4. is faster than numerical integration methods that compute $\boldsymbol{\Lambda}_{\nu}\left[S_{\nu}\right]$ directly from $S_{\nu}\left(\tau_{\nu}\right)$ with the exponential integral in (4.20i). Thus, a differential solver (Feautrier method) may advantageously be used as an integral operator $\left(\boldsymbol{\Lambda}_{\nu}\right)$.

\subsection{Lambda iteration}

\subsubsection{Classical Lambda iteration}

The Feautrier method solves the boundary-condition problem in order to derive $J_{\nu}$ from a given $S_{\nu}$. The next question is how to derive $S_{\nu}$ itself.

Formal solution. Substitution of the $\boldsymbol{\Lambda}_{\nu}$ operator with

$$
J_{\nu}\left(\tau_{\nu}\right)=\mathbf{\Lambda}_{\nu}\left[S_{\nu}(t)\right]
$$

in the two-level coherent-scattering line source function gives:

$$
S_{\nu}^{l}\left(\tau_{\nu}\right)=\left(1-\varepsilon_{\nu}\left(\tau_{\nu}\right)\right) \boldsymbol{\Lambda}_{\nu}\left[S_{\nu}^{l}\left(t_{\nu}\right)\right]+\varepsilon_{\nu}\left(\tau_{\nu}\right) B_{\nu}\left(\tau_{\nu}\right) .
$$

This expression shows directly that $S_{\nu}$ must be known non-locally in order to find $S_{\nu}$ locally. The combination of localness and non-localness in the same variable makes this a 
conceptually difficult relation $n_{-\mathbf{r}}^{\pi}$

Discretization. The source function $S_{\nu}$ is a continuous function of the location; it depends only on $\nu$ and $\tau_{\nu}$ since we assume axial symmetry and source function isotropy. In practice, it is discretized and therefore a spatial vector per $\nu$, typically with 100 or so elements. That makes $\boldsymbol{\Lambda}_{\nu}$ a matrix operator, integrating per $\tau_{\nu}$ value over all other $\tau_{\nu}$ values when used on $S_{\nu}$.

Lambda iteration. Rewriting (5.30) by dropping all indices $\left(\nu, \tau_{\nu}\right.$ and $\left.l\right)$ and arguments $\left(\tau_{\nu}, t_{\nu}\right.$ and $\left.S_{\nu}\right)$ into

$$
S=(1-\varepsilon) \boldsymbol{\Lambda}_{\nu}[S]+\varepsilon B
$$

shows that the direct solution is given by

$$
S=(1-(1-\varepsilon) \boldsymbol{\Lambda})^{-1}[\varepsilon B],
$$

where the brackets [ ] still mean "operated upon" so that the exponent -1 describes inverse operation. This solution requires matrix inversion which is computationally expensive. It is a typical example of the general matrix equation $\mathbf{A}[\mathbf{x}]=\mathbf{b}$ that is difficult to solve directly from $\mathbf{x}=\mathbf{A}^{-1}[\mathbf{b}]$ when $\mathbf{A}$ is very large or hard to invert (having many off-diagonal elements). If computation of $\mathbf{A}[\mathbf{x}]$ is significantly easier than $\mathbf{A}^{-1}[\mathbf{b}]$ one should use iteration. For example, for 3D radiative transfer through an $N$-point grid the computational cost of matrix inversion scales as $N^{9}$ while iteration takes $n_{\text {it }} N^{3}$ and is much cheaper if one manages to keep the number of required iteration steps $n_{\text {it }}$ small. The classical alternative to $(5.32 i)$ is therefore to "Lambda-iterate" as

$$
S^{(n+1)}=(1-\varepsilon) \boldsymbol{\Lambda}\left[S^{(n)}\right]+\varepsilon B,
$$

starting with a first guess $S^{(1)}$, for example $S^{(1)}=B$.

Multi-level iteration. The principle of iteration is not restricted to the didactic case of a two-level atom. In the general multi-level case, Lambda iteration may proceed as

$$
\begin{aligned}
J_{\nu}^{(n)} & =\boldsymbol{\Lambda}_{\nu}\left[S_{\nu}^{(n)}\right] \\
\mathbf{n}^{(n)} & =f_{1}\left(J^{(n)}\right) \\
S_{\nu}^{(n+1)} & =f_{2}\left(\mathbf{n}^{(n)}\right) .
\end{aligned}
$$

The first step may use the Feautrier method to obtain $J_{\nu}(i j)$ for each frequency needed to sample each pertinent transition $i j$ at each depth from the total source function $S_{\nu}(i j)$. The second step then determines, per depth, the corresponding level populations $\mathbf{n}$ (a vector constituting many levels for all pertinent transitions) from the radiation fields, using the statistical equilibrium equations as schematically defined here by the functional $f_{1}$. In the third step, new source functions are determined for each transition (or for each frequency point) from the populations, for example using the line source function (2.7.7i) on page $2 \overline{2} \overline{5}_{1}$ as functional $f_{2}$ for lines in complete redistribution and mixing that according to

\footnotetext{
${ }^{4}$ Just as the $\sqrt{\varepsilon}$ law which is a surface representation of this depth-dependent relation. Hubeny $(1987 \mathrm{~b})$ calls $\left(15.30_{1}^{\prime}\right)$ the Rybicki equation and elaborates on his interpretative experiments of the $\sqrt{\varepsilon}$ law in (1987a) to explain it.
} 
$\left(\overline{2} 2 \overline{2} 3_{i}\right)$ with $S_{\nu}^{c}$ for the background continuum per frequency. The latter may also depend on scattering processes for which $J_{\nu}$ is needed, but the thermal part $\left(S_{\nu}^{c}=B_{\nu}\right)$ may be evaluated once and for all at the start.

An exact linear form was required in (5.27) to close the transport equation for (5.17i), but thanks to the iteration employed here the functionals $f_{1}$ and $f_{2}$ in $(5.351)-(5.36 i)$ may be approximate rather than exact. The errors will then be corrected in the successive iterations (if these converge). The technique is discussed in more detail in Section 5.5 below.

Convergence. The problem of Lambda iteration is that it converges very slowly at large optical depth (Mihalas 1970 page 46; 1978 page $147 \mathrm{ff}$ ). Near the surface, Lambda iteration affects appreciable changes per step as seen for a linear $S(\tau)=a+b \tau$ from:

$$
\begin{aligned}
\boldsymbol{\Lambda}_{\tau}[a+b \tau] & =a+b \tau+\frac{1}{2}\left[b E_{3}(\tau)-a E 2(\tau)\right] \\
\boldsymbol{\Lambda}_{\tau=0}[S] & =\frac{a}{2}+\frac{b}{4}
\end{aligned}
$$

since $E_{2}(0)=1$ and $E_{3}(0)=1 / 2$ (Table $\overline{4} .1$ in page $S / 2=I / 2$ for a constant $-S$ atmosphere with $b=0$; in that case, the $\boldsymbol{\Lambda}_{\nu}$ operator produces the required halving from $S$ to $J$ (no incoming photons) in a single step. At large depth in a scattering atmosphere, however, the $\boldsymbol{\Lambda}_{\nu}$ operator achieves only much smaller corrections. The exponential integrals drop as $(1 / \tau) \exp (-\tau)$ for $\tau>1$ in (4.13i) on page $\overline{1} \overline{8}_{1}^{\prime}$, so rapidly that their weighting of $S(|t-\tau|)$ in $\boldsymbol{\Lambda}_{\nu}$ around $t=\tau$ tends to produce a value close to $S(\tau)$ as in (4.43i): $J(\tau)=\boldsymbol{\Lambda}_{\nu}[S] \approx S(\tau)$ for $\tau>1$. The convergence speed given by the change per step

$$
S^{(n+1)}-S^{(n)}=(1-\varepsilon) \boldsymbol{\Lambda}_{\nu}\left[S^{(n)}\right]+\varepsilon B-S^{(n)}
$$

is therefore small for $\varepsilon \ll 1$ and $\tau>1$ :

$$
S^{(n+1)}-S^{(n)} \approx \boldsymbol{\Lambda}_{\nu}\left[S^{(n)}\right]-S^{(n)} \approx S^{(n)}-S^{(n)} \approx 0 .
$$

Procedurally, the reason is that $(5.33$ in $)$ solves the nonlocal radiative transfer equation $J=\boldsymbol{\Lambda}[S]$ and the local population equation $S=(1-\varepsilon) J+\varepsilon B$ (statistical equilibrium for two-level atoms) sequentially instead of combining them and solving them together. Physically, the reason is that $\boldsymbol{\Lambda}_{\nu}$ follows photons in its propagation of corrections per step since it transfers the old $S$ into a new one using the photons in $J$. Therefore, the corrections propagate with $\Delta \tau=1$ steps just as photons do. Mathematically, $E_{1}(\Delta \tau) \sim$ $\exp (-\Delta \tau) / \Delta \tau$ for $\Delta \tau \gg 1$ as shown by (4.13). For strong scattering the distance between $\tau=1$ and the thermalization depth $\tau=\Lambda$ measures many $\Delta \tau=1$ steps, of order $1 / \sqrt{\varepsilon}$ for constant $\varepsilon$ in the case of coherent scattering and much larger yet for complete redistribution, of order $1 / \varepsilon$ for a Gaussian line and of order $1 / \varepsilon^{2}$ for a Lorentzian as given by $(4.106 i)-(4.107)$ on page 1100 . Starting with $S^{(1)}=B$ then means that the successive $S^{(n)}$ estimates barely differ from $S=B$ for depths $\tau>1$. An illustrative example is shown in the upper-left panel of Figure 5.2 .12 on page $1 \overline{1} 2 \overline{2}$. The corrections per step have become very small after 20 iterations, especially for large $\tau$, but the correct result is still far away, of order $10^{3}$ iterations.

In the older literature the smallness of the successive corrections was often taken as proof that LTE holds in cases where it actually doesn't. The relative change $\Delta S / S$ per iteration is not a valid precision indicator; one should instead require that $\Delta S / S \ll 1 / \Lambda$ 
before stopping the iteration (Auer 1991 ), but that takes as many as $\varepsilon^{-2}$ iterations for a Lorentzian line, $10^{3}-10^{8}$ for strong scattering. Lambda iteration therefore got a very bad name ("it doesn't converge"). It actually remains useful for situations where photons travel far between successive extinctions, for example in hot-star winds.

\subsubsection{Approximate Lambda iteration}

Operator perturbation. 'C'annon- $(1 \overline{9} \overline{7} \overline{3})_{1}^{\prime}$ started the currently blooming industry of operator perturbation methods with approximate operators. The basic idea is to use a $\boldsymbol{\Lambda}_{\nu}$-like operator that also produces $J_{\nu}$ from $S_{\nu}$ but does so inaccurately rather than exact. The approximate Lambda operator $\boldsymbol{\Lambda}^{*}$ should then be a simple one, permitting very fast implementation, but sufficiently precise that iteration makes up for its deficiencies in not too many iteration steps. When splitting $\boldsymbol{\Lambda}_{\nu}$ in

$$
\boldsymbol{\Lambda}_{\nu}=\boldsymbol{\Lambda}^{*}+\left(\boldsymbol{\Lambda}_{\nu}-\boldsymbol{\Lambda}^{*}\right)
$$

the mean intensity given by

$$
J_{\nu}=\boldsymbol{\Lambda}_{\nu}^{*}[S]+\left(\boldsymbol{\Lambda}_{\nu}-\boldsymbol{\Lambda}_{\nu}^{*}\right)[S]
$$

is still exact because the second term on the righthand side represents a correction to the approximate result in the first term. For the illustration case of two-level atoms with coherent scattering an iteration scheme may now be set up as

$$
S^{(n+1)}=(1-\varepsilon) \boldsymbol{\Lambda}^{*}\left[S^{(n+1)}\right]+(1-\varepsilon)\left(\boldsymbol{\Lambda}_{\nu}-\boldsymbol{\Lambda}^{*}\right)\left[S^{(n)}\right]+\varepsilon B
$$

where the new $(n+1)^{\text {th }}$ estimate of $S$ is also entered in the first term on the righthand side in order to make the correction term in the remainder similar to the righthand side of (5.33) on page $12 \overline{2} \overline{3}^{1}$. Reshuffling gives

$$
\begin{aligned}
S^{(n+1)}-(1-\varepsilon) \boldsymbol{\Lambda}^{*}\left[S^{(n+1)}\right] & =(1-\varepsilon) \boldsymbol{\Lambda}_{\nu}\left[S^{(n)}\right]+\varepsilon B-(1-\varepsilon) \boldsymbol{\Lambda}^{*}\left[S^{(n)}\right] \\
& =S^{\mathrm{FS}}-(1-\varepsilon) \boldsymbol{\Lambda}^{*}\left[S^{(n)}\right],
\end{aligned}
$$

where $S^{\mathrm{FS}} \equiv(1-\varepsilon) \boldsymbol{\Lambda}_{\nu}\left[S^{(n)}\right]+\varepsilon B$ is the formal solution $(\overline{5} . \overline{30})$ obtained from exact $\boldsymbol{\Lambda}_{\nu}$ operation on the known source function $S^{(n)}$. The new estimate is then found from inverting as in ( $(5.32)$ :

$$
S^{(n+1)}=\left(1-(1-\varepsilon) \boldsymbol{\Lambda}^{*}\right)^{-1}\left[S^{\mathrm{FS}}-(1-\varepsilon) \boldsymbol{\Lambda}^{*}\left[S^{(n)}\right]\right] .
$$

An important difference with $\left(\overline{5} \cdot \overline{3}^{*} i\right)$ is that only the approximate operator $\boldsymbol{\Lambda}^{*}$ is inverted, not the full $\boldsymbol{\Lambda}_{\nu}$; much less work if $\boldsymbol{\Lambda}^{*}$ is cleverly chosen. In addition, the convergence at large depth is much faster than for classical Lambda iteration. In the latter case, the convergence is given by $\left(\overline{5} . \overline{3} \overline{7}_{i}\right)$ as

$$
S^{(n+1)}-S^{(n)}=S^{\mathrm{FS}}-S^{(n)},
$$

while subtraction of $S^{(n)}$ from both sides of $(5.42 i)$ and re-ordering gives for the approximate $\boldsymbol{\Lambda}_{\nu}$ iteration

$$
\begin{aligned}
& S^{(n+1)}-(1-\varepsilon) \boldsymbol{\Lambda}^{*}\left[S^{(n+1)}\right]+(1-\varepsilon) \boldsymbol{\Lambda}^{*}\left[S^{(n)}\right]-S^{(n)}=S^{\mathrm{FS}}-S^{(n)} \\
& \left(1-(1-\varepsilon) \boldsymbol{\Lambda}^{*}\right)\left[S^{(n+1)}\right]-\left(1-(1-\varepsilon) \boldsymbol{\Lambda}^{*}\right)\left[S^{(n)}\right]=S^{\mathrm{FS}}-S^{(n)}
\end{aligned}
$$

\footnotetext{
${ }^{5}$ If one would enter $S^{(n)}$ in the first term on the righthand side, $(\overline{5}-\overline{4} \overline{1})$ remains identical to $\left(\overline{5} . \overline{3} \overline{3}_{i}\right)$ so that the splitting has no effect.
} 
so that

$$
S^{(n+1)}-S^{(n)}=\left(1-(1-\varepsilon) \boldsymbol{\Lambda}^{*}\right)^{-1}\left[S^{\mathrm{FS}}-S^{(n)}\right] .
$$

At large depth, $J_{\nu} \approx S_{\nu}$ so that $\boldsymbol{\Lambda}^{*} \approx 1$ just as $\boldsymbol{\Lambda}_{\nu} \approx 1$. The inverted operator

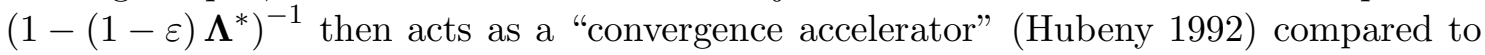
$(15.44)$ since it has

$$
\left(1-(1-\varepsilon) \Lambda^{*}\right)^{-1} \approx 1 / \varepsilon,
$$

giving considerable step size increase for $\varepsilon \ll 1$. This is the reason that "ALI" is often taken to mean "accelerated Lambda iteration".

How to define the approximate operator $\Lambda^{*}$ ? The two extremes are $\boldsymbol{\Lambda}^{*}=\boldsymbol{\Lambda}_{\nu}$, equal to the accurate but laborious direct inversion in (5.32i), and $\boldsymbol{\Lambda}^{*}=0$, equal to the classical Lambda iteration in (5.33), without costly inversion but with bad convergence. Therefore, the two contrary conditions one seeks to fulfill in $\boldsymbol{\Lambda}^{*}$ are:

$-\boldsymbol{\Lambda}^{*}$ should contain the basic physical properties of $\boldsymbol{\Lambda}_{\nu}$ in order to cut down the number of iterations;

- $\boldsymbol{\Lambda}^{*}$ should be simple (meaning fast in computer time) to construct and should be simple to invert.

Such operators are discussed below. The iteration scheme of (5.4i) employs once again the special property of the two-level line source function that it is linear in $J_{\nu}$, but as we will see

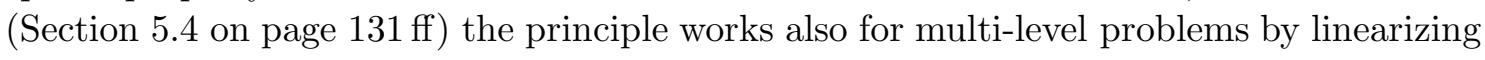
all actual dependences and letting the iterations correct for these simplifications.

\subsubsection{Approximate Lambda operators}

This section discusses three different approximate $\boldsymbol{\Lambda}$ operators, respectively the core saturation $\boldsymbol{\Lambda}_{\nu}$ operator, the Scharmer $\boldsymbol{\Lambda}_{\mu \nu}$ operator, and the OAB diagonal operator, in the order in which they have appeared in the literature and came into use.

Core saturation operator. Classical Lambda iteration converges slowly when there is much scattering in optically thick regimes ${ }^{5 !}$ because the iteration proceeds like photons. The worst photons are those in the center of a thick line with large $\eta$; they scatter a

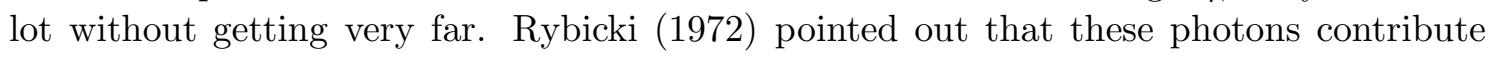
most to the slow convergence of $\boldsymbol{\Lambda}$ iteration, but are also the photons that carry least information about non-local conditions. At sufficient depth, the local intensity saturates to:

$$
I_{\nu}(z) \approx S_{\nu}^{\mathrm{tot}}(z)
$$

This approximation does not hold near the surface where photons escape. The surface regime is at a different location for an opaque line core and for transparent wings. The core saturation approximation is given by

$$
I_{\nu}(z)=S_{\nu}(z) \text { for } \tau_{\nu}(z)>\gamma
$$

\footnotetext{
${ }^{6}$ The problem posed by scattering in thick regimes is what this whole chapter is about. For optically thin objects no radiative transfer is needed; for LTE conditions the source function is known so that the Feautrier solution produces the radiation field in a single pass. In actual optically-thick objects, multi-level coupling across frequencies is usually important and photon conversion sequences as in planetary nebulae (Zanstra mechanism) may be important. The illustration case of coherent two-level scattering gets so much attention here and in the literature because it is the simplest one in which the non-local nature of radiation is taken into account, without having to bother with non-monochromatic coupling between different wavelength domains.
} 

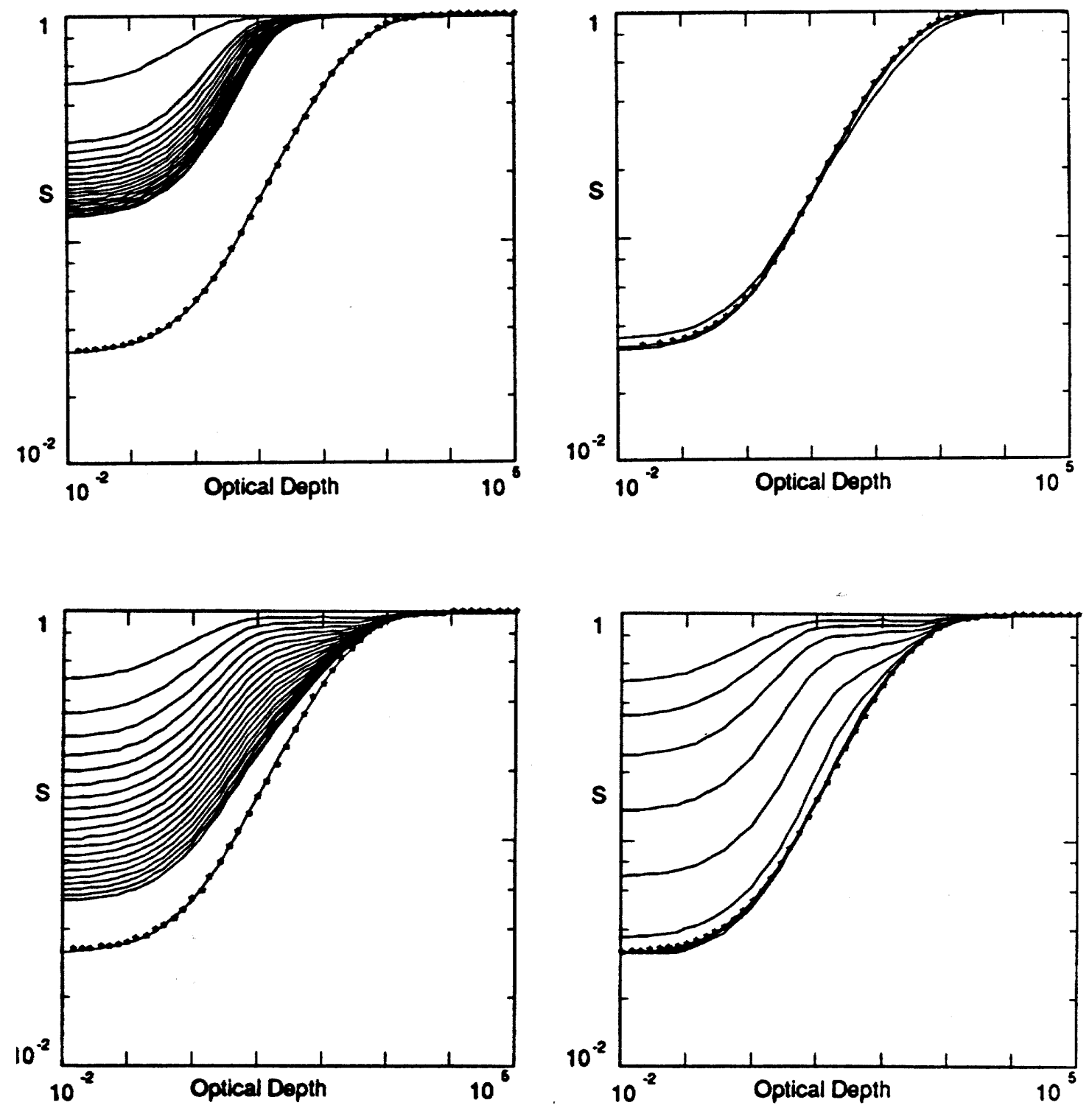

Figure 5.2: Examples of convergence for Lambda iteration with different operators, for the classic test case of a semi-infinite isothermal atmosphere with constant $\varepsilon_{\nu_{0}}=10^{-3}$ and complete redistribution over a Doppler profile (Gaussian line broadening, the same as Figure 4.12 on page 108i). Each panel plots twenty successive source function estimates $S^{(n)}$ with $n=1, \ldots, 20$ against optical depth, starting from $S=B$. The correct solution is given by the curve with superposed dots in each panel. The scales are $\operatorname{logarithmic}$; the x-axis extends from $\log \tau=-2$ to $\log \tau=5$, the y-axis from $S=10^{-2}$ to $S=1$. The mean intensity $\bar{J}$ has $\bar{J} \approx S$. The radiation field thermalizes near $\tau=\Lambda_{\nu_{1}} \approx 10^{3}$ corresponding to (4.106i) on page 110 . Upper left: classical $\boldsymbol{\Lambda}$ iteration as discussed on page 124. The twenty $\boldsymbol{\Lambda}$ iterations don't reach the converged solution (bottom curve) since of order $\Lambda_{\nu_{0}} \approx 10^{3}$ iterations are required to do so. However, the increments have become deceptively small percentage-wise; it is easy to believe mistakenly that the right $S$ is close already at $n=20$. Upper right: iteration with the Scharmer operator (page 128i). Its convergence is excellent: it reaches the correct curve already at $n=2$. Lower left: iteration with the local OAB operator (page $130 \mathrm{j})$, consisting of the diagonal of the $\boldsymbol{\Lambda}$ matrix. Reasonable convergence. Lower right: iteration with the same one-point operator using "conjugate vector" acceleration. From iAuer" i. - (1991) 
with $\gamma=2-5$ a number that defines per $z$ whether $\nu$ is in the "core" (optically thick regime) or in the "wings" (optically thin regime). It effectively determines whether photons may escape and defines the escape probability. At each height $z$ all frequencies (and directions) with $\tau_{\nu}(z)>\gamma$ belong to the core, while those with $\tau_{\nu}<\gamma$ sit in the wings and may describe escaping photons. The accuracy of this approximation increases with $\gamma$.

'Scharmer $(19 \overline{1} \overline{1})_{1}^{1}$ defined a simple approximate $\boldsymbol{\Lambda}_{\nu}^{*}$ using the core saturation approximation for the optically thick regime:

$$
\boldsymbol{\Lambda}_{\nu}^{*}\left[S\left(\tau_{\nu}\right)\right]=S\left(\tau_{\nu}\right) \text { or } \boldsymbol{\Lambda}_{\nu}^{*}=1 \text { for } \tau_{\nu} \geq \gamma
$$

with $\gamma=2-5$. For the optically thin regime (surface layers, line wings in deeper layers) he used the Eddington-Barbier-like approximation

$$
J_{\nu} \approx \frac{1}{2} S_{\nu}\left(\tau_{\nu}=\gamma\right)
$$

so that

$$
\boldsymbol{\Lambda}_{\nu}^{*}\left[S\left(\tau_{\nu}\right)\right]=\frac{1}{2} S\left(\tau_{\nu}=\gamma\right) \text { or } \boldsymbol{\Lambda}_{\nu}^{*}=\frac{1}{2} \int \delta\left(\tau_{\nu}-\gamma\right) \mathrm{d} \tau_{\nu} \text { for } \tau_{\nu}<\gamma
$$

This simple operator represents numerical integration by one-point quadrature. Șcharmen - $(\overline{1} 9 \overline{8} \overline{1})$, found that it is a stable and fast method for two-level-like resonance lines well with $\gamma$ as small as $\gamma=2$. It has been employed successfully in various variants in various codes (Werner and Husfeld $19 \overline{8} \overline{8}$, Werner $19 \overline{8} 6$, Hamann 1985, 1986), but a drawback of this operator is that the somewhat arbitrary choice of the parameter $\gamma$ affects the speed of convergence. Its optimum value depends on the nature of the problem and is

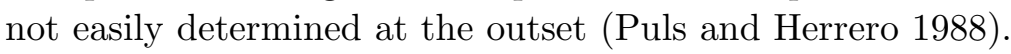

Scharmer operator. The core saturation operator $\Lambda_{\nu}^{*}$ in $(5.49)-(5.51)$ is a sort of Eddington-Barbier approximation, exact for a constant source function with $J(\tau=0)=$ $I / 2$ (cf. 5.50$]$ ). It produces $J_{\nu}$, while the actual Eddington-Barbier relation produces $I_{\nu}(0, \mu)$ rather than $J_{\nu}(0)$. 'Scharmer $(19 \overline{9} \overline{1})_{1}^{1}$ continued by defining an alternative approximate operator $\boldsymbol{\Lambda}_{\nu \mu}^{*}$ that produces $I_{\nu}\left(\tau_{\nu}, \mu\right)$ rather than $J_{\nu}\left(\tau_{\nu}\right)$ from $S_{\nu}\left(\tau_{\nu}\right)$ per angle $\mu$ at every depth $\tau_{\nu}$. The Eddington-Barbier-like character of $\boldsymbol{\Lambda}_{\nu}^{*}$ and the simplicity of the one-point quadrature in $(5 . \overline{4})-(5.51)$ made him search for quantities $W_{\nu \mu}^{ \pm}$and $\tau_{\nu \mu}^{ \pm}$in the one-point quadrature expressions

$$
I_{\nu}\left(\tau_{\nu \mu}, \mu\right) \equiv I_{\nu \mu}^{ \pm}=\Lambda_{\nu \mu}^{*}\left[S_{\nu}\left(\tau_{\nu \mu}\right)\right] \approx W_{\nu \mu}^{ \pm}\left(\tau_{\nu \mu}\right) S_{\nu}\left(f_{\nu \mu}^{ \pm}\left(\tau_{\nu \mu}\right)\right)
$$

${ }^{7}$ Scharmer (1981)!' used it to treat the illustration case of a two-level atom with complete redistribution in the presence of a thermal background continuum as discussed on page 104. In that case, (15.43') delivers the NLTE line source function $S^{l}$ without having to specify and linearize the rate equations in detail. It is applicable when the NLTE effects from resonance scattering in the line source function are more important than the NLTE effects from other processes in the line opacity, as is the case for the solar Ca II $\mathrm{H} \& \mathrm{~K}$ lines or more in general, for resonance lines from the dominant ionization stage. For these, NLTE ionization effects are not important and the two-level approximation holds very well, with $b_{l} \approx 1$ and no large changes in the line opacity between successive iterations. Resonance lines of minority stages, such as the solar Na ID lines, have two-level-atom source function behavior in $b_{u} / b_{l}$, but $b_{u}$ and $b_{l}$ are together sensitive to the NLTE ionization balance because most sodium particles are ionized in the solar atmosphere (see Section 10.1 , in particular Figure 10.21 on page 2151 ). In such minority-stage cases, both the line source function and the line opacity may change considerably between iterations. 
i.e., Eddington-Barbier-like approximations at any depth. The + variables are again for outgoing beams with $\mu>0$, the - variables for incoming directions with $\mu<0$, and the optical depth $\tau_{\nu \mu}$ is again measured inwards along or against the beam, with

$$
\mathrm{d} \tau_{\nu \mu}=\left(1+\eta_{\nu}\right) \frac{\mathrm{d} \tau_{\nu}^{c}}{|\mu|}
$$

where $\tau_{\nu}^{c}$ is the radial continuum optical depth

What are suitable $W_{\nu \mu}^{ \pm}$and $f_{\nu \mu}^{ \pm}$? Let's once again derive the Eddington-Barbier relation. The transport equation

$$
\frac{\mathrm{d} I_{\nu \mu}}{\mathrm{d} \tau_{\nu \mu}}=I_{\nu \mu}-S_{\nu}
$$

has $(\overline{4} . \overline{2} \bar{i})$ on page $\overline{8} \overline{1}$, as solution for $\mu>0$

$$
I_{\nu \mu}^{+}=\mathrm{e}^{\tau_{\nu \mu}} \int_{\tau_{\nu \mu}}^{\infty} S_{\nu} \mathrm{e}^{-t_{\nu \mu}} \mathrm{d} t_{\nu \mu}
$$

and $(4 . \overline{3} 1)$ for $\mu<0$

$$
I_{\nu \mu}^{-}=\mathrm{e}^{-\tau_{\nu \mu}} \int_{0}^{\tau_{\nu \mu}} S_{\nu} \mathrm{e}^{t_{\nu \mu}} \mathrm{d} t_{\nu \mu}
$$

again putting $I_{\nu \mu}^{-}\left(\tau_{\nu \mu}=0\right)=0$. The assumption of linear depth-dependence of the source function along the beam

$$
S_{\nu}=a+b \tau_{\nu \mu}
$$

produces with $\int x \exp (-x) \mathrm{d} x=-(x+1) \exp (-x)$ for $\mu>0$

$$
I_{\nu \mu}^{+}=a+b+b \tau_{\nu \mu}
$$

and with $\int x \exp (x) \mathrm{d} x=(x-1) \exp (x)$ for $\mu<0$

$$
I_{\nu \mu}^{-}=a-b+b \tau_{\nu \mu}-(a-b) \mathrm{e}^{-\tau_{\nu \mu}} .
$$

These approximate Eddington-Barbier solutions hold at any depth. Scharmer's trick is to use them also at depth, not only at the surface, to define the integration weights and points in (5.5). For $\mu>0$ they become

$$
\begin{aligned}
W_{\nu \mu}^{+} & =1 \\
f_{\nu \mu}^{+} & =\tau_{\nu \mu}+1
\end{aligned}
$$

to produce

$$
I_{\nu \mu}^{+}\left(\tau_{\nu \mu}\right)=1 \cdot S_{\nu}\left(\tau_{\nu \mu}+1\right)=a+b\left(\tau_{\nu \mu}+1\right)
$$

which is a local Eddington-Barbier approximation saying that at any depth the local intensity in an outgoing beam comes from deeper layers and is represented by the source function $\Delta \tau=1$ deeper along the beam. Very simple and very clever ${ }_{-1}^{\mathbf{8}}$. Similarly, for

\footnotetext{
8 Like Scharmer's vacuum solar telescope on La Palma. The design paper in Applied Optics by Scharmer iet al. (1985)' was surprising: who built a refractor in the twentieth century? The clever trick was that the imaging doublet at the entrance of the telescope doubles as a vacuum window. It avoids the problems that plane vacuum windows have from internal stresses, by being designed to take its proper shape under 1 bar pressure differential. Since then, Scharmer has developed clever hardware and software systems to grab and hold the sharpest images out of the many bad ones that are spoiled by atmospheric seeing. Another trick was to use phase diversity image registration, in focus and out of focus simultaneously, to restore spoiled wavefronts by sampling the spoiling ('Löfdahl and Scharmer 1994 , 'Paxman et al. 1996). Currently, he is revamping his telescope into what is likely to become the first adaptively corrected one with angular resolution at $0.1^{\prime \prime}$.
} 
ingoing beams with $\mu<0$ he sets

$$
\begin{aligned}
W_{\nu \mu}^{-} & =1-\mathrm{e}^{-\tau_{\nu \mu}} \\
f_{\nu \mu}^{-} & =\frac{\tau_{\nu \mu}}{1-\mathrm{e}^{-\tau_{\nu \mu}}}-1
\end{aligned}
$$

which, when used in (5.52), produces (5.59). At large depth core saturation occurs automatically since $W_{\nu \mu}^{+}=W_{\nu \mu}^{-}=1$ and $I_{\nu \mu}=S_{\nu}$. At small depth $W_{\nu \mu}^{+} \rightarrow 1$ and $f_{\nu \mu}^{+}=1$, recovering the Eddington-Barbier approximation for the emergent intensity, while $W_{\nu \mu}^{-} \rightarrow 0$ recovers the outer boundary condition. Thus, these generalized Eddington-Barbier approximations produce a new approximate operator $\boldsymbol{\Lambda}_{\nu \mu}^{*}$ called the Scharmer operator. It is an excellent one, as shown in the upper-right panel of Figure 5.2. by SScharmer and Carlsson (19985); with a Newton-Raphson complete linearization scheme (Section

Partial redistribution. An advantage of the Scharmer operator is that it is $\mu$ and $\nu$ dependent so that complexities having to do with $\mu$ and $\nu$ variations may be accommodated, including anisotropy in $S_{\nu}$. 'Uitenbroek $\overline{1} \overline{1989)}$, has expanded the method for the case of partial frequency redistribution (a frequency-dependent prescription of how photons change frequency in resonance scatterings, see Section $3 . \overline{4} . \overline{3}$ on page $\left.{ }_{1}^{7} \overline{2}{ }_{1}^{\prime}\right)$. This is done, following another Scharmer idea, by inserting a redistribution iteration loop between successive Scharmer operator iterations.

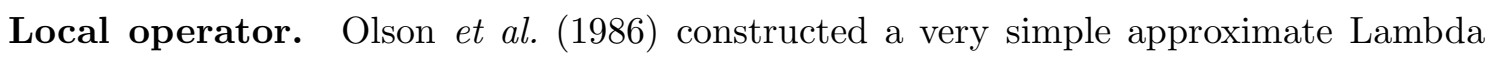
operator (often called the $\mathrm{OA} \overline{\mathrm{B}}$ operator) by using only the diagonal of the matrix which describes the exact Lambda operator. Its inversion is trivial. This (very) approximate operator takes only the local influences from $S$ on $J$ into account. In the exact Lambda operator the matrix elements that are farthest from the diagonal describe photons that travel farthest through the atmosphere. Elimination of all these is similar to the core saturation approximation. It implies that more iterations are required to obtain the correct radiation field, as seen in the lower-left panel of Figure, 5.2 . For large matrices (many depth points) or a large number of matrices (many lines, therefore many frequencies, and/or many angles) its advantage over the Scharmer operator is the much faster inversion.

Finding the $\boldsymbol{\Lambda}$ diagonal is less trivial than using it; an efficient recipe is given in Appendix B of 'Rybicki and Hummer $(\overline{1} \overline{9} \overline{9} \overline{1})^{\prime}$. It is based on the Feautrier scheme and permits simultaneous determination of the formal solution and of the diagonal of the inverse $\boldsymbol{\Lambda}_{\mu \nu}$ matrix. Their formalism makes it easy also to include side bands to the diagonal, incorporating some non-local cross-coupling.

Local operators are particularly useful for multi-dimensional radiative transfer in which the local intensity can be influenced by very different source functions in different directions. The Scharmer operator works best when only the source function variation along the line of sight counts, as in plane-parallel (1D) geometry; it is not easily generalized to multi-dimensional geometry.

Convergence acceleration The rather slow convergence of the local operator in Figure ". $2 . " 1$ and of other iteration sequences can be improved by acceleration methods. One of these is to estimate the change needed to get close to the final solution by extrapolating a least-square fit to the previous changes between successive iterations. An example from 
another scheme is shown in the lower-right panel of Figure 5.2. Convergence acceleration is now the frontier of ALI methodology

\subsection{Multi-level iteration}

For simplicity, the Feautrier method, classical Lambda iteration and approximate Lambda iteration have been presented above for the single-frequency case of two-level coherent scattering. We now turn to more general multi-level situations which require such solutions at many frequencies (across many spectral lines and bound-free edges) and also require simultaneous solution of the rate equations $(\hat{2} \cdot \overline{1} \overline{0} \overline{0})$ on page $\overline{3}_{2} \overline{2}_{r}^{\prime}$ If the height-dependent atmospheric state parameters $\left(T_{\mathrm{e}}, N_{\mathrm{e}}\right.$, chemical composition) and all atomic cross-sections are known $n_{-}^{\prime 10 !}$, we need to solve for all pertinent population densities and radiation fields. The basic problem is that their coupling through the radiation fields $J_{\nu}$ is highly non-linear.

Equivalent two-level atom method. The simplicity and transparency of the two-level problem is to some extent maintained in the "equivalent two-level" approach in which one transition is iterated at a time, keeping the transition rates in all other transitions that may take part in the problem constant until the solution for the iterated transition is sufficiently converged. One by one the other transitions then follow, and when all are done one starts all over again with the new solutions in an overall iteration step. The procedure can be fast and endlessly expanded, but has the disadvantage that both the convergence and the consistency can go wrong. The famous PANDORA code of iAvretet and Loeser $(\overline{1} \overline{9} \overline{9} \overline{2})$ : uses this technique.

Complete linearization. All equations are linearized by cutting all dependences on radiation to the first-order term in the complete linearization approach of Auer and Mihāàs $1(1969 \bar{a})_{i}^{1}$ The simple linear versions are then used within an iterative solution scheme. The solution per step can never be exact since the dependences are imprecise; therefore the whole problem is iterated until the results have converged to the desired precision at all depths. Thus, the idea is basically the same as for approximate operator iteration: the approximation makes the equations easier to solve but the solution inaccurate; successive iteration then reduces the errors to the desired small size (if convergence occurs).

Newton-Raphson iteration. Complete linearization represents multi-dimensional Newton-Raphson iteration for systems of equations. For a simple function $f(x)$ NewtonRaphson iteration is based on the Taylor expansion

$$
\begin{aligned}
f(x) & =\sum_{n=0}^{\infty} \frac{1}{n !} f^{(n)}\left(x_{0}\right)\left(x-x_{0}\right)^{n} \\
& =f\left(x_{0}\right)+\left[\frac{\partial f}{\partial x}\right]_{x_{0}}\left(x-x_{0}\right)+\mathcal{O}\left[\left(x-x_{0}\right)^{2}\right]
\end{aligned}
$$

\footnotetext{
${ }^{9}$ In keeping with Hubeny's (1992) feeling that the development of ALI methods has passed from physics to mathematics.

${ }^{10}$ Atomic cross-sections are usually badly known. The Sven-Erik Johansson conservation law says that the funding allocated_in physics to measure cross-sections times the astrophysical need for them is a constant (Rutten 1990bi); For this reason, astronomy is taking over. In the international Opacity Project started by Seaton (1987) transition probabilities for 1.6 million lines between 52000 levels in 18 elements (atoms and ions) have been computed quantummechanically already, and more are coming. The Opacity Project database and similar other databases (molecules) are accessible via Internet.
} 

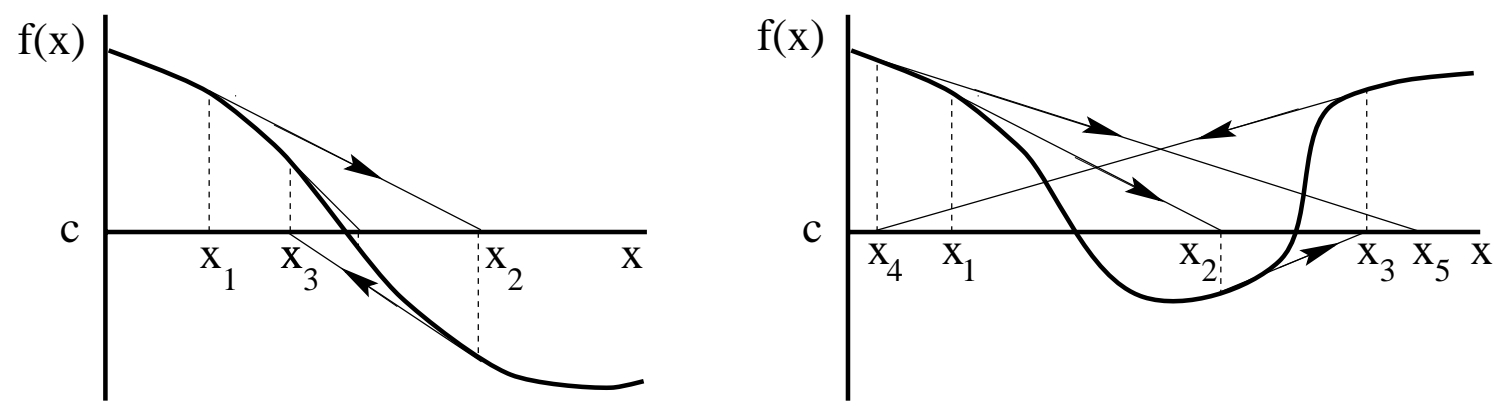

Figure 5.3: Newton-Raphson iteration to find the $x$ for which $f(x)=c$. Find the tangent to $f(x)$ at the first estimate $x=x_{1}$, find its intersection $x=x_{2}$ with the constant $c$, find the tangent to $f(x)$ there, locate its intersection at $x=x_{3}$, and so on. It works well at left but won't find either solution at right. The convergence region around the solution is small.

so that in first-order approximation ("linearization")

$$
f\left(x_{0}+\Delta x\right)-f\left(x_{0}\right) \approx\left[\frac{\partial f}{\partial x}\right]_{x_{0}} \Delta x
$$

Suppose we want to find the $x$ for which $f(x)=c$. Starting from a first guess $x=x^{(1)}$, substitution in (5.64)

$$
c-f\left(x^{(1)}\right) \approx\left[\frac{\partial f}{\partial x}\right]_{x^{(1)}} \Delta x^{(1)}
$$

delivers the approximation

$$
\Delta x^{(1)}=\frac{c-f\left(x^{(1)}\right)}{[\partial f / \partial x]_{x^{(1)}}}
$$

so that the next approximation is given by $x^{(2)}=x^{(1)}+\Delta x^{(1)}$. Und so weiter! Figure $\overline{3} .3$ illustrates the process. The convergence is fast, typically gaining one or more digits precision per step, if the starting value is sufficiently close to the correct value.

Newton-Raphson iteration is a more complex process when used for systems of equations, where chaotic flipping between different solutions or total lack of convergence may easily occurili... When, however, the starting solution is sufficiently close to the correct value in all parameters, also a multi-equation multi-dimensional Newton-Raphson iteration converges stably and fast. A trick to ensure sufficient proximity of the starting values in stellar atmosphere modeling is described on page $1 \overline{1} \overline{3} \overline{5}$.

An important advantage of Newton-Raphson iteration is that, when convergence occurs, the current error does not exceed the current increment $\Delta x^{(n)}$. You may see this graphically by playing games as in Figure 15.3 . Note that the error flips sign between successive steps.

\footnotetext{
${ }^{11}$ Two quotes from Numerical Recipes by Press et $\overline{a l} . \overline{(19 \overline{6})}$ ' "There are no good, general methods for solving systems of more than one nonlinear equation. Furthermore, it is not hard to see why (very likely) there never will be any good, general methods." and "[...] the simplest multidimensional root finding method, Newton-Raphson. This method gives you a very efficient means of converging to a root, if you have a sufficiently good initial guess. It can also fail spectacularly to converge, indicating (though not proving) that your putative root does not exist nearby".
} 
Rate equations. Complete linearization is used in nearly all multi-level radiative transfer codes. I illustrate it here following Scharmer and Carlsson (1985)! If $n_{i}^{(n)}$ is the current estimate, in the $(n)^{\text {th }}$ iteration step, of the population density $n_{i}$ of level $i$ and if the current estimates do not satisfy statistical equilibrium exactly, then the rate equations (2.100i) become:

$$
n_{i}^{(n)} \sum_{j \neq i}^{N} P_{i j}^{(n)}-\sum_{j \neq i}^{N} n_{j}^{(n)} P_{j i}^{(n)}=E_{i}^{(n)}
$$

where $N$ is the number of levels in the problem and $E_{i}^{(n)}$ is the current error. The rates $P_{i j}$ per particle in state $i$ or $j$ are:

$$
P_{i j}=R_{i j}+C_{i j}
$$

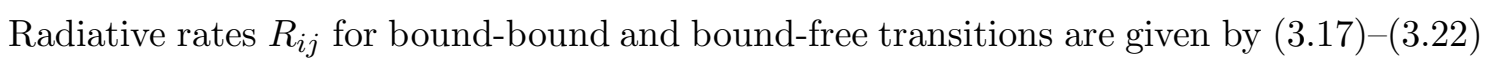

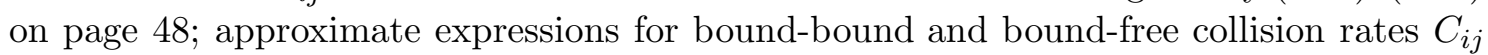
are given in (3.32i $)-(3.37)$ on page $1 . \overline{1}$. For given $n_{i}, n_{j}, \varphi(\Delta \nu)$ and $J_{\nu}$ they can be evaluated directly. To get these values they must be combined with the mass conservation equation (total number of particles per atomic species equal to the abundance fraction of the local density) and many radiative transfer equations, one for each frequency that is needed to cover all pertinent transition profiles sufficiently well and for each direction needed to integrate $I_{\nu \mu}$ to $J_{\nu}$.

After each iteration $(n)$ the equations (5.6.i) can be evaluated to obtain the current error $E_{i}$. It specifies the overall imbalance between transitions into level $i$ and out of it; the net rate is, in this iteration step, not zero but has an excess of $E_{i}$ transitions leaving $i$. Knowing this overall net rate error shows how far the current iteration is from the exact value in a global sense, but it doesn't show how each specific rate fails and does not deliver corrections that one might apply directly to the individual rates.

Perturbations. To obtain a better solution the current estimates $n_{i}^{(n)}$ and $P_{i j}^{(n)}$ are linearly perturbed as

$$
\begin{aligned}
& n_{i}^{(n+1)}=n_{i}^{(n)}+\delta n_{i}^{(n)} \\
& P_{i j}^{(n+1)}=P_{i j}^{(n)}+\delta P_{i j}^{(n)}
\end{aligned}
$$

with the requirement that the new values make $E_{i}^{(n)}$ go away:

$$
n_{i}^{(n+1)} \sum_{j \neq i}^{N} P_{i j}^{(n+1)}-\sum_{j \neq i}^{N} n_{j}^{(n+1)} P_{j i}^{(n+1)}=0 .
$$

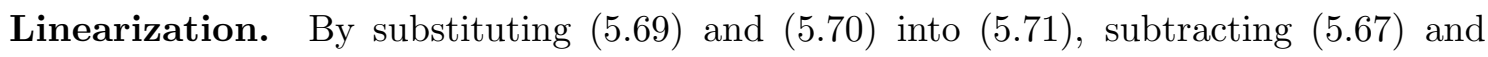
setting all nonlinear products $\delta n \cdot \delta P=0$ we obtain a set of linearized equations that express the current $\delta n_{i}$ in the current $\delta P_{i j}$ :

$$
\delta n_{i}^{(n)} \sum_{j \neq i}^{N} P_{i j}^{(n)}+n_{i}^{(n)} \sum_{j \neq i}^{N} \delta P_{i j}^{(n)}-\sum_{j \neq i}^{N} \delta n_{j}^{(n)} P_{j i}^{(n)}-\sum_{j \neq i}^{N} n_{j}^{(n)} \delta P_{j i}^{(n)}=-E_{i}^{(n)} .
$$

This is not yet a closed system since the $\delta P$ 's must be known to find the $\delta n_{i}$ 's or vice versa. The $\delta P$ 's follow from perturbations $\delta \bar{J}$ because the $\delta C_{i j}$ are zero (the collisions 
follow the local temperature without bothering with the radiation field) and $\delta \varphi\left(\nu-\nu_{0}\right)$ can be neglected, so that:

$$
\begin{aligned}
\delta P_{i j}^{(n)} & =B_{i j} \delta \bar{J}_{i j}^{(n)} \\
& =\frac{B_{i j}}{2} \int_{-1}^{+1} \int_{0}^{\infty} \varphi\left(\nu-\nu_{0}\right) \delta I_{\nu}^{(n)}(\mu) \mathrm{d} \nu \mathrm{d} \mu .
\end{aligned}
$$

Auer-Mihalas second-order solution. In the method of 'Âuer and Mihalas (1969) described by Mihalas (1978, p. $396 \mathrm{ff}$ ) the the radiation field $\bar{J}_{i j}$ are taken as the unknowns. Equation (15.73) is used to express the $\delta n_{i}$ into corrections $\delta \bar{J}_{i j}$ within a Feautrier scheme to solve the second-order transport equation

$$
\frac{\mathrm{d}^{2} f_{\nu} J_{\nu}\left(\tau_{\nu}\right)}{\mathrm{d} \tau_{\nu}^{2}}=J_{\nu}\left(\tau_{\nu}\right)-S_{\nu}\left(\tau_{\nu}\right)
$$

where $f_{\nu}$ is called the variable Eddington factor. It has $f_{\nu}=1 / 3$ in (4.59i) on page $\overline{9} \overline{2} \overline{2}$, and it makes this equation general by being variable. The population corrections are effectively written as

$$
\delta n_{i}=\sum_{k} \frac{\partial n_{i}}{\partial J_{k}} \delta J_{k}
$$

and a solution is then obtained for the $\delta J_{k}$ using the next-higher and next-deeper locations in a Feautrier-type forward elimination - backward substitution scheme.

This method formed the basis for a famous code named LINEAR described by Auer, iet. al. (1972)', that was the workhorse of NLTE spectral line modeling in the 1970's. In the 1980 's it was replaced by LINEAR-B following Rybicki's reformulation of the Feautrier method for lines with complete redistribution ${ }_{-}^{122 !}$ discussed on page $12 \overline{1} \overline{1}_{1}^{\prime}$.

Scharmer-Carlsson first-order solution. Scharmer and Carlsson $(1985)$, take the populations $n_{i}$ as the unknowns and employ the first-order transport equation for the intensity to find corrections $\delta n_{i}$. They use the Scharmer approximate operator in

$$
\delta I_{\nu}^{(n)}(\mu)=\boldsymbol{\Lambda}_{\nu \mu}^{*}\left[\delta S_{\nu}^{(n)}\right]
$$

on a perturbed source function $\delta S_{\nu}^{(n)}$ to determine the $\delta I_{\nu}^{(n)}$ in $(5.74)$. The source function perturbation is expressed into the population perturbations through perturbing and linearizing ( $\left.22^{-} \cdot \overline{3}\right)$ on page $\overline{2} \overline{5}_{\mathbf{r}}^{\prime}$ The complete system of linear equations is then closed and delivers population corrections $\delta n_{i}$ that should reduce the overall error $E_{i}$. The corrections are not exact in their combined effect since they are derived from $E_{i}$ through linearization, making further iteration necessary.

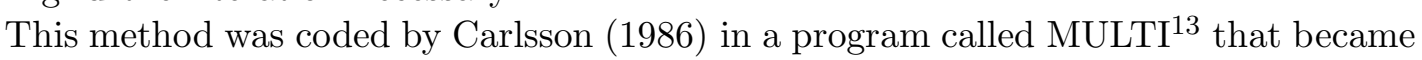
the workhorse in cool-star NLTE line formation. It is about 10 times faster than LINEAR$\mathrm{B}$ and handles atoms with up to a few hundred levels and lines. For large setups, it employs the $\mathrm{OAB}$ diagonal operator rather than the Scharmer one.

\footnotetext{
${ }^{12}$ I ran a version of LINEAR-B in the late 1970's on a CDC computer that was the only computer for all of Utrecht University. It was slower than your PC and it had only a few Mbyte memory. A run typically sat a full week in that computer before results came out, mostly just waiting for smaller jobs to get done. I monitored the progress with a $50 \mathrm{~kg}$ dumb terminal at home via a $110 \mathrm{bps}$ modem. The cost was 1000 Freudenthalers/job, paper guilders that I didn't have to pay but that did express the real price of the computer and the computer center personnel servicing it, some 30 white-coated persons. My largest model atom (for Fe II) had 5 levels and 5 lines.

${ }^{13}$ MULTI: http://www.astro.uio.no/ matsc/mul22.
} 
A yet more sophisticated and more general code which originates from hot-star modeling, combines stellar spectrum evaluation with the construction of stellar-atmosphere stratification assuming radiative and hydrostatic equilibrium, and also handles accretion disks is TLUSTY' of "Hubeny and Lanz

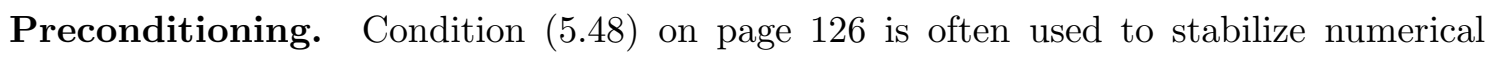
radiative transfer codes by eliminating all photons that are not taking an active part in the propagation of NLTE non-localness and anisotropy. The wing photons, with their much longer travel distance between successive extinctions, are much more important in defining these but are far fewer in number. Cutting the core photons out improves the numerical stability of the solution schemes (for example on computers with poor accuracy in single precision). This is called preconditioning. Of course, the advantage decreases when $\gamma$ is chosen large for better precision in the approximation; a best balance must be found.

'Scharmer and Carlsson $(1985)$, simply replace the Scharmer operator for actual application by

$$
\boldsymbol{\Lambda}_{\nu \mu}^{\dagger} \equiv \boldsymbol{\Lambda}_{\nu \mu}^{*}-1
$$

to achieve such preconditioning by cutting out all the saturated-core photons. In addition, they simply take $W_{\nu \mu}^{-}=0$ for $\tau_{\nu \mu}<0.1$ and use the Rosseland approximation for $\tau_{\nu}>10$.

Start-up trick. These multi-dimensional Newton-Raphson iterations do not converge if the starting guess is not sufficiently close to the correct solution. One usually starts with $S^{(1)}=B_{\nu}$ but this may be quite wrong. A classic problem case is to solve for the H I spectrum where the Lyman continuum and Ly $\alpha$ are very thick, scatter tremendously, and also set the structure of the atmosphere (which must therefore be recomputed along with the radiative transfer in each iteration step). Another one is the computation of radiative transfer in stellar atmospheres near the Eddington limit, where radiation pressure nearly blows the top off so that the amount of radiation may not be overestimated during the iteration. In general, such problems occur when the initial LTE estimate lies too far from the correct NLTE solution for the iteration to find its way towards the latter.

A simple, elegant remedy is the "collisional-radiative switching" of 'Hummer and Voès' (1985): They enforce the correctness of LTE at the start by setting all collisional crosssections to enormous values. The error $E^{(1)}$ after starting with $S_{\nu}=B_{\nu}$ is than small by definition; the solution is sufficiently close that proper convergence is ensured. They then gradually reduce the collisional cross-sections back to their real values, smoothly guiding the radiative transfer solution along. Thus, the trick is to compute physically correct but unrealistic solutions that move towards realism in small steps. It may be a slow process, but it always worksit!.

\footnotetext{
${ }^{14}$ TLUSTY: http://tlusty.gsfc.nasa.gov.

${ }^{15}$ The procedure may be used initially to come up with a better starting solution for subsequent setups and projects that represent variations on the initial one. The code should therefore be able to restart from a previously converged solution.
} 


\section{Chapter 6}

\section{Polarised Radiative Transfer}

$\prod$ his chapter discusses polarised transfer — but mostly not yet...

\subsection{Stokes parameters}

When the radiation in a beam is fully or partially polarized, three more quantities are required in addition to the monochromatic intensity $I_{\nu}$ to specify it completely. The wave representation of electromagnetic radiation provides an appropriate description. Two parameters are needed to describe the time-dependent orientation of the electric wave vector $\vec{E}$ in the vibration plane perpendicular to the propagation direction; the orientation of the magnetic vector $\vec{B}$ then follows because $|\vec{E}|=|\vec{B}|$ and $\vec{E} \perp \vec{B}$. The third parameter specifies the degree of polarization. In practice, this information is split differently between the Stokes parameters which furnish a description in observable quantities.

Stokes parameters for a single wave. Let us decompose the harmonic vibration of the electric field vector $\vec{E}_{\text {rad }}$ of a monochromatic light wave which propagates along the $z$-axis into its $x$ and $y$ components (Fig. $\overline{6} \cdot \overline{1}_{1}$ ):

$$
\begin{aligned}
& E_{x}=A_{x} \cos \left(\omega t-\phi_{x}\right) \\
& E_{y}=A_{y} \cos \left(\omega t-\phi_{y}\right),
\end{aligned}
$$

where $A_{x}$ and $A_{y}$ are the amplitudes, $\phi_{x}$ and $\phi_{y}$ the phase offsets and $\omega=2 \pi \nu$ is the circular frequency. For a fully polarized wave, the four Stokes parameters are defined by:

$$
\begin{aligned}
I_{\nu} & \equiv A_{x}^{2}+A_{y}^{2} \\
Q_{\nu} & \equiv A_{x}^{2}-A_{y}^{2} \\
U_{\nu} & \equiv 2 A_{x} A_{y} \cos \left(\phi_{x}-\phi_{y}\right) \\
V_{\nu} & \equiv 2 A_{x} A_{y} \sin \left(\phi_{x}-\phi_{y}\right),
\end{aligned}
$$

with $I_{\nu}^{2}=Q_{\nu}^{2}+U_{\nu}^{2}+V_{\nu}^{2}$. "Fully polarized" means that the vector $\vec{E}$ is well-behaved, its tip harmonically traveling along a line, ellipse or circle in the $(x, y)$ plane. In these cases the wave is said to be linearly polarized, elliptically polarized, or circularly polarized. Depending on whether the vector tip travels clockwise or counterclockwise, the elliptical and circular polarizations are called left-handed or right-handed. Usually right-handed implies clockwise as seen by the observer towards whom the beam travels, looking back along the line of sight, but sometimes the reverse definition is used. (Polarization theory is fraught with sign convention problems, see $\left.\bar{R}^{-} \overline{-} \overline{1} \overline{1} \bar{g} \overline{8} \bar{\tau}_{1}\right)$. 
Stokes parameters for actual radiation. Radiation fields that one actually detects and measures tend to consist of many superimposed polarization staes. An unpolarized contribution may also be present, and the total polarization generally varies with time. If the temporal changes are slow, the Stokes parameters for actual radiation are:

$$
\begin{aligned}
I_{\nu} & =I_{\nu}^{\text {unpol }}+<A_{x}^{2}+A_{y}^{2}> \\
Q_{\nu} & =<A_{x}^{2}-A_{y}^{2}> \\
U_{\nu} & =<2 A_{x} A_{y} \cos \left(\phi_{x}-\phi_{y}\right)> \\
V_{\nu} & =<2 A_{x} A_{y} \sin \left(\phi_{x}-\phi_{y}\right)>,
\end{aligned}
$$

where Stokes $I$ is the sum of the unpolarized and polarized contributions and where the

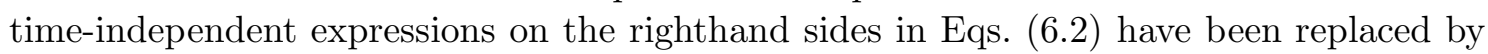
temporal averages.

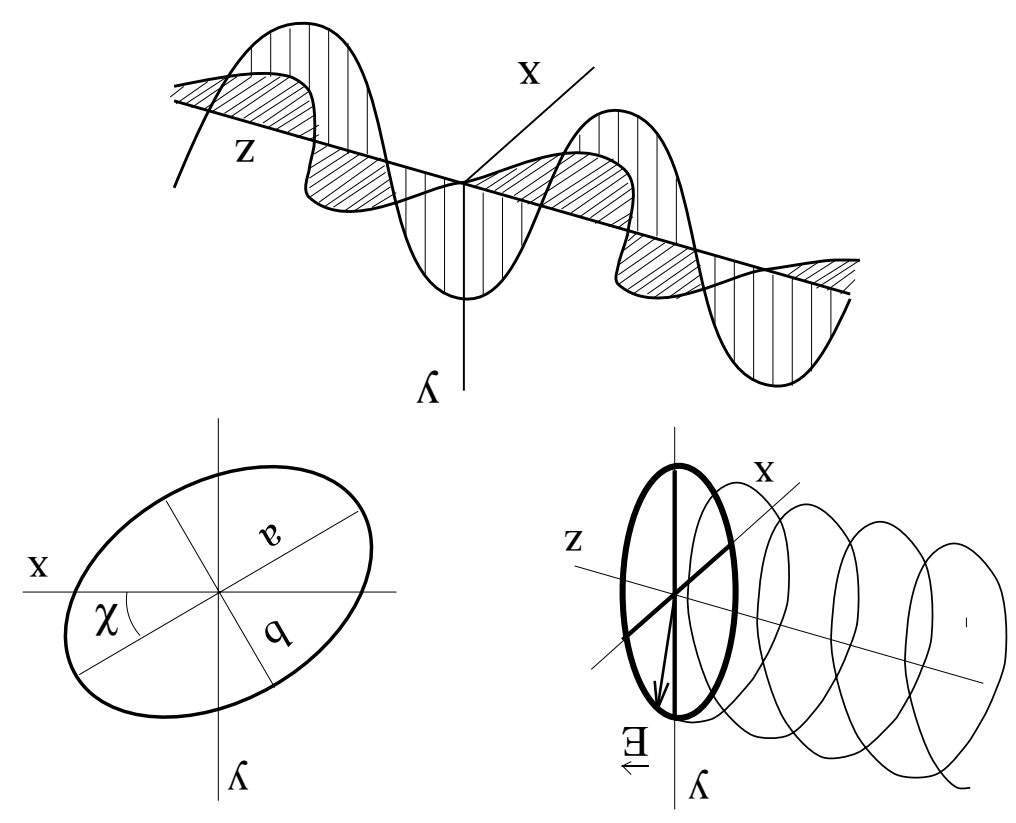

Figure 6.1: Elliptical polarization. Top: decomposition of the electric wave vector $\vec{E}$ into two sinusoidal components $E_{x}$ and $E_{y}$. The two amplitudes $A_{x}$ and $A_{y}$ are unequal; there is a $90^{\circ}$ phase lag $\phi_{x}-\phi_{y}$ between them. In that case, the tip of $\vec{E}$ describes an ellipse in the $(x, y)$ plane of which the axes are aligned with $x$ and $y$ (bottom left). For arbitrary amplitudes and phase lag, the tip of $\vec{E}$ travels clockwise or counterclockwise along an $(x, y)$ ellipse of which the axes are offset over an angle $\chi$ (bottom right).

Stokes parameters for observations. Figure $\vec{b}$ ishows $\vec{E}$-tip orbits in the $(x, y)$ plane. The angle $\chi$ measures the rotation of the ellipse axes from the $x$ and $y$ axes. The ratio of the semi major-axis $a$ and the semi minor-axis $b$ defines an angle $\beta$ with $\tan \beta=a / b$. With these quantities the Stokes parameters for fully polarized radiation become:

$$
\begin{aligned}
I_{\nu} & =A_{x}^{2}+A_{y}^{2} \equiv A^{2} \\
Q_{\nu} & =A^{2} \cos 2 \beta \cos 2 \chi \\
U_{\nu} & =A^{2} \cos 2 \beta \sin 2 \chi \\
V_{\nu} & =A^{2} \sin 2 \beta .
\end{aligned}
$$



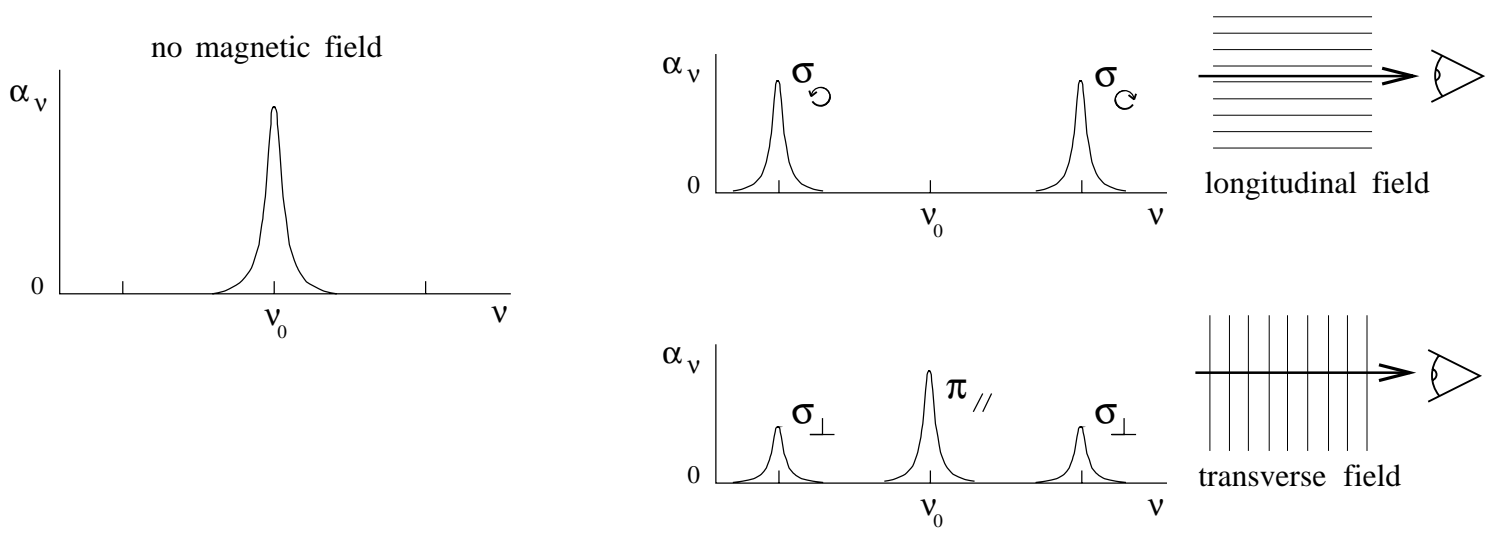

Figure 6.2: Zeeman triplet. Left: extinction profile for a medium without magnetic field. The other two graphs are for a medium that is pervaded by a strong, homogeneous magnetic field, respectively showing the longitudinal Zeeman pattern (upper right) and the transverse Zeeman pattern (lower right). In the presence of a magnetic field, the extinction coefficient differs for radiation with different Stokes vector orientations. The normal Zeeman effect (the magnetic splitting of the energy levels of a hydrogen-like atom with a single valence electron)_splits the extinction_profile_into__onultiple peaks depending on the circumstances (e.g., § II.3 of 'Herzberg 1944,', § V.10 of 'Condon and Shortley 1964,'). When the line of sight is along the field lines ("longitudinal" Zeeman effect), the extinction profile consists of two symmetrically displaced $\sigma$ components, applying to lefthand and righthand circularly polarized light, respectively. When the line of sight crosses the field at right angles, the "transverse" Zeeman effect produces three extinction peaks, one at line center which applies to linearly polarized radiation with the Stokes vector parallel to the field vector (the $\pi$ component), and two displaced $\sigma$ components that extinguish linearly polarized radiation with the Stokes vector perpendicular to the field direction. The separation of the $\sigma$ peaks scales with the magnetic field strength. Astrophysical fields are often too weak to separate the $\sigma$ components fully from the central $\pi$ component (bottom), or from the peak that is present when there is also non-magnetic plasma within the field of view (left).

These relations help to interpret the Stokes parameters in observational terms. They were originally defined as:

$$
\begin{aligned}
I_{\nu} & \equiv \text { total intensity } \\
Q_{\nu} & \equiv I_{0}^{\text {linear }}-I_{90}^{\text {linear }} \\
U_{\nu} & \equiv I_{+45}^{\text {linear }}-I_{-45}^{\text {linear }} \\
V_{\nu} & \equiv I_{\text {right }}^{\text {circular }}-I_{\text {left }}^{\text {circular }} .
\end{aligned}
$$

so that Stokes $Q$ and $U$ describe intensity differences between measurements with crossed linear polarizers, while Stokes $V$ specifies the difference between the amounts of righthanded and left-handed circularly polarized radiation in a beam.

These four parameters are often combined into the Stokes vector for use in matrix transformations (Mueller calculus) which quantitatively describe all effects of optical devices such as lenses, beam splitters, polarizers, retarders etc. on a beam of light. For more on polarization and polarized radiative transfer, see Section 2.4 of Rybicki and Light-

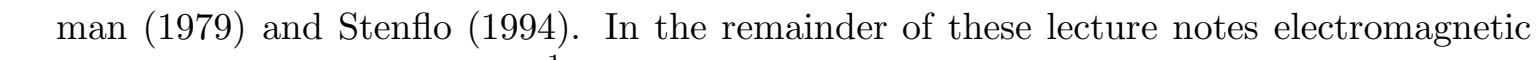
radiation is treated as quantar.

\footnotetext{
${ }^{1}$ With the convention, generally assumed implicitly in radiative transfer texts, that "photons" retain their identity in two-level scattering sequences even while they are redistributed over the line profile. The quantum that emerges in radiative deexcitation is then called the same photon as the one that excited the atom radiatively (with "atom" meaning any particle susceptible to radiative excitation).
} 
Astronomical polarimetry in general, from an observationally oriented point of view, is discussed by Tinbergen $(1996)^{1}$. In the context of stellar atmospheres, the most important application is the measurement of solar and stellar magnetic fields from the Zeeman splitting of spectral lines. The latter is illustrated in Fig. '6.2. The techniques and the underlying theory are treated by 'Stenflo $(199 \overline{9})$, and idel Toro Iniesta $(20 \overline{0} \overline{3})$.

\subsection{More detail}

This chapter stops here. Better see Landi Degl'Innocenti $1 \overline{1} 9 \overline{9})_{1}^{\prime}$ and 'dè Toro Iniestà $(2003)$ : 


\section{Chapter 7}

\section{Atmospheres of Plane-Parallel Stars}

S tellar atmospheres are described by models $s_{-1}^{\mathrm{I}_{1}}$ in a narrow sense of the word, usually just Stemperature-depth relations. Such "classical" atmosphere models are plane-parallel paradigms of stellar spectra interpretation that represent nothing more than a first-order description of the temporally and horizontally averaged radial stratification of the layers of a star where the bulk of the radiative energy escapes. Solar models tend to be empirical, using observed spectral diagnostics to constrain the $T_{\mathrm{e}}\left(\tau_{0}\right)$ relation. Stellar models tend to be theoretical, based on the requirement that the total outward energy flux remains constant with height.

\subsection{Classical modeling}

Assumptions. The simplifications that define "standard" stellar atmospheres are (Baschek and Scholz 1982 p. 92):

- the atmosphere is spherically symmetric (excluding close binaries, rapid rotators, magnetic fields, spotted surfaces);

- the element mixture is homogeneous with depth;

- the atmosphere is in hydrostatic equilibrium (no large-scale motions);

- the atmosphere is time-independent (statistical equilibrium);

- the mass of the atmosphere is small compared with the total stellar mass;

- there are no sources or sinks of energy;

- energy transport takes place by radiation and convection (no heat conduction, acoustic waves, MHD waves);

\footnotetext{
${ }^{1}$ Of course, all our astrophysical explanations are models - all explanations are models. Astronomical models are perhaps a bit more Platonic shadow-on-the-wall conjectures than medical or biological or fashion ones because we cannot touch distant stars. A quote from Gray (1992, p. 146): "It would seem logical to take our stellar observations and deduce from them the physical conditions existing in the atmosphere of the star - somewhat like a parallax measurement yields the distance to a star. Alas, the formation of the stellar spectrum is not so simply related to the physical state of the atmosphere as distance is to parallax. There are many physical variables, and a rigorous deductive interpretation cannot be made in most instances. Indeed we are led to hypothesize a model to organize and relate the details conveyed in the starlight." More formally, astronomers' models result from inversions of undersampled data; such inversions tend to be unstable unless rigorously limited in variables (Craig and Brown 1986i).
} 
- the free electrons as well as the free heavier particles obey the Maxwell distribution with local kinetic temperature $T_{\mathrm{e}}$.

Model parameters. Classical stellar model atmospheres are fully determined by

- the stellar luminosity $L$;

- the stellar radius? $R$;

- the element mixture, usually parameterized by the metallicity $[\mathrm{Fe} / \mathrm{H}]$ defined by $(\overline{9} \cdot \overline{9}-4)$ on page 203

- the microturbulence $\xi_{\text {micro }}$, an ad-hoc line-width fitting parameter defined by (3.80i) on page $\overline{6} 2$. It supposedly describes the most probable velocity of small-scale nonthermal, isotropic, depth-independent motions with a gaussian velocity distribution along the line of sight.

Any two of the three parameters $L, M$ and $R$ may be replaced by

- the effective temperature $T_{\text {eff }}=\left(L / 4 \pi \sigma R^{2}\right)^{1 / 4}$;

- the surface gravity $g_{s}=G M / R^{2}$.

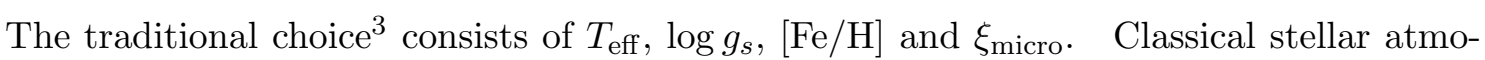
sphere models thus consist of tables per combination of these four parameters that specify the temperature and gas pressure and/or density as functions of depth, often the Rosseland depth $\tau_{\mathrm{R}}$ (defined on page $1 \overline{1} \overline{6}_{1}^{\prime}$ ) or the continuum optical depth $\tau_{0}$ at $\lambda=500 \mathrm{~nm}$. An informative standard paper with tables for nearly 300 stellar models is the one by Kurucz

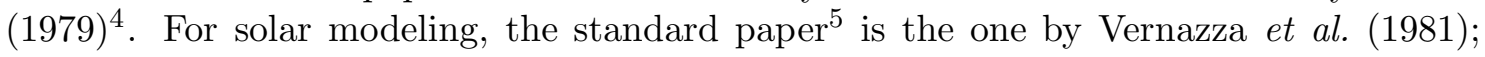
their VALIII model is specified on page $18 \overline{2}$.

\subsection{Pressure stratification}

\subsubsection{Gas law}

The ideal gas law generally holds in stellar photospheres. It exists in various disguises (Kourganoff 1973; the treatment on Gray's p. 10 has errors). The classical version is

$$
P_{\mathrm{g}} V=n_{\text {mole }} \mathcal{R} T
$$

with $n_{\text {mole }}$ the number of moles and $\mathcal{R}=8.314 \times 10^{7} \mathrm{erg} \mathrm{mole}^{-1} \mathrm{~K}^{-1}$ the gas constant with

$$
\mathcal{R}=k N_{\mathrm{A}}=k / m_{\mathrm{H}}
$$

\footnotetext{
${ }^{2}$ For extended atmospheres the radius $R$, effective temperature $T_{\text {eff }}$ and surface gravity $g_{s}$ depend on where exactly the surface is located. Often, this is defined at $\tau_{\mathrm{R}}=1$ or $\tau_{\mathrm{R}}=2 / 3$ with the Rosseland depth $\tau_{\mathrm{R}}$ given by $\left({ }^{7} \cdot 62_{1}^{\prime}\right)$ on page 160 !

${ }^{3}$ This choice leaves one stellar parameter free, for example the radius $R$ in keeping with the notion of a plane-parallel atmosphere of infinite horizontal extent. One may therefore compute surface fluxes (outward directed energy through a $\mathrm{cm}^{2}$ of the stellar surface) from such models, but needs $R$ in addition to link atmospheric modeling to stellar evolution modeling. Reversely, by determining $T_{\text {eff }}$ through matching observed spectral characteristics (continuum distribution, spectral lines) to model-computed ones and measuring the angular diameter or the absolute monochromatic irradiance (flux at Earth) one obtains $R$ (Chapter 15 of Gray 1992').

${ }^{4}$ Robert L. Kurucz at the Harvard-Smithsonian Center for Astrophysics (Cambridge Mass.) has been the main provider of stellar model atmospheres over the past decades. Currently, he delivers them per CD-ROM, as well as gigantic lists of spectral line opacities and other atomic data.

${ }^{5}$ Also from the Center for Astrophysics, where solar modeling efforts center around Eugene H. Avrett.
} 
with $k=1.38 \times 10^{-16} \mathrm{erg} \mathrm{K}^{-1}$ the Boltzmann constant, $N_{\mathrm{A}}=6.02 \times 10^{23} \mathrm{~mole}^{-1}$ Avogadro's number of particles per mole and $m_{\mathrm{H}}=1.66 \times 10^{-24} \mathrm{~g}$ the mass of unit atomic weight on the ${ }^{12} \mathrm{C}=12$ scale, approximately the mass of a hydrogen atom. Other versions are, with the gas particle density $N_{\mathrm{g}}=n_{\text {mole }} N_{\mathrm{A}} / V$ counting all particles $\mathrm{cm}^{-3}$, the mean "molecular" weight per free particle (including free electrons) $\mu \equiv \bar{m} / m_{\mathrm{H}}$ in atomic units and the gas mass density $\rho$ in $\mathrm{g} \mathrm{cm}^{-3}$ with $\rho=N_{\mathrm{g}} \mu m_{\mathrm{H}}$ :

$$
P_{\mathrm{g}}=\frac{n_{\mathrm{mole}} N_{\mathrm{A}}}{V} \frac{\mathcal{R}}{N_{\mathrm{A}}} T=N_{\mathrm{g}} k T=\frac{\rho k T}{\mu m_{\mathrm{H}}}=\frac{\rho \mathcal{R} T}{\mu} .
$$

The total gas pressure is the sum of all partial pressures with $P_{\mathrm{g}}=\sum_{i} P_{i}=\sum_{i} N_{i} k T$ with $i$ specifying all types of particles including free electrons. In particular for the partial electron pressure

$$
P_{\mathrm{e}}=N_{\mathrm{e}} k T \text {. }
$$

The conversion between $P_{\mathrm{g}}$ and $P_{\mathrm{e}}$ for given chemical composition and $T_{\mathrm{e}}$ is discussed on page $1 \overline{1} \overline{4} \overline{6}_{6}^{\prime}$. With the gas law two of the three parameters $T, P_{\mathrm{g}}$ and $N_{\mathrm{g}}$ remain unknowns. The temperature and density are the independent ones but pressure is usually chosen in atmospheric modeling because it follows from pressure equilibrium.

\subsubsection{Particle densities}

Chemical composition. Table the specifies the solar abundances of the more important elements, with their first and second ionization energies. Larger tables are given in Gray's appendices; complete tables are found with lots of other useful astrophysical data in 'Álien $(\overline{1} \overline{9} \overline{7} 6)_{1}^{1}$. Usually, abundances are given relative to the hydrogen abundance on a logarithmic scale with $A_{12}(\mathrm{H})=12$, for element E given by $A_{12}(\mathrm{E}) \equiv{ }^{10} \log \left(N_{\mathrm{E}} / N_{\mathrm{H}}\right)+12$

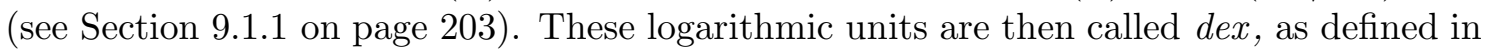
$\S 5$ of Âllen $(1976)^{1}$. For example, there is a discussion at the moment whether the solar iron abundance is 0.2 dex $(60 \%)$ higher than the meteoritic value or not. This is a characteristic uncertainty for abundance values. NLTE effects are often neglected in abundance studies $s_{L^{\prime}}^{\mathfrak{b}^{\prime \prime}}$ but tend to produce corrections to LTE estimates in the $\pm 0.1-0.3$ dex range. Most stars have solar-like abundances. Metal deficiencies ("metal-poor stars") occur compared with the solar metal content when stars started their life in an earlier phase of galactic evolution (Population II halo dwarfs). Enrichments may be present when stars have evolved far enough to show CNO products (carbon stars), or when a companion perturbs its evolution (barium stars).

Electron donors. The continuous extinction in the hottest stellar atmospheres is dominated by Thomson scattering from free electrons. In cool stellar atmospheres the extinction is dominated by $\mathrm{H}^{-}$transitions. Both extinction providers scale with the electron density $N_{\mathrm{e}}$. Electrons come either from hydrogen (and helium) or from the metals, meaning elements that combine relatively large abundance with relatively low first ionization energy as listed in Table

\footnotetext{
${ }^{6}$ Stellar abundance determiners make up a relatively large astronomical community. The most hotly debated abundance is that of lithium, presumably made in the Big Bang and depleted in stars through low-temperature $\operatorname{Li}(\mathrm{p}, \alpha)$ He reactions. Its abundance varies over three dex (orders of magnitude) between stars. For a review see Carlsson et al. $(1994)$. Abundance determination is discussed in Section $19.1{ }^{\prime}$ on page $203 \mathrm{ff}$.
} 


\begin{tabular}{|c|c|c|c|c|c|c|c|c|c|}
\hline $\mathrm{E}$ & $A_{\mathrm{E}}$ & $A_{12}$ & $\chi_{0}$ & $\chi_{1}$ & $\mathrm{E}$ & $A_{\mathrm{E}}$ & $A_{12}$ & $\chi_{0}$ & $\chi_{1}$ \\
\hline $\mathrm{H}$ & 1.000 & 12.0 & 13.60 & - & $\mathrm{Al}$ & $2.5 \times 10^{-6}$ & 6.4 & 5.99 & 18.83 \\
\hline $\mathrm{He}$ & $7.9 \times 10^{-2}$ & 10.9 & 24.59 & 54.42 & $\mathrm{Si}$ & $3.2 \times 10^{-5}$ & 7.5 & 8.15 & 16.35 \\
\hline $\mathrm{C}$ & $3.2 \times 10^{-4}$ & 8.5 & 11.26 & 24.38 & S & $1.6 \times 10^{-5}$ & 7.2 & 10.36 & 23.33 \\
\hline $\mathrm{N}$ & $1.0 \times 10^{-4}$ & 8.0 & 14.53 & 29.60 & $\mathrm{~K}$ & $1.0 \times 10^{-7}$ & 5.0 & 4.34 & 31.63 \\
\hline $\mathrm{O}$ & $6.3 \times 10^{-4}$ & 8.8 & 13.62 & 35.12 & $\mathrm{Ca}$ & $2.0 \times 10^{-6}$ & 6.3 & 6.11 & 11.87 \\
\hline $\mathrm{Na}$ & $2.0 \times 10^{-6}$ & 6.3 & 5.14 & 47.29 & $\mathrm{Cr}$ & $7.9 \times 10^{-7}$ & 5.9 & 6.77 & 16.50 \\
\hline $\mathrm{Mg}$ & $2.5 \times 10^{-5}$ & 7.4 & 7.65 & 15.04 & $\mathrm{Fe}$ & $4.0 \times 10^{-5}$ & 7.6 & 7.87 & 16.16 \\
\hline
\end{tabular}

Table 7.1: Abundances by number and ionization energies for major elements. The abundances $A_{\mathrm{E}} \equiv$ $N_{\mathrm{E}} / N_{\mathrm{H}}$ and $A_{12} \equiv \log N_{\mathrm{E}}-\log N_{\mathrm{H}}+12$ are mainly determined from the solar photospheric spectrum but generally correspond closely to the meteoritic values for the heavier particles and are assumed to express cosmic standard values. The corresponding composition mix by mass is: $X=0.73$ (fraction made up by hydrogen), $Y=0.25$ (fraction in helium, not a well-known number), $Z=0.017$ (remaining elements including "metals" = all the ones heavier than neon). The energies $\chi_{0}$ and $\chi_{1}$ are in eV and measure ionization energy between the ground level and its bound-free edge for the neutral and singly-ionized stage, respectively. The elements with large abundance and low first ionization energy ( $\mathrm{Si}, \mathrm{Fe}, \mathrm{Al}, \mathrm{Mg}, \mathrm{Ca}$, $\mathrm{Na}$ ) provide most free electrons in cool atmospheres where hydrogen is not ionized (see Figure $\mathbf{1}_{-1}^{1}$ below

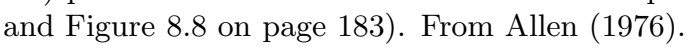
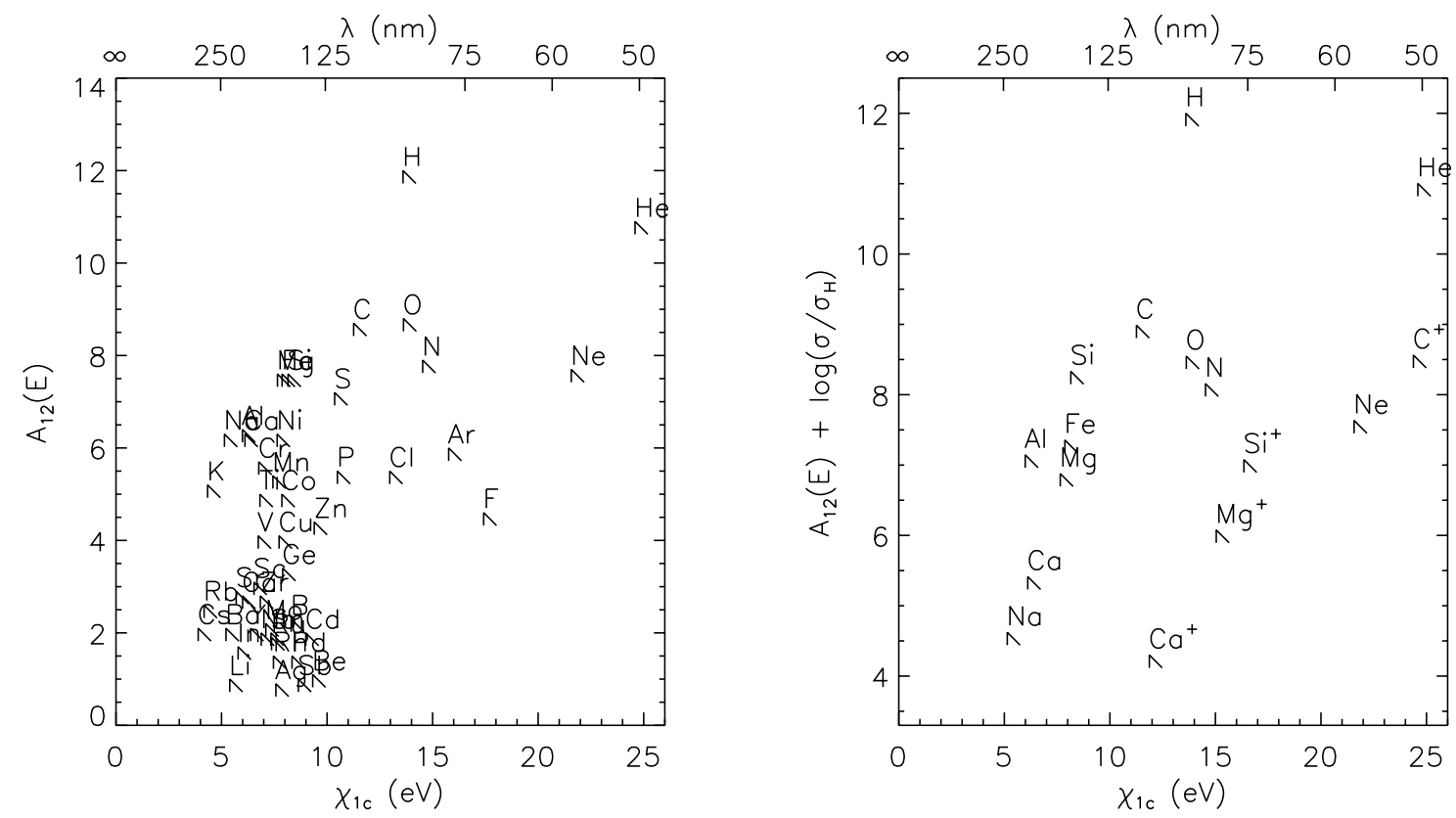

Figure 7.1: Ionization edges for a selection of abundant elements. The triangular symbols depict boundfree continuum edges in the form of schematic hydrogenic $\nu^{-3}$ decay functions above each ionization threshold. The lefthand plot shows the edge distribution over ionization energy $\chi_{1 c}$ (along the bottom) or threshold wavelength (along the top) and logarithmic abundance $A_{12}$ (vertically). Each symbol corresponds to the bound-free threshold for the ground state of the neutral stage of the indicated element. The righthand plot weights some abundance values with the bound-free cross-section at threshold by plotting $A_{12}(\mathrm{E})+\log \left(\sigma / \sigma_{\mathrm{H}}\right)$ along_the y_axis. The plus signs indicate important_first-ion edges. The abundance values come from 'Engvold (1977)', the ionization energies from 'Novotny_(1973)', the cross-sections from Baschek and Scholz (1982)! Thijs Krijger production following E.H. Ávrett's lecture notes. 
An instructive example (from Mihalas, p. 73 in the 1970 edition, p. 116 in the 1978 edition) is to take a gas that consists only of hydrogen and one electron-donor metal $M$ with abundance $A_{\mathrm{M}}=N_{\mathrm{M}} / N_{\mathrm{H}} \ll 1$ and ionization energy well below the $13.6 \mathrm{eV}$ value of hydrogen. Using $f_{\mathrm{H}}$ for the fraction of hydrogen particles that is ionized and $f_{\mathrm{M}}$ for the ionized fraction of metal particles (single ionization only) the total particle number density is:

$$
N_{\mathrm{g}}=N_{\mathrm{H}}+A_{\mathrm{M}} N_{\mathrm{H}}+f_{\mathrm{H}} N_{\mathrm{H}}+f_{\mathrm{M}} A_{\mathrm{M}} N_{\mathrm{H}}
$$

where the first two terms count nuclei (atoms and ions) and the last two count the free electrons as

$$
N_{\mathrm{e}}=f_{\mathrm{H}} N_{\mathrm{H}}+f_{\mathrm{M}} A_{\mathrm{M}} N_{\mathrm{H}}
$$

The density ratio is:

$$
\frac{N_{\mathrm{e}}}{N_{\mathrm{g}}}=\frac{f_{\mathrm{H}}+f_{\mathrm{M}} A_{\mathrm{M}}}{1+f_{\mathrm{H}}+\left(1+f_{\mathrm{M}}\right) A_{\mathrm{M}}}
$$

with

$$
\begin{aligned}
f_{\mathrm{H}} \approx 1 & \rightarrow \frac{N_{\mathrm{e}}}{N_{\mathrm{g}}} \approx \frac{1}{2} \\
A_{\mathrm{M}} \ll f_{\mathrm{H}} \ll 1 & \rightarrow \frac{N_{\mathrm{e}}}{N_{\mathrm{g}}} \approx f_{\mathrm{H}} \\
f_{\mathrm{H}} \approx 0 & \rightarrow \frac{N_{\mathrm{e}}}{N_{\mathrm{g}}} \approx f_{\mathrm{M}} A_{\mathrm{M}} .
\end{aligned}
$$

The first case is for high temperatures with hydrogen fully ionized. In that case the metals do not matter, nor their state of ionization. At intermediate temperatures where hydrogen is partially ionized, the metal is fully ionized with $f_{\mathrm{M}} \approx 1$ due to its lower ionization energy. As long as hydrogen donates many more electrons than the metal does $\left(A_{\mathrm{M}} \ll f_{\mathrm{H}}\right)$, the fractional electron density equals the hydrogen ionization fraction. Finally, at low temperatures with $f_{\mathrm{H}} \approx 0$ but $f_{\mathrm{M}}$ still appreciable the electron fraction is made up by the metal. It now plays the dominant role. The upper $N_{e}$ panels of

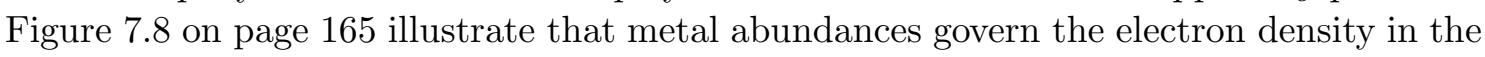
atmospheres of cool stars.

Electron and gas pressure. For a given chemical composition relations are needed between the gas pressure $P_{\mathrm{g}}=N_{\mathrm{g}} k T$ (given by the model of the atmosphere, see Sec-

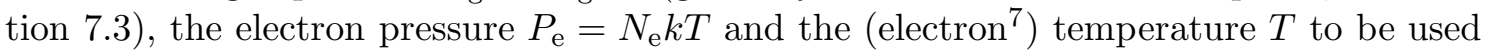
in the Saha-Boltzmann equations. The simplification in (1.7) must be generalized. Start with a given $T_{\mathrm{e}}$ and a first estimate for $P_{\mathrm{e}}$. Neglect all elements and ionization stages that do not contribute electrons, i.e., include the ones in Table $\overline{7}$. For example, do not include neon although $A_{\mathrm{Ne}} \approx 10^{-4}$ because $\chi_{0}^{\mathrm{Ne}}=21.6 \mathrm{eV}$; but do include potassium for cool stars, although its abundance is small, because it ionizes at low energy. The Saha distribution (assuming LTE) then delivers per metal z the ratios $N_{\text {II }} / N_{\text {I }}$ and $N_{\text {III }} / N_{\text {II }}$ with $N_{\mathrm{z}}=N_{\mathrm{I}}+N_{\mathrm{II}}+N_{\mathrm{III}}$. The ionization fractions $f_{\mathrm{II}}=N_{\mathrm{II}} / N_{\mathrm{z}}$ and $f_{\mathrm{III}}=N_{\mathrm{III}} / N_{\mathrm{z}}$ may be

\footnotetext{
${ }^{7}$ Formally, each particle species has its own kinetic temperature: $P_{\mathrm{e}}=N_{\mathrm{e}} k T_{\mathrm{e}}$ for electrons, $P_{\text {ion }}=$ $N_{\text {ion }} K T_{\text {ion }}$ for ions, etc. In stellar atmospheres one may usually assume the Maxwell distribution with the same kinetic temperature for the different species, even when LTE (Saha-Boltzmann statistics) is not valid. Thus, $T_{\mathrm{e}}$ and $T$ are used without distinction.
} 
evaluated from these ratios as (Novotny 1973; p. 163; Gray p. 158-159)

$$
\begin{aligned}
\frac{1}{f_{\mathrm{II}}} & =\frac{N_{\mathrm{I}}}{N_{\mathrm{II}}}+\frac{N_{\mathrm{II}}}{N_{\mathrm{II}}}+\frac{N_{\mathrm{III}}}{N_{\mathrm{II}}} \\
\frac{1}{f_{\mathrm{III}}} & =\frac{N_{\mathrm{I}}}{N_{\mathrm{II}}} \frac{N_{\mathrm{II}}}{N_{\mathrm{III}}}+\frac{N_{\mathrm{II}}}{N_{\mathrm{III}}}+\frac{N_{\mathrm{III}}}{N_{\mathrm{III}}} .
\end{aligned}
$$

With the ionization fractions known, the mean number of free electrons per nucleus $E$ is found from

$$
E=\frac{N_{\mathrm{e}}}{N_{\text {nuclei }}}=\frac{\sum_{\mathrm{z}} N_{\mathrm{z}} f_{\mathrm{II}}(z)+2 \sum_{\mathrm{z}} N_{\mathrm{z}} f_{\mathrm{III}}(z)}{\sum_{\mathrm{z}} N_{\mathrm{z}}},
$$

so that

$$
\frac{P_{\mathrm{g}}}{P_{\mathrm{e}}}=\frac{\left(N_{\text {ions }}+N_{\text {atoms }}+N_{\mathrm{e}}\right) k T}{N_{\mathrm{e}} k T}=\frac{\left(N_{\text {nuclei }}+N_{\mathrm{e}}\right) k T}{N_{\mathrm{e}} k T}=\frac{E+1}{E}
$$

or

$$
P_{\mathrm{e}}=P_{\mathrm{g}} \frac{E}{E+1} .
$$

If this result disagrees with the initial estimate then iterate the evaluation until sufficient precision is reached. The full rate equations must be solved if LTE is not valid. For cool stars molecules must be taken into account as well (iMihalas 1970. $74 \mathrm{ff}$ ).

\subsubsection{Hydrostatic equilibrium}

Atmospheric stationarity requires (Gray p. 147)

$$
\frac{\mathrm{d} P}{\mathrm{~d} z}=-g \rho
$$

or, on a reference optical depth scale with $\mathrm{d} \tau_{0}=-\kappa_{0} \rho \mathrm{d} z$

$$
\frac{\mathrm{d} P}{\mathrm{~d} \tau_{0}}=\frac{g}{\kappa_{0}} .
$$

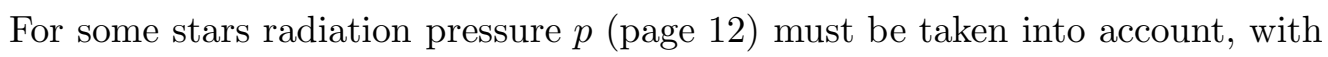

$$
\frac{\mathrm{d} p}{\mathrm{~d} \tau_{0}}=\frac{4 \pi}{c} \int_{0}^{\infty} \frac{\mathrm{d} K_{\nu}}{\mathrm{d} \tau_{0}} \mathrm{~d} \nu=\frac{4 \pi}{c} \int_{0}^{\infty} \frac{\mathrm{d} K_{\nu}}{\mathrm{d} \tau_{\nu}} \frac{\mathrm{d} \tau_{\nu}}{\mathrm{d} \tau_{0}} \mathrm{~d} \nu=\frac{1}{c} \int_{0}^{\infty} \mathcal{F}_{\nu} \frac{\kappa_{\nu}}{\kappa_{0}} \mathrm{~d} \nu
$$

which may be estimated from the diffusion approximation in deep layers but requires lineby-line evaluation higher up. For other stars one should include rotational acceleration and/or turbulent pressures. Here, we simply set $P=P_{\mathrm{g}}$.

\footnotetext{
${ }^{8}$ In the case that the measured "microturbulence" is interpreted as real turbulence, or at least as smallscale motions of a stochastic nature. A likely candidate for hot stars (De Jager) is given by internal gravity waves since these stars have large observed microturbulence, but no (outer) convection zones. Convection and internal gravity waves are mutually exclusive, with the square of the Brunt-Väisälä frequency $N^{2}=$ $-g\left[(1 / \rho)(\mathrm{d} \rho / \mathrm{d} z)+g / c_{s}^{2}\right]=(g / T)[(\mathrm{d} T / \mathrm{d} z)-(\mathrm{d} T / \mathrm{d} z)$ ad $\left.)\right]$ respectively positive and negative. Another option (Lamers) is that the gradient of the stellar wind causes apparent line broadening wrongly interpreted as turbulence.
} 
Model completion. Integration of ( $\overline{7} \overline{\overline{1}} \overline{1} \bar{i})$ requires knowledge of the extinction $\kappa_{0}\left(\tau_{0}\right)$ at the reference frequency. That extinction scales with the densities of the extincting particles and therefore depends on chemical composition, electron pressure $P_{\mathrm{e}}$ and temperature $T_{\mathrm{e}}$, for LTE via the Saha-Boltzmann laws and otherwise in more complicated fashion. The three quantities $P_{\mathrm{g}}, P_{\mathrm{e}}$ and $T_{\mathrm{e}}$ may be expressed in each other as discussed on page $1{ }^{1} \overline{4} \overline{6}_{1}^{\prime}$ but we need to know two of these quantities to obtain the third. The condition of hydrostatic equilibrium furnishes the second, so that only a single input parameter is required in principle. It takes the form of a given $T\left(\tau_{0}\right)$ temperature stratification; all other state parameters are derived from it.

There are various procedures to obtain $P_{\mathrm{g}}\left(\tau_{0}\right)$ from $T\left(\tau_{0}\right)$. Gray (p. 149) describes an iteration writing $\left(\overline{1}: \overline{1} \overline{1}_{1}\right)$ in the form

$$
P_{\mathrm{g}}^{1 / 2} \frac{\mathrm{d} P_{\mathrm{g}}}{\mathrm{d} \tau_{0}}=P_{\mathrm{g}}^{1 / 2} \frac{g}{\kappa_{0}}
$$

with formal solution

$$
P_{\mathrm{g}}\left(\tau_{0}\right)=\left(\frac{3 g}{2} \int_{0}^{\tau_{0}} \frac{P_{\mathrm{g}}^{1 / 2}\left(t_{0}\right)}{\kappa_{0}\left(t_{0}\right)} \mathrm{d} t_{0}\right)^{2 / 3} .
$$

Guess $P_{\mathrm{g}}\left(\tau_{0}\right)$ for all $\tau_{0}$ initially and then numerically evaluate the integral on the right for each $\tau_{0}$ to obtain a better estimate of $P_{\mathrm{g}}\left(\tau_{0}\right)$ on the lefthand side. Iterate this procedure. At each step, $\kappa_{0}\left(\tau_{0}\right)$ must be determined along with $N_{\mathrm{e}}$ from $P_{g}\left(\tau_{0}\right)$ and $T\left(\tau_{0}\right)$.

Mihalas (1970, p.149-151) describes another method in which (7.17) is integrated step-by-step inward, starting at the outside from a known asymptotic solution. Iteration

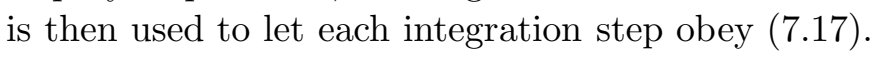

Plane-parallel layers. The simple case of an isothermal atmosphere with constant

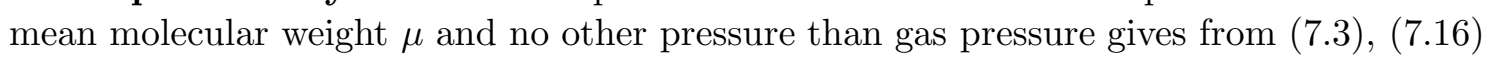
and the pressure scale height $H_{\mathrm{P}} \equiv \mathcal{R} T / \mu g$

$$
\frac{\mathrm{d} P_{\mathrm{g}}}{\mathrm{d} z}=-\frac{\mu g}{\mathcal{R} T} P_{\mathrm{g}}=-\frac{P_{\mathrm{g}}}{H_{\mathrm{P}}}
$$

with as solution the standard barometric exponential decay law

$$
P_{\mathrm{g}}(z)=P_{\mathrm{g}}(0) \mathrm{e}^{-z / H_{\mathrm{P}}} .
$$

The scale height is a good indicator for the radial extent of a stellar atmosphere; the spectra that we observe come from layers spanning a few times $H_{\mathrm{P}}$ at most. Figure on page $1 \overline{1} 8 \overline{1}$ i' shows that the whole photosphere is only $500 \mathrm{~km}$ thick. The assumption of plane-parallel layers rather than spherical-parallel layers holds well if

$$
\frac{H_{\mathrm{P}}}{R_{*}}=\frac{\mathcal{R} T}{\mu g R_{*}}=\frac{\mathcal{R} T R_{*}}{\mu G M_{*}}=4.4 \times 10^{-8} \frac{T_{\mathrm{eff}}\left(R_{*} / R_{\odot}\right)}{\mu\left(M_{*} / M_{\odot}\right)} \ll 1
$$

with the mean molecular weight $\mu$ of order 1 . It holds for all stars except the largest supergiants. The sun has $R_{\odot}=7 \times 10^{5} \mathrm{~km}, T_{\text {eff }}=5770 \mathrm{~K}$ and $H_{\mathrm{P}} \approx 150 \mathrm{~km}$. However, this test for sphericity doesn't say anything about the effect of horizontal inhomogeneities on the validity of the plane-parallel-layer assumption. 

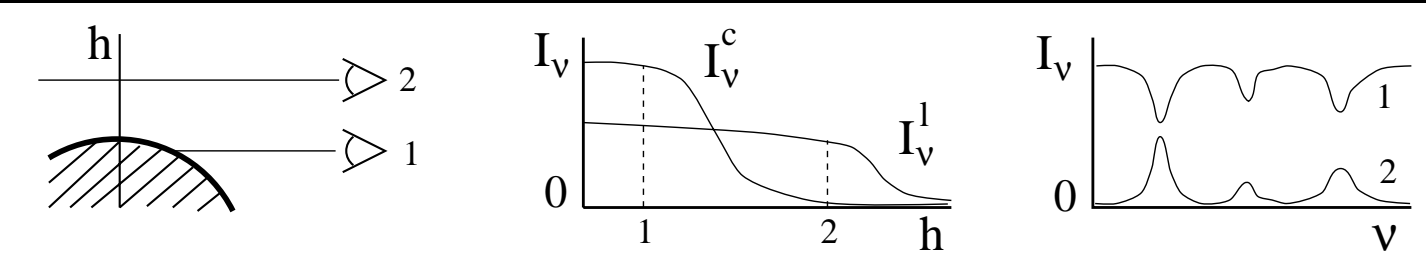

Figure 7.2: The solar "flash" spectrum. During a total solar eclipse, while the lunar limb progresses over the solar limb, the absorption-line spectrum observed from the last edge of the disk (along line of sight 1) abruptly converts into an emission-line spectrum (along line of sight 2) because the sun becomes optically thin a little bit further out in strong spectral lines $\left(I_{\nu}^{l}\right)$ than in the adjacent continuum $\left(I_{\nu}^{c}\right)$. In the continuum, the "limb" (location with $\tau_{\nu}^{\tan }(h) \approx 1$ along the whole line of sight, close to the inflection point in $\left.I_{\nu}(h)\right)$ is just outside line of sight 1 , while in the strongest line the limb is just outside line of sight 2. This schematic diagram neglects the integration over height outside the lunar limb, transverse to the line of sight, that is caused by turbulence in the earth's atmosphere ("seeing").

Solar limb. The small scale height in the solar atmosphere also makes the limb of the apparent solar disk sharp. Integration along tangential lines of sight skirting the limb shows that the total optical thickness of the Sun drops from $\tau_{\nu}^{\tan } \approx 10$ to $\tau_{\nu}^{\tan } \approx 0.1$ in the visible for an outward shift of the tangential line of sight over only $300 \mathrm{~km}$, about two scale heights. The limb, say between $\tau_{\nu}^{\tan } \approx 3$ and $\tau_{\nu}^{\tan } \approx 0.3$, extends only over about a hundred $\mathrm{km}$ in the radial direction?t.

Across this narrow limb the source function does not change dramatically, but the emergent spectrum does. Its intensity drops from thick formation with $I_{\nu} \approx S_{\nu}\left(\tau_{\nu}^{\mathrm{tan}}=1\right)$ to thin formation with $I_{\nu} \approx S_{\nu} \tau_{\nu}^{\text {tan }}$ over the hundred $\mathrm{km}$ in radial distance. This steep drop occurs first in the continuum, and appreciably further out in strong lines. The flash spectrum therefore shows emission lines that are roughly the reverse of the absorption lines seen on the solar disk (Figure $\left.\overline{7} \overline{2}^{2}\right)$.

The off-limb line reversal led to the Schuster-Schwarzschild reversing layer concept

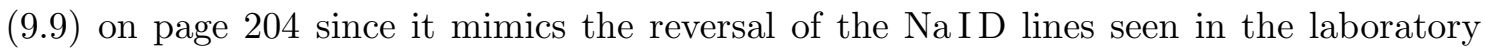
from an optically thin flame with and without a bright background source. However, the solar line reversal has more to do with the solar thickness flip between thick and thin. (Figure $\overline{7} . \overline{2}$ ).

\subsection{Temperature stratification}

\subsubsection{Empirical models}

Empirical $T\left(\tau_{0}\right)$ relationships are in principle derived by inversion of observed intensities $I_{\nu}$ into a $S_{\nu}\left(\tau_{\nu}=\mu\right)$ description of their formation, with $S_{\nu}$ a function of the temperature (simply $S_{\nu}=B_{\nu}(T)$ when one assumes LTE) and the Eddington-Barbier depth $\tau_{\nu}=\mu$

\footnotetext{
${ }^{9} \mathrm{~A}$ second of arc measures $725 \mathrm{~km}$ on the Sun; the limb width is therefore well below the usual observational resolution of about $1^{\prime \prime}$. Higher spatial resolution may be obtained during solar eclipses. The Moon then covers the solar disk at a projected speed of about $300 \mathrm{~km} \mathrm{~s}^{-1}$. Taking $0.1 \mathrm{~s}$ exposures therefore produces height resolution of $30 \mathrm{~km}$ on the Sun. However, one usually takes slitless spectrographs to eclipses so that the narrow remaining solar crescent itself is imaged as spectral line. This implies also integration in the radial direction outward from the lunar limb, in addition to the integration along the line of sight. The double integration makes interpretation of eclipse spectra difficult; a double differentiation is required that tends to cause instability in the results. Eclipse expeditions are out of fashion since the glimpses one gets are too brief, too risky and too costly. Their place has been taken by space observation, also risky, much more costly, but more productive.
} 


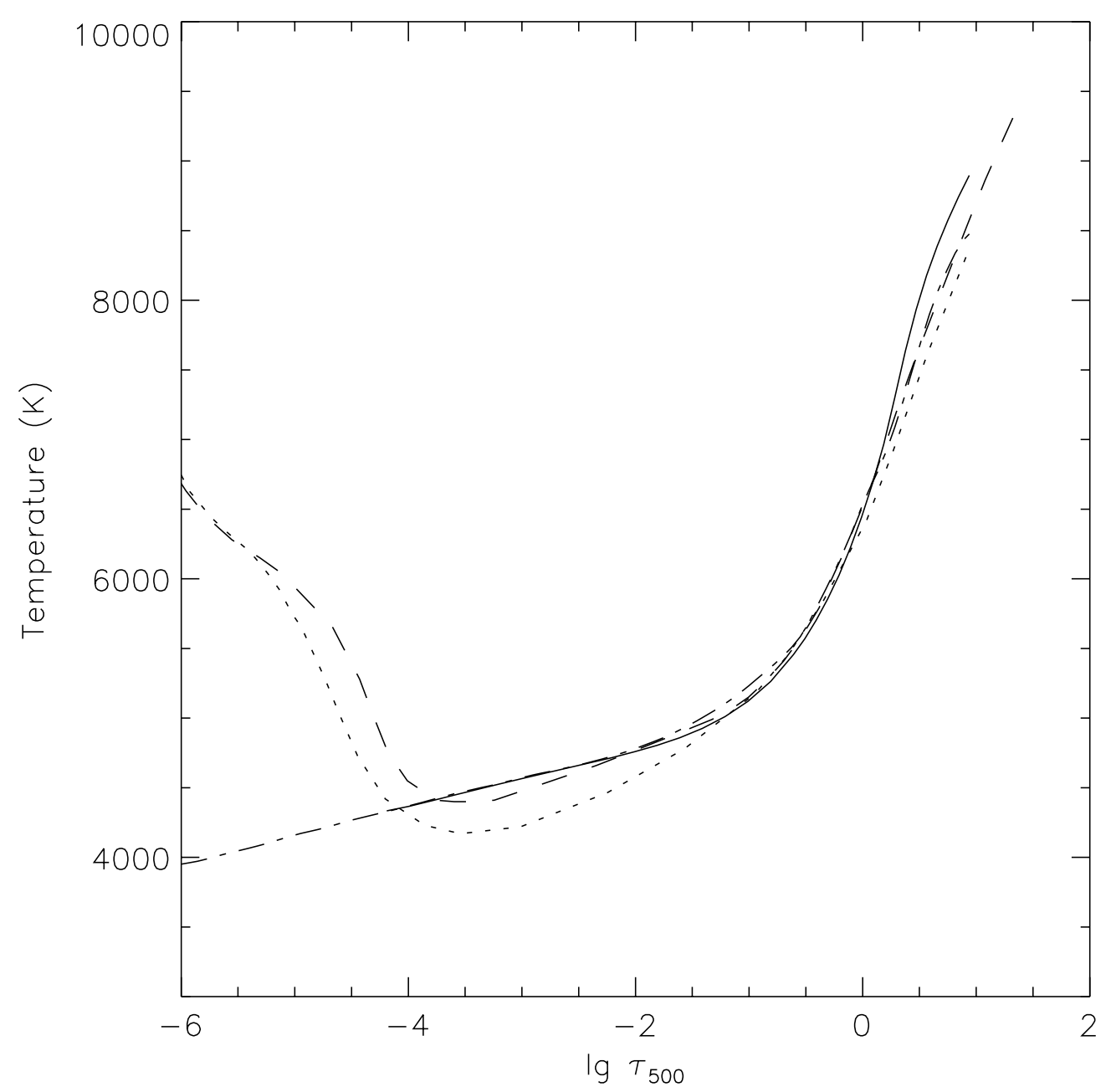

Figure 7.3: Solar model atmospheres. The x-axis measures the logarithm $(\mathrm{lg} \equiv \log$ in Scandinavia) of the continuum optical depth at $\lambda=500 \mathrm{~nm}$. Dotted: the VALIII model discussed in Section 18.24 on page $180 \mathrm{ff}$ and specified in Table $18.2 \overline{2}$ on page'182. It is based on the observed brightness temperatures of the solar continua throughout the spectrum, as shown in Figs. 8.21 .51 on page 173 of VALIII by 'Maltby_et al. (1986)! called MACKKL, having a somewhat higher_temperature minimum between photosphere and chromosphere. Dot-dashed: the HOLMUL model of Holweger and Müller (1974) based on LTE interpretation of observed FeI line-center intensities. It has no chromospheric temperature rise because solar Fe I lines do not have emission peaks in their centers. This model is the standard choice of solar abundance determiners. Solid: the T5780 model, a theoretical LTE-RE one from Uppsala. It is also shown in Figure 165 together with comparable stellar models. Its close agreement with the HOLMUL and MACKKL models in the middle and upper photosphere demonstrates that the solar photosphere obeys radiative equilibrium to high precision (cf. Table 17.21 on page 159 . the T5780 model takes convective flux transport into account. From Carlsson et al. (1992). 
providing the location at which that $S_{\nu}$ holds. The trick is to obtain variation along $\tau_{0}$ from variation in $\tau_{\nu}=\mu$. Examples for the solar atmosphere are shown in Figure

Center-limb variation. For the Sun the observed center-to-limb variation provides

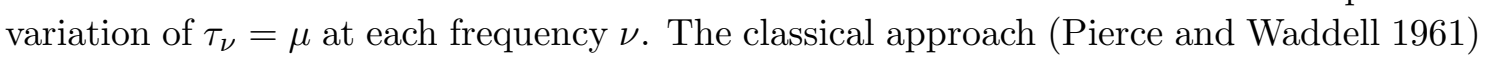
is to fit the observed limb darkening per frequency $\nu$ with coefficients $a_{\nu}, b_{\nu}$ and $c_{\nu}$ in

$$
\frac{I_{\nu}(0, \mu)}{I_{\nu}(0,1)}=a_{\nu}+b_{\nu} \mu+c_{\nu}\left(1-\mu \ln \left(1+\frac{1}{\mu}\right)\right)
$$

because this expression is the $\boldsymbol{\Lambda}_{\tau}$ transform of

$$
S_{\nu}\left(\tau_{\nu}\right)=a_{\nu}+b_{\nu} \tau_{\nu}+c_{\nu} E_{2}\left(\tau_{\nu}\right)
$$

with $E_{2}(x)$ defined by (4.12) on page $\overline{1} \overline{8} \overline{1}$, and its $\boldsymbol{\Lambda}_{\tau}$ transform given in Eq. (17.7) of Kourganoff $(1952)$. The exponential term delivers more accurate least-square fitting of typical limb-darkening curves from just three coefficients than a third-order polynomial expansion would give.

At different frequencies one samples the same locations $z$ at other $\mu$ values. The optical depth scales have

$$
\frac{\mathrm{d} \tau_{\nu}}{\mathrm{d} \tau_{0}}=\frac{\kappa_{\nu} \rho \mathrm{d} z}{\kappa_{0} \rho \mathrm{d} z}=\frac{\kappa_{\nu}}{\kappa_{0}} \quad \tau_{\nu}\left(\tau_{0}\right)=\int_{0}^{\tau_{0}} \frac{\kappa_{\nu}}{\kappa_{0}} \mathrm{~d} t_{\nu}
$$

and can be converted into each other when $\tau_{\nu}\left(T, P_{\mathrm{e}}\right)$ is known. If they are not, as was the case historically, the transformation may be found empirically from the redundancy available from measuring the center-limb variation at different frequencies. The procedure is sketched in Figure

Center-limb inversions of this type have been important historically in showing the importance of $\mathrm{H}^{-}$extinction, but do not deliver very accurate models. Per frequency only a single decade in $\tau_{\nu}$ is sampled because $I_{\nu}(0, \mu)$ can only be measured accurately $y_{1}^{1} I_{1}^{\prime}$ for $0.1 \lesssim \tau_{\nu} \leq 1$. In addition, the inhomogeneity of the actual solar surface spoils the precision. For other stars the technique is obviously impossible (except for some rare eclipsing binaries with known center-to-limb variation).

Line intensities. A larger range in $\tau_{\nu}$ is reached by inversion of the intensity profile of strong spectral lines. Solar and stellar models have been constructed from the extended wings of the Ca II H \& K lines: - Another example is the HOLMUL model of Holweger and

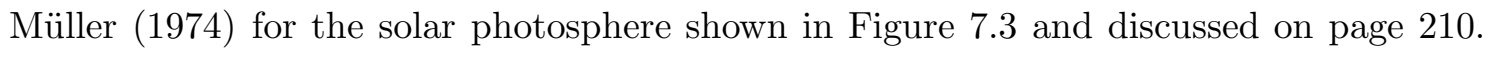
It was constructed by converting the observed line-center intensities of photospheric Fe I

\footnotetext{
${ }^{10}$ Viewing angle $\mu=\cos \theta=0.1$ corresponds to $r / R_{\odot}=\sin \theta=\sqrt{1-\mu^{2}}=0.995$ on the apparent disk, only $5^{\prime \prime}$ from the solar limb. Even if the seeing and the telescope permit spatial resolution better than $1^{\prime \prime}$, stray light spoils intensity measurements of source variations on longer angular modulation scales. The resolution is given by the halfwidth of the point-spread function for small-scale large-contrast structures, but the measured intensity depends also on the wide wings of the point-spread profile. They are made up by stray light due to scattering in the Earth's atmosphere and to imperfections in the telescope including vignetting. They usually decay slowly, roughly as $I(\Delta \theta) / I(0) \sim 1 / \Delta \theta^{2}$. Post-detection correction for stray light is not easy, even more difficult than it was for the aberrated HST because the point-spread function is not known and highly_variable.

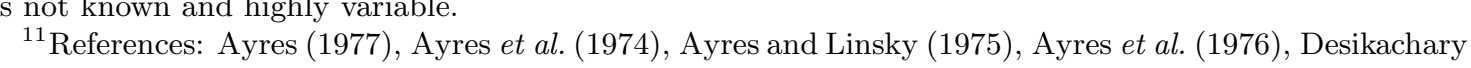
ind Gray (1978), Kelch and Linsky (1978), Kelch et al. (1979).
} 

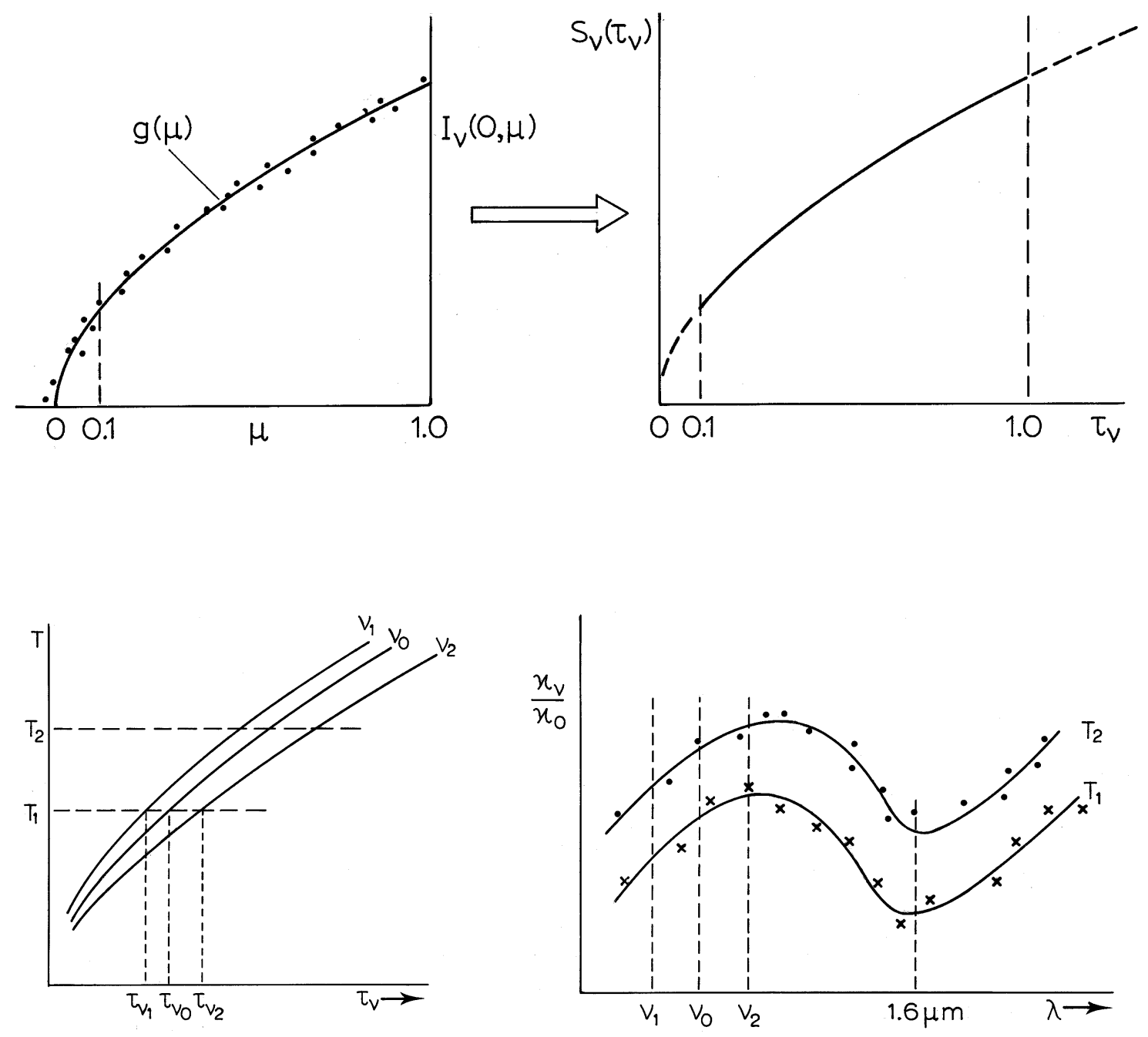

Figure 7.4: Determination of the wavelength variation of the solar continuum extinction from observed limb darkening. Upper graphs: at each frequency, the observed limb darkening (left) produces a corresponding $S_{\nu}\left(\tau_{\nu}\right)$ relation (right). Lower graphs: assuming LTE each $S_{\nu}\left(\tau_{\nu}\right)$ relation transforms into a $T\left(\tau_{\nu}\right)$ relation. A given value of $T$ describes a given depth in the atmosphere; per $T$ one finds the relative wavelength dependence of the extinction coefficient by determining the horizontal offsets between the various curves. The resulting curves show $\mathrm{H}^{-}$-like behavior (right). This procedure was used by 'C̄'Chalonge and Kourganoff - 1946$)^{\prime}$, to demonstrate that $\mathrm{H}^{-}$dominates the continuous extinction in the solar photosphere at visible and near-infrared wavelengths. From 'Zwaan $(1993)$ ! 

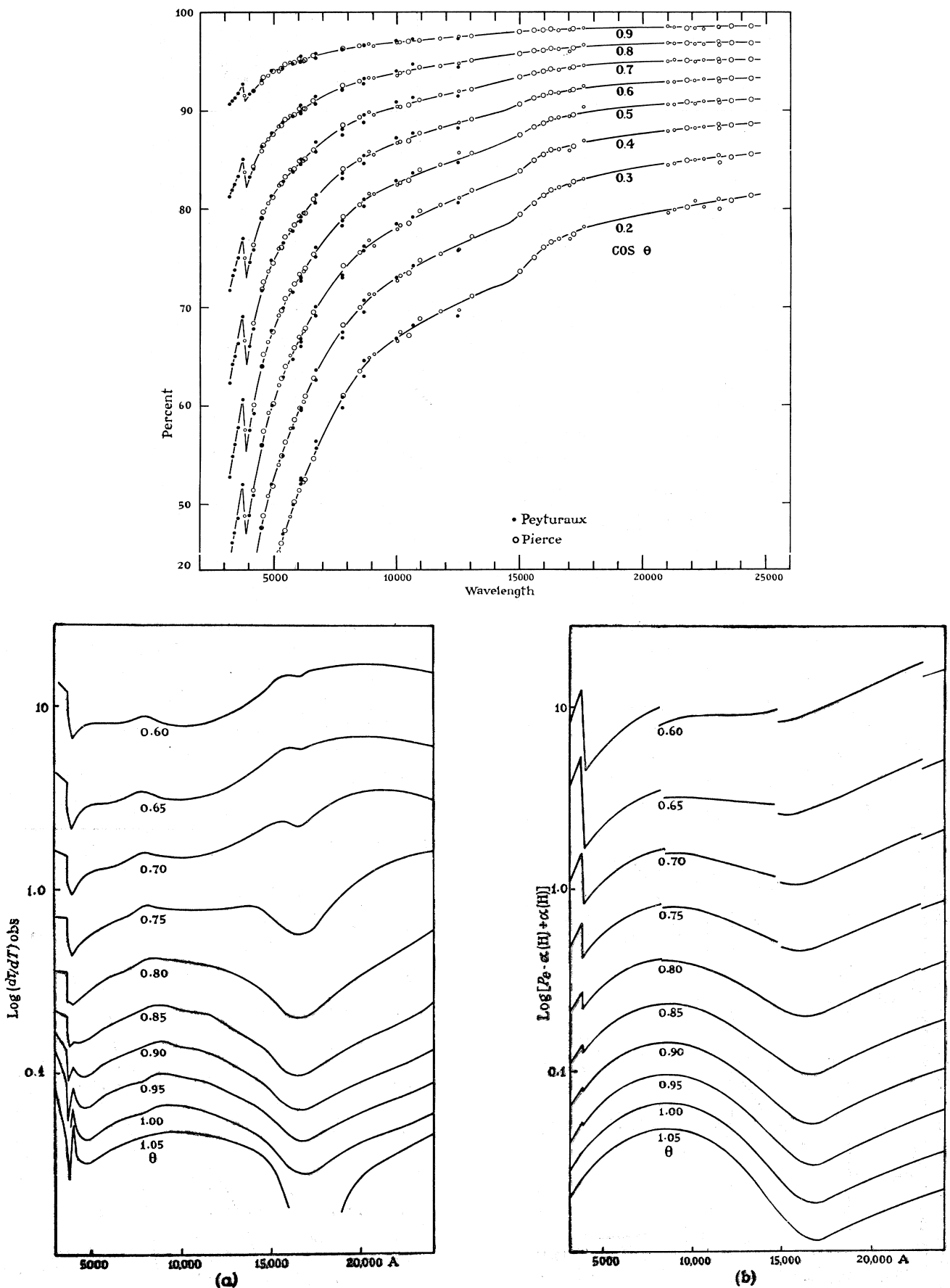

Figure 7.5: Classical empirical model fitting to the observed solar limb darkening. The upper graph shows observed solar continua $I_{\lambda}(0, \mu) / I_{\lambda}(0,0)$ for different values of $\mu=\cos \theta$. These curves were fit with the coefficients $a_{\nu}, b_{\nu}$ and $c_{\nu}$ in $\left(\overline{7}_{2} .24_{1}^{\prime}\right)$ and produced, with the assumption of LTE, the observationally determined gradients $\mathrm{d} \tau_{\lambda} / \mathrm{d} T$ shown in the lefthand lower panel for different temperatures (now with $\theta=5040 / T$ and at arbitrary vertical offsets). The righthand lower panel shows comparable curves obtained from the solar model that was derived by fitting these observations (plus the absolute disk-center intensity value to set the zero point) assuming LTE, $\mathrm{HE}$ and only $\mathrm{H}$ and $\mathrm{H}^{-}$continuous extinction. The resulting curves correspond best to the observed ones for $\theta \approx 0.85$ or $T \approx 5930 \mathrm{~K}$. The discrepancies at left and the unsharpness of the observed Paschen and Brackett edges were attributed to line crowding; the lack of agreement at other temperatures to surface inhomogeneity. From Pierce and Waddell $(1961)_{r}^{\prime}$ 
lines of varying strength into brightness temperature assuming LTE. This method has also been used with lines in the flux spectrum of other stars. For these, stellar limb darkening and stellar rotation present problems that do not occur for the solar disk-center intensity spectrum ${ }_{-}^{1 / 2}$ !.

Continuum intensities. The third approach to empirical $T(\tau)$ determination is to use the observed continuum intensity throughout the spectrum. This technique was used

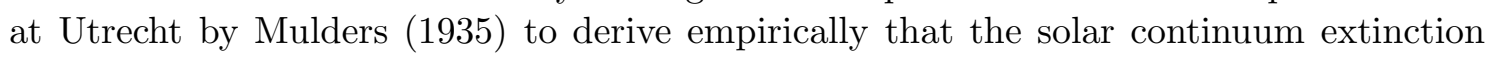
has a dip below $\lambda=500 \mathrm{~nm}$, a maximum around $\lambda=1 \mu \mathrm{m}$ and a deep minimum near $\lambda=1.7 \mu \mathrm{m}$, well before the Wildt-Chandrasekhar (page $\left.1 \overline{1} \overline{1} \overline{8}_{1}^{\prime}\right)$ identification of $\mathrm{H}^{-}$ as its cause and the similar Chalonge-Kourganoff continuum measurement illustrated in Figure $\overline{7} \cdot \overrightarrow{4}$.

More recently, this technique formed the basis for the standard VALIII model of the

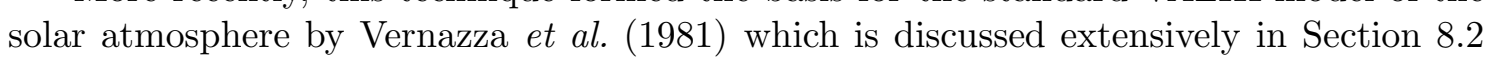
on page $1 \overline{1} \overline{8} \overline{0}_{1}^{\prime} f f$. The VALIIII modeling combined the observed brightness temperature throughout the disk-center solar spectrum, from X-ray to infrared (shown on page $1 \overline{1} \overline{7}_{-}^{-}$ , $8.5 i)$, with NLTE modeling to obtain the optical depth scales at each wavelength.

Such modeling is difficult for other stars because fluxes must then be measured on absolute energy scales throughout the spectrum. Absolute calibration is usually not achieved even for the fluxes detected at Earth (irradiance). Even if the stellar irradiance is calibrated, the distance to the star (or its radius) must be known for conversion into stellar surface flux.

\subsubsection{Radiative equilibrium}

Flux constancy. Stellar model atmospheres are rarely made by empirical methods as the ones discussed above. The distinction between empirical and theoretical modeling is effectively also a distinction between solar and stellar modeling $g_{-}^{13}$. The solution for stellar modeling is to take the empirical solar result that the photosphere obeys radiative equilibrium (page $\left.1{ }^{1} \overline{5} \overline{8}_{1}^{\prime}\right)$ for granted and to require it as a basis to derive stellar $T\left(\tau_{0}\right)$ stratifications. The general version is to require flux constancy:

$$
\nabla \cdot \mathbf{F}_{\text {tot }}(\mathbf{r})=\nabla \cdot\left[\mathbf{F}_{\text {rad }}(\mathbf{r})+\mathbf{F}_{\text {conv }}(\mathbf{r})+\mathbf{F}_{\text {cond }}(\mathbf{r})+\mathbf{F}_{\text {mech }}(\mathbf{r})\right] \equiv 0,
$$

\footnotetext{
${ }^{12}$ Even in the solar case the method is problematic because FeI lines, favored because they are numerous and have negligible broadening from hyperfine structure and isotope splitting, suffer from NLTE overionization due to $J_{\nu}>B_{\nu}$ excesses in the ultraviolet that affect their opacity. A better option is to use the $\mathrm{CO}$ rotation bands in the infrared of which the lines probably obey LTE with high precision. They have recently been measured by the ATMOS shuttle experiment (Atmospheric Trace Molecule Spectroscopy). This is a Fourier spectrometer mapping the infrared spectrum of the Earth's atmosphere, in ozone-hole-type trace-element studies. It uses the Sun as background source and measures the extinction of the terrestrial atmosphere at sunrise and sunset as seen from the Space Shuttle. In order to calibrate the background source, ATMOS has also mapped the solar infrared spectrum without intervening terrestrial atmosphere. CO line-depth models are presently being constructed by E.H. Avrett (CfA).

${ }^{13}$ I apologize for using "solar" as "non-stellar" and "stellar" as "non-solar". This usage is fairly commonplace, leading to book titles as "The Sun as a Star" and "The Solar-Stellar Connection". There are many solar-stellar connections, the essential one being that the Sun is a star - as advocated by Giordano Bruno who was burned to death in 1600 for claiming that stars are suns. In the twentieth century, the development of stellar physics and radiative transfer as treated in this course constituted the major solar-stellar connection. A more recent one is the study of stellar magnetic activity using solar activity as guideline. A connection in the making is between helioseismology and asteroseismology.
} 
saying that the total radial energy flux traveling outward through the atmosphere should be constant in time and along any vector $\mathbf{r}$. For plane-parallel atmospheres the requirement simplifies to:

$$
\frac{\mathrm{d} F_{\text {tot }}}{\mathrm{d} z}=0 .
$$

The first three energy fluxes, respectively by radiation, convection and conduction, are set by the radial temperature gradient. The fourth describes "mechanical" energy transport by waves of any type (acoustic waves, internal gravity waves, MHD waves, plasma instabilities). It is important for cool-star chromospheres and coronae, but not for photospheres. Conduction is only important in coronae and in degenerate stellar interiors. Convection is important in the deeper layers of the solar atmosphere and other cool stars. It should be noted that the term "radiative equilibrium model" or "RE model" is often used for stellar atmospheric models that do include convective energy transport, so that "flux-constant model" is a better name.

Radiative equilibrium (RE). By setting the total energy flux equal to the radiative flux through the atmosphere, the stability requirement becomes a radiative equilibrium condition:

$$
\mathcal{F}_{\text {rad }}(z) \equiv \int_{0}^{\infty} \mathcal{F}_{\nu}(z) \mathrm{d} \nu=\mathcal{F}
$$

at every $z$, with $\mathcal{F}=\pi F$ at every height $z$ equal to the observed stellar surface flux

$$
\mathcal{F} \equiv \sigma T_{\text {eff }}^{4}=\frac{L_{*}}{4 \pi R_{*}^{2}}
$$

We may also write the RE condition as

$$
\frac{\mathrm{d} \mathcal{F}_{\text {rad }}(z)}{\mathrm{d} z}=0
$$

or using $F_{\nu}=4 H_{\nu}$ and equation $\left(\overline{4}_{4} \cdot \overline{7}_{1}\right)$ on page ${ }_{1}^{1} \overline{\sigma_{1}^{1}}$ rewrite it into the Strömgren equation

$$
\int_{0}^{\infty} \kappa_{\nu}(z) \rho(z) J_{\nu}(z) \mathrm{d} \nu=\int_{0}^{\infty} \kappa_{\nu}(z) \rho(z) S_{\nu}(z) \mathrm{d} \nu
$$

and with the total radiative flux divergence $\Phi_{\text {tot }}(z)$ in $\operatorname{erg~cm}^{-3} \mathrm{~s}^{-1}$ as

$$
\Phi_{\text {tot }}(z) \equiv \frac{\mathrm{d} \mathcal{F}_{\text {rad }}(z)}{\mathrm{d} z}=4 \pi \int_{0}^{\infty} \alpha_{\nu}(z)\left[S_{\nu}(z)-J_{\nu}(z)\right] \mathrm{d} \nu=0,
$$

or more generally in Hubený notation

$$
\Phi_{\text {tot }}(z) \equiv \frac{\mathrm{d} \mathcal{F}_{\text {rad }}(z)}{\mathrm{d} z}=\frac{1}{2} \int_{0}^{\infty} \int_{-1}^{+1}\left[j_{\nu \mu}(z)-\alpha_{\nu \mu}(z) I_{\nu \mu}(z)\right] \mathrm{d} \mu \mathrm{d} \nu=0 .
$$

Thus, in RE the flux divergence integral is zero at all heights. At every height all emitted energy (written as $j_{\nu}=\alpha_{\nu} S_{\nu}$ when the emissivity $j_{\nu \mu}$ is isotropic) must equal all extincted energy (with $J_{\nu}$ the average over all directions when the extinction $\alpha_{\nu \mu}$ is isotropic) to obtain local balancing without enhancing or diminishing the flux. The monochromatic integrand $\alpha_{\nu}(z)\left[S_{\nu}(z)-J_{\nu}(z)\right]$ is called the net radiative cooling rate because an overdose of radiation is produced locally when $S_{\nu}>J_{\nu}$, representing an energy loss for this location. 
Reversely, when $J_{\nu}>S_{\nu}$ there is net radiative heating at this location since more photons are extincted than emitted or re-emitted.

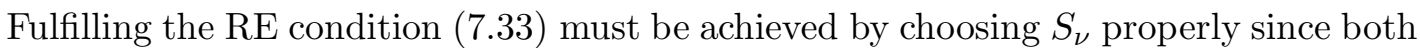
$F_{\nu}\left(\tau_{\nu}\right)=\boldsymbol{\Phi}_{\tau_{\nu}}\left[S_{\nu}\left(t_{\nu}\right)\right]$ and $J_{\nu}\left(\tau_{\nu}\right)=\boldsymbol{\Lambda}_{\tau_{\nu}}\left[S_{\nu}\left(t_{\nu}\right)\right]$ represent exponential-integral transforms of $S_{\nu}$ as defined by the operator definitions (page $\left.\overline{8}^{\prime} \bar{I}^{\prime}\right)$. This conditioning of $S_{\nu}\left(\tau_{\nu}\right)$ is far from straightforward because it requires integration over the full spectrum: the equality $\left(1 \overline{3} 3{ }^{3}\right)$ is an integrated one. At each frequency, local radiative cooling or heating may occur as long as it is balanced by the reverse at other frequencies. The flux divergence $\mathrm{d} \mathcal{F}_{\text {rad }}(z) / \mathrm{d} z$ is the sum over all such monofrequent $S_{\nu}-J_{\nu}$ divergences; only the total needs to be zero.

Discussion. An obvious way to fulfill (77.33) is to have $J_{\nu}=S_{\nu}$ at every frequency $\nu$, as is the case for TE. It is not realistic for stellar atmospheres, however, since these harbor a net radiative energy flux (stars shine) that cannot exist in TE. Take the LTE case $S_{\nu}=B_{\nu}$ and inspect the $\boldsymbol{\Lambda}$ operator graphs in Figure 4.4 . They show that $J_{\nu}=B_{\nu}$ requires a $B_{\nu}$ slope $a \approx 1.5$. The same is seen for the $\boldsymbol{\Phi}$ operator diagrams in Figure 4.5 . If the stellar temperature stratification $T(z)$ is such that that happens at a frequency $\nu=\nu_{0}$, then $J_{\nu}$ drops steeper than $B_{\nu}$ at larger frequency and less steeply at smaller frequency due to the variation in Planck function sensitivity to temperature across the spectrum. Thus, LTE flux constancy requires $a \approx 3 / 2$ but that cannot be the case at multiple frequencies simultaneously for a given $T(z)$ relation. What about NLTE? The equality $S_{\nu}=J_{\nu}$ indeed holds at all frequencies when all extinction processes consist of coherent scattering so that no photons are created locally. That may describe an irradiated optically-thin planetary atmosphere like the one outside your window, but we cannot make a self-radiating optically-thick stellar atmosphere or stellar interior that way. That requires a thermal source term $\varepsilon_{\nu} B_{\nu}$ to create the photons that make up $J_{\nu}$ initially. The source may be small but it can't be zero. For very small $\varepsilon_{\nu}$ the problem is simply transported deeper, to the thermalization depth.

Line cooling. A bound-bound transition may contribute local cooling or heating (again erg $\mathrm{cm}^{-3} \mathrm{~s}^{-1}$ ) according to its net radiative cooling rate given by

$$
\begin{aligned}
\Phi_{u l} & =4 \pi \alpha_{\nu_{0}}^{l}\left(S_{\nu_{0}}^{l}-\bar{J}_{\nu_{0}}\right) \\
& =4 \pi j_{\nu_{0}}^{l}-4 \pi \alpha_{\nu_{0}}^{l} \bar{J}_{\nu_{0}} \\
& =h \nu_{0}\left[n_{u}\left(A_{u l}+B_{u l} \bar{J}_{\nu_{0}}\right)-n_{l} B_{l u} \bar{J}_{\nu_{0}}\right] \\
& =h \nu_{0}\left[n_{u} R_{u l}-n_{l} R_{l u}\right],
\end{aligned}
$$

dropping the $z$ dependences and using $(\overline{2}-\overline{6} \overline{2}),\left(\overline{2}_{2}-\overline{6}_{1}\right)$ and $\int \varphi\left(\nu-\nu_{0}\right) \mathrm{d} \nu=\int \chi\left(\nu-\nu_{0}\right) \mathrm{d} \nu=$ $\int \psi\left(\nu-\nu_{0}\right) \mathrm{d} \nu=1$ to express the bound-bound contribution to the flux divergence integral (1733i) in terms of the net radiative rate $\left[n_{u} R_{u l}-n_{l} R_{l u}\right]$ that was evaluated in (3.29) on page 491 in the reverse of this derivation. For $n_{u} R_{u l}>n_{l} R_{u l}$ the line cools the medium at the location $z$ by adding an excess of photons into the net flux; it feeds on the flux in the reverse case.

In the Wien limit and using the population departure coefficients $b_{i}$ defined by (2.104)

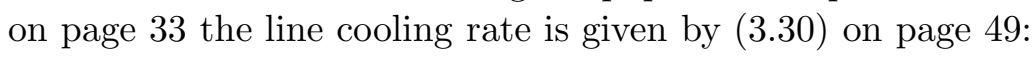

$$
\Phi_{u l}=h \nu_{0}\left[n_{u} R_{u l}-n_{l} R_{l u}\right] \approx 4 \pi b_{u}\left[\alpha_{\nu_{0}}^{l}\right]_{\mathrm{LTE}}\left(B_{\nu_{0}}-\frac{b_{l}}{b_{u}} \bar{J}_{\nu_{0}}\right) .
$$


Continuum cooling. A similar expression follows from (3.23) on page $4 \overline{4} \overline{3}$ for the contribution to the flux divergence integral given by a bound-free transition:

$$
\Phi_{c i}=4 \pi n_{i}^{\mathrm{LTE}} b_{c} \int_{\nu_{0}}^{\infty} \sigma_{i c}(\nu)\left[B_{\nu}\left(1-\mathrm{e}^{-h \nu / k T}\right)-\frac{b_{i}}{b_{c}} J_{\nu}\left(1-\frac{b_{c}}{b_{i}} \mathrm{e}^{-h \nu / k T}\right)\right] \mathrm{d} \nu .
$$

In the Wien limit this expression simplifies to:

$$
\Phi_{c i}=4 \pi n_{i}^{\mathrm{LTE}} b_{c} \int_{\nu_{0}}^{\infty} \sigma_{i c}(\nu)\left(B_{\nu}-\frac{b_{i}}{b_{c}} J_{\nu}\right) \mathrm{d} \nu
$$

\subsubsection{The grey approximation}

The easiest way to solve the RE condition ( by assuming the extinction in the atmosphere to be independent of frequency. This is called the grey case. Since Thomson scattering is the only source of extinction which is indeed frequency-independent this isn't a very realistic approximation. Nevertheless, it has received much attention in the older literature where it served to obtain analytical approximations before computers permitted more realistic modeling $\mathbf{1}_{-}^{174}$.. It is summarized here briefly.

The grey approximation replaces the infinite number of transport equations $\left(2 . \overline{4} 0_{1}\right)$ on page independent optical depth scale $\tau(z)$ (Gray p. $120 \mathrm{ff}$ ):

$$
\begin{aligned}
\int_{0}^{\infty} \mu \frac{\mathrm{d} I_{\nu}\left(\tau_{\nu}, \mu\right)}{\mathrm{d} \tau_{\nu}} \mathrm{d} \nu & =\int_{0}^{\infty}\left[I_{\nu}\left(\tau_{\nu}, \mu\right)-S_{\nu}\left(\tau_{\nu}\right)\right] \mathrm{d} \nu \\
\mu \frac{\mathrm{d} I(\tau, \mu)}{\mathrm{d} \tau} & =I(\tau, \mu)-S(\tau) .
\end{aligned}
$$

The RE condition becomes simply

$$
S(\tau)=J(\tau)
$$

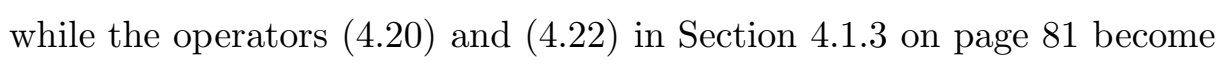

$$
J(\tau)=\boldsymbol{\Lambda}_{\tau}[S(t)]
$$

and

$$
F(\tau)=\boldsymbol{\Phi}_{\tau}[S(t)]=F .
$$

Thus, when RE holds $S(\tau)$ is its own $\boldsymbol{\Lambda}$ transform and its $\boldsymbol{\Phi}$ transform is constant with $\tau$.

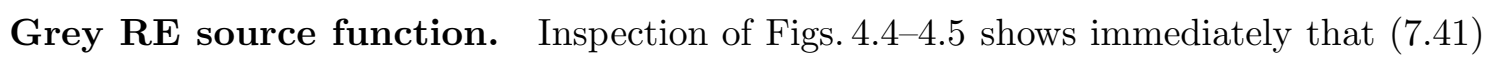
and (7.72i) require a source function with:

$$
S(\tau) \approx c\left(1+\frac{3}{2} \tau\right) .
$$

\footnotetext{
${ }^{14}$ Gray (what's in a name) comments: "The usefulness of the grey case is small when it comes to interpreting real stellar spectra. It is worthwhile recalling Eddington's (1926) comment concerning the grey case: 'This, however, is a lazy way of handling the problem and it is not surprising that the result fails to accord with observation. The proper course is to find the spectral distribution of the emergent radiation by treating each wave-length separately using its own proper value of $j$ and $\kappa$ '." (Page 123 of 'Gray 1992').
} 
A formal derivation follows from the $\chi$ operator in $(4 . \overline{2} 3)$ and $(\overline{4} . \overline{2} 4)$ :

$$
F=\boldsymbol{\Phi}_{\tau}[S(t)]=\frac{\mathrm{d}}{\mathrm{d} \tau} \chi_{\tau}[S(\tau)]=4 \frac{\mathrm{d} K(\tau)}{\mathrm{d} \tau},
$$

showing $K(\tau)$ to be linear in $\tau$ with $K(\tau)=(1 / 4) F \tau+a$. The first Eddington approximation and (7. $\overline{4} 0 i)$ give $K(\tau) \approx(1 / 3) J(\tau)=(1 / 3) S(\tau)$ so that $S(\tau) \approx(3 / 4) F \tau+3 a$. Thus, $S(\tau)$ is indeed approximately linear. In the literature the exact result is written as

$$
S(\tau)=\frac{3}{4}(\tau+q(\tau)) F
$$

with $q(\tau)$ the Hopf function which obeys

$$
\tau+q(\tau)=\boldsymbol{\Lambda}_{\tau}[\tau+q(\tau)]
$$

It varies slowly with $\tau$. Eddington used his second approximation $S(0)=J(0) \approx F / 2$ to find from (i7. $\overline{4} \overline{5})$ that $q(\tau)=2 / 3$ when assumed constant, giving the Milne-Eddington approximation for a grey $\mathrm{RE}$ atmosphere:

$$
S(\tau) \approx \frac{3}{4}\left(\tau+\frac{2}{3}\right) F=\left(\frac{3}{4} \tau+\frac{1}{2}\right) F=\frac{1}{2}\left(1+\frac{3}{2} \tau\right) F
$$

with $F=(\sigma / \pi) T_{\text {eff. }}^{4}$. The last version confirms the $\mathrm{d} S / \mathrm{d} \tau=3 / 2$ flux constancy seen in Figure 4.5 , on page

Grey RE temperature stratification. The assumption of LTE gives with the StefanBoltzmann law for the total Planck intensity $S(\tau)=B(\tau)=(\sigma / \pi) T^{4}$ and with (77.4.

$$
T(\tau) \approx T_{\mathrm{eff}}\left(\frac{3}{4} \tau+\frac{1}{2}\right)^{1 / 4}
$$

with $T_{\text {eff }}=T(\tau=2 / 3)$ as it should. Knowing the temperature-depth relation and still assuming LTE implies that the monochromatic source function is known as well:

$$
S_{\nu}(\tau)=B_{\nu}[T(\tau)]
$$

with $T(\tau)$ given by (7.78).

Grey RE scattering. The LTE assumption above is wrong for hot stars in which Thomson scattering dominates over continuous processes (Section 8 scattering is frequency-independent. How does such grey scattering affect the temperature stratification? The discussion on page $10 \overline{1} \overline{6}_{1}^{\prime}$ shows that for Thomson scattering the source function may be written as:

$$
S_{\nu}=\left(1-\varepsilon_{\nu}\right) J_{\nu}+\varepsilon_{\nu} B_{\nu}
$$

with $\varepsilon_{\nu}$ given by $(4.100)$ as the probability per extinction that a photon is destroyed by a non-scattering bound-free or free-free interaction. If we assume the latter extinction to be grey as well, $\varepsilon$ is also independent of frequency and the RE condition ( $7 . \overline{3} 2)$ becomes (dropping the $\tau$ dependences):

$$
\begin{aligned}
\int_{0}^{\infty} \kappa_{\nu} \rho J_{\nu} \mathrm{d} \nu & =\int_{0}^{\infty} \kappa_{\nu} \rho S_{\nu} \mathrm{d} \nu \\
\kappa \rho J & =\kappa \rho[(1-\varepsilon) J+\varepsilon B] \\
\varepsilon J & =\varepsilon B \\
J & =B=S,
\end{aligned}
$$




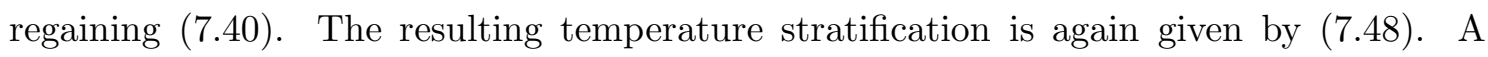
grey atmosphere doesn't care what processes cause its extinction; the RE condition fixes its temperature stratification $T(\tau)$ regardless of the $(1-\varepsilon) J$ part that is contributed by scattering.

However, the monochromatic $J_{\nu}$ and $S_{\nu}$ do depend on the nature of the extinction. For strong scattering (small $\varepsilon$ ) the radiation at long wavelengths has $J_{\nu}<S_{\nu}<B_{\nu}$ near

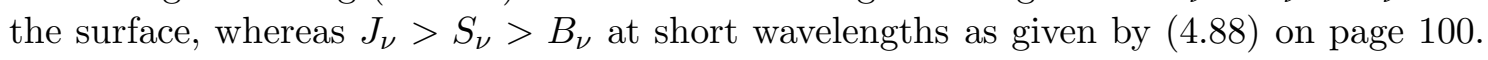
These splits extend inwards to $\tau \approx \Lambda=1 / \sqrt{\varepsilon}$. They have the same sign but smaller magnitude when $\varepsilon=1$, as given by (4.87i). They add up across the spectrum to produce zero split $S=J=B$ in the total, whatever the value of $\varepsilon$ as long as $\kappa$ and $\varepsilon$ are grey. There are no optical depth scale effects as in $(4.90 \%)$ on page $103^{\prime \prime}$ because the $\tau$ scale is the same at all frequencies, without spectral lines.

Grey RE limb darkening. The center-limb variation of a grey star follows from (7.7) with the Eddington-Barbier relation ${ }_{-1}^{15 !}(2-\overline{2} \cdot \overline{3})$ :

$$
\frac{I(0, \mu)}{I(0,1)}=\frac{3}{5}\left(\mu+\frac{2}{3}\right) .
$$

'S̄chwarzschild $(1906)$ ' introduced the concept of radiative equilibrium and derived a similar expression for the limb darkening ${ }_{-}^{1} \bar{l}_{1}^{1}$. His paper (reprinted in English in very readable) ends with a table in which he compared the bolometrically observed solar limb darkening with predictions from this RE result and from an adiabatic temperature gradient that would hold for convective flux constancy. It is copied here in Table The comparison showed clearly that the photosphere obeys radiative equilibrium, and not convective equilibrium as was expected from the existence of solar granulation. The adiabatic gradient is much steeper and produces much too strong limb darkening.

Grey extinction and mean extinction. Real atmospheres are not grey; their extinction varies strongly with wavelength. However, the results for a grey atmosphere may be useful to describe radiative transfer in terms of a frequency-averaged mean extinction coefficient $\bar{\kappa}$ with corresponding optical depth scale $\bar{\tau}$. There are various possibilities to define such mean extinction (see 'Minhalas 1970' p. $37 \mathrm{ff}$ ); one possibility is to require that

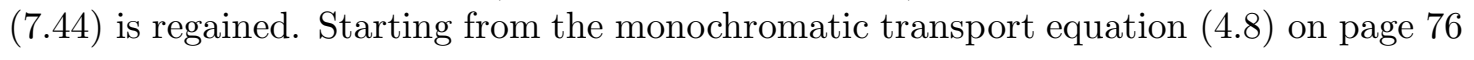

$$
4 \frac{\mathrm{d} K_{\nu}(z)}{\mathrm{d} \tau_{\nu}}=4 \frac{\mathrm{d} K_{\nu}(z)}{-\kappa_{\nu}(z) \rho(z) \mathrm{d} z}=F_{\nu}(z)
$$

we may require the integrated version to obey:

$$
\int_{0}^{\infty} 4 \frac{\mathrm{d} K_{\nu}(z)}{-\kappa_{\nu}(z) \rho(z) \mathrm{d} z} \mathrm{~d} \nu \equiv \frac{1}{\bar{\kappa}(z)} \int_{0}^{\infty} 4 \frac{\mathrm{d} K_{\nu}(z)}{-\rho(z) \mathrm{d} z} \mathrm{~d} \nu
$$

\footnotetext{
${ }^{15}$ The derivation of the Eddington-Barbier approximation holds also for the total source function. In this case it is not an approximation but a relation since $\left(\overline{7} .4 \overline{7}^{\prime}\right)$ is linear in $\tau$.

${ }^{16}$ Namely $I(0, \mu) / I(0,1)=(2 / 3)(\mu+1 / 2)$. It differs from $\left(7.52_{1}^{\prime}\right)$ because he didn't know the Eddington approximation yet, and obtained $T=T_{\text {eff }}(\tau / 2+1 / 2)^{1 / 4}$ rather than (1.48) mass" and assumed that the solar photosphere consists of air at $6000 \mathrm{~K})$. As a result, his convective stability criterion, formulated in the same brief paper, compares the adiabatic gradient $\mathrm{d} T / \mathrm{d} h=[(\gamma-1) / \gamma] M / R$ with the radiative gradient $\mathrm{d} T / \mathrm{d} h=(1 / 4)\left[1-2 T_{\text {eff }}^{4} / T^{4}\right] M / R$ which is always satisfied for $\gamma>4 / 3$ rather than $\gamma>5 / 3$.
} 


\begin{tabular}{cc|r|lc|lc}
\hline & & \multicolumn{5}{|c}{ Limb darkening $I(0, \mu) / I(0,1)$} \\
$r / R_{\odot}$ & $\mu$ & Observed & \multicolumn{2}{|c}{ Radiative equilibrium } & \multicolumn{2}{l}{ Convective equilibrium } \\
\hline 0.00 & 1.00 & 1.00 & 1.00 & 1.00 & 1.00 & 1.00 \\
0.20 & 0.98 & 0.99 & 0.99 & 0.99 & 0.98 & 0.97 \\
0.40 & 0.92 & 0.97 & 0.95 & 0.95 & 0.92 & 0.87 \\
0.60 & 0.80 & 0.92 & 0.87 & 0.88 & 0.80 & 0.70 \\
0.80 & 0.60 & 0.81 & 0.73 & 0.76 & 0.60 & 0.44 \\
0.90 & 0.44 & 0.70 & 0.63 & 0.66 & 0.44 & 0.27 \\
0.98 & 0.20 & 0.49 & 0.47 & 0.52 & 0.20 & 0.08 \\
1.00 & 0.00 & $\approx 0.40$ & 0.33 & 0.40 & 0.00 & 0.00 \\
\hline
\end{tabular}

Table 7.2: Solar limb darkening. First two columns: viewing angle, respectively the apparent solar radius given by $\underline{r} L_{-} \underline{R}_{\odot}=\sin \theta=\left(1-\mu^{2}\right)^{1 / 2}$ and $\mu=\cos \theta$. Third column: bolometric observations taken by 'Schwarzschild $(\overline{1} 906)$,' from a book by G. Müller. Next two columns: grey atmosphere in radiative equilibrium. The lefthand column is for Schwarzschild's result $I(0, \mu) / I(0,1)=(2 / 3)(\mu+1 / 2)$; the righthand one for $I(0, \mu) / I(0,1)=(3 / 5)(\mu+2 / 3)$. Final two columns: atmosphere in convective equilibrium according to Schwarzschild's own criterion having $I(0, \mu) / I(0,1)=\mu^{4(\gamma-1) / \gamma)}$. The lefthand column is for Schwarzschild's choice of $\gamma=4 / 3$. The righthand column is for $\gamma=5 / 3$. The observed solar limb darkening is much closer to radiative equilibrium than to convective equilibrium. From îMenzel 1 - $(1993)$

The lefthand side has with (7.53i)

$$
\int_{0}^{\infty} 4 \frac{\mathrm{d} K_{\nu}(z)}{-\kappa_{\nu}(z) \rho(z) \mathrm{d} z} \mathrm{~d} \nu=\int_{0}^{\infty} F_{\nu}(z) \mathrm{d} \nu=F(z)
$$

so that the definition of mean extinction $\bar{\kappa}$ in $(7 . \overline{5} \overline{4})$ is equivalent, using $(\overline{7} .53 i)$ again in the righthand side of $(17.54)$, to the flux-weighted mean extinction

$$
\bar{\kappa}(z) \equiv \frac{\int_{0}^{\infty} \kappa_{\nu}(z) F_{\nu}(z) \mathrm{d} \nu}{\int_{0}^{\infty} F_{\nu}(z) \mathrm{d} \nu}=\int_{0}^{\infty} \kappa_{\nu}(z) \frac{F_{\nu}(z)}{F(z)} \mathrm{d} \nu .
$$

On the other hand, the righthand side of (17.54) has

$$
\frac{1}{\bar{\kappa}(z)} \int_{0}^{\infty} 4 \frac{\mathrm{d} K_{\nu}(z)}{-\rho(z) \mathrm{d} z} \mathrm{~d} \nu=\frac{1}{\bar{\kappa}(z)} 4 \frac{\mathrm{d} K(z)}{-\rho(z) \mathrm{d} z}
$$

so that the definition of $\bar{\kappa}$ in $(\overline{7} .5 \overline{4})$ may also be reordered into the harmonic average

$$
\overline{\frac{1}{\kappa(z)}} \equiv \frac{\int_{0}^{\infty}\left[1 / \kappa_{\nu}(z)\right]\left(\mathrm{d} K_{\nu}(z) / \mathrm{d} z\right) \mathrm{d} \nu}{\int_{0}^{\infty}\left(\mathrm{d} K_{\nu}(z) / \mathrm{d} z\right) \mathrm{d} \nu}=\int_{0}^{\infty} \frac{1}{\kappa_{\nu}(z)} \frac{\mathrm{d} K_{\nu}(z) / \mathrm{d} z}{\mathrm{~d} K(z) / \mathrm{d} z} \mathrm{~d} \nu .
$$

Flux-weighted mean and Rosseland mean. The $1 / \kappa$ in $(7,58)$ rather than $\kappa$ results from the $\kappa_{\nu}$ in the denominator of (7.53i). The reversal resembles the Rosseland extinction

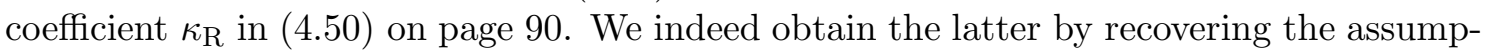
tions of the diffusion approximation through adopting the first Eddington approximation, LTE and (4.47):

$$
K_{\nu} \approx \frac{1}{3} J_{\nu} \approx \frac{1}{3} B_{\nu}
$$


so that $\mathrm{d} K_{\nu} / \mathrm{d} z \approx(1 / 3) \mathrm{d} B_{\nu} / \mathrm{d} z=(1 / 3)\left(\mathrm{d} B_{\nu} / \mathrm{d} T\right)(\mathrm{d} T / \mathrm{d} z)$ and (17.58.) gives:

$$
\frac{1}{\bar{\kappa}} \approx \int_{0}^{\infty} \frac{1}{\kappa_{\nu}} \frac{\mathrm{d} B_{\nu} / \mathrm{d} T}{\mathrm{~d} B / \mathrm{d} T} \mathrm{~d} \nu \equiv \frac{1}{\kappa_{\mathrm{R}}} .
$$

Thus, at sufficiently large depth the Rosseland extinction acts as flux-weighted mean extinction that regains the simple grey form $(\overline{7} \cdot \overline{4} \overline{4})$ of the transport equation for radiative equilibrium. Stellar RE-LTE interiors therefore have temperature stratification

$$
T\left(\tau_{\mathrm{R}}\right)=T_{\text {eff }}\left[\frac{3}{4} \tau_{\mathrm{R}}+\frac{3}{4} q\left(\tau_{\mathrm{R}}\right)\right]^{1 / 4}
$$

where the Rosseland optical depth $\tau_{\mathrm{R}}$ has

$$
\mathrm{d} \tau_{\mathrm{R}}=-\kappa_{\mathrm{R}} \rho \mathrm{d} z .
$$

This equation regains the simple stratification of the grey RE atmosphere in $(\overline{7} . \overline{4} \overline{5})$ for stellar interiors, but at the cost of having to evaluate all $\kappa_{\lambda}$ in the computation of $\tau_{R}$. The radiation has depth dependence

$$
J\left(\tau_{\mathrm{R}}\right)=S\left(\tau_{\mathrm{R}}\right)=B\left(\tau_{\mathrm{R}}\right)=\frac{\sigma}{\pi} T^{4}\left(\tau_{\mathrm{R}}\right)=\frac{3}{4}\left[\tau_{\mathrm{R}}+q\left(\tau_{\mathrm{R}}\right)\right] F,
$$

again with the Hopf function $q\left(\tau_{\mathrm{R}}\right) \approx 2 / 3$.

\subsubsection{Line blanketing}

Actual stellar atmospheres are not grey except for Thomson scattering. They contain narrow-band spectral lines and edges in which the extinction varies rapidly over huge amounts. How do these affect the actual temperature stratification of an atmosphere in radiative equilibrium?

Backwarming. The simplest effect is the line blocking in the deep layers where the observed continuum originates. The presence of strong lines means that much less flux is transported at their frequencies through this layer, because the radiation field remains (nearly) isotropic for large $\tau_{\nu}$ and locally enclosed at the line frequencies. To first order, it doesn't matter whether the line extinction consists of true absorption or scattering; in both cases the overlying layer is more opaque at the line frequency than it would have been otherwise. A few photons leak through when the line is a scattering one, making the blocking slightly less effective for scattering lines.

Compared to a star without lines there is less frequency bandwidth available for the flux. The spectrum-averaged flux per unit bandwidth must be larger, which implies a larger local temperature. In addition, the temperature gradient must be slightly steeper to push the radiation through the atmosphere employing the remaining continuum windows. In the emergent spectrum the continuum between the lines is therefore higher than for a star without lines. An estimate of the effect is given by defining $f$ as the fraction of the total flux that is blocked by lines. The ratio of the flux $F^{\prime}$ for the blocked case to the unblocked flux $F$ is then:

$$
\frac{\int_{0}^{\infty} F_{\nu}^{\prime} \mathrm{d} \nu}{\int_{0}^{\infty} F_{\nu} \mathrm{d} \nu}=\frac{F^{\prime}}{F}=\frac{(\sigma / \pi) T_{\text {eff }}^{\prime 4}}{(\sigma / \pi) T_{\text {eff }}{ }^{4}}=1-f
$$

so that

$$
T_{\text {eff }}=(1-f)^{-1 / 4} T_{\text {eff }}^{\prime} \approx(1+f / 4) T_{\text {eff }}^{\prime} .
$$


The Sun has $f \approx 14 \%$ so that its effective temperature is higher by $3.5 \%$ or $200 \mathrm{~K}$ than it would be without lines. Its flux distribution between lines looks as a $6000 \mathrm{~K}$ black body, rather than one with temperature equal to the effective solar temperature $T_{\text {eff }}^{\prime}=5770 \mathrm{~K}$ as determined from the bolometric flux spectrum (Böhshows the effect schematically.
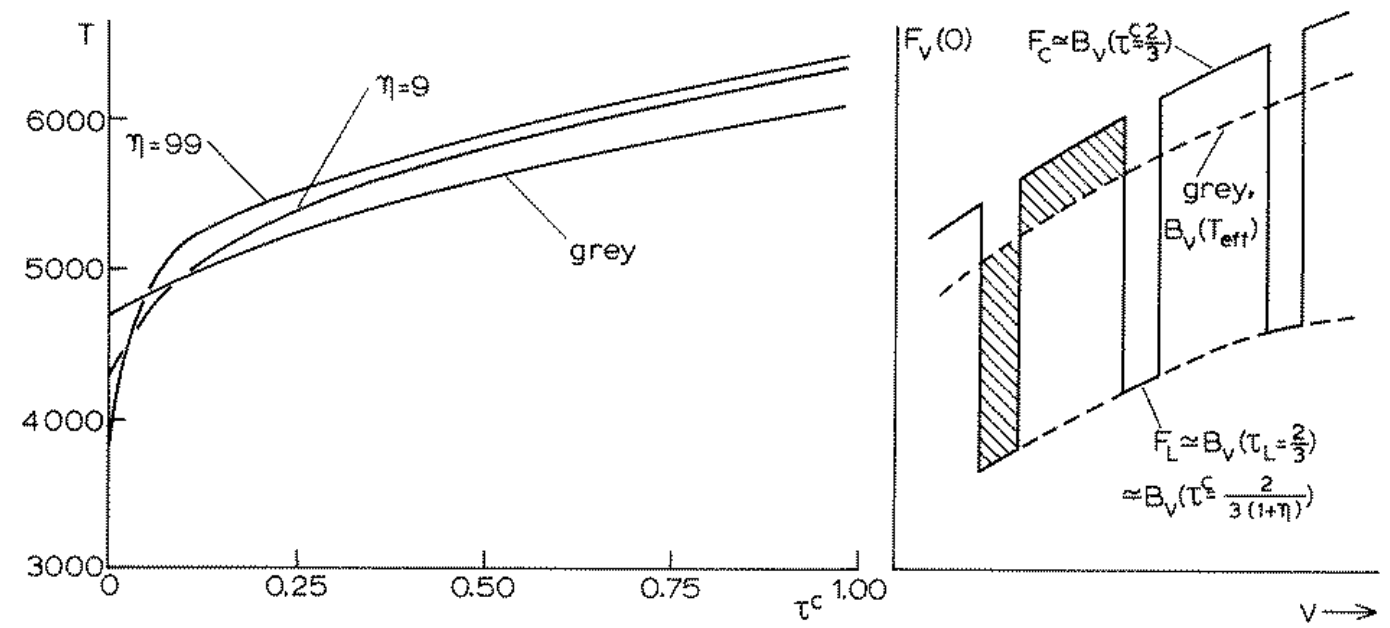

Figure 7.6: LTE backwarming and surface cooling using a schematic "picket fence" model in which the flux-blocking fraction $f$ consists of equidistantly-spaced rectangular lines, all with the same extinction and resembling pickets in a fence. They occupy $20 \%$ of the spectral bandwidth; their strength is given by $\eta=$ $\kappa_{\nu}^{l} / \kappa_{\nu}^{c}$. The lines are formed in LTE, as is the continuum. The blocking causes backwarming and a higher flux for the continuum between the lines than in the grey case. At the surface the lines cause appreciable cooling because they are in LTE; their photon losses deplete the thermal pool locally. The righthand graph shows the spectrum. The Eddington-Barbier depths for the emergent flux are $\tau_{\nu}^{c}=2 / 3$ for the continuum windows and $\tau_{\nu}^{c}=(2 / 3)\left(1 / 1+\eta_{\nu}\right)$ for the lines, with total optical depth $\mathrm{d} \tau_{\mathrm{L}}=\mathrm{d} \tau_{\nu}^{c}+\mathrm{d} \tau_{\nu}^{l}=\left(1+\eta_{\nu}\right) \mathrm{d} \tau_{\nu}^{c}$. From iZwaan $(1993)$.

Surface effects. The effects at the surface are more complicated. For each line the net cooling rate $(7 . \overline{3} 5)$ is given by

$$
\Phi_{u l}(z)=4 \pi \alpha_{\nu_{0}}^{l}(z)\left[S_{\nu_{0}}^{l}(z)-\bar{J}_{\nu_{0}}(z)\right] ;
$$

a similar expression holds per bound-free edge.

Whether these contributions are positive or negative depends on the sign of the $S_{\nu_{0}}^{l}-$ $\bar{J}_{\nu_{0}}$ split; its size is scaled by the extinction coefficient. Even in the case of LTE with $S_{\nu_{0}}^{l}=B_{\nu}$ such surface splits are present, with $J_{\nu}(0)>B_{\nu}(0)$ on the high-frequency side of the peak in the emergent flux spectrum and $J_{\nu}(0)<B_{\nu}(0)$ on the low-frequency side, due to the change in $B_{\nu}$ steepness for a given $T\left(\tau_{0}\right)$ relation as discussed on page $10 \overline{0} \overline{2}_{r}$

When the lines are coolers with $S_{\nu_{0}}>\bar{J}_{\nu_{0}}$ they contribute an excess of photons to the net outward flux. This must be compensated by a decrease of the photon production 
compared with the absence of lines to achieve flux constancy. Reversely, lines with $S_{\nu_{0}}<$ $\bar{J}_{\nu_{0}}$ contribute insufficiently. They must be compensated by increased production of other photons.

Strong LTE lines. For very strong lines with large $\eta_{\nu}$ the corresponding optical depth scaling has $\mathrm{d} \tau_{\nu}^{\text {tot }}=\mathrm{d} \tau_{\nu}^{c}+\mathrm{d} \tau_{\nu}^{l}=\left(1+\eta_{\nu}\right) \mathrm{d} \tau_{\nu}^{c}$ so that their Eddington-Barbier $\tau_{\nu}^{\text {tot }}=1$ depth is very shallow, at $\tau_{\nu}^{c}=1 /\left(1+\eta_{\nu}\right)$. This depth rescaling makes the Planck function gradient appear flat measured in $\tau_{\nu}^{\text {tot }}$, just as in $(4.90)$ on page $10 \overline{1}$. Their case is therefore like the isothermal one, in which $J_{\nu}$ drops down to $J_{\nu}(0)=(1 / 2) B_{\nu}$ for LTE. Strong LTE lines therefore contribute a positive value of $(7.6 \overline{6})$. They cool the surface. This is directly seen from the LTE-RE condition

$$
\int_{0}^{\infty} \kappa_{\nu}(0) J_{\nu}(0) \mathrm{d} \nu=\int_{0}^{\infty} \kappa_{\nu}(0) B_{\nu}(0) \mathrm{d} \nu
$$

in which the $J_{\nu}$ deficiencies due to lines on the lefthand side must be compensated by smaller $B_{\nu}(0)$ on the righthand side to achieve RE.

Physically, the reason for the surface cooling is that LTE lines loose photons from the thermal pool at the location of the last interaction, a photon-creation process by definition. The escaping photons take thermal energy away, adding that directly to the net flux. Fluxconstancy requires that fewer other photons should be created, requiring lower temperature than for the case without lines. Figures

Strong scattering lines. Strongly scattering strong lines also "feel" the atmosphere as isothermal. At the surface they therefore obey the $\sqrt{\varepsilon}$ law and have $J_{\nu_{0}}(0) \approx S_{\nu_{0}}(0) \approx$ $\sqrt{\varepsilon_{\nu_{0}}} B_{\nu_{0}}(0) \ll B_{\nu_{0}}(0)$ at any wavelength, from (14.80i) and (4.8. also for two-level scattering with complete redistribution, as mentioned above (4.105i) on page $1 \overline{1} 1 \overline{1} \hat{O}_{r}^{\prime}$ Their $J_{\nu_{0}}(0)$ drops to very low values, but they do not contribute much cooling because $S_{\nu}(0)$ drops along, with

$$
S_{\nu_{0}}^{l}(0)-\bar{J}_{\nu_{0}}(0) \approx \frac{\varepsilon_{\nu_{0}}}{1+\sqrt{\varepsilon_{\nu_{0}}}} B_{\nu_{0}} \approx \varepsilon_{\nu_{0}} B_{\nu_{0}}
$$

The cooling rate in a two-level-atom transition is

$$
\begin{aligned}
\Phi_{u l} & =4 \pi \alpha_{\nu_{0}}^{l}\left(S_{\nu_{0}}^{l}-\bar{J}_{\nu_{0}}\right) \\
& =4 \pi \alpha_{\nu_{0}}^{l}\left[\left(1-\varepsilon_{\nu_{0}}\right) \bar{J}_{\nu_{0}}+\varepsilon_{\nu_{0}} B_{\nu_{0}}-\bar{J}_{\nu_{0}}\right] \\
& =4 \pi \alpha_{\nu_{0}}^{l} \varepsilon_{\nu_{0}}\left(B_{\nu_{0}}-\bar{J}_{\nu_{0}}\right)
\end{aligned}
$$

and reduced by $\varepsilon_{\nu_{0}}$ compared to LTE cooling.

The physical reason is that the escaping flux is made up by photons that were taken out of the thermal pool in much deeper layers; the surface temperature has no knowledge of these scattered photons. Figure the influence of strong LTE and scattering lines on the RE temperature stratification.

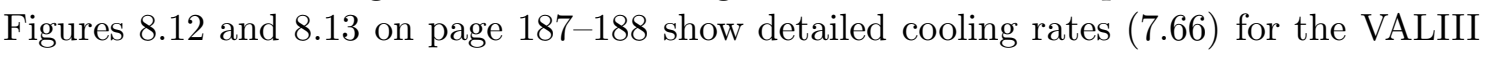
model of the solar atmosphere.

Scattering continua. Bound-free transitions have bound-bound character for the fixedenergy part below the ionization limit and free-free character for the kinetic energy apart 


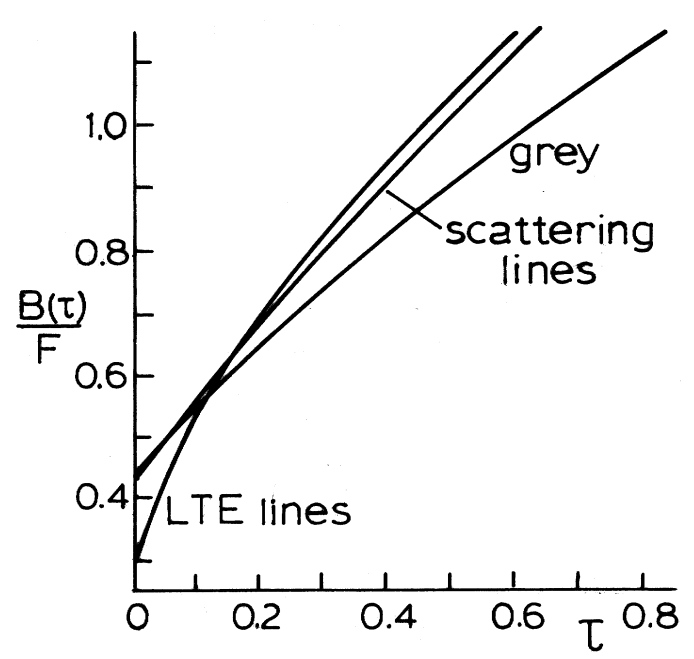

Figure 7.7: NLTE backwarming and surface cooling. Results from a schematic "picket fence" model computation. LTE and scattering lines cause similar backwarming, consisting of a slightly steeper gradient and a higher value at the depth where the continuum escapes than for the grey case. At the surface, strong LTE lines cause large cooling compared with the grey case, whereas the temperature is not affected by the presence of scattering lines. From ZZwaan (1993), who took it from 'Mihalas (1970),', who took it from 'Münch! $(1946)$

above the ionization limit. Bound-free transitions in which the fixed part is relatively small tend towards LTE process conditions with $S_{\nu}=B_{\nu}$. This is generally the case for $\mathrm{H}^{-}$ bound-free. It has $E_{\infty}-E_{1}=0.75 \mathrm{eV}$ or $\lambda_{\text {edge }}=1.65 \mu \mathrm{m}$ and such a wide edge profile for $\sigma_{1 c}(\nu)$ (with the maximum not at all at the edge but near $\lambda=800 \mathrm{~nm}$ ) that scattering-like behavior is negligible because there is no discrete memory effect.

At shorter wavelengths, the edges behave more as resonance lines, with complete redistribution over the edge but with a sufficiently narrow edge profile that resonance scattering may influence the source function. This is the case in the solar ultraviolet bound-free edges. Their collisional photon destruction probability is not very high because their edge energy $h \nu_{0}$ is large and a three-body collision is required (the factor $N_{\mathrm{e}}^{2}$ in (3.37it)). Their source function therefore follows $J_{\nu}$ and departs from $B_{\nu}$ well below their $\tau_{\nu}=1$ escape depth, just as in the case of a strongly scattering resonance line. However, unlike strong resonance lines, they have $S_{\nu}>B_{\nu}$ in the upper photosphere; see the mid-

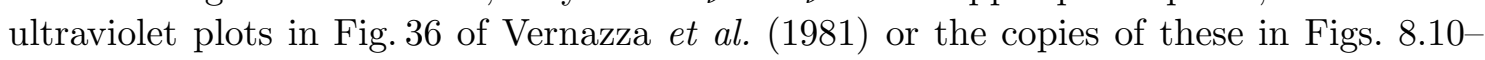

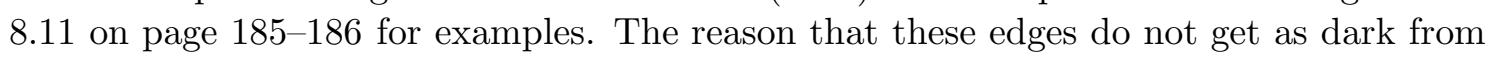
scattering as strong resonance lines tend to do is that the bound-free opacity increase at the edge is not as enormous, per unit of bandwidth, as in a strong resonance line ${ }_{-}^{1} T_{1}$. The $\tau_{\nu}$-scale compression that makes strong lines "see" the atmosphere as isothermal over their thermalization depth in $(4.90)$ on page $10 \overline{3}$, does not occur as effectively in bound-free edges.

\footnotetext{
${ }^{17}$ For example, the extinction increase at the solar H I Balmer edge in Figure $18.66^{\prime \prime}$ on page '179' is two orders of magnitude, but it vanishes in the total extinction which is dominated by the much larger $\mathrm{H}^{-}$ bound-free component. The solar H I Lyman edge (Figure $8.1 \overline{7}_{1}$ on page 194) has six orders of magnitude extinction increase but only 3 or so in the total extinction coefficient. The metal edges with $J_{\nu}>B_{\nu}$ in Figs. 8.10
} 


\subsection{Numerical modeling}

The discussion above makes clear that the computation of non-grey model atmospheres from the radiative equilibrium condition is not simple, especially when using actual NLTE radiation fields. The bound-free edges at short wavelengths cause surface heating while those at long wavelengths cause surface cooling; strong lines cause surface cooling when they have LTE source functions but no surface cooling or heating when they are scattering; the monochromatic optical depth scale may be sensitive to NLTE processes at widely different wavelengths, etcetera.

In principle, the numerical approach follows the methods and tricks treated in Chapter and frequencies that are important for the atmosphere must be solved together with the hydrostatic equilibrium equation, the $P_{\mathrm{g}}-P_{\mathrm{e}}-T$ conversion equations, and the radiative equilibrium (or convective equilibrium) equation. This is again done by rigorous linearization and solution in a giant Newton-Raphson iteration scheme comparable to the methods described in Section combined complete linearization with Feautrier-like solution methods to connect the outer and inner boundary conditions. At present the emphasis shifts to ALI methods, as has been the case for the simpler problem of line formation in a given atmosphere. Hübeny, and Lanz $(1995)$ describe the state of the art. Since the basic tricks have already been treated in Chapter section treats some examples.

\subsubsection{LTE-RE modeling of cool stars}

Sample models. A review of photosphere modeling for cool stars is given by iGustaftafs 'son and Jørgensen $(\overline{1} 9 \overline{9} 4)$ '. Such modeling is nearly always based on LTE and radiative equilibrium. Figure 7.8 shows state-of-the-art LTE-RE models for cool-star atmospheres. The upper part shows the temperature stratifications. Actually, convection is taken into account using mixing-length theory (with $l / H_{\mathrm{P}}=1.5$ ) so that these are flux-constant models rather than RE models. Convection affects their deeper layers; radiative equilibrium holds in the upper layers.

The line blanketing was treated with the LTE ODF approximation discussed below. The metallicity affects the temperature stratification in the deepest layers for the cooler stars, especially for dwarfs where the line opacities influence the convection. The temperature gradient flattens in the upper layers of the warmer stars (lower panels upper part) for low metallicity (dashed curves), because these become transparent for the bulk of the flux at low line opacity so that radiative equilibrium requires constant temperature.

The corresponding electron densities are plotted in the lower part of Figure 7.8. giants have much lower densities above $\tau_{500}=1$ than the dwarfs. The electron density at a given optical depth goes down appreciably with metallicity for the cool stars (upper panels at right), up to two order of magnitudes as predicted by (7.

Line haze. For solar-type stars, the enormous quantity of spectral lines in the nearultraviolet has not been modeled properly yet. The line crowding (especially from Fe I for $\lambda=300-400 \mathrm{~nm}$ and Fe II for $\lambda=200-300 \mathrm{~nm}$ ) is so large that the millions of lines represent a quasi-continuous source of extinction called the line haze. It is hard to treat them in detail even if one adopts LTE - which is bound to be wrong since the photon 

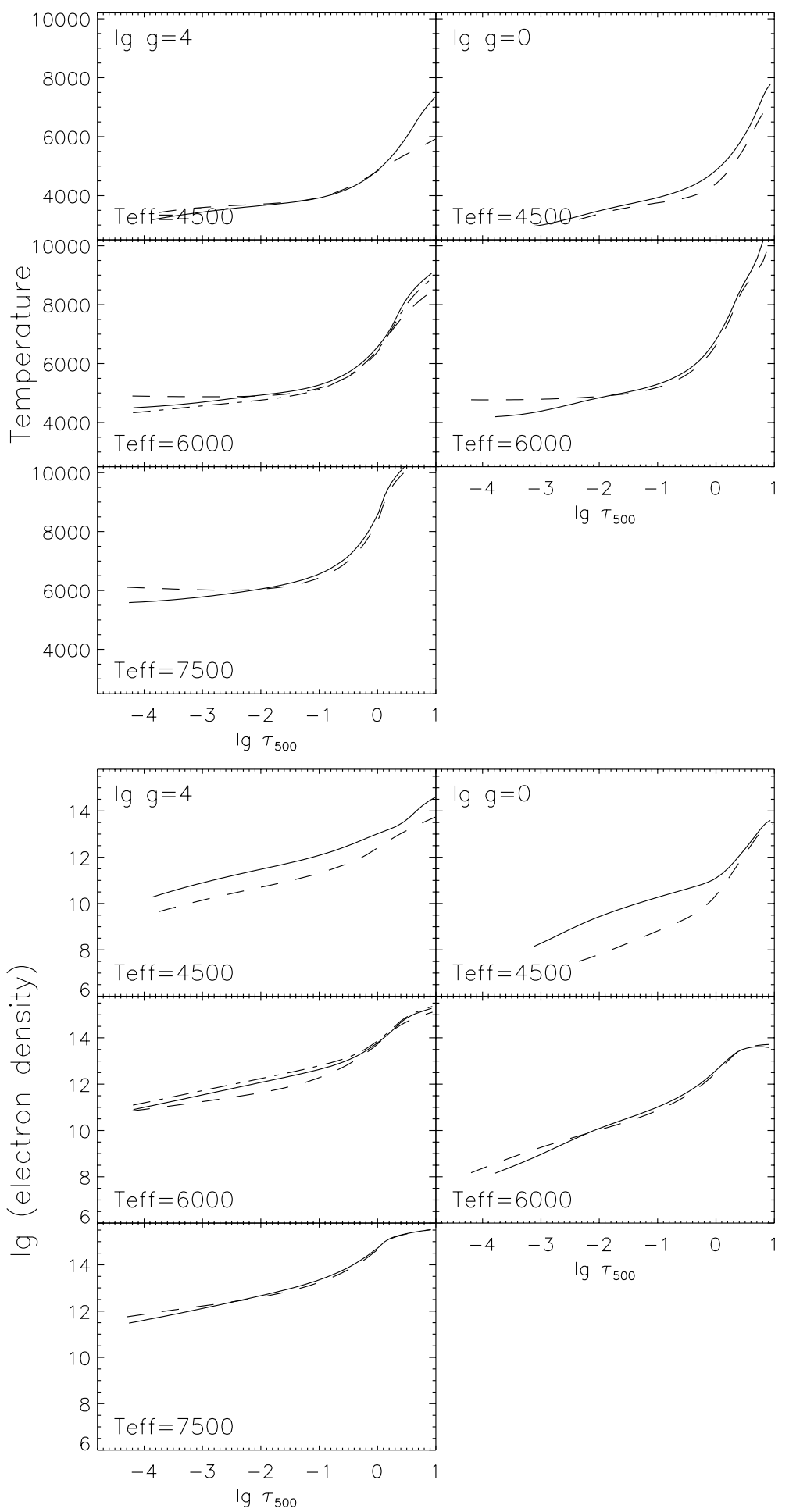

Figure 7.8: Flux-constant models for late-type stars_computed with the Uppsala MARCS code of 'Gustafs-1

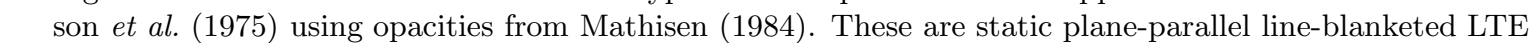
models. The upper part shows the temperature stratifications. the lower part displays the electron density stratifications (electrons $\mathrm{cm}^{-3}$ ) on logarithmic scales. The $\mathrm{x}$ axes measure continuum optical depth at $\lambda=500 \mathrm{~nm}$. The effective temperature $T_{\text {eff }}$ is specified per panel. The two columns are for different surface gravity $\lg g \equiv \log g$, with $\log g=4$ describing dwarfs and $\log g=0$ giants. Solid curves: solar abundances with metallicity $[\mathrm{Fe} / \mathrm{H}]=0$ (defined by $\left(\begin{array}{l}19.4) \\ -4\end{array}\right)$ on page 203i). Dashed curves: metal-poor stars with $[\mathrm{Fe} / \mathrm{H}]=-2$. The dot-dashed curve in the middle panels of the $\log g=4$ columns is a solar LTE-RE model for $T_{\text {eff }}=5780 \mathrm{~K}$ also shown in Figure if 3 on page 149. From iCarlsson et al. 

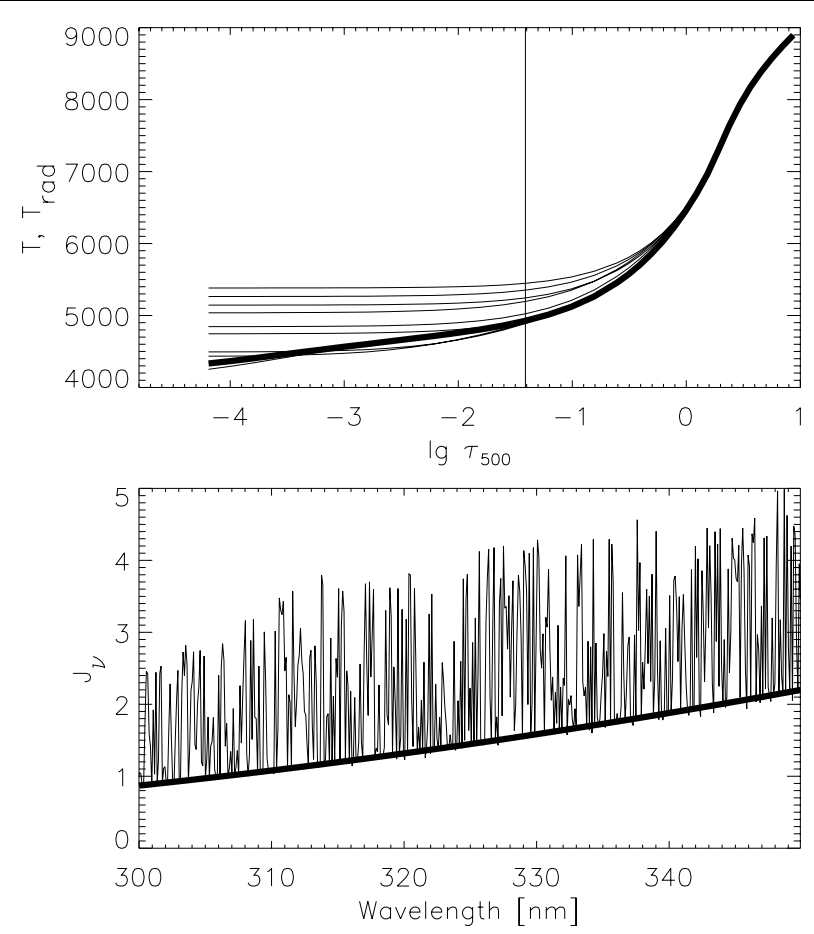

Figure 7.9: The solar near-ultraviolet line haze in LTE approximation. The upper panel shows $J_{\nu}$ against continuum optical depth at $\lambda=500 \mathrm{~nm}$ in the form of the corresponding brightness temperature $T_{\text {rad }}$ with $B_{\nu}\left(T_{\mathrm{rad}}\right) \equiv J_{\nu}$. The thick curve is the model temperature. The thin curves show $T_{\text {rad }}$ for a selection of wavelengths sampling the variation seen in the lower panel. The vertical line marks the depth for which the lower panel holds. It shows $J_{\nu}$ at that depth as a function of wavelength, in units $10^{6}$ erg $\mathrm{cm}_{-}^{-2} \mathrm{~s}_{-}^{-1} \mathrm{ster}^{-1} \mathrm{~Hz}^{-1}$. The thick boundary line is the Planck function at the same depth. From Carlsson et al. $(1994)$.

energy is $3-5 \mathrm{eV}$ at ultraviolet wavelengths and exceeds the mean kinetic energy of $1-2 \mathrm{eV}$.

For LTE the source function simply has $S_{\nu}=B_{\nu}$ and the problem is simplified to adding all the line opacities into the modeling. This is done by Opacity Sampling (OS) or Opacity Distribution Functions (ODF). In the first technique the lines are sampled in say $10^{4}$ frequency points. In the second, the actual rapid variations of $\kappa_{\nu}$ with $\nu$ are redistributed into bins of similar $\kappa_{\nu}$ across a not too wide wavelength interval, and the bins are then used instead. Tables containing many millions of lines are used in these procedures. For cooler stars, molecule formation enhances these problems.

Figure $\overline{7} \bar{g}_{1}$ shows an example of the solar line haze, computed assuming LTE and using opacity sampling. The upper panel shows that $J_{\nu}>B_{\nu}$ in the near-ultraviolet even for LTE conditions, again due to the $\boldsymbol{\Lambda}$ operator properties in Figure $\overline{4} . \overline{4}$ on page $\overline{8} \overline{2} \overline{3}$ and the relatively steep slope of the Planck function in the ultraviolet for a given temperature gradient. The lower panel shows the corresponding near-ultraviolet spectrum of $J_{\nu}$ at the location of the vertical line in the upper panel. This is the actual near-ultraviolet radiation field "seen" by bound-free transitions in this wavelength region. The LTE assumption causes the sharp lower boundary at $J_{\nu} \approx S_{\nu} \equiv B_{\nu}$. It is undoubtedly an artifact. NLTE resonance scattering causes much darker line cores, with $J_{\nu} \approx S_{\nu} \ll B_{\nu}$. Other NLTE effects arise from cross-talk between transitions with common upper levels (interlocking). They include photon pumping which may cause $S_{\nu} \approx J_{\nu}>B_{\nu}$. Sophisticated attempts to model the solar line haze without assuming LTE have been made by Anderson (1 $198 \overline{8} \overline{5}^{\prime}$, 


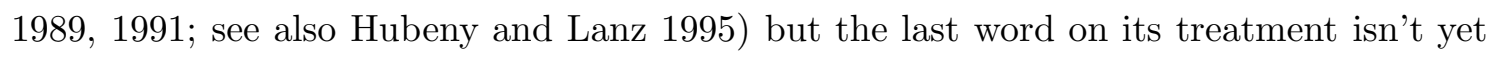
in.

Worse, these broad-band line formation problems need to be also addressed in 3D time-dependent radiation hydrodynamics code where solving radiative transfer can easily eat up huge amounts of computing power. Usually such codes assume LTE and sample the whole spectrum in only a few wavelength points and directions. A better technique in which opacities are grouped with a scattering term in the source function is discussed by Skartien $(2000)$ !'
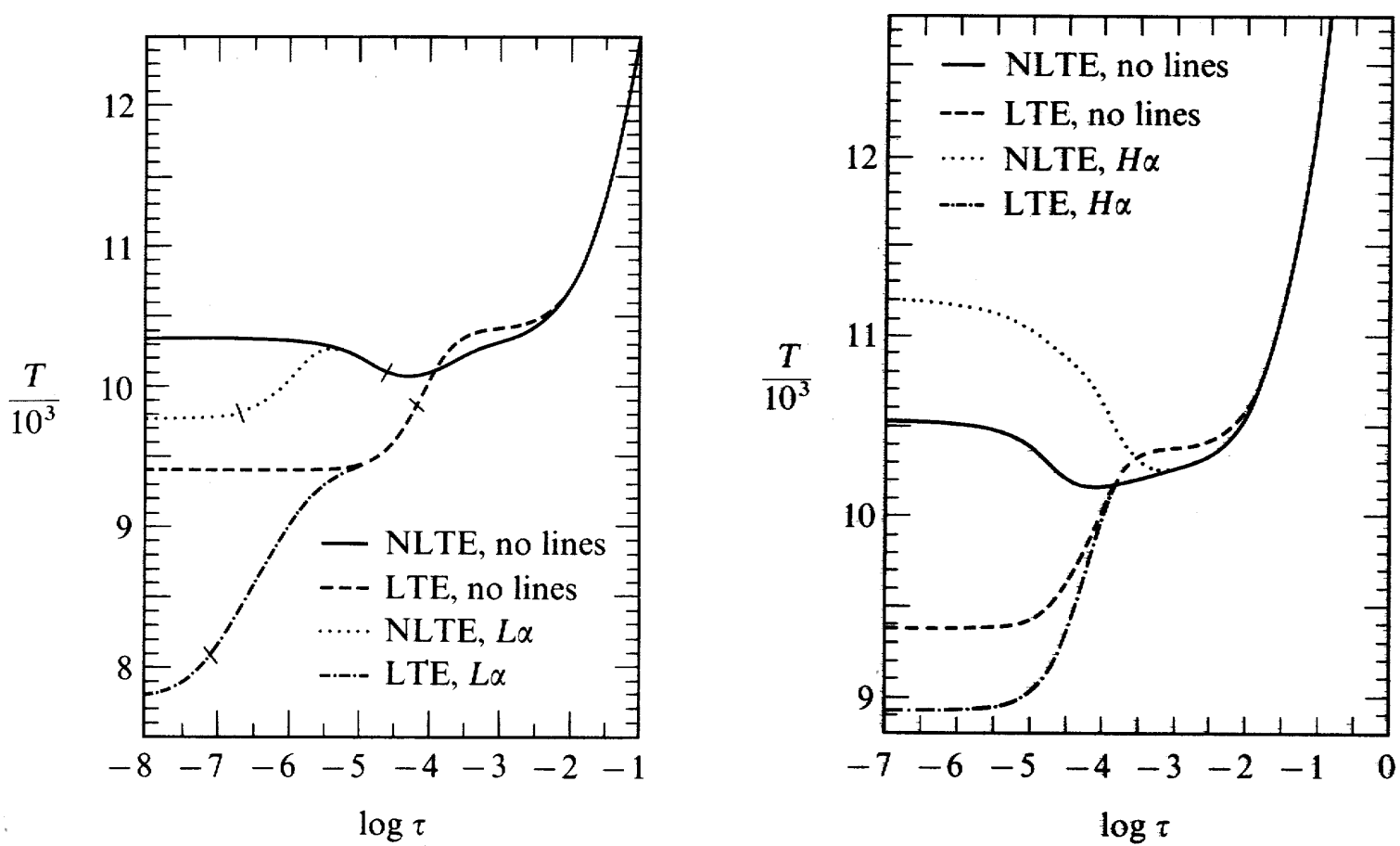

Figure 7.10: NLTE-RE modeling for a star with $T_{\text {eff }}=15000 \mathrm{~K}$ and $\log g=4$ by Auer and Mihalas. The $\mathrm{x}$ axes specify continuum optical depth at $\lambda=400 \mathrm{~nm}$. Left: temperature stratifications for LTE and NLTE modeling with and without H I Lyman- $\alpha$. "No lines" means no Lyman- $\alpha$, which is the only line in the two-level plus continuum H I model atom. The tick marks indicate $\tau_{\nu}=1$ locations for the center of Lyman- $\alpha$ (outer ones) and the Lyman continuum (inner ones). Right: temperature stratifications for LTE and NLTE modeling with and without H I Balmer- $\alpha$, using a three-level plus continuum H I model atom with the 3-2 Balmer- $\alpha$ line as the only bound-bound transition. In this case "no lines" means no Balmer- $\alpha$. From iMihalas (1970)!'

\subsubsection{NLTE-RE modeling of hot stars}

The task is somewhat less daunting for hot stars because their atmospheres are dominated by hydrogen radiative transfer, with a sniff of helium. Their spectra contain far fewer lines, mainly of hydrogen and helium, and the electrons come from hydrogen ionization rather than metal ionization as in $(\overline{7} . \overline{8})$ on page $1 \overline{1} \overline{4} \overline{1}$.

Hot-star NLTE-RE modeling therefore simplifies in first order down to H I NLTE modeling in conjunction with the HE-RE conditions. The hydrogen atom is well known and relatively simple; a few levels and lines suffice to treat its influence on the atmosphere. This was done in the early days of numerical NLTE modeling in classic papers 


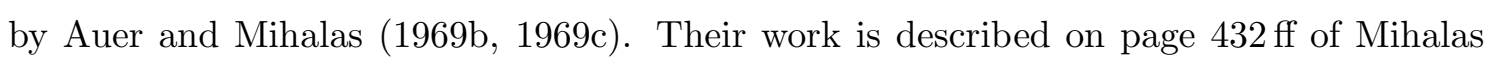
- _ (1970). Newer results, using opacity distribution functions in which many levels and lines are grouped together in "superlevels" and "superlines", are given by "Hubeny and Lanz - $(\overline{1} \overline{9} \overline{9} \overline{5})$ I I describe some key Auer-Mihalas results here to illustrate the effects of NLTE line formation on atmospheric structure. More detail is found in Exercise 112 2. on page 23

Two-level atom with Lyman alpha. The lefthand panel of Figure i7.10 shows AuerMihalas results for a two-level plus continuum hydrogen model atom, with and without Lyman- $\alpha$. In each case, the surface temperature is well below the grey-model prediction

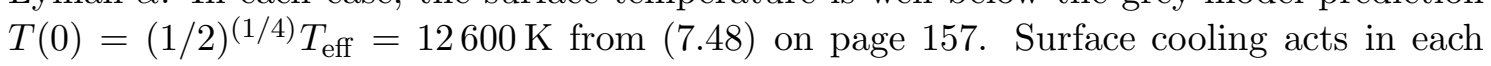
computation.

The dashed curve is for LTE without Lyman- $\alpha$. The downward slope from $\log \tau=-3$ to $\log \tau=-5$, around the $\tau_{\mathrm{LyC}}=1$ location in the Lyman continuum, results from the strong surface cooling in the $n=1-\infty$ Lyman continuum. When the condition of LTE is relaxed (solid curve), the strong scattering in this continuum results in slightly stronger initial cooling around $\log \tau=-3$, where the scattering photons are created, and much less cooling in higher layers because the extinction is from scattering without coupling to the temperature.

The solid curve even shows a slight outward rise which is due to absorption in the $n=2-\infty$ Balmer continuum. The Balmer continuum (edge at $\lambda=364.6 \mathrm{~nm}$ ) escapes near $\log \tau=-1.4$ (the scale is for $\lambda=400 \mathrm{~nm}$, just longward of the Balmer edge) and irradiates the layers around $\log \tau=-5$ from below with $J_{\nu}>B_{\nu}$. In order to maintain radiative equilibrium, the absorption and corresponding overionization are compensated through a higher temperature, giving more radiative recombination. The effect is the same as the backwarming from spectral lines: the additional extinction enforces a higher temperature. It is the reverse of surface cooling because the absorption feeds on radiation from below instead of adding to $\mathrm{it}_{1}^{118{ }^{\prime}}$.

When Lyman- $\alpha$ is switched on it causes large surface cooling in the LTE case around its $\tau_{\mathrm{Ly} \alpha}=1$ formation height (split between dot-dashed and dashed curves). It also cools the atmosphere when scattering is taken into account (split between dotted and solid curves), but much less than for the LTE case.

Three-level atom with Balmer alpha. The righthand panel of Figure results for a three-level plus continuum hydrogen model atom, now with and without the 3-2 Balmer- $\alpha$ line as the only bound-bound transition. 'Auer and Mihalas $(\overline{1} \overline{6} \overline{6} \bar{c})$ 'd did not include the 2-1 Lyman- $\alpha$ line and the 3-1 Lyman- $\beta$ line in this analysis because these are in detailed balance (net radiative rate zero) at the height where Balmer- $\alpha$ influences the atmosphere. The curves in Figure lefthand panel for the Lyman- $\alpha$ curves $(\log \tau<-5)$.

The no-line LTE curve (dashed) is the same as in the lefthand panel. In the no-line NLTE case (solid) the boundary temperature is slightly higher because the heating in the Balmer continuum is enhanced by the addition of some Paschen extinction.

Switching on the Balmer- $\alpha$ line in LTE (dot-dashed curve) produces surface cooling, similarly to the effect of switching on Lyman- $\alpha$ in LTE at left. The bound-bound photon losses lower the temperature appreciably. The effect occurs deeper for Balmer- $\alpha$ because

\footnotetext{
${ }^{18}$ Such temperature rise is called the "Cayrel effect" after 'Cayrel (1963)!' In the extreme case, the electron temperature in the outer atmosphere rises to the color temperature of the photospheric radiation.
} 
this line is weaker, resulting in a steeper gradient. The temperatures level off to constant $J_{\nu}$ in the line for $\log \tau<-5$.

However, the NLTE effect of Balmer- $\alpha$ (dotted curve) is the reverse of the effect of Lyman- $\alpha$ in the lefthand panel. Balmer- $\alpha$ heats the upper atmosphere considerably, compared with the NLTE no-line case; the boundary temperature increases from $10500 \mathrm{~K}$ to $11200 \mathrm{~K}$. The line is strong and a net radiative cooler with $J_{\nu}<S_{\nu}$. How can it cause heating?

It is caused by the strong photon losses in Balmer- $\alpha$. These deplete the $n=3$ level, feeding electrons into $n=2$. The resulting overpopulation of this level (about a factor 2) greatly enhances the Balmer continuum heating since the heating contribution to the flux divergence integral scales with the $n=2$ overpopulation as shown in $(\overline{7} . \overline{3} 7)$ on page $1 \overline{1} \overline{5} \overline{6}$. Amazingly, the photon losses in the subordinate Balmer- $\alpha$ line, located in the low-energy red part of the spectrum, cause heating of the whole outer atmosphere of this hot star. 


\section{Chapter 8}

\section{Continua from Plane-Parallel Stars}

$\mathrm{T}$ his chapter discusses the continuous spectra that emerge from classical stellar atmospheres, divided between the Sun, a solar-like paradigm star called VALIII, and non-solar-like stars. The presentation is mostly graphical, marking a transition from the more formal treatment in the previous chapters to discussion of real stars (although mostly computationally existing ones). The exercises on page $2 \overline{3} \overline{1} \overline{1}, \mathrm{ff}$ are intended to assist in graph interpretation.

\subsection{Solar continua}

Observations. Figure $\overline{8}$ in gives an overview of the continuous solar spectrum. Details are given in Figs. 8.28 The wavelengths of the major spectral features are listed in Table $\overline{1} \cdot \overline{1}$.

Continuous extinction. The basic continuum processes that take place in the solar atmosphere are:

- Free-free transitions. See Gray Chapter 8, Rybicki \& Lightman $\S 5.2-5.3$. H I free-free is important at long wavelengths ( $\mathrm{mm}$ to $\mathrm{m}$ ) in solar-type stars. At these wavelengths the radiation comes from the chromosphere, transition region and corona, above the height of the classical temperature minimum so that the rising $T_{\mathrm{e}}$ and low density reduce the amount of $\mathrm{H}^{-}$extinction and emission by "ionization" to $\mathrm{HI}$. $\mathrm{H}^{-}$free-free dominates the infrared continuum from $1.6 \mu \mathrm{m}$ to $160 \mu \mathrm{m}$. See also page $1 \overline{1} \bar{L} \overline{8} \mathrm{~g}$.

- Bound-free transitions. Gray Chapter 8, Rybicki \& Lightman p. $282 \mathrm{ff} . \mathrm{H}^{-}$boundfree peaks in the visible. The metals Al I, Mg I, Si I, C I and Fe I cause a sequence of edges in the near to middle ultraviolet. The H I and He I Lyman continua dominate the extinction/emission at wavelengths below their edges. In the X-ray regime, the edges of highly ionized metal ions contribute continuum extinction/emission.

- Cyclotron radiation, synchrotron radiation, plasma radiation. Only at the far ends of the spectrum, from flares and other activity. Rybicki \& Lightman.

- Thomson scattering. Gray p. 139 ff, Rybicki \& Lightman $\S 3.4$. It causes the solar $\mathrm{K}$ corona. 


\section{THE SOLAR SPECTRUM}

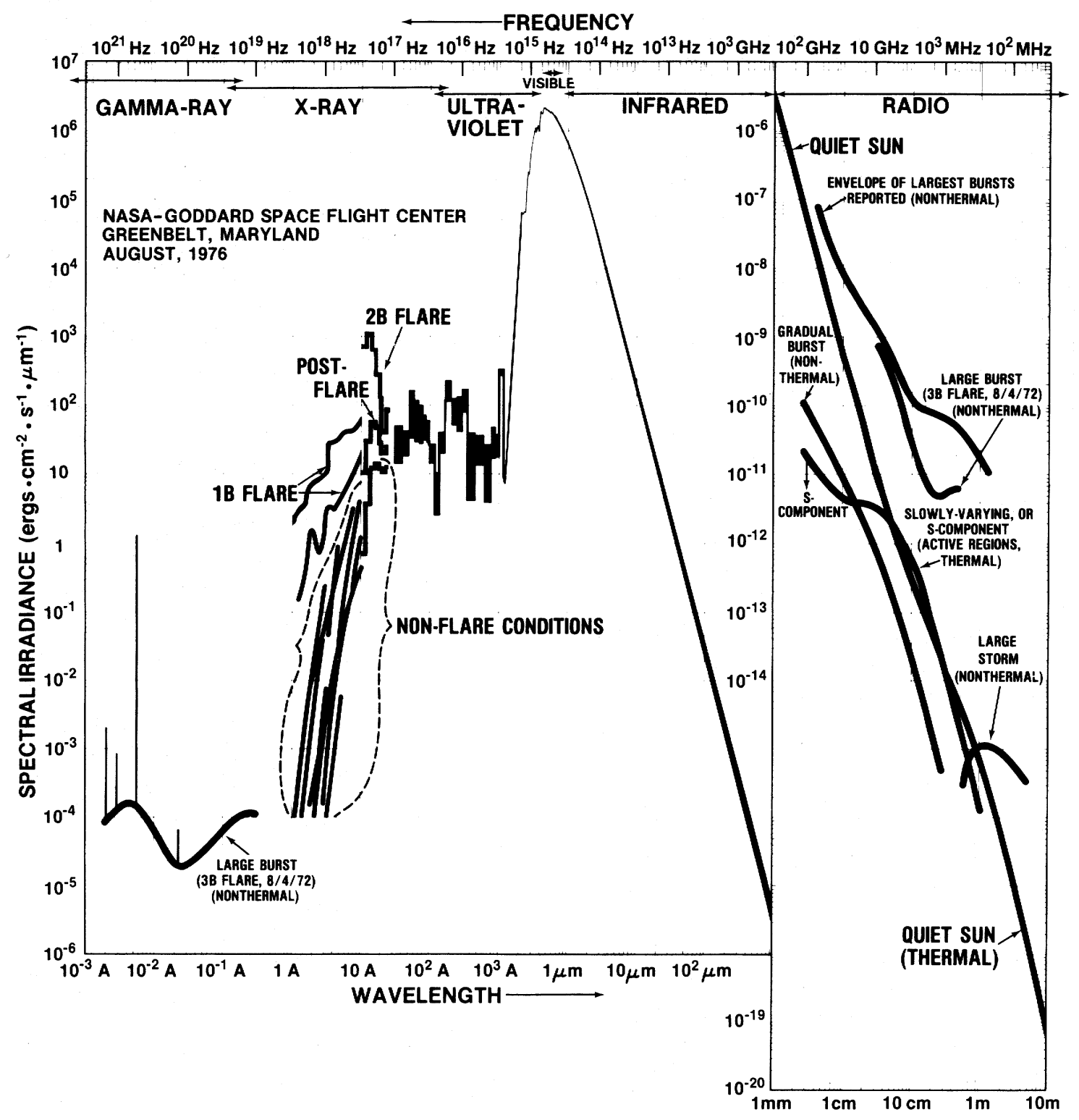

Figure 8.1: The solar irradiance spectrum, compiled by Harriet H. Malison from data in iWhite $(\overline{1} 9 \overline{7}) \bar{i}$ The peak in the visible is stable to within $10^{-3}$ with regards to solar activity modulation and $10^{-6^{-}}$with regards to solar oscillations. The outer ends of the flux distribution are highly_sensitive to activity which produces nonthermal cyclotron and synchrotron radiation. From Rutten and Cram (1981)! 


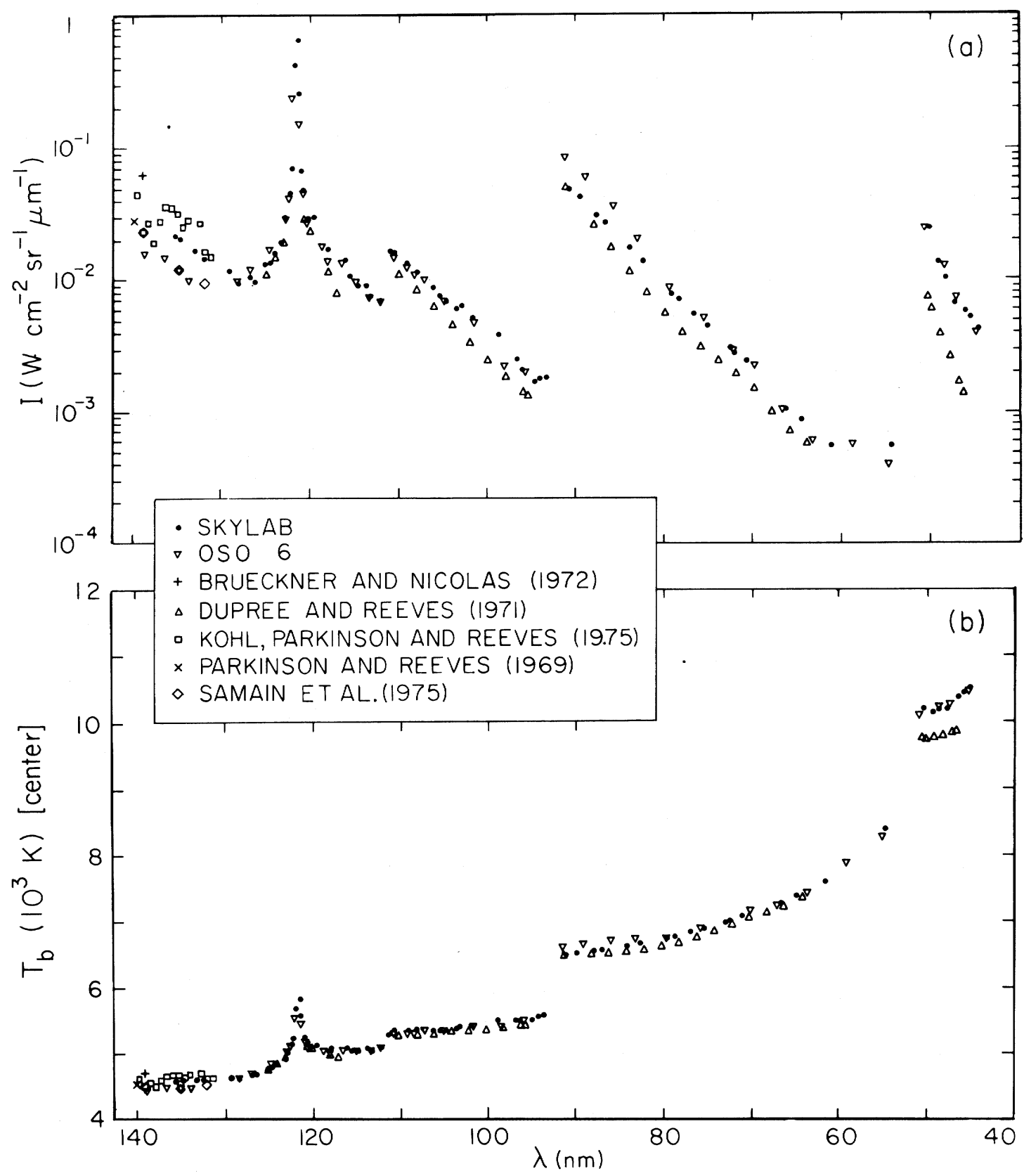

Figure 8.2: Top: observed solar disk-center continuum intensities in the far ultraviolet. Bottom: corresponding brightness temperatures. Note that the wavelength scale along the $\mathrm{x}$ axis in this figure and in the following figures is reversed. The data points represent continuum "windows" between the many emission lines in this part of the spectrum; the only line shown is Ly $\alpha$ at $\lambda=121.6 \mathrm{~nm}$. Bound-free threshold wavelengths: H I Lyman continuum (1c) at $91.2 \mathrm{~nm}$, He I at $50.4 \mathrm{~nm}$, C I at $110 \mathrm{~nm}$, Si I at $131.8 \mathrm{~nm}$ as

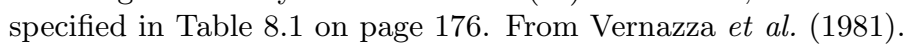



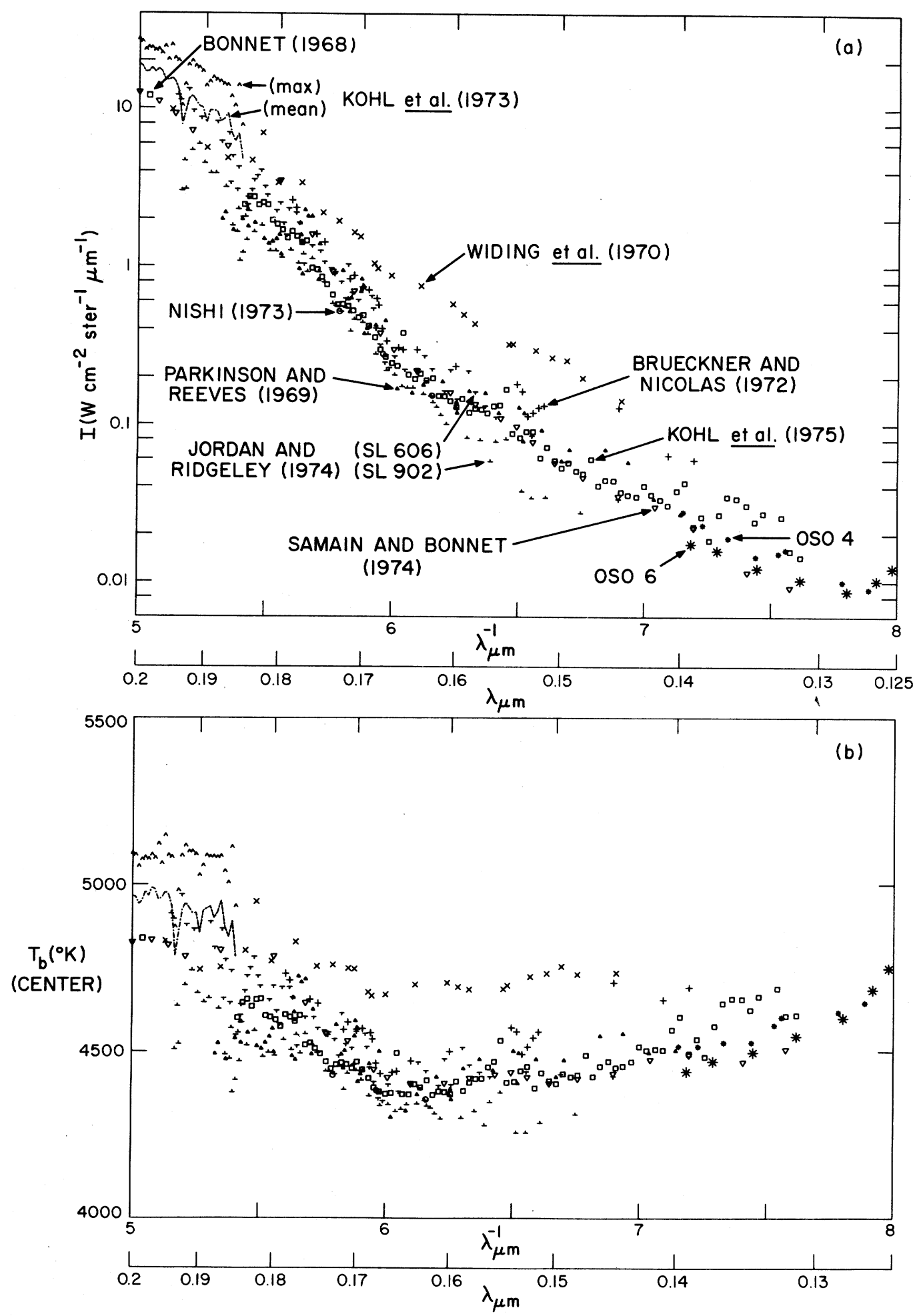

Figure 8.3: Top: observed solar disk-center continuum intensities in the mid ultraviolet. Bottom: corresponding brightness temperatures. The data points are for continuum windows between lines. Bound-free thresholds in this region: CI 144.4nm, Si I $152.5 \mathrm{~nm}$, FeI $157.5 \mathrm{~nm}, \mathrm{Mg}$ I $162.2 \mathrm{~nm}$, Si I $168.2 \mathrm{~nm}$, Fe I $176.8 \mathrm{~nm}$, Si I $198.6 \mathrm{~nm}$. From ivernazza et al. $(1976)_{1}^{\prime}$. 

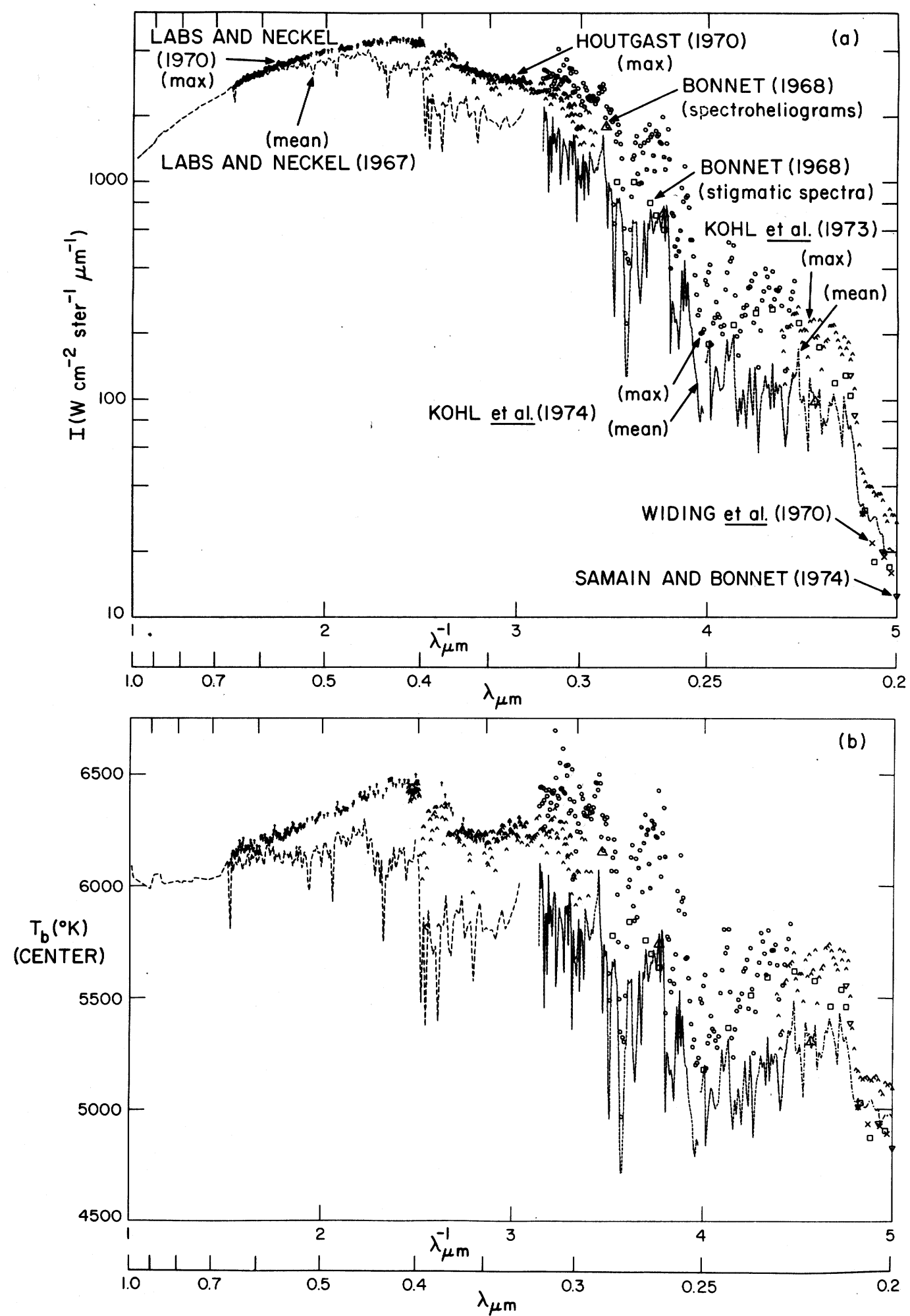

Figure 8.4: Top: observed solar disk-center intensities in the near ultraviolet, visible and near infrared. The values labeled $(\max )$ are for the highest points between lines; (mean) denotes averages including lines. Bottom: corresponding brightness temperatures. Bound-free thresholds in this region: Al I $207.6 \mathrm{~nm}, \mathrm{Mg} I$ 251_4 nm, HI_ 364.7_nm. Note that the solar spectrum does not show a distinct Balmer jump. From 'Vernazza et al. $(1976)_{\mathrm{r}}^{\prime}$ 


\begin{tabular}{|c|c|c|c|c|c|c|c|c|}
\hline \multirow[t]{9}{*}{$\mathrm{HI}$} & $1-2 \operatorname{Ly} \alpha$ & $121.5 \mathrm{~nm}$ & $\mathrm{Li} \mathrm{I}$ & $1-2$ & $670.7 \mathrm{~nm}$ & $\mathrm{HI}$ & $1-\mathrm{c}$ & $91.2 \mathrm{~nm}$ \\
\hline & $1-3$ Ly $\beta$ & $102.6 \mathrm{~nm}$ & C I & $1-5$ & $165.5 \mathrm{~nm}$ & & $2-\mathrm{c}$ & $364.6 \mathrm{~nm}$ \\
\hline & $1-4 \operatorname{Ly} \gamma$ & $97.2 \mathrm{~nm}$ & & $1-7$ & $155.9 \mathrm{~nm}$ & & $3-\mathrm{c}$ & $820.4 \mathrm{~nm}$ \\
\hline & $2-3 \mathrm{H} \alpha$ & $656.3 \mathrm{~nm}$ & & $2-6$ & $193.0 \mathrm{~nm}$ & & $4-\mathrm{c}$ & $1.458 \mu \mathrm{m}$ \\
\hline & $2-4 \mathrm{H} \beta$ & $486.1 \mathrm{~nm}$ & C II & $1-2$ & $133.5 \mathrm{~nm}$ & & $5-\mathrm{c}$ & $2.279 \mu \mathrm{m}$ \\
\hline & $2-5 \mathrm{H} \gamma$ & $434.0 \mathrm{~nm}$ & $\mathrm{OI}$ & $1-5$ & $130.2 \mathrm{~nm}$ & $\mathrm{HeI}$ & $1-\mathrm{c}$ & $50.4 \mathrm{~nm}$ \\
\hline & $3-4 \operatorname{Pa} \alpha$ & $1.875 \mu \mathrm{m}$ & $\mathrm{NaI}$ & $1-2 \mathrm{D}_{2}$ & $589.0 \mathrm{~nm}$ & He II & $1-\mathrm{c}$ & $22.8 \mathrm{~nm}$ \\
\hline & $4-5 \operatorname{Br} \alpha$ & $4.051 \mu \mathrm{m}$ & & $1-2 \mathrm{D}_{1}$ & $589.6 \mathrm{~nm}$ & & $2-\mathrm{c}$ & $91.1 \mathrm{~nm}$ \\
\hline & $5-6 \operatorname{Pf} \alpha$ & $7.458 \mu \mathrm{m}$ & $\mathrm{Mg} \mathrm{I}$ & $1-2$ & $285.2 \mathrm{~nm}$ & & $3-\mathrm{c}$ & $205.0 \mathrm{~nm}$ \\
\hline \multirow[t]{3}{*}{$\mathrm{He} \mathrm{I}$} & $1-2$ & $58.4 \mathrm{~nm}$ & & $2-4 b_{1}$ & $518.4 \mathrm{~nm}$ & & $4-\mathrm{c}$ & $364.4 \mathrm{~nm}$ \\
\hline & $2 s-2 p$ & $1.083 \mu \mathrm{m}$ & & $2-4 b-2$ & $517.3 \mathrm{~nm}$ & & $5-\mathrm{c}$ & $569.4 \mathrm{~nm}$ \\
\hline & $2 s-3 p$ & $501.5 \mathrm{~nm}$ & & $2-4 b_{3}$ & $516.7 \mathrm{~nm}$ & C I & $1-\mathrm{c}$ & $109.8 \mathrm{~nm}$ \\
\hline \multirow[t]{10}{*}{ He II } & $1-2$ & $30.3 \mathrm{~nm}$ & Mg II & $1-2 \mathrm{k}$ & $279.5 \mathrm{~nm}$ & & $2-\mathrm{c}$ & $123.9 \mathrm{~nm}$ \\
\hline & $1-3$ & $25.6 \mathrm{~nm}$ & & $1-2 \mathrm{~h}$ & $280.3 \mathrm{~nm}$ & & $3-\mathrm{c}$ & $144.4 \mathrm{~nm}$ \\
\hline & $1-4$ & $24.3 \mathrm{~nm}$ & K I & $1-2$ & $766.5 \mathrm{~nm}$ & Mg I & $1-\mathrm{c}$ & $162.2 \mathrm{~nm}$ \\
\hline & $2-3$ & $164.0 \mathrm{~nm}$ & & $1-2$ & $769.9 \mathrm{~nm}$ & & $2-\mathrm{c}$ & $251.4 \mathrm{~nm}$ \\
\hline & $2-4$ & $121.5 \mathrm{~nm}$ & $\mathrm{CaI}$ & $1-5$ & $422.7 \mathrm{~nm}$ & $\mathrm{Al} \mathrm{I}$ & $1-\mathrm{c}$ & $207.6 \mathrm{~nm}$ \\
\hline & $2-5$ & $102.5 \mathrm{~nm}$ & $\mathrm{Ca} I I$ & $1-3 \mathrm{~K}$ & $393.4 \mathrm{~nm}$ & Si I & $1-\mathrm{c}$ & $152.5 \mathrm{~nm}$ \\
\hline & $3-4$ & $468.6 \mathrm{~nm}$ & & $1-3 \mathrm{H}$ & $396.9 \mathrm{~nm}$ & & $2-\mathrm{c}$ & $168.2 \mathrm{~nm}$ \\
\hline & $3-5$ & $320.3 \mathrm{~nm}$ & & $2-3 \mathrm{IR}$ & $854.2 \mathrm{~nm}$ & & $3-\mathrm{c}$ & $198.6 \mathrm{~nm}$ \\
\hline & $4-5$ & $1.012 \mu \mathrm{m}$ & & $2-3$ IR & $866.2 \mathrm{~nm}$ & $\mathrm{FeI}$ & $1-\mathrm{c}$ & $157.0 \mathrm{~nm}$ \\
\hline & $4-6$ & $656.0 \mathrm{~nm}$ & & $2-3$ IR & $849.8 \mathrm{~nm}$ & & $2-\mathrm{c}$ & $176.1 \mathrm{~nm}$ \\
\hline
\end{tabular}

Table 8.1: Selected spectral features. Left: line-center wavelengths for bound-bound transitions of hydrogen and helium. Middle: bound-bound wavelengths for some other spectra. Right: threshold wavelengths of bound-free transitions. The H I and He II multiplets (left) have unsplit wavelengths. For example, $\mathrm{H} \alpha$ has three degenerate components at $\lambda=656.3 \mathrm{~nm}$ (plus many more Stark components that seperate in the presence of an electric field). For the non-hydrogenic species, the transitions are identified in a simple level numbering scheme, from the bottom up and without multiplet splitting. Mostly taken from 'Állenı' i. (1976)', and Vernazza et al. $(1976,1981)$. Much more complete tabulations are found in the partial (but highly useful) Grotrian diagrams of Moore and Merrill (1968)', in the more complete Grotrian diagrams of 'Bashkin and Stoner $(1975)$ ', in Charlotte Moore's monumental tabulations (Moore 1949, 1952, 1958, 1959,

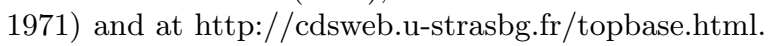

- Rayleigh scattering. Important in sunspot umbrae which are cool enough to contain many molecules. Also appreciable in the near ultraviolet due to the $\nu^{4}$ dependence of the cross-section, especially in cool components of the chromosphere where hydrogen is not ionized.

- Line haze (Gray p. 142-143). Lines are formally not a source of continuum extinction/emission, but in practice they represent one in the solar violet and ultraviolet where the line haze is so crowded that it acts as quasi-continuous extinction (page !1 $1 \overline{6} \overline{6} \overline{4})$.

Vitense diagram. Some of the solar extinction providers are evaluated in Figure 8.6, , a classic confusogram copied from Novotny $(1 \overline{9} \overline{7} \overline{3}) \bar{i}$; who took it from the standard paper by 


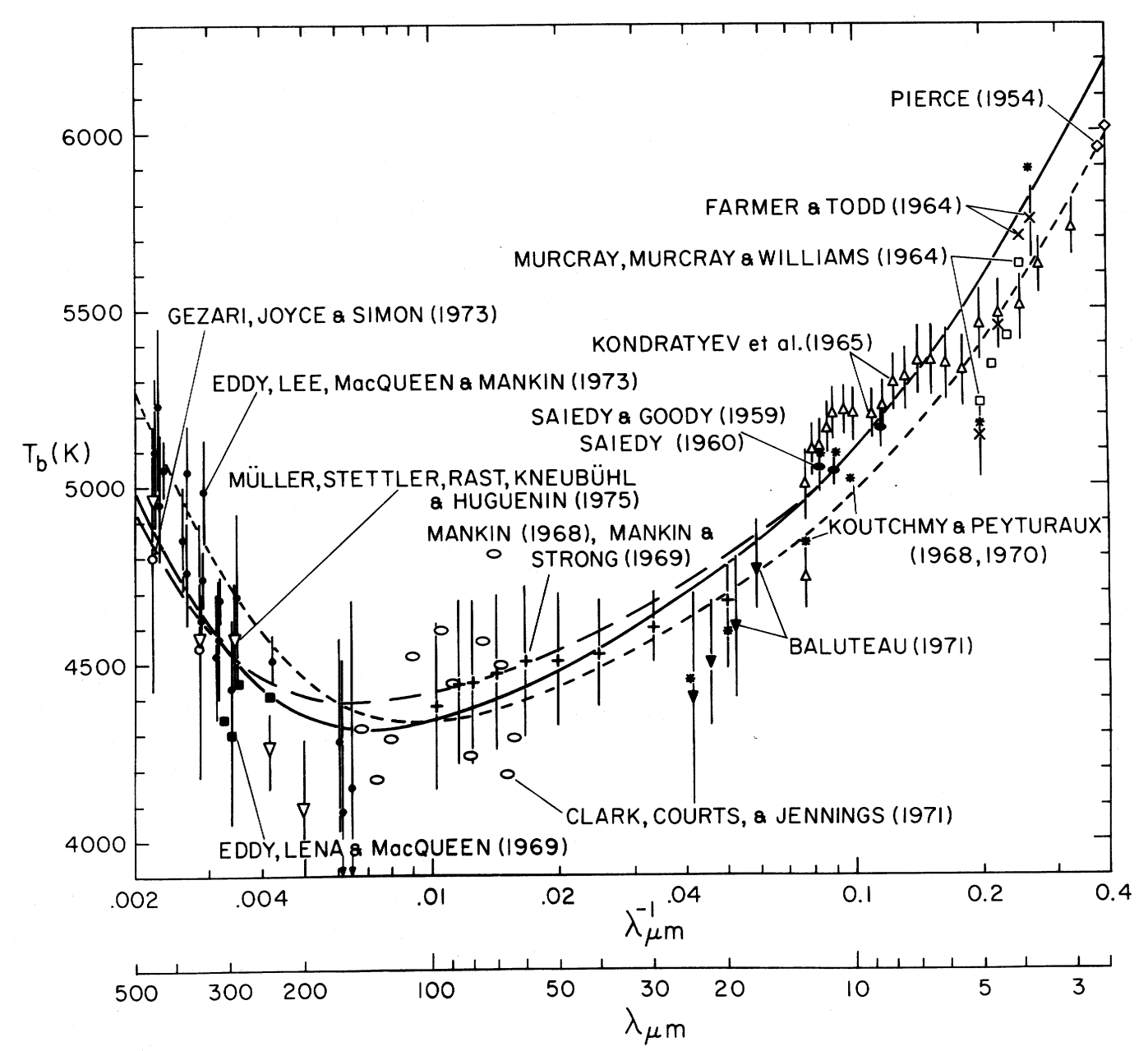

Figure 8.5: Observed solar intensity and flux $F$ in the infrared, plotted as brightness temperature. No distinction has been made between disk-center intensity and full-disk flux (disk-averaged intensity, $F_{\lambda}=\bar{I}_{\lambda}$ ) because the scatter between different measurements is larger than the intensity-flux difference. Some of the measurements are from mountain tops, others from balloon platforms. The curves are model predictions. From 'Vernazza et al. $(1976)^{\prime}$

Vitense $(1951)$ : Both publications contain similar plots for other temperatures, of which a few are shown in Figure 8.15 .18 on page $19 \overline{2} 2 \mathrm{ff}$. They specify continuous extinction coefficients for solar-like chemical composition (given in Novotny's Table 3-7), a range of temperatures (different plots) and a range of electron pressures $P_{\mathrm{e}}$ (different curves per plot). The Vitense plot in Figure $\overline{8} \cdot \bar{\prime}$ is for stellar atmospheres with solar effective temperature, from dwarf (large pressure) to giants (low pressure).

The function $G$ is the Rosseland weighting function defined by

$$
G \equiv \frac{\mathrm{d} B_{\nu} / \mathrm{d} T}{\mathrm{~d} B / \mathrm{d} T}
$$

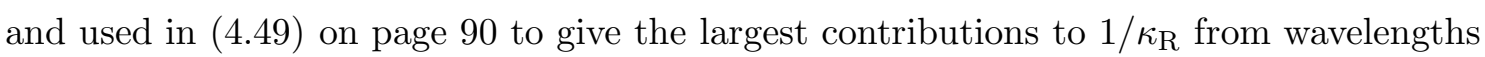
where the extinction is smallest and $G$ largest. The function $G$ is shaped as the Planck function but peaks at $h \nu / k T \approx 3.8$ rather than $h \nu / k T \approx 2.8$. The peak tends to coincide 
with minimum extinction because stellar photospheres tend to obey radiative equilibrium with constant $\mathcal{F}(z)$ (Chapter i'i).

The zig-zag curve near the bottom of the plot specifies the H I bound-free extinction. The variation with wavelength differs from Figure $2 . \overline{2}$. is now measured per gram (and per unit pressure but that only causes an offset of the logarithmic scale). The numbers per particle in Figure $\overline{2} . \overline{6}^{\prime}$ have been multiplied with the lower-level Boltzmann populations and the different $\mathrm{HI}$ edges are now added together.

The solid curves with $\log P_{\mathrm{e}}=1,2\left(\mathrm{cgs}\right.$, dyne $\left.\mathrm{cm}^{-2}\right)$ in Figure $\overline{8} .6$ are representative of the total extinction for depths $\tau_{\nu} \approx 1$ in the solar photosphere in the visible and the near infrared. Their shape shows that $\mathrm{H}^{-}$is the main contributor to the extinction above $\log \lambda=3.5(\AA)$. The H I edges only survive noticeably in the upper curve, for the very small electron pressure that designates a giant rather than a solar-type dwarf. Towards the ultraviolet the actual solar continuum forms in higher layers (curves $\log P_{\mathrm{e}}=0,-1$ ). The larger edges seen in the $\log P_{\mathrm{e}}=3$ curve are from right to left $\mathrm{Mg}$ I $251.4 \mathrm{~nm}(\log \lambda=3.40)$, Si I $152.5 \mathrm{~nm}(\log \lambda=3.18)$, C I $110 \mathrm{~nm}(\log \lambda=3.04)$ and the H I Lyman edge at $91.2 \mathrm{~nm}$

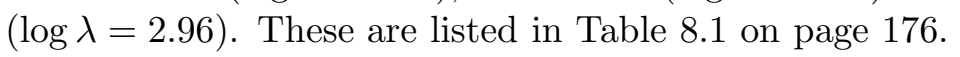

Note that Rayleigh scattering equals $\kappa_{\lambda}$ around $\log \lambda=3.1$ for $\log P_{\mathrm{e}}=-1$ in Figure $\bar{s}$. It is less important in the actual solar ultraviolet because that originates from higher temperatures than 5040/T =0.9. A $\log P_{\mathrm{e}}=-1$ curve in Figure 88.17.; on page 1994; would be a better approximation for the solar chromosphere (but the lowest pressure shown there is $\left.\log P_{\mathrm{e}}=0.5\right)$.

Dominance of $\mathbf{H}^{-}$. The suggestion that $\mathrm{H}^{-}$extinction might be the solution to the long-standing problem of the missing stellar-atmosphere opacity (e.g., Eddington 192.

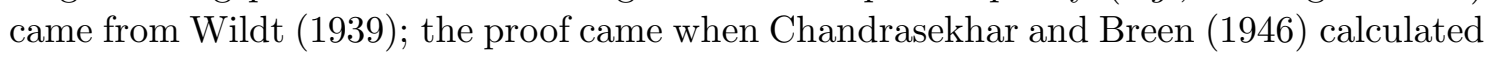
its extinction coefficient quantummechanically (a nasty two-electron system).

Let's quantify the ratio of $\mathrm{H}^{-}$ions over $\mathrm{HI}$ atoms in the solar photosphere as illustration (Novotny p. 162), taking $\log P_{\mathrm{e}}=1.3$ and $T_{\mathrm{e}}=6000 \mathrm{~K}$. Evaluate the Saha equation

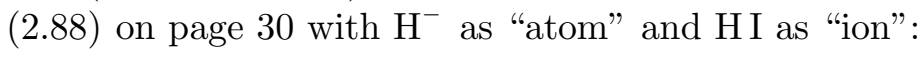

$$
\log \frac{N(\mathrm{HI})}{N\left(\mathrm{H}^{-}\right)}=-0.1761-\log P_{\mathrm{e}}+\log \frac{U(\mathrm{HI})}{U\left(\mathrm{H}^{-}\right)}+2.5 \log T_{\mathrm{e}}-\theta \chi=7.64
$$

where $\chi=0.754 \mathrm{eV}, U(\mathrm{HI}) \approx g_{1}=2$ (because the electron in the hydrogen atom ground state may flip its spin, $m= \pm 1 / 2)$ while $U\left(\mathrm{H}^{-}\right)=1$. The quantity $\theta \equiv 5040 / T_{\mathrm{e}}$ facilitates such logarithmic evaluation ${ }_{1-1}^{\mathrm{TI}}$ of the Boltzmann factor (Novotny p. 109):

$$
\mathrm{e}^{-h \nu / k T}=10^{-1.6021 \times 10^{-12} \log (\mathrm{e})(\chi / k T)}=10^{-(5040 / T) \chi}=10^{-\theta \chi}
$$

with the excitation energy $h \nu$ in erg, $\chi$ in $\mathrm{eV}$ and $T$ in $\mathrm{K}$. The result $N(\mathrm{HI}) / N\left(\mathrm{H}^{-}\right)=$ $4 \times 10^{7}$ illustrates the scarcity of free electrons when hydrogen is neutral. The bulk of the photosphere consists of neutral hydrogen atoms. However, these produce only a small amount of extinction in the visible and near infrared. It is set by the HI excitation fraction $n_{3} / N(\mathrm{HI})$ since the Paschen continuum provides H I bound-free extinction at

\footnotetext{
${ }^{1}$ Logarithmic equations of this type are often seen in the older literature, when log tables and slide rules (a logarithmic instrument converting multiplication into addition) rather than computer windows made up the astronomer's desktop. Logarithmic equation notation is still useful for quick order-of-magnitude estimates. Be glad, however, that you were born after the pocket calculator. All logarithms written log in these lecture notes are ${ }^{10} \log$. Natural logs are written ln.
} 


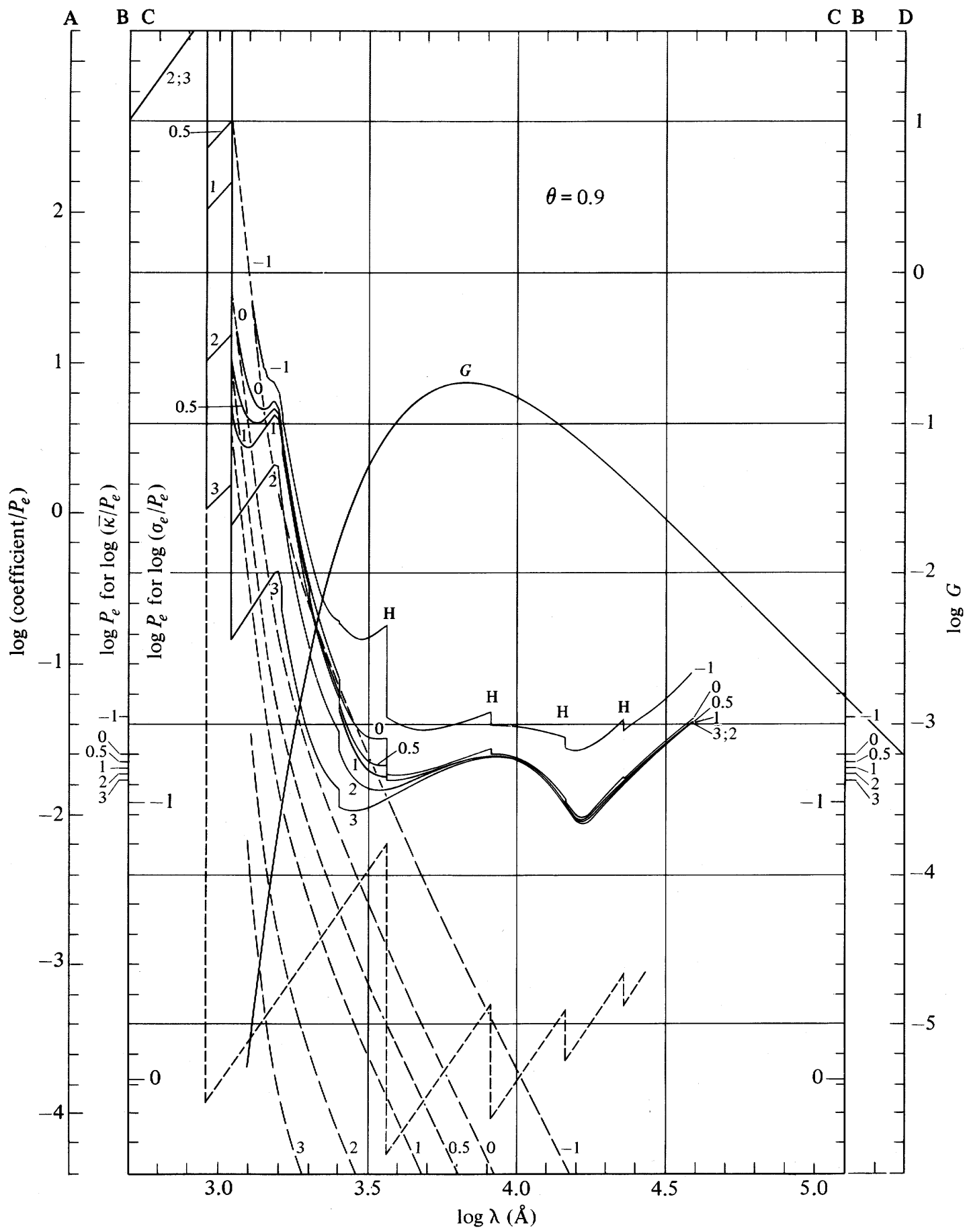

Figure 8.6: Vitense diagram of the continuous extinction in the atmospheres of stars with solar effective temperature $\left(\theta \equiv 5040 / T_{\mathrm{e}}=0.9\right.$ or $\left.T_{\mathrm{e}}=T_{\mathrm{eff}}^{\odot}=5600 \mathrm{~K}\right)$. Solid curve marked G: Rosseland weighting factor in $\left(\$ .1_{1}^{\prime}\right)$. Solid curves marked by numbers: base-10 logarithm of total continuous extinction coefficient $\kappa_{\lambda}$ $\left(\mathrm{cm}^{2} \mathrm{~g}^{-1}\right)$ divided by the electron pressure $P_{\mathrm{e}}$ as specified along scale A on the lefthand side. These curves are labeled with $\log P_{\mathrm{e}}$. The hydrogen bound-free edges are labeled along the $\log P_{\mathrm{e}}=-1$ curve. Jagged dashed curve: total H I extinction coefficient (bound-free plus free-free) on a shifted scale. Smooth dashed curves: $\log \left(\sigma_{\mathrm{H}} / P_{\mathrm{e}}\right)$ with $\sigma_{\mathrm{H}}$ the extinction from Rayleigh scattering, also labeled with $\log P_{\mathrm{e}}$. Horizontal axis: logarithm of the wavelength in Angstrom. Vertical axes: scale A specifies $\log \left(\kappa_{\lambda} / P_{\mathrm{e}}\right)$, except for the jagged H I curve which is offset by factors given by Novotny on p. 135. Scale B specifies the value of $\log P_{\mathrm{e}}$ at which scale $\mathrm{A}$ is to be read to obtain $\log \bar{\kappa} / P_{\mathrm{e}}$ where $\bar{\kappa}$ is the wavelength-averaged extinction coefficient. Scale $\mathrm{C}$ defines the value of $\log P_{\mathrm{e}}$ at which scale $\mathrm{A}$ is to be read to obtain $\log \sigma_{\mathrm{e}} / P_{\mathrm{e}}$ where $\sigma_{\mathrm{e}}$ is the extinction coefficient for Thomson scattering in $\mathrm{cm}^{2} \mathrm{~g}^{-1}$. Scale D (on the right) specifies $\log G$. See also Exercise 10 in on page 232 . From Novotny $(1973)$. 
these wavelengths, whereas the Balmer and Lyman continua don't. The $n=3$ level has (Boltzmann):

$$
\frac{n_{3}}{N(\mathrm{HI})} \approx \frac{n_{3}}{n_{1}}=\frac{g_{3}}{g_{1}} \mathrm{e}^{-h \nu / k T}=\frac{18}{2} 10^{-\theta \chi}=9 \times 10^{-10.1556}=6.2 \times 10^{-10}
$$

with $\chi_{3}=12.09 \mathrm{eV}$ and $g_{n}=2 n^{2}$ for hydrogen. At this $P_{\mathrm{e}}$ and $T_{\mathrm{e}}$ there are $10^{-7.6} / 6.2 \times$ $10^{-10}=40$ times more $\mathrm{H}^{-}$ions available to cause continuous extinction than $\mathrm{HI}$ atoms in the $n=3$ state.

Thus, the solar photosphere consists nearly exclusively of neutral hydrogen atoms

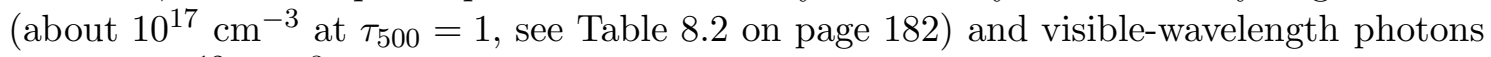
(roughly $10^{12} \mathrm{~cm}^{-3}$ from (2.1.1) on page million of the atoms may experience the presence of the photons, either by having caught a rare free electron which it may loose through extincting a passing photon, or, even less likely, by sitting excitedly in its $n=3$ level, a briefly occupied perch from which it may also extinct a passing photon.

\subsection{VALIII continua}

VALIII modeling. The standard analysis of the solar continuous spectrum is the excellent 90-page VALIII paper of 'Vernazza et al. $\overline{1}(\overline{1} \overline{8} \overline{1})$.'. It uses the observed disk-center solar continuum from X-rays to radio to obtain the temperature-height relation in the solar atmosphere from the deep photosphere (seen in the $\lambda=1.6 \mu \mathrm{m}$ opacity window) to the transition region between chromosphere and corona (sampled by $\operatorname{Ly} \alpha$ ). Basically, the VALIII procedure was a $T_{b}=B_{\nu}^{-1}\left[I_{\nu}\right]$ inversion of all observed intensities shown in Figs. $8.2-\overline{8} .5$ into brightness temperatures. The complexity lies in having to establish the extinction coefficient to determine the $\tau_{\nu}$ scales (and so the $\tau_{\nu} \approx 1$ Eddington-Barbier location to which each $T_{b}$ applies) at all frequencies. NLTE corrections are required to obtain correct $\tau_{\nu}$ scales; NLTE corrections are also required to convert the measured $T_{b}$ into $T_{e}$. In such a procedure the densities follow from imposing hydrostatic equilibrium, in a giant iteration $\mathbf{L}_{-1}^{\mathbf{Z}_{1}}$. In this case, the NLTE ionization and excitation equilibria of $\mathrm{HI}, \mathrm{H}^{-}$, CI, Si I, FeI, MgI, Al I, HeI, HeII, Ca II and Mg II were all solved simultaneously. The resulting description forms the standard model of the solar atmosphere $\mathbf{L}^{3}$ in terms of the steady-state plane-parallel paradigm. 'Vernazza et $\bar{a} \bar{l}$. locations where the Sun is hotter or cooler than average, as seen in Skylab images taken in $\operatorname{Ly} \alpha$.

\footnotetext{
${ }^{2}$ The technique has been described by

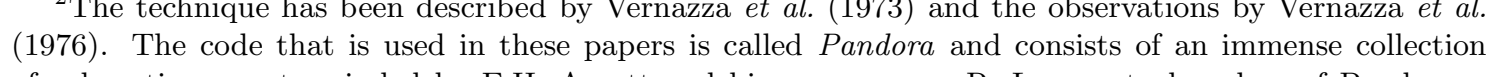
of subroutines masterminded by E.H. Avrett and his programmer R. Loeser, truly a box of Pandora. See http://cfa-www.harvard.edu/ rloeser/pandora.html. It doesn't use the techniques described in Chapter "5it but equivalent two-level approach in which each transition is described in terms of a simple two-level atom one, and the results are then iterated in an enormous loop_over all transitions to correct for the actual multi-level crosstalk between transitions. It is described by Auvrett and Loeser (1987)!

${ }^{3}$ It has been modified slightly since. 'Avrett $(1985)$ and Maltby et al. $(1986)$ raised the value of the temperature minimum a little bit; subsequently Fontenla et al. Currently, CO line observations suggest an appreciable higher location of the time-averaged temperature

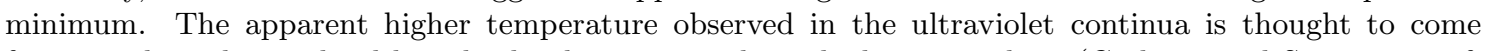
from non-linearly weighted hot shocks that run up through the atmosphere ('Carlsson and Stein 1995, cf. Section, 10.21$)$.
} 


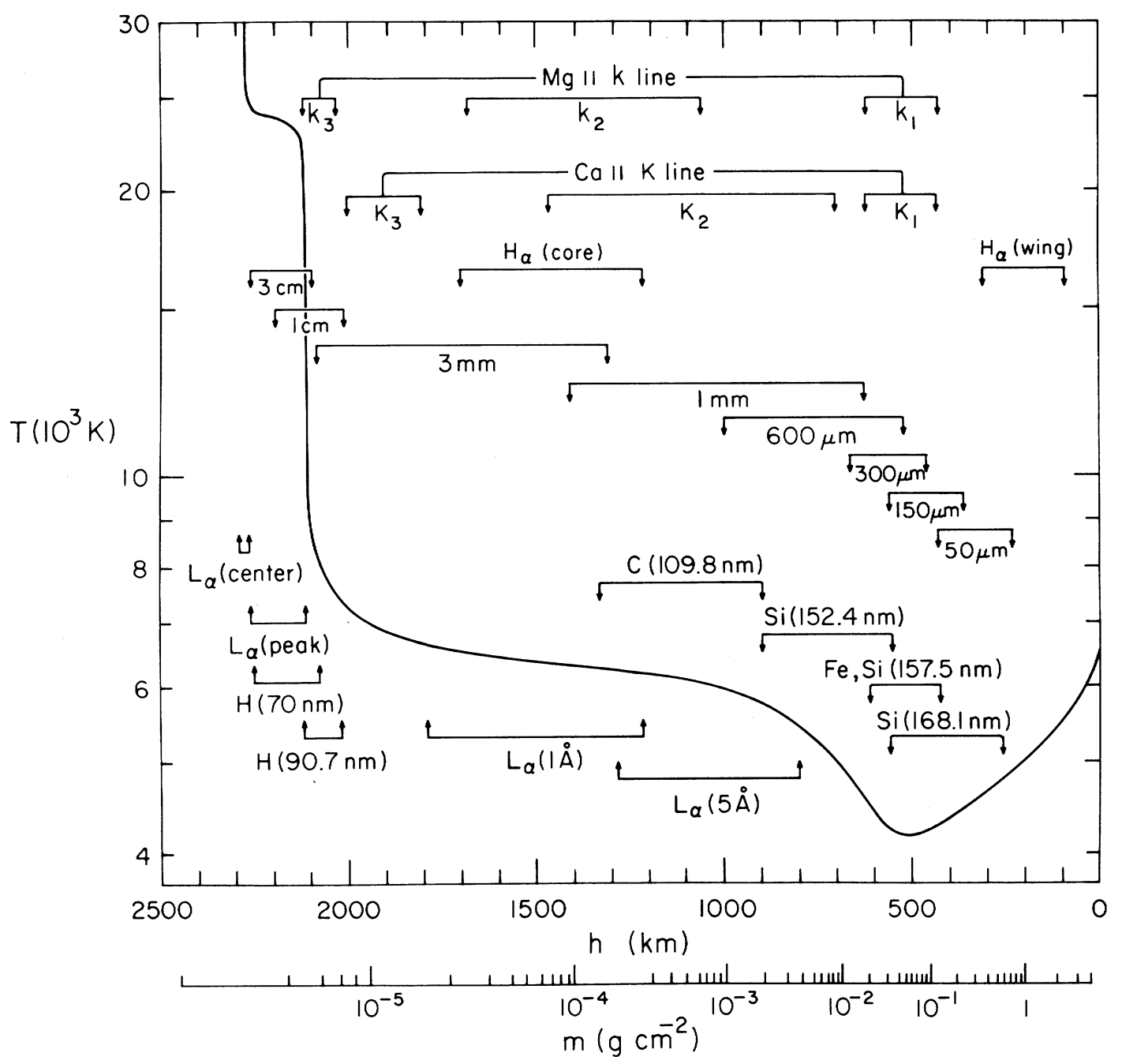

Figure 8.7: The radial temperature distribution in the VALIII atmosphere according to the canonical model

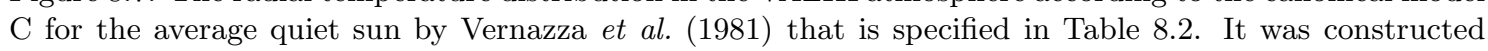
by fitting the observed solar continua. Plane-parallel geometry and hydrostatic equilibrium were assumed, but neither LTE nor RE. The height scale has its zero value at $\tau_{\nu}^{c}=1$ for $\lambda=500 \mathrm{~nm}$ and increases to the left; the solar center is (very) far to the right. The bottom scale measures column mass density. The outward decline on the right is called the photosphere; the increase over $h=500-2000 \mathrm{~km}$ the chromosphere, the steep temperature increase above $h=2000 \mathrm{~km}$ the transition region. The approximate depths where various continua and lines originate are indicated, covering major spectral features listed in Table ' $8 . \overline{1}$ ' on page $17 \overline{6}$. The plots in Figs. 8.9 .11 ' diagnose VALIII continum formation at a selection of these wavelengths. The heights sampled by the $\mathrm{Mg}$ II $\mathrm{k}$ line and the Ca II K lines are indicated at the top of the plot. They are split between $\mathrm{K}_{1}, \mathrm{~K}_{2}$ and $\mathrm{K}_{3}$ for CaII $\mathrm{K}$ and $\mathrm{k}_{1}, \mathrm{k}_{2}$ and $\mathrm{k}_{3}$ for $\mathrm{Mg}$ II $\mathrm{k}$. This is a traditional notation with 3 designating line center, 2 the emission peaks on each side and 1 the dips further away from line center. The $\mathrm{Mg}$ II $\mathrm{h} \& \mathrm{k}$ lines are much stronger than Ca II $\mathrm{H} \& \mathrm{~K}$ and have high $\mathrm{h}_{2}$ and $\mathrm{k}_{2}$ peaks on each side of their center. In the infrared, the $\lambda=160 \mu \mathrm{m}$ radiation originates from the temperature minimum, as does the ultraviolet radiation around $\lambda=160 \mathrm{~nm}$. The elements indicated for the ultraviolet wavelengths identify the major bound-free edges, as listed in Table $\bar{\phi} .1_{-1}^{1}$. The spans at bottom left define formation ranges for the H I Lyman- $\alpha$ line at various locations in the line and for two wavelengths in the Lyman continuum (symbol H). From 'Vernazza et al. $(1981)^{\prime}$ 
Atmospheric Parameters for Model C

\begin{tabular}{|c|c|c|c|c|c|c|c|c|c|c|}
\hline & $\begin{array}{c}\mathrm{h} \\
(\mathrm{km})\end{array}$ & $\begin{array}{c}\mathrm{m} \\
\left.\mathrm{g} \mathrm{cm}^{-2}\right)\end{array}$ & $\tau_{500}$ & $\begin{array}{c}T \\
(K)(k\end{array}$ & 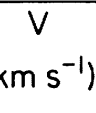 & $\begin{array}{c}n_{H} \\
\left(\mathrm{~cm}^{-3}\right)\end{array}$ & $\begin{array}{c}n_{e} \\
\left(\mathrm{~cm}^{-3}\right)\end{array}$ & $\begin{array}{c}P_{\text {total }} \\
\left(\text { dyn } \mathrm{cm}^{-2}\right)\end{array}$ & $\frac{P_{\text {gos }}}{P_{\text {total }}}$ & $\begin{array}{c}\sigma \\
\left(g \mathrm{~cm}^{-3}\right)\end{array}$ \\
\hline $\begin{array}{l}1 \\
2 \\
3 \\
4 \\
5\end{array}$ & $\begin{array}{l}2543 \\
2298 \\
2290 \\
2280 \\
2274\end{array}$ & $\begin{array}{l}5.257-06 \\
5.365-06 \\
5.373-06 \\
5.389-06 \\
5.404-06\end{array}$ & $\begin{array}{l}0 . \\
3.712-08 \\
3.969-08 \\
4.491-08 \\
4.952-08\end{array}$ & $\begin{array}{r}447000 \\
141000 \\
89100 \\
50000 \\
37000\end{array}$ & $\begin{array}{r}11.28 \\
9.87 \\
9.82 \\
9.76 \\
9.73\end{array}$ & $\begin{array}{l}1.005+09 \\
3.205+09 \\
5.041+09 \\
9.038+09 \\
1.201+10\end{array}$ & $\begin{array}{l}1.205+09 \\
3.839+09 \\
5.961+09 \\
9.993+09 \\
1.318+10\end{array}$ & $\begin{array}{l}1.440-01 \\
1.470-01 \\
1.472-01 \\
1.477-01 \\
1.481-01\end{array}$ & $\begin{array}{l}.9896 \\
.9752 \\
.9614 \\
.9318 \\
.9102\end{array}$ & $\begin{array}{l}2.349-15 \\
7.494-15 \\
1.179-14 \\
2.113-14 \\
2.808-14\end{array}$ \\
\hline $\begin{array}{r}6 \\
7 \\
8 \\
9 \\
10\end{array}$ & $\begin{array}{l}2271 \\
2267 \\
2263 \\
2255 \\
2230\end{array}$ & $\begin{array}{l}5.413-06 \\
5.427-06 \\
5.443-06 \\
5.476-06 \\
5.583-06\end{array}$ & $\begin{array}{l}5.234-08 \\
5.657-08 \\
6.124-08 \\
7.110-08 \\
1.030-07\end{array}$ & $\begin{array}{l}32000 \\
28000 \\
25500 \\
24500 \\
24200\end{array}$ & $\begin{array}{l}9.71 \\
9.70 \\
9.68 \\
9.64 \\
9.49\end{array}$ & $\begin{array}{l}1.378+10 \\
1.567+10 \\
1.718+10 \\
1.797+10 \\
1.862+10\end{array}$ & $\begin{array}{l}1.498+10 \\
1.677+10 \\
1.812+10 \\
1.881+10 \\
1.943+10\end{array}$ & $\begin{array}{l}1.483-01 \\
1.487-01 \\
1.491-01 \\
1.500-01 \\
1.530-01\end{array}$ & $\begin{array}{l}.8976 \\
.8840 \\
.8738 \\
.8698 \\
.8718\end{array}$ & $\begin{array}{l}3.222-14 \\
3.665-14 \\
4.017-14 \\
4.203-14 \\
4.355-14\end{array}$ \\
\hline $\begin{array}{l}11 \\
12 \\
13 \\
14 \\
15\end{array}$ & $\begin{array}{l}2200 \\
2160 \\
2129 \\
2120 \\
2115\end{array}$ & $\begin{array}{l}5.716-06 \\
5.902-06 \\
6.055-06 \\
6.101-06 \\
6.128-06\end{array}$ & $\begin{array}{l}1.426-07 \\
1.977-07 \\
2.427-07 \\
2.562-07 \\
2.640-07\end{array}$ & $\begin{array}{l}24000 \\
23500 \\
23000 \\
22500 \\
21000\end{array}$ & $\begin{array}{l}9.33 \\
9.08 \\
8.87 \\
8.81 \\
8.78\end{array}$ & $\begin{array}{l}1.932+10 \\
2.051+10 \\
2.163+10 \\
2.231+10 \\
2.403+10\end{array}$ & $\begin{array}{l}2.009+10 \\
2.120+10 \\
2.219+10 \\
2.276+10 \\
2.402+10\end{array}$ & $\begin{array}{l}1.566-01 \\
1.617-01 \\
1.659-01 \\
1.672-01 \\
1.679-01\end{array}$ & $\begin{array}{l}.8645 \\
.8778 \\
.8801 \\
.8789 \\
.8710\end{array}$ & $\begin{array}{l}4.517-14 \\
4.795-14 \\
5.058-14 \\
5.216-14 \\
5.619-14\end{array}$ \\
\hline $\begin{array}{l}16 \\
17 \\
18 \\
19 \\
20\end{array}$ & $\begin{array}{l}2113 \\
2109 \\
2107 \\
2104 \\
2090\end{array}$ & $\begin{array}{l}6.140-06 \\
6.172-06 \\
6.193-06 \\
6.228-06 \\
6.416-06\end{array}$ & $\begin{array}{l}2.674-07 \\
2.754-07 \\
2.801-07 \\
2.877-07 \\
3.243-07\end{array}$ & $\begin{array}{r}18500 \\
12300 \\
10700 \\
9500 \\
8440\end{array}$ & $\begin{array}{l}8.77 \\
8.74 \\
8.72 \\
8.71 \\
8.60\end{array}$ & $\begin{array}{l}2.732+10 \\
4.092+10 \\
4.673+10 \\
5.239+10 \\
6.127+10\end{array}$ & $\begin{array}{l}2.620+10 \\
3.306+10 \\
3.535+10 \\
3.705+10 \\
3.799+10\end{array}$ & $\begin{array}{l}1.682-01 \\
1.691-01 \\
1.697-01 \\
1.706-01 \\
1.758-01\end{array}$ & $\begin{array}{l}.8539 \\
.7839 \\
.7552 \\
.7277 \\
.6986\end{array}$ & $\begin{array}{l}6.390-14 \\
9.569-14 \\
1.093-13 \\
1.225-13 \\
1.433-13\end{array}$ \\
\hline $\begin{array}{l}21 \\
22 \\
23 \\
24 \\
25\end{array}$ & $\begin{array}{l}2080 \\
2070 \\
2050 \\
2016 \\
1990\end{array}$ & $\begin{array}{l}6.564-06 \\
6.722-06 \\
7.066-06 \\
7.732-06 \\
8.322-06\end{array}$ & $\begin{array}{l}3.507-07 \\
3.770-07 \\
4.299-07 \\
5.203-07 \\
5.903-07\end{array}$ & $\begin{array}{l}8180 \\
7940 \\
7660 \\
7360 \\
7160\end{array}$ & $\begin{array}{l}8.55 \\
8.50 \\
8.42 \\
8.22 \\
8.01\end{array}$ & $\begin{array}{l}6.541+10 \\
6.960+10 \\
7.705+10 \\
9.075+10 \\
1.033+11\end{array}$ & $\begin{array}{l}3.780+10 \\
3.783+10 \\
3.792+10 \\
3.811+10 \\
3.858+10\end{array}$ & $\begin{array}{l}1.798-01 \\
1.842-01 \\
1.936-01- \\
2.118-01 \\
2.280-01\end{array}$ & $\begin{array}{l}.6891 \\
.6808 \\
.6701 \\
.6616 \\
.6600\end{array}$ & $\begin{array}{l}1.530-13 \\
1.628-13 \\
1.802-13 \\
2.122-13 \\
2.417-13\end{array}$ \\
\hline $\begin{array}{l}26 \\
27 \\
28 \\
29 \\
30\end{array}$ & $\begin{array}{l}1925 \\
1785 \\
1605 \\
1515 \\
1380\end{array}$ & $\begin{array}{l}1.015-05 \\
1.647-05 \\
3.407-05 \\
5.144-05 \\
1.012-04\end{array}$ & $\begin{array}{l}7.717-07 \\
1.212-06 \\
1.958-06 \\
2.420-06 \\
3.286-06\end{array}$ & $\begin{array}{l}6940 \\
6630 \\
6440 \\
6370 \\
6280\end{array}$ & $\begin{array}{l}7.63 \\
6.92 \\
5.85 \\
5.26 \\
4.51\end{array}$ & $\begin{array}{l}1.380+11 \\
2.601+11 \\
6.386+11 \\
1.048+12 \\
2.273+12\end{array}$ & $\begin{array}{l}4.028+10 \\
4.771+10 \\
6.005+10 \\
6.456+10 \\
7.600+10\end{array}$ & $\begin{array}{l}2.780-01 \\
4.511-01 \\
9.334-01 \\
1.409+00 \\
2.774+00\end{array}$ & $\begin{array}{l}.6620 \\
.6772 \\
.7262 \\
.7595 \\
.8051\end{array}$ & $\begin{array}{l}3.227-13 \\
6.082-13 \\
1.493-12 \\
2.450-12 \\
5.315-12\end{array}$ \\
\hline $\begin{array}{l}31 \\
32 \\
33 \\
34 \\
35\end{array}$ & $\begin{array}{r}1280 \\
1180 \\
1065 \\
980 \\
905\end{array}$ & $\begin{array}{l}1.747-04 \\
3.112-04 \\
6.299-04 \\
1.098-03 \\
1.840-03\end{array}$ & $\begin{array}{l}4.084-06 \\
5.075-06 \\
6.861-06 \\
9.148-06 \\
1.239-05\end{array}$ & $\begin{array}{l}6220 \\
6150 \\
6040 \\
5925 \\
5755\end{array}$ & $\begin{array}{l}3.92 \\
3.48 \\
2.73 \\
2.14 \\
1.70\end{array}$ & $\begin{array}{l}4.200+12 \\
7.865+12 \\
1.711+13 \\
3.147+13 \\
5.546+13\end{array}$ & $\begin{array}{l}7.486+10 \\
8.108+10 \\
9.349+10 \\
1.041+11 \\
1.049+11\end{array}$ & $\begin{array}{l}4.786+00 \\
8.527+00 \\
1.726+01 \\
3.008+01 \\
5.043+01\end{array}$ & $\begin{array}{l}.8423 \\
.8694 \\
.9136 \\
.9440 \\
.9628\end{array}$ & $\begin{array}{l}9.822-12 \\
1.839-11 \\
4.000-11 \\
7.359-11 \\
1.297-10\end{array}$ \\
\hline $\begin{array}{l}36 \\
37 \\
38 \\
39 \\
40\end{array}$ & $\begin{array}{l}855 \\
755 \\
705 \\
655 \\
605\end{array}$ & $\begin{array}{l}2.632-03 \\
5.577-03 \\
8.333-03 \\
1.276-02 \\
2.013-02\end{array}$ & $\begin{array}{l}1.553-05 \\
2.537-05 \\
3.288-05 \\
4.452-05 \\
7.022-05\end{array}$ & $\begin{array}{l}5650 \\
5280 \\
5030 \\
4730 \\
4420\end{array}$ & $\begin{array}{r}1.53 \\
1.23 \\
1.09 \\
.96 \\
.83\end{array}$ & $\begin{array}{l}8.135+13 \\
1.864+14 \\
2.935+14 \\
4.794+14 \\
8.119+14\end{array}$ & $\begin{array}{l}1.064+11 \\
8.838+10 \\
7.664+10 \\
8.085+10 \\
1.112+11\end{array}$ & $\begin{array}{l}7.210+01 \\
1.528+02 \\
2.283+02 \\
3.495+02 \\
5.516+02\end{array}$ & $\begin{array}{l}.9691 \\
.9784 \\
.9821 \\
.9852 \\
.9881\end{array}$ & $\begin{array}{l}1.902-10 \\
4.358-10 \\
6.864-10 \\
1.121-09 \\
1.899-09\end{array}$ \\
\hline $\begin{array}{l}41 \\
42 \\
43 \\
44 \\
45\end{array}$ & $\begin{array}{l}555 \\
515 \\
450 \\
350 \\
250\end{array}$ & $\begin{array}{l}3.270-02 \\
4.878-02 \\
9.378-02 \\
2.481-01 \\
6.172-01\end{array}$ & $\begin{array}{l}1.456-04 \\
3.014-04 \\
1.017-03 \\
5.626-03 \\
2.670-02\end{array}$ & $\begin{array}{l}4230 \\
4170 \\
4220 \\
4465 \\
4780\end{array}$ & $\begin{array}{l}.70 \\
.60 \\
.53 \\
.52 \\
.63\end{array}$ & $\begin{array}{l}1.382+15 \\
2.096+15 \\
3.989+15 \\
9.979+15 \\
2.315+16\end{array}$ & $\begin{array}{l}1.733+11 \\
2.495+11 \\
4.516+11 \\
1.110+12 \\
2.674+12\end{array}$ & $\begin{array}{l}8.958+02 \\
1.336+03 \\
2.569+03 \\
6.798+03 \\
1.691+04\end{array}$ & $\begin{array}{r}.9912 \\
.9934 \\
.9949 \\
.9954 \\
.9936\end{array}$ & $\begin{array}{l}3.232-09 \\
4.902-09 \\
9.327-09 \\
2.334-08 \\
5.413-08\end{array}$ \\
\hline $\begin{array}{l}46 \\
47 \\
48 \\
49 \\
50\end{array}$ & $\begin{array}{r}150 \\
100 \\
50 \\
0 \\
-25\end{array}$ & $\begin{array}{l}1.433+00 \\
2.118+00 \\
3.056+00 \\
4.279+00 \\
4.991+00\end{array}$ & $\begin{array}{l}1.117-01 \\
2.201-01 \\
4.395-01 \\
9.953-01 \\
1.683+00\end{array}$ & $\begin{array}{l}5180 \\
5455 \\
5840 \\
6420 \\
6910\end{array}$ & $\begin{array}{l}1.00 \\
1.20 \\
1.40 \\
1.60 \\
1.70\end{array}$ & $\begin{array}{l}4.917+16 \\
6.866+16 \\
9.203+16 \\
1.166+17 \\
1.261+17\end{array}$ & $\begin{array}{l}6.476+12 \\
1.066+13 \\
2.122+13 \\
6.433+13 \\
1.547+14\end{array}$ & $\begin{array}{l}3.926+04 \\
5.804+04 \\
8.274+04 \\
1.172+05 \\
1.368+05\end{array}$ & $\begin{array}{l}.9854 \\
.9801 \\
.9748 \\
.9702 \\
.9688\end{array}$ & $\begin{array}{l}1.150-07 \\
1.606-07 \\
2.152-07 \\
2.727-07 \\
2.949-07\end{array}$ \\
\hline $\begin{array}{l}51 \\
52 \\
\end{array}$ & $\begin{array}{l}-50 \\
-75\end{array}$ & $\begin{array}{l}5.747+00 \\
6.534+00 \\
\end{array}$ & $\begin{array}{l}3.338+00 \\
7.445+00 \\
\end{array}$ & $\begin{array}{l}7610 \\
8320 \\
\end{array}$ & $\begin{array}{l}1.76 \\
1.80 \\
\end{array}$ & $\begin{array}{l}1.317+17 \\
1.365+17 \\
\end{array}$ & $\begin{array}{l}4.645+14 \\
1.204+15 \\
\end{array}$ & $\begin{array}{l}1.575+05 \\
1.790+05 \\
\end{array}$ & $\begin{array}{l}.9697 \\
.9711 \\
\end{array}$ & $\begin{array}{l}3.080-07 \\
3.192-07 \\
\end{array}$ \\
\hline
\end{tabular}

Table 8.2: The VALIII atmosphere. The third column $(m)$ species a depth scale in the form of column mass density: the mass in a solar column with $1 \mathrm{~cm}^{2}$ cross-section that extends upwards from the height $h$. The next column gives the standard continuum optical depth $\tau_{500}$ at $\lambda=500 \mathrm{~nm}$. The value $\tau_{500}=1$ defines the zero point of the height scale $h$. The temperature stratification (next column) is plotted in Figure $18.7_{-}^{\prime}$ The column $V$ specifies the microturbulence $V \equiv \xi_{\text {micro. }}$ It is assumed to contribute turbulent pressure $P_{\text {turb }}=(1 / 2) \rho V^{2}$ so that $P_{\text {total }}=P_{\mathrm{g}}+P_{\text {turb }}$; the ratio $P_{\mathrm{g}} / P_{\text {total }}$ is specified in the next to last column. The hydrogen density $n_{\mathrm{H}}$ specifies the total hydrogen nuclei number density, summing neutral atoms, $\mathrm{H}^{-}$ ions and free protons. The last column is labeled $\sigma$ by mistake; it is the mass density $\rho$. The temperature minimum between the VALIII photosphere and chromosphere is at line 42 . The VALIII atmosphere closely resembles the solar atmosphere as it would be if it emitted the actual solar continua from plane-parallel layers in hydrostatic equilibrium. The VALIII paper contains five more tables like this one, for hotter and cooler plane-parallel stars of which the spectrum resembles solar locations that are hotter and cooler than average. From ivernazza et al. $(1981)$. 

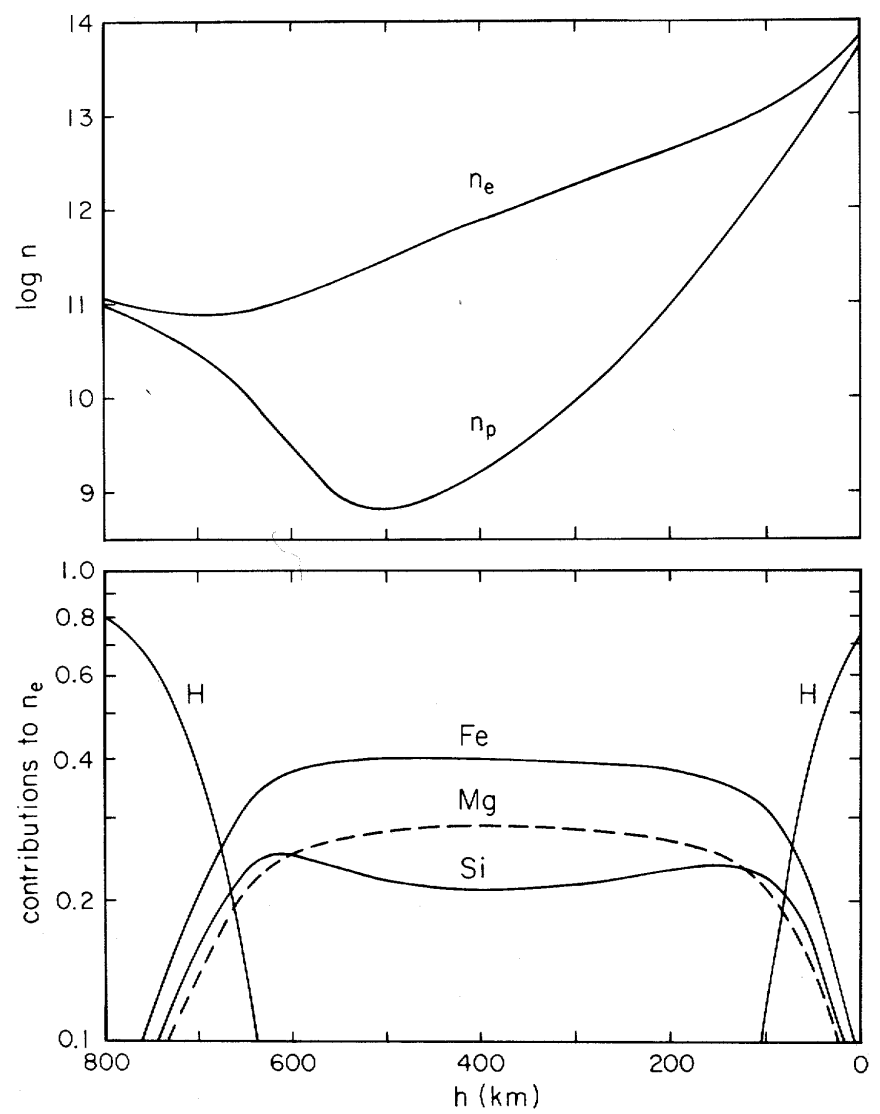

Figure 8.8: Electron densities and donors in the VALIII temperature-minimum region. The upper panel shows the electron density $n_{\mathrm{e}}=N_{\mathrm{e}}$ and the free-proton density $n_{p}$. The dip of the latter in the temperature minimum at $h=500 \mathrm{~km}$ shows that free electrons in that region are not from ionized hydrogen. The lower panel shows the major contributions to $N_{\mathrm{e}}$ as fractions of the total per height. Iron, magnesium and silicon - are the most important donors. Hydrogen dominates both deeper down and higher up. From 'Vernazzal et al. $(1981)$

VALIII as a star. The real Sun is far more complex than the VALIII models indicate; the latter may even fail as a description of the spatial and temporal average of the real atmosphere. Nevertheless, they represent a self-consistent, physically correct model of how the solar atmosphere might have worked if the sun were a static plane-parallel stari:

\footnotetext{
${ }^{4}$ I have heard a leading Dutch astronomer wonder whether astronomy wouldn't have been better off if all stars were VALIII-type stars, truly obeying static plane-parallel geometry. Their radiative transfer and this course would have been identical. The Hertzsprung-Russell diagram, color-color diagrams, the theory of stellar interior structure and the theory of stellar evolution would also be the same. What would be missed are sunspots, granules, spicules, floccules, plage, prominences, flares, X-ray bright points, $\mathrm{K}_{2 V}$ bright points, coronal loops, magnetic fluxtubes, noise storms, jets, explosive events, coronal mass ejections and all the other solar "dermatology" phenomena that require radiation hydrodynamics, MHD and plasma physics rather than just plane-parallel HE-RE-LTE modeling. They make solar physics quite complicated and make stellar astronomers fear that actual stellar physics is also quite complicated. A VALIII-type Sun would sit easier on their conscience. Indeed, the Sun was papally ordained to be a radiating sphere of unquestionable beauty $\equiv$ homogeneity until Galileo saw spots on it. Nowadays, such infernal blemishes make up most of solar physics. Perhaps nature strives for maximum detail with complexity $\equiv$ beauty, just as in biology or Bach fugas (Rutten 1990ai). Solar physics illustrates the ingenuity with which nature converts simple
} 

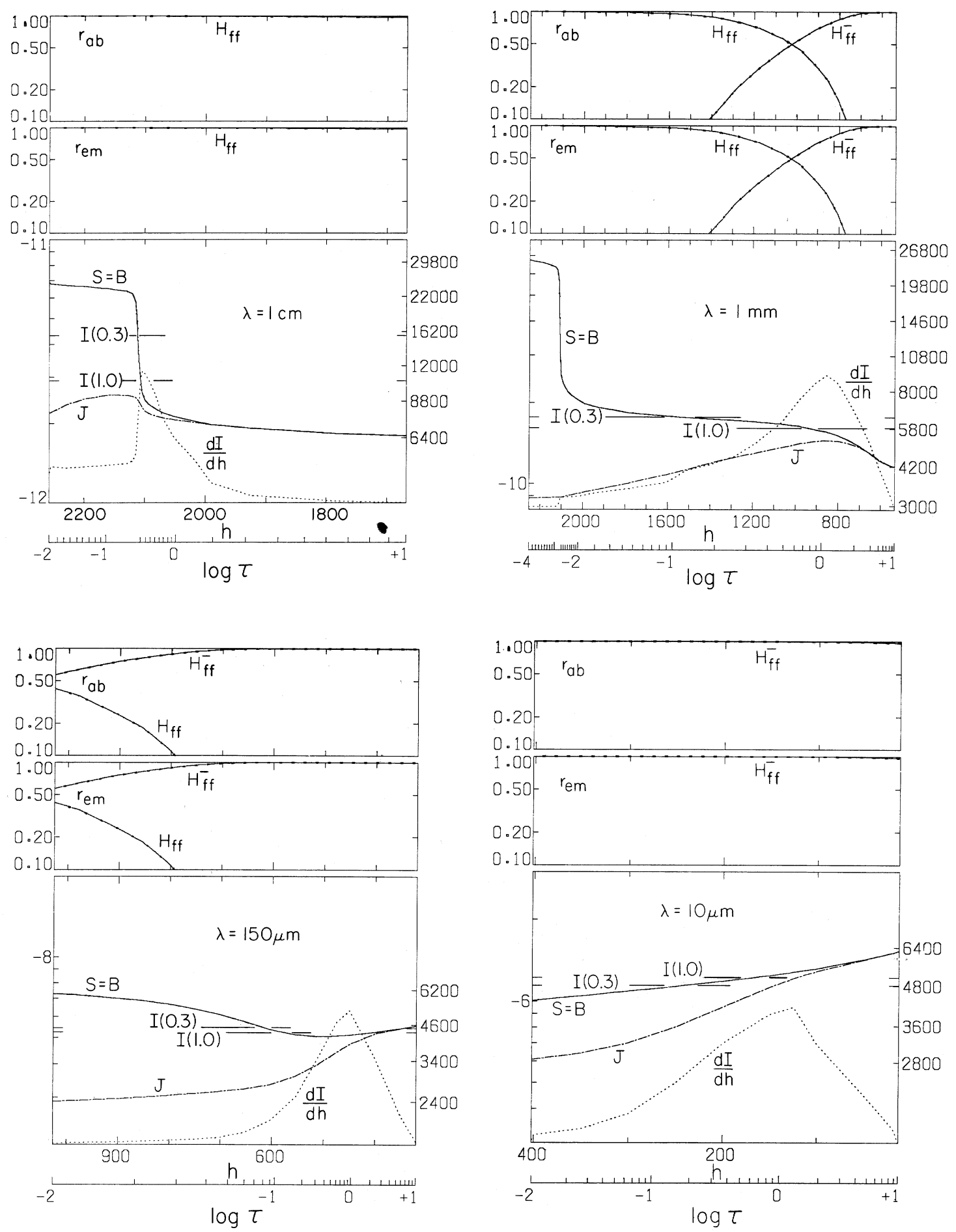

Figure 8.9: Physics of the formation of VALIII continua at selected wavelengths. Radio to infrared. The upper panel of each plot specifies the relative contributions $r_{\mathrm{ab}}$ at each height $h$ to the continuum extinction by the processes marked along each curve. Element symbols denote bound-free transitions unless otherwise labeled. The second panel shows the relative contributions $r_{\mathrm{em}}$ to the local emissivity (with the exception of scattering in spectral lines, present on the next two pages). The lower panel shows $B_{\nu}, J_{\nu}$ and $S_{\nu}$ as a function of height for the indicated wavelength. $I(1.0)$ and $I(0.3)$ are the emergent intensities for $\mu=1$ and $\mu=0.3$. They are marked by horizontal dashes where the $S_{\nu}$ curve equals their values and

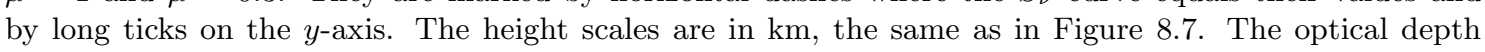
scales are monochromatic, for the specified wavelength. The ordinates measure $\bar{B}_{\nu}, J_{\nu}, S_{\nu}$ and $I_{\nu}$ on logarithmic scales in units erg $\mathrm{cm}^{-2} \mathrm{~s}^{-1} \mathrm{ster}^{-1} \mathrm{~Hz}^{-1}$. The scales on the right show corresponding radiation temperatures; the temperature minimum between photosphere and chromosphere is at $4200 \mathrm{~K}$. The axis ranges differ between different wavelength plots. Exercise on page 231 may be helpful in interpreting these plots. From 'Vernazza et al. $(1981)_{\mathrm{r}}^{\prime}$ 

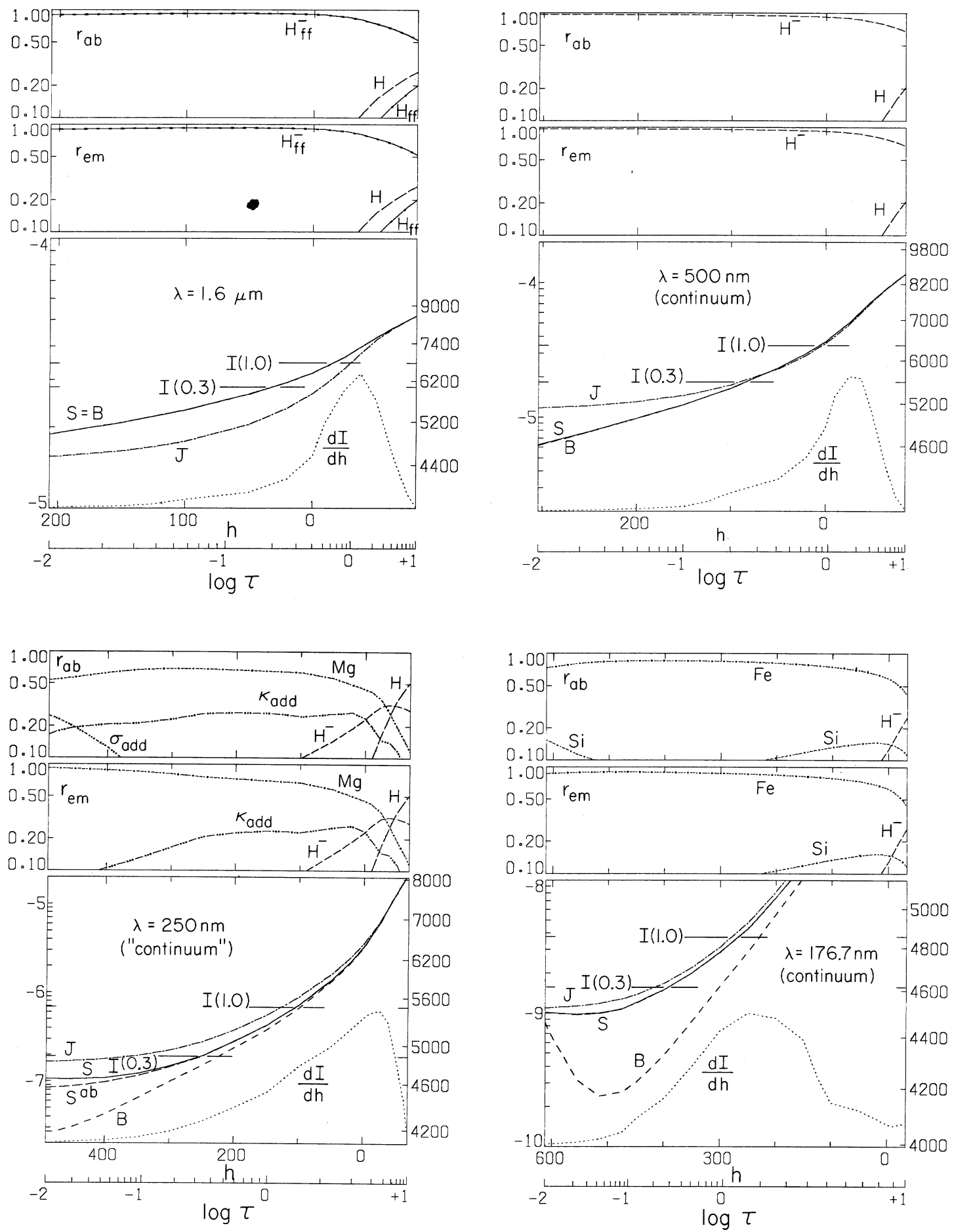

Figure 8.10: The formation of VALIII continua, near-infrared to mid-ultraviolet. The label "continuum" specifies that the plot is for the high points in the spectrum, between the spectral lines and not for the average intensity including lines. The continuous extinction at $\lambda=250 \mathrm{~nm}$ (lower-left plot) is dominated by

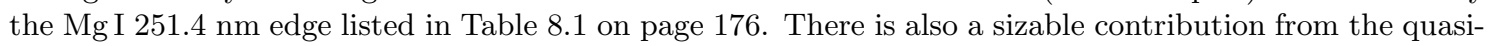
continuous "line-haze" made up by the myriads of lines in the ultraviolet. The VALIII modeling assumed (rather ad-hoc) that their source functions change in smooth fashion from purely thermal $\left(S_{\nu}=B_{\nu}\right)$ in deep layers to pure scattering $\left(S_{\nu}=J_{\nu}\right)$ at large height. The curves labeled $\kappa_{\text {add }}$ give the LTE part of the line haze contribution; their difference between the $r_{\mathrm{ab}}$ and $r_{\mathrm{em}}$ panels marks the increasing contribution by line-haze scattering. The latter emerges at the left in the top panel (curve labeled $\sigma_{\text {add }}$ ) but is not specified in the $r_{\mathrm{em}}$ panel. The source function curve labeled_ $S_{-}^{\mathrm{ab}}$ does not contain line-haze scattering. The fourth plot is at the FeI $176.8 \mathrm{~nm}$ edge. From 'Vernazza et al. $(1981)$ r 

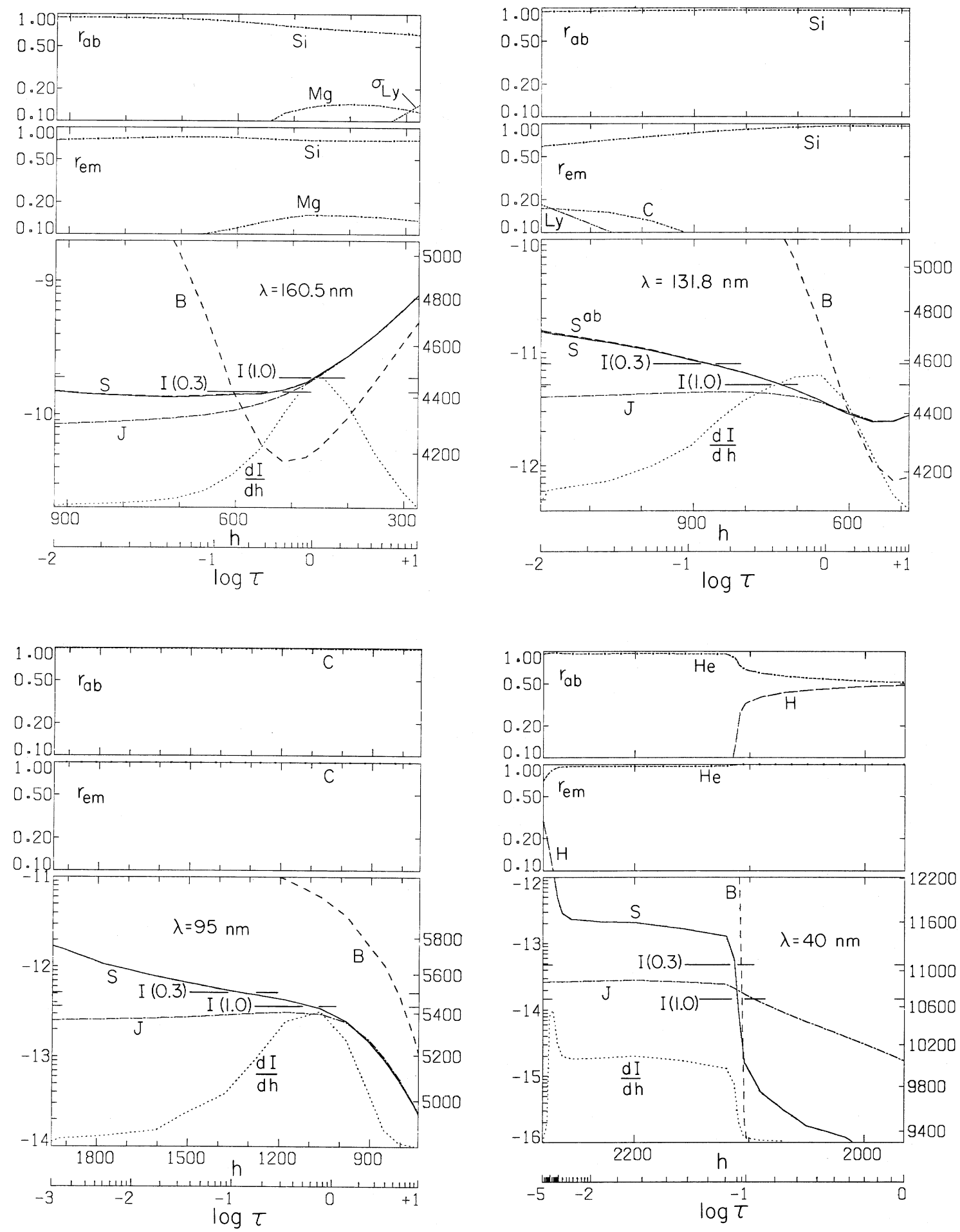

Figure 8.11: The formation of VALIII continua, mid to far ultraviolet. The $\lambda=131.8 \mathrm{~nm}$ wavelength (Si I

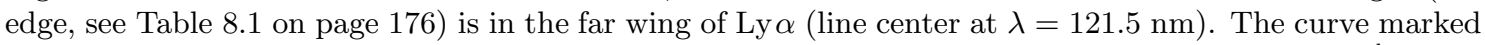
Ly in the top panel marks its contribution to the (quasi-)continuous opacity. The curve marked $S^{\text {ab }}$ in the $131.8 \mathrm{~nm}$ plot excludes scattering in Ly $\alpha$. From 'Vernazza et al. $(1981)_{\mathrm{r}}^{\prime}$ 


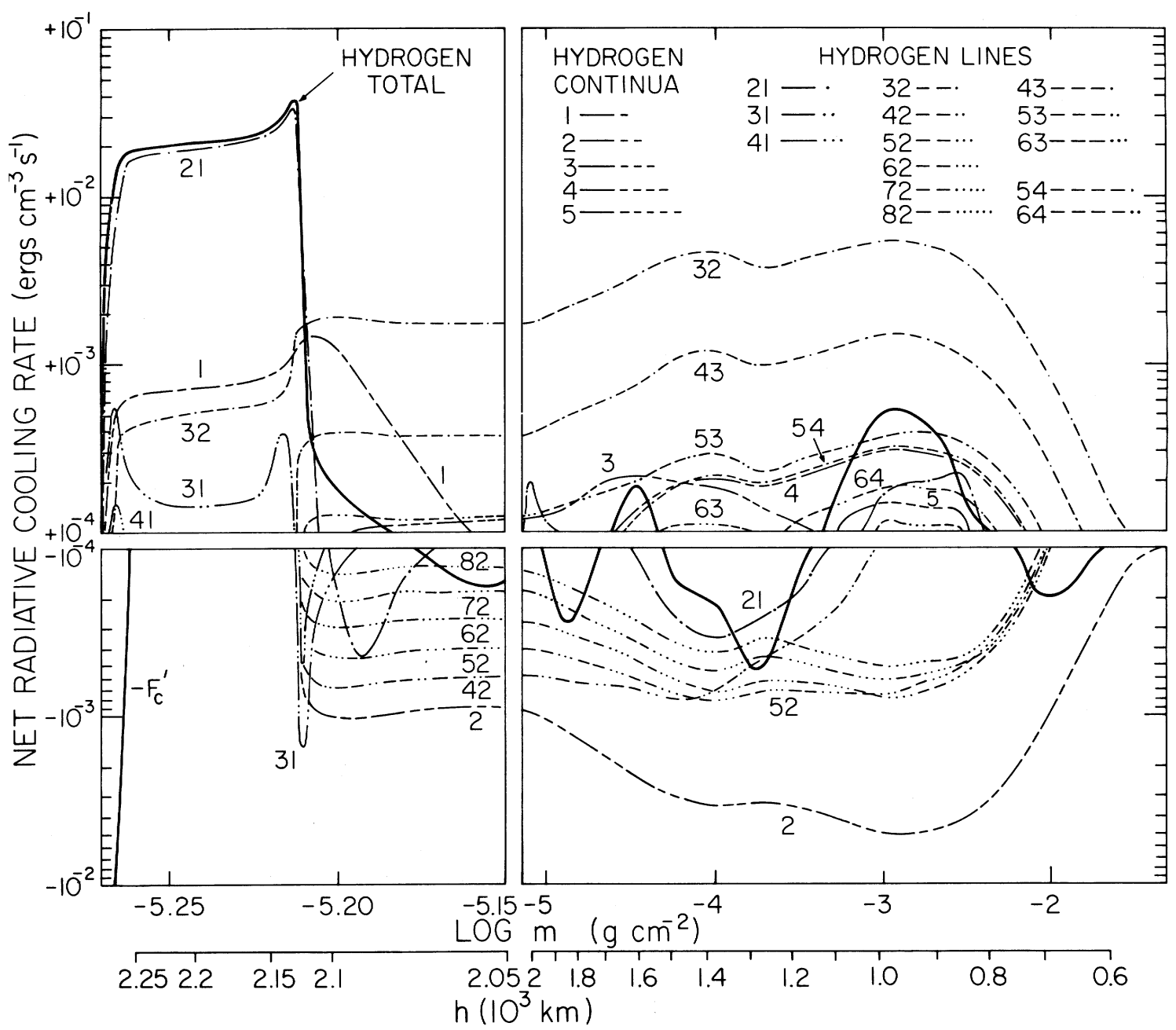

Figure 8.12: Hydrogen balances of the VALIII atmosphere. Net radiative cooling rates defined by $\left(\overline{7} .3 \overline{5}_{i}^{\prime}\right)$ on page 155 and $\left(17.3 \overline{7}_{1}\right)$ on page 156 are plotted per feature of the VALIII H I spectrum. The upper and lower panels plot radiative cooling and heating, respectively. The lefthand panels are on an expanded log $m$ scale spanning the transition region. The solid curve is the combined net cooling rate in all $\mathrm{HI}$ lines and continua. In the transition region (left) it is dominated by radiative cooling in Ly $\alpha$ (label 21). Downward conduction takes over at the far left as shown by the negative conductive flux gradient $-F_{\mathrm{c}}^{\prime}$. Lower in the atmosphere, the total cooling rate oscillates around zero due to a rough balancing between radiative $J_{\nu}>S_{\nu}$ heating in the Balmer continuum (level $n=2$, label 2) and photon losses in the Balmer- $\alpha$ (label 32 ) line. The much stronger Lyman- $\alpha$ line (label 21 ) is roughly in detailed_balance up to $\log m \approx-5.20$ and then causes large uncompensated photon-loss imbalance. From ivernazza et al. $(1981)$.'. 


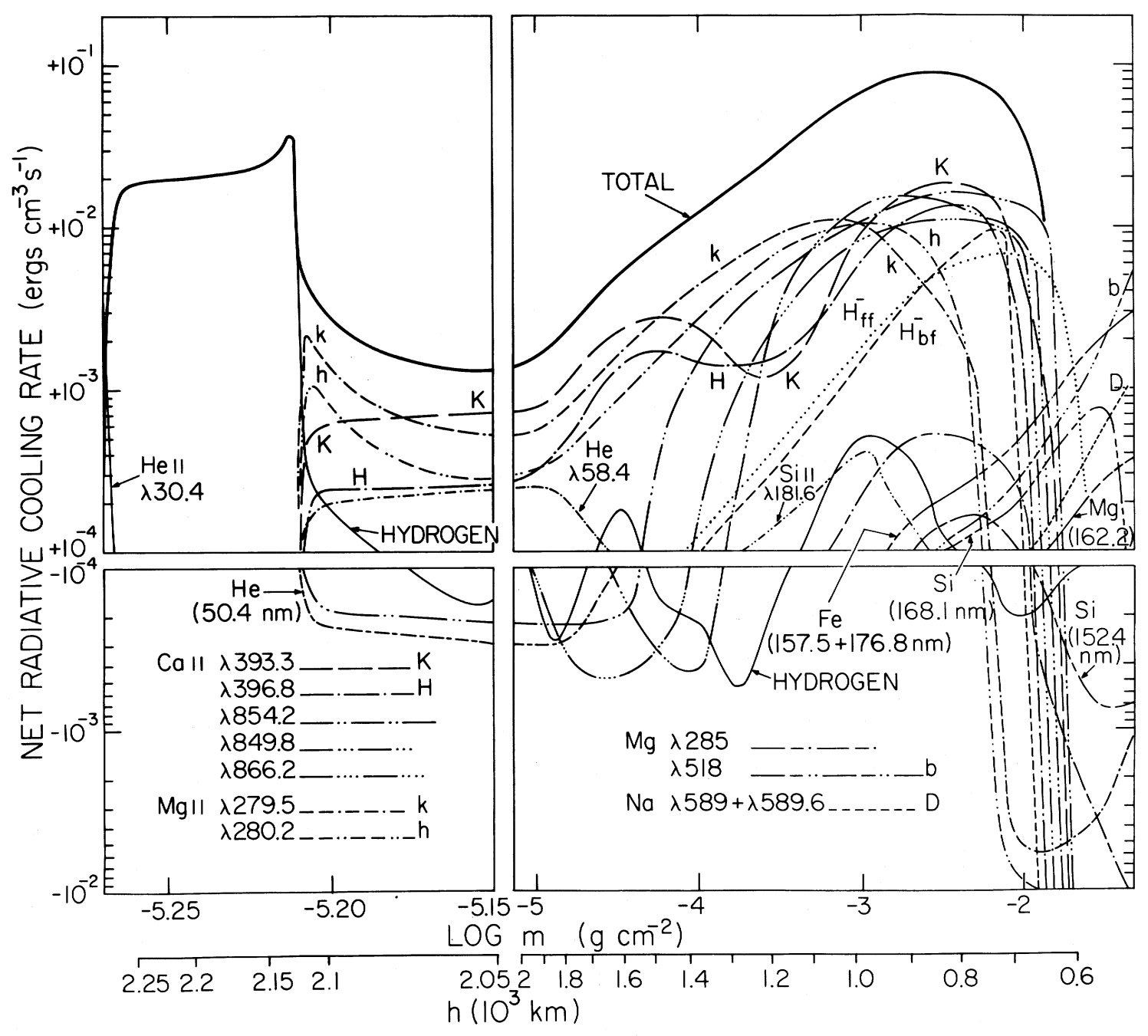

Figure 8.13: Total energy budget of the VALIII atmosphere. Net radiative cooling rates defined by $(\overline{7} . \overline{3} \overline{5})$ on page $15 \overline{5}$ and $\left(\overline{7}_{3} \cdot \overrightarrow{7}_{1}\right)$ on page 156 spectrum. The curve marked HYD $\bar{R}$ OGEN is the combined net cooling rate in all H I lines and continua shown solid in Figure 18.12, The solid curve marked TOTAL specifies the total radiative losses of the VALIII chromosphere at every height. They are dominated by the $\mathrm{Mg}$ II $\mathrm{h} \& \mathrm{k}$ and $\mathrm{Ca}$ II $\mathrm{H} \& \mathrm{~K}$ resonance lines over most of the chromosphere. In the temperature minimum region (around $h=500 \mathrm{~km}$, far right) these lines are net heaters, feeding on (ultra)violet continua from below. The subordinate Ca II "infrared" lines near $\lambda=850 \mathrm{~nm}$ share in this heating because they share their upper levels with_ $\underline{H}_{\mathbf{L}} \underline{K}_{-} \underline{H}_{-}^{-}$bound-free transitions also contribute net heating in the temperature minimum. From 'Vernazza et al. 
I use the quiet-Sun VALIII model here to illustrate the physics of bound-free continua as it was derived by the computer. The physics is good physics even if the real Sun works differently. The results may be interpreted as holding for a computationally existing star called VALIII which faithfully portrays the theory of Chapters is remarkably like the Sun in its temporally and spatially averaged continuous spectral distribution, but in contrast to the Sun it does obey hydrostatic equilibrium and static plane-parallel geometry, and it contains only those atoms, ions and electrons that were specified in the Pandora code, fortunately with just the corresponding cross-sections. Its modeling is exact. The advantage of studying the star VALIII rather than the star Sol is that the physics of VALIII radiation is fully understandable. Also, it keeps adhering to these lecture notes ad infinitum while solar physics evolves to more complexity.

VALIII atmosphere. Figure $\overline{8}$. temperature-height distribution. It is compared with other models in Figure $\overline{7} . \overline{3}$ on page $1{ }^{1} 4 \overline{9}^{\prime}$. The characteristic range of formation of many diagnostics is illustrated by the bracketed arrows. Table 8.24 specifies the VALIII quiet-Sun model in detail. Figure illustrates that at low temperatures where hydrogen is neutral the electrons come from elements with lower first ionization energy.

VALIII radiative transfer. Figures 8 nazza et al. $(1981)$; there are more in the paper for other wavelengths. These plots detail the relative contributions to the extinction coefficient $\left(r_{\mathrm{ab}} ; \sigma\right.$ for scattering, "add" for additional line extinction that simulates the ultraviolet line haze) and the emissivity $\left(r_{\mathrm{em}}\right)$ in the upper panels. The lower panels show the height variations of $B_{\nu}, J_{\nu}$ and $S_{\nu}$, the emergent intensities for $\mu=1$ and $\mu=0.3$, and the intensity contribution function $\mathrm{d} I / \mathrm{d} h=(\mathrm{d} / \mathrm{d} h) \int S \exp (-\tau) \mathrm{d} \tau$. Since VALIII strictly obeys the laws of plane-parallel radiative transfer treated in these lecture notes, you should be able to interpret every detail of these plots. Exercise $\underline{9}_{1}^{\overline{9}}$ on page $\overline{2} \overline{3} \overline{3} \overline{1}, 1$ may help you to achieve this.

VALIII energy budget. Figure $\overline{8}, \overline{1} 2$ and Figure $\overline{8} \cdot \overline{1}$, show the major radiative imbalances in the VALIII atmosphere by $(7,35)$ on page $115 \overline{5}$ for the hydrogen lines and continua; Figure 8.13 shows cooling rates for $\mathrm{H}^{-}$and the strongest non-hydrogen spectral lines. The VALIII energy budget is not balanced because the VALIII atmosphere is not in radiative equilibrium. It was determined empirically from inversion of the observed solar disk-center brightness temperatures shown in Figs. 8.2 .5 assuming plane-parallel stratification and hydrostatic equilibrium, without requiring energy conservation. The total radiative losses in Figure 8.13 therefore represent a deficit that is implicitly balanced by the non-specified mechanical energy input from below ("chromospheric heating"). Thus, the star VALIII possesses a chromosphere and transition region that are empirically constrained.

The strongest lines in the VALIII chromosphere are CaII $\mathrm{H} \& \mathrm{~K}$ and $\mathrm{Mg}$ II $\mathrm{h} \& \mathrm{k}$. They have resonance-line NLTE photon losses which represent appreciable energy loss

and well-known laws of physics into staggering arrays of beautiful and interesting phenomena. Like the human genome: just 100000 variables permitting already $2^{100000} \approx 10^{30000}$ combinations when regarded as simple on/off switches - whereas the universe contains only about $10^{80}$ particles. The building blocks are simple but nature makes much out of them.

${ }^{5}$ Additional rate plots have been published by Avrett $(1985)$ for the MACKKL model of Figure page 149 . It is a slightly modified VALIII-like atmosphere with a warmer temperature minimum. 
to the chromosphere. The losses extend far down from their $\tau \approx 1$ Eddington-Barbier heights marked in Figure $\overline{8}$. loses photons, but its contribution to the cooling is balanced by heating in the Balmer continuum (Figure 8.12). The latter feeds on the $J_{\nu}>B_{\nu}$ imbalance seen for nearultraviolet wavelengths in Figure 8.10 . The transition region (far left) is dominated by H I Lyman- $\alpha$.

\subsection{Stellar continua}

Time for the stars, finally. Having discusssed the solar and VALIII continua in detail, this section is limited to graphical displays of the variation that results from changes in effective temperature and gravity. This is first done for the continuous extinction, than for the emergent spectrum.

Stellar classification. Appendix B of Gray the basic stellar parameters $T_{\text {eff }}, M_{v}, M / M_{\odot}, R / R_{\odot}, \log g$ and $\langle v \sin i\rangle$ as well as the corresponding colors $B-V, b-y$ and $V-R$ as function of the spectral type and luminosity classification.

Continuous extinction. Hot stars (spectral types $\mathrm{O}$ and $\mathrm{B}$ ) have photospheres in which hydrogen is fully ionized, making $\mathrm{H}^{-}$negligible and Thomson scattering the major opacity source. In O stars helium is ionized so that He II bound-free and free-free transitions are

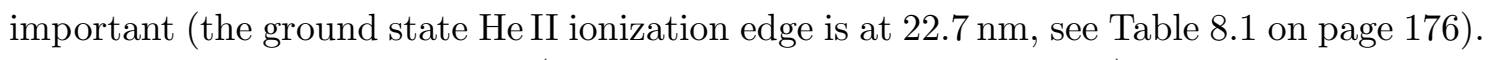

He I bound-free transitions (ground-state threshold at $50.4 \mathrm{~nm}$ ) constitute an important extinction contributor in the photospheres of A stars, as do ions of C, N, O etc. and $\mathrm{HI}$ bound-free and free-free transitions.

In the photospheres of stars cooler than the Sun, negative molecular ions comparable to $\mathrm{H}^{-}$become important: $\mathrm{H}_{2}^{-}, \mathrm{CN}^{-}, \mathrm{C}_{2}^{-}, \mathrm{H}_{2} \mathrm{O}^{-}$. There is also Rayleigh scattering from neutral $\mathrm{H}$ atoms and $\mathrm{H}_{2}$ molecules.

Figure 18.14 , from to $\mathrm{HI}, \mathrm{H}^{-}$and $\mathrm{He}^{-}$in the photospheres of three dwarf stars with wavelength. The extinction coefficients are plotted per neutral hydrogen atom in whatever level, so that the

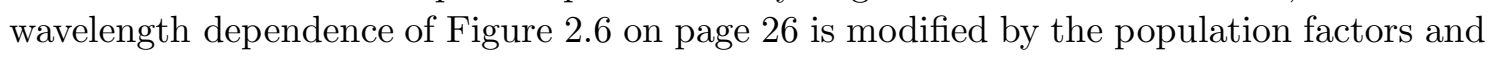
their temperature dependence. The different bound-free contributions are also added up in this case. In the top panel (a solar-type dwarf) the extinction in the longer-wavelength $\mathrm{HI}$ edges is reduced considerably by their small Boltzmann factors and is dwarfed by the

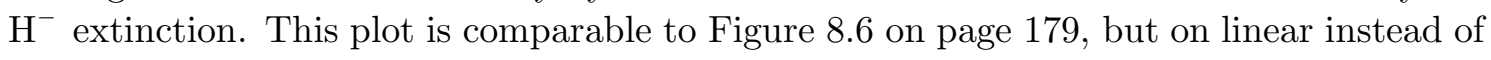
logarithmic scales, less rich and less confusing. The A dwarf (middle panel) has roughly equal contributions from $\mathrm{H}^{-}$and $\mathrm{HI}$ in the visible. The $\mathrm{B}$ dwarf (bottom) gets all its continuous extinction from HI bound-free transitions. It isn't hot enough yet to have a significant contribution from He II edges.

Vitense diagrams. Figures 8.15 , confusograms for different $T_{\mathrm{e}}$ (with $\theta \equiv 5040 / T_{\mathrm{e}}$ ). More are to be found in

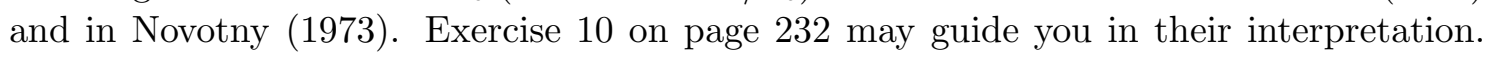
$M$ stars are not represented because molecular extinction was not included in Vitense's calculations. 

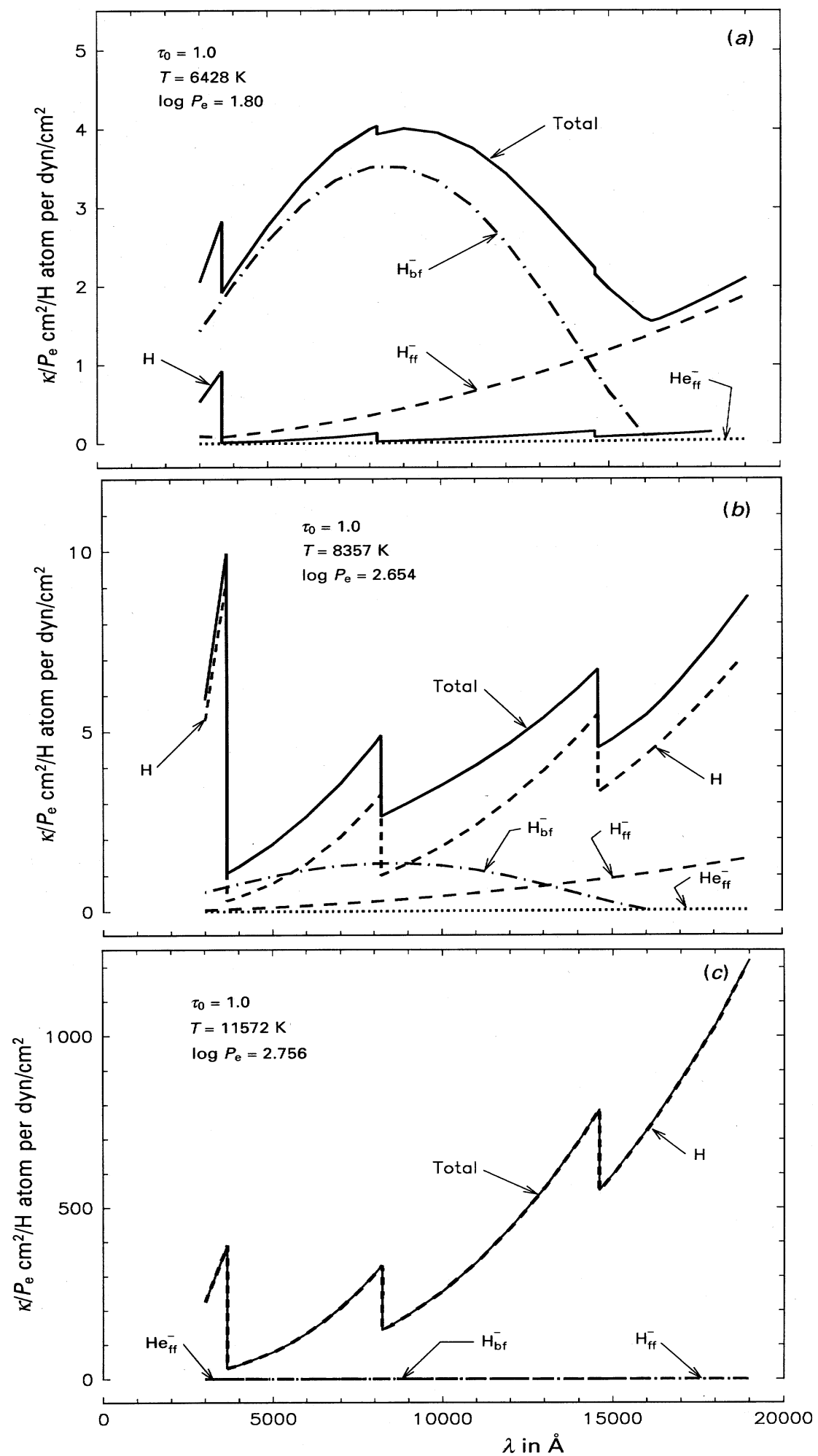

Figure 8.14: Continuous extinction coefficients $\kappa_{\nu}^{c}$ from hydrogen and helium, per neutral hydrogen atom and per unit electron pressure, for the depth $\tau_{0}=1$ (continuum optical depth at $\lambda=5000 \AA$ ) in the photospheres of three dwarf stars. The coefficients $\kappa$ are here measured per neutral hydrogen atom in whatever state of excitation, assuming Boltzmann population ratios, and normalized by the electron pressure because the $\mathrm{H}^{-} / \mathrm{H}$ density ratio scales with $P_{\mathrm{e}}$. The cross-sections are in units of $10^{-26} \mathrm{~cm}^{2}$, not $\mathrm{cm}^{2}$ as specified in the $y$-axis labels. Panel (a) is for the Sun, panel (b) for a late A dwarf, panel (c) for a late B dwarf. The curves do not extend beyond the Balmer edge at left where the neglected metal edges become important. From 'Gray $(1992)$ '. 


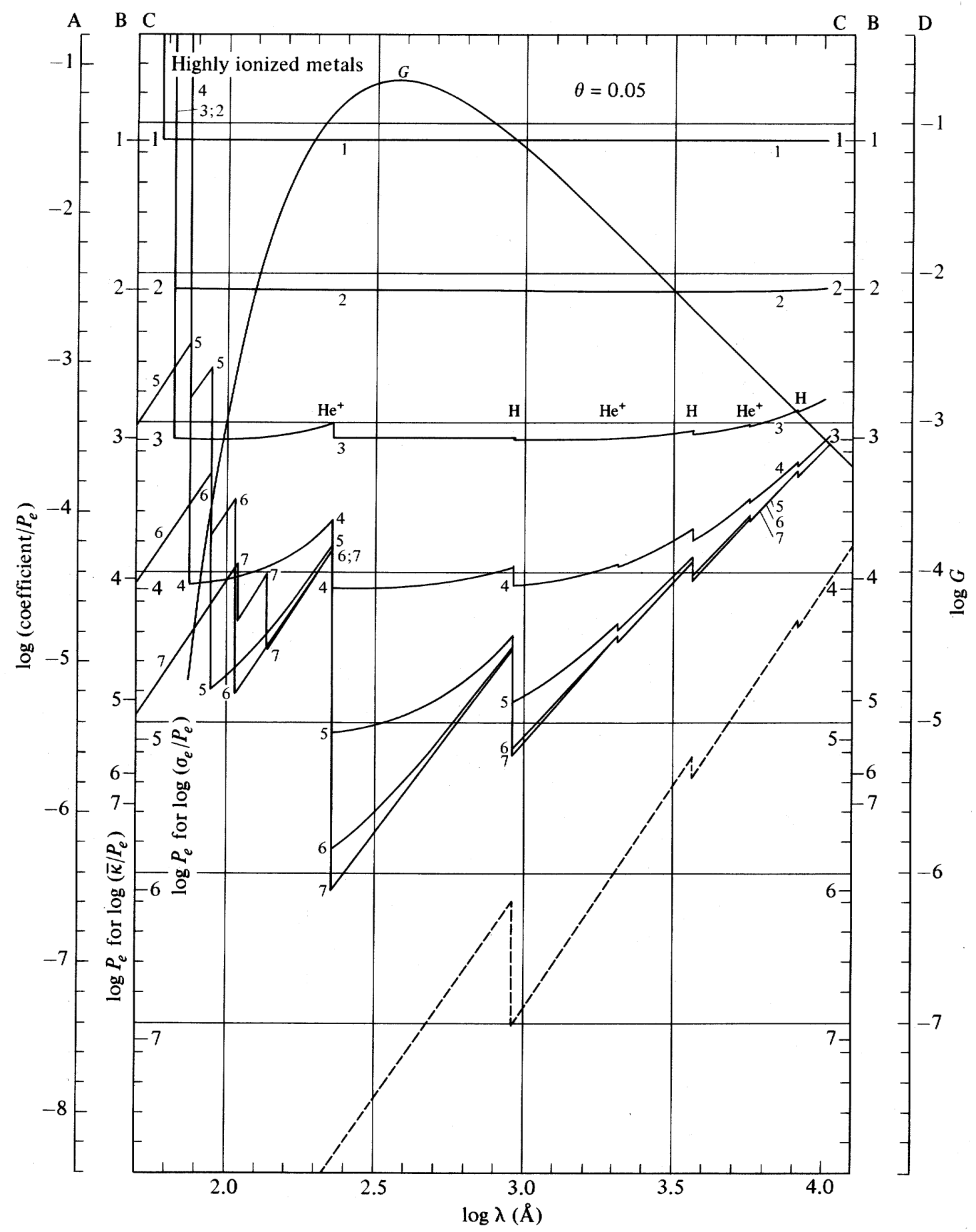

Figure 8.15: Stellar extinction curves from Nitense $(1951)$. Labeling as in Fig. $\overline{8} \cdot \overline{6}$ (note the shift of the $\mathrm{x}$

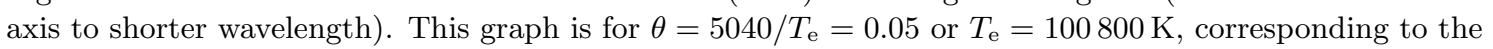
effective temperature of the hottest O stars. From Novotny $(1973)$. 


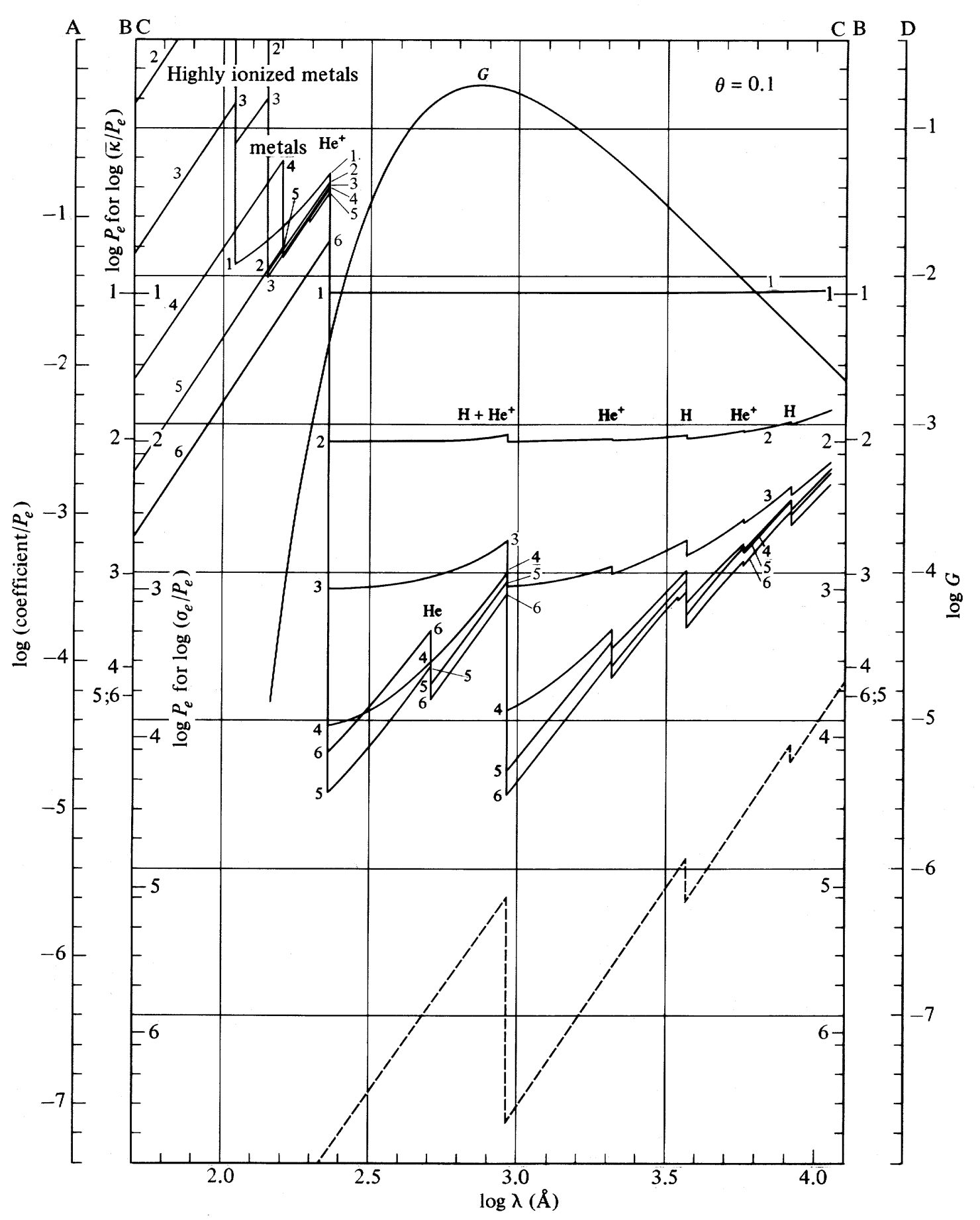

Figure 8.16: Stellar extinction curves from $\theta=0.1$ or $T_{\mathrm{e}}=50400 \mathrm{~K}$, representing 05 stars. From Novotny $(1973) !$ 


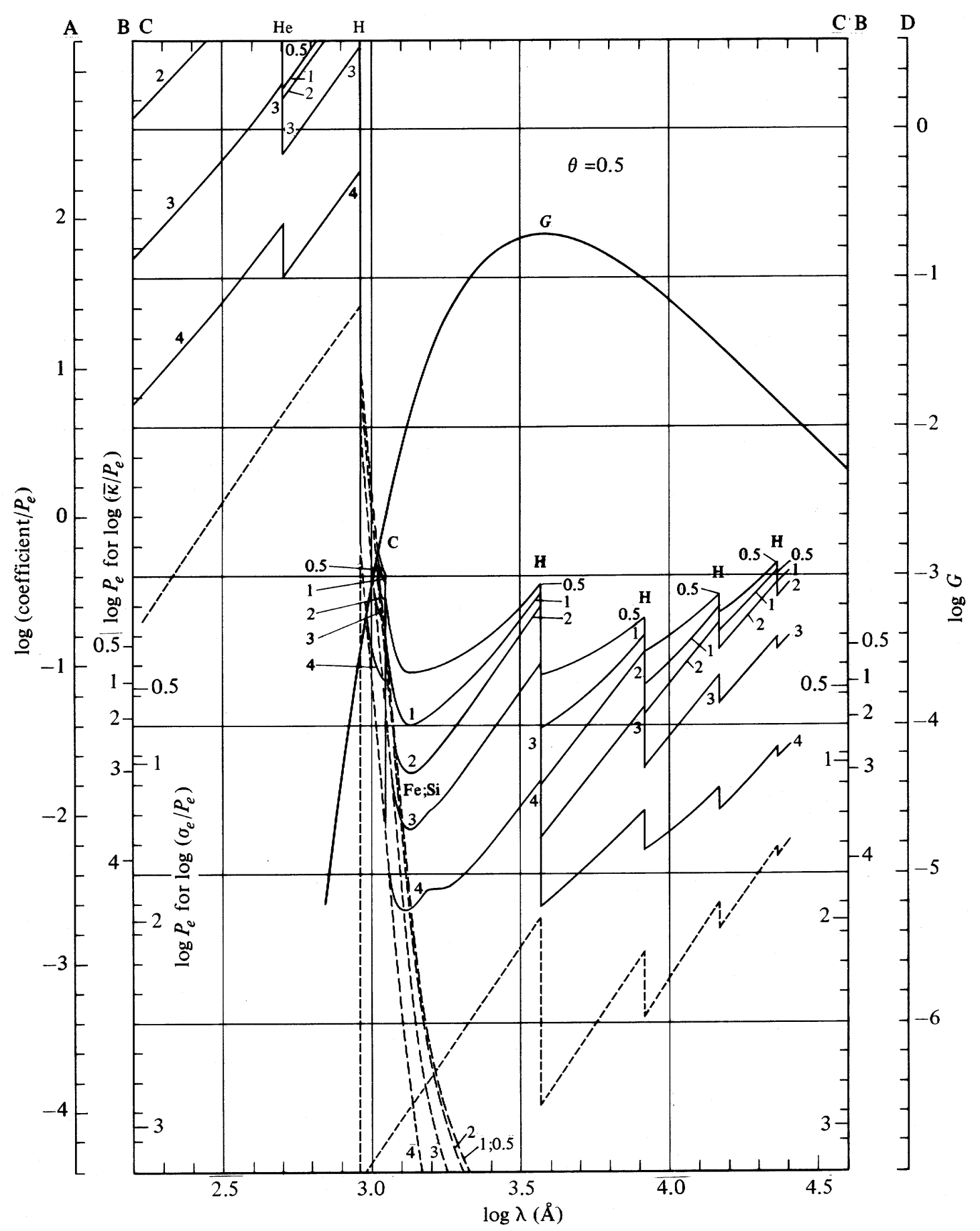

Figure 8.17: Stellar extinction curves from $\underline{\theta}=\underline{0.5}$ or $T_{\mathrm{e}}=10080 \mathrm{~K}$, representing the photosphere of B9.5 stars and the solar chromosphere. From Novotny $(1973)$ 


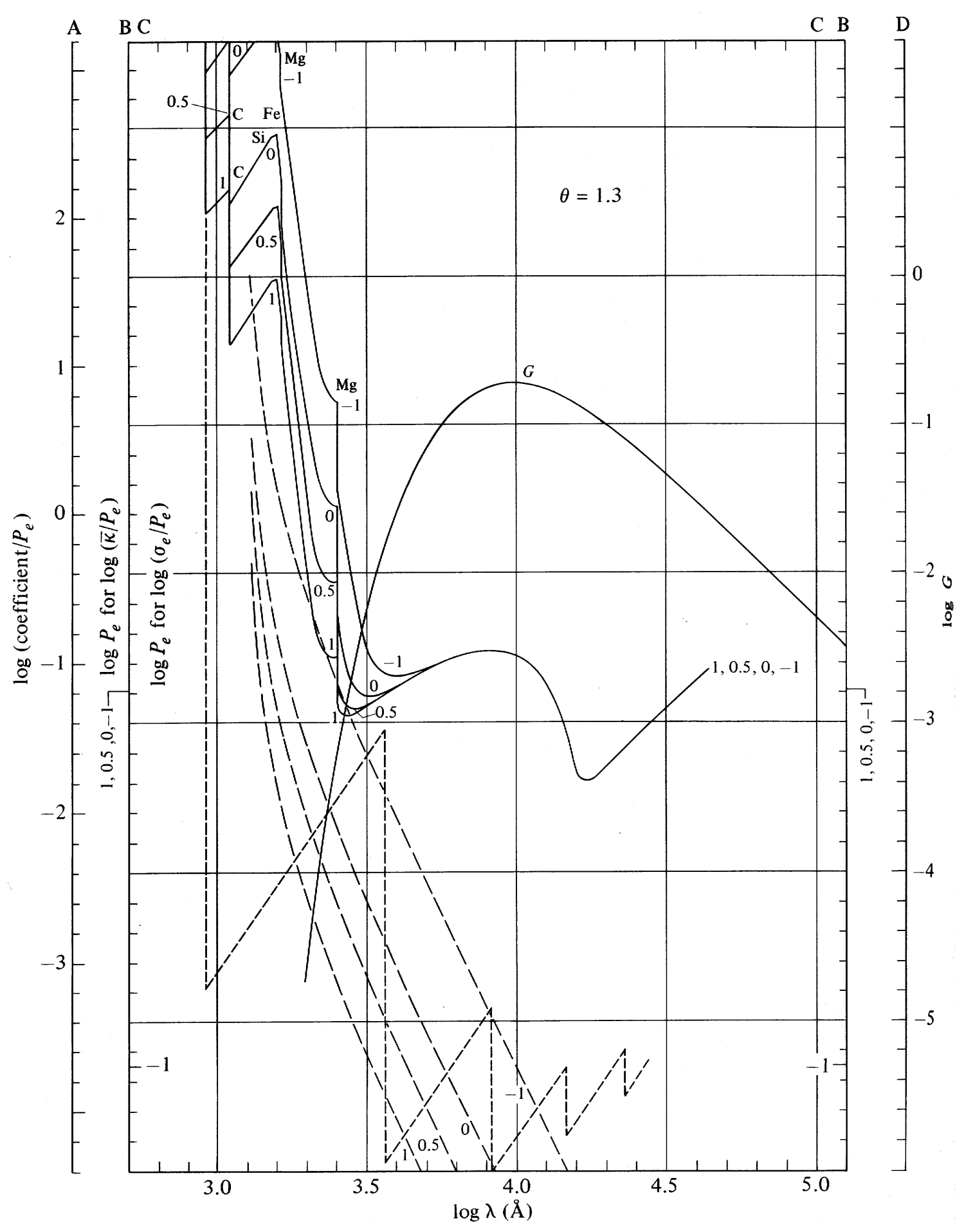

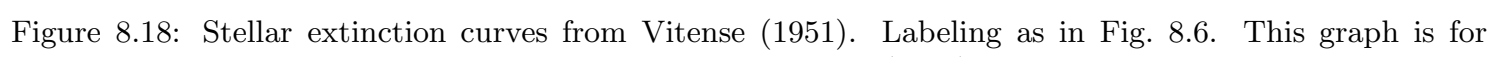
$\theta=1.3$ or $T_{\mathrm{e}}=3880 \mathrm{~K}$, representing $\mathrm{K} 8$ stars. From Novotny $(1973)_{\mathrm{L}}$ 


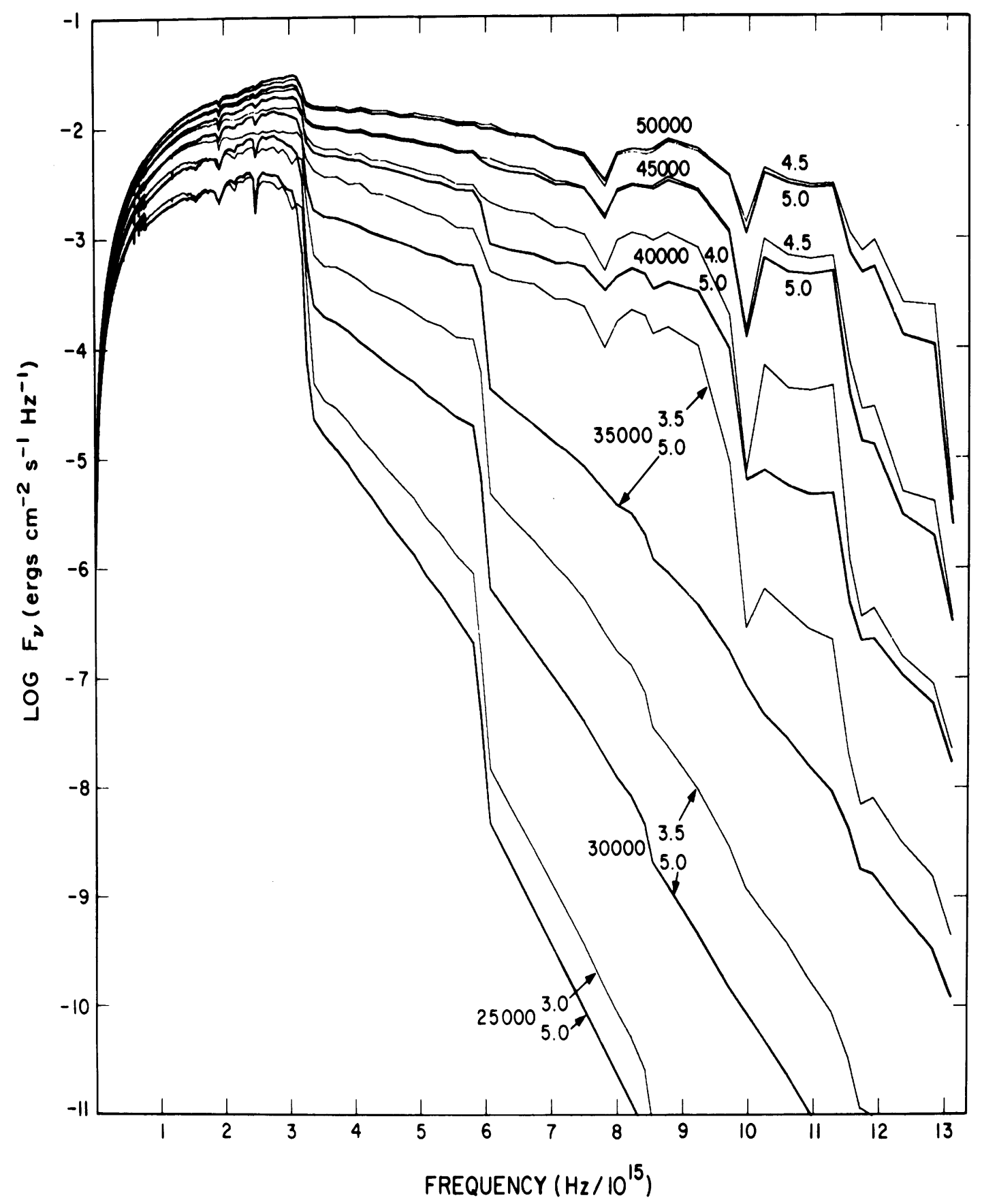

Figure 8.19: Computed LTE-RE flux spectra $\log F_{\nu}$ against $\nu$ for solar abundances and the indicated values of $T_{\text {eff }}$ and $\log g$, for hot stars with $T_{\text {eff }}=25000-50000 \mathrm{~K}$. The x axis specifies the frequency $\nu$ in units of $10^{15} \mathrm{~Hz}$, with the wavelength in nm given by $\lambda=300 / \nu$. The H I Balmer and Lyman bound-free thresholds are at $\nu=0.8$ and $\nu=3.3$, respectively, the H I Ly $\alpha$ line (plus the overlapping He II 2-4 line) is at $\nu=2.5$, the HeI bound-free threshold is at $\nu=6.0$, the He II threshold is at $\nu=13.0$, the He II 1-2 line is at $\nu=9.9$, the HeII $1-3$ line is at $\nu=11.7$. From Turucz $(1979)^{\prime}$ 

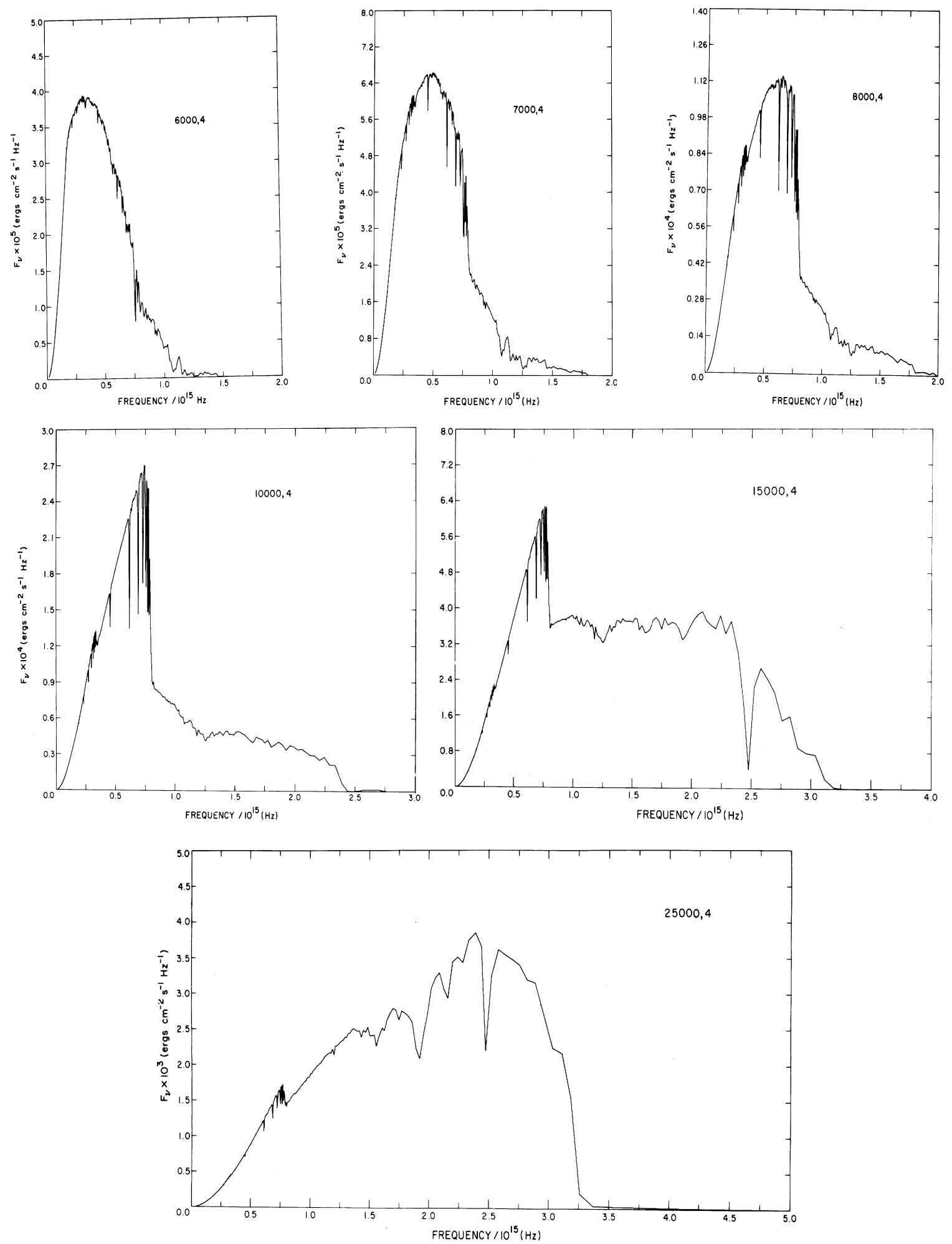

Figure 8.20: Computed LTE-RE flux spectra $F_{\nu}$ against $\nu$ for solar abundances, $\log g=4$ and $T_{\text {eff }}=$ $6000,7000,8000,10000,15000,25000 \mathrm{~K}$. In the x-axis units of $10^{15} \mathrm{~Hz}$ the Lyman, Balmer and Paschen bound-free thresholds are at $\nu=3.3, \nu=0.8$ and $\nu=0.4$, respectively. The H I Ly $\alpha$ line is at $\nu_{-}=2.5_{2}$ the Balmer series extends over $\nu=0.25-0.8$ and the Paschen series over $\nu=0.25-0.4$. From TKurucz $(1979)^{\prime}$ 

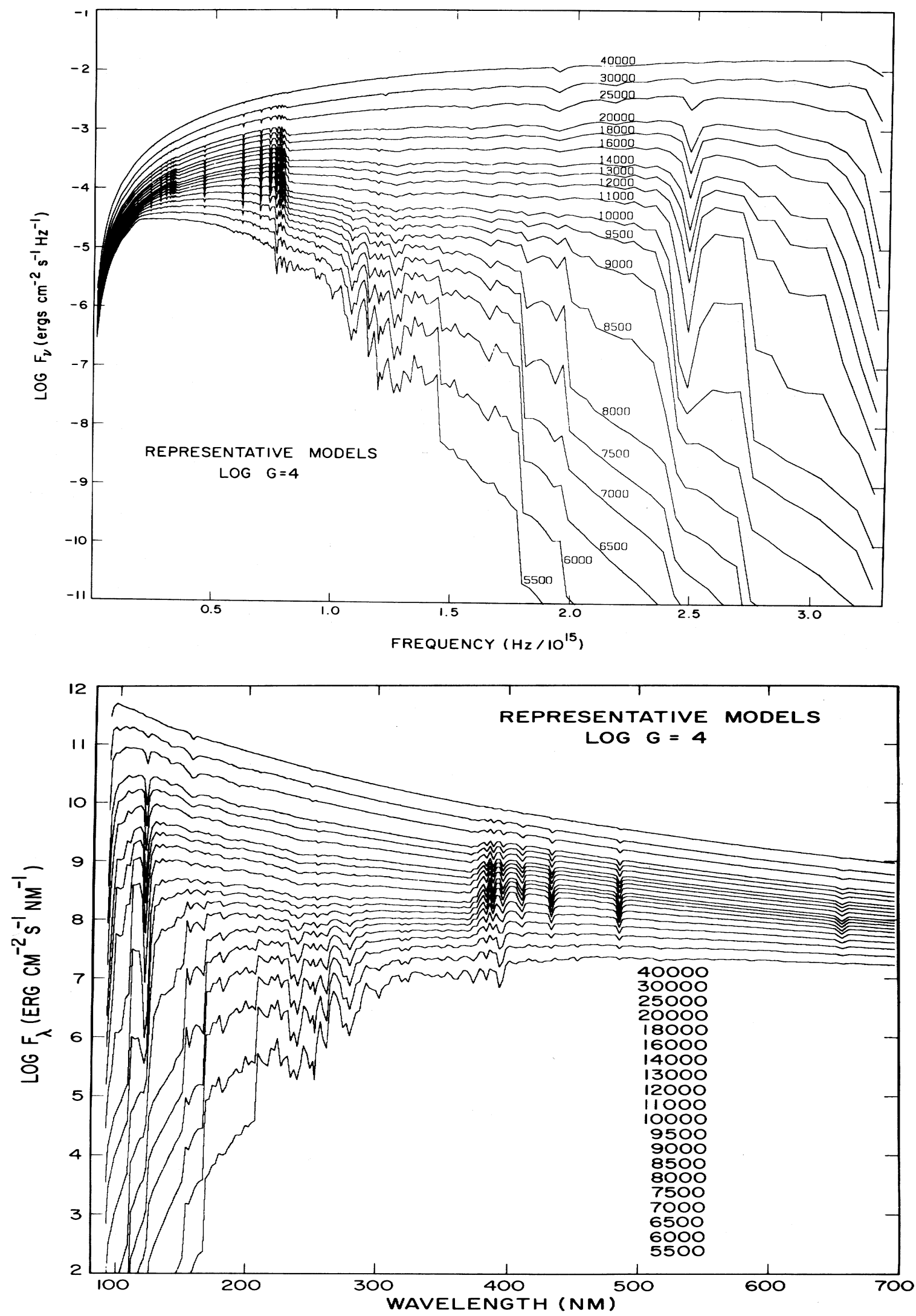

Figure 8.21: Computed LTE-RE flux spectra $F_{\nu}$ against $\nu$ and $F_{\lambda}$ against $\lambda$ for solar abundances, $\log g=4$ and the indicated values of $T_{\text {eff }}$, for the part of the spectrum redward of the Lyman ionization limit. From 'Kurucz_(1979)' 

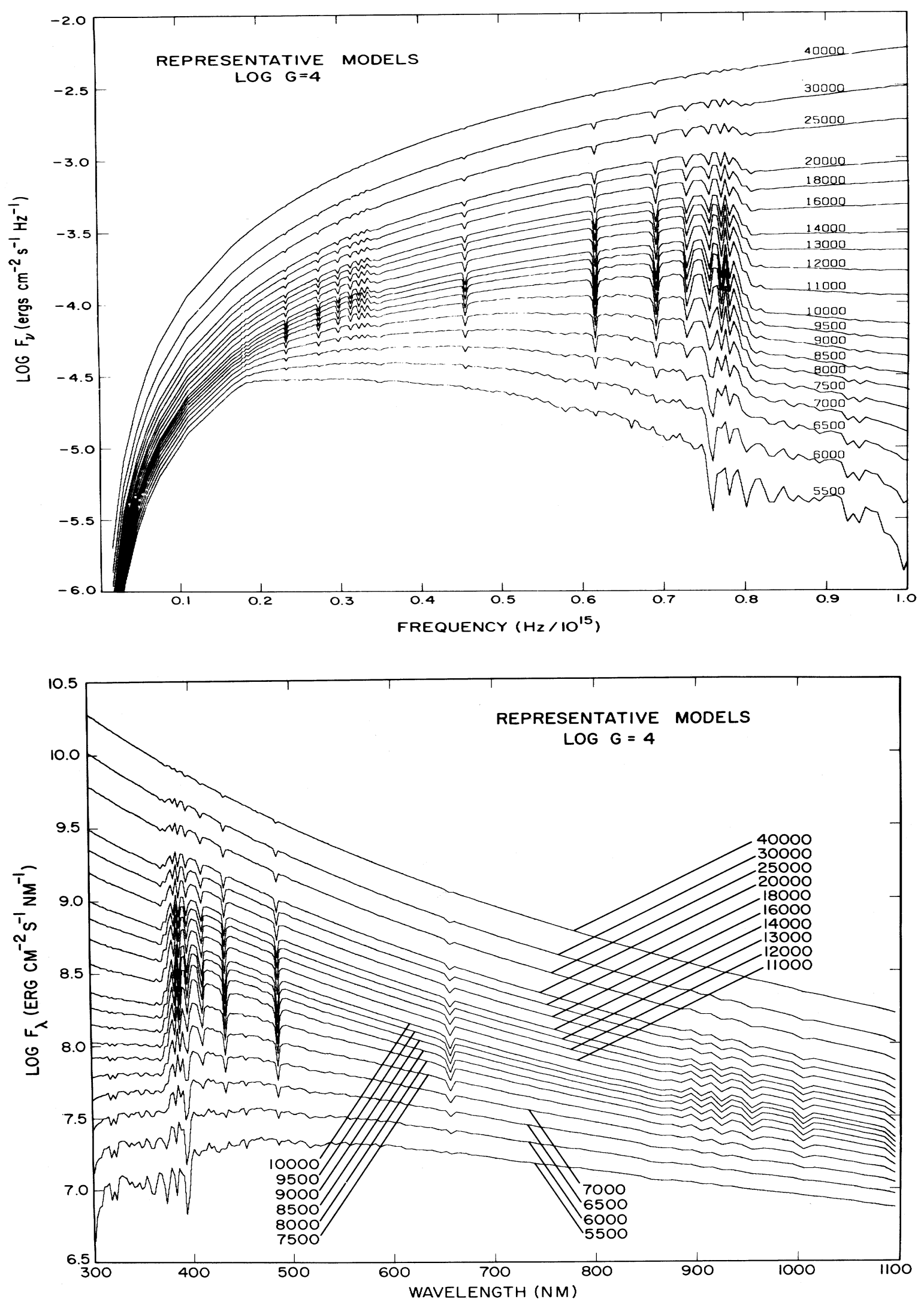

Figure 8.22: Computed LTE-RE flux spectra $F_{\nu}$ against $\nu$ and $F_{\lambda}$ against $\lambda$ for solar abundances, $\log g=4$ and the indicated values of $T_{\mathrm{eff}}$, for the optical part of the spectrum. From Kurucz $(1979)^{\prime}$. 


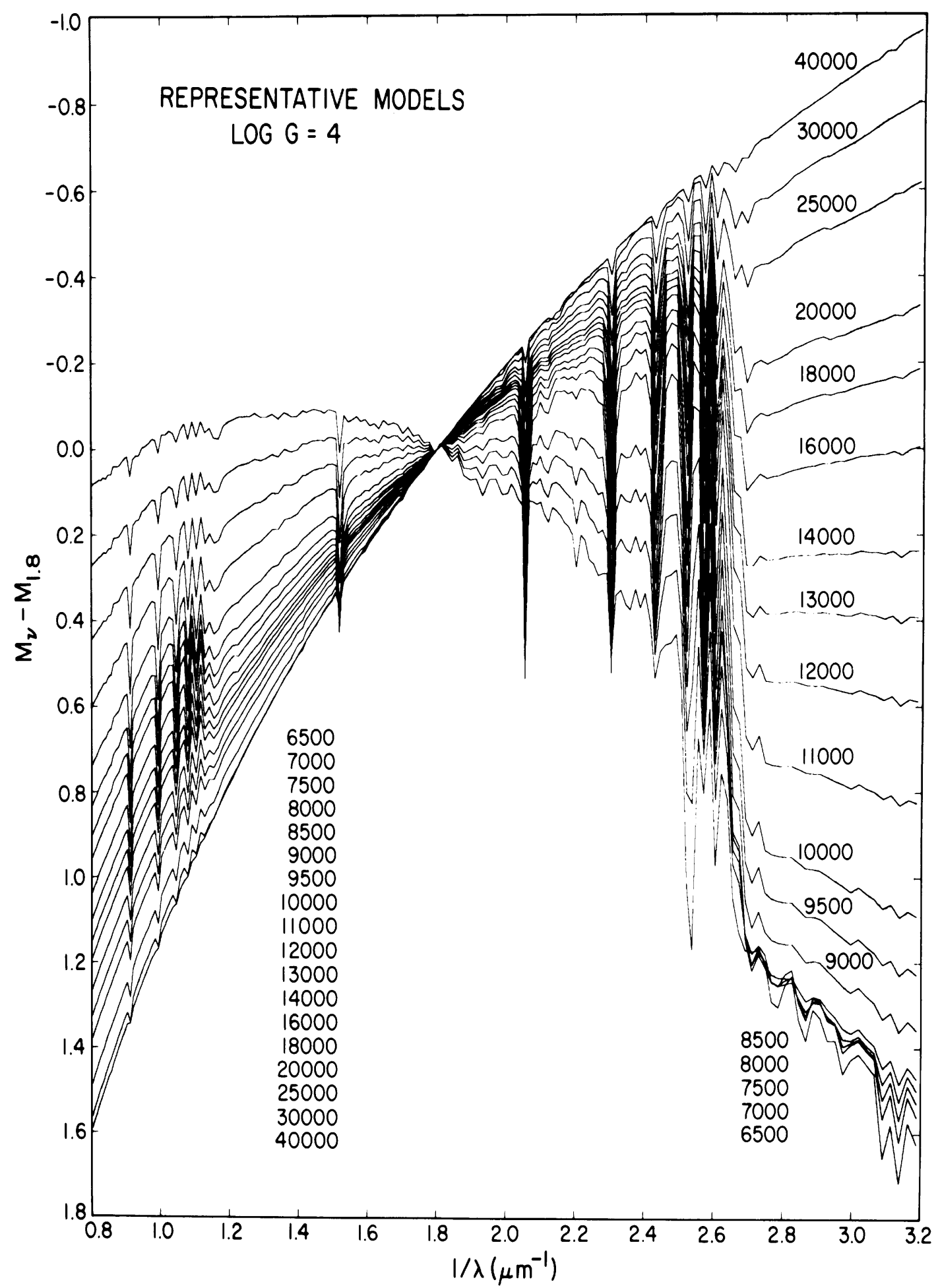

Figure 8.23: Computed color $M_{\mathrm{V}}-M_{1.8}$ against inverse wavelength $1 / \lambda$ in the optical part of the spectrum for the indicated values of $T_{\text {eff }}$. The inverse wavelength $1 / \lambda=1.8 \mu \mathrm{m}^{-1}(\lambda=0.555 \mu \mathrm{m}=555 \mathrm{~nm})$ corresponds approximately to the passband of the $V$ filter in the $U B V$ photometry system. The H I Paschen and_Balmer series limits are at $1 / \lambda=1.22 \mu \mathrm{m}^{-1}$ and $1 / \lambda=2.74 \mu \mathrm{m}^{-1}$, respectively. From 'Kurucz $(1979)^{\prime}$. 
Hydrogen and helium edges. The graphs in 8.15 hydrogen bound-free extinction at high temperature and high pressure, for which the total extinction displays the H I edges faithfully. Note the occurrence of He II edges at and between the HI edges (most coincident edges in the graphs are labeled only by $\mathrm{H}$ ). This pattern is due to the hydrogen-like atomic structure of $\mathrm{He}^{+}$. For hydrogen-like ions, the series limits obey the Rydberg formula:

$$
\lambda_{n} \sim 91.16 \frac{n^{2}}{Z^{2}}
$$

with $\lambda_{n}$ the edge wavelength in $\mathrm{nm}, n$ the principal quantum number and $Z$ the atomic number of the nucleus. Therefore Table 18 shows alternate equalities. They are not exact because the reduced masses $\left(m_{\text {nucleon }} m_{\mathrm{e}}\right) /\left(m_{\text {nucleon }}+m_{\mathrm{e}}\right)$ of H I and He II are not equal, but very close. The Pickering series of He II bound-bound transitions corresponds similarly in alternate lines with the H I Balmer series (Table 8.11 vanish for lower temperatures where the total extinction is set by $\mathrm{H}^{-}$for $\log \lambda>3.5$.

\begin{tabular}{lc|ccccccccc}
\hline$n$ & & 1 & 2 & 3 & 4 & 5 & 6 & 7 & 8 & $\ldots$ \\
\hline H I & $n^{2} / Z^{2}$ & 1 & 4 & 9 & 16 & 25 & 36 & 49 & 64 & $\ldots$ \\
He II & $n^{2} / Z^{2}$ & $1 / 4$ & 1 & $9 / 4$ & 4 & $25 / 4$ & 9 & $49 / 4$ & 16 & $\ldots$ \\
\hline
\end{tabular}

Table 8.3: H I and He II edge wavelengths divided by 91.16. After iNovotny (1973)L

Balmer jump. The Balmer jump at $\lambda=364.7 \mathrm{~nm}$ had special importance in classical stellar spectrometry because it is the only $\mathrm{H} \mathrm{I}$ edge in the visible. The jump-like extinction increase at the edge may result in very different height of formation of the continuum just above and just below the edge wavelength (Gray Fig. 9.13). In addition, the increase has different sensitivity to the state parameters between different spectral types. $\mathrm{F}$ and $\mathrm{G}$ stars have (Böhm-Vitense 1989 p. $89 \mathrm{ff})$ :

$$
\frac{\kappa(\lambda>364.7)}{\kappa(\lambda<364.7)}=\frac{\sigma_{\lambda}\left(\mathrm{H}^{-}\right) N\left(\mathrm{H}^{-}\right)}{\sigma_{\lambda}\left(\mathrm{H}^{-}\right) N\left(\mathrm{H}^{-}\right)+\sigma_{\lambda}^{\mathrm{B}} N_{\mathrm{H}}(n=2)}<1,
$$

with $\sigma_{\lambda}^{\mathrm{B}}$ the Balmer bound-free extinction per particle below $\lambda=364.7 \mathrm{~nm}$. The difference in the correction for stimulated emission between the two wavelengths is neglected. The density of $\mathrm{H}^{-}$ions $N\left(\mathrm{H}^{-}\right)$is related to the neutral hydrogen density $N_{\mathrm{H}} \approx N_{\mathrm{H}}(n=1)$ through the Saha equation as in $\left(\overline{8} . \overline{2}^{2}\right)$ on page $1 \overline{1} \bar{z}_{1}^{\prime}$. It is sensitive to both the electron density $N_{\mathrm{e}}$ and the electron temperature $T_{\mathrm{e}}$. The density of Balmer-extinguishing H I atoms is related to $N_{\mathrm{H}}(n=1)$ through the Boltzmann factor similarly to the Paschen ratio in (1, $\left.\overline{1}^{-}\right)$on page $\overline{1} 8 \overline{0}_{1}^{\prime}$, sensing only $T_{\mathrm{e}}$. For very cool stars, the $\mathrm{H}^{-}$contribution dominates so that the opacity ratio is unity; they show no Balmer jump. With increasing temperature the $\mathrm{H}^{-}$contribution decreases while the Balmer contribution increases; the opacity ratio drops below unity and the spectrum shows a Balmer jump.

For F stars $\left(T_{\text {eff }}=6000-7500 \mathrm{~K}\right)$ the Balmer contribution dominates over the $\mathrm{H}^{-}$ contribution so that

$$
\frac{\kappa(\lambda>364.7)}{\kappa(\lambda<364.7)} \sim \frac{\sigma_{\lambda}\left(\mathrm{H}^{-}\right) N_{\mathrm{H}}(n=1)}{\sigma_{\lambda}^{\mathrm{B}} N_{\mathrm{H}}(n=2)} N_{\mathrm{e}} T_{\mathrm{e}}^{-3 / 2} \mathrm{e}^{h \nu / k T} \sim \frac{\sigma_{\lambda}\left(\mathrm{H}^{-}\right)}{\sigma_{\lambda}^{\mathrm{B}}} N_{\mathrm{e}} T_{\mathrm{e}}^{-3 / 2} \mathrm{e}^{2 h \nu / k T} .
$$


The F-star Balmer jump varies ${ }_{1-1}^{\sigma_{1}}$ with both $T_{\mathrm{e}}$ and $N_{\mathrm{e}}$. For increasing $N_{\mathrm{e}}$ the ratio increases towards unity, reducing the observed jump; the jump increases for increasing $T_{\mathrm{e}}$.

In yet hotter stars with $T_{\text {eff }}>9000 \mathrm{~K}$ (A2 and earlier) the $\mathrm{H}^{-}$extinction is negligible on both sides of the Balmer edge; the $\kappa(\lambda>364.7)$ then comes from the H I $n=3$ Paschen continuum so that:

$$
\frac{\kappa(\lambda>364.7)}{\kappa(\lambda<364.7)} \sim \frac{\sigma_{\lambda}^{\mathrm{P}} N_{\mathrm{H}}(n=3)}{\sigma_{\lambda}^{\mathrm{B}} N_{\mathrm{H}}(n=2)} \sim \mathrm{e}^{-h \nu / k T_{\mathrm{e}}} .
$$

For these, the jump depends on the temperature alone, decreasing for increasing temperature since the ratio increases towards unity. The jump also vanishes when Thomson scattering takes over. A graphical display of the Balmer jump sensitivity to temperature is given in Figure 8.20 on page $20 \overline{0}$. It illustrates that the jump increases over the $T_{\text {eff }}=6500-9000 \mathrm{~K}$ range and decreases for higher temperature.

Thomson scattering. At high temperature and low pressure the extinction is dominated by Thomson scattering. For example, the equality between the top parts of B and $\mathrm{C}$ ordinate scales in the $\theta=0.05$ plot in Figure 8.15 shows that Thomson scattering makes up all of the wavelength-averaged extinction for $\log P_{\mathrm{e}}<3$. The same is seen from the extinction curves. The top curve, marked with $\log P_{\mathrm{e}}=1$, is constant across the spectrum and sits at the ordinates $A=B=1$, meaning that $\sigma_{\mathrm{e}}$ makes up all the monochromatic extinction at every wavelength. The curve is constant because Thomson scattering is wavelength-independent. At these low pressures, hydrogen and helium are too far ionized to contribute significant opacity. Only at much higher pressure are the hydrogen and helium edges not fully washed out by the electron scattering.

Thomson scattering is a NLTE process as discussed in Section $\overline{4} . \overline{3} \overline{5}$ on page $\overline{1} \overline{0} \overline{6}_{r}^{\prime}$ Its source function equals $J_{\nu}$; the parameter $\varepsilon_{\nu}$ measures the relative probability of photon extinction by thermal bound-free or free-free continuum processes.

Kurucz flux spectra. Figures 8.190 .23 are taken from the 340 -page standard paper by i.jurucz (1979)!. They show stellar flux spectra in various formats that have been computed from LTE-RE model atmospheres tabulated in the same paper. Figure 8.19 gives a $\log F_{\nu}-\nu$ overview for hot stars, showing the influence of the surface gravity on the emergent spectra. Figures $8.20,82$, show flux spectra for dwarf stars $(\log g=4)$ in various representations. Figure 8.23 displays the spectral sensitivity of two-color photometric temperature determination. It illustrates the behavior of the Balmer jump discussed on

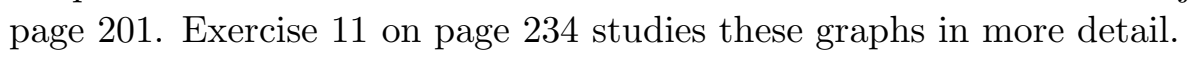

\footnotetext{
${ }^{6}$ The size of the Balmer jump is affected by NLTE effects in H I if these change the $n=2$ and/or $n=3$ populations. A likely situation in cool stars is that $n=2$ is underpopulated through overionization from a $J_{\nu}>B_{\nu}$ excess in the Balmer continuum (gradient steeper than the radiative-equilibrium gradient set in the visible as described on page 102i) whereas the $n=3$ level is overpopulated (from $J_{\nu}<B_{\nu}$ in the Paschen continuum and stronger collisional coupling to the overpopulated continuum $N_{\mathrm{p}}$ as discussed in Section 3.2.5il). A sensitive NLTE diagnostic is therefore given by the ratio of the Balmer and Paschen jumps in the observed spectrum (Section 7-5 of Mihalas 1970).

${ }^{7}$ Most of the 340 pages are tables specifying stellar atmospheric models. At the time, Kurucz made them available on punched cards and magnetic tape; as noted before, he now supplies model atmospheres on CD-ROM together with the programs that generate them and with enormous lists detailing atomic and molecular lines. The main change between his newer models and the 1979 grid is the inclusion of much more line opacity.
} 


\section{Chapter 9}

\section{Lines from Plane-Parallel Stars}

$\mathrm{T}$ his chapter treats spectral line formation in classical stellar atmospheres, more or less in the order of its historical development.

\subsection{Classical abundance determination}

In this section I treat classical methods for LTE abundance determination. These are still in heavy use by stellar spectroscopists who seek to determine the chemical composition of varied sorts of stars by adopting radiative-equilibrium model photospheres and LTE line formation, employing micro- and macroturbulence plus collisional damping enhancement factors as fitting parameters, and taking the relative area that spectral lines "absorb" out of the continuum as their yardstick. See 'Gustafsson and Jørgensen (1994), for a critical review. This industry started in the first half of this century; the concepts of the equivalent width of a spectral line and the curve of growth to measure its dependence on the amount of extinction were introduced by Minnaert and coworkers at Utrecht.

\subsubsection{Abundance}

The abundance $A_{\mathrm{E}}$ of element $\mathrm{E}$ (including all ionization stages) is measured as its nuclei number density $N_{\mathrm{E}}$ relative to the total hydrogen nuclei density $N_{\mathrm{H}}$ (including free protons and counting $\mathrm{H}_{2}$ double)

$$
A_{\mathrm{E}} \equiv \frac{N_{\mathrm{E}}}{N_{\mathrm{H}}}
$$

but it is usually specified on a logarithmic scale $A_{12}(\mathrm{E})$ with $A_{12}(\mathrm{H})=12$ :

$$
A_{12}(\mathrm{E}) \equiv \log N_{\mathrm{E}}-\log N_{\mathrm{H}}+12 .
$$

Stellar spectroscopists often relate stellar abundances to solar values, using square brackets to denote the logarithm of a stellar quantity minus the logarithm of that quantity in the Sun:

$$
[X] \equiv \log X_{\text {star }}-\log X_{\text {Sun }} .
$$

This is done particularly for the iron abundance of which the logarithmic ratio difference

$$
[\mathrm{Fe} / \mathrm{H}]=\log \left(N_{\mathrm{Fe}} / N_{\mathrm{H}}\right)_{\text {star }}-\log \left(N_{\mathrm{Fe}} / N_{\mathrm{H}}\right)_{\text {Sun }}
$$

is called the metallicity of a star. Young stars in the galactic disk have solar metallicity $[\mathrm{Fe} / \mathrm{H}] \approx 0$ while "metal poor" stars have $[\mathrm{Fe} / \mathrm{H}] \approx-1$ and Population II halo dwarfs that date back to really ancient times are "extreme metal deficient" with $[\mathrm{Fe} / \mathrm{H}] \approx-3$. 
The atmospheric abundance $A_{\mathrm{E}}$ of an element $\mathrm{E}$ enters the line extinction coefficient as a ratio to the hydrogen density in the lower-level population

$$
n_{l}=b_{l} n_{l}^{\mathrm{LTE}}=b_{l} \frac{n_{l}^{\mathrm{LTE}}}{N_{\mathrm{E}}} N_{\mathrm{H}} A_{\mathrm{E}},
$$

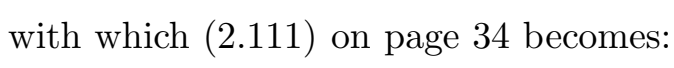

$$
\alpha_{\lambda}^{l}=\frac{\sqrt{\pi} e^{2}}{m_{\mathrm{e}} c} \frac{\lambda^{2}}{c} b_{l} \frac{n_{l}^{\mathrm{LTE}}}{N_{\mathrm{E}}} N_{\mathrm{H}} A_{\mathrm{E}} f_{l u} \frac{H(a, v)}{\Delta \lambda_{\mathrm{D}}}\left[1-\frac{b_{u}}{b_{l}} \mathrm{e}^{-h c / \lambda k T}\right]
$$

where the LTE population fraction $n_{l}^{\mathrm{LTE}} / N_{\mathrm{E}}$ is given by the Saha and Boltzmann distributions and the microturbulence $\xi_{\text {micro }}$ is hidden in the Dopplerwidth $\Delta \lambda_{\mathrm{D}}$ defined by $\left(\overline{3} . \overline{8} 0_{1}^{*}\right)$ on page $\overline{6} \overline{2}$.

\subsubsection{Curve of growth methods}

Traditionally, curves of growth are employed to derive element abundances. This usage is

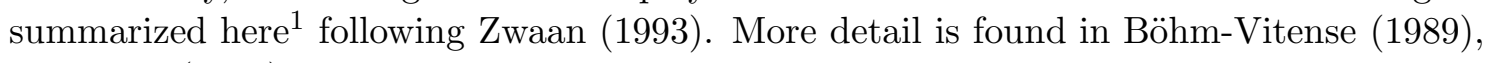
and 'GGray

Equivalent width. The equivalent width is defined for intensity by

$$
W_{\lambda}=\int_{\text {line }} \frac{I_{c}-I_{\lambda}^{l}}{I_{c}} \mathrm{~d} \lambda
$$

and for flux by

$$
W_{\lambda}=\int_{\text {line }} \frac{\mathcal{F}_{c}-\mathcal{F}_{\lambda}^{l}}{\mathcal{F}_{c}} \mathrm{~d} \lambda
$$

It measures the integrated dip of an absorption line (Figure 1.12 on page "equivalent width" because its value is the same as the width of a rectangular piece of spectrum that blocks the emergent intensity (flux) completely. The idea was that this area is a direct measure of the number of absorbing atoms that cause an absorption line. Obviously, this principle portrays an optically thin notion of line formation. The "curve of growth" represents a method to correct for optical thickness.

Schuster-Schwarzschild atmosphere. The original description for the formation of the Fraunhofer absorption lines in the solar spectrum interpreted these with a reversing layer that caused line extinction, much as a flame with sodium vapor shows dark NaID lines against a bright continuum source. The Schuster-Schwarzschild model has two layers: a deep one that emits the solar continuum, given by a Planck function, and a second one higher up that is the reversing layer and that causes the spectral lines. It is homogeneous and obeys LTE. The intensity of the continuum irradiation is given by $I_{c}=B_{\lambda}\left(T_{\mathrm{R}}\right)$ with $T_{\mathrm{R}}$ the radiation temperature, equal to the temperature of the deeper layer. The radiation that we observe is given by $(2 . \overline{4})$ as:

$$
I_{\lambda}=B_{\lambda}\left(T_{\mathrm{R}}\right) \mathrm{e}^{-\tau_{\lambda}}+B_{\lambda}\left(T_{\mathrm{L}}\right)\left(1-\mathrm{e}^{-\tau_{\lambda}}\right)
$$

\footnotetext{
${ }^{1}$ Although I concluded a 1984 paper with Ed van der Zalm by the following statement: "With this paper, on solar equivalent widths and the curve of growth, ends a Utrecht tradition which started 50 years ago when Minnaert and coworkers introduced these concepts". Curves of growth are indeed no longer used in solar physics, but they are still useful in stellar studies.
} 
with $T_{\mathrm{L}}$ the temperature of the reversing layer and $\tau_{\lambda}$ its optical thickness in a spectral line, given by (neglecting stimulated emission)

$$
\tau_{\lambda}=\sigma_{\lambda} N_{\mathrm{i}}=\frac{\sqrt{\pi} \mathrm{e}^{2}}{m_{\mathrm{e}} c} \frac{\lambda_{0}^{2}}{c} \frac{f}{\Delta \lambda_{\mathrm{D}}} N_{\mathrm{i}} H(a, v) \approx \tau_{\lambda_{0}} H(a, v)
$$

with $N_{i}$ the integrated column density in $\mathrm{cm}^{-2}$ of the line-absorbing particles in the lower level $i$ along the line of sight through the reversing layer, $\sigma_{\lambda}$ the extinction coefficient per particle in level $i, f$ the oscillator strength of the transition, $\Delta \lambda_{\mathrm{D}}$ the Doppler width and $a$ and $v$ the Voigt parameters defined by $(3.7 \overline{1})$ and $(3.70)$. The $\approx$ sign signifies that $H(a, v) \approx 1-a$ at $\lambda=\lambda_{0}$ rather than unity. The relative line depression $D_{\lambda}$ is

$$
D_{\lambda} \equiv \frac{I_{\mathrm{c}}-I_{\lambda}}{I_{\mathrm{c}}}=\frac{B_{\lambda}\left(T_{\mathrm{R}}\right)-B_{\lambda}\left(T_{\mathrm{L}}\right)}{B_{\lambda}\left(T_{\mathrm{R}}\right)}\left(1-\mathrm{e}^{-\tau_{\lambda}}\right)=D_{\max }\left(1-\mathrm{e}^{-\tau_{\lambda}}\right)
$$

with

$$
D_{\max } \equiv \frac{B_{\lambda}\left(T_{\mathrm{R}}\right)-B_{\lambda}\left(T_{\mathrm{L}}\right)}{B_{\lambda}\left(T_{\mathrm{R}}\right)}
$$

the maximal depression for very strong lines. The equivalent width is:

$$
W_{\lambda}=D_{\max } \int_{\text {line }}\left(1-\mathrm{e}^{-\tau_{\lambda}}\right) \mathrm{d} \lambda
$$

The righthand graph in Figure 1 in shows how this result depends on the line-center optical thickness $\tau_{\lambda_{0}}$ of the reversing layer. The lefthand part illustrates the shapes of corresponding spectral lines.
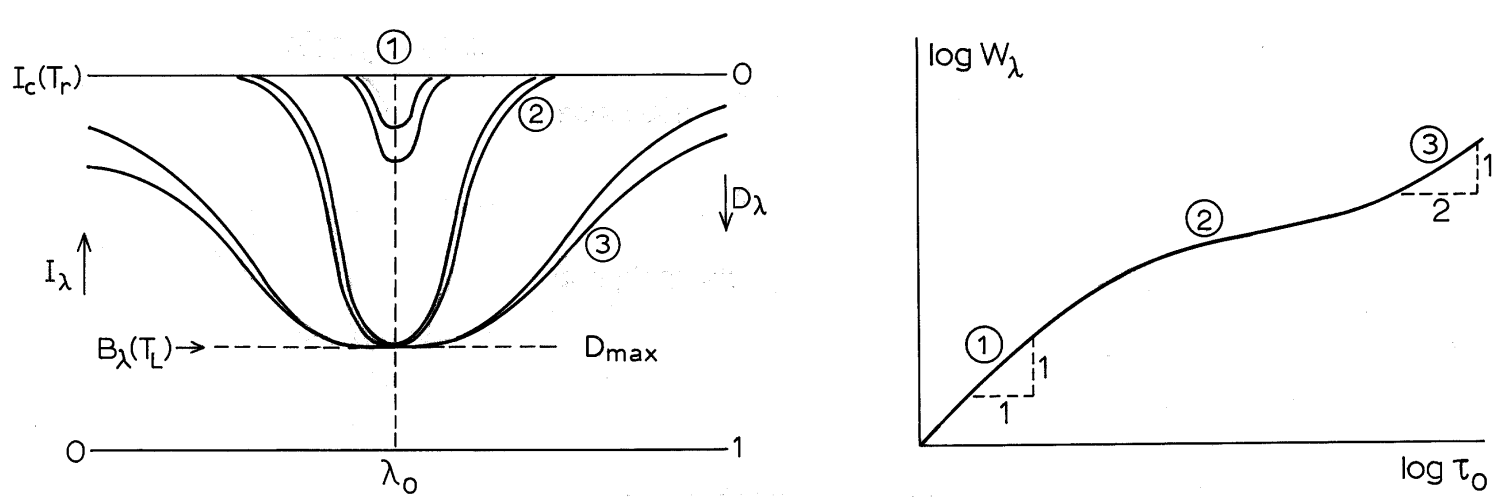

Figure 9.1: Line profiles and curve of growth in the Schuster-Schwarzschild model. Weak lines have Gaussian shapes. Their area grows linearly with the amount of extinction. The curve of growth flattens when lines become saturated because the reversing layer becomes optically thick at line center. The curve of growth rises again when optically thin damping wings develop. This plot is for absorption lines with $T_{\mathrm{L}}<T_{\mathrm{R}}$. From Zwaan $(1993) !$

Weak lines. For $\tau_{\lambda} \ll 1$ we may use $\exp \left(-\tau_{\lambda}\right) \approx 1-\tau_{\lambda}$ so that $D_{\lambda} \approx D_{\max } \tau_{\lambda}$. The Voigt profile may be approximated by a Doppler profile because the opacity is too small to map the damping wings into the emergent spectrum. Say that we measure the line depth with a precision of $10^{-3}$ of the continuum intensity and that $a=0.1$ so that the Doppler core in Figure 1.1 extends to $H(a, v) \approx 0.01$; a weak line with $D_{\lambda}=0.1$ then reaches the 
measurement noise just where its wings should become noticeable. Replacing $H(a, v)$ by $\exp \left[-\left(\Delta \lambda / \Delta \lambda_{\mathrm{D}}\right)^{2}\right]$ with area $\sqrt{\pi} \Delta \lambda_{\mathrm{D}}$ gives:

$$
\begin{aligned}
D_{\lambda} & \approx D_{\max } \tau_{\lambda_{0}} \mathrm{e}^{-\left(\Delta \lambda / \Delta \lambda_{\mathrm{D}}\right)^{2}} \\
W_{\lambda} & \approx D_{\max } \tau_{\lambda_{0}} \sqrt{\pi} \Delta \lambda_{\mathrm{D}}=\frac{\pi \mathrm{e}^{2}}{m_{e} c} \frac{\lambda_{0}^{2}}{c} f D_{\max } N_{\mathrm{i}}
\end{aligned}
$$

The equivalent width increases linearly with the product of the particle density $N_{i}$ and the oscillator strength $f$. The curve of growth plotting $\log W_{\lambda}$ against $\log \tau_{\lambda_{0}}$ has an initial slope 1:1 called the Doppler part (Figure $\overline{9} \cdot \overline{1})$. The linear increase results from the optical thinness of the reversing layer $\mathfrak{L}_{\mathbf{r}}^{\mathcal{Z}_{1}}$

Saturated lines. For $\tau_{\lambda_{0}}>1$ the line cannot grow deeper than the saturation depth $D_{\max }$ with $I_{\nu_{0}}=B_{\nu}\left(T_{\mathrm{L}}\right)$ at line center. The width of the line increases for increasing $\tau_{\lambda_{0}}$ and therefore:

$$
W_{\lambda} \approx Q D_{\max } \Delta \lambda_{\mathrm{D}}
$$

with the factor $Q$ roughly estimated from Figure ${ }^{1}$ to be $Q=2-4$. This is the shoulder of the curve of growth.

Strong lines. For $\tau_{\lambda_{0}} \gg 1$ the core doesn't change anymore. The line-center contribution is fixed at $D_{\max }$ in (9.13). However, the far wings have $\tau_{\lambda}<1$. These may yet grow in optically-thin fashion and so contribute additional equivalent width. For sufficiently large layer thickness $\tau_{\lambda_{0}}$ the optically thin far wings contribute appreciably because they then map the damping part of $H(a, v) \approx a /\left(\sqrt{\pi} v^{2}\right)=(a / \sqrt{\pi})\left(\Delta \lambda_{\mathrm{D}} / \Delta \lambda\right)^{2} \sim 1 / \Delta \lambda^{2}$, a drop-off with $\Delta \lambda$ that its much less steep than the exponential decay of the Doppler core. In the damping part of $H(a, v)$ we may write:

$$
\tau_{\lambda}=\tau_{\lambda_{0}} \frac{a}{\sqrt{\pi} v^{2}}=\tau_{\lambda_{0}} \frac{a}{\sqrt{\pi}} \frac{\Delta \lambda_{\mathrm{D}}^{2}}{\Delta \lambda^{2}}
$$

and with the transformation $u^{2}=\Delta \lambda^{2} /\left(\tau_{\lambda_{0}}(a / \sqrt{\pi}) \Delta \lambda_{\mathrm{D}}^{2}\right)$

$$
\begin{aligned}
W_{\lambda} & =D_{\max } \int_{\text {line }}\left(1-\mathrm{e}^{-\tau_{\lambda}}\right) \mathrm{d} \lambda \\
& =D_{\max } \Delta \lambda_{\mathrm{D}} \sqrt{\tau_{\lambda_{0}}(a / \sqrt{\pi})} \int_{\text {line }}\left(1-\mathrm{e}^{-1 / u^{2}}\right) \mathrm{d} u \\
& \sim D_{\max } \Delta \lambda_{\mathrm{D}} \sqrt{\tau_{\lambda_{0}} a}
\end{aligned}
$$

\footnotetext{
${ }^{2}$ In bad lingo, stellar spectroscopists often call a line itself "optically thin" or "optically thick", for example "H $\alpha$ from $\alpha$ Cen is thick". In the Schuster-Schwarzschild picture that means $\tau_{\mathrm{L}}<1$ or $\tau_{\mathrm{L}}>1$. In general, it means that the line-center optical thickness of the material between the observer and the depth $\tau_{\nu_{0}}^{c} \approx 1$ where the continuum originates has $\tau_{\nu_{0}}^{l}<1$ or $\tau_{\nu_{0}}^{l}>1$. The usage is sloppy because it is not the line that possesses opacity but the gas, and because the radiation on its way out doesn't care whether it is blocked by line or continuous extinction, it senses the total extinction. A star has optical thickness $\tau \simeq 10^{11}$ (page 190 ) and is therefore thick at the frequency of any line whatsoever. Sometimes even a continuum is called "optically thin"; for example the X-ray emission of the solar corona in YOHKOH images. Bad lingo again since the Sun is not optically thin for $\mathrm{X}$ rays either, only for neutrinos. However, the corona is optically thin. The X-ray intensity from the layer with $\tau \approx 1$ is very low due to the low temperature of the sub-coronal layers; $T_{\mathrm{R}}$ in $(1.9 .9-1)$ is therefore negligible compared to the coronal $T_{\mathrm{L}}$. In this case the Schuster-Schwarzschild reversing-layer picture is a good one - except that the corona doesn't reverse, is very far from LTE, and is very inhomogeneous.
} 
where $a$ and $\Delta \lambda_{\mathrm{D}}$ are taken out of the integral because it is limited to the narrow line (and therefore finite). Thus, the damping part of the curve of growth scales as $W_{\lambda} \sim \sqrt{\tau_{\lambda_{0}} a} \sim$ $\sqrt{f N_{i} \gamma}$, producing the $1: 2$ slope of the righthand part of the $\log -\log$ curve in Figure

This description was evidently derived from absorption-tube experiments in physics laboratories. It holds equally for layers with higher temperature than the background temperature. The limiting intensity $B_{\lambda}\left(T_{\mathrm{L}}\right)$ then lies above the continuum intensity $B_{\lambda}\left(T_{\mathrm{R}}\right)$ and the lines are in emission. It is indeed a good description for flame experiments or absorption-tube experiments as long as the emitting or absorbing gas may be assumed homogeneous and produces no continuous background ${ }_{-\mathbf{L}}^{33_{1}}$ It is generally a bad description for stellar line formation.

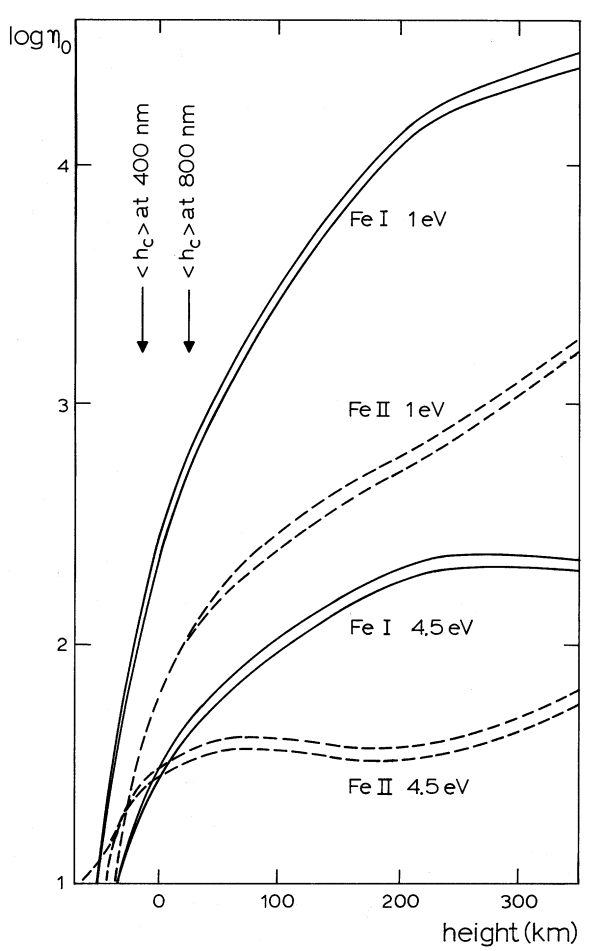

Figure 9.2: The logarithm of the line-center line-to-continuous extinction ratio $\eta_{0}=\kappa_{\lambda_{0}}^{l} / \kappa_{\lambda_{0}}^{c}$ for iron lines with lower-level excitation energy $\chi=1 \mathrm{eV}$ and $\chi=4.5 \mathrm{eV}$ in the solar photosphere. Solid: Fe I lines; dashed: Fe II lines. The upper curve of each pair is for $\lambda=800 \mathrm{~nm}$, the lower for $\lambda=400 \mathrm{~nm}$. The arrows mark the two mean heights of continuum formation at the two wavelengths. Each pair of curves has been shifted vertically over an arbitrary amount. The curves are representative for most metal lines in the visible part of the solar spectrum. From "Rutten and van der Zalm $(1984)^{\prime}$

Milne-Eddington atmosphere. We now know that the solar gas is well mixed. Line extinction and continuum extinction occur everywhere, not split between two distinct lay-

\footnotetext{
${ }^{3}$ Such laboratory gases are used to measure the oscillator strengths needed for stellar abundance analysis. The same assumptions and the same problems affect these that also affect stellar spectrometry. For example, the solar iron abundance had for a long time a strange factor ten difference between its value in the photosphere and its value in the corona. It turned out that departures from LTE in the laboratory sources had caused errors in the $g f$ determinations for the photospheric lines. Nowadays, iron oscillator strengths are measured from radiative decay times with laser excitation, a more secure method. The precision gets to the 0.1 dex level.
} 
ers. Weak lines originate from the same layer where the continuum escapes, whereas stronger lines have a representative Eddington-Barbier formation depth $\tau_{\nu}=1$ that moves outward with increasing line extinction. The classical alternative to the SchusterSchwarzschild model is to assume that there is no local variation between line and continuum extinction by requiring that the extinction ratio $\eta_{\lambda} \equiv \kappa_{\lambda}^{l} / \kappa_{\lambda}^{c}$ and the profile function $\varphi\left(\lambda-\lambda_{0}\right)$ do not vary with height. This is the Milne-Eddington approximation. It is also rough (see Figure $\overline{9} .2 \overline{2})$, but for most lines a better description than the Schuster-Schwarzschild simplification. Assuming LTE and a linear Planck function $B_{\lambda}\left(\tau_{c}\right)=B_{0}+b_{c} \tau_{c}$ then gives for the depression of the flux profile:

$$
\begin{aligned}
B_{\lambda}\left(\tau_{\lambda}\right) & =B_{0}+\frac{b_{\mathrm{c}}}{1+\eta_{\lambda}} \tau_{\lambda} \\
F_{\lambda}(0) & =B_{0}+\frac{b_{\mathrm{c}}}{1+\eta_{\lambda}} \frac{2}{3} \\
D_{\lambda} & \equiv \frac{F_{\mathrm{c}}(0)-F_{\lambda}(0)}{F_{\mathrm{c}}(0)} \\
& =\frac{(2 / 3) b_{\mathrm{c}} \eta_{\lambda} /\left(1+\eta_{\lambda}\right)}{B_{0}+(2 / 3) b_{\mathrm{c}}} \\
& =D_{\max } \frac{\eta_{\lambda}}{1+\eta_{\lambda}}
\end{aligned}
$$

with $D_{\max }=(2 / 3) b_{\mathrm{c}} /\left(B_{0}+(2 / 3) b_{\mathrm{c}}\right)$. The equivalent width of the flux profile has, with $\eta_{v}=\eta_{0} H(a, v)$ and the derivation given on page $332 \mathrm{ff}$ of iMinăas

$$
\begin{aligned}
W_{\lambda} & =\int_{\text {line }} D_{\lambda} \mathrm{d} \lambda=D_{\max } \Delta \lambda_{\mathrm{D}} \int_{\text {line }} \frac{\eta_{v}}{1+\eta_{v}} \mathrm{~d} v \\
\frac{W_{\lambda}}{D_{\max } \Delta \lambda_{\mathrm{D}}} & =\int_{\text {line }} \frac{\eta_{0} H(a, v)}{1+\eta_{0} H(a, v)} \mathrm{d} v \\
\frac{W_{\lambda}}{D_{\max } \Delta \lambda_{\mathrm{D}}} & =\sqrt{\pi} \eta_{0} \quad \text { for } \eta_{0} \ll 1 \\
\frac{W_{\lambda}}{D_{\max } \Delta \lambda_{\mathrm{D}}} & =2-4 \text { for } \eta_{0}>1 \\
\frac{W_{\lambda}}{D_{\max } \Delta \lambda_{\mathrm{D}}} & =\sqrt{\pi^{3 / 2} a \eta_{0}} \quad \text { for } \eta_{0} \gg 1 .
\end{aligned}
$$

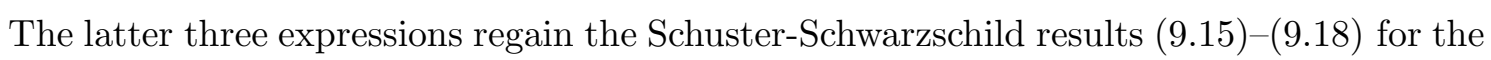
Doppler, shoulder and damping parts of the curve of growth, respectively, by substituting $\eta_{0}$ for $\tau_{\lambda_{0}}$.

Curve of growth fitting. The curve of growth stratagem is to simultaneously find the abundance $A_{\mathrm{E}}$, the damping parameter $a$, a representative excitation temperature $T_{\text {exc }}$ and the microturbulence $\xi_{\text {micro }}$. One plots the measured equivalent widths along a $\log \left(W_{\lambda} / \lambda\right)$ ordinate (dividing $W_{\lambda}$ by $\lambda$ because $\Delta \lambda_{\mathrm{D}}=\lambda \xi_{0} / c$ ) against

$$
\log X=\log C+\log \left(g f \lambda_{0}\right)-\chi 5040 / T_{\text {exc }}
$$

where $g f=g_{i} f$ and the excitation energy $\chi$ is in $\mathrm{eV}$. The statistical weight $g$ and the last term come from the Boltzmann population factor

$$
N_{\mathrm{i}} \sim g_{\mathrm{i}} \mathrm{e}^{-E_{\mathrm{i}} / k T}=g_{\mathrm{i}} 10^{-\chi_{\mathrm{i}} 5040 / T_{\text {exc }}}
$$


while the parameter $C$ contains unknowns as $D_{\max }$, microturbulence, the Saha population factor, the continuous extinction and the element abundance $A_{\mathrm{E}}$. Pieces of the curve of growth are now obtained by plotting $\log \left(W_{\lambda} / \lambda\right)$ against $\log (g f \lambda)$ for sets of lines that all belong to the same multiplet and therefore differ in $g f$ but not in the other parameters ${ }_{\llcorner}^{7 !}$. The horizontal shifts between the different pieces of curve that one obtains for different multiplets are then ascribed to the excitation term $\chi 5040 / T_{\text {exc }}$ where $\chi$ is known. By shifting all the pieces together so that the scatter is minimized, and then shifting the result to a computed model curve of growth, one derives a mean excitation temperature $T_{\text {exc }}$, the value of the microturbulence from the height of the shoulder, the value of the damping parameter $\gamma$ from the location of the damping part, and finally the element abundance $A_{\mathrm{E}}$ from $C$.

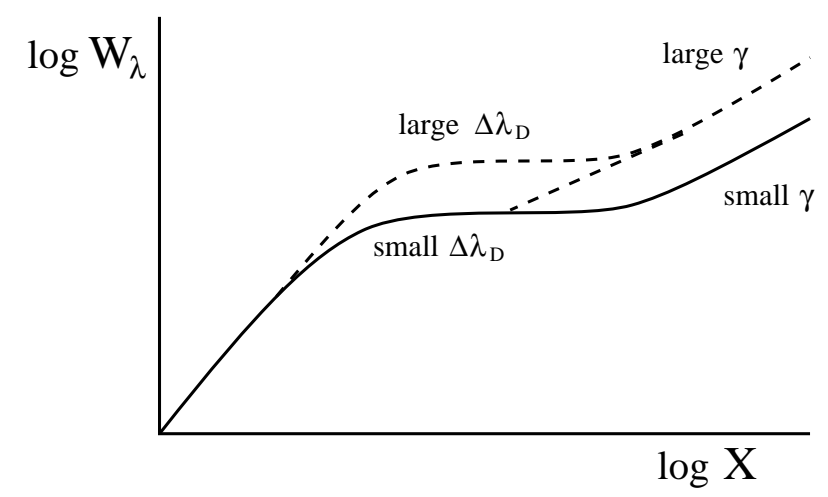

Figure 9.3: Schematic sensitivities of the curve of growth. Increasing the Doppler width raises the shoulder because the width of the line increases so that saturation occurs at larger line area. Increasing the damping width $\gamma$ results in earlier onset of the 1:2 damping part because the optically-thin wings add line area without saturation. After 'Böhm-Vitense $(1989) !$

An extensive description of how these procedures work in practice is given by '- Vitense (1989), while 'Gray (1992)', discusses the dependences of the curve of growth to various parameters in detail. Figure width (microturbulence) and the damping part for larger $\gamma$ (or Van der Waals fudge factor). Figure $\overline{9} . \overline{4}$ shows a measured curve of growth.

\subsubsection{LTE line synthesis}

Parameters. Most stellar spectroscopists fit observed equivalent widths nowadays per line, using a spectral line synthesis code and a theoretical LTE-RE model such as the ones in Figure 7 to compute each line. They then vary the element abundance until it fits the observed line strength $W_{\lambda} / \lambda$. A few use NLTE codes, but most stick to LTE for simplicity. The parameters in such an LTE-RE procedure are limited to the choice of model, the element abundance, the microturbulence and the Van der Waals damping enhancement factor. This is a rather limited set of parameters; no wonder that the contestants tend to dispute each other's choices like the sails of a windmill chasing each other (iKostik et al.'. $\left.19966^{\prime}\right)$.

\footnotetext{
${ }^{4}$ This role of the product $g f$ explains why curve-of-growth abundance determiners always list boundbound transition probabilities in the form of "gf-values".
} 


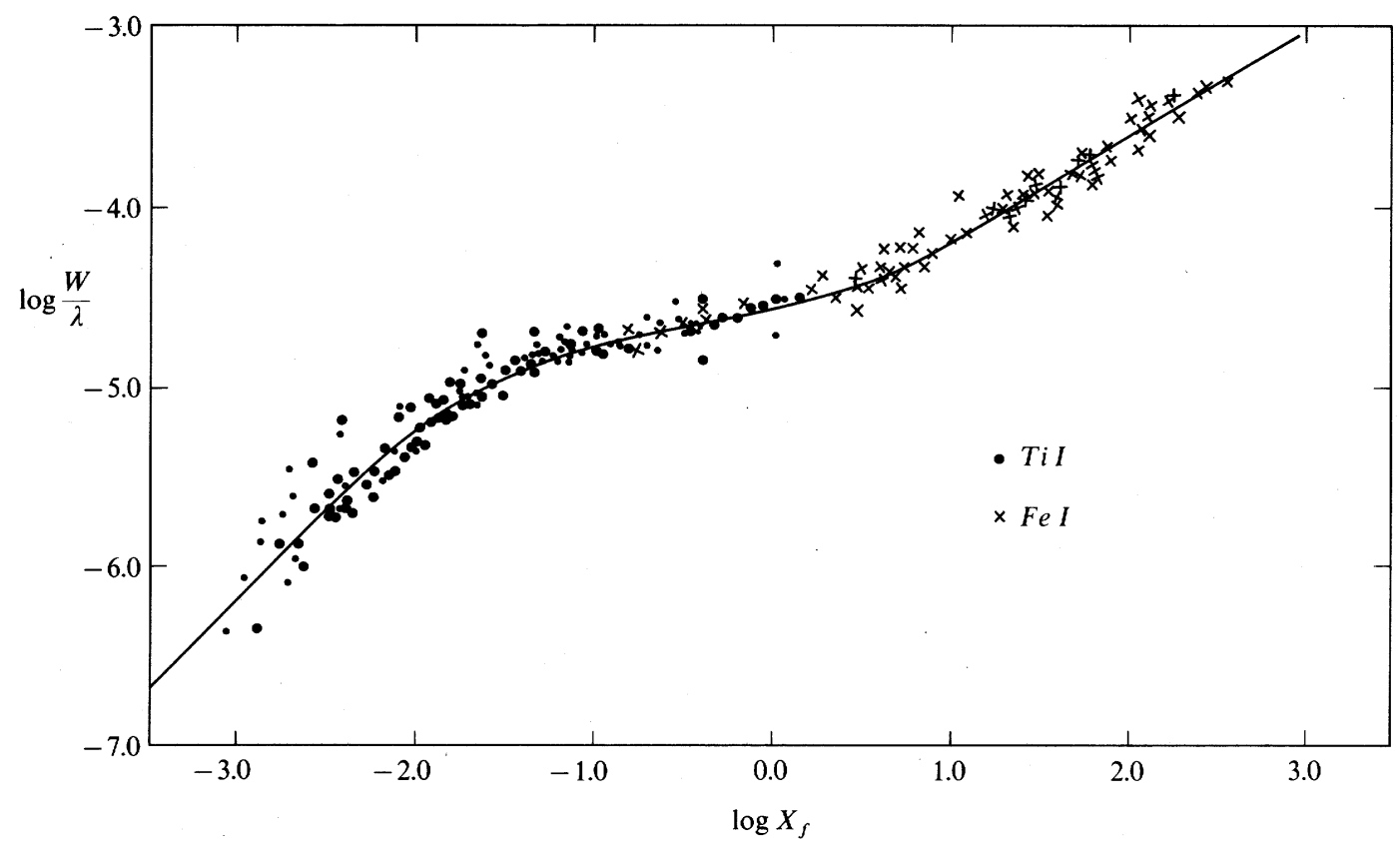

Figure 9.4: Empirical curve of growth for solar Fe I and Ti I lines. Taken from iMihalas (1970), who took it from 'Wright $(1948)$. Wright measured the equivalent widths of 700 lines in the Utrecht Átlas.

HOLMUL photosphere. Solar abundance determiners invariably use the HOLMUL model of the solar photosphere rather than VALIII. It is shown in Figure $\overline{7} \cdot \bar{\prime}$ on page $1 \overline{4} \bar{q}$. It was determined by Holweger and Müller $(1974)$, by inverting the observed line-center brightness temperatures of optical Fe I lines into $T(\tau)$ relations assuming LTE. It does not possess a chromospheric temperature rise because solar Fe I lines do not have self-reversed emission peaks in their cores $s_{1 \cdot r}^{51}$ It represents a characteristic Fe I excitation temperature as defined by $(2 . \overline{1} \overline{2} \overline{8})$ on page $\overline{3} \overline{7}_{1}$ rather than the electron temperature. The strongest Fe I lines have NLTE scattering source functions, rather like the NaID ones discussed in Section $1 \overline{1} 01$. The drop sufficiently deep below the Planck function that they do not feel the chromospheric temperature rise present in VALIII. The absence of a chromosphere makes the HOLMUL model suited to LTE abundance determination using lines that suffer similar photon losses and therefore have similar excitation temperature.

Validity. Such simple LTE spectral line modeling works remarkably well in the visible for cool-star metal lines such as Fe I lines. The reason is that these tend to come from complex spectra with much interlocking between the members of rich multiplets. The strongest members of each multiplet are in the ultraviolet. These suffer photon losses and have $S_{\nu}^{l} \ll B_{\nu}$ at their $\tau \approx 1$ escape depth. The weaker lines in the visible part of the spectrum share their upper and lower level populations with the stronger ones. At the height in the atmosphere where a weak line has $\tau_{\nu} \approx 1$, the strongest members of its

\footnotetext{
${ }^{5}$ The only lines in the solar visible that have emission reversals in their core are the Ca II H \& K lines. They are the strongest lines in the visible spectrum. Their tiny peaks are another story - a long one, given in Section 10.2. One of my better (but never cited) papers concerns an even tinier emission peak of an Fe II line close to Ca II H line center that sometimes appears in emission right at the center of the solar disk due to ultraviolet pumping (Cram et al. 1980i).
} 
multiplet (or of another multiplet at similar upper and lower energy to which it is coupled collisionally) are still close to detailed balance. The weaker multiplet members follow their population control, and therefore tend to have LTE line source functions at their height of formation.

Invalidity. Even if one restricts the analysis to weak multiplet members for which the assumption of LTE source functions $\left(b_{u}=b_{l}\right)$ is likely to be reasonable, departures from LTE may yet affect the optical depth scaling of the line, or the stratification of the LTE-RE model atmosphere. The first is likely for non-dominant ionization stages with bound-free edges in the ultraviolet. The second is likely for atmospheres in which the ultraviolet line haze contributes quasi-continuous extinction.

\subsection{NLTE line synthesis}

Obviously, LTE should never be assumed without checking its validity. That means that generally, NLTE computation is required at least initially. With the advent of codes as MULTI (cool stars) and TLUSTY (hot stars), there is no reason not to do so in any study employing stellar spectrometry, whether aiming at simple abundance determination or at diagnosing stellar atmospheric physics. Since these lecture notes as well as Mihalas' (1970, 1978) books are all about providing background for NLTE stellar atmosphere studies, no further elaboration of this point is needed at this point. The remainder of this section therefore provides a pictorial summary of NLTE mechanisms in the form of cartoon-wise type casting, using a fictitious element tailored to the purpose. Solar examples are added as illustrations.

\subsubsection{Pictorial guide to solar NLTE mechanisms}

Not yet... 


\section{Chapter 10}

\section{Lines from Non-Plane-Parallel Stars}

$\mathrm{T}$ his final chapter presents some examples of solar and stellar line formation. It illustrates the usage of spectral lines as diagnostics of atmospheric structure beyond the limited goal of abundance determination and the plane-parallel treatment discussed sofar, building on many of the points made in the earlier chapters. It is not the end of the story, but it is the final chapter here because it presents recent analyses. Coming this far, you have caught up - your turn now.

\subsection{The solar $\mathrm{NaD}$ lines}

In this section I take the solar NaID lines as an example of NLTE line formation, using results from the 1992 Utrecht thesis $\underline{L}_{-1}^{\mathrm{T}_{1}}$ of Jo Bruls that serve here as illustration, a boundbound counterpart to the continuum modeling in Section 2.1 . In addition, they serve to introduce departures from plane-parallel modeling in the form of granulation, and to show that the frontier lies in multi-dimensional radiative transfer.

Atomic structure. Figure 10.1 shows the NaI D Grotrian diagram? Its structure is characteristic for the alkalis ( $\mathrm{Li} \mathrm{I}, \mathrm{NaI}, \mathrm{KI}$ ). The ionization energy is very low, only $5.1 \mathrm{eV}$ (see Table atmosphere: the fraction $N(\mathrm{NaI}) / N(\mathrm{Na}$ II) is very small. The single valence electron causes hydrogen-like spectra. This term diagram is complete up to $n=7$ but with all $n=7$ states "collapsed" into a single level because they are very similar. The Na ID lines are the $3 p^{2} \mathrm{P}^{o}-3 s^{2} \mathrm{~S}^{e}$ doublet. Bruls entered this model $]_{-1}^{31}$ into Carlsson's code MULTI

\footnotetext{
${ }^{1}$ Published in 'Bruls et al. (1992)! and 'Bruls and Rutten (1992)', exactly 150 years after Becquerel took the first photograph of the solar Na I D lines.

${ }^{2}$ Formally, the term term diagram means an energy-level diagram with energy levels only, while a Grotrian diagram contains both energy levels and radiative bound-bound transitions. A large collection of both is found in 'Bashkin and Stoner $(1975)^{1}$. A handy selection containing only levels and lines that are important for stellar spectroscopy (near-ultraviolet and visible) is given in Partial Grotrian Diagrams of Astrophysical Interest of 'Moore and Merrill (1968)!. Other important tabulations from Mrs. Moore are the Multiplet Table ('Moore 1959' and the Atomic Énergy Levels (Moore 1949, 1952, 1958, 1971).

${ }^{3}$ Transition probabilities and photoionization cross-sections needed to be found for all 68 lines and 17 bound-free transitions; in addition, collisional cross-sections were needed for every level combination. This used to be a matter of delving into the library dungeons; Bruls' set-up article has 150 references, mostly to hard-to-read experimental physics papers. Fortunately, model-atom setup is now becoming easier via
} 


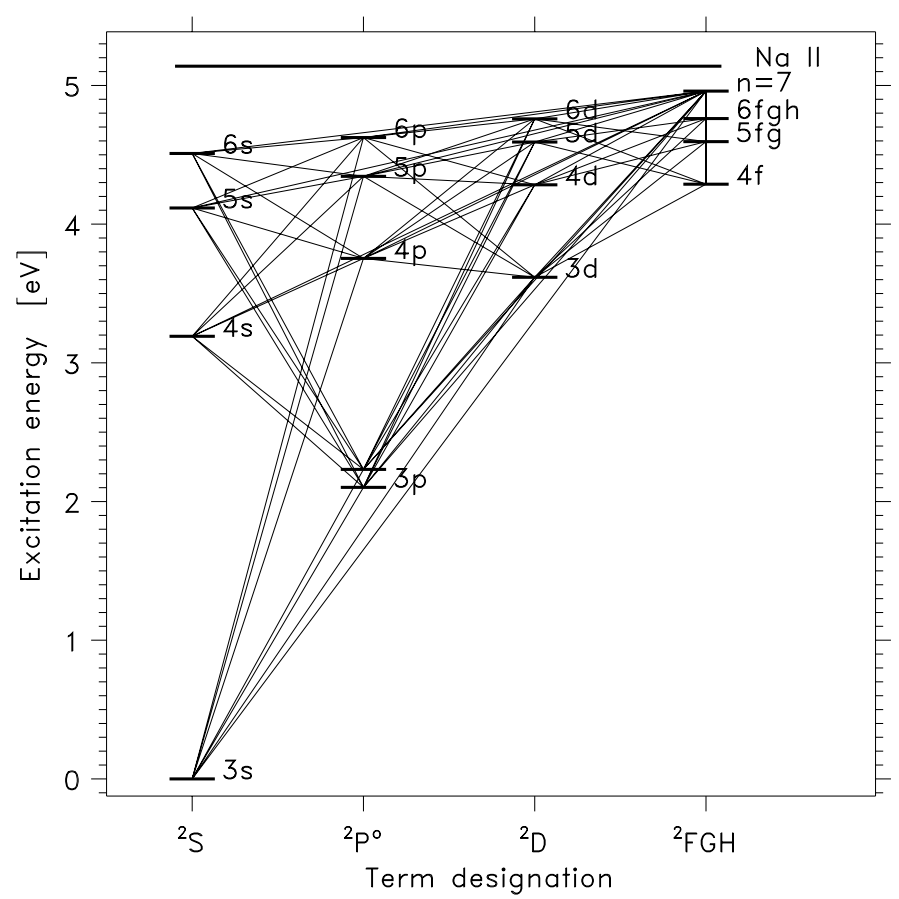

Figure 10.1: Partial Grotrian diagram for Na I. All $n=7$ states and the ${ }^{2} \mathrm{~F},{ }^{2} \mathrm{G}$ and $2 \mathrm{H}$ levels are collapsed into representative levels. The bound levels above $n=7$ are neglected, as is all fine-structure splitting except for $3 p^{2} \mathrm{P}^{o}$. The two $3 p-3 s$ transitions produce the $\mathrm{NaI} \mathrm{D}_{1}$ and $\mathrm{D}_{2}$ lines.

(see Section 15.

VALIII formation. Figure, $10 \overline{0} 2$ shows results for the NLTE VALIII atmosphere of Section $\overline{8} .2 \overline{2}$ on page $1 \overline{1} \overline{8} \overline{0}_{1}^{\prime}$ at left and for the HOLMUL atmosphere discussed on page $\overline{2} \overline{1} \bar{Q}$ at right. The VALIII model has a chromosphere, here seen as outward rise of $B_{\nu}$ in the lefthand middle panel. The HOLMUL model has none. The departure coefficient $t_{1-1}^{\pi_{1}}$ curves in the upper panels show what populations the sodium particles in the solar atmosphere need to possess in various levels of their term structure in order to achieve statistical equilibrium, given these atmospheric models. These panels show the populations as departures from Saha-Boltzmann populations. They represent the basic physics, the way solar sodium atoms feel things. The middle panels show the resulting NLTE source functions, with the model Planck function for reference. The Eddington-Barbier depths for $\mu=1$ and $\mu=0.2$ are marked by tick marks. The bottom panels show the line profiles that follow from the source functions that follow from the populations.

First inspect the ground state $3 s$ curve in the upper lefthand panel (solid). It has a slight hump around $h=300 \mathrm{~km}$ (upper photosphere) followed by a slight dip in the temperature minimum and a steep increase in the low chromosphere. The steep increase is due to imbalance in the ionization equilibrium as given by $(\overline{3} . \overline{4} \overline{4})$ on page $\overline{4} \overline{8}_{\mathbf{r}}^{\prime \prime}$ The ionizing radiation field $J_{\nu}^{\mathrm{bf}}$ escapes for all $\mathrm{NaI}$ levels from the photosphere and is constant with height in the chromosphere (see Figure 10 on page $\left.10 \overline{8} \overline{5}_{1}^{\prime}\right)$. The temperature rise

Internet access to the Opacity Project database.

${ }^{4}$ With $\underline{\beta}=b^{\text {Zwaan }} \equiv b$ the definition we use throughout these lecture notes, see page ' 36 '. 'Wijbenga and Zwaan (1972), wrote their $b^{\text {Zwaan }}$ as $\beta$ to distinguish them from the Menzel $b$ 's in the literature. 

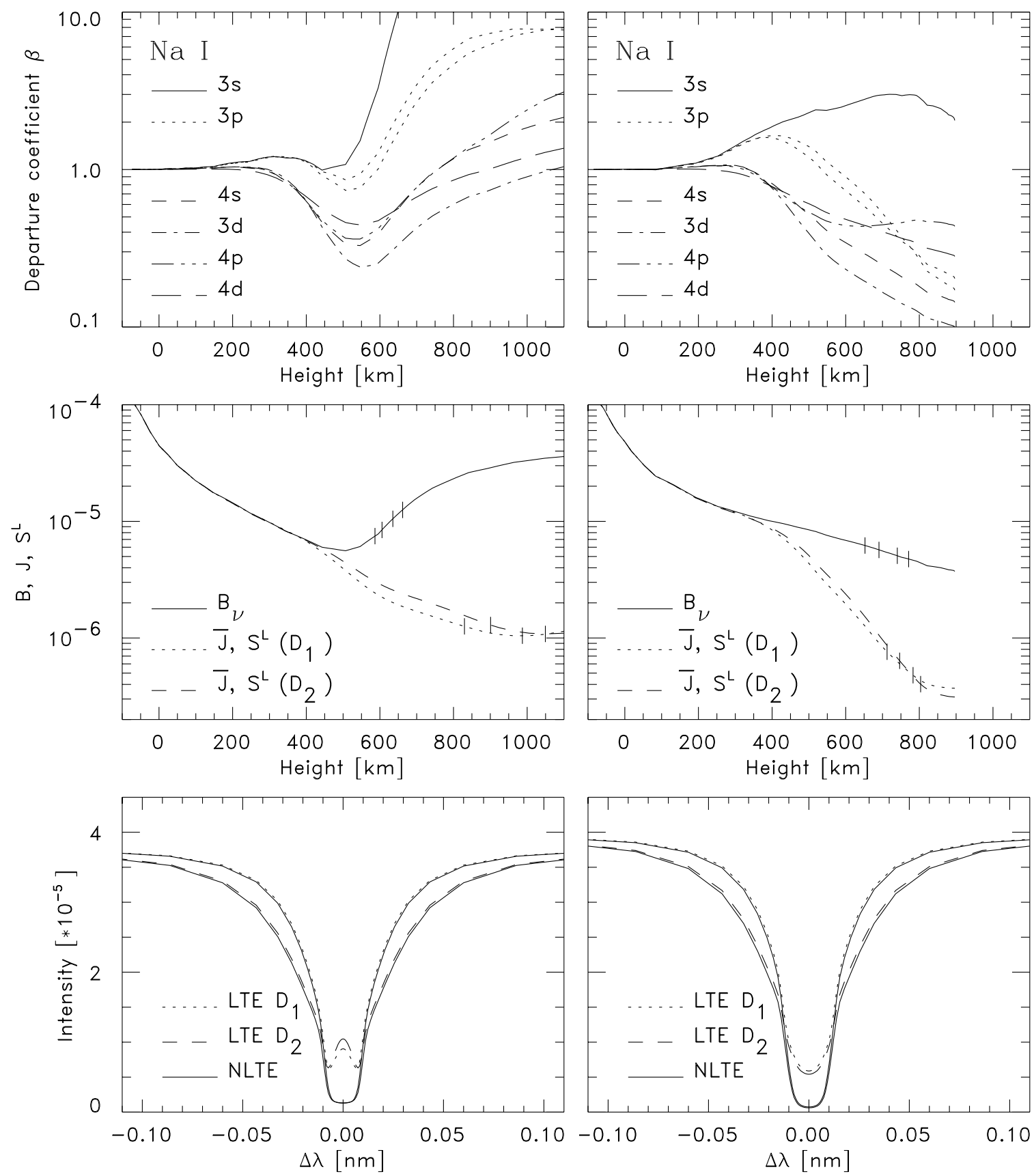

Figure 10.2: NaI results for the VALIII solar model (lefthand panels) and the HOLMUL solar model (righthand panels). Top panels: departure coefficients $\beta$ with $\beta=b^{\text {Zwaan }}$ in $(\overline{2}-\overline{1} 4)$. Middle panels: Planck functions $B_{\nu}$, line source functions $S^{\mathrm{L}}$ and mean intensities $\bar{J}$ for $\mathrm{NaI} \mathrm{D}_{1}{ }^{-}$and $\mathrm{NaI}_{2}$. The tick marks on the curves indicate the heights with total optical depth at line center $\tau_{\nu_{0}}=1$ and $\tau_{\nu_{0}}=0.2$, respectively on the $B_{\nu}$ curve for LTE line formation and on the $S^{\mathrm{L}}$ curve for NLTE line formation. Bottom panels: corresponding emergent line profiles, for LTE and NLTE line formation respectively. 
there translates into a corresponding $\beta$ rise because the actual ionization equilibrium follows $J_{\nu}<B_{\nu}$ in the edges, and not $B_{\nu}$ because the electron density is too low in the chromosphere to produce much collisional ionization. The dip at the temperature minimum is similarly due to $J_{\nu}>B_{\nu}$ (see Figure 8.11 ). The slight hump at lower height is discussed below.

The two dotted $3 p$ curves are for the upper levels of the NaID lines. They drop below the ground state $3 s$ curve in the chromosphere. The ratio $\beta_{3 p} / \beta_{3 s}$ translates via $S^{\mathrm{L}} \approx\left(\beta_{u} / \beta_{l}\right) B_{\nu}$ into the $S^{\mathrm{L}}<B_{\nu}$ splits seen in the middle panel. The drop is due to photon losses in the NaID lines. Note that their thermalization depth is near $h=500 \mathrm{~km}$, well below their $\tau_{\nu_{0}}=1$ Eddington-Barbier photon-escape depth. Thus, these lines are strongly scattering, as expected for resonance lines.

The $\beta$ 's of the higher levels drop below the $3 s$ curves already at lower height in the atmosphere. This is also due to photon losses, in the higher lines in the Grotrian diagram. They have large transition probability but they are weaker in the spectrum because their lower-level population is smaller by about the Boltzmann factor. The radiation in these lines thermalizes/escapes deeper in the atmosphere. These lines also cause the slight hump in the $3 s$ curve. Their photon losses produce slight overpopulation of the $3 p$ states, and these share that with the $3 s$ ground state.

The bottom panels illustrate that LTE line formation from VALIII would produce selfreversed line cores with central emission peaks. Note also (middle panel) that the LTE opacity (ticks on $B_{\nu}$ curve) is much smaller than the NLTE opacity because the assumption of LTE ionization does away with the steep rise of the $3 s$ curve in the top panel. The LTE assumption equates all departure coefficients to unity and therefore depletes neutral sodium an order of magnitude more around $h=600 \mathrm{~km}$ than is the case for the actual NLTE ionization.

HOLMUL formation. The righthand panels show results of LTE and NLTE modeling for the HOLMUL model. The latter is intended (page $\overline{2} \overline{1}$ ) for LTE modeling and has no chromospheric temperature rise. Therefore, the outward rise of the $3 s$ curve in the top HOLMUL panel is not caused by a chromospheric temperature rise but due to the photon losses in the various Na I bound-bound transitions. They cause appreciable underpopulation for their upper levels, especially $3 d$ (dot-dashed). This level is the most important one in the ionization equilibrium; its small $\beta<1$ draws a net recombination rate down from the large population reservoir in the Na II ground state (see (3.23i) on page $\left.\overline{4}^{\overline{4}} \overline{8_{1}^{\prime}}\right)$. These recombined electrons tend to cascade down and to end up in the ground state, producing $\beta_{3 s}>1$. This photon-loss-incited population increase drawn from the reservoir in the next ionization stage is similar to the Lyman continuum effect discussed in Exercise $1{ }_{2}^{1} 2$

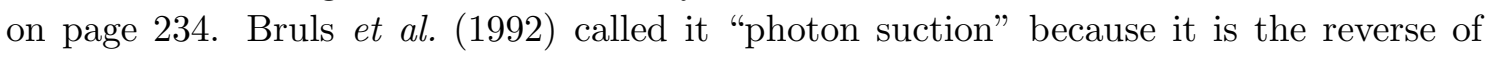
"photon pumping" in which overly intense radiation $\left(J_{\nu}>B_{\nu}\right)$ causes overexcitation or overionization.

The resonance-line split between the $3 p$ and $3 s$ curves is about the same as for VALIII, and therefore also the split between $S^{\mathrm{L}}$ and $B_{\nu}$ in the middle panel. However, since $B_{\nu}$ itself is lower at the $\tau_{\nu_{0}}=1$ height, the emergent NLTE profiles (bottom panel) are deeper. The LTE profiles have no self-reversals, of course.

Atom-size experiments. The results in Figure 10.21 depict statistical equilibrium reached in the computer when using the model atom of Figure 10.11. Figure 10.3 shows four other solutions. I include these to illustrate that a complete NLTE simulation of stel- 

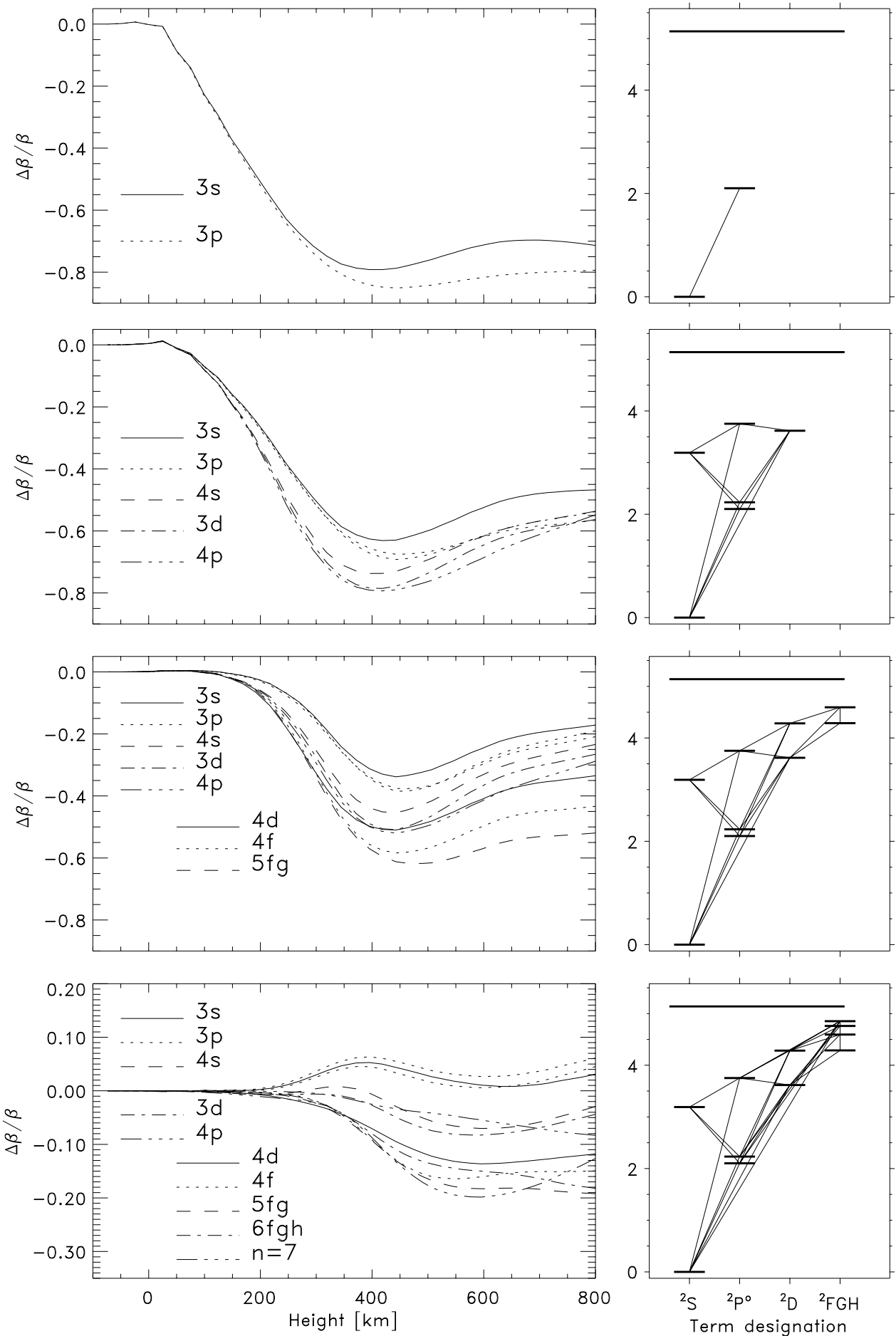

Figure 10.3: Departure coefficient changes for four simplified NaI model atoms (right-hand panels) for VALIII. The relative changes $\Delta \beta / \beta$ measure the difference with the results from the comprehensive model atom (Figure 10.1') shown in the top-left panel of Figure 10.2. 
lar radiative transfer is strongly dependent on the input. The fact that the model atom, cross-sections and atmospheric model permit convergence to a statistical-equilibrium solution does not guarantee that that is the correct one since it is model-dependent. In this type of numerical research one needs to experiment with one's model and its solution to gain confidence in their reliability

Figure 10.3 shows simple experiments where solutions that are obtained for the sequence of model atoms depicted at right are compared with the comprehensive result using the full model atom of Figure 10.21 . The lefthand panels plot the fractional change of the departure coefficients relative to those plotted in the upper-left panel of Figure 10.2 .

The upper experiment is for a simple two-level-plus-continuum Na I atom. The changes in the result are large in the sense that the overall $\mathrm{NaI}$ population changes appreciably. The source function of the resonance line, however, is not much affected since the $3 s$ and $3 p$ departure coefficients vary in tandem. Adding more and more levels and lines, from top to bottom, effectively constructs a "ladder" from the lowest levels to the continuum. Remember that the Na II ground state contains most of the sodium particles. The ladder helps to bring electrons down from that reservoir to the $\mathrm{NaI}$ ground state, and so fills in the underpopulation seen in the top panel. The bottom panel (note the y-axis scale change) represents a reasonably good approximation to the comprehensive result.

The simple model atom at the top approximates the resonance-line source function quite well because that senses only the ratio $\beta_{u} / \beta_{l}=\beta_{3 p} / \beta_{3 s}$, demonstrating that the twolevel atom approximation suits very well to describe the Na I D source function. However, this model underestimates the line opacity (which scales with $\beta_{l}=\beta_{3 s}$ ) by $80 \%$ in the line-forming region.

Solar granulation. The actual Sun isn't static plane-parallel. The deeper layers of the photosphere are in violent turmoil from turbulent convection, seen as granulation. It is no accident that we just see into the top of the convection zone: we see into the depths where the bulk of the flux leaves the Sun and because the gas gets transparent at that depth, the radiative flux takes over the outward energy transport from the convection which handles it in the opaque subsurface layers of cool stars. The layer where the visible spectrum escapes from the Sun therefore has to be the upper surface of the convection zone. For stars of different effective temperature, our eyes are not so well adjusted to this intrinsic surface.

The solar granulation was discovered by William Herschel. It has been much studied ever since. A recent image is shown in Figure 10.4 .

The granulation is by and large understood, thanks to detailed numerical simulations

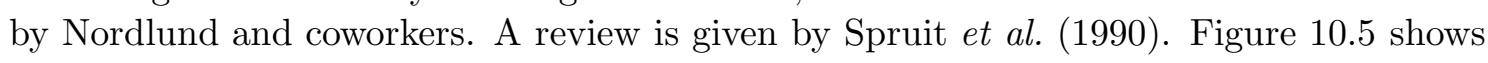
the surface of a sample simulation result that is used below for NaID line synthesis. Figure 10.6 shows the temperature, vertical velocity and optical depth in a vertical plane through the simulation cube.

Quasi-plane-parallel formation. Figures 100.40 demonstrate that the solar photosphere is not plane-parallel, at least not in the deeper layers. The next step is to model the formation of the NaID lines in the solar spectrum by computing them for different

\footnotetext{
${ }^{5}$ That holds of course for any type of computational physics. The current-day possibility to run realistic simulations without tractability shortcuts is the greatest breakthrough in astrophysics since the spectrograph. The most important advantage is that one can tinker with the code to find out what actually is going on - in the computer and in the universe.
} 


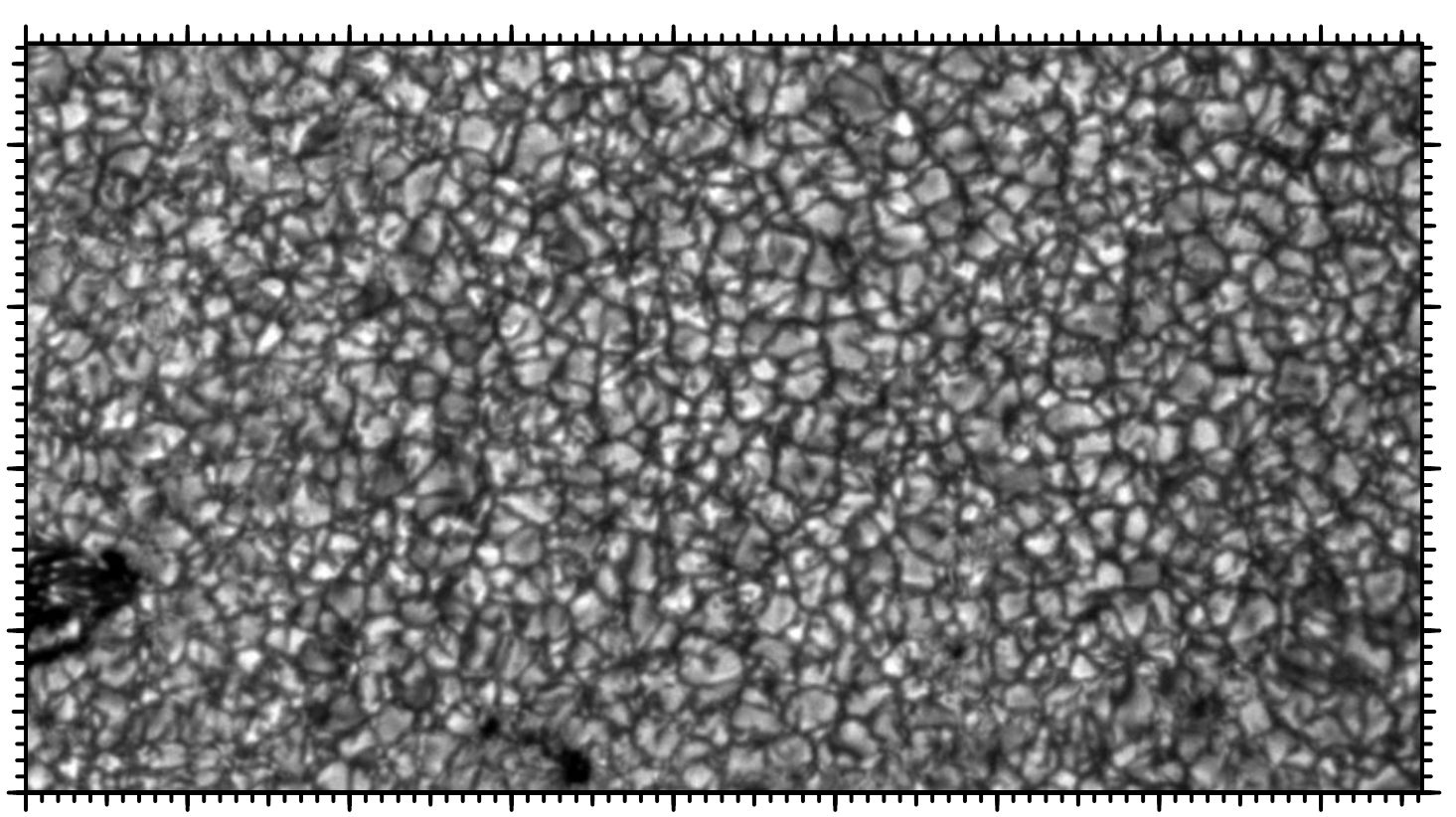

Figure 10.4: Observed solar granulation. A high-quality image of the solar surface taken with the Swedish Vacuum Solar Telescope on La Palma. The smallest axis divisions are $1^{\prime \prime}$ or $725 \mathrm{~km}$ on the Sun; the field measures $46^{\prime \prime} \times 86^{\prime \prime}$. The larger granules have diameters just over one arcsecond. Granules are blobs of hot gas that have risen to the surface and there lose their energy by radiation. The larger ones develop dark centers and break into pieces within 5-10 minutes because insufficient heat comes up to maintain their stability against the radiation losses and adiabatic expansion cooling. The smallest bright specks in the dark intergranular lanes, just at the $0.2^{\prime \prime}$ resolution of the telescope, are magnetic elements, commonly called fluxtubes since 'Spruit (1977)', laid down the basic physics of idealized evacuated strong-field tubes. Courtesy of G.W. Simon, copied from 'Rutten and Schrijver $(1994)$.'

columns in the simulation cube as if each column represents a plane-parallel atmosphere by itself

The lefthand side of Figure 10.726 different vertical computed stratifications (making up the cuts through the simulation cube marked in Figure 110.5$)$ with the VALIII and HOLMUL standard models. The dotted curves show the outer boundaries of the spread in the granular stratifications; the solid curves are the spatial mean. They are in rather good agreement with the standard models, showing that these are roughly the

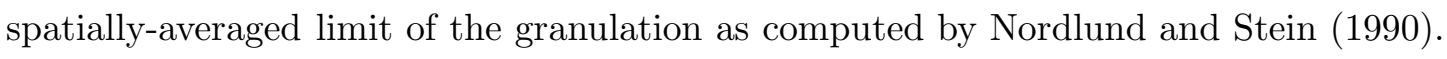

The righthand side of Figure 10.7 portrays NaID line formation for two extreme stratifications, one from a bright granule and one from a dark intergranular lane. The middle panel shows that the temperature contrast reverses sign above $h \approx 150 \mathrm{~km}$; this reversal is also observed in the actual solar granulation. The two line source functions are not very different in the upper layers. The bright granule is much hotter in the deep layers. As a result, it produces brighter continua, not only for the $\lambda=590 \mathrm{~nm}$ wavelength of the NaID lines but also in the near-ultraviolet where the major $\mathrm{NaI}$ ionization edges are. The hot ionizing radiation depletes the population hump seen for the dotted $3 \mathrm{~s}$ and $3 p$ curves around $h=400 \mathrm{~km}$ in the upper panel. The line profiles in the bottom panel

\footnotetext{
${ }^{6}$ But not a static atmosphere because the densities do not follow from hydrostatic equilibrium but from the simulation.
} 


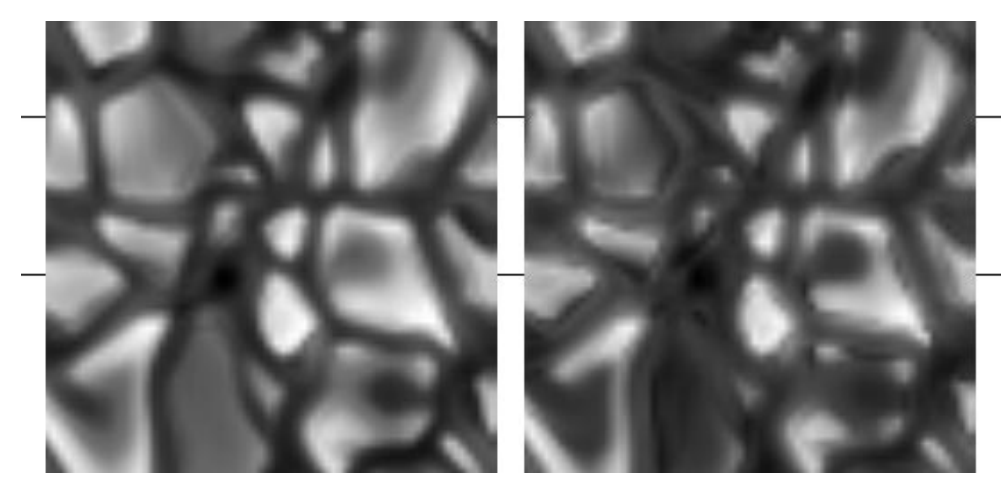

Figure 10.5: Computed solar granulation. A snapshot of a solar granulation simulation by 'Nordlundì and Stein (1990). They computed time-dependent turbulent convection including radiative transfer for a volume of $6000 \times 6000 \times 1150 \mathrm{~km}^{3}$. This image shows the computed solar surface for a single time step. Left: emergent continuum intensity at $\lambda=500 \mathrm{~nm}$. Right: $T_{\mathrm{e}}$ at optical depth $\tau_{500}=1$. The marks indicate the upper cut through the data cube shown in Figure 10.6 . These computed granules are much like the observed one. Magnetic elements (the tiniest white specks in Figure 10.4.) are not present in the intergranular lanes here because no magnetic fields were present in the simulation. Magnetoconvection is the current frontier in numerical simulation. An informative description of the state of the art is given by

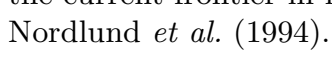

differ primarily in their continuum background intensity.

Figure 10.8 plots the ratio of the upper and lower departure coefficients for the Na ID lines computed from 63 different stratifications. The near-equality of the curves demonstrates that the NaID lines act as two-level-atom scattering lines. Their line source function $S^{\mathrm{L}} \approx\left(\beta_{3 p} / \beta_{3 s}\right) B_{\nu}$ is almost insensitive to the stratification.

Finally, Figure 10.9 shows emergent $\mathrm{NaI} \mathrm{D}_{1}$ line profiles. The lefthand panel shows the profile from 126 different stratifications in the simulation cube. The variations are large, especially in the continuum intensity. The righthand panel compares the spatial average over the 126 stratifications with profiles computed from VALIII and with the spatially averaged profile observed at the center of the apparent solar disk. The computed average is smeared with three different macroturbulent velocities, as an indication of the effect of the neglect of acoustic waves in the computations.

Non-plane-parallel formation. The agreement between the computed average and the observed $\mathrm{NaI} \mathrm{D}_{1}$ profiles in Figure $10.9 \mathrm{i}$ is reasonable but not perfect. The computed line core is too deep, or to pointed when smeared with large macroturbulence. The line wings are too dark, just the reverse of the VALIII computation where they are too bright.

Thus, the solar NaI D lines are not yet reproduced to perfection. That is not surprising because the quasi-plane-parallel modeling done here from the simulation cube is not physically realistic. The horizontal structuring in the solar granulation is well below $1^{\prime \prime}$ in scale size, close to the $50-100 \mathrm{~km} \mathrm{~s}^{-1}$ mean free path of photons in the photosphere and equal to the distances scattering photons travel between creation and destruction if they don't escape. Therefore, proper modeling with pluri-dimensional radiative transfer is required. At any location, the source function is influenced laterally by structures that differ in temperature, density and velocity. The simplification of taking each column of the simulation cube as an independent plane-parallel atmosphere must be wrong. The 

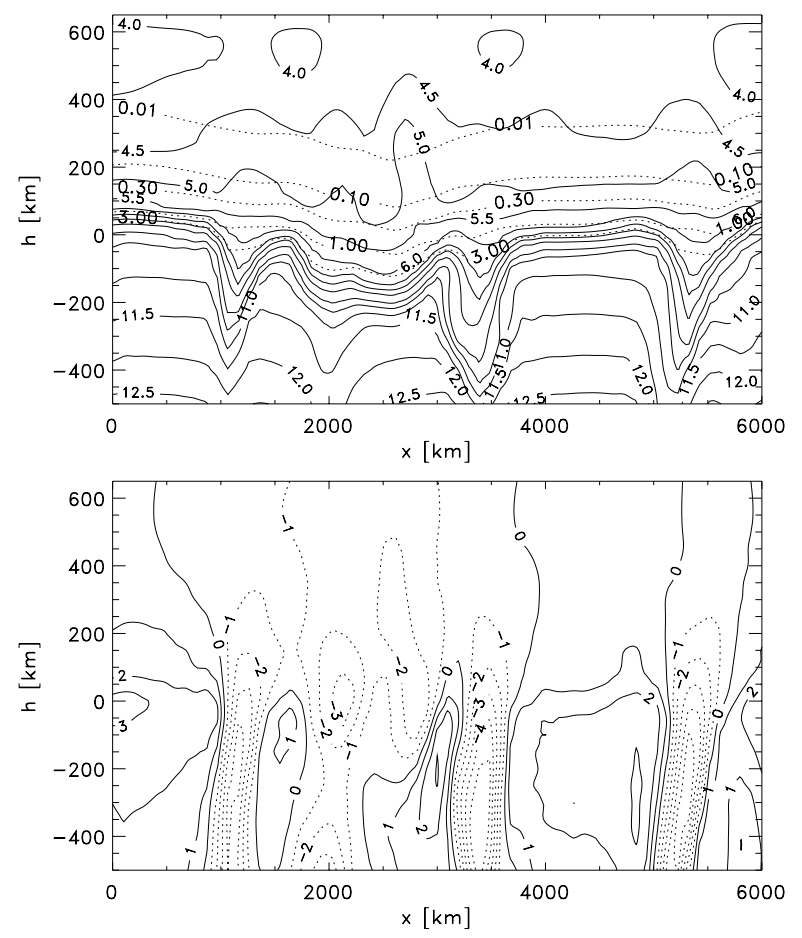

Figure 10.6: Parameters in a vertical cut through the simulation cube indicated by the upper marks in Figure 10.5. Upper panel: temperature in $10^{3} \mathrm{~K}$ (solid; smaller numbers). The dotted contours with the larger annotation specify the continuum optical depth. Lower panel: vertical velocity in $\mathrm{km} \mathrm{s}^{-1}$. Upward motions solid, downward motions dotted. The rising granules are broad gentle upwellings. The dark intergranular lanes are fast-sinking "fingers". At larger depth then shown here the fingers connect together in progressively larger-scale patterns.

next thesis on this subject (yours?) should employ pluri-dimensionalili' radiative transfer to model the solar Na ID lines.

\subsection{Solar and stellar CaII $\mathrm{H}$ and $\mathrm{K}$ lines}

Not yet...

\subsection{Coronal lines}

Not yet....

\footnotetext{
${ }^{7}$ Multi-dimensional radiative transfer is required, but $2 \mathrm{D}$ rather than $3 \mathrm{D}$ suffices for most situations. This is not the case in hydrodynamics or MHD in which the third dimension adds intrinsically new types of instability. In radiative transfer the essential addition of a second dimension is that the local source function along the line of sight feels photons from aside, laterally impinging with information from regions with other characteristics in the neighborhood. The quasi-plane-parallel modeling discussed above is often called "1.5D radiative transfer". The atmosphere is structured (as in the simulation cube) but the radiation fields that contribute to the source function along the line of sight (a column for radial viewing as above, or slanted if desired) are only evaluated along the line of sight.
} 

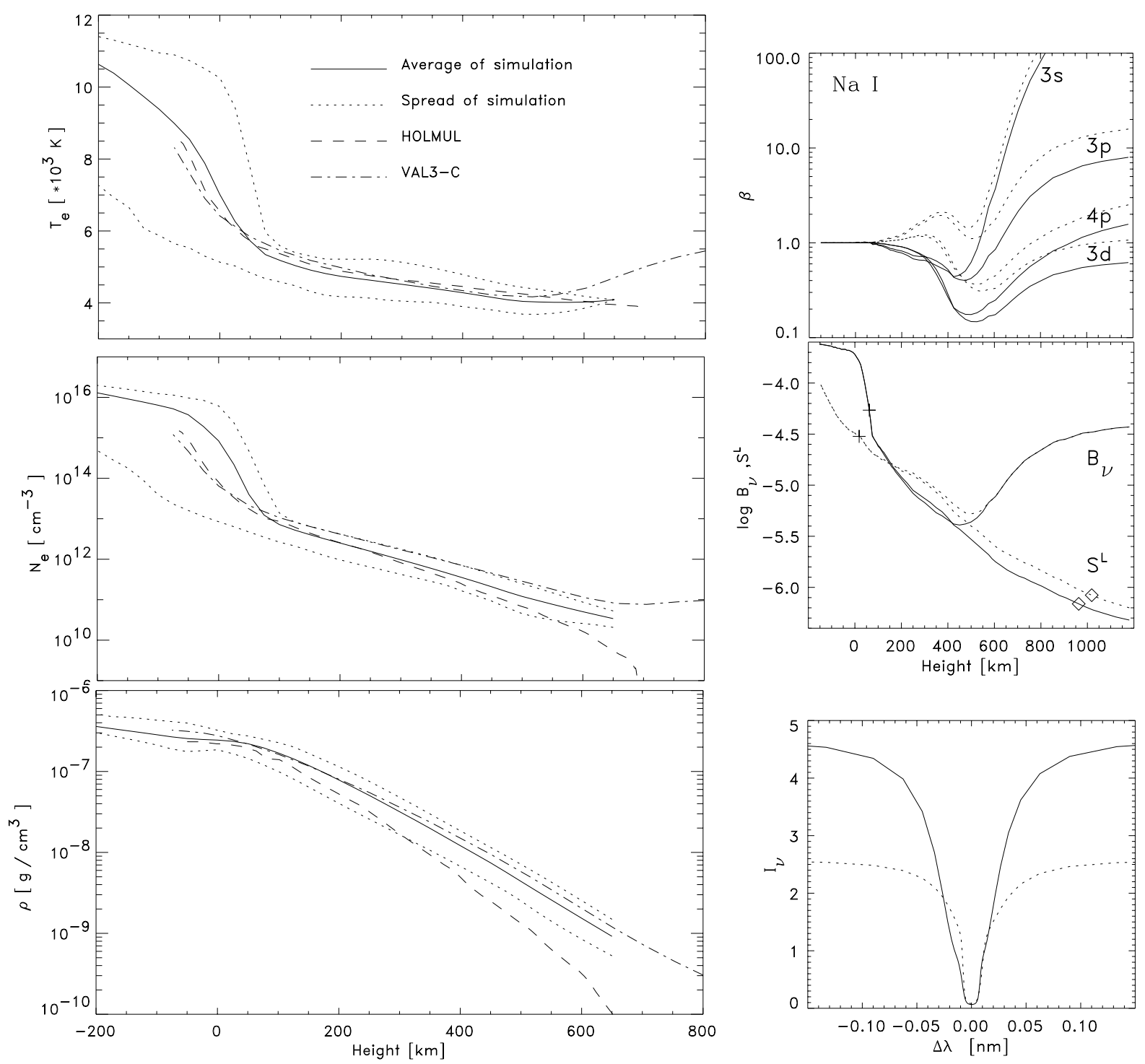

Figure 10.7: Lefthand column: mean and spread of granular stratifications compared with standard planeparallel models of the solar atmosphere, respectively for the temperature $T_{\mathrm{e}}$ (top), the electron number density $N_{\mathrm{e}}$ (middle) and the gas mass density $\rho$ (bottom). The horizontal averages for the granulation simulation are similar to the standard plane-parallel models, but the spread between different simulation locations is large. Righthand column: NaI D line formation for hot (solid curves) and cool (dotted curves) granular stratifications. The panel layout is the same as for Figure $\overline{1} \overline{0} \cdot \overline{1}$ on page $\overline{2} \overline{1} \overline{\mathrm{i}}$. The squares in the middle panel specify $\tau=1$ locations for $\mathrm{NaI}_{1}$ at line center; the crosses specify $\dot{\tau}^{c}=1$ depths in the continuum near the line. Units of $I_{\nu}, S^{\mathrm{L}}$ and $B_{\nu}: 10^{-5} \mathrm{erg} \mathrm{cm}^{-2} \mathrm{~s}^{-1} \mathrm{~Hz}^{-1} \mathrm{sr}^{-1}$. 


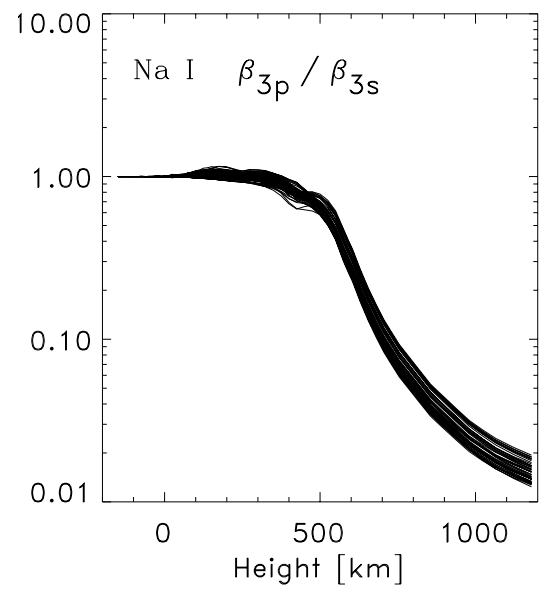

Figure 10.8: Ratio of upper to lower departure coefficients for the Na I D lines in each of the 63 stratifications in the vertical cut through the simulation cube marked by the upper ticks in Figure 10.5.
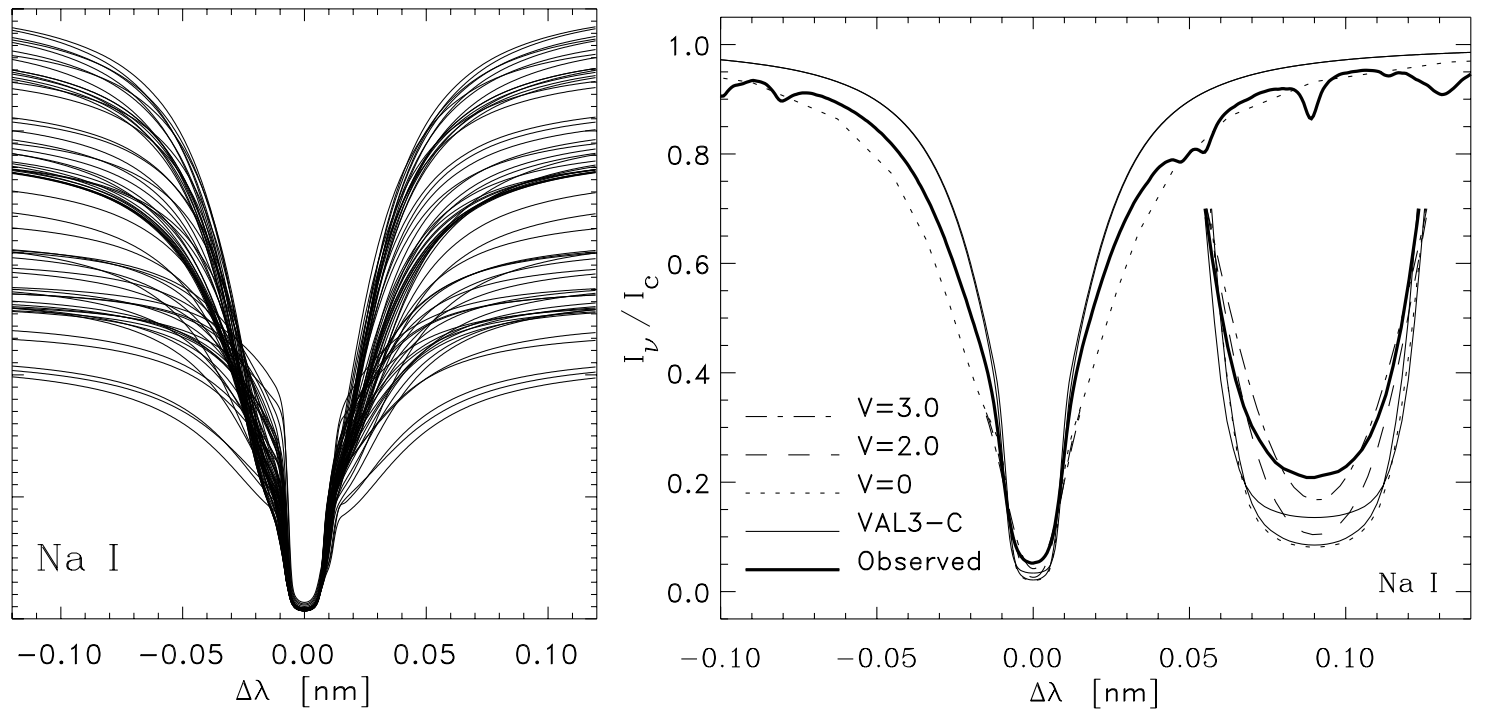

Figure 10.9: Left: emergent profiles per granular stratification, on an arbitrary intensity scale. Right: spatial average over 126 granular stratifications, smeared with various macroturbulent velocities $V$ and normalized to the averaged continuum intensity. The thick solid profiles are spatially-averaged disk-center observations. The thin solid profiles were computed from the static plane-parallel VALIII model with and without macroturbulent broadening. Inset: line core on $4 \times$ expanded scales.

\subsection{Wind lines}

Not yet.... 


\section{Exercises}

\begin{tabular}{ll}
\hline Planck constant $h=6.63 \times 10^{-27} \mathrm{erg} \mathrm{s}$ & hydrogen ionization energy $\chi_{\mathrm{H}}=13.6 \mathrm{eV}$ \\
Boltzmann constant $k=1.38 \times 10^{-16} \mathrm{erg} \mathrm{K}^{-1}$ & energy of $1 \mathrm{eV}=1.602 \times 10^{-12} \mathrm{erg}$ \\
$=8.62 \times 10^{-5} \mathrm{eV} \mathrm{K}^{-1}$ & photon energy in eV $E=1239.85 / \lambda(\mathrm{in} \mathrm{nm})$ \\
Stefan-Boltzmann const. $\sigma=5.67 \times 10^{-5} \mathrm{erg} \mathrm{cm}^{-2} \mathrm{~K}^{-4} \mathrm{~s}^{-1}$ & solar radius $R_{\odot}=6.96 \times 10^{10} \mathrm{~cm}$ \\
speed of light $c=3.00 \times 10^{10} \mathrm{~cm} \mathrm{~s}^{-1}$ & solar mass $M_{\odot}=1.99 \times 10^{33} \mathrm{~g}$ \\
electron mass $m_{\mathrm{e}}=9.11 \times 10^{-28} \mathrm{~g}$ & solar luminosity $L_{\odot}=3.86 \times 10^{33} \mathrm{erg} \mathrm{s}^{-1}$ \\
hydrogen atom mass $m_{\mathrm{H}}=1.67 \times 10^{-24} \mathrm{~g}$ & solar effective temperature $T_{\odot}^{\mathrm{eff}}=5780 \mathrm{~K}$ \\
\hline
\end{tabular}

Abundance values and ionization energies are given in Table $\overline{\overline{7}} \overline{\overline{1}} \overline{\mathrm{i}}$ on page $\mathbf{i} \overline{4} \overline{4}$.

Wavelengths of principal spectral features are given in Table '8.1' on page $\mathbf{1} \mathbf{1}_{\mathbf{1}}^{\prime}$

The VALIII model is specified in Table 8.2 on page 182.

\section{Bound-free edges (Section 2.21 on page 114}

These questions concern the appearance of bound-free edges in the spectrum of a constant- $S$ object. Assume source function equality $S_{\nu}^{\mathrm{bb}}=S_{\nu}^{\mathrm{bf}}=S_{\nu}^{\mathrm{cont}}=S$, where "cont" identifies other continuous processes than the given bound-free transition, and assume $S$ invariant both with location within the object and with frequency.

a) Sketch a figure similar to Figure $\overline{2} \cdot \overline{2}$ on page $\overline{1} \overline{6}_{1}^{1}$ for a hydrogenic bound-free ionization edge.

b) A homogeneous cloud of pure hydrogen shows the H I Lyman lines in emission. The cloud is optically thick at the Lyman bound-free threshold. Is the H I Lyman edge in emission, in absorption, or absent? What is the shape of the edge? And of Ly $\alpha$ ? Which of these two reaches the highest intensity?

c) Devise a spherical star which shows the Lyman edge in emission in its irradiance spectrum even though $S$ is invariant as defined above (hint: extended atmosphere). Is the Lyman edge also in emission in its intensity spectrum?

d) Now drop the assumption of homogeneity and source function constancy. Imagine yourself to be the first astronomer taking an near-infrared spectrum of an unknown unresolved object. It shows the Paschen edge and the Paschen lines in emission. Formulate at least three possibilities and suggest corresponding diagnostics.

\section{Linear source function (Section 2.21 on page 1}

Assume that the source function in a plane-parallel atmosphere obeys $S_{\nu}\left(\tau_{\nu}\right)=a_{0}+a_{1} \tau_{\nu}$ and that there is no irradiation from outside.

a) Express $I_{\nu}^{+}\left(\tau_{\nu}, \mu\right), J_{\nu}\left(\tau_{\nu}\right)$ and $F_{\nu}\left(\tau_{\nu}\right)$ for $\tau_{\nu}=0$ in $a_{0}$ and $a_{1}$. 
b) Use the results to derive the Eddington-Barbier approximations for these quantities.

c) Compare the outer-boundary values $I_{\nu}(0,1), J_{\nu}(0)$ and $F_{\nu}(0)$ with $S_{\nu}(0)$ for $a_{1}=0$ and $a_{1}=5 a_{0}$.

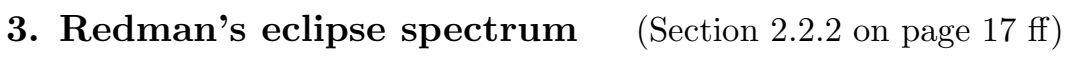

This problem illustrates the transition from optically thick to optically thin line formation near the solar limb. R.O. Redman obtained a spectrum of the Ca II K line at $\lambda_{\mathrm{K}}=393.3 \mathrm{~nm}$ during a total solar eclipse. A tracing made from his plate is sketched in Fig. $10 . \overline{1} \overline{1} \frac{1}{1}$. The K line shows four peaks which we try to explain.

The density in the solar atmosphere drops with height $h$ as $N \sim \exp (-h / H)$, with scale height $H \approx 100 \mathrm{~km}$. Assume that the ratio of line-center extinction over continuum extinction $\eta=\alpha^{l} / \alpha^{c}$ has $\eta_{\mathrm{K}}=10^{6}$ at all heights. Assume also, for the moment, that the atmosphere is isothermal with $T=6000 \mathrm{~K}$ for all $h$ and that LTE holds.

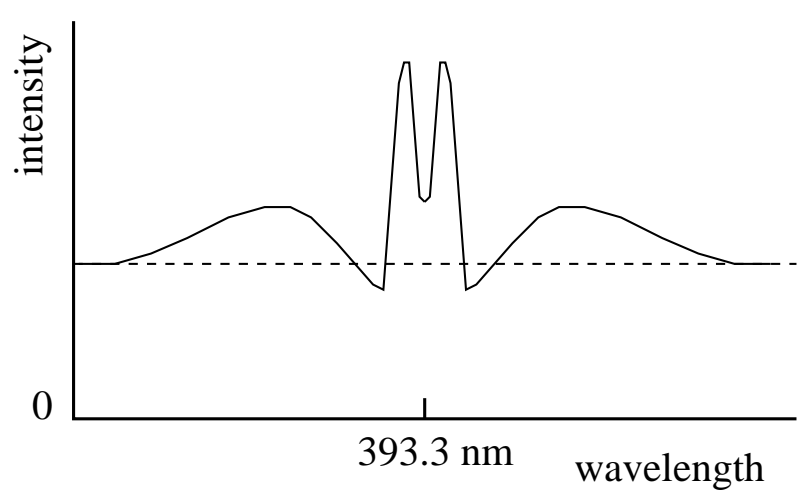

Figure 10.10: Sketch of a spectrum obtained by R.O. Redman in 1952 in Khartoum.

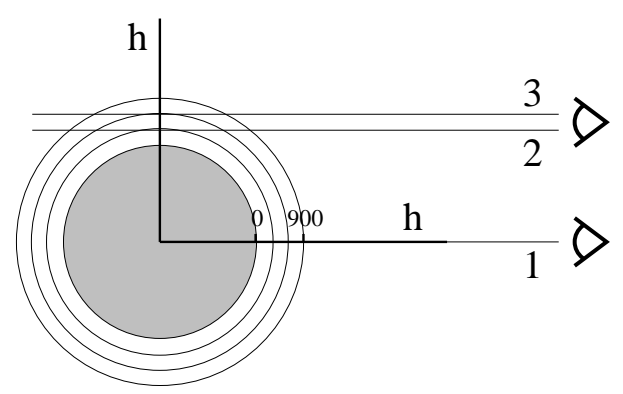

Figure 10.11: Geometry, with lines of sight towards the center of the disk (ignore the presence of the moon during the eclipse) and towards the limb, tangential at $h=300 \mathrm{~km}$ and $h=600 \mathrm{~km}$.

a) First the instrument. Redman used a heliostat mirror and a lens of $20 \mathrm{~cm}$ diameter to project a $10 \mathrm{~cm}$ solar image on the spectrograph slit (width $20 \mu \mathrm{m}$ ). The spectrograph consisted of a slit, a collimation mirror, a reflection grating and a camera mirror. It projected the slit monochromatically 1:1 on a photographic plate. Give a rough estimate of the fraction of the intensity that leaves the sun radially that was irradiating his photographic plate when the telescope was pointed at disk center.

b) Now the geometry (Fig. $\left.10 . \overline{1} \overline{1}_{1}^{\prime}\right)$. Let us use $\tau$ for radial optical depth and $t$ for optical 
thickness along the beam. The zero point of the radial height scale $h=0 \mathrm{~km}$ is defined as the location with radial optical depth $\tau_{1}^{c}=1$ in the continuum close to the $\mathrm{K}$ line, i.e., measured along line of sight number 1 . Why is this a logical definition? Where is $\tau_{1}^{c}=0$ ? What is the optical thickness of the sun along this line of sight?

The lines of sight 2 and 3 are tangential to the sun. The solar limb is defined to be the location $h_{i}$ at which the continuum optical thickness $t_{i}^{c}$ of the sun along a tangential line of sight $i$ equals unity: that direction $i$ for which $t_{i}^{c}=1$. In the continuum near the $\mathrm{K}$ line this is number 2, cutting through shells with $h>300 \mathrm{~km}$ and touching the limb at $h_{\mathrm{L}}=h_{2}=300 \mathrm{~km}$. Why isn't the limb at $h=0 \mathrm{~km}$ ?

Are there wavelengths in the spectrum with $h_{\mathrm{L}}<300 \mathrm{~km}$ ?

Line of sight 3 is tangential to the shell with $h_{3}=600 \mathrm{~km}$. Estimate the continuum optical thickness $t_{3}^{c}$ of the sun for this viewing direction.

c) Give equations which express the observed intensity in the Planck function and optical depth $\tau$ or thickness $t$, for the lines of sight 1,2 and 3. Estimate the fractions of $B_{\nu}$ observed along 1, 2 and 3 .

Is the Eddington-Barbier approximation valid along 1, 2 and 3 ?

d) Sketch the observed spectral profiles $I-\lambda$ for lines with $\eta=0.1$ and $\eta=10^{6}$ along 1,2 and 3 .

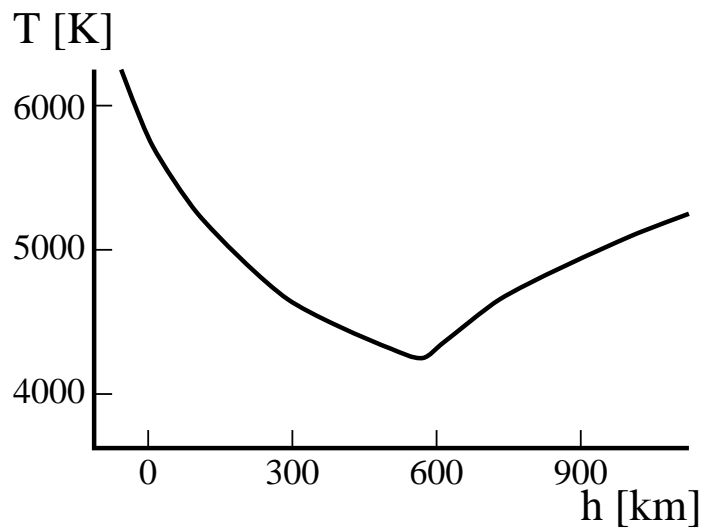

Figure 10.12: Temperature distribution in the solar atmosphere.

In reality the solar atmosphere is not isothermal and the assumption of LTE is often wrong. We now drop these assumptions. Fig. 10.12 10 specifies the temperature distribution $T(h)$.

e) Give a sketch of the spectral profile which the $\mathrm{K}$ line should show along direction 2 if LTE were valid.

f) Now explain all humps and dips in Redman's tracing in Fig. 10.10. What is the physical cause of the central dip?

The viewing direction was not exactly known. Argue that it must have had $300<h_{i}<$ $600 \mathrm{~km}$.

g) Is the assumption that $\eta_{\mathrm{K}}$ does not vary with $h$ realistic? What are the height dependences of $\alpha^{l}$ and $\alpha^{c}$ ?

h) Why was a total eclipse necessary to obtain this tracing? May such just-off-the-limb spectra also be obtained outside eclipses? How? 


\section{Bound-free radiation through a hydrogen shell (Section 2.6 .3 on page $33^{\prime}$ ff)}

A homogeneous shell consists of tenuous, cold hydrogen gas at temperature $T$ and density $N$. It sits in vacuum and encloses a vacuum. The shell thickness $d$ and radius $R$ have $d \ll R$. The shell is optically thin in all directions for the Balmer continuum and the Balmer lines. It is optically thick but effectively thin for Lyman continuum radiation and the Lyman lines. The bound-free Balmer and Lyman extinction and emissivities are $\alpha_{\nu}^{\mathrm{BaC}}, j_{\nu}^{\mathrm{BaC}}, \alpha_{\nu}^{\mathrm{LyC}}$ and $j_{\nu}^{\mathrm{LyC}}$, respectively.

a) Sketch the total extinction as function of wavelength with the help of Table $8.1 \mathrm{i}$ on page ${ }^{1} \overline{7} \overline{6}_{\mathbf{z}}^{\prime}$ At which wavelengths do the Balmer and Lyman continua overlap? Are there spectral lines in the overlap region?

Express the total source function in this wavelength region in the coefficients above, for locations within the shell layer, outside the shell, and at the shell center.

Express $I_{\nu}, J_{\nu}$ and $\mathcal{F}_{\nu}$ at the shell center in the coefficients above for both continua. Do these expressions include radiation that was emitted by one part of the shell towards another part and then scattered towards the shell center?

b) Sketch the angular intensity distributions observed by a distant observer across the shell at $\lambda=364.5 \mathrm{~nm}$ and $\lambda=91.1 \mathrm{~nm}$. Are the Lyman lines in emission, in absorption or absent?

c) The Lyman bound-free processes may be described by a "one-level-plus-continuum" approximation resembling the two-level simplification for resonance lines. What is neglected in this approximation? What processes take the place of resonance scattering? Show that stimulated emission is negligible and argue that there is complete redistribution over the Lyman continuum.

d) Discuss the collisional photon destruction probability $\varepsilon$ for Lyman continuum photons. What particles take part in such collisions? Why may one expect that $\varepsilon \ll 1$ ? Is $(\overline{2} \cdot \overline{2} \cdot \overline{4} \overline{4})$ (page $\left.\overline{4}_{1} \overline{1}_{1}\right)$ valid or should you use some analogy of $\left(\overline{2} . \overline{1} \overline{4} \overline{5}_{1}^{\prime}\right)$ with the averaged radiation field $\overline{J_{\nu}}$ ? Averaged over what? And what about $\varepsilon_{\nu_{0}}$ ?

e) Explain that $\alpha_{\nu}^{\mathrm{BaC}} \ll \alpha_{\nu}^{\mathrm{LyC}}$ and explain that $\alpha_{\nu}^{\mathrm{BaC}} \ll\left[\alpha_{\nu}^{\mathrm{BaC}}\right]_{\mathrm{LTE}}$. Give an estimate for the ratio $\alpha_{\nu}^{\mathrm{LyC}} /\left[\alpha_{\nu}^{\mathrm{LyC}}\right]_{\mathrm{LTE}}$.

f) Assume that there is a (rather strange) star at the center of the shell which emits the Ly $\alpha$ line exclusively, Lambert-wise and with brightness temperature $T_{b}^{\mathrm{Ly} \text { star }}$. Its radius is $r=0.001 R$. Express the flux $\mathcal{F}_{\nu}^{\mathrm{Ly} \alpha}$ emitted by the shell in $T_{b}^{\mathrm{Ly} \text { star }}$ on the assumption that all impinging $\operatorname{Ly} \alpha$ photons scatter through the shell eventually $\left(\varepsilon_{\nu_{0}}^{\operatorname{Ly} \alpha}=0\right)$. Is this assumption realistic?

Will the shell reach $T_{b}^{\mathrm{Ly} \text { star }}$ if it is effectively thick for the Ly $\alpha$ irradiation?

g) Now assume that the central star emits the Lyman continuum as well, also with $T_{b}^{\text {Lystar }}$. Explain that the shell produces much stronger Balmer line emission in this case.

\section{The Eddington and two-stream approximations (Section $\overline{4} . \overline{3}$ on page $\left.\overline{9} 2_{1}^{\prime} \mathrm{ff}\right)$}

This problem illustrates the two-stream method mentioned on page 94 i. It is copied from Rybicki and Lightman (1979; their problem 1.10).

A semi-infinite isothermal atmosphere contains material that absorbs and emits thermally and scatters radiation monochromatically, with constant collision destruction probability $\varepsilon_{\nu}$. Assume that the radiation field can be described as radiation traveling through the 
atmosphere at only two angles, respectively outward going intensity $I_{\nu}^{+}=I_{\nu}(\mu=+1 / \sqrt{3})$ and inward going intensity $I_{\nu}^{-}=I_{\nu}(\mu=-1 / \sqrt{3})$.

a) Write the moments $J_{\nu}, H_{\nu}$ and $K_{\nu}$ in terms of $I_{\nu}^{+}$and $I_{\nu}^{-}$. Show that the Eddington approximation holds. Is this also true if one would choose a different set of angles than $\mu= \pm 1 / \sqrt{3}$ ?

b) Write $I_{\nu}^{+}$and $I_{\nu}^{-}$in terms of $J_{\nu}$ and $\mathrm{d} J_{\nu} / \mathrm{d} \tau_{\nu}$.

c) Derive the boundary conditions:

$$
\begin{aligned}
& J_{\nu} \rightarrow B_{\nu} \quad \text { for } \tau_{\nu} \rightarrow \infty \\
& \left.\frac{1}{3} \sqrt{3} \frac{\mathrm{d} J_{\nu}}{\mathrm{d} \tau_{\nu}}\right|_{\tau_{\nu}=0}=J_{\nu}(0) .
\end{aligned}
$$

d) Solve the second-order transport equation for $J_{\nu}$ using these boundary conditions. Give an expression for the emergent flux.

e) Use the solution for $J_{\nu}\left(\tau_{\nu}\right)$ to obtain an explicit expression for $S_{\nu}\left(\tau_{\nu}\right)$. Use the EddingtonBarbier approximation to show that:

$$
\begin{array}{cc}
I_{\nu}^{+}(0)=B_{\nu} & \text { for } \varepsilon_{\nu}=1 \\
I_{\nu}^{+}(0) \approx 2 \sqrt{\varepsilon_{\nu}} B_{\nu} & \text { for } \varepsilon_{\nu} \ll 1 .
\end{array}
$$

You have now derived the $\sqrt{\epsilon}$ law. Discuss it.

\section{Formation of resonance lines (Section '4. 3.5 in page}

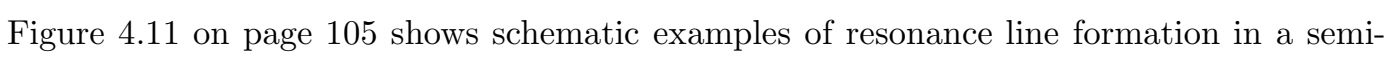
realistic atmosphere with a radiative-equilibrium photosphere and a hotter chromosphere. The scattering is coherent.

a) The $\tau^{c}=1$ depth is at the same location in all panels. Where? What is the EddingtonBarbier prediction for the emergent continuum intensity?

b) All $J$ curves flatten towards small $\tau^{\text {tot }}$. Why? Do they remain flat for yet smaller $\tau^{\text {tot}}$ ?

c) Locate the thermalization depth in each panel.

d) The $\sqrt{\varepsilon}$ law $(\bar{A} . \overline{1})$ does not hold at all. Why not? A much better approximation is given for many panels by $S(0) \approx \sqrt{\varepsilon} B\left(\tau^{\text {tot }}=\Lambda\right)$. Why? A yet better approximation, for example in the top-left panel, consists of combining $\left(\mathbf{A}^{-6} \mathbf{0}^{\prime}\right)$ with a similar adaptation of $\left(\underline{4} .8 \overline{0} \bar{y}^{\prime}\right): J(0) \approx \sqrt{\varepsilon} /(1+\sqrt{\varepsilon}) B\left(\tau^{\text {tot }}=\Lambda\right)$. Why?

e) Thermalization occurs at $B \approx 1$ in both the middle and bottom panels of the righthand column, but the value of $J(0)$ is higher in the bottom panel. Why?

f) The chromosphere is optically thin for the panels in the upper rows. Estimate its effect on the emergent intensity by describing it as an optically thin irradiated shell using (2.2.36i) on page $1 \overline{1} \overline{1}$, (the "thin cloud model").

g) Each column may be seen as sampling a line profile with line-center $\eta=10^{6}$ and coherent scattering with the same $\varepsilon$ at every wavelength. Assume that $S^{c}=B$ and sketch the 
shape of the emergent line profile per column using the Eddington-Barbier approximation. Assume a depth-independent Voigt profile with the cross-over between Doppler core and damping wings at $\eta=10^{4}$, as for $a=0.001$ in Figure $\overline{3} \cdot \overline{1}$ on page $6 \overline{0}_{1}^{\prime}$

h) This demonstration plot was made by using ( $\left(\mathbf{A}^{-6} \cdot \overline{0} 0^{\prime}\right)$ and approximate Lambda iteration, with $B$ as starting guess. It therefore shows the total source function $S^{\text {tot }}$ (why?) and assumes that $S^{l}=S^{c}=S^{\text {tot }}=S$ (why?), with the same degree of scattering in both the continuous and the bound-bound processes. Sketch the differences that would occur if the background continuum is formed in LTE.

i) Why are these plots schematic in comparison with resonance line formation in a more realistic plane-parallel atmosphere such as VALIII one? (The virtual star VALIII is defined to possess a strictly plane-parallel atmosphere on page 183 ; its temperature

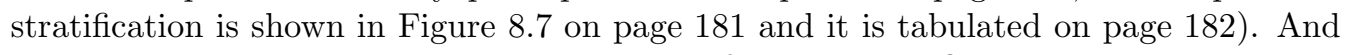
what about real resonance lines in the spectra from real stars?

\section{The VALIII NaID source function (Section $1 . \overline{4} .5$ in page}

Figure 10.13 , shows, against height in the VALIII atmosphere, the total source function $S_{\nu}$ (solid) and the Planck function $B_{\lambda}$ (dotted upper curve) at the wavelength of the $\mathrm{NaI} \mathrm{D}_{1}$ line $(\lambda=589.6 \mathrm{~nm})$, and also the mean intensity $J_{\nu}$ and continuum source function $S_{\nu}^{c}$. The latter two quantities are plotted twice, not only at line center but also for a location in the far wing. Complete redistribution and a two-level atom are excellent approximations for the $\mathrm{Na} I \mathrm{D}_{1}$ line.

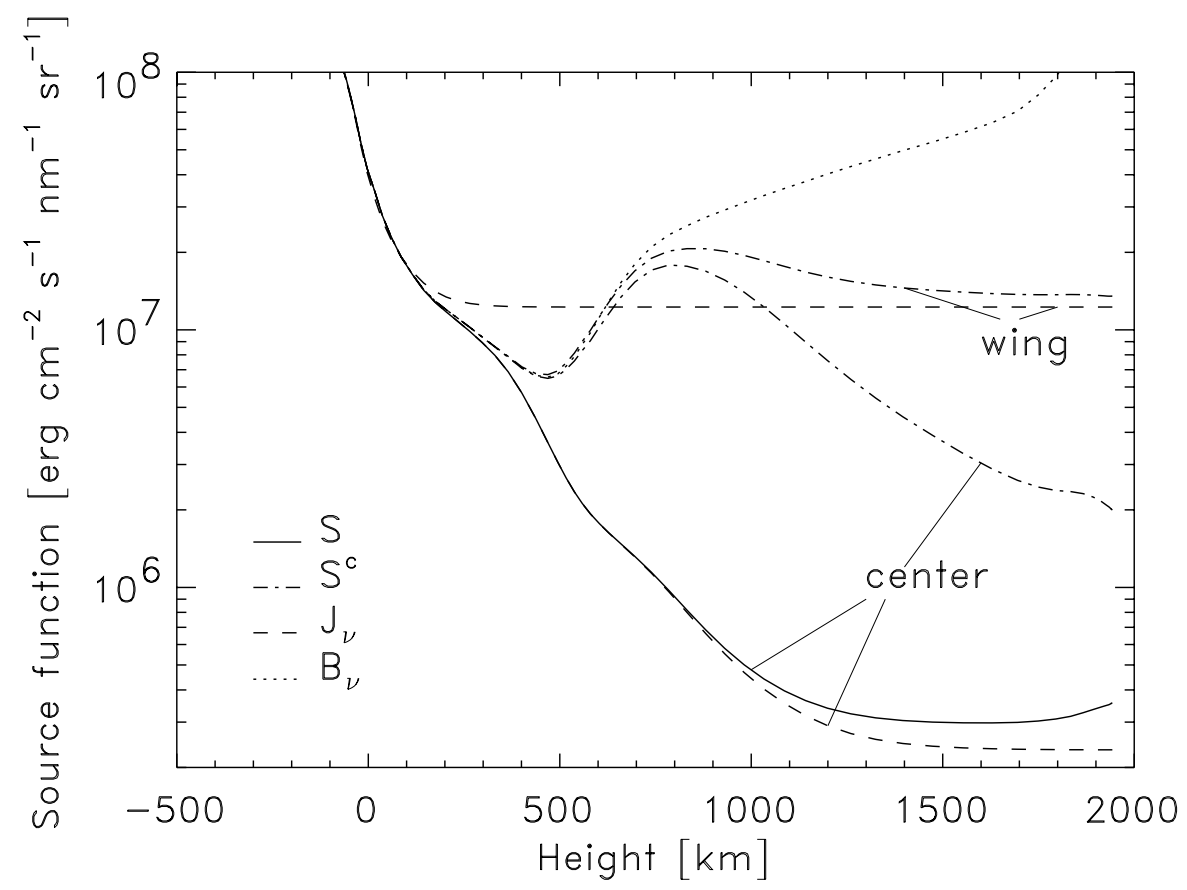

Figure 10.13: Source function structure of the VALIII Na I D line. From 'Uitenbroek and Bruls (1992)!

a) Estimate the radially emergent intensities at line center and in the far wing from the boundary values of $J$. Estimate the locations where $\tau=1$ at the two wavelengths. Is 
the $\mathrm{NaI} \mathrm{D}_{1}$ line a photospheric or a chromospheric one?

b) Show that the NaI $\mathrm{D}_{1}$ line is in absorption. What would it look like for LTE?

c) Explain that $S$ follows $J$ rather than $B$ above $300 \mathrm{~km}$. What does the temperature minimum have to do with this separation of $S$ from $B$ ? Would the separation be at the same place for much larger sodium abundance? Estimate the value of $\varepsilon_{\nu_{0}}$ at $h=1500 \mathrm{~km}$.

d) The background continuum is dominated by $\mathrm{H}^{-}$bf bound-free interactions. Describe this process and argue that LTE is valid for it. In addition, Thomson scattering is increasingly important above $h=500 \mathrm{~km}$. Why is that and how does that follow from the figure?

e) Explain that the line-center and wing continuum source functions diverge with height.

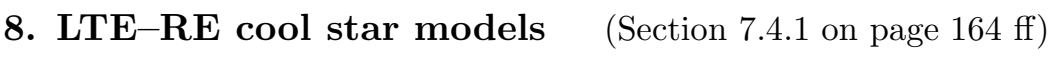

These questions concern the cool-star models shown in Figure 7.8 on page 165

a) First inspect the three solar models in the second panels of the lefthand columns. The low-metallicity model has constant $T(\tau)$ in the upper layers while the other models still decline outwards. Why?

b) In the deepest layers the low-metallicity model is cooler than the other two. Why?

c) Explain that the cool dwarf $\left(T_{\text {eff }}=4500 \mathrm{~K}, \log g=4\right)$ suffers a much larger depletion of electrons from low metallicity than the solar atmosphere (lower part of Figure $\left.7 .{ }^{7}{ }^{i}\right)$.

d) The electron depletion is yet larger for the cool giant. Why?

e) The metallicity has not much effect on the electron density in the hot dwarf atmosphere. Why?

f) The difference between the solar and low metallicity models extends to the deepest layers for the cool dwarf, but vanishes in the deepest layers for the cool giant. Why?

\section{VALIII continuum formation graphs (Section $8.2 \overline{2}$ on page $1 \overline{180} \mathrm{ff}$}

These questions concern the formation of the VALIII continua in Figures 18.918 on page 184i

a) First study the formation of the solar spectrum at $\lambda=10 \mu \mathrm{m} . \mathrm{H}^{-}$free-free dominates, why? It produces LTE, how do you see that? And why?

The dotted curve specifies the contribution function $\mathrm{d} I_{\nu} / \mathrm{d} h$ to the emergent intensity $I_{\nu}$ at $\mu=1$. Express it in $\tau_{\nu}$ and the emissivity $j_{\nu}$ and use this expression to explain the two sides of the curve.

Check the validity of the Eddington-Barbier approximation.

Why does $J_{\nu}$ drop below $B_{\nu}$ ?

b) Now look at the $\lambda=150 \mu \mathrm{m}$ plot. VALIII has limb brightening at this wavelength, why? Is the Eddington-Barbier approximation valid? 
The contribution function has a steeper outward drop, compared with the $\exp \left(-\tau_{\nu}\right)$ inward decay, than in the $\lambda=10 \mu \mathrm{m}$ plot. Use Figure

c) At $\lambda=1 \mathrm{~cm}$ the dotted contribution curve has an abrupt drop on its outward side. Why?

d) The $\lambda=1.6 \mu \mathrm{m}$ wavelength is very special. In what sense? How do you see that from its plot?

e) The $\lambda=500 \mathrm{~nm}$ plot is labeled "continuum" because it is for the high points between the lines in the spectrum. It shows that the photosphere is close to radiative equilibrium. How?

Does the $B_{\nu}$ slope obey $\mathrm{d} B_{\nu} / \mathrm{d} \tau_{\nu}=1.5 B_{\nu, 0}$ as in $(1 \overline{7} . \overline{4} \overline{3})$ on page $11 \overline{5} \overline{5}_{-1}$ ?

f) The $\lambda=250 \mathrm{~nm}$ plot has curves marked $\kappa_{\text {add }}$ specifying the contribution by added "line haze" quasi-continuous opacity made up from line distribution tables. How do you see that the lines are supposed to behave thermally in deep layers and to scatter higher up? This is the first panel where $S_{\nu}$ tends towards $J_{\nu}, S_{\nu}$ a bit more than $S_{\nu}^{a b}$. Why?

g) The $\lambda=176.7 \mathrm{~nm}$ and $\lambda=160.5 \mathrm{~nm}$ plots display formation near the temperature minimum. $S_{\nu}$ follows $J_{\nu}$, and $J_{\nu}$ departs from $B_{\nu}$ well below the $\tau_{\nu}=1$ location. What is the reason for this $J_{\nu}>B_{\nu}$ split? Estimate the thermalization depth at these two wavelengths. Is this coherent or redistributed scattering?

The expression $S_{\nu}=\left(1-\varepsilon_{\nu}\right) J_{\nu}+\varepsilon_{\nu} B_{\nu}$ requires that $S_{\nu}$ lies between $J_{\nu}$ and $B_{\nu}$ and that $S_{\nu}$ and $J_{\nu}$ intersect $B$ at the same point. This is not the case just above the temperature minimum in the $\lambda=160.5 \mathrm{~nm}$ plot. Why does this expression fail?

Does the expression $J_{\nu}=\boldsymbol{\Lambda}\left(S_{\nu}\right)$ fail as well?

h) The wavelength $\lambda=131.8 \mathrm{~nm}$ sits in the far wing of Ly $\alpha$. There is a lot of scattering, but it is not produced by Ly $\alpha$. How do you see that? What is the scattering agent?

Where does the radiation field thermalize in this plot?

The dotted $\mathrm{d} I_{\nu} / \mathrm{d} h$ curve in the $\lambda=131.8 \mathrm{~nm}$ plot is still fairly high at heights where $J_{\nu}$ is already flat. Show how this flatness results from $\boldsymbol{\Lambda}$ weighting of the outward rise of $S_{\nu}$.

i) The final plot is for a wavelength in the HeI and H I 1-c continua. The upper panels are the first in which the major contribuants are not similar for $r_{\mathrm{ab}}$ and $r_{\mathrm{em}}$. The HI Lyman continuum supplies half of the extinction below $2100 \mathrm{~km}$ but less than $10 \%$ of the emission. What does that signify?

This plot also shows funny $\mathrm{d} I_{\nu} / \mathrm{d} h$ behavior. This integrand does not at all peak near $\tau_{\nu}=1$ as in all other plots. Why not?

Is this is a case of a thin layer with or without irradiation from behind? Can you apply $(\overline{2} . \overline{3} \overline{6} \bar{i})$ on page $\overline{1} \bar{s}_{1}$ to obtain the emergent intensity?

This layer results from the small plateau around $h=2200 \mathrm{~km}$ in Figure $\mathrm{Can}$ you come up with a reason why empirical modeling of the solar spectrum requires this plateau within the VALIII approximations? Do you think it realistic?

\section{Vitense extinction diagrams (Section 8.}

These questions concern the Vitense diagrams of the continuous extinction coefficient in stellar atmospheres in Figure 18.6 on page 1 
Each plot is for the given value of $T_{\mathrm{e}}$ and a range of values of the electron pressure $P_{\mathrm{e}}$ as indicated by $\log P_{\mathrm{e}}$ values. These two parameters plus the given composition (element mixture) define the gas pressure as well as all Saha ionization ratios in the gas.

The y-axis scales A, B and $\mathrm{C}$ are normalized by $P_{\mathrm{e}}$ so that linear dependence on $P_{\mathrm{e}}$ produces curve equality in the logarithmic plot. The computation of the extinction coefficients (units $\mathrm{cm}^{2}$ gram $^{-1}$ ) included neutral $\mathrm{H}, \mathrm{H}^{-}, \mathrm{H}_{2}$, neutral $\mathrm{He}, \mathrm{He}^{+}$and several heavier elements (electron donors), plus Thomson scattering by electrons and Rayleigh scattering by $\mathrm{H}$ atoms. A more extensive description, with similar plots for other values of $\theta=5040 / T_{\mathrm{e}}$, is given in Novotny $(19 \overline{7} \overline{3})$ :

a) First study the solar plot on page 1.799. Identify the axis meanings.

Check: for $\log P_{\mathrm{e}}=-1$ and $\log \lambda=4.0, \log \kappa=-1.4-1.0=-2.4$.

Identify the edges in the dashed $\mathrm{H}$ curve (it has an offset scale so that it fits in the figure; the offsets are specified in Novotny $\left.1973^{i}\right)$. If the atmosphere contained only $\mathrm{H}$ atoms, the solid curves would equal the $\mathrm{H}$ curve, shifted up or down. Why does it simply shift? How would the extinction in such an atmosphere change with $\log P_{\mathrm{e}}$ ? How would the corresponding curves in a Vitense graph shift for different $\log P_{\mathrm{e}}$ ?

Why do all hydrogen edges share the same slope? What is its value? Why do their peaks for increasing wavelength first drop tremendously, but then increase again?

The shape of the solid curves shows that $\mathrm{H}^{-}$dominates at longer wavelengths except at low electron pressure. Why?

Show from the VALIII model specified in Table 8.21 on page $i \overline{1} \overline{2} 2$ that the electron pressure in the low photosphere has $\log P_{\mathrm{e}} \approx 1$. What type of star is modeled by the $\log P_{\mathrm{e}}=-1$ curve?

The solid curves for $\log P_{\mathrm{e}}=0-3$ nearly coincide for $\log \lambda>4$. What does that imply for the sensitivity of the extinction to the electron pressure? Why is this the case?

The $\log P_{\mathrm{e}}=-1$ lies higher in the figure. Why? It shows contribution from neutral $\mathrm{H}$ but this contribution does not dominate. How do you see this?

Rayleigh scattering is only important near $\log \lambda=3.1$ for $\log P_{\mathrm{e}}=-1$. Why? Explain the shapes of the Rayleigh curves and the shift between them. Actually, Rayleigh scattering is not important for the formation of the solar spectrum at this wavelength. Why?

Thomson scattering has its own scale $\mathrm{C}$ in complex notation. Check: for $\log P_{\mathrm{e}}=-1$ this solar plot has $\log \sigma_{\mathrm{e}}=-1.9-1.0=-2.9$. This is the highest value in the plot, why? Check that it never dominates in all $P_{\mathrm{e}}$ cases. What fraction is Thomson scattering of the spectrum-averaged mean extinction for the various $\log P_{\mathrm{e}}$ values?

b) Now look at the cool-star plot on page 195 . Explain the changes compared with the solar plot, those between the two $\mathrm{H}$ curves, in Rayleigh and Thomson scattering, in relative $\mathrm{H}^{-}$contribution and $\mathrm{H}$ contribution.

Why are the solid curves (and their means) at higher extinction than in the solar plot?

c) Now turn to the hottest Vitense diagram, on page '192.'. Why is there no curve for $\log P_{\mathrm{e}}=0$ or $\log P_{\mathrm{e}}=-1$ ?

Thomson scattering dominates fully for $\log P_{\mathrm{e}}=1$. How do you see that immediately from scales B and C? And from the corresponding solid curves? And from the shifts between them?

The $\mathrm{H}$ curve on the bottom is very different from the solar case. The edges are much smaller and overall, the extinction increases rapidly with wavelength. Why?

Do the solid curves contain $\mathrm{He}^{+}$edges at the wavelengths of the $\mathrm{H}$ edges, coinciding with the latter, or not? And reversely? 


\section{Kurucz flux plots (Section $8 . \overline{3}$ on page, 190 if)}

These questions concern the Kurucz plots of computed LTE-RE stellar flux spectra in Fig-

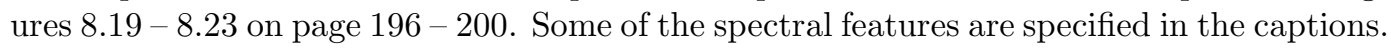
Additional ones are listed in Table on page '1 $14 \overline{4}$ '.

a) First study Figure 1.19 . Locate all features listed in the caption. On which side of each edge is the extinction largest? How can you see that from the flux behavior?

Compare the two bound-free edges at $\nu=3.3$ and $\nu=6.0$ (x-axis units). The latter edge vanishes for the hottest stars, but the $\nu=3.3$ edge does not. The caption suggests that they are due to HI and HeI, respectively. Show from the corresponding ionization energies that this cannot be the whole story. Which species contributes additionally to which edge, and why?

Now turn to the line present in the upper curves of Figure $18 . \overline{8} \bar{g}_{1}$ at $\nu=7.8$. Its presence varies just as the He II 2-1 line at $\nu=9.9$, but HeII has no line in this spectral region (Table 8.1 . . Use Table 7 to argue that this line is probably C III ??-?? because C III mimics He II behavior closely.

b) The lowest curves in Figure $8 . \overline{1}$ ing gradually bend over with increasing $\nu$ towards the Lyman limit at $\nu=3.3$, whereas the upper curves don't. What causes this difference?

The intermediate $T_{\text {eff }}=35000$ curves show appreciable difference below the Lyman limit between low and high $\log g$. Why?

At $\nu \approx 6$ the $T_{\text {eff }}=35000$ curve drops much more for larger $\log g$. A similar split sets in at the Lyman limit. What causes these?

To the right of the $\nu \approx 6$ edge, the upper curve has a flatter gradient than the lower one. Why?

c) Now inspect Figure $\overline{8} . \overline{2} \overline{0}$. The $T_{\text {eff }}=15000$ panel has a prominent peak at $\nu=7.5$ in the x-axis units. Why doesn't it coincide with the Balmer limit?

What is the deep line at this frequency in the $T_{\text {eff }}=6000$ panel. Why is this the strongest spectral line in the whole spectrum at this temperature?

d) The following questions concern Figure $8 . \overline{2} \overline{3}_{-i}$ Why is the hottest curve nearly without $\mathrm{HI}$ lines and edges?

What sets the steep increase with $1 / \lambda$ for the hottest model?

Why is the curve stacking inverse to $T$ on the left?

Why do the Paschen lines diminish for the lowest $T$ (top curve)?

Why do the Paschen lines diminish for the highest $T$ (bottom curve)?

Why is the curve stacking conform to $T$ on the right?

Why are the lowest curves on the right about equal?

\section{NLTE-RE hot star models (Section i:}

This final big-whopper exercise concerns the hot-star models computed by Auer and Mihalas

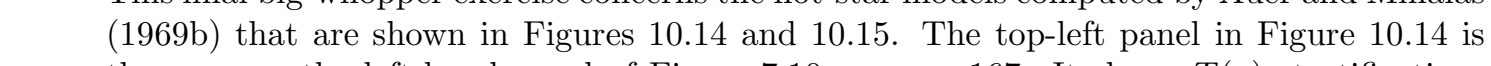
the same as the left-hand panel of Figure $t_{-1}^{1} 0_{1}^{1}$ on page $167_{1}$. It shows $T(\tau)$ stratifications of the atmosphere of a hot dwarf star that result from $\bar{H}_{\bar{E}}-\mathrm{SE}-\mathrm{RE}$ modeling with various assumptions. The major one is that the atmosphere consists exclusively of hydrogen gas. This is largely ionized, so that there are only protons, electrons and neutral hydrogen atoms. 

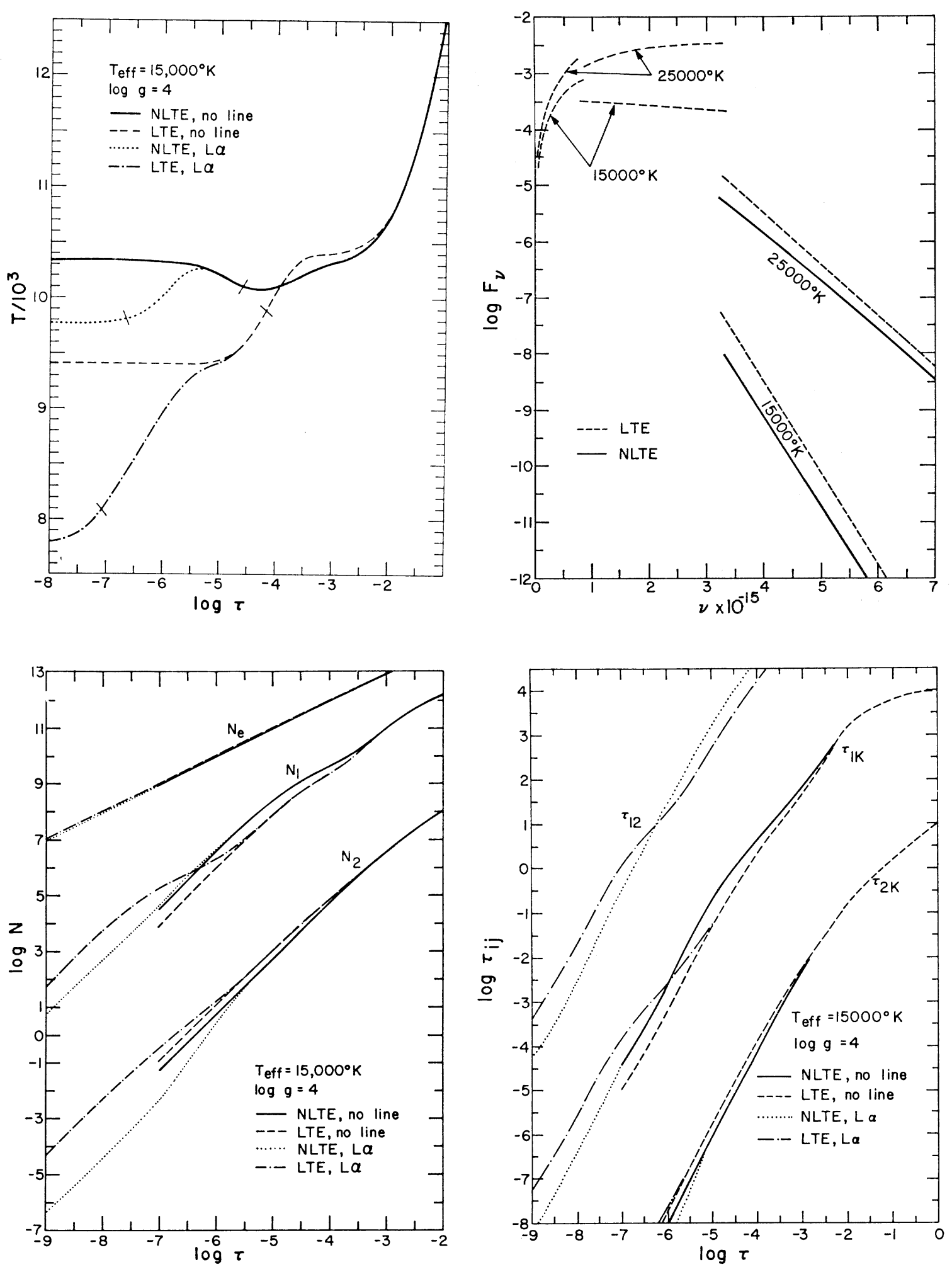

Figure 10.14: Results for HE-RE-NLTE_modeling_of the atmosphere of a hot dwarf star with $T_{\text {eff }}=$ $15000 \mathrm{~K}$ and $\log g=4$ by Auer and Mihalas $(1969 \mathrm{~b})_{1}^{\prime}$. The $\log \tau$ scales along the $\mathrm{x}$ axes are measured in the H I free-free continuum at $\bar{\lambda}=400 \mathrm{~nm}$, just longward of the Balmer jump. The $\tau_{i j}$ values in the lower right panel are given for each feature separately, without summing. The corresponding Ly $\alpha$ departure coefficients and source functions are shown in the next figure. The upper right panel also contains AuerMihalas results for a dwarf with $T_{\text {eff }}=25000 \mathrm{~K}$. 

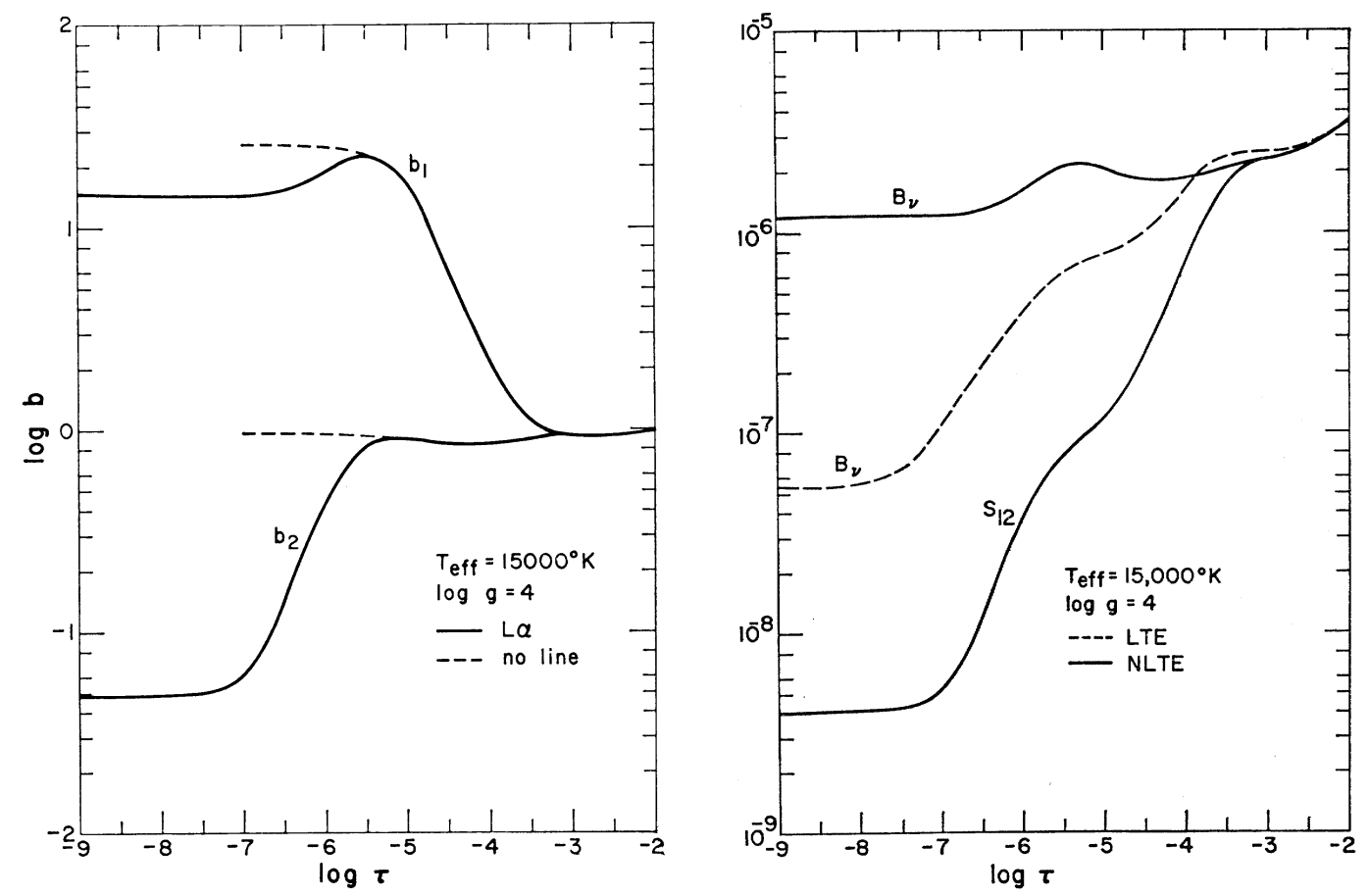

Figure 10.15: More Auer-Mihalas results for the same star. The logarithmic divisions along the $y$ axes have no ticks for $7 \times 10^{n}$ and $9 \times 10^{n}$.

The different models serve to illustrate the influence of the NLTE effects in the Lyman and Balmer continua and the $\operatorname{Ly} \alpha$ line on the resulting atmospheric temperature structure.

These six graphs show results for the case that the hydrogen atoms contain only bound levels 1 and 2 and the ion state (labeled K). The nice thing about this highly simplified twolevel-plus-continuum hydrogen atom is that the remaining extinction features differ so much in opacity that their effects on the resulting model are fairly well spread in height. (This is not the case in the righthand panel of Figure 17.10 on page $16 \%$ in which the Balmer $\mathrm{H} \alpha$ line affects the $T(\tau)$ stratification at just the height where the Lyman continuum originates and where Balmer continuum heating is most effective.)

There are four sets of computations, for LTE and NLTE with Ly $\alpha$ absent or present. The top-left panel of Figure $10 . \overline{1} \overline{4}_{1}^{1}$ shows the resulting RE temperature stratifications. The Ly $\alpha$ line was computed with a Doppler profile assuming complete redistribution.

a) First sketch the appropriate $\mathrm{HI}$ term diagram for future reference. It has $E_{21}=10.2 \mathrm{eV}$, $E_{\mathrm{K} 1}=13.6 \mathrm{eV}$. Calculate the frequencies and wavelengths of all spectral features (or look them up).

b) Panel 3 of Figure $10 . \overline{1}$, shows the particle densities for level 1, level 2 and free electrons. How large is the proton density $N_{\mathrm{K}}$ ?

What is the ionization fraction $N_{\mathrm{H} \text { II }} / N_{\mathrm{H} \text { I }}$ at $\log \tau=-3$ ? Give two reasons why the ratio increases with height. Does the ratio increase further for $\log \tau<-9$ ?

At the top of the atmosphere the electron densities $N_{\mathrm{e}}$ differ only slightly between the NLTE and LTE models including Ly $\alpha$. The dotted NLTE curve in panel 3 falls just below the dot-dashed LTE curve. Why whould you expect rather the reverse, if anything, from panel 1 ?

The reason for this reversed sign is that the atmosphere obeys hydrostatic equilibrium.

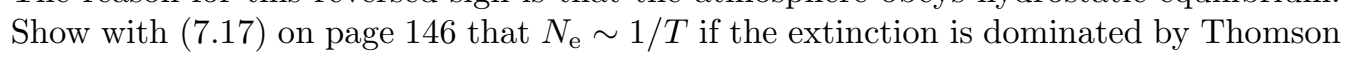


scattering at large hydrogen ionization. Why is the latter assumption valid at sufficiently small $\tau$ ?

c) The outer-layer density drop from cooler to hotter model is much larger for $N_{1}$ and is yet larger for $N_{2}$. Why?

d) Next come the optical depths. The $\log \tau$ scale along the $x$ axes is for $\lambda=400 \mathrm{~nm}$, just longward of the Balmer jump (the wavelengths of the various H I features are given in

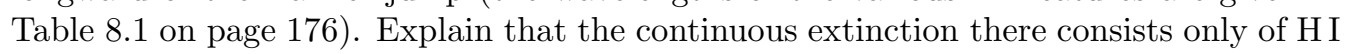
free-free transitions and Thomson scattering. Why is there $\mathrm{H}^{-}$extinction negligible?

Other $\tau$ scales are given in panel 4 , for each feature separately (not summed together). The $\tau_{12}$ scale holds for the center of $\operatorname{Ly} \alpha$; the $\tau_{1 \mathrm{~K}}$ and $\tau_{2 \mathrm{~K}}$ Lyman and Balmer continuum scales are at the head (maximum extinction) of each edge. Why do these curves follow those in panel 3 ?

The vertical offset between the $\tau_{12}$ and the $\tau_{1 \mathrm{~K}}$ curves is to some extent a measure of the ratio between the bound-free and bound-bound extinction coefficients per particle in the $n=1$ ground state $\sigma_{12}$ and $\sigma_{1 \mathrm{~K}}$. Why? Precisely or not precisely?

We will now quantify these extinctions. The bound-free H I $n=1$ extinction coefficient per particle at threshold is $\sigma_{1 \mathrm{~K}}=6.3 \times 10^{-18} \mathrm{~cm}^{-2}$. Why is this number independent of pressure and temperature? Check its value from (12.74) on page 2 Figure $\overrightarrow{2} \cdot \vec{i}$

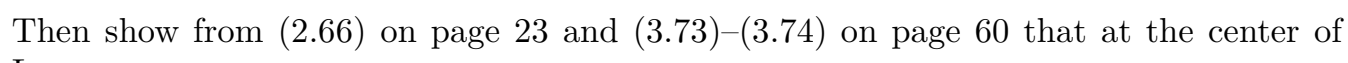
$\operatorname{Ly} \alpha$

$$
\sigma_{12}\left(\lambda=\lambda_{0}\right) \approx 0.02654(1-a) \frac{\lambda^{2}}{c} \frac{f_{12}}{\sqrt{\pi} \Delta \lambda_{\mathrm{D}}} \mathrm{cm}^{2} .
$$

Evaluate this number, using $f_{12}=0.416, T=10^{4} \mathrm{~K}$ and $a=0$. (Auer and Mihalas assumed a Doppler profile and did not include microturbulence; the Doppler width $\Delta \lambda_{\mathrm{D}}$

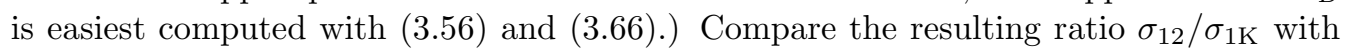
the offset between the $\tau_{12}$ and $\tau_{1 \mathrm{~K}}$ curves in panel 4 .

Figure $\overline{2} . \overline{1}$ on page $\overline{2}_{1}^{\prime}$ shows that $\sigma_{2 \mathrm{~K}} / \sigma_{1 \mathrm{~K}} \approx 2$. What causes this increase?

The $\tau_{1 \mathrm{~K}}$ and $\tau_{2 \mathrm{~K}}$ curves in panel 4 show a reverse ratio, with much larger difference. Why?

The $\tau_{12}=1$ locations are specified by the two tick marks on the Ly $\alpha$ curves. The low-temperature tick is further out. Why?

The other two ticks mark the $\tau_{1 \mathrm{~K}}=1$ locations on the two no-line curves. These are at similar temperature and show a reversed shift, with the NLTE tick further out. This shift results from NLTE overpopulation of level 1 at heights above $\log \tau_{400}=-4$ which is shown in the lefthand panel of Figure $10 . \overline{1} \overline{1}$. How does such overpopulation affect the $\tau_{1 \mathrm{~K}}=1$ location? Linearly or nonlinearly? Should the warning about different definitions of departure coefficients on page $\bar{B}_{1}^{\prime}$ be heeded here? What is the value of $b_{\mathrm{K}}$ ?

e) We will now inspect the spectral features due to H I, first in the spectrum. Use the offsets at $\log \tau_{400}=-4$ in panel 4 to sketch the variation of $\log \alpha_{\nu}^{\text {tot }}$ with wavelength over the whole spectrum at that height. How do you fix the y scale?

Identify the corresponding features in the emergent flux spectra in panel 2. (This panel also contains Auer-Mihalas results for another star with $T_{\text {eff }}=25000 \mathrm{~K}$.) Which feature is missing? Is it in absorption or emission? Why are the slopes of the continua different from the slopes in your plot?

The $\tau_{1 \mathrm{~K}}=1$ ticks in panel 1 indicate that, due to the outward rise of the solid curve, the emergent flux samples a higher temperature in the NLTE case than in the LTE case. However, the NLTE Lyman continuum in panel 2 is lower than the LTE Lyman 
continuum. Why?

f) Let us now inspect the influence of the H I spectral features on the atmosphere. The LTE curve including Ly $\alpha$ (dot-dashed) in panel 1 shows three steep outward declines, each levelling of to a horizontal plateau. Each steep part marks something getting optically thin. What somethings? The ticks on the curves mark the corresponding $\tau_{\nu}=1$ locations, again per feature. Check them from panel 4 .

First the effect of Ly $\alpha$. The model that results for LTE without this line (dashed curve in panel 1) has a final drop that ends at $\log \tau_{400} \approx-5$. Why is it flat at larger height? This value represents the boundary temperature $T(\tau=0)$.

Why is $T(\tau=0)$ much lower if Ly $\alpha$ is taken into account LTE-wise (dot-dashed curve)?

Why is $T(\tau=0)$ considerably larger when the assumption of LTE is dropped (dotted curve)? Why does the dotted curve flatten sooner than the dot-dashed curve with respect to the $\tau_{12}=1$ tick marks?

Why is the boundary temperature drop between the two NLTE curves much smaller than between the two LTE curves?

The Ly $\alpha$ behavior is illustrated by the departure coefficients $b_{1}$ and $b_{2}$ and the LTE and NLTE source functions in Figure $10 . \overline{1} \overline{5}_{1}$. What happens to the $b_{1}$ and $b_{2}$ curves for LTE? The offsets between the solid curves at the outer boundary are the same in the two panels (2.6 units in the log). Why?

The lefthand panel of Figure $10 . \overline{1} \overline{5}_{1}$ shows that $b_{2}$ drops down from $b_{2} \approx 1$ above (higher in the atmosphere then) $\log \tau_{400}=-5.5$. What mechanism causes this loss of level 2 population? There is an an outward rise for $b_{1}$ already in the layers below $\log \tau_{400}=-5$ (where "below" means deeper) which does not have to do with Ly $\alpha$ as can be seen from panel 1 in Figure $10.14^{1}$. How would $b_{1}$ behave if Ly $\alpha$ strictly obeyed the two-level approximation?

Can you estimate the location $\tau_{12}^{*}=1$ (effective optical depth unity) for Ly $\alpha$ radiation from the two $b_{2}$ curves in Figure 10.1 $5_{1}$ ? And from the $S_{12} / B_{\nu}$ behavior in the righthand panel? What about the thermalization depth $\Lambda_{12}$ for Ly $\alpha$ radiation? And the value of the collisional destruction probability $\varepsilon_{12}$ ?

g) The next item is the Balmer continuum. The location of $\tau_{2 \mathrm{~K}}=1$ is not marked with a tick in panel 1 of Figure $10 . \overline{1}$ but panel 4 shows that it lies near $\log \tau_{400}=-1.4$. Why is it so much deeper than the location with $\tau_{1 \mathrm{~K}}=1$ ? How does panel 1 illustrate that the Balmer continuum formation obeys LTE? How can you estimate this location from the curves in panel 1? Sketch the $T\left(\log \tau_{400}\right)$ curve in panel 1 that would result if the Balmer bound-free transitions are the only H I photon transitions (no Ly $\alpha$ and no Lyman continuum, only the Balmer and free-free continua plus Thomson scattering).

Panel 2 shows that the Balmer continuum gives the largest contribution to the flux integral. The RE condition (flux divergence integral zero) is very sensitive to this continuum and vice-versa. The value $T_{\text {eff }}=15000 \mathrm{~K}$ implies a boundary temperature $T(\tau=0)=12600 \mathrm{~K}$ for the grey case. How? Why is $T(\tau=0)$ much lower with the Balmer bound-free transitions present? How does the RE condition come in?

h) The most complex result in panel 1 is the upturn of the solid curve. This curve results from admitting NLTE in the Lyman and Balmer continua (with or without Ly $\alpha$ ). It has a small outward rise around the tick mark for $\tau_{1 \mathrm{~K}}=1$ that results from local heating in the Balmer continuum and absence of local cooling in the Lyman continuum. We will explore these processes in a few steps.

Start with the free-free continuum. Longward of $\lambda=400 \mathrm{~nm}$ this has boundaray radiation $J_{\nu}(\tau=0)<B_{\nu}(\tau=0)$. Show that this inequality represents a cooling contribution to the 
flux divergence integral in layers that still have some free-free extinction.

In contrast, the Balmer continuum has $J_{2 \mathrm{~K}}(\tau=0)>B_{2 \mathrm{~K}}(\tau=0)$ above $\log \tau_{400} \approx-3$. Thus, the Balmer continuum supplies a net heating contribution along the curve that you have drawn in panel 1. Explain the reason for the sign difference between these two $J_{\nu}-B_{\nu}$ splits. What do you expect for $J_{2 \mathrm{~K}}-B_{2 \mathrm{~K}}$ in deeper layers?

The Lyman continuum is much further shortward from $\lambda=400 \mathrm{~nm}$ than the Balmer continuum, but it is nevertheless a strong coolant in LTE as shown by the dashed curve in panel 1. Why?

The cooling effect of the Lyman continuum vanishes in the NLTE case (solid curve in panel 1) because the Lyman bound-free transitions act rather as bound-bound resonance transitions. Use your HI term diagram to explain that Lyman continuum transitions have larger bound-bound character than Balmer continuum transitions. Why is there complete redistribution over the edge profile?

Why is the bound-free collisional destruction probability defined by $\overline{3} \cdot \overline{1} \overline{1} \overline{1})$ on page $\overline{7}$ an approximation? Why may you expect it to be smaller for the Lyman bound-free transitions than for the Balmer bound-free transitions? Additional destruction of Lyman and Balmer continuum photons occurs through free-free interactions. How? Why is its probability also smaller for Lyman continuum photons than for Balmer continuum photons?

Thus, in the NLTE case the Lyman continuum acts as a scattering resonance line, with

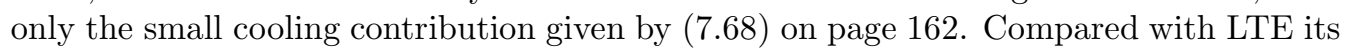
cooling vanishes while the Balmer heating remains; the temperature goes up to maintain the RE condition as shown by the solid curve in panel 1.

Let us consider this process in more detail. We have already seen that $b_{\mathrm{K}} \approx 1$ everywhere, without sensitivity to the spectral features that we discuss. The departure coefficient of level 2 is strongly coupled to this population reservoir and it therefore also has NLTE departure coefficient $b_{2} \approx 1$ until the onset of Ly $\alpha$ losses ( $b_{2}$ curve in Figure $\left.10.101-15\right)$. The collisional coupling of level 1 to the reservoir is far smaller so that its population may therefore depart much more. The overpopulation of the ground state reaches $b_{1} \approx 10$ already at $\log \tau_{400}=-5$. This increase must be due to the Lyman bound-free transitions alone, without influence from $\operatorname{Ly} \alpha$. Now do a thought experiment $\bigsqcup_{-1}^{\overline{8}}$ in which all

\footnotetext{
${ }^{8}$ Such thought experiments are interesting exercises when trying to understand the mechanisms that control NLTE level populations and radiation fields. Auer \& Mihalas discuss another one in their paper in order to understand the limiting of the amount of Balmer continuum heating by Ly $\alpha$. However, such experiments are also dangerous. It is usually difficult or impossible to distinguish causes and effects from each other. The statistical equilibrium solution established by the computer represents a closed multi-loop system in which all mechanisms act and counteract to obtain a stable time-independent overall pattern in which any net rate is balanced by other net rates. Even in this simplified HI case, with only a few mechanisms at work and those mostly separated in their major depth of operation, the final solution is complex. None of the me to do so. The Auer and Mihalas (1969b) paper that this exercise is built on and its sequel on the effect_of including Balmer- $\alpha$ rather than Ly $\alpha$ (second panel in Figure 7.10 ' on page '167, 'Auer and Mihalas 1969c) have established the mechanism of "Balmer continuum heating" - but it might also be called "absence of Lyman continuum cooling". Let me digress by way of example to the Atlantic climate control system. The Gulf Stream, the evaporation in the northern Atlantic by westerly storms (dry from passing over Canada and depositing the picked-up humidity in northern Europe, on Holland at the moment of my writing this), the gigantic underwater waterfall southwest of Iceland where the resulting heavy salt water plunges down, the deep-sea salt-water flow to Cape Horn and into a loop around the Indian Ocean, the mixing with cold fresh water melted from the Antarctic ice sheet, and the re-emergence at the surface in the Easterly winds near the Caribbean all together represent a rather similar closed-loop flow that obeys stationarity = statistical equilibrium (hopefully). One might take the Gulf Stream, the North-Atlantic wind forcing, the salt-water waterfall, the cold fresh Antarctic water supply or the Mid-Atlantic trade winds each as the major driver and then describe the others as the resulting effects. And, on a longer time scale, one
} 
populations have LTE values initially. The LTE photon losses in the Lyman continuum then pull population from the reservoir down to the ground state: each photon that is lost to space leaves one more surplus atom in $n=1$ behind. The proton reservoir is too large to be affected by this drain so that $b_{\mathrm{K}}$ remains close to unity, but level 1 gets overpopulated. Its departure coefficient $b_{1}$ grows until photoionization and/or collisional ionization have sufficiently increased to balance the photon-loss drain so that statistical equilibrium is reached. Why do these upward rates increase with $b_{1}$ ? Do the collisional ionization and recombination rates balance each other? How does the Lyman continuum cooling depend on $b_{1}$ ?

might declare the current absence of high mountains in north-east Canada, the presence of a snow-catching ice-laden continent at the South Pole, the land-locked confinement of the Arctic sea, the global weather pattern, or even solar activity each as the essential boundary condition making northern Europe just wet rather than frozen in our era. Clearly, each of these mechanisms and boundary conditions is of considerable interest and must be diagnosed in detail, but actual solutions, including the one demonstrated by the earth itself, can only be simulated by numerical modeling of the whole system. 


\section{References}

Abramowitz, M. and Stegun, I.: 1964, Handbook of Mathematical Functions, U.S. Dept. of Commerce, Washington

Allen, C. W.: 1976, Astrophysical Quantities, Athlone Press, Univ. London

Aller, L. H.: 1952, Astrophysics. The Atmospheres of the Sun and Stars, Ronald Press Co., New York

Anderson, L.: 1991, in L. Crivellari, I. Hubeny, and D. G. Hummer (Eds.), Stellar Atmospheres: Beyond Classical Models, NATO ASI Series C 341, Kluwer, Dordrecht, p. 29

Anderson, L. S.: 1985, Astrophys. J. 298, 848

Anderson, L. S.: 1989, Astrophys. J. 339, 558

Athay, R. G.: 1972, Radiation Transport in Spectral Lines, Reidel Publ. Co., Dordrecht

Athay, R. G. and Lites, B. W.: 1972, Astrophys. J. 176, 809

Auer, L.: 1967, Astrophys. J. 150, L53

Auer, L.: 1991, in L. Crivellari, I. Hubeny, and D. G. Hummer (Eds.), Stellar Atmospheres: Beyond Classical Models, NATO ASI Series C 341, Kluwer, Dordrecht, p. 9

Auer, L. H., Heasley, J. N., and Milkey, R. W.: 1972, A Computational Method for the Solution of Non-LTE Transfer Problems by the Complete Linearization Method, Contribution No. 555, Kitt Peak National Observatory

Auer, L. H. and Mihalas, D.: 1969a, Astrophys. J. 158, 641

Auer, L. H. and Mihalas, D.: 1969b, Astrophys. J. 156, 157

Auer, L. H. and Mihalas, D.: 1969c, Astrophys. J. 156, 681

Avrett, E. H.: 1965, in E. H. Avrett, O. J. Gingerich, and C. A. Whitney (Eds.), The formation of spectral lines, Procs. Second Harvard-Smithsonian Conf. on Stellar Atmospheres, Smithsonian Astrophys. Obs. Special Report 174, Cambridge, Mass., p. 101

Avrett, E. H.: 1985, in B. W. Lites (Ed.), Chromospheric Diagnostics and Modeling, National Solar Observatory Summer Conference, Sacramento Peak Observatory, Sunspot, p. 67

Avrett, E. H. and Hummer, D. G.: 1965, Mon. Not. R. Astron. Soc. 130, 295

Avrett, E. H. and Loeser, R.: 1987, in W. Kalkofen (Ed.), Numerical Radiative Transfer, Cambridge University Press, U.K., p. 135

Avrett, E. H. and Loeser, R.: 1992, in M. Giampapa and J. M. Bookbinder (Eds.), Cool Stars, Stellar Systems and the Sun, Proc. Seventh Cambridge Workshop, Astron. Soc. Pacific Conf. Series26, p. 489

Ayres, T. R.: 1977, Astrophys. J. 213, 296

Ayres, T. R. and Linsky, J. L.: 1975, Astrophys. J. 200, 660

Ayres, T. R., Linsky, J. L., Rodgers, A. W., and Kurucz, R. L.: 1976, Astrophys. J. 210, 199

Ayres, T. R., Linsky, J. L., and Shine, R. A.: 1974, Astrophys. J. 192, 93

Barklem, P. S. and O'Mara, B. J.: 1997, Mon. Not. Roy. Astron. Soc. 290, 102

Baschek, B. and Scholz, M.: 1982, in K.-H. Hellwege (Ed.), Landolt-Börnstein New Series, Stars and Star Clusters, Group VI Vol. 2b, Astronomy and Astrophysics, Springer, Heidelberg, p. 91

Bashkin, S. and Stoner, J. O.: 1975, Atomic Energy Levels and Grotrian Diagrams, North-Holland Publ. Co., Amsterdam

Böhm-Vitense, E.: 1989, Introduction to stellar astrophysics. II. Stellar Atmospheres, Cambridge Univ. Press, Cambridge UK

Bowers, R. L. and Deeming, T.: 1984, Astrophysics I. Stars, Jones and Bartlett, Boston 
Bransden, B. H. and Joachain, C. J.: 1989, Introduction to quantum mechanics, Longman Scientific \& Technical, Harlow, U.K.

Bruls, J. H. M. J. and Rutten, R. J.: 1992, Astron. Astrophys. 265, 257

Bruls, J. H. M. J., Rutten, R. J., and Shchukina, N. G.: 1992, Astron. Astrophys. 265, 237

Cannon, C. J.: 1973, Astrophys. J. 185, 621

Cannon, C. J.: 1985, The transfer of spectral line radiation, Cambridge Univ. Press, Cambridge UK

Carlsson, M.: 1986, A Computer Program for Solving Multi-Level Non-LTE Radiative Transfer Problems in Moving or Static Atmospheres, Report No. 33, Uppsala Astronomical Observatory

Carlsson, M., Rutten, R. J., Bruls, J. H. M. J., and Shchukina, N. G.: 1994, Astron. Astrophys. $\mathbf{2 8 8}, 860$

Carlsson, M., Rutten, R. J., and Shchukina, N. G.: 1992, Astron. Astrophys. 253, 567

Carlsson, M. and Stein, R. F.: 1995, Astrophys. J. 440, L29

Cayrel, R.: 1963, Comptes Rendus Acad. Sci. Paris 257, 3309

Chalonge, D. and Kourganoff, V.: 1946, Ann. d'Astrophys. 9, 69

Chandrasekhar, S.: 1939, An Introduction to the Study of Stellar Structure, University of Chicago, reprinted Dover Pub., 1957

Chandrasekhar, S.: 1950, Radiative Transfer, Clarendon Press, Oxford, reprinted Dover Pub., 1960

Chandrasekhar, S. and Breen, F.: 1946, Astrophys. J. 104, 430

Condon, E. U. and Shortley, G. H.: 1964, The Theory of Atomic Spectra, Cambridge Univ. Press, Cambridge UK

Corliss, C. and Bozman, W. R.: 1962, Experimental Transition Probabilities for Spectral Lines of Seventy Elements, NBS Monograph 53, Government Printing Office, Washington DC

Craig, I. J. D. and Brown, J. C.: 1986, Inverse problems in astronomy, Hilger, Bristol

Cram, L. E., Rutten, R. J., and Lites, B. W.: 1980, Astrophys. J. 241, 374

Crivellari, L., Hubeny, I., and Hummer, D. G. (Eds.): 1991, Stellar Atmospheres: Beyond Classical Models, NATO ASI Series C 341, Kluwer, Dordrecht

del Toro Iniesta, J. C.: 2003, Introduction to Spectropolarimetry, Cambridge Univ. Press, Cambridge UK

Desikachary, K. and Gray, D. F.: 1978, Astrophys. J. 226, 907

Dijksterhuis, E. J.: 1950, De mechanisering van het wereldbeeld, Meulenhoff, Amsterdam

Dijksterhuis, E. J.: 1969, The mechanization of the world picture, Galaxy Books, London

Dorfi, E. A. and Drury, L. O.: 1987, J. Comp. Phys. 69, 175

Eddington, A. S.: 1916, Mon. Not. R. Astron. Soc. 77, 16

Eddington, A. S.: 1926, The Internal Constitution of the Stars, Dover Pub., New York

Engvold, O.: 1977, Physica Scripta 16, 48

Feautrier, P.: 1964, Comptes Rendus Acad. Sci. Paris 258, 3189

Fontenla, J. M., Avrett, E. H., and Loeser, R.: 1993, Astrophys. J. 406, 319

Forbes, J. D.: 1836, Edinburgh Trans. II, 453

Foukal, P.: 1990, Solar Astrophysics, Wiley and Sons, New York

Gray, D. F.: 1992, The Observation and Analysis of Stellar Photospheres, Cambridge Univ. Press, U.K. (second edition)

Griem, H. R.: 1974, Spectral Line Broadening by Plasmas, Acad. Press, New York

Gustafsson, B., Bell, R. A., Eriksson, K., and Nordlund, Å..: 1975, Astron. Astrophys. 42, 407

Gustafsson, B. and Jørgensen, U. G.: 1994, Astron. Astrophys. Rev. 6, 19

Halenka, J. and Grabowski, B.: 1984, Astron. Astrophys. Suppl. Ser. 57, 43

Hamann, W.-R.: 1985, Astron. Astrophys. 148, 364

Hamann, W.-R.: 1986, Astron. Astrophys. 160, 347

Harwit, M.: 1988, Astrophysical Concepts, Springer-Verlag, New York (second edition)

Hearnshaw, J. B.: 1986, The analysis of starlight. One hundred and fifty years of astronomical spectroscopy, Cambridge Univ. Press, Cambridge UK

Herzberg, G.: 1944, Atomic spectra and atomic structure, Dover Pub., New York

Holweger, H. and Müller, E. A.: 1974, Solar Phys. 39, 19 
Houtgast, J.: 1942, The variations in the profiles of strong Fraunhofer lines along a radius of the solar disc, $\mathrm{PhD}$ thesis, Utrecht University

Hubeny, I.: 1987a, Astron. Astrophys. 185, 332

Hubeny, I.: 1987b, Astron. Astrophys. 185, 336

Hubeny, I.: 1992, in U. Heber and C. S. Jeffery (Eds.), The Atmospheres of Early-Type Stars, Lecture Notes in Physics 401, Springer, Berlin, p. 377

Hubeny, I. and Lanz, T.: 1995, Astrophys. J. 439, 875

Hummer, D. G.: 1968, Mon. Not. R. Astron. Soc. 138, 73

Hummer, D. G. and Voels, S. A.: 1988, Astron. Astrophys. 192, 279

Ivanov, V. V.: 1973, Transfer of Radiation in Spectral Lines, English Language Edition of Radiative Transfer and the Spectra of Celestial Bodies, Special Publication 385, National Bureau of Standards, Washington

Jefferies, J. T.: 1968, Spectral Line Formation, Blaisdell, Waltham, Mass.

Kalkofen, W. (Ed.): 1984, Methods in Radiative Transfer, Cambridge Univ. Press, Cambridge UK Kalkofen, W. (Ed.): 1987, Numerical Radiative Transfer, Cambridge Univ. Press, Cambridge UK Kelch, W. L. and Linsky, J. L.: 1978, Solar Phys. 58, 37

Kelch, W. L., Linsky, J. L., and Worden, S. P.: 1979, Astrophys. J. 229, 700

Kirchhoff, G.: 1859, Monatsberichter Berliner Akad. 783

Kirchhoff, G. and Bunsen, R.: 1860, Poggendorff's Ann. 110, 160

Kostik, R. I., Shchukina, N. G., and Rutten, R. J.: 1996, Astron. Astrophys. 305, 325

Kourganoff, V.: 1952, Basic methods in transfer problems, Clarendon Press, Oxford (Dover 1963)

Kourganoff, V.: 1973, Introduction to the physics of stellar interiors, Reidel, Dordrecht

Kurucz, R. L.: 1979, Astrophys. J. Suppl. Ser. 40, 1

Landi Degl'Innocenti, E.: 1992, in F. Sánchez, M. Collados, and M. Vázquez (Eds.), Solar Observations: Techniques and Interpretation, First Canary Islands Winter School, Cambridge Univ. Press, Cambridge UK, p. 71

Lang, K. R.: 1974, Astrophysical Formulae, Springer, Berlin

Löfdahl, M. G. and Scharmer, G. B.: 1994, Astron. Astrophys. Suppl. Ser. 107, 243

Maltby, P., Avrett, E. H., Carlsson, M., Kjeldseth-Moe, O., Kurucz, R. L., and Loeser, R.: 1986, Astrophys. J. 306, 284

Marsden, J. E. and Tromba, A. J.: 1976, Vector Calculus, W. H. Freeman, New York

Mathisen, R.: 1984, Oslo Inst. Theor. Astrophys. Publ. Series 1, 1

Menzel, D. H. (Ed.): 1966, Selected Papers on the Transfer of Radiation, Dover Pub., New York

Menzel, D. H. and Cillié, G. G.: 1937, Astrophys. J. 85, 88

Mihalas, D.: 1970, Stellar Atmospheres, W. H. Freeman and Co., San Francisco (first edition)

Mihalas, D.: 1978, Stellar Atmospheres, W. H. Freeman and Co., San Francisco (second edition)

Mihalas, D. and Mihalas, B. W.: 1984, Foundations of Radiation Hydrodynamics, Oxford Univ. Press, New York

Milne, E. A.: 1928, Mon. Not. R. Astron. Soc. 88, 493

Milne, E. A.: 1930, Handbuch der Astrophysik 3 Part 1, Chapt. 2, 65

Minnaert, M.: 1954, The nature of light and colour in the open air, Dover Pub., New York

Minnaert, M. G. J., Mulders, G. F. W., and Houtgast, J.: 1940, Photometric Atlas of the Solar Spectrum 3332 A to 8771 A, Schnabel, Amsterdam

Moore, C. E.: 1949, Atomic Energy Levels, ${ }^{1} \mathrm{H}-22$ V, NSRDS-NBS Circular 467/vol. I, Natl. Bur. Standards, Washington

Moore, C. E.: 1952, Atomic Energy Levels, ${ }^{24} \mathrm{Cr}-{ }^{41} \mathrm{Nb}$, NSRDS-NBS Circular 467/vol. II, Natl. Bur. Standards, Washington

Moore, C. E.: 1958, Atomic Energy Levels, ${ }^{42}$ Mo- ${ }^{57}$ La \& ${ }^{72} \mathrm{Hf}-{ }^{89}$ Ac, NSRDS-NBS Circular 467/vol. III, Natl. Bur. Standards, Washington

Moore, C. E.: 1959, A Multiplet Table of Astrophysical Interest, Nat'l Bur. Standards Technical Note 36, Washington

Moore, C. E.: 1971, Atomic Energy Levels, NSRDS-NBS 35/vol III, Natl. Bur. Standards, Washington

Moore, C. E. and Merrill, P. W.: 1968, Partial Grotrian Diagrams of Astrophysical Interest, 
NSRDS-NBS 23, Natl. Bur. Standards, Washington

Moore, C. E., Minnaert, M. G. J., and Houtgast, J.: 1966, The Solar Spectrum 2935 A to 8770 A. Second Revision of Rowland's Preliminary Table of Solar Spectrum Wavelengths, NBS Monograph 61, National Bureau of Standards, Washington

Mulders, G. W. F.: 1935, Zeitschr. für Astrophys. 11, 132

Münch, G.: 1946, Astrophys. J. 104, 87

Nordlund, Å., Galsgaard, K., and Stein, R. F.: 1994, in R. J. Rutten and C. J. Schrijver (Eds.), Solar Surface Magnetism, NATO ASI Series C 433, Kluwer, Dordrecht, p. 471

Nordlund, Å. and Stein, R. F.: 1990, Comp. Phys. Comm. 59, 119

Novotny, E.: 1973, Introduction to stellar atmospheres and interiors, Oxford Univ. Press, New York

Olson, G. L., Auer, L. H., and Buchler, J. R.: 1986, J. Quant. Spectrosc. Radiat. Transfer 35, 431

Osterbrock, D. E.: 1974, Astrophysics of gaseous nebulae, W. H. Freeman and Co., San Francisco

Pannekoek, A.: 1951, De groei van ons wereldbeeld. Een geschiedenis van de sterrekunde, Wereldbibliotheek, Amsterdam

Pannekoek, A.: 1961, A History of Astronomy

Paxman, R. G., Seldin, J. H., Löfdahl, M. G., Scharmer, G. B., and Keller, C. U.: 1996, Astrophys. J. 466, 1087

Pierce, A. K. and Waddell, J. H.: 1961, Mem. Roy. Astr. Soc. 58, 89

Pomraning, G. C.: 1973, The equations of radiation hydrodynamics, Pergamon press, Oxford

Press, W. H., Flannery, B. P., Teukolsky, S. A., and Vetterling, W. T.: 1986, Numerical Recipes, Cambridge Univ. Press, Cambridge UK

Puls, J. and Herrero, A.: 1988, Astron. Astrophys. 204, 219

Rees, D. E.: 1987, in W. Kalkofen (Ed.), Numerical Radiative Transfer, Cambridge University Press, Cambridge, Great Britain, p. 213

Rosseland, S.: 1924, Mon. Not. R. Astron. Soc. 1924, 525

Rutten, R. J.: 1976, Solar Eclipse Observations and Ba II Line Formation, Ph.D. Thesis, Utrechtse Sterrekundige Overdrukken 365, Utrecht University

Rutten, R. J.: 1990a, in J.-O. Stenflo (Ed.), Solar Photosphere: Structure, Convection and Magnetic Fields, IAU Symp. 138 (Kiev), Kluwer, Dordrecht, p. 501

Rutten, R. J.: 1990b, in G. Wallerstein (Ed.), Cool Stars, Stellar Systems and the Sun, Proc. Sixth Cambridge Workshop, Astron. Soc. Pacific Conf. Series, Vol. 9, p. 91

Rutten, R. J. and Carlsson, M.: 1994, in D. M. Rabin, J. T. Jefferies, and C. Lindsey (Eds.), Infrared Solar Physics, Proc. Symp. 154 IAU (Tucson), Kluwer, Dordrecht, p. 309

Rutten, R. J. and Cram, L. E.: 1981, in S. D. Jordan (Ed.), The Sun as a Star, CNRS-NASA Monograph Series on Nonthermal Phenomena in Stellar Atmospheres, NASA SP-450, Washington, p. 473

Rutten, R. J. and Schrijver, C. J. (Eds.): 1994, Solar Surface Magnetism, NATO ASI Series C 433, Kluwer, Dordrecht

Rutten, R. J. and van der Zalm, E. B. J.: 1984, Astron. Astrophys. Suppl. 55, 143

Rybicki, G. B.: 1971, J. Quant. Spectrosc. Radiat. Transfer 11, 589

Rybicki, G. B.: 1972, in R. G. Athay, L. L. House, and G. Newkirk (Eds.), Line Formation in the Presence of Magnetic Fields, High Altitude Observatory, NCAR, Boulder, p. 145

Rybicki, G. B.: 1991, in L. Crivellari, I. Hubeny, and D. G. Hummer (Eds.), Stellar Atmospheres: Beyond Classical Models, NATO ASI Series C 341, Kluwer, Dordrecht, p. 1

Rybicki, G. B. and Hummer, D. G.: 1991, Astron. Astrophys. 245, 171

Rybicki, G. B. and Hummer, D. G.: 1992, Astron. Astrophys. 262, 209

Rybicki, G. B. and Hummer, D. G.: 1994, Astron. Astrophys. 290, 553

Rybicki, G. B. and Lightman, A. P.: 1979, Radiative Processes in Astrophysics, John Wiley \& Sons, Inc., New York

Scharmer, G., Brown, D., Petterson, L., and Rehn, J.: 1985, Appl. Opt. 24, 2558

Scharmer, G. B.: 1981, Astrophys. J. 249, 720

Scharmer, G. B. and Carlsson, M.: 1985, J. Comput. Phys. 59, 56

Schatzman, E. L. and Praderie, F.: 1993, The Stars, Springer, Berlin 
Schrijver, C. J. and Zwaan, C.: 2000, Solar and Stellar Magnetic Activity, Cambridge Univ. Press, Cambridge, UK

Schuster, A.: 1905, Astrophys. J. 21, 1

Schwarzschild, K.: 1906, Nachrichten Königlichen Gesellschaft Wissensch. Göttingen. Math.-Phys. Klasse 195, 41

Schwarzschild, K.: 1914, Sitzungsberichte Königlichen Preussichen Akad. Wissensch. 1183-1200

Seaton, M. J.: 1987, J. Phys. B: At. Mol. Phys. 20, 6363

Shu, F. H.: 1991, The Physics of Astrophysics I. Radiation, University Science Books, Mill Valley

Skartlien, R.: 2000, Astrophys. J. 536, 465

Sobelman, I. I., Vainshtein, L. A., and Yukov, E. A.: 1981, Excitation of Atoms and Broadening of Specral Lines, Springer, Berlin

Spruit, H. C.: 1977, Magnetic flux tubes and transport of heat in the convection zone of the Sun, $\mathrm{PhD}$ thesis, Utrecht University

Spruit, H. C., Nordlund, Å., and Title, A. M.: 1990, Ann. Rev. Astron. Astrophys. 28, 263

Stenflo, J. O.: 1994, Solar Magnetic Fields, Polarized Radiation Diagnostics, Kluwer, Dordrecht

Stix, M.: 1989, The Sun. An Introduction, Springer, Berlin

Thomas, R. N.: 1957, Astrophys. J. 125, 260

Tinbergen, J.: 1996, Astronomical Polarimetry, Cambridge Univ. Press, Cambridge UK

Uitenbroek, H.: 1989, Astron. Astrophys. 213, 360

Uitenbroek, H.: 1990, Partial redistribution modeling of the CaII K line, PhD thesis, Utrecht University

Uitenbroek, H. and Bruls, J. H. M. J.: 1992, Astron. Astrophys. 265, 268

Unsöld, A.: 1955, Physik der Sternatmosphären, Springer Verlag, Berlin (second edition)

van de Hulst, H. C.: 1957, Light Scattering by Small Particles, Wiley \& Sons, New York

van de Hulst, H. C.: 1980, Multiple Light Scattering, Academic Press, New York

Van Regemorter, H.: 1962, Astrophys. J. 136, 906

Vernazza, J. E., Avrett, E. H., and Loeser, R.: 1973, Astrophys. J. 184, 605

Vernazza, J. E., Avrett, E. H., and Loeser, R.: 1976, Astrophys. J. Suppl. Ser. 30, 1

Vernazza, J. E., Avrett, E. H., and Loeser, R.: 1981, Astrophys. J. Suppl. Ser. 45, 635

Vitense, E.: 1951, Zeitschr. für Astrophys. 28, 81

Warner, B.: 1967, Mon. Not. R. Astron. Soc. 136, 381

Werner, K.: 1986, Astron. Astrophys. 161, 177

Werner, K. and Husfeld, D.: 1985, Astron. Astrophys. 148, 417

White, O. R. (Ed.): 1977, The Solar Output and Its Variations, Colorado Assoc. Univ. Press, Boulder

Wijbenga, J. W. and Zwaan, C.: 1972, Solar Phys. 23, 265

Wildt, R.: 1939, Astrophys. J. 89, 295

Wright, K. O.: 1948, Pub. Dominion Astrophys. Obs. 8, 1

Zirin, H.: 1988, Astrophysics of the Sun, Cambridge Univ. Press, Cambridge UK

Zwaan, C.: 1993, Steratmosferen, Lecture Notes, Sterrekundig Instituut Utrecht (in Dutch) 


\section{Index}

absorption

true, $\overline{1} \overline{3}$

abundance, $1143,1,144$,

classical determination, $\overline{1} 20 \overline{3}$

definitions, '203'

iron, 1431,144

lithium, 143

LTE determination, 209

ALI, 114

approximate operators, $1 \overline{2} 25$

approximation

conduction, $\overline{9} \overline{0}$

core saturation, 126

diffusion, $\overline{8}^{\prime} \overline{9}_{1}^{\prime}$

Eddington-Barbier, $\overline{1} \overline{8}_{1}^{\prime}, \overline{2} \overline{0}_{n}^{\prime}, \overline{8} \overline{6}_{1}^{\prime}, 128_{1}^{\prime}, 129_{1}^{\prime}$

equivalent two-level, 180

first Eddington, 191,

grey, 156

impact, 55

Milne-Eddington, $1 \overline{1} \overline{5} \overline{1}$

quasi-static, $\overline{5} \overline{5}_{1}$

Rayleigh-Jeans, $3 \overline{1}_{1}^{\prime}$

Rosseland, $\overline{8} \overline{9}_{1}^{\prime}$

second Eddington, $\overline{8} \overline{7}$,

two-stream, ${ }^{\prime} \overline{9} \overline{4}$,

Wien, $\overline{3} \overline{1}$

ATMOS spectrometer, 1153

atmosphere

classic assumptions, 'i $14 \overline{1}_{i}^{\prime}$

atoms

multi-level, $\overline{4} \overline{1}$

Auer-Mihalas

modeling of hot stars, 1167',

solution, 134i:

autoionization, $\overline{4} \overline{3}$

Avogadro's number, '14 143

axial symmetry, 10

background continuum, '104'

backwarming, 160'

Balmer alpha

in hot stars, '167\%, '168'

Balmer jump, $\overline{2} 0 \overline{1}$

in the solar spectrum, 175

Boltzmann distribution, $\overline{2} \overline{9}_{1}^{1-}$ bound-bound

collisions, $\overline{50}$

cooling rate, $15 \overline{5}$

process pairs, $\overline{3} \overline{1}$

transitions, 18

bound-free

collisions, 50

extinction, $\overline{2} \overline{5}, \overline{3} \overline{5}$

H-min, 191'

hydrogen extinction, $19 \overline{1}$

in the solar atmosphere,

rates, $\overline{4} \overline{6}$

source function, ' $\overline{1} \overline{5} \bar{\prime}$

boundary conditions

for Eddington approximation, $\overline{9} \overline{3} \overline{1}$

for Feautrier solution, $11 \overline{1}$

problems, $1 \overline{117}$,

broadening,,$\overline{5} \overline{2}$

as a convolution, $\overline{6} \overline{1}_{1}^{\prime}$

collisional, $\overline{5} \overline{4}_{1}^{\prime}$

Doppler, $5 \overline{7_{1}}$

hyperfine structure, $\overline{6} \overline{3}$

isotope splitting, 163

linear Stark, $\overline{5} \overline{5}$,

quadratic Stark, $15 \overline{6}$

radiative, $15 \overline{3}$

resonance, 156

rotation, 60

thermal, $5 \overline{7}_{1}$

turbulent, $62_{1}^{1}$

Van der Waàls, $\overline{5} \overline{6}_{1}^{\prime \prime}$

Voigt function, 5

CaII H and K, '150', '189,', '221'

schematic formation, 20

chemical composition, '143145.'

Chi operator, $18 \overline{1}$

CO lines, 153

collisions

bound-bound, $\overline{50}$

bound-free, $\overline{5} \overline{1}_{1}^{1}$

coupling, 52

electrons, $22_{1}^{1}$

frequency, 50

line broadening, 
LTE, $\overline{5} \overline{2}_{1}^{\prime \prime}$

net rates, $\overline{5} \overline{1}$,

rates, $\overline{5} \overline{0}$

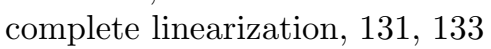

complete redistribution, $\overline{\overline{7}} \overline{0_{1}^{1}}$

line source function, 25

conduction

approximation,

continuum

background, $10 \overline{1}$

photon destruction, i104'

cooling

by solar hydrogen lines, $18 \overline{7}$

by solar lines, '18̄'

in lines, 155

core saturation, 126

corona, 'ín'

conditions, $\overline{4} \overline{4} \bar{i}$

dielectronic recombination, $\overline{4} \overline{3}$

creation

of photons, $\overline{3} \overline{8}_{1}^{\prime}$

cross-sections

collisional, $\overline{2} \overline{2}$

curve of growth

for abundance determination, $120 \overline{3}$

cyclotron radiation, 'i $1 \overline{7} \overline{1} \mid$

damping

broadening, 53

constant, 19

profile, 153

radiative, 119

deexcitation

collisional, $\overline{2} \overline{2}_{1}^{\prime}$

induced, $\overline{2} 2$

net rates, $\overline{1} 4 \overline{9}$,

spontaneous, ${ }_{1}^{1} \overline{1} \overline{1}$

density

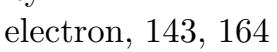

in the solar atmosphere, 183

gas, , i $\overline{4} \bar{\prime}$

photon, $1 \overline{1}_{1}^{\prime}$

radiation energy, $1 \overline{1}_{1}^{\prime}$

scale height, il

departure coefficients

definition, $\overline{3} 3, \overline{3} \overline{6}$

depth

effective optical, $\overline{4} \overline{0}$

optical, $177_{0}^{\prime}, 1-161$

radial optical, $\overline{1} \overline{7}, \overline{7} \overline{1}$

reference optical, i1 16

Rosseland, $1 \overline{1} \overline{2}$

thermalization, $99_{1}^{1}, 106{ }^{\prime}, 110$

destruction continuum, 'ī04'

of photons, $\overline{3} \overline{3} \overline{1}^{\prime}$

probability, $39_{1}^{1}, 169_{1}^{1}, 10 \bar{t}_{1}$

dex, $14 \overline{4}$

in Einstein coefficients, $6 \overline{6}$

diffusion

approximation, 8

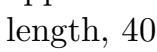

discrete ordinates,

discretization, i1 14

adaptive, 116

angles, i114'-

depths, $11 \overline{1}$

frequencies, 116

source function, $1233_{1}^{\prime}$

divergence

flux, $15 \overline{4}$

Doppler broadening, $\overline{5} \overline{7}$

Doppler profile, $, \overline{5} \overline{8}, \overline{1}$

Doppler shift, $\overline{5} \overline{7}$

thermal, $57_{1}$

Doppler width, 121

Dopplerwidth, 116

eclipse

flash spectrum, $1 \overline{1} \overline{4} \overline{8}$ '

Eddington

first approximation, $\overline{9} \overline{9} \overline{1}, \overline{9} \overline{5_{1}}$

flux, 12

limit, 135

second approximation, 8

variable factor, 13

Eddington approximation

boundary conditions, 9

isothermal atmosphere, 94

solutions, $\overline{9} \overline{3}{ }_{1}^{\prime}$

split, i ioo'

validity, $\overline{9} \overline{1}$

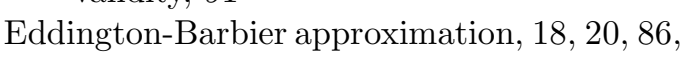

$$
\text { '1 } 128,1,129 \text { ' }
$$

for flux, $18_{1}^{\top}$

Einstein

coefficients, $\overline{1} \overline{1} \overline{1}_{1}^{\prime}$

relations, ${ }^{2}, \overline{1}$,

Einstein-Milne equations, ${ }^{4} \overline{6} \bar{w}_{1}^{\prime}$

electron

density, $\overline{1} \overline{1} \overline{4} \overline{1}$

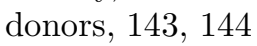

emission

induced scattering, $\overline{6} \overline{6} \overline{6}_{1}^{4}$

induced thermal, $65_{1}$

spontaneous thermal, $6 \overline{5}^{1}, 6 \overline{6}_{1}^{1}$

emissivity

for sharp-line atoms, $\overline{6} \bar{z}_{1}^{\prime}$ 
monochromatic, $\overline{1} \overline{2}$

spontaneous, '"' $\overline{2} \overline{4}$,

energy density

of radiation, ${ }^{1} \overline{1} \overline{1}_{1}^{1}$

equilibrium

hydrostatic, 146 '

radiative, 153

equivalent two-level approximation, 1 equivalent width

definition, 2004

Milne-Eddington model, '208'

Schuster-Schwarzschild model, 205

excitation

collisional, $\overline{2} \overline{2}_{i}^{\prime}$

radiative, $21_{4}^{1-}$

excitation temperature, $\overline{3} \overline{\overline{7}}$,

exponential integrals, $7 \overline{7}_{1}^{2}$

extinction

induced scattering, $\overline{6} \overline{5}$

Rosseland mean, 90

spontaneous scattering, ${ }^{\prime} 6 \overline{5}$,

thermal, $\overline{6} \overline{5}$

extinction coefficient

bound-free, $\overline{2} \overline{5_{1}}, \overline{3} \overline{4}, \overline{3} \overline{5_{1}^{\prime}}$

for sharp-line atoms, $6 \overline{7}$,

free-free, $\overline{2} \overline{6}, \overline{1}, \overline{3} \overline{1}$

grey, '158'

H-min, 150 153 , $19 \overline{1}$

H-min bound-free, $1 \overline{1} \overline{1}$ ',

hydrogen bound-free, $2 \sigma_{6}^{\prime \prime}, 19 \underline{1}$

in stars, 19001

in the solar atmosphere, $1799_{1}^{\prime}$

line, $\overline{2} \overline{3}$

line in LTE, $3 \overline{1}$

line, per particle, $\overline{2} 3$

mean, 158,159

per cm, 13

per gram, 13

per particle, 13

Rosseland, 159

Rosseland mean, i izt.

scattering, $\overline{6} \overline{\bar{t}_{\mathrm{t}}}$

thermal, $6 \overline{z_{1}}$

total line, $\overline{1} \overline{2} \overline{1}$

total line, per particle, $\overline{2} \overline{3}$

with departure coefficients, 34

Feautrier solution, $\overline{1} \overline{1} \overline{1} \overline{7}^{\prime} \overline{1} \overline{3} \overline{4}$,

and Lambda operator, $185,1,121$,

Rybicki modification, 12121,

first Eddington approximation, $\overline{9} \overline{1}, \overline{9}, \overline{9}$

flash spectrum, $148_{1}^{\prime}$

flux, 12,

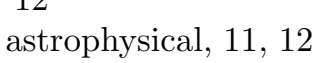

at Earth, '1' $\overline{1} \overline{1}$

constancy, 153

Eddington, 112

Eddington-Barbier approximation, $1 \overline{1}$

emergent, $8 \overline{1}_{1}^{1}, \overline{8} \overline{6}_{1}^{\prime}, \overline{9} \overline{5}_{1}^{\prime}$

from a star, 1 1,

incoming, '11',

Milne equation, $\overline{7} \overline{9}_{1}^{\prime}$

monochromatic, '10

operator, "82,", 84

outgoing, 11 ',

solar infrared, i17\%.

flux divergence, 154

Fokker-Planck equation, $\overline{6} \mathbf{6} \overline{9}$

formal solution, $\overline{7} \overline{\overline{1}}$,

formal solutions, 17

forward-backward solution, 121

free-fee

in the solar atmosphere,

free-free

extinction, $\overline{2} \overline{6}_{1}^{1}, \overline{3} \overline{6}_{1}^{\prime}$

frequency dependence, $\overline{2} \overline{2} \bar{\psi}_{1}$

H-min, 19-',

Freudenthalers, 134

G function, $1 \overline{1} \overline{1} 7 \overline{\mathrm{T}}$

gas

density, $14 \overline{3}$

ideal, 142

law, 142

mass density, 143

pressure, 142

total pressure, it43i

Gauss profile, 110

Gauss-Laguerre integration, '114';

Gauss-Legendre integration, ${ }_{11}^{1} \overline{4}$

Gaussian profile, 'i10

geometry

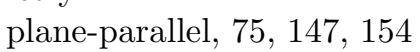

spherical, $\overline{7} \overline{5}, 14 \overline{1}, 1$

granulation, 218

gravity, surface, $1 \overline{1} \overline{4}$

grey approximation, 156

grey scattering, $15 \overline{1}$

H-min

bound-free extinction, $\overline{1} \overline{19} \overline{1}$,

free-free extinction, 191

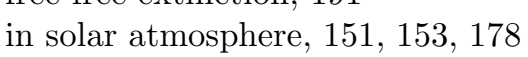

Heisenberg, $\overline{1} \overline{9}_{1}^{\prime}$

helium

Hmin edges,

in solar atmosphere, 150

HOLMUL model, '149,', '210,', 216 
Holtsmark profile, 55

homogeneous medium, $\overline{9}_{4}^{4}$

Hummer

collisional-radiative switching, $13 \overline{3} \overline{3}$,

hydrodynamics radiation, $\overline{3} \overline{3}$, , '1- $\overline{1} \overline{6}$

hydrogen

bound-free extinction,

edges, '201'

in hot stars, 167

net rates in the solar atmosphere, $1 \overline{1} \mathbf{1} \overline{4}$

hydrostatic equilibrium, '146i

hyperfine structure, $\overline{6} \overline{3}$

impact approximation, $\overline{5} \overline{5}_{1}^{\prime}$

induced emission

cancelation, $\overline{6} \overline{9}_{1}^{\prime}, \overline{7} \overline{0}_{1}^{\prime}$

correction for, $31_{1}^{1}$

Inglis-teller estimate, 64

integrals

exponential, $\bar{i} \overline{\bar{\tau}_{\mathrm{i}}}$

integration

Euler, 1114

Gauss-Laguerre, 'īi4',

Gauss-Legendre, $11 \overline{4}$

Newton-Cotes, $1 \overline{14}$

numerical, 1514,

Simpson, 114

trapezoidal,

intensity

averaged mean, $\overline{2} \overline{1}_{1}^{\prime}$

emergent, $181,81,861,95$

mean, ${ }_{1}^{1} 10_{1}^{1}, 121$

at the surface, $\overline{8} \overline{6}_{1}^{\prime}, \overline{9} \overline{5}_{1}^{\prime}$

moments, 12

monochromatic,

Schwarzschild equation for mean, $\overline{7} \overline{8}$,

solar disk-center, '173, $17 \overline{7}, 1,17 \overline{7}$,

specific, $\overline{\mathbf{p}_{1}}$

total,

ionization

dielectronic, $\overline{4} \overline{3}$

temperature, $37_{1}$

ionization temperature, $\overline{3} \overline{2} \bar{z}_{1}$

iron

solar abundance, $1 \overline{1} \overline{4} 3,1,14 \overline{4}$

solar line formation, $207_{1}$

irradiance, $\overline{1} \overline{1}$, ',

solar spectrum, $17 \overline{2}$

isothermal atmosphere, $\overline{9} \overline{4}, 1,1 \overline{4}, \overline{7}$,

isotope splitting, 63

iteration

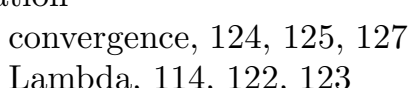

linear perturbation, $13 \overline{1}$

multi level, '153',

multi-dimensional, $13 \overline{1}$

multi-level, 'isìi,

Newton-Raphson, '131', '132", '164,'

starting solution, 135

with local operator, 1130

with Scharmer operator, 1픈

K integral, $\overline{1} \overline{2}$

Kompaneets equation, $\overline{6} \overline{9}_{1}^{\prime}$

Kramers law, $2 \overline{2}$

Lambda iteration, 'i $\overline{2} \overline{2}$

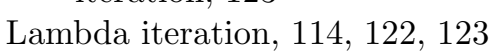
convergence, $124,125,127$

Lambda operator, 122,155 and Feautrier method, 185, '12 12

and formal solution, 85

approximate, 125

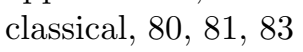

generalized, 182

Laplace operator, $8 \overline{1}_{1}^{1}$

length

limb

thermalization,

lunar, '140"'

solar, $1 \overline{1} \overline{4} 8_{1}^{\top}$

limb darkening, $1 \overline{1}, 1,150$,

continuum extinction, 15151,152

line

grey case, $15 \overline{1}$ ',

blanketing, 1501

blocking, 1600

broadening, 152

cooling rate, $1 \overline{1} \overline{1}$,

haze, 164 , 166 it

redistribution, $72_{1}^{1}$

line formation

schematic diagrams,

LINEAR, '134

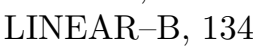

lithium

abundance, $14 \overline{1}$

local operator, 130

Lorentz profile, $1199_{1}^{\overline{1}}, 53_{1}^{1}, 110$

LTE

blanketing, '1-162',

definition, 28

interpretation, $3 \overline{1}$

line synthesis, '209"

model computation, 1164:

within stars, $3 \overline{2}^{\prime \prime}$

luminosity, stellar, 14 


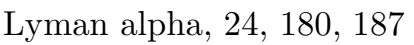

in hot stars, 1167,168

in the solar spectrum,

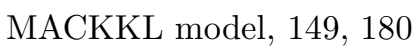

macroturbulence, $\overline{62}$

Maxwell distribution, i1 $141_{1}^{\prime}$

for speed, $2 \overline{2}_{1}^{\prime}$

per velocity component, $\overline{2} \overline{8}$

Menzel b definition, 36

metal-poor, i1 $4 \overline{3}$

metallicity, 142

definition, 203

MgII $h$ and $k, 189$

microturbulence, $62,1,142$,

Milne equation, $7 \underline{1}_{1}^{-1}$

Milne-Eddington approximation, 115

Milne-Eddington atmosphere, 207

molecular weight, mean, 'i143'

molecules, $1+146$

moments

of the intensity, $112 \overline{2}_{1}^{\prime}$

MULTI, '134i,', '213i'

$\mathrm{NaD}$ lines

from a flame, 'ī $1 \overline{4} \bar{c}$

from granulation, 'i218'

from HOLMUL, ' $2 \overline{1} \overline{6}$

from VALIII, '2014]

Grotrian diagram, 213

schematic formation, 20

solar, $21 \overline{1}$

neutrino, $144_{1}^{i}$

Newton-Cotes integration, $1 \overline{1} 1 \overline{1}$

Newton-Raphson, '131', 'i 322,164 starting solution, $1355^{-1}$

NLTE

definition, $3 \overline{2}$

in bound-free continua, 162

model computation, 1ㄴ.

NLTE-RE

modeling of hot stars, 1

ODF, '1-164;

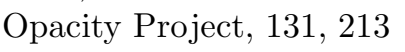

operator

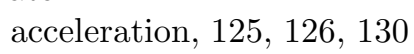

approximate, $\overline{1} \overline{2} \overline{2} \overline{5}$

approximate Lambda, 125

Chi, $8 \overline{2}_{1}^{\prime \prime}$

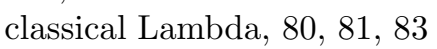

convergence, $1 \overline{1} 30$

core saturation, 126

Feautrier method, $\overline{85}$

flux, $\overline{8} \overline{2}_{-1}^{1}, \overline{8} \bar{u}_{1}^{\prime}$ formal solution, $\overline{8} \overline{5}$

generalized Lamb̄a $\bar{d}$, ${ }^{18} \overline{2}$

Lambda, $1 \overline{1} \overline{2} \overline{1}$

Laplace, $\overline{81_{1}^{\prime}}-$

local, 1130

$\mathrm{OAB}, 130$

perturbation, ,i114, , 125i

Phi, 82

Scharmer, i128, 'is4'

oscillator strength, $23_{-1}^{\top}$

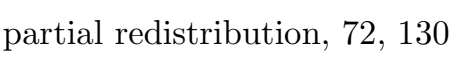

partition function, ${ }^{1} \underline{0}{ }_{1}^{\prime}$

path

effective, $\overline{4} 4 \overline{0}$

geometrical, $1 \overline{1}$

mean free, $155_{1}^{\prime}, 40$

optical, $14_{1}^{\prime}$

personal computer,

Phi operator, $82_{2}^{\prime}, 155$

photoionization, $46_{1}^{-}$

photoionization rate, 4

photon

as boson, $1 \overline{1} \overline{4}_{1}^{\prime}$

creation, 38

density,

destruction, $\overline{3} \overline{8}$

scattering, $38_{1}^{1}$

Planck function, 301

plasma radiation, '1 $17 \overline{1}$ ',

polar coordinates

solid angle, $1 \overline{1} \overline{0}^{\prime}$

polarization

circular, 'i $1 \overline{3} \overline{1}$

elliptical, 138

linear, $1399_{1}$

population

equations, $\overline{3} \overline{2}_{1}^{\prime \prime}$

preconditioning, 135

for Scharmer operator, 135

pressure

broadening, 154

electron, 145,147

equilibrium, $146^{-}$

gas, 'i-142,', 'i-155, 147,

radiation, 12,146

scale height, $1, \overline{4} \vec{\gamma}_{1}^{-}$

stratification,

total gas, $143_{1}^{1-1}$

processes

continuum, 'i $1 \overline{7} \overline{1}$ '

profile

two-electron, 43

Doppler, $5 \underline{5}^{\prime}$ 
emission, i1 $\overline{9}_{1}^{\prime}$

extinction, $2 \overline{1}_{1}^{1}$

Gaussian, 58

Holtsmark, 55

induced emission, $\overline{2} \overline{2}_{-1}^{\prime}$

Lorentz, 53

Voigt,, $599_{1}^{1}$

quasi-static approximation, 55

radiation

energy density, i1

photon density, $111_{1}^{\prime}$

pressure, $\overline{1} 1 \overline{2}_{1}^{\prime}$

radiative equilibrium, i 153

grey case, $1 \overline{1} 5 \overline{1}$,

solar atmosphere, 'i $149 \mathbf{9}_{1}^{\prime}$

stellar models, 1165

radius, stellar, 142

rate

rates

equations, $132 \underline{2}, 13$

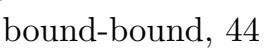

bound-bound collision, $5 \overline{0}_{1}^{\prime}$

bound-free, $\overline{4} \overline{5}_{1}^{\prime}, \overline{4} \overline{6}_{1}^{\prime}$

bound-free collision, 51

collision, 50

hydrogen in solar atmosphere, is

in the solar atmosphere, $188_{1}^{\prime}$

induced recombination, $\overline{4} \overline{7}_{1}^{-}$

net bound-bound radiative, $4 \overline{9}_{1}^{\prime}$

net collision, $5 \overline{1}_{1}^{\prime}$

net deexcitation, $\overline{4} \overline{9}$

net radiative recombination, $\overline{4} \overline{8}_{1}^{\prime}$

net recombination, $\overline{4} \overline{\mathbf{4}}$

per particle, $\overline{3} \overline{2}, 1 \overline{3} \overline{3}$

photoionization, $45_{1}^{1}, 466_{1}^{1}$

radiative recombination, $\overline{4} \overline{1}, \overline{4} \overline{4}$

spontaneous recombination, 46

total radiative recombination, 4

unified radiative, $\overline{4} \overline{8}$

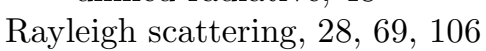

in the solar atmosphere, $171_{1}^{\prime}$

Rayleigh-Jeans approximation, ${ }^{1} 1_{1}^{\prime}$

recombination

dielectronic, 43

induced, $\overline{4} \overline{7}_{1}$

net rates, $4 \overline{4}$

radiative, $4 \overline{5}$

spontaneous, $\overline{1}^{4} \overline{6}_{1}^{\prime}$

redistribution

bound-free, ' 110 '

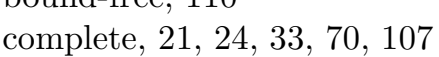

partial, $722_{1}^{1}, 130$

resonance lines, 104,

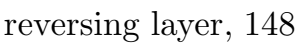

Rosseland

approximation, $\overline{8} \overline{8}$

mean extinction, $90,1,159$

weighting function, $17 \overline{7}$

Rosseland depth, 1른

rotational broadening, i $6 \bar{a}$ as a convolution, $61_{1}^{\top}$

Rybicki

core saturation, $12-6$ i

equation, 11231

reordered Feautrier solution, 'i21.'

Saha distribution

for full stages, $\overline{30}$

for ground levels, $30_{1}^{\prime}$

partition function, 30

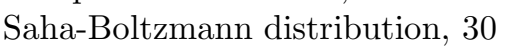

scale height, i $1 \overline{4} \bar{z}$

scattering

coherent, $\overline{2} \overline{2} \overline{7}, \overline{19}$

continuum, 106

for two-level atoms,

grey, 'i 157,

in Eddington approximation, $\overline{9} \overline{5}$

line blanketing, $\overline{1} \overline{1} \overline{2}{ }_{1}^{\prime}$

probability, $39_{1}^{\prime}$

Rayleigh, 20

resonance, 38,104,

Thomson, $227,6996,106,171,202$

two-level, 38

Scharmer

core saturation operator, 1i2 281

operator, i128, 'i34

phase diversity, 12

telescope, 'i129'

Scharmer-Carlsson solution, 1134

Schuster-Schwarzschild atmosphere, i'i 14

Schwarzschild, 'i 158 '

Schwarzschild-Milne equations, $\overline{7} \overline{8} \overline{8}_{1}^{\prime}$

SE, $\overline{3} \overline{2}_{1}^{\prime}$

SE (Statistical Equilibrium), $3 \overline{2}_{1}^{\prime \prime}$

second Eddington approximation, $\overline{1} \overline{8} \overline{7}$,

sharp-line atoms, ${ }^{\prime} 6 \overline{1}_{1}^{\prime}$

up-down sequences, $\overline{6} \overline{5}_{1}^{\prime}$

solar limb, 'i $1 \overline{4} \overline{8}$ '

solar models, $\overline{1} \overline{4} \overline{\mathbf{g}_{1}}$

empirical, $1488^{\prime}$

HOLMUL, $149_{n}^{\prime}, 2101,21 \bar{c}_{1}^{\prime}$

MACKKL, $\overline{1} \overline{4} \overline{9}_{\mathrm{v}}^{\prime} \overline{1} \overline{\mathrm{B}} \overline{\mathrm{O}}$

T5780, '1 149

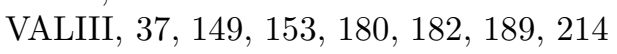

solid angle

in polar coordinates, $10 \underline{1}^{\prime}$ 
solutions

for Eddington approximation, $\overline{9} \overline{3}$

source function, $\overline{1} \overline{3}$,

anisotropy, $\frac{171_{1}^{1}}{1}$

bound-free, $355_{1}^{\prime}$

coherent scattering, '4i ',

for redistributed scattering, $\overline{1} \overline{1} \overline{\overline{7}}$,

for sharp-line atoms, $68^{\prime}, 69$

for two-level atoms with redistribution, 70

grey, 156

line, $\overline{24}$

pure scattering, $\overline{4} 0$

total, $\overline{1} \overline{3_{1}^{\prime}}$

true absorption, $\overline{4} \overline{4}$

two-level atom, 40

with departure coefficients, $\overline{3} \overline{3}$

spectrograph

double pass, $\overline{p_{1}^{\prime}}$

ghosts, $\overline{1}$

slitless, $14 \overline{1}$

spectrum

split

$$
\text { features, } 17 \overline{6}
$$

in Eddington approximation, 1000

in resonance lines, $10 \overline{1}$

producing NLTE, 102 ;

Stark effect

linear, $\overline{5} \overline{5}$

quadratic, $\overline{1} \overline{\underline{6}} \overline{6}_{1}^{\prime}$

statistical equilibrium, $32 \overline{2}, 70$

Stefan-Boltzmann constant, $3 \overline{1}_{1}^{1}$

Stefan-Boltzmann law, $3 \overline{1}_{-1}$

stellar models, 165,167 ,

stellar wind, '223'

stimulated emission, $\overline{2} \overline{2}$

Stokes parameters, 137 ,

for a single wave, $\overline{1} \overline{3} \overline{1}$

for complete polarization, '138'

for mixed waves, i138'

original definition, 139

Strömgren equation, 1154

stray light, 150 '

in spectrograph,

symmetry

axial, 1010

synchrotron radiation, $\overline{1} \overline{1} \overline{1} \overline{1}$

T5780 model, 'i $\overline{1} \overline{4} \overline{9}$

temperature

effective, 'i $\overline{4} \overline{4}$

excitation, $3 \overline{3}$

ionization, 3

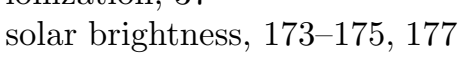

thermalization

depth, $9 \bar{g}_{1}^{\prime}, 106$, , i1

length, 40

thick

effectively, $\overline{9} \overline{9}_{1}^{\prime}$

optically, 100

thickness

effective optical, $\overline{4}^{\prime}$

optical, $\overline{1} \overline{5}$

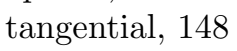

thin

effectively, '100'.'

optically, 100

Thomson scattering, $\overline{2} \overline{2}$, $\overline{6} \overline{9}_{1}^{\prime}, 10 \overline{6}_{1}^{\prime}$

at high temperature, 69.

in stars, 202

in the solar atmosphere, $1 \overline{1} \overline{1}$

time dependence, $\overline{3} \overline{3}_{1}^{1}$

TLUSTY, '1 $135 \overline{1}$

transfer equation, see transport equation

transition rates, ${ }^{4} \overline{4}^{\prime}$

transitions

bound-bound, $\overline{1} \overline{1}$

transport equation, $144_{1}^{1}, 1 \overline{1}$

along the beam, 11

coupled, $\overline{1} \overline{2} \bar{\prime}$

Eddington-Barbier approximation, $1 \overline{1}$

Feautrier form, '11

for flux, $\overline{7} \overline{6}_{1}^{1}$

for homogeneous medium, $1 \overline{1}$

for $\mathrm{K}$ integral, $\overline{7} \bar{\sigma}_{1}^{\prime}$

for sharp-line atoms, $\overline{6} \overline{\mathbf{b}}$

for two-level atom, $41_{1}^{1}$

for two-level atoms, 41

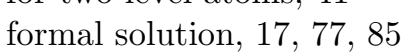

general, $\overline{7} \overline{5}$

in Eddington approximation,

in standard form, $\overline{1} \overline{\bar{T}_{1}}$

schematic solutions, '20"

second order, "92", 1119

solutions for homogeneous medium, $\overline{1} \overline{6}_{1}^{\prime}$

spherical geometry, $\overline{1} \overline{5} \overline{5}$

trapezium rule, 'i114'

two-electron transitions, $\overline{4} \overline{3_{1}}$

two-stream approximation, 94.

Utrecht Atlas,

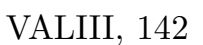

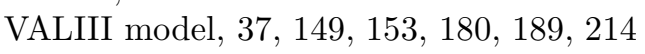

as a star, $18 \overline{3}$

continuum formation, '154' '- 186 '

hydrogen radiation balances, 187,

plot of temperature distribution, $181_{1}^{1}$ specified in table, $15 \overline{2}$ 
Van der Waals

broadening, $5 \overline{5}$

enhancement factor, $\overline{5} 5 \overline{7}_{1}$

variable Eddington factor, 134

Voigt function, $\overline{1} \overline{9}_{v}^{\prime} \overline{2} \overline{1}_{n}^{1} \overline{5} \overline{9}_{1}^{\prime}$

weight, mean molecular, 1ín

Wien approximation, $31_{1}^{1}$

\section{Zeeman}

longitudinal effect, $1 \overline{1} 3 \overline{9}_{1}^{\prime}$

transverse effect, $1399_{1}^{i}$

triplet, 'i 139 '

Zeeman effect, 63

Zwaan b definition, $\overline{3} \overline{3}, \overline{3} \overline{6}_{1}^{\prime}$ 


\section{Radiative Transfer Rap}

specific intensity

$I_{\nu}(\vec{r}, \vec{l}, t)$ erg $\mathrm{cm}^{-2} \mathrm{~s}^{-1} \mathrm{~Hz}^{-1}$ ster $^{-1}$

emissivity

$j_{\nu} \quad \operatorname{erg~} \mathrm{cm}^{-3} \mathrm{~s}^{-1} \mathrm{~Hz}^{-1} \mathrm{ster}^{-1}$

extinction coefficient

$\begin{array}{lllll}\alpha_{\nu} \mathrm{cm}^{-1} & \sigma_{\nu} & \mathrm{cm}^{2} \operatorname{part}^{-1} \quad \kappa_{\nu} & \mathrm{cm}^{2} & \mathrm{~g}^{-1}\end{array}$

source function

$S_{\nu}=\Sigma j_{\nu} / \Sigma \alpha_{\nu}$

radial optical depth

$\tau_{\nu}\left(z_{0}\right)=\int_{z_{0}}^{\infty} \alpha_{\nu} \mathrm{d} z$

plane-parallel transport

incoming rays

$\mu \mathrm{d} I_{\nu} / \mathrm{d} \tau_{\nu}=I_{\nu}-S_{\nu}$

outgoing rays

$I_{\nu}^{-}\left(\tau_{\nu}, \mu\right)=-\int_{0}^{\tau_{\nu}} S_{\nu}\left(t_{\nu}\right) \mathrm{e}^{-\left(t_{\nu}-\tau_{\nu}\right) / \mu} \mathrm{d} t_{\nu} / \mu$

emergent intensity

$I_{\nu}^{+}\left(\tau_{\nu}, \mu\right)=+\int_{\tau_{\nu}}^{\infty} S_{\nu}\left(t_{\nu}\right) \mathrm{e}^{-\left(t_{\nu}-\tau_{\nu}\right) / \mu} \mathrm{d} t_{\nu} / \mu$

Eddington-Barbier

$I_{\nu}^{+}(0, \mu)=\int_{0}^{\infty} S_{\nu}\left(t_{\nu}\right) \mathrm{e}^{-t_{\nu} / \mu} \mathrm{d} t_{\nu} / \mu$

$I_{\nu}^{+}(0, \mu) \approx S_{\nu}\left(\tau_{\nu}=\mu\right)$

$\mathcal{F}_{\nu}^{+}(0) \approx \pi S_{\nu}\left(\tau_{\nu}=2 / 3\right)$

mean intensity

$J_{\nu}(z)=\frac{1}{2} \int_{-1}^{+1} I_{\nu} \mathrm{d} \mu$

flux

$H_{\nu}(z)=\frac{1}{2} \int_{-1}^{+1} \mu I_{\nu} \mathrm{d} \mu=\mathcal{F}_{\nu} / 4 \pi=F_{\nu} / 4$

mean mean intensity

$\bar{J}_{\nu_{0}}^{\varphi}=\frac{1}{2} \int_{0}^{\infty} \int_{-1}^{+1} I_{\nu} \varphi\left(\nu-\nu_{0}\right) \mathrm{d} \mu \mathrm{d} \nu$

Schwarzschild equation

$J_{\nu}\left(\tau_{\nu}\right)=\frac{1}{2} \int_{0}^{\infty} S_{\nu}\left(t_{\nu}\right) E_{1}\left(\left|t_{\nu}-\tau_{\nu}\right|\right) \mathrm{d} t_{\nu}$

lambda operator

$\boldsymbol{\Lambda}_{\nu}\left[S_{\nu}\left(t_{\nu}\right)\right]=J_{\nu}\left(\tau_{\nu}\right)$

photon destruction

$\varepsilon_{\nu}=\alpha_{\nu}^{\mathrm{a}} /\left(\alpha_{\nu}^{\mathrm{a}}+\alpha_{\nu}^{\mathrm{s}}\right)$

coherent scattering

$S_{\nu}^{l}=\left(1-\varepsilon_{\nu}\right) J_{\nu}+\varepsilon_{\nu} B_{\nu}$

two-level atoms

$\varepsilon_{\nu_{0}}=C_{21} /\left(C_{21}+A_{21}+B_{21} B_{\nu}\right)$

complete redistribution

$S_{\nu_{0}}^{l}=\left(1-\varepsilon_{\nu_{0}}\right) \bar{J}_{\nu_{0}}^{\varphi}+\varepsilon_{\nu_{0}} B_{\nu_{0}}$

isothermal atmosphere

$S_{\nu}(0)=\sqrt{\varepsilon_{\nu}} B_{\nu}$ 